\title{
Zweikernige Zinkkomplexe als Modellverbindungen für Hydrolasen
}

\author{
Dissertation \\ zur Erlangung des Doktorgrades \\ der Mathematisch-Naturwissenschaftlichen Fakultäten \\ der Georg-August-Universität zu Göttingen \\ vorgelegt von \\ Diplom-Chemiker \\ Bernhard Bauer-Siebenlist \\ aus München
}

Göttingen, den 16.03.2004 


\section{D7}

Referent: Prof. Dr. Franc Meyer

Korreferent: Prof. Dr. Herbert W. Roesky

Tag der mündlichen Prüfung: 27.04.2004 
„Felix, qui potuit rerum cognoscere causas.”

(Glücklich ist der, der den Dingen auf den Grund gehen konnte.) Vergil (70-19 v. Chr.), Georgica 2, 490 

Ich erkläre hiermit an Eides statt, dass ich diese Arbeit selbständig und ohne unerlaubte Hilfsmittel angefertigt habe. 
Die vorliegende Arbeit entstand im Zeitraum von März 2001 bis März 2004 unter Anleitung von Herrn Professor Dr. Franc Meyer am Anorganisch-Chemischen Institut der Ruprecht-Karls-Universität Heidelberg und am Anorganisch-Chemischen Institut der Georg-August-Universität Göttingen. 


\section{Inhalt}

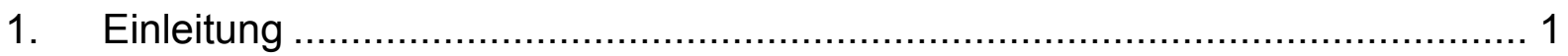

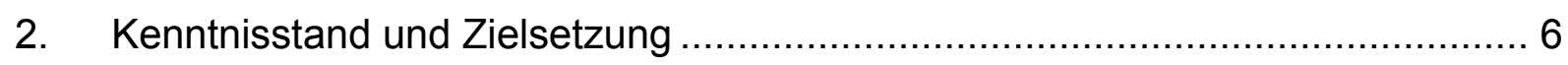

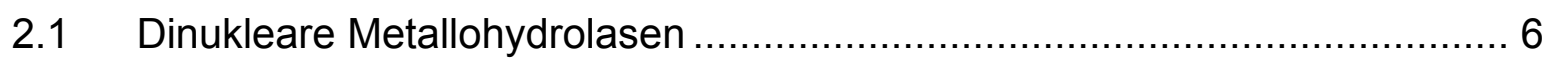

2.2 Dinukleare Phosphatasen und Nukleasen ......................................... 7

2.2.1 Alkalische Phosphatase (AP) …................................................ 7

2.2.2 P1 Nuklease ...................................................................... 10

2.2.3 Violette saure Phosphatasen (PAP) ........................................... 11

$2.3 \beta$-Lactam-Antibiotika und $\beta$-Lactamasen ..................................... 12

2.4 Ligandsysteme für dinukleare Komplexe ..................................... 16

2.5 Dinukleare Pyrazolatometallkomplexe ............................................ 17

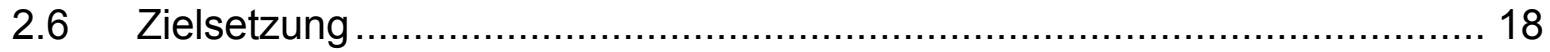

3. Ligandsynthese und Ligandeigenschaften .......................................... 19

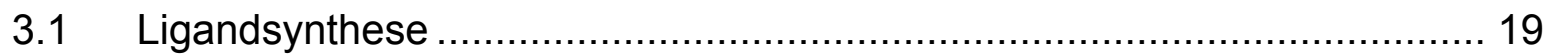

3.1.1 Synthese von Bis(aminomethyl)pyrazol-Liganden......................... 19

3.1.2 Synthese von bisfacialen Pyrazolatliganden ................................ 25

3.2 Potentiometrische Charakterisierung der Liganden............................ 27

4. Darstellung und Eigenschaften von Pyrazolatodizinkkomplexen ................... 36

4.1 Dizinkpyrazolatokomplexe - Synthesen und Festkörperstrukturen ............ 36

4.1.1 Komplexe der bis-tetradentaten Liganden $\mathrm{L}^{1}, \mathrm{~L}^{3}, \mathrm{~L}^{5}, \mathrm{~L}^{6}$ und $\mathrm{L}^{8} \ldots \ldots \ldots . . .36$

4.1.2 Komplexe der bis-tridentaten Liganden $L^{2}, L^{4}, L^{7}$ und $L^{9} \ldots \ldots \ldots \ldots \ldots . . . . . . .46$

4.1.3 Komplexe bisfacialer Liganden........................................... 57

4.2 Potentiometrische Charakterisierung von Zink(II)-Pyrazolatokomplexen ... 59

5. Aktivität der Pyrazolatodizinkkomplexe in der Phosphatesterhydrolyse ........... 72

$5.1 \quad$ Modellsubstrate für die Spaltung von Phosphatestern ........................... 72

5.2 Zweikernige Zinkkomplexe als Modelle für Hydrolasen........................... 73

$5.3 \quad$ Kinetische Untersuchung der Hydrolyse von BNPP ........................... 77

5.3.1 pH-Abhängigkeit der BNPP-Hydrolyse ...................................... 78

5.3.2 Abhängigkeit der BNPP-Hydrolyse von der Komplexkonzentration .... 82

5.3.3 Abhängigkeit der BNPP-Hydrolyse von der Substratkonzentration ..... 83

5.4 Untersuchungen zur Anbindung von Phosphaten an Dizinkzentren ......... 87

$5.4 .1 \quad{ }^{31}$ P-NMR-Spektroskopie ........................................................ 87

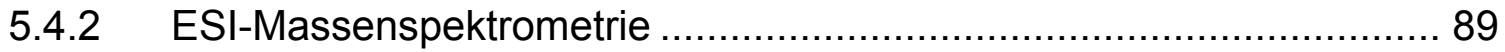


5.5 Vergleichende Diskussion der Phosphatesterhydrolysen.......................102

6. $\beta$-Lactamase-Aktivität

6.1 Modellkomplexe für $\beta$-Lactam-Metallkomplexe und Dizink-

Metallolactamasen

6.2 Verwendete $\beta$-Lactamsubstrate.

6.3 Kinetische Untersuchungen der $\beta$-Lactamhydrolyse durch IR-Spektroskopie

6.4 Untersuchungen zur Bindungssituation von $\beta$-Lactamen an Dizinkkomplexe 124

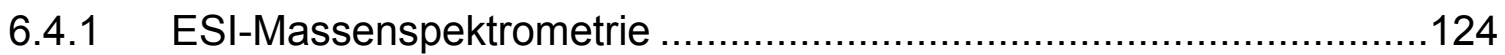

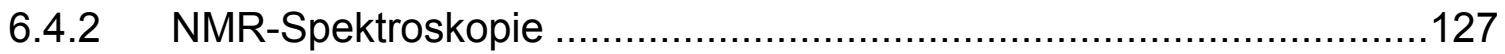

6.4.3 Darstellung von $\beta$-Lactamaddukten ...................................... 130

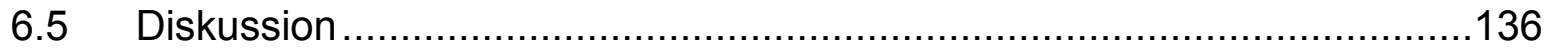

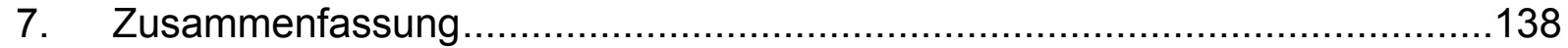

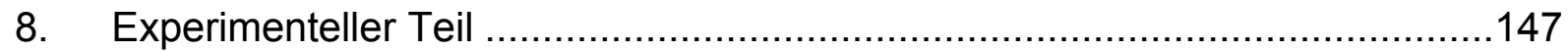

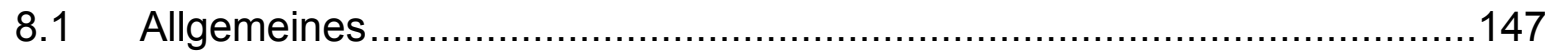

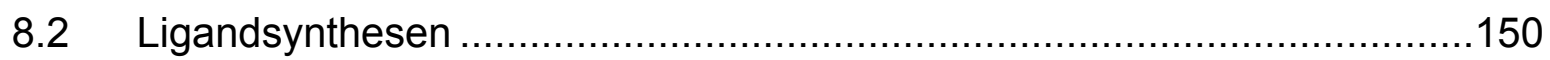

8.2.1 Synthese von N-(2-Picolyl)methylamin XIV ..............................150

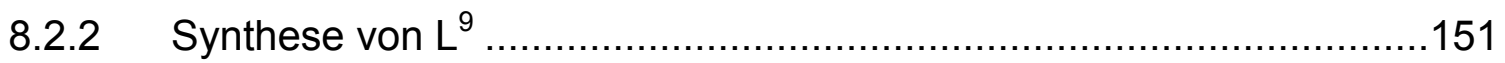

8.2.3 Synthese von 3,5-Bis(bis(1-methyl)imidazol-2-yl-hydroxymethyl)-1tetrahydropyran-2-yl-1H-pyrazol XXIla

8.2.4 Synthese von 3,5-Bis(bis(1-methyl)imidazol-2-yl-methoxymethyl)-1tetrahydropyran-2-yl-1H-pyrazol XXIIla

8.2.5 Synthese von 3,5-Bis(bis(1-methyl)imidazol-2-yl-methoxymethyl)-1Hpyrazol XXIVa.

8.2.6 Synthese von 3,5-Bis(dipyridin-2-yl-hydroxymethyl)-1-tetrahydropyran-

2-yl-1H-pyrazol XXIIb

8.2.7 Synthese von 3,5-Bis(dipyridin-2-yl-methoxymethyl)-1-tetrahydropyran2-yl-1H-pyrazol XXIIIb 156

8.2.8 Synthese von 3,5-Bis(dipyridin-2-yl-methoxymethyl)-1-H-pyrazol XXIVb

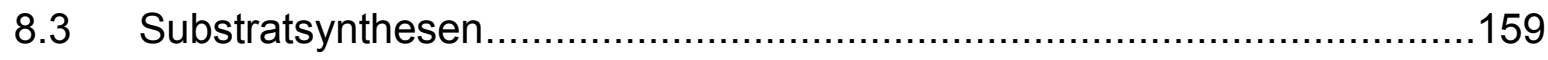

8.3.1 Synthese von tert-Butyl-2-(2-Oxoazetidin-1-yl)acetat .....................159

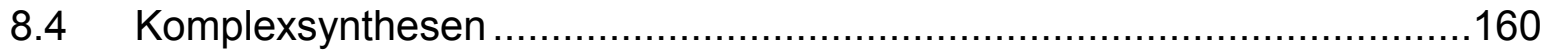




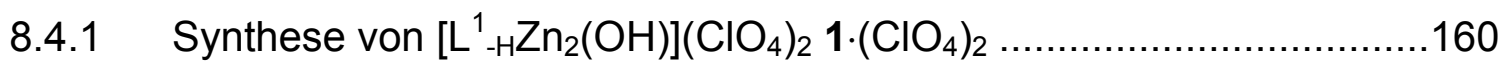

8.4.2 Synthese von $\left[\mathrm{L}_{-}^{3} \mathrm{Zn}_{2}(\mathrm{MeOH})(\mathrm{OH})\right]\left(\mathrm{ClO}_{4}\right)_{2} \mathbf{2 b} \cdot\left(\mathrm{ClO}_{4}\right)_{2} \ldots \ldots \ldots \ldots \ldots \ldots \ldots . . . . . . . .161$

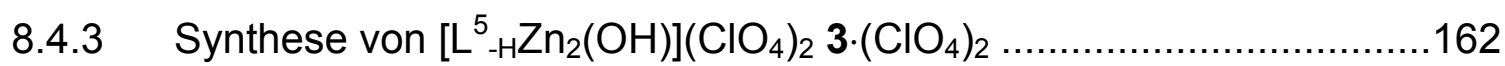

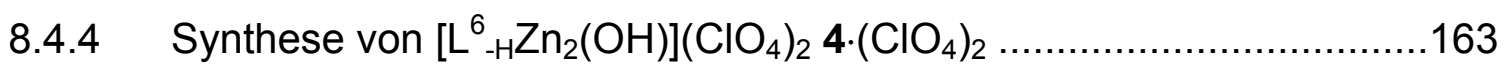

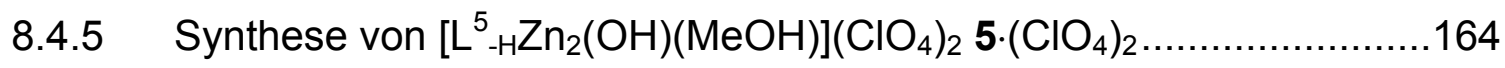

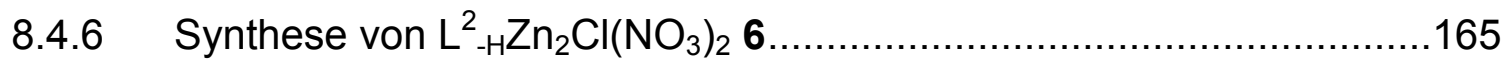

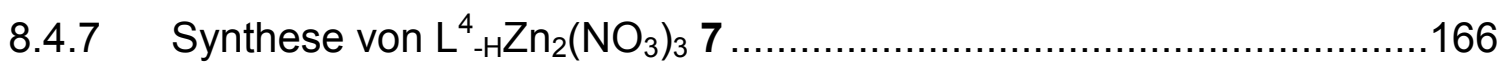

8.4.8 Synthese von $\left[\left(\mathrm{L}^{4}{ }_{-\mathrm{H}}\right)_{2} \mathrm{Zn}_{4}(\mathrm{OH})_{2}\left(\mathrm{CO}_{3}\right)\right]\left(\mathrm{ClO}_{4}\right)_{2} \mathbf{8} \cdot\left(\mathrm{ClO}_{4}\right)_{2} \ldots \ldots \ldots \ldots \ldots \ldots . . . . . . . . .167$

8.4.9 Synthese von $\left[\left(\mathrm{L}^{4}{ }_{-\mathrm{H}}\right)_{2} \mathrm{Zn}_{4}(\mathrm{OH})_{2}\left(\mathrm{CO}_{3}\right)\right]\left(\mathrm{BPh}_{4}\right)_{2} 8 \cdot\left(\mathrm{BPh}_{4}\right)_{2} \ldots \ldots \ldots \ldots \ldots \ldots . . . . . . . . .169$

8.4.10 Synthese von $\mathrm{L}^{9}{ }_{-\mathrm{H}} \mathrm{Zn}_{2}\left(\mathrm{NO}_{3}\right)_{3} \mathbf{9}_{\ldots \ldots \ldots \ldots \ldots \ldots \ldots \ldots \ldots \ldots \ldots \ldots \ldots \ldots \ldots \ldots \ldots \ldots \ldots \ldots \ldots \ldots \ldots \ldots \ldots \ldots \ldots \ldots \ldots \ldots \ldots \ldots}$

8.5 Komplexsynthesen - Phosphatesterkomplexe.................................172

8.5.1 Synthese von $\left[\mathrm{L}_{-}^{3} \mathrm{Zn}_{2}\left\{\mathrm{O}_{2} \mathrm{P}(\mathrm{OMe})_{2}\right\}\right]\left(\mathrm{ClO}_{4}\right)_{2} 10 \mathrm{a} \cdot\left(\mathrm{ClO}_{4}\right)_{2} \ldots \ldots \ldots \ldots \ldots \ldots . . . . . . . . .172$

8.5.2 Synthese von $\left[\mathrm{L}^{6}{ }_{-\mathrm{H}} \mathrm{Zn}_{2}\left\{\mathrm{O}_{2} \mathrm{P}(\mathrm{OMe})_{2}\right\}\right]\left(\mathrm{ClO}_{4}\right)_{2} \mathbf{1 1 a} \cdot\left(\mathrm{ClO}_{4}\right)_{2} \ldots \ldots \ldots \ldots \ldots \ldots . . . \ldots . \ldots . .173$

8.5.3 Synthese von $\left[\mathrm{L}^{8}{ }_{-\mathrm{H}} \mathrm{Zn}_{2}\left\{\mathrm{O}_{2} \mathrm{P}(\mathrm{OMe})_{2}\right\}\right]\left(\mathrm{ClO}_{4}\right)_{2} \mathbf{1 2 a} \cdot\left(\mathrm{ClO}_{4}\right)_{2} \ldots \ldots \ldots \ldots \ldots \ldots . . . \ldots \ldots$

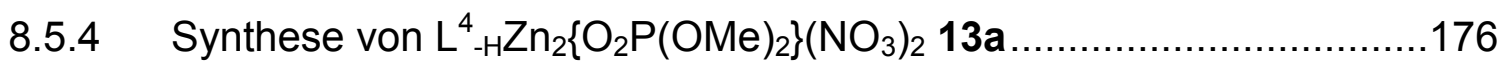

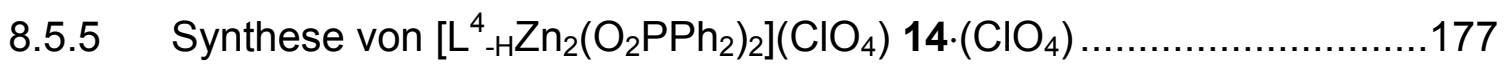

8.5.6 Synthese von $\left[\left(\mathrm{L}^{4}{ }_{-H}\right)_{2} \mathrm{Zn}_{4}(\mathrm{OH})_{2}(\mathrm{MNPP})\right]\left(\mathrm{ClO}_{4}\right)_{2}$ 15. $\left(\mathrm{ClO}_{4}\right)_{2} \ldots \ldots \ldots \ldots \ldots . . .178$

8.6 Komplexsynthesen $-\beta$-Lactamaddukte .......................................180

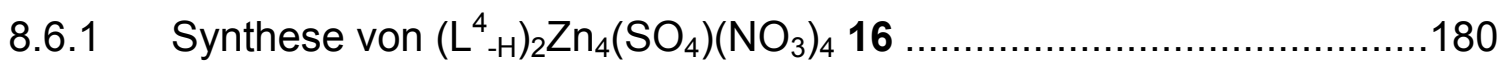

8.6.2 Synthese von $\left[\mathrm{L}^{4}{ }_{-H} \mathrm{Zn}_{2}\left(\mathrm{OH}_{2}\right)\left(\mathrm{C}_{5} \mathrm{H}_{6} \mathrm{NO}_{3}\right)\right]_{2}\left(\mathrm{ClO}_{4}\right)_{4}$ 17. $\left(\mathrm{ClO}_{4}\right)_{4} \ldots \ldots \ldots \ldots . . .181$

8.6.3 Synthese von Penicillin G-Addukten............................................ 182

8.7 ${ }^{1} \mathrm{H}-\mathrm{NMR}-\mathrm{Parameter}$ für die Pyridylliganden und -komplexe.......................182

8.8 Potentiometrische Messungen ........................................................

8.9 Untersuchungen zur BNPP-Hydrolyse ......................................... 187

8.9.1 Kinetische Messungen der BNPP-Hydrolyse mittels UV/Vis-

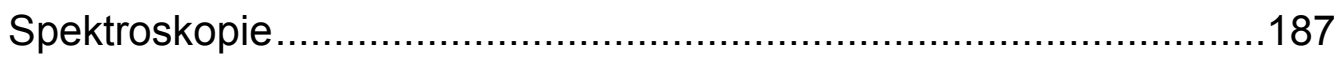

8.9.2 ${ }^{31}$ P-NMR-spektroskopische Untersuchung der BNPP-Hydrolyse .......189

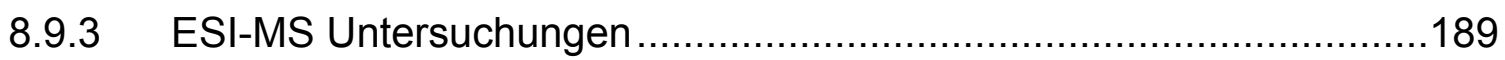

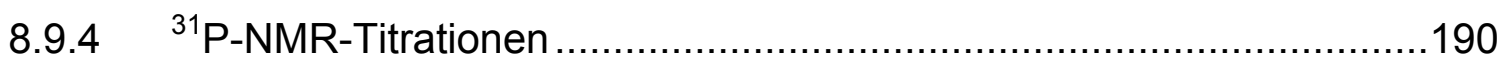

8.10 Untersuchungen zur $\beta$-Lactam Hydrolyse .......................................190

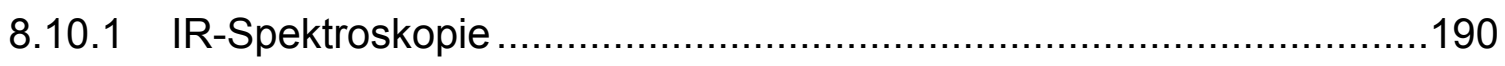

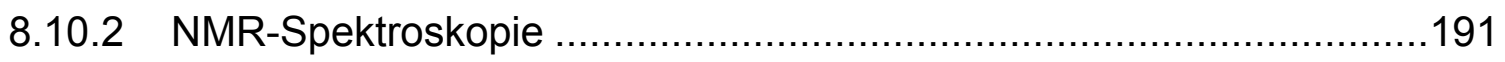

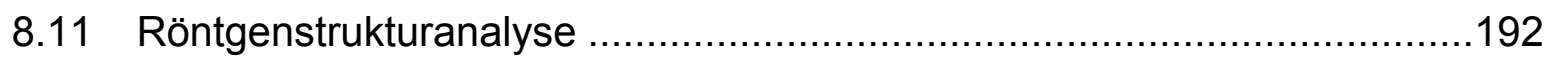




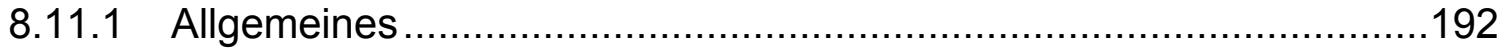

8.11.2 Kristalldaten und Angaben zur Struktur im Festkörper .....................193

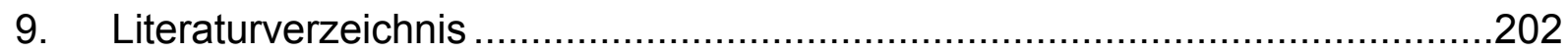




\section{Verwendete Abkürzungen}

AP Alkalische Phosphatase

BNPP Bis(4-nitrophenyl)phosphat

bpta N,N-Bis(2-pyridylmethyl)-tert-butylamin

Cp Cyclopentadienyl

cyclen 1,4,7,10-Tetraazacyclododecan

DMF Dimethylformamid

DMP Dimethylphosphat

DMSO Dimethylsulfoxid

DNA Desoxyribonukleinsäure

El electron ionization

ESI electro spray ionization

FAB fast atom bombardment

HPNPP 2-Hydroxypropyl(4-nitrophenyl)phosphat

Imid Imidazol

${ }^{i} \mathrm{Pr} \quad$ iso-Propyl

Me Methyl

MNPP Mono(4-nitrophenyl)phosphat

MS Massenspektrometrie

NMR Kernresonanz (nuclear magnetic resonance)

NP 4-Nitrophenol

NPMP 4-Nitrophenylmethylphosphat

PDB Protein database

Ph Phenyl

Py Pyridin

$\mathrm{Pz} \quad$ Pyrazol

RNA Ribonukleinsäure

tacn 1,4,7-Triazacyclononan

$\mathrm{TBABr} \quad$ Tetra(n-butyl)ammoniumbromid

${ }^{t} \mathrm{Bu} \quad$ tert-Butyl

THF Tetrahydrofuran

THP Tetrahydropyranyl

TNPP Tris(4-nitrophenyl)phosphat

Ts Tosyl (4-Toluolsulfonyl) 


\section{Legende für die abgebildeten Kristallstrukturen}

$\begin{array}{ll}\text { hellgrau } & \text { Wasserstoff } \\ \text { grau } & \text { Kohlenstoff } \\ \text { blau } & \text { Stickstoff } \\ \text { rot } & \text { Sauerstoff } \\ \text { rosa } & \text { Phosphor } \\ \text { gelb } & \text { Schwefel } \\ \text { grün } & \text { Chlor } \\ \text { türkis } & \text { Zink }\end{array}$




\section{Einleitung}

Basis allen Lebens sind die zahlreichen in jedem Lebewesen ablaufenden chemischen Prozesse. Um diese Vorgänge unter den Reaktionsbedingungen lebender Organismen bewerkstelligen zu können, hat die Evolution seit dem Beginn des Lebens vor ungefähr 3.5 Milliarden Jahren eine außerordentliche Vielfalt an Biokatalysatoren hervorgebracht. Diese Enzyme beeindrucken in ihren Umsetzungen durch ihre bisher von Menschenhand unerreicht hohen Geschwindigkeiten und Selektivitäten. Das Verstehen der Wirkungsweisen von Enzymen kann in zweierlei Hinsicht von bedeutendem Nutzen für die Menschheit sein. Zum Ersten ermöglicht nur dieses Verständnis, die in vivo ablaufenden Prozesse für den Menschen positiv zu beeinflussen; sei es um Krankheiten zu heilen, das Wachstum von Pflanzen zu kontrollieren oder pathogenen Organismen zu schaden. Zum Zweiten können die Reaktionsmechanismen der Natur durch den Menschen kopiert werden, um dann in der Katalyse interessanter Reaktionen für industriell und medizinisch wichtige oder neuartige Substanzen genutzt zu werden.

So ist es nicht verwunderlich, dass sich ein großer Teil der heutigen Forschung auf die molekularbiologische und biochemische Prozesse in Organismen, insbesondere dem Aufbau und der Funktionsweise von Enzymen, konzentriert, und bereits Tausende von Enzymen strukturell charakterisiert wurden. ${ }^{[1]}$ Dabei zeigt sich deutlich, dass für viele Reaktionen eine Proteinumgebung allein nicht ausreichend aktiv ist, sondern dass erst der Einbau eines oder mehrerer Metallionen in ein Proteingerüst hohe Aktivitäten ermöglicht. Solche Metallionen im Bereich des aktiven Zentrums können die Reaktion durch eine direkte Beteiligung oder aber durch gezielte strukturelle Funktionen beeinflussen. ${ }^{[2,3]}$

Es ist die bioanorganische Chemie, die sich mit den Rollen der Metalle in biologischen Systemen beschäftigt und versucht, direkt aus der Untersuchung solcher Metalloenzyme Erkenntnisse zu erlangen oder durch den geschickten Aufbau von Modellsystemen biologische Fragen zu beantworten. Diese Modelle sollen entweder die gleiche Funktionalität aufweisen wie die Metallzentren im Enzym oder aber strukturelle Modelle für die Art der Koordination des Metallions im Protein darstellen, wobei sich aber letztendlich beide Zielsetzungen nicht gänzlich voneinander trennen lassen. ${ }^{[4-6]}$ 
Die Vereinfachung des Systems von der komplexen natürlichen Umgebung des Metalls im Enzym hin zu einem einfachen, synthetischen Modell des aktiven Zentrums (Abbildung 1) bringt mehrere Vorteile.

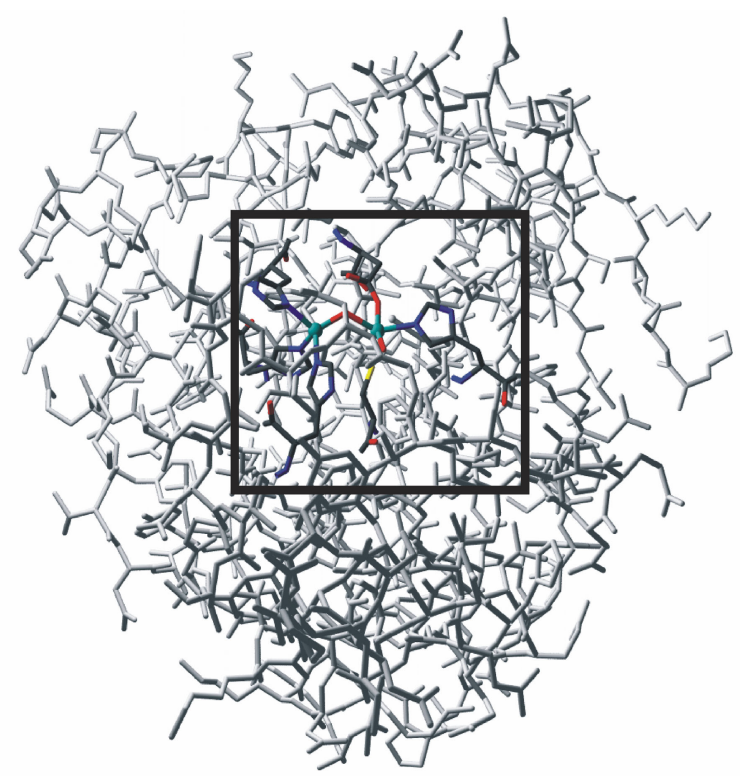

$\Downarrow$

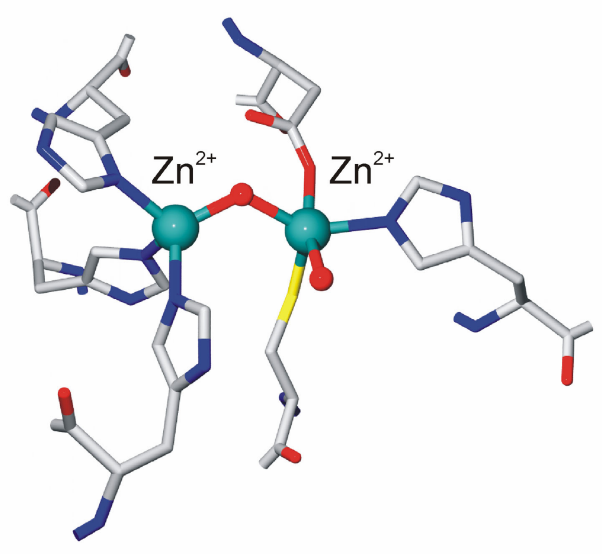

$\Downarrow$

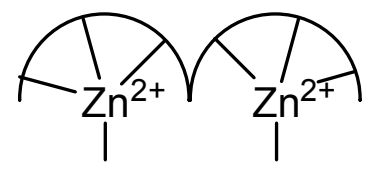

Abbildung 1: Vereinfachung eines Enzyms (oben) durch ein Modellsystem (unten) des aktiven Zentrums (Mitte) am Beispiel einer Metallo- $\beta$-Lactamase ${ }^{[7]}$. 
Zum Einen wird die Untersuchung der Rolle der Metallionen stark vereinfacht, da die das Metallion umgebende Matrix eines Proteins aus typischerweise mehreren Hundert Aminosäuren entfernt wird und so das Metallzentrum unmittelbar untersucht werden kann.

Zum anderen sind Veränderungen an einem kleinen Modellsystem - vergleicht man sie mit Änderungen am Aufbau von Proteinen - mit sehr viel weniger Aufwand vorzunehmen. Die Modifikation und Optimierung eines Modells und somit die Korrelation zwischen seinen strukturellen Parametern und der beobachteten Reaktivität ist so sehr viel leichter zu erreichen.

Aus der schier unendlichen Zahl von Enzymen ist die Gruppe der Hydrolasen besonders interessant. In dieser Gruppe finden sich als Hauptklassen die Esterasen (Abbildung 2, A), die die Spaltung von Carbonsäureestern bewirken, die Peptidasen (B), die die Spaltung von Peptidbindungen katalysieren sowie die Phosphatasen und Nukleasen (C), welche katalytisch phosphorylierte Aminosäuren, Zucker und Lipide sowie Nukleotide, RNA und DNA spalten. ${ }^{[2,3]}$

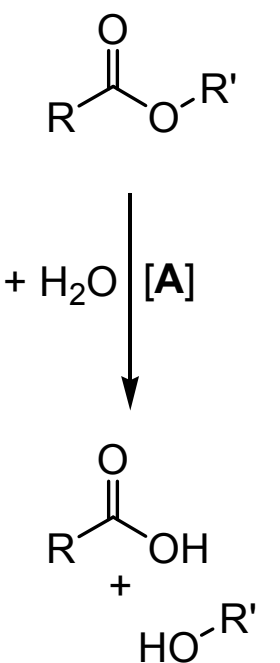<smiles>[R]NC([R])=O</smiles><smiles>[R]O[P+]([O-])([O-])O[R]</smiles><smiles></smiles><smiles>[R]O[18O][18O]</smiles><smiles>[R]O[PH3+]P(=O)([O-])O</smiles>

Abbildung 2: Hydrolyse von Carbonsäureestern, Peptiden und Organophosphaten.

Denn obwohl Peptid- als auch Phosphatesterbindungen thermodynamisch instabil sind, weisen sie hohe kinetische Barrieren auf. Da diese Enzyme an vielen Stellen des Aufbaus und der Funktion lebenswichtiger Zellprozesse und der Signalübertragung eine unentbehrliche Rolle spielen, sind sie ubiquitär und für alle Lebewesen essentiell. ${ }^{[2]}$ 
Hydrolasen sind schon jetzt aus dem Altag industrieller Prozesse und der chemischen und biologischen Forschung nicht mehr wegzudenken. Als spezifische DNA-Hydrolasen sind Restriktionsendonukleasen unabdingbare Werkzeuge in der Biotechnologie; für die kinetische Racemattrennung von Enantiomeren in der organischen Synthese sind Hydrolasen von großer Bedeutung und Nützlichkeit, und die eigentlich für die Proteinverdauung notwendigen Peptidasen können auch dazu genutzt werden, um die Rückreaktion - die Peptidsynthese - zu bewerkstelligen.

Das Interesse an artifizielle Peptidasen und Nukleasen für Anwendungen in Biochemie und Biotechnologie, beispielsweise bei der sequenzspezifischen Spaltung von Nukleinsäuren oder der Sequenzierung großer Peptide, ist groß, da sie leichter zugänglich und einfacher modifizierbar als die natürlichen Enzyme sind. ${ }^{[8,9]}$ Auch Anwendungen als Pharmaka für eine selektive Kontrolle der Genexpression wurden vorgeschlagen. ${ }^{[10]}$

So wurden viele Hydrolasen im Detail studiert und eine Vielzahl von Strukturen, funktionellen Gruppen und chemischen Mechanismen enthüllt. Man findet Hydrolasen ohne Metallionen im aktiven Zentrum; in ihnen wirkt meist ein Serinrest als angreifendes Nukleophil. Die Mechanismen der Enzyme mit einem mononuklearen Metallzentrum, wie der Carboxypeptidase, wurden bereits intensiv untersucht; jedoch wurden in letzter Zeit vermehrt dinukleare Metalloenzyme entdeckt, deren Mechanismen bis jetzt noch wenig erforscht und verstanden sind. In diesen dinuklearen Enzymen ist oft ein Zinkion neben einem anderen Metallion, manchmal sind auch zwei an der Reaktion beteiligt. ${ }^{[2,3]}$

Zink scheint aufgrund dieser Beobachtung besonders geeignet für die Beteiligung an Hydrolysereaktionen zu sein, denn es weist einige charakteristische Merkmale auf. Es ist durch seine $d^{10}$-Elektronenkonfiguration unter den Bedingungen, wie sie in Organismen vorherrschen, redoxinaktiv und in seiner Koordinationsumgebung sehr flexibel. So findet man Zink(II) in Komplexen mit Koordinationszahlen von 4, 5 oder $6 .^{[11]}$

Zink(II) bildet kinetisch labile Komplexe, bei denen ein schneller Ligandenaustausch möglich ist. Es weist eine hohe Lewis-Acidität auf, so dass es sehr gut Substrate zu aktivieren vermag. Zuletzt zeigt es eine vergleichsweise mittlere Polarisierbarkeit; es können also die unterschiedlichsten Substrate angebunden werden. 
Zink erfült jedoch nicht nur funktionelle, sondern auch regulatorische ${ }^{[12]}$ und strukturelle ${ }^{[13]}$ Aufgaben, so dass es nach Eisen das wichtigste Übergangsmetall in höheren Lebewesen ist.

Das Zusammenwirken zweier Metalle kann allgemein vielfältiger Natur sein. Deshalb sei hier nur allgemein das Prinzip einer Zweizentrenkatalyse erläutert (Abbildung 3). Koordiniert an ein Metallion das Edukt E und an das andere Metallion das Reagens $\mathrm{R}$, so kommt es durch die erfolgte Aktivierung der beiden Reaktionspartner durch die Metallionen und die gleichzeitige räumliche Nähe von Edukt und Reagens zu einer höheren Reaktionsgeschwindigkeit.

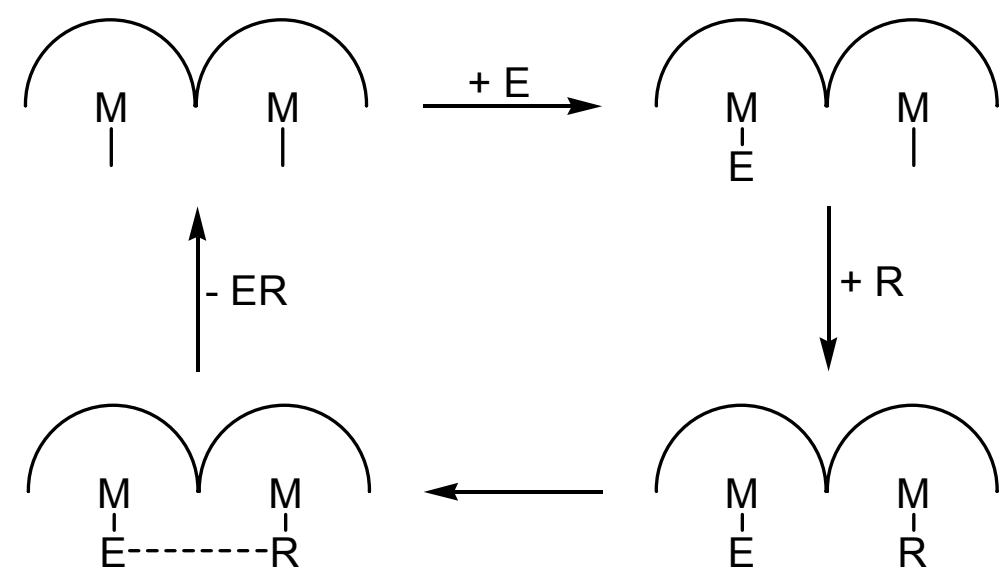

Abbildung 3: Schema einer Zweizentrenkatalyse.

In einigen wenigen Fällen ist es bereits gelungen, diese Prinzipien aus der Natur auf die Katalyse industriell interessanter Reaktionen zu übertragen. ${ }^{[14-17]}$ 


\section{Kenntnisstand und Zielsetzung}

\subsection{Dinukleare Metallohydrolasen}

Eine Möglichkeit, wie die Natur die stabilen Ester-, Peptid- und Phosphatesterbindungen effizient zu spalten vermag, ist - wie erwähnt - die vereinte Kraft zweier (oder mehrerer) Metallionen zu nutzen. So beträgt die Halbwertszeit von DNA bei $25^{\circ} \mathrm{C}$ in neutralem Wasser einige 10 bis 100 Milliarden Jahre, die Halbwertszeit der „instabileren“ RNA 110 Jahre. Enzyme, die DNA in Sekunden spalten, bewirken demnach eine Geschwindigkeitssteigerung um den Faktor $10^{17} .[5,18,19]$

Prinzipiell bestehen für Metallionen drei Möglichkeiten, die Reaktionsgeschwindigkeiten in Hydrolysen zu beschleunigen. Dies ist die Aktivierung (i) des Substrats als Lewis-Säure (Abbildung 4, A), (ii) eines Nukleophils durch Koordination des Nukleophils wie Hydroxid an das Metallion (B) und (iii) der Abgangsgruppe (C). ${ }^{[5]}$<smiles>[Y4]OP([R])(=O)O[2H]</smiles>

A<smiles>[M]OP(=O)(O)OCO</smiles>

B<smiles>[M]OP([R])(=O)O[2H]</smiles>

C

Abbildung 4: Aktivierung durch Metallionen am Beispiel von Phosphatdiestern als Substrat.

In dinuklearen Komplexen kann jedes Metallion mehrere dieser Aufgaben während der Reaktion übernehmen, so dass auch mehrere mechanistische Modelle des nukleophilen Angriffs plausibel sind. Dabei unterscheiden sie sich im Wesentlichen in der Anbindung von Nukleophil und Substrat an das dimetallische Zentrum. So ist ein Angriff eines an ein Metallion gebundenen, terminalen Nukleophils an ein monodentat (Abbildung 5, B) oder bidentat gebundenes Substrat (A, C) als auch der Angriff eines verbrückenden Hydroxids an ein bidentat gebundenes Substrat (D) denkbar. Auch eine allgemeine Basenkatalyse durch ein metallgebundenes Hydroxid (E) ist möglich. 
<smiles>[R]O[PH]([R])(=O)(O[M])O[M]</smiles>

A<smiles>[M]OC[P+]([R])([O-])[O-]</smiles>

B<smiles>[M]O[P+]([R])(O[R])O[M]</smiles>

C<smiles>[M]OP([R6])([R])([O-])O[M]</smiles>

D<smiles>[M]O[P+]([R])(O[R])O[W]</smiles>

E

Abbildung 5: Vereinfachte Darstellung von verschiedenen Bindungsmodi eines Phosphatdiesters und eines Nukleophils an ein Dimetallzentrum.

\subsection{Dinukleare Phosphatasen und Nukleasen}

\subsubsection{Alkalische Phosphatase (AP)}

Phosphatasen mit Zink im aktiven Zentrum und einem pH-Optimum bei höherem $\mathrm{pH}$ (> 7.5) und geringer Substratspezifität für Phosphatmonoester werden in Eukaryonten und Prokaryonten gefunden; ihre physiologische Rolle ist jedoch weitgehend unbekannt. Die bestuntersuchte dieser alkalischen Phosphatasen ist die alkalische Phosphatase aus Escherichia coli. ${ }^{[2,20]}$ Sie ist eine homodimere Phosphomonoesterase aus 449 Aminosäuren ( 47 kDa) pro Monomer (pdb 1ALK). Die flache Tasche des aktiven Zentrums besitzt anscheinend keine Bindungsstelle für einen Phosphatesterrest, woraus sich die Unspezifität der AP erklärt. ${ }^{[1]}$ Jedes aktive Zentrum weist drei räumlich eng benachbarte Metallbindungsstellen M1, M2 und M3

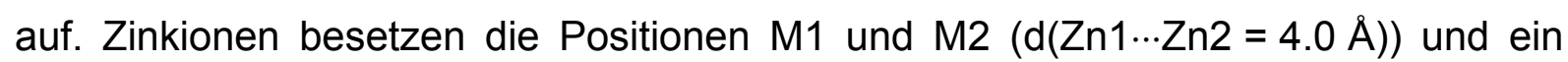
Magnesiumion M3. Die Phosphatesterhydrolyse erfordert die Besetzung von M1; eine Besetzung von M2 erhöht die Hydrolysegeschwindigkeit beträchtlich. ${ }^{[22,23]}$ Die enzymkatalysierte Reaktion hat ein Maximum im alkalischen $\mathrm{pH}-$ Bereich und ist diffusionskontrolliert. ${ }^{[24]}$ 


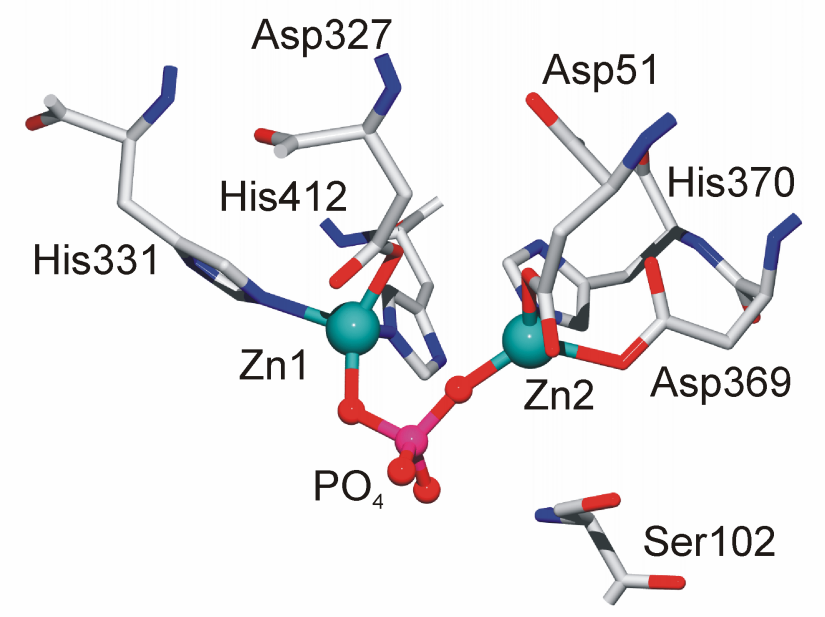

Abbildung 6: Kristallstruktur von E. coli AP mit gebundenem Phosphat (PDB-ID 1ED8). ${ }^{[25]}$

E. coli AP ist ein Prototyp für alle alkalischen Phosphatasen, speziell für alkalische Phosphatasen der Säugetiere. Sequenzvergleiche zwischen alkalischer Phosphatase aus E. coli, Hefe und Säugetieren zeigen nur eine Sequenzhomologie von $25-30 \%$; $^{[26-29]}$ das aktive Zentrum jedoch ist ein hoch konservierter Bereich mit einer Sequenzabweichung von maximal drei Aminosäuren. ${ }^{[30,31]}$ Auch verwandte Phosphoesterasen wie Phospholipase $C$ aus Bacillus cereus ${ }^{[32]}$ und P1 Nuklease aus Penicillium citrinum ${ }^{[33]}$ besitzen ähnliche Metalltriaden wie die AP in ihren aktiven Zentren.

Auf der Basis kristallographischer Untersuchungen und der Beobachtung, dass die Hydrolyse unter Retention am Phosphoratom abläuft, wurde ein Hydrolysemechanismus über eine Zwei-Metallionen-Katalyse mit einem durch Serin phosphorylierten Intermediat aufgestellt (Abbildung 7). ${ }^{[34]}$ 


\section{$\mathrm{Zn} 1$}

$$
\mathrm{H}_{-\mathrm{N}}^{\mathrm{H}-\mathrm{N}}=\mathrm{N}^{-\mathrm{Arg}^{166}} \frac{+\mathrm{ROPO}_{3}{ }^{2-}}{-\mathrm{ROPO}_{3}{ }^{2-}}
$$<smiles>[R17]O[Se][Ba]</smiles>

E

$$
+\mathrm{PO}_{4}^{2-} \|-\mathrm{PO}_{4}^{2-}
$$

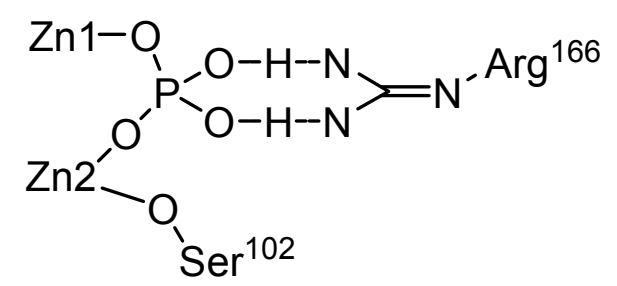

\section{$E \cdot P_{i}$}

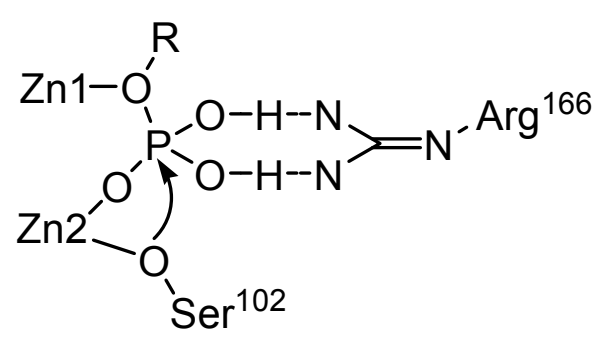

E-ROP

$$
\begin{array}{l|l}
+\mathrm{RO}^{-} & -\mathrm{H}_{2} \mathrm{O}
\end{array} \|+\mathrm{RO}^{-} \mathrm{O}
$$

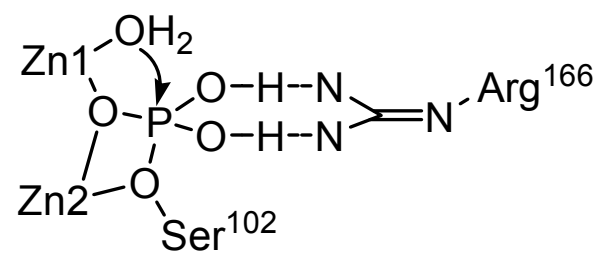

Abbildung 7: Vorgeschlagener Mechanismus einer Zwei-Metallionen-Katalyse bei der Hydrolyse von Phosphatmonoestern durch AP.

Aus der ermittelten Geometrie der Metallbindungsstellen ist die Position von Zn2 geeignet, um die Hydroxygruppe von Ser102 zu koordinieren und das Sauerstoffatom für einen nukleophilen Angriff an das Phosphoratom eines Phosphats zu aktivieren (E-ROP). Die Position von Zn1 steht im Einklang mit seiner zweifachen Rolle: Zuerst wird die negativ geladene Abgangsgruppe des Substrats als Alkoxidion koordiniert und dann wird im zweiten Schritt ein Wassermolekül aktiviert (E.P). Dieses Wassermolekül konnte kristallographisch in einer Mutante der AP lokalisiert werden. ${ }^{[35]}$ In dieser Struktur ist das Zn1-gebundene Wasser in optimaler Lage für einen apikalen nukleophilen Angriff auf das Phosphatzentrum des Phosphoserylintermediats.

Die Aufgaben der beiden Zinkionen der AP lassen sich also wie folgt zusammenfassen:

- Anbindung des Substrats

- Aktivierung des Serin-Nukleophils 
- Stabilisierung der trigonal-pyramidalen Übergangszustände

- Erleichterung der Loslösung des Alkohols

- Anbindung und Aktivierung eines Wassermoleküls durch Senken des $\mathrm{pK}_{\mathrm{S}^{-}}$ Werts. ${ }^{[2]}$

Das Magnesiumion spielt nur eine untergeordnete Rolle, indem es die aktivste Konformation des Proteins stabilisiert. ${ }^{[20]}$

\subsubsection{P1 Nuklease}

Die P1 Nuklease ist ein 36 kDa großes Glycoprotein, das aus Penicillium citrinum isoliert wurde. Es katalysiert vorzugsweise die Hydrolyse einsträngiger DNA und RNA, ist aber auch in der Lage als Phosphomonoesterase 5'-terminale Phosphatgruppen zu entfernen. ${ }^{[36-39]}$ Für maximale Aktivität erfordert es drei Zinkionen, und es hydrolysiert Phosphatester unter Inversion des Phosphorzentrums. ${ }^{[40]}$ Die bestimmte Kristallstruktur $^{[33,41]}$ zeigt zwei Zinkionen Zn1 und Zn3, die von einem Aspartat (Asp120) verbrückt werden und sich in einem Abstand von etwa $3.2 \AA$ befinden. Das dritte Zinkion $Z n 2$ ist weiter entfernt $(d(Z n 2 \cdots Z n 3)=4.7 \AA)$ und weist eine fünffache Koordinationsumgebung aus zwei Solvensmolekülen, zwei Histidin- und einem Aspartatliganden auf.

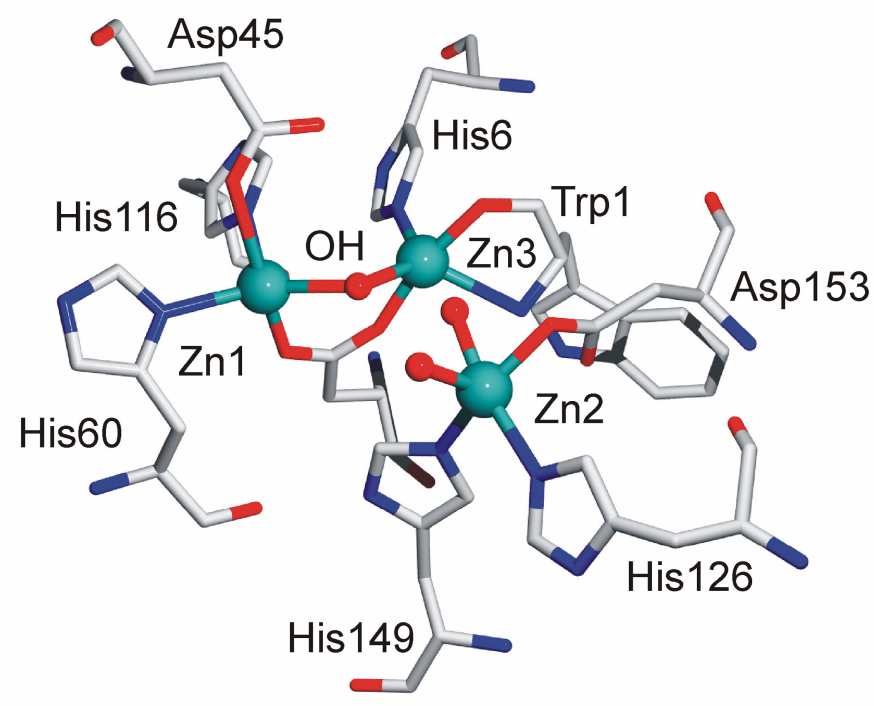

Abbildung 8: Struktur der P. citrinum P1 Nuklease (PDB-ID 1AKO). 
Neuere Untersuchungen legen nahe, dass das Zn1 und Zn3 verbrückende Hydroxid das Phosphat attackiert (Abbildung 9). ${ }^{[41]}$ Im resultierenden fünffach koordinierten Übergangszustand besetzen das angreifende Hydroxid und das als Abgangsgruppe fungierende O3'-Oxyanion apikale Positionen. Zn2 stabilisiert das O3'-Oxyanion, während die Guanidingruppe eines Arginins die zusätzliche negative Ladung des Übergangszustands neutralisiert.

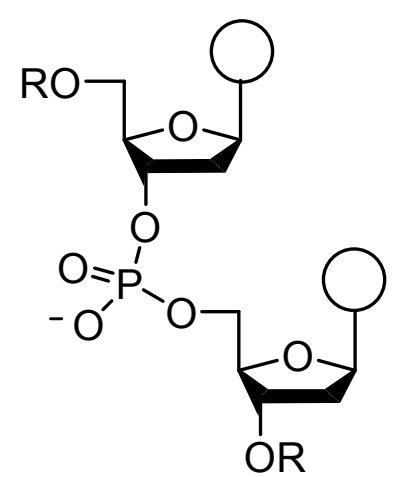

$s D N A$

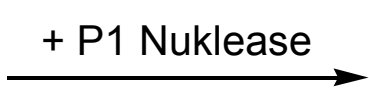

Abbildung 9: Mechanismus der Spaltung von sDNA durch P1 Nuklease.

\subsubsection{Violette saure Phosphatasen (PAP)}

Enzyme, die eine Phosphatesterhydrolyse bei sauren pH-Werten (Optimum zwischen 4.9 und 6.0) katalysieren, durch Tartrat nicht inhibiert werden, zwei Eisenionen oder ein Eisen und ein anderes, leicht austauschbares Metall, beispielsweise $\mathrm{Zn}^{2+}$, enthalten und eine charakteristische violette Farbe in ihrer oxidierten, inaktiven Form aufzeigen bzw. in ihrer aktiven Form eine rosa Farbe besitzen, werden als violette saure Phosphatasen (PAP) bezeichnet. ${ }^{[42-45]}$ Diese homologen Proteine mit $\sim 35$ kDa wurden nicht nur in zahlreichen unterschiedlichen Säugetiergeweben gefunden, sondern auch in Pflanzen oder bestimmten Bakterien. ${ }^{[2]}$ Obwohl die physiologische Funktion dieser Enzyme nicht genau bekannt ist, vermutet man eine Rolle als intrazelluläre Monophosphatasen ${ }^{[43,45]}$ und auch eine Beteiligung im Eisenmetabolismus von Säugetieren. ${ }^{[46]}$ Die aktive, reduzierte $\mathrm{Fe}(\mathrm{II}) / \mathrm{Fe}(\mathrm{III})$-Form der Säugetier-PAP konnte bisher aufgrund der Labilität des $\mathrm{Fe}^{2+}$ nicht röntgenographisch charakterisiert werden, jedoch die PAP der Weißen Bohne (kbPAP). ${ }^{[47,48]}$ Diese enthält ein $\mathrm{Fe}(\mathrm{III})$ und ein $\mathrm{Zn}(\mathrm{II})$ in ihrem dimetallischen aktiven Zentrum. Jedes Metallion wird von vier Proteinliganden koordiniert: Tyr167, His325, 
Asp135 und Asp 164 für Fe(III) und His286, His323, Asn201 und Asp164 für Zn(II) (Abbildung 10). Das Asp164 dient als Carboxylatbrücke zwischen den beiden Metallionen und fixiert diese in einem Abstand von $3.3 \AA$. Aufgrund der bestimmten Geometrie wurde ein verbrückendes Hydroxid, ein terminales Wasser am Zink und ein terminales Hydroxid am Fe(III) hinzugefügt; diese wurden kristallographisch jedoch nicht lokalisiert.

Der Befund, dass die Hydrolyse zu einer Inversion am Phosphor führt, ${ }^{[49]}$ macht ein phosphoryliertes Intermediat sehr unwahrscheinlich, ${ }^{[50]}$ lässt auf einen direkten Transfer der Phosphatgruppe auf ein Wassermolekül schließen und unterstützt einen Mechanismus über ein trigonal-bipyramidales Phosphatintermediat. Es wird derzeit der in Abbildung 10 gezeigte Mechanismus vorgeschlagen, bei dem das Monophosphat an das labile $\mathrm{M}(\mathrm{II})$ Zentrum bindet und durch ein an $\mathrm{Fe}(\mathrm{III})$ gebundenes Hydroxid angegriffen wird. ${ }^{[48]}$
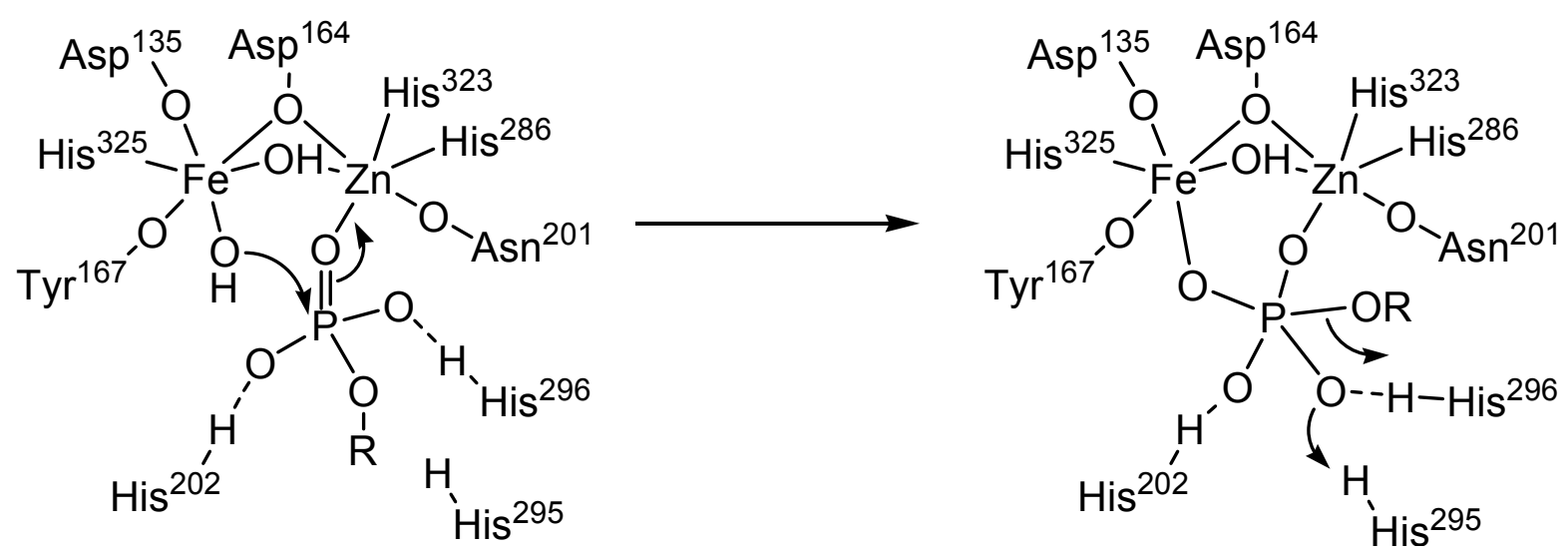

Abbildung 10: Vorgeschlagener Mechanismus für die Phosphatesterhydrolyse an kbPAP.

\section{$2.3 \beta$-Lactam-Antibiotika und $\beta$-Lactamasen}

$\beta$-Lactam-Antibiotika wie Penicilline, Cephalosporine und Carbapeneme sind in der Medizin weit verbreitet im Kampf gegen bakterielle Infektionen. Durch die Hemmung des Aufbaus des Peptidoglykangerüsts, dem Hauptbestandteil bakterieller Zellwände, wirken sie für wachsende und sich vermehrende Keime bakterizid. 

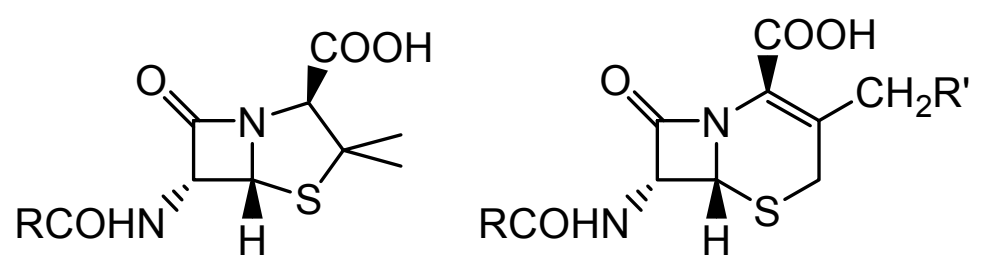<smiles>[R7]C1=C(C(=O)O)N2C(=O)[C@@H](C(C)O)[C@@H]2C1[R]</smiles>

Abbildung 11: Klassen von $\beta$-Lactam-Antibiotika: Penicilline, Cephalosporine, Carbapeneme.

Die bakterielle Resistenz gegenüber diesen Antibiotika durch das vermehrte Auftreten von $\beta$-Lactamasen stellt eine ernste Gefahr für die Behandlung bakterieller Erkrankungen dar. Der Mechanismus der metallfreien Serin- $\beta$-Lactamasen der Klassen $A, C$ und $D$ ist schon länger bekannt, und geeignete Hemmstoffe stehen zur Verfügung; hingegen hydrolysieren die in letzter Zeit entdeckten Metallo- $\beta$ Lactamasen ein breites Spektrum der $\beta$-Lactamantibiotika, ohne dass ein brauchbarer Inhibitor zur Verfügung steht. Dieses Problem verschärft sich noch dadurch, dass diese Enzyme oft auf Plasmiden codiert sind und so leicht auch zwischen verschiedenen Bakterienstämmen ausgetauscht werden können.

Die Mehrzahl der Metallo- $\beta$-Lactamasen weisen mononukleare Enzymzentren mit einem Zinkion im aktiven Zentrum auf. Jedoch stehen für einige Vertreter der Zink- $\beta$ Lactamasen Kristallstrukturen zur Verfügung, die zeigen, dass im aktiven Zentrum zwei Zinkionen und ein metallgebundenes Hydroxid, das die beiden Metalle entweder verbrückt oder terminal gebunden ist, enthalten sind (Abbildung 12). ${ }^{[7,51,52]}$ Die Rolle des zweiten Zinkions und die Art des Zusammenwirkens beider Metallionen ist jedoch noch größtenteils unbekannt. ${ }^{[53]}$

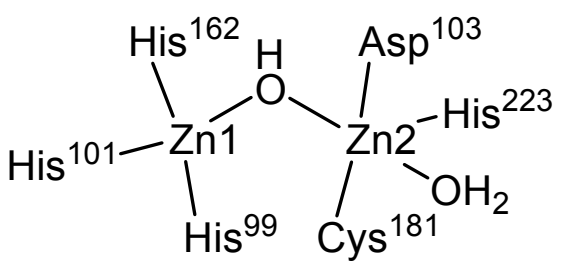

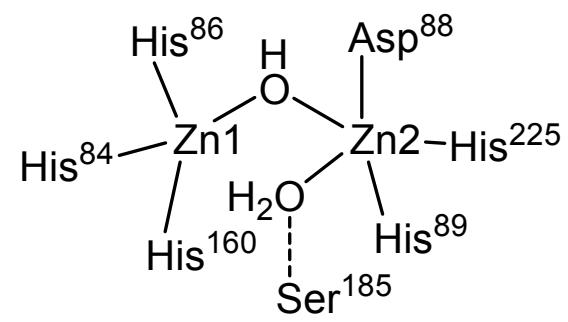

Abbildung 12: Aktive Zentren der Metallo- $\beta$-Lactamasen aus Bacteroides fragilis und Stenotrophomonas maltophilia (PDB-Code 1ZNB, 1SML). 


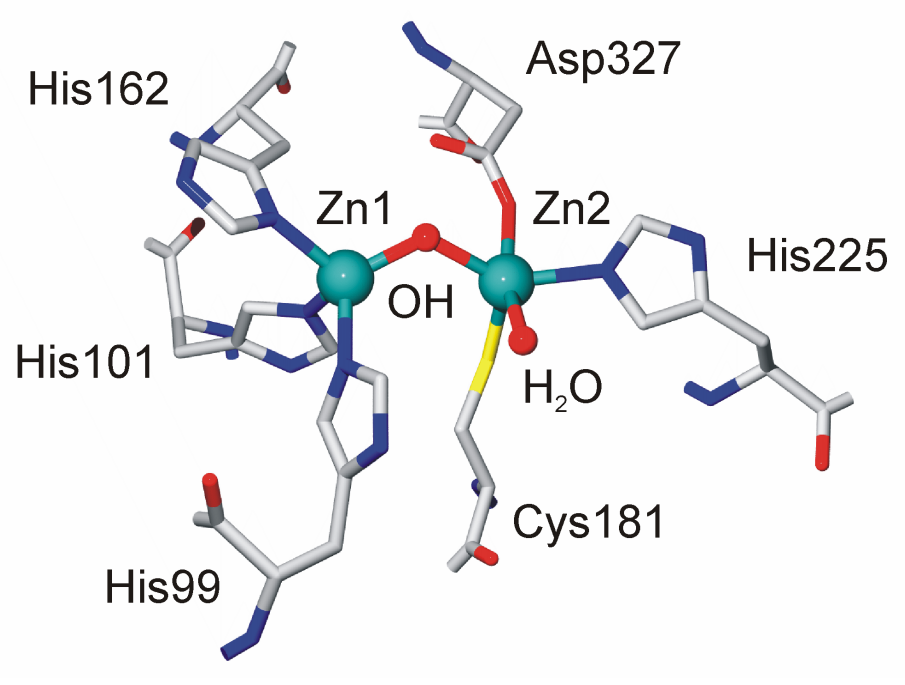

Abbildung 13: Struktur des aktiven Zentrums der Metallo- $\beta$-Lactamase aus B. fragilis (PDB-Code 1ZNB). ${ }^{[7]}$

Im Zentrum der Metallo- $\beta$-Lactamase aus $B$. fragilis befindet sich $\mathrm{Zn} 1$ in einer tetraedrischen $\mathrm{His}_{3}(\mathrm{OH})$-Umgebung und $\mathrm{Zn2}$ in einer trigonal-bipyramidalen (Asp)(Cys)(His) $\left(\mathrm{OH}_{2}\right)(\mathrm{OH})$-Umgebung; das Hydroxid verbrückt $\mathrm{Zn} 1$ und $\mathrm{Zn2}$ mit einem $\mathrm{Zn} \cdots \mathrm{Zn}$-Abstand von $4.0 \AA$.

Die Aufklärung von Struktur und Reaktivität dieser Zink- $\beta$-Lactamasen ist Gegenstand der aktuellen Forschung; so steht ein detailliertes Bild des Mechanismus der metallvermittelten $\beta$-Lactam-Hydrolyse noch aus, speziell auch der Rolle des zweiten Zinkions. ${ }^{[53,54]}$

Aus den durchgeführten Studien wird für die $\beta$-Lactam-Hydrolyse durch die $B$. fragilis Metallo- $\beta$-Lactamase der in Abbildung 14 dargestellte Mechanismus vorgeschlagen ${ }^{[55]}$ Das nukleophile Hydroxid wird bereits an $\mathrm{Zn} 1$ oder als $\mu-\mathrm{OH}$ mit einem sehr niedrigen $\mathrm{pK}_{\mathrm{S}}(<5,25)$ vorgebildet. ${ }^{[56]}$ Zudem dient $\mathrm{Zn} 1$ zusammen mit einem Asparaginrest (Asn193) als Oxyanionbindungsstelle, um die Carbonylgruppe des $\beta$-Lactams zu polarisieren. Die Carboxylatgruppe des $\beta$-Lactams wird genutzt, um das Substrat über Bindung an Zn2 oder an ein benachbartes Lysin (Lys184) zu fixieren, so dass der $\beta$-Lactam-Stickstoff in der Lage ist, das apikale Wassermolekül zu ersetzen und an Zn2 zu koordinieren. Das Hydroxid greift dann aus einer terminalen Position an Zn1 oder aus verbrückender Position die Carbonylgruppe des Substrats an. Die Spaltung der C-N-Bindung der tetraedrischen Zwischenstufe führt zu einer negativ 
geladenen Stickstoff-Abgangsgruppe, die durch eine elektrostatische Wechselwirkung mit Zn2 stabilisiert wird. Dieses Intermediat konnte durch stopped-flowExperimente charakterisiert werden. ${ }^{[57]}$ Das Produkt bildet sich darauf im geschwindigkeitsbestimmenden Schritt aus dem Zerfall des Intermediats durch Protonierung des negativ geladenen Stickstoffs und Ligandenaustausch an Zn1, d.h. durch Austausch der Carboxylatgruppe des Produkts mit Wasser aus der Umgebung.
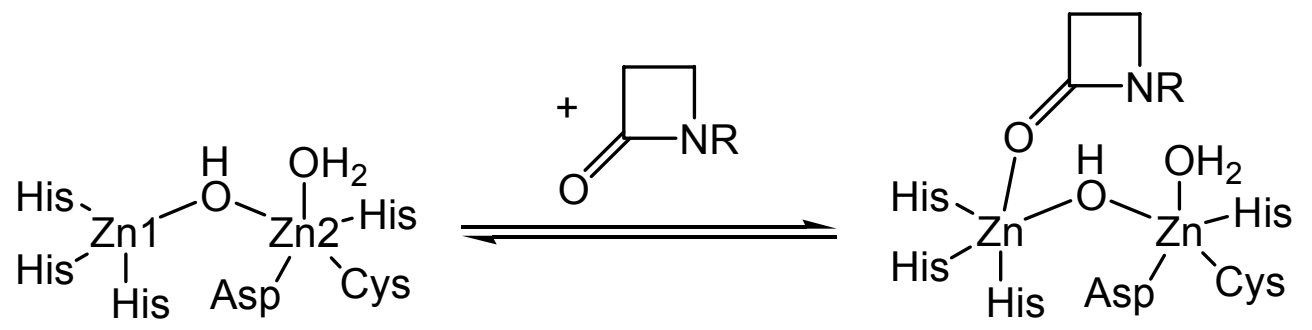<smiles>[R]NCCC(=O)O</smiles>

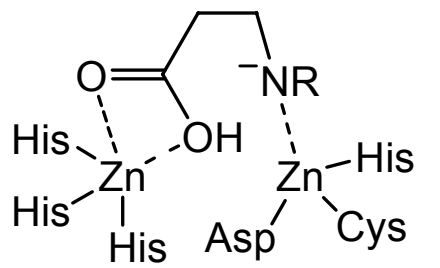

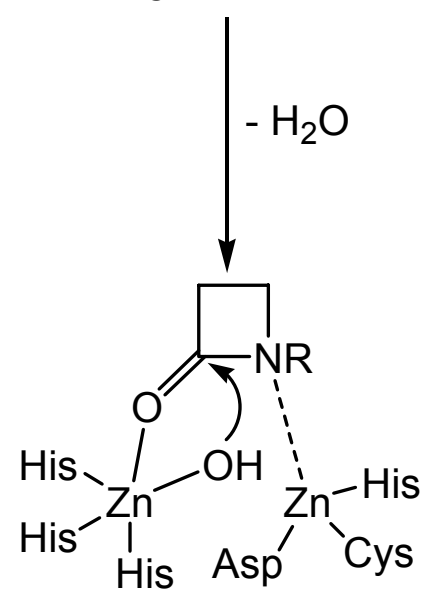

Abbildung 14: Vorgeschlagener Mechanismus für die Hydrolyse von $\beta$-Lactamantibiotika durch $B$. fragilis Metallo- $\beta$-Lactamase .

Die Mechanismen anderer dinuklearer Metall- $\beta$-Lactamasen folgen wahrscheinlich einem ähnlichen Reaktionspfad. Jedoch führen die kleinen Unterschiede in den aktiven Zentren zu leicht differenzierten Reaktionswegen, die die unterschiedlichen Aktivitäten und Selektivitäten begründen. ${ }^{[55]}$

Die Vorteile dinuklearer Metallzentren in Hydrolysen lassen sich trotz der aufgezeigten mechanistischen Vielfalt demnach wie folgt zusammenfassen:

- Verteilung der Ladung

- kleinere Aktivierungsbarrieren durch die delokalisierte Ladung

- Möglichkeit zur Bindung größerer Substrate an das Metallzentrum 
- einfachere elektrostatische Aktivierung von Substraten oder lonisierung eines Wassermoleküls. ${ }^{[58]}$

Weiterhin sind jedoch noch mehrere Fragen nach dem Mechanismus dinuklearer Hydrolasen offen. Diese betreffen vor allem die Identität des Nukleophils, den Details der Substratanbindung und die Reihenfolge der ablaufenden Elementarschritte.

Eine Möglichkeit, Einblicke in die Mechanismen dinuklearer Hydrolasen zu erhalten, ist die Modellierung der aktiven Zentren durch kleine, wohldefinierte Modellkomplexe.

\subsection{Ligandsysteme für dinukleare Komplexe}

Ein Ligandsystem für den Aufbau dinuklearer Komplexe sollte als zentrales Element eine als Brücke zwischen zwei Metallionen geeignete Funktionalität aufweisen. Anders als dinukleare Komplexe aus zwei monomeren Einheiten bieten solche Ligandgerüste die Möglichkeit, die beiden Metallionen als zentralen Bestandteil zu fixieren und unabhängig davon Veränderungen an der Peripherie zu ermöglichen. Der Ligand sollte des weiteren die beiden Metallionen in einem Abstand voneinander positionieren, der den Metall-Metall-Abständen in den zu untersuchenden Enzymen entspricht. Nur so kann eine realistische Modellierung von dinuklearen aktiven Zentren ermöglicht werden. Zum dritten sollte das Ligandsystem einfach verändert werden können, so dass sich über systematische Veränderungen an den Koordinationsumgebungen der Metallionen Zusammenhänge zwischen Struktur und beobachteter Reaktivität finden lassen. Schließlich sollten die gebildeten Komplexe in wässriger Lösung hinreichend stabil sein, um Untersuchungen der Reaktivität in Hydrolysereaktionen durchführen zu können.

Für den Aufbau der Dizink-Modellsysteme in dieser Arbeit wurden deshalb präorganisierende Kompartimentliganden verwendet, in denen die beiden Koordinationseinheiten durch eine Pyrazolatbrücke verbunden sind (Abbildung 15).

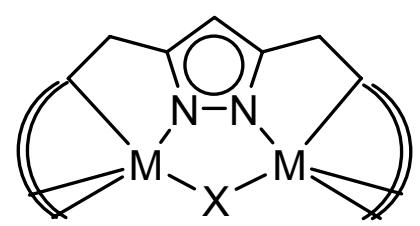

Abbildung 15: Schema eines dinuklearen Pyrazolatokomplexes. 
Man erhält in deren Komplexen typischerweise Metall-Metallabstände im Bereich von 3.0 bis $4.6 \AA$ - Abstände, wie man sie oft in dinuklearen Enzymen findet. ${ }^{[3]}$ In 3- und 5-Position des Pyrazols können über zwei flexible, modular aufgebaute und sehr effiziente Syntheserouten unterschiedlichste Seitenarme angebracht werden. Die dadurch mögliche hohe Variabilität der strukturellen und elektronischen Eigenschaften der Liganden erlaubt eine Feineinstellung des Ligandgerüsts. Durch das Anbringen chelatisierender Seitenarme können außerdem hohe Komplexstabilitäten mit Zink(II) erreicht werden. Verwendet man chelatisierende Seitenarme mit ungeladenen N-Donoren, so erhält man eine Gesamtladung von +2 , die sich zusammen aus dem negativ geladenen Pyrazolatring, der meist einfach negativ geladenen Zweitbrücke $\mathrm{X}$ und den beiden $\mathrm{Zn}^{2+}$-Ionen ergibt. Dadurch wird ein Angriff an die negativ geladenen oder zumindest negativ polarisierten Substrate durch die elektrostatische Anziehung erleichtert. Dies steht auch im Einklang mit neueren theoretischen Untersuchungen, nach denen das aktive Zentrum in der betrachteten $\beta$ Lactamase, einer Dizinkpeptidase, eine formale Ladung von +2 besitzen sollte. ${ }^{[59,60]}$

\subsection{Dinukleare Pyrazolatometallkomplexe}

In vorangegangenen Arbeiten über zweikernige Nickel- und zweikernige Kupfersysteme zeigte sich, dass sich durch die Variation des Liganden der MetallMetall-Abstand steuern lässt, und dieser einen wesentlicher Parameter in den beobachteten Reaktivitäten darstellt. ${ }^{[61-64]}$

Erste Analysen zweikerniger Pyrazolatodizinkkomplexe zeigten, dass durch die Länge der Seitenarme auch hier der Metall-Metall-Abstand beeinflusst werden kann. Lange Propylentriaminseitenarme in 1 lassen einen kurzen Zn...Zn-Abstand von $3.613 \AA$ und die Ausbildung einer monoatomaren Zweitbrücke zu. Hingegen ziehen die kurzen Ethylentriaminseitenarme in $\mathbf{2 a}$ die Metallionen auseinander $(\mathrm{d}(\mathrm{Zn} \cdots \mathrm{Zn})=4.374 \AA)$ und bewirken den Einbau eines zusätzlichen Solvensmoleküls in die Zweitbrücke. ${ }^{[65]}$ 


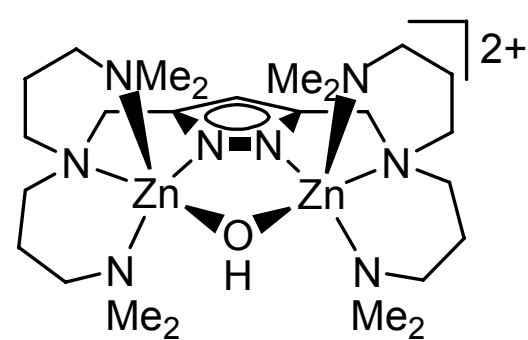

1

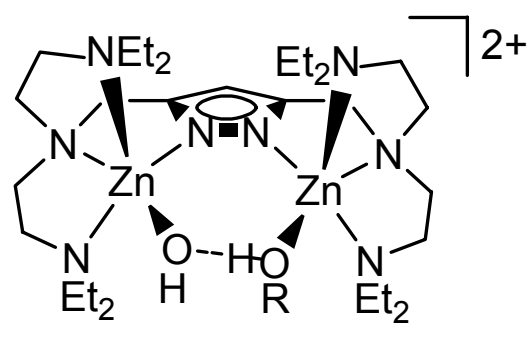

$2 \mathrm{a}(\mathrm{R}=\mathrm{H})$

Abbildung 16: Pyrazolatodizinkkomplexe mit verbrückendem und terminalem Hydroxid.

Komplex 1 ist so der Grundtyp eines metallgebundenen, verbrückenden Hydroxids, wohingegen 2a als Grundtyp eines terminalen, metallgebundenen Hydroxids in einem Dizinkzentrum betrachtet werden kann.

\subsection{Zielsetzung}

Ziel dieser Arbeit ist es, durch den Aufbau einfacher Modellsysteme für Dizinkkomplexe und durch die Untersuchung ihrer Eigenschaften, ihrer Reaktivität und ihrer Hydrolyseaktivität gegenüber typischen Substraten einen Beitrag zum Verständnis grundlegender Reaktionsmechanismen dinuklearer Hydrolasen zu liefern.

Insbesondere sollen Unterschiede in der Reaktivität von terminal metallgebundenem Hydroxid in Dizinkzentren und zwei Zinkionen verbrückendem Hydroxid herausgearbeitet werden. Dazu muss eine Reihe neuer Dizinkkomplexe aus präorganisierenden Pyrazolat-basierten Kompartimentliganden synthetisiert und sowohl in fester Form als auch in Lösung charakterisiert werden.

Um die Auswirkung von strukturellen Veränderungen im Modell zu untersuchen, sollten diese als Modellverbindungen für Hydrolasen auf ihre Reaktivität gegenüber Phosphatestern und auf ihre katalytische Aktivität in der Hydrolyse der Amidbindung in $\beta$-Lactamen hin getestet werden.

Um die Ursachen der beobachteten Reaktivitätsunterschiede zu ermitteln, ist es des weiteren notwendig, eine Reihe spektroskopischer Studien über die Anbindung von Substraten und Substratanaloga an die verwendeten Modellkomplexe durchzuführen. 


\section{Ligandsynthese und Ligandeigenschaften}

\subsection{Ligandsynthese}

\subsubsection{Synthese von Bis(aminomethyl)pyrazol-Liganden}

Als Grundbausteine für die Synthese symmetrischer Pyrazolatliganden mit chelatisierenden Aminomethylseitenarmen stehen das in einer Stufe aus Pyrazol-3,5dicarbonsäure I zugängliche Pyrazol-3,5-dicarbonsäurechlorid II und das in fünf Stufen aus 3,5-Dimethylpyrazol zugängliche N-THP-geschützte 3,5-Bis(chlormethyl)pyrazol X zur Verfügung (Abbildung 17). Die chelatisierenden Seitenarme können dann je nach ihrer chemischen Beschaffenheit an einen dieser Grundbausteine gekuppelt werden. Die Synthesen der verwendeten Liganden folgen dabei prinzipiell literaturbekannten Methoden.<smiles>[R]N([R])Cc1cc(CN([R])[R])[nH]n1</smiles>

Abbildung 17: Bis(aminomethyl)pyrazol-Liganden.

Die aliphatischen Liganden $\mathrm{L}^{1}, \mathrm{~L}^{2}, \mathrm{~L}^{3}, \mathrm{~L}^{4}$ und $\mathrm{L}^{5}$ wurden aus dem 3,5Pyrazoldicarbonsäurechlorid II und den entsprechenden sekundären Aminen nach Literaturvorschriften synthetisiert. ${ }^{[62,66-68]}$ Zunächst wurde die Pyrazol-3,5-dicarbonsäure I mit Thionylchlorid in das Pyrazol-3,5-dicarbonyl-dichlorid II umgewandelt. Anschließend wurde dieses in Gegenwart eines Überschusses der Hilfsbase Triethylamin mit dem jeweiligen aliphatischen Amin $H N R^{1} R^{2}$ umgesetzt, und dann das entstandene Amid III mit Lithiumaluminiumhydrid zum Liganden IV reduziert (Abbildung 18). 


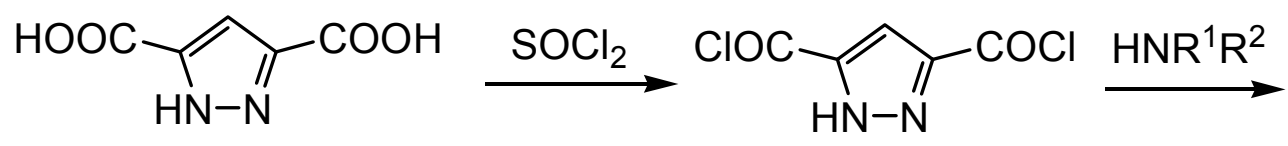

I<smiles>[R1]NCc1cc(CN=[R1])[nH]n1</smiles>

III

$$
\left.\mathrm{NMe}_{2}\right)_{2}^{\mathrm{L}^{1}} \mathrm{R}_{\mathrm{R}^{2}}^{1}
$$

\section{IV}

Abbildung 18: Synthese der Pyrazolliganden mit aliphatischen Seitenarmen.

Diese Syntheseroute erweist sich für Liganden mit aromatischen Pyridin-haltigen Seitenarmen als ungünstig, da es hier zu Nebenreaktionen bei der Reduktion kommt. Die aromatischen Liganden $L^{6}, L^{7}, L^{8}$ und $L^{9}$ sind jedoch über das N-THP geschützte 3,5-Bis(chlormethyl)-pyrazol $\mathbf{X}$ und die entspechenden Seitenarme zugänglich. ${ }^{[63]}$ Zunächst wird hier nach Literaturvorschrift das 3,5-Bis(chlormethyl)-1-(tetrahydropyran-2-yl)pyrazol hergestellt. ${ }^{[69-71]}$ Über Oxidation des kommerziell erhältlichen 3,5-Dimethylpyrazols $\mathbf{V}$ durch $\mathrm{KMnO}_{4}$ zum Pyrazol-3,5-dicarbonsäuremonokaliumsalz VI und anschließender Veresterung zum Dimethylester VI lässt sich nach Reduktion 3,5-Dihydroxymethylpyrazol VIII darstellen. Dieses wird durch Thionylchlorid in das 3,5-Dichlormethylpyrazol IX überführt und anschließend mit 3,4Dihydropyran zu 3,5-Bis(chlormethyl)-1-(tetrahydropyran-2-yl)pyrazol $\mathbf{X}$ umgesetzt (Abbildung 19). 

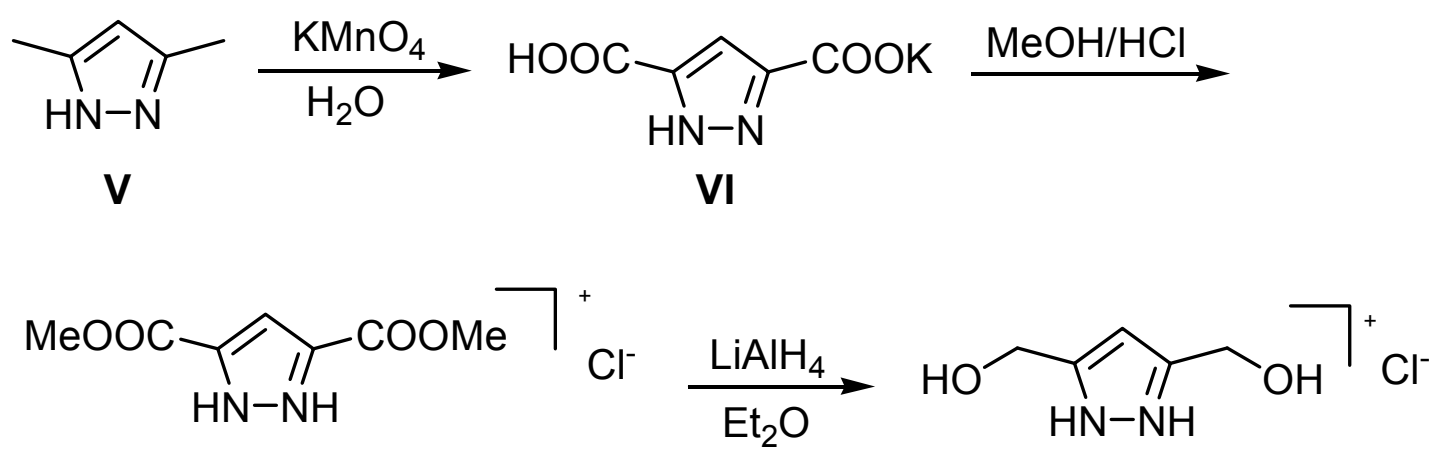

VII

VIII

$\stackrel{\mathrm{SOCl}_{2}}{\longrightarrow} \mathrm{Cl} \underset{\mathrm{HN}-\mathrm{NH}}{\stackrel{\mathrm{CCM}}{\mathrm{Cl}^{+}}} \stackrel{\widehat{\mathrm{O}^{-}}}{\stackrel{(1)}{\longrightarrow}}$

IX<smiles>ClCc1cc(CCl)n(C2CCCCO2)n1</smiles>

$\mathbf{X}$

Abbildung 19: Synthese von 3,5-Bis(chlormethyl)-1-(tetrahydropyran-2-yl)pyrazol.

Zur Synthese der Liganden mit Pyridin-Seitenarmen wurden zunächst das jeweilige Amin und die geschützte Pyrazolverbindung $\mathbf{X}$ über $\mathrm{Na}_{2} \mathrm{CO}_{3}$ in Acetonitril unter Rückfluss erhitzt. Die Schutzgruppe wird unter sauren Bedingungen durch $\mathrm{EtOH} / \mathrm{HCl}$ abgespalten und das entstandene Hydrochlorid mit wässriger $\mathrm{NaOH}$ zum freien Liganden deprotoniert (Abbildung 20). 


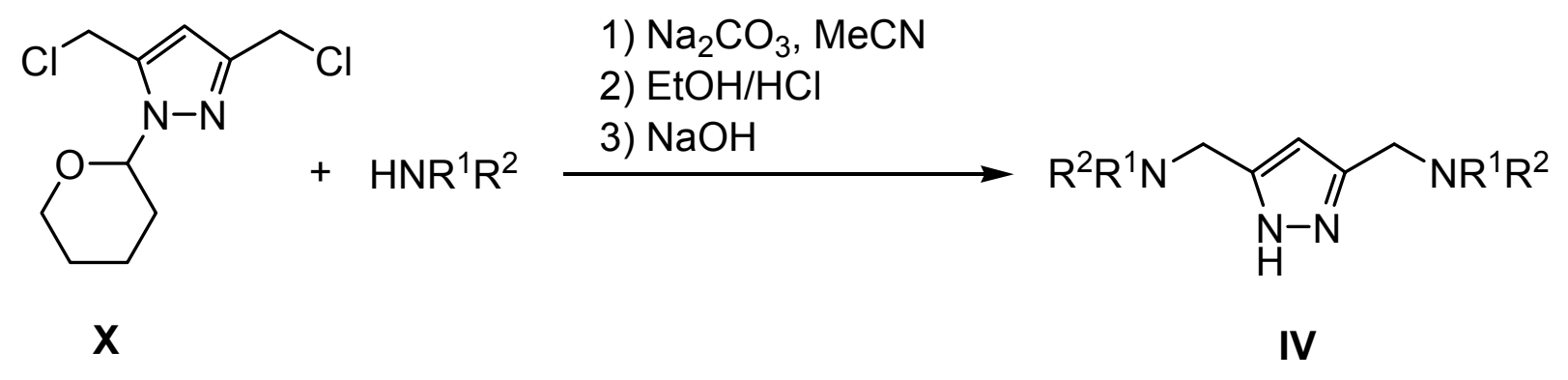<smiles>[R]#N</smiles>

$L^{6}$<smiles>CNCCc1ccccn1</smiles>

$\mathrm{L}^{7}$<smiles>CN=Cc1ccccn1</smiles>

$L^{8}$<smiles>CNCc1ccccn1</smiles>

$L^{9}$

Abbildung 20: Synthese der Pyrazolliganden mit Pyridinseitenarmen.

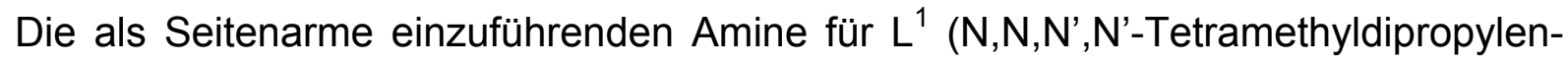
triamin), $L^{2}$ (N,N,N'-Trimethylpropylendiamin), $L^{3}$ (N,N,N',N'-Tetraethyldiethylentriamin), $L^{4}$ (N,N,N'-Trimethylethylendiamin), $L^{7}$ (Methyl-2-pyridyl-2-ethylamin) und $L^{8}$ (Bis[(2-pyridyl)methyl]amin) sind kommerziell erhältlich. Die Seitenarme für $L^{5}\left(N, N^{\prime}-\right.$ Diisopropyl-1,4,7-triazacyclononan $\mathbf{X V I}$ ), $L^{6}$ (Bis[2-(2-pyridyl)ethyl]amin XVIII) und $\mathrm{L}^{9}$ (Methyl-2-pyridyl-methylamin XX) wurden nach literaturbekannten Methoden hergestellt.

Der tacn-Makrozyklus XVI wurde in einer sechsstufigen Syntheseroute nach literaturbekannter Vorschrift aus Diethylentriamin und Ethylenglykol synthetisiert (Abbildung 21). ${ }^{[72]}$ Schlüsselschritt ist dabei die selektive Abspaltung von nur zwei der drei N-Tosylgruppen in XIII. 


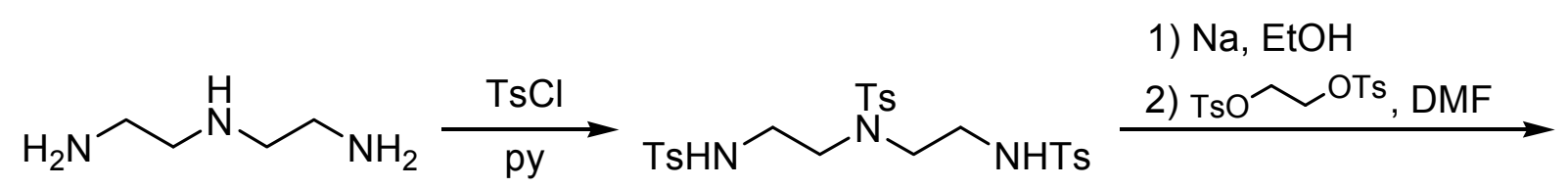

$\mathbf{X I}$

XII

$L_{T s}^{T s}$

XIII

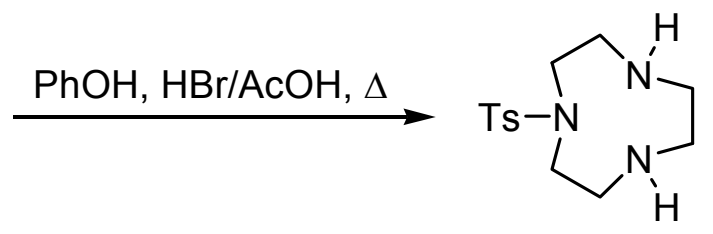

XIV<smiles>CCCN1CCN([As])CCN(CCC)CC1</smiles>

XV<smiles>CCCN1CCNCCN(CCC)CC1</smiles>

XVI

Abbildung 21: Synthese des tacn-Seitenarms XVI.

Bis[2-(2-pyridyl)ethyl]amin XVIII kann durch Addition von Ammoniumchlorid an 2-Vinylpyridin XVII in Methanol gewonnen werden (Abbildung 22; oben). ${ }^{[73]}$ Methyl-2pyridyl-methylamin $\mathbf{X X}$ wurde durch eine reduktive Aminierung aus Pyridin-2-carboxaldehyd XIX und Methylammoniumhydrochlorid mit Natriumcyanoborhydrid in Methanol synthetisiert (Abbildung 22; unten). ${ }^{[74]}$ 
<smiles>COC(O)(Cl)Cl</smiles>

XVII<smiles>c1ccc(CCNCCc2ccccn2)nc1</smiles>

XVIII

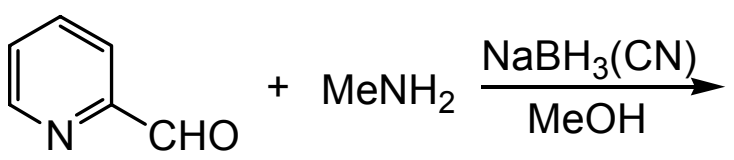

XIX<smiles>CNCc1ccccn1</smiles>

$\mathbf{X X}$

Abbildung 22: Synthese der Seitenarme XVIII und XX mit PyridyIresten.

Da diese Liganden auch potentiometrisch charakterisiert werden sollten, war es notwendig, einige Modifikationen an den in der Literatur beschriebenen Synthesen vorzunehmen, um eine größtmögliche Reinheit der Liganden zu erzielen. Dabei war das Ziel, die Liganden schon möglichst rein aus der Reaktion zu erhalten, da eine spätere Reinigung der Liganden durch Umkristallisation oder chromatographische Methoden sehr aufwendig ist und oft nicht zum erwünschten Erfolg führt. Für die Synthese der aliphatischen Liganden war es ausreichend, die Aminseitenarme vor der Verwendung zu destillieren. Im Fall der Seitenarme mit Pyridylresten wurde die Destillation auch für $L^{7}, L^{8}$ und $L^{9}$ durchgeführt. Zusätzlich wurde bei der Entschützung der Liganden $L^{6}$ und $L^{8}$ mittels ethanolischer Chlorwasserstofflösung die Nachfällung mit Diethylether unterlassen, um die Mitfällung von Verunreinigungen zu verhindern. Dadurch konnten sehr saubere Liganden isoliert werden, die im NMR wie auch bei der späteren potentiometrischen Charakterisierung keine oder nur sehr geringe Mengen stickstoffhaltiger und damit störender Verunreinigungen aufwiesen. Zusätzlich wurde für die potentiometrischen Messungen der tripodale Ligand $\mathrm{L}^{10}$ verwendet, um die die dinuklearen Systeme mit einem mononuklearen System vergleichen zu können. 


\title{
3.1.2 Synthese von bisfacialen Pyrazolatliganden
}

Durch ihren Aufbau über die 3,5-Bis(aminomethyl)pyrazolfunktionen bieten die bisher vorgestellten Ligandsysteme den Metallionen eine doppelt trigonal-bipyramidale Koordinationsumgebung bzw. eine doppelt quadratisch-pyramidale oder quadratischplanare Koordinationsumgebung. Die Koordinationssphären vieler dinuklearer Zinkenzyme weisen jedoch für eines der beiden Zinkionen oft eine pseudotetraedrische Geometrie mit drei Histidinresten und einer meist durch Wasser besetzten "freien" Bindungsstelle auf. Deshalb wurde in dieser Arbeit ein neues Ligandsystem geschaffen, welches durch eine faciale Anordnung dreier Donoren in jedem Kompartiment zwei tetraedrische Koordinationsumgebungen ermöglicht.
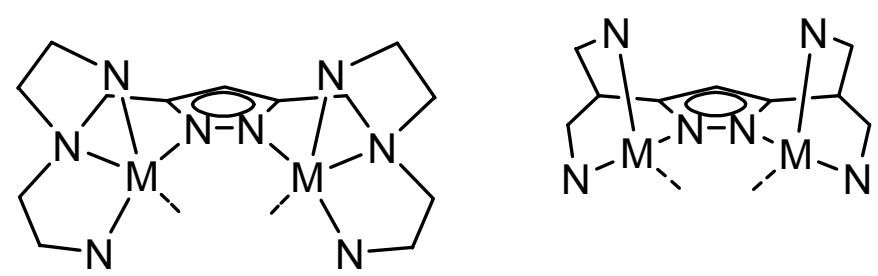

\begin{abstract}
Abbildung 23: Vergleich der Metallkoordination zwischen Bis(aminomethyl)pyrazolliganden und bisfacialen Liganden.
\end{abstract}

Diese Liganden sollten eine hohe Komplexstabilität auch bei nur dreifacher Koordination je Kompartiment sicherstellen und zusätzliche freie Koordinationsstellen an den beiden Metallionen anbieten. Bei einer nur vierfachen Koordination sollte sich der Elektronenzug des Metallions vom Liganden der "freien" Koordinationsstelle erhöhen, und so z. B. im Fall von Zink(II) zu Metall-gebundenen Wasserspezies mit noch deutlich geringeren $\mathrm{pK}_{\mathrm{S}}$-Werten zu kommen.

Als Ersatz für die Imidazolringe des Histidins wurden für die Synthese N-Methylimidazol- oder Pyridinringe gewählt, da sie die größte elektronische Ähnlichkeit zum Imidazolring des Histidins aufweisen.

Synthetisch lassen sich solche Bis(aryl)systeme durch Addition von Aryllithiumverbindungen an Carbonsäureester aufbauen. ${ }^{[75]}$ Als Grundbaustein für die Synthese wurde deshalb der THP-geschützte Pyrazol-3,5-dicarbonsäuremethylester ${ }^{[76]} \mathbf{X X I}$ verwendet. N-Methylimidazol bzw. 2-Brompyridin wurden jeweils bei $-78^{\circ} \mathrm{C}$ mit $n$-Butyllithium in Tetrahydrofuran lithiiert und dann der Diester zugefügt. 


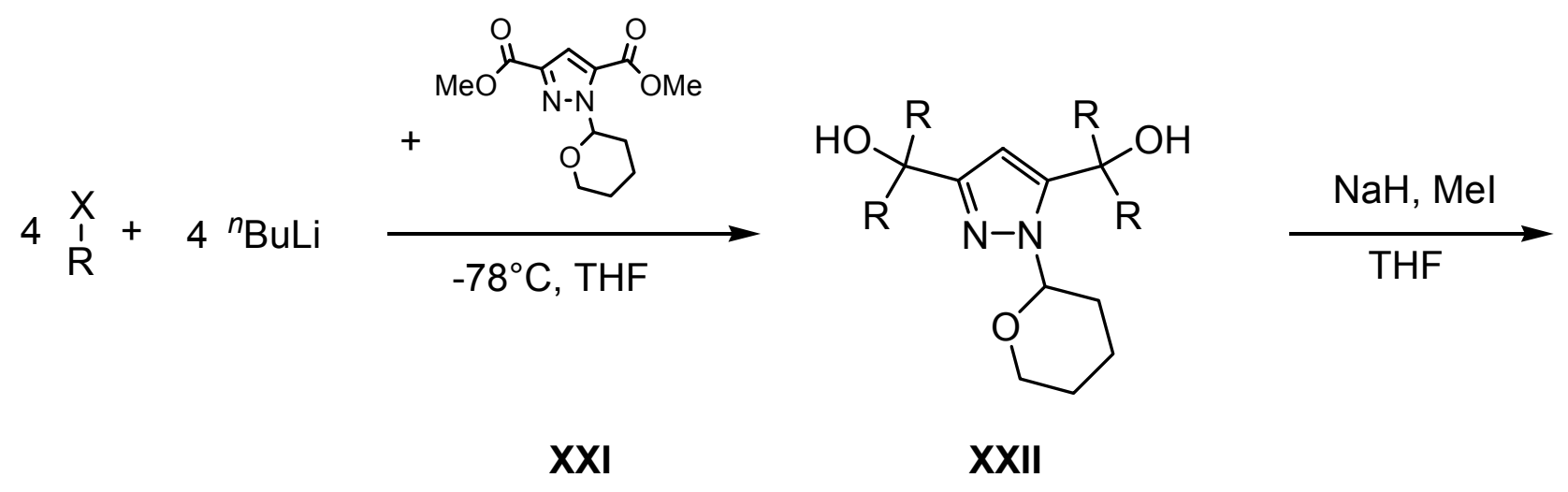<smiles>[R]C([R])(OC)c1cc(C([R])([R])OC)n(C2CCCCO2)n1</smiles>

XXIII

XXIV

$$
\text { a } \quad \mathrm{R}=\mathrm{R}=
$$

Abbildung 24: Synthese von Bis(diarylmethoxymethyl)pyrazolen.

Da ausschließlich eine Koordination über die heterozyklischen Stickstoffatome erwünscht ist, wurden die Hydroxyfunktionen anschließend durch Deprotonieren mit Natriumhydrid und Umsetzen mit Methyliodid verethert. Die isolierten Ausbeuten über beide Stufen sind mit $21 \%$ für das N-Methylimidazolderivat XXIIIa und $11 \%$ für das Pyridinderivat XXIIIb relativ niedrig, da die Trennung der Produkte von unerwünschten Nebenprodukten nicht durch Umkristallisieren erfolgen konnte, und die säulenchromatographische Reinigung dieser Polyamine ebenfalls problematisch ist. 
Die THP-Schutzgruppe wurde anschließend mit $\mathrm{HCl} / \mathrm{EtOH}$ abgespalten, und das Hydrochlorid mit wässriger Natriumhydroxid-Lösung zum freien Liganden XXIV deprotoniert.

\subsection{Potentiometrische Charakterisierung der Liganden}

Die Liganden $L^{1}, L^{3}, L^{4}, L^{5}, L^{7}, L^{8}, L^{9}$ und $L^{10}$ wurden in Zusammenarbeit mit Frau Professor Etelka Farkas in Debrecen/Ungarn potentiometrisch charakterisiert. Wässrige Lösungen der Liganden wurden dazu mit Salzsäure versetzt und mit Kaliumhydroxidlösung im Bereich von pH 2 bis 11.5 titriert. Anhand der aufgenommenen Titrationskurven wurden dann mittels des Programms SUPERQUAD ${ }^{[77]}$ die pK $_{\mathrm{S}}$-Werte und die Bildungskonstanten $\beta$ bzw. lg $\beta$ errechnet. Die Titrationskurven sind zusammen mit den exakten potentiometrischen Bedingungen in Kapitel 8 zu finden.

Die erhaltenen Daten sind in Tabelle 1 zusammengefasst. Zum Vergleich sind zusätzlich die in der Arbeitsgruppe von Kaden et al. bestimmten Werte für $L^{3}{ }^{[78]}$ und $\mathbf{X X V}{ }^{[79]}$, einem dem hier verwendeten Liganden $L^{5}$ ähnlichen, ebenfalls von Kaden et al. charakterisierten Triazacyclononanliganden, und in Tabelle 2 die pKs-Werte einiger einfacher Polyamine aufgeführt.<smiles>c1cc(CN2CCNCCNCC2)n[nH]1</smiles>

\section{XXV}

Abbildung 25: Triazacyclononan-Ligand von Kaden et al.

\begin{tabular}{llllllll}
\hline Spezies & & {$\left[\mathrm{LH}_{6}\right]^{6+}$} & \multicolumn{1}{c}{$\left[\mathrm{LH}_{5}\right]^{5+}$} & \multicolumn{1}{c}{$\left[\mathrm{LH}_{4}\right]^{4+}$} & \multicolumn{1}{c}{$\left[\mathrm{LH}_{3}\right]^{3+}$} & \multicolumn{1}{c}{$\left[\mathrm{LH}_{2}\right]^{2+}$} & \multicolumn{1}{c}{$[\mathrm{LH}]^{+}$} \\
\hline \multirow{2}{*}{$\mathrm{L}^{1}$} & $\lg \beta$ & $46.61(4)$ & $42.55(2)$ & $37.05(3)$ & $28.50(1)$ & $19.49(1)$ & $9.99(2)$ \\
& $\mathrm{pK}$ & 4.05 & 5.50 & 8.55 & 9.01 & 9.50 & 9.99 \\
\hline \multirow{2}{*}{$\mathrm{L}^{3}$} & $\lg \beta$ & & & $38.08(3)$ & $29.46(3)$ & $20.15(2)$ & $10.39(3)$ \\
& $\mathrm{pK}$ & & & 8.58 & 9.34 & 9.76 & 10.39 \\
\hline \multirow{2}{*}{$\mathrm{L}_{\mathrm{S}}$} & $\lg \beta$ & & $(\sim 28)$ & $26.31(1)$ & $22.78(1)$ & $17.82(1)$ & $9.24(1)$ \\
& $\mathrm{pK}$ & & $(\sim 1.7)$ & 3.53 & 4.96 & 8.58 & 9.24 \\
\hline
\end{tabular}




\begin{tabular}{|c|c|c|c|c|c|c|}
\hline \multirow{2}{*}{$L^{5}$} & $\lg \beta$ & $(\sim 9.35)$ & $8.82(1)$ & \multicolumn{3}{|l|}{$4.87(1)$} \\
\hline & $\mathrm{pK}_{\mathrm{s}}$ & $(\sim 0.53)$ & 3.95 & 4.87 & & \\
\hline \multirow{2}{*}{$L^{6}$} & $\lg \beta$ & $23.8(1)$ & $21.18(7)$ & $17.68(7)$ & $13.55(4)$ & $7.61(2)$ \\
\hline & $\mathrm{pK}_{\mathrm{s}}$ & 2.62 & 3.50 & 4.13 & 5.94 & 7.61 \\
\hline \multirow{2}{*}{$L^{8}$} & $\lg \beta$ & & 19.18(1) & $15.73(1)$ & 11.61(1) & $6.26(1)$ \\
\hline & $\mathrm{pK}_{\mathrm{s}}$ & & 3.45 & 4.12 & 5.35 & 6.26 \\
\hline \multirow{2}{*}{$\mathrm{L}^{9}$} & $\lg \beta$ & & & & $12.96(1)$ & $7.21(1)$ \\
\hline & $\mathrm{pK}_{\mathrm{s}}$ & & & & 5.75 & 7.21 \\
\hline \multirow{2}{*}{$L^{10}$} & $\lg \beta$ & & & & $18.82(2)$ & $9.95(1)$ \\
\hline & $\mathrm{pK}_{\mathrm{s}}$ & & & & 8.87 & 9.95 \\
\hline \multirow{2}{*}{$X X V$} & $\lg \beta$ & & 34.67 & 28.59 & 21.79 & 11.25 \\
\hline & $\mathrm{pK}_{\mathrm{s}}$ & & 6.08 & 6.80 & 10.54 & 11.25 \\
\hline
\end{tabular}

Tabelle 1: Protonierungskonstanten der Liganden bei $25^{\circ} \mathrm{C}, I=0.2 \mathrm{M}(K C l)$ für $L^{1}, L^{3}, L^{4}, L^{5}, L^{6}, L^{8}, L^{9}$ und $L^{10}$ und $I=0.5 \mathrm{M}\left(\mathrm{KNO}_{3}\right)$ für $L^{2}$.

\begin{tabular}{|c|c|c|c|c|c|}
\hline Abkürzung & Ligand & $\begin{array}{l}\mathrm{pK}_{\mathrm{S}} \\
{[\mathrm{LH}]^{+}}\end{array}$ & $\begin{array}{l}\mathrm{pK}_{\mathrm{S}} \\
{\left[\mathrm{LH}_{2}\right]^{2+}}\end{array}$ & $\begin{array}{l}\mathrm{pK}_{\mathrm{S}} \\
{\left[\mathrm{LH}_{3}\right]^{3+}}\end{array}$ & Lit. \\
\hline tmeda & Tetramethylethylendiamin & 9.1 & 5.7 & & [80] \\
\hline tren & Pentamethyldiethylentriamin & 9.4 & 8.4 & 2.4 & [80] \\
\hline tmpda & Tetramethylpropylendiamin & 9.8 & 7.7 & & [80] \\
\hline trpn & Pentamethyldipropylentriamin & 10.0 & 9.0 & 6.4 & [80] \\
\hline \multirow[t]{2}{*}{ bpma } & Bis(2-pyridylmethyl)-amin & 7.28 & 2.60 & 1.13 & {$[81]$} \\
\hline & & 7.30 & 2.60 & 1.12 & [82] \\
\hline
\end{tabular}


Tabelle 2: pKs-Werte einfacher Polyamine.

Im Fall des einfachsten Liganden $\mathrm{L}^{4}$ mit Ethylendiamin-Seitenarmen können vier Deprotonierungsschritte im gemessenen pH-Bereich von 2 bis 11.5 gefunden werden. Eine weitere Protonierung/Deprotonierung kann bei einem $\mathrm{pH}-$ Wert unter 2 aus den vorliegenden Daten vermutet werden, ist jedoch gerade außerhalb des zugänglichen Bereichs. Dieser erste Schritt kann der Deprotonierung des sehr aciden protonierten Pyrazolrings zugeordnet werden.

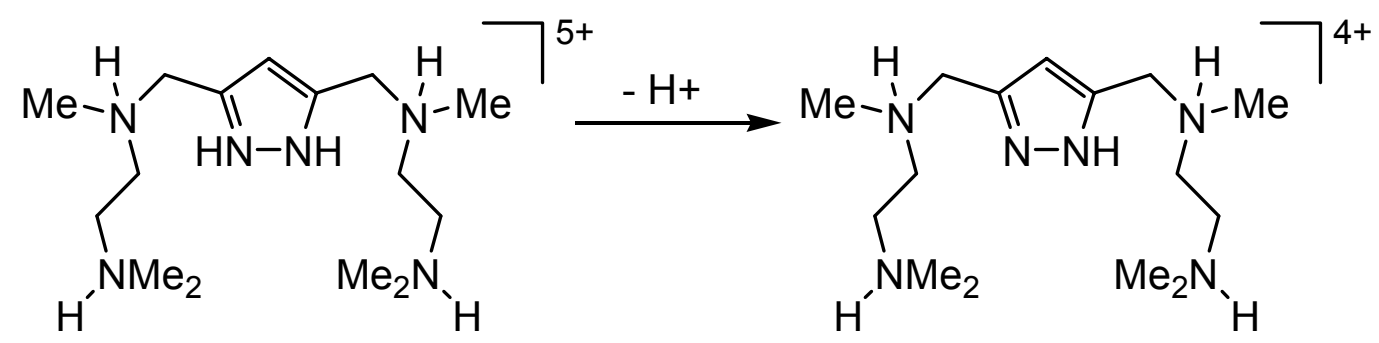

Abbildung 26: Deprotonierung des protonierten Pyrazolrings von $L^{4}$.

In den folgenden zwei Schritten wird ein Proton pro Seitenarm entfernt, und die beiden letzten Deprotonierungen sind jeweils wieder an einem der beiden Seitenarme lokalisiert. Den genauen Ort der jeweiligen Deprotonierungen zu bestimmen, gestaltet sich jedoch äußerst schwierig, da die chemischen Umgebungen aller vier Aminfunktionen relativ ähnlich sind. Jedoch ist der Unterschied der $\mathrm{pK}_{\mathrm{S}^{-}}$Werte von $\left[\mathrm{L}^{4} \mathrm{H}_{2}\right]^{2+}$ und $\left[\mathrm{L}^{4} \mathrm{H}\right]^{+}$mit 0.82 nahe an dem statistischen Wert, der unabhängige Deprotonierungen der beiden letzten Schritte nahe legt. Dies kann am einfachsten durch eine Abfolge erklärt werden, in der jeder Ethylendiamin-Seitenarm jeweils ein Proton verliert. Demzufolge müssen $\left[\mathrm{L}^{4} \mathrm{H}_{4}\right]^{4+}$ und $\left[\mathrm{L}^{4} \mathrm{H}_{3}\right]^{3+}$ auch Protonierungen/Deprotonierungen an verschiedenen Seitenarmen entsprechen. Ein Vergleich mit den pKs-Werten des einfachen tmeda-Liganden, der einem Seitenarm entspricht, stützt diese Annahme und macht eine Beteiligung des Pyrazolkerns bei den letzten beiden Deprotonierungen unwahrscheinlich (Tabelle 2). 


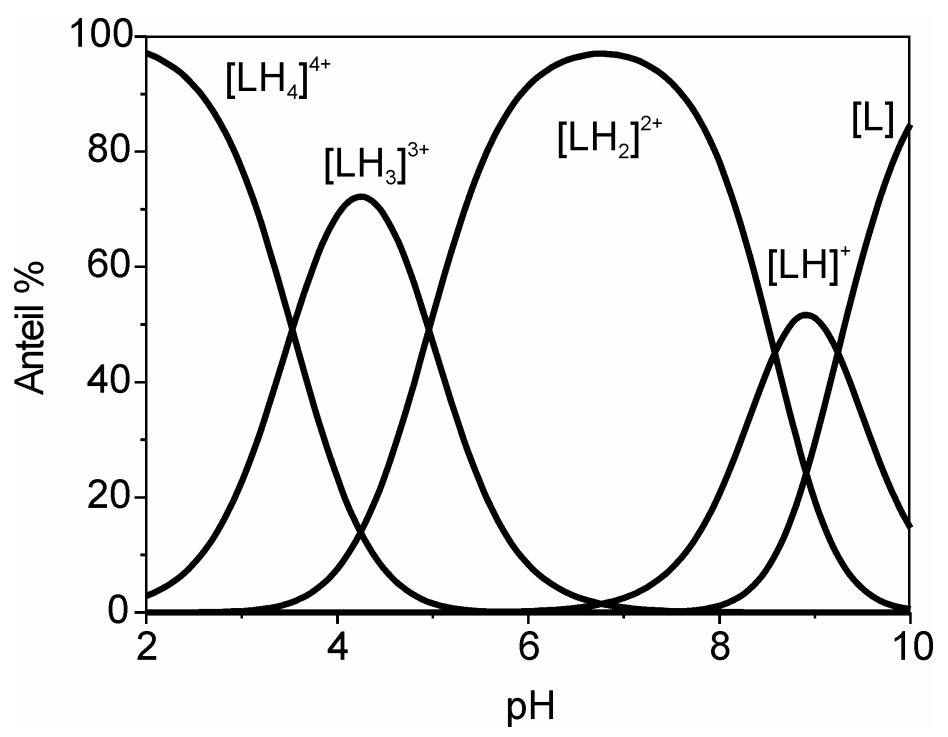

Abbildung 27: Speziesverteilung für $L^{4}$ im Bereich von $p H 2$ bis 10.

Im Fall von $\mathrm{L}^{5}$ konnten nur zwei Deprotonierungen aus der Titrationskurve abgeleitet werden. Diese entsprechen dem Entfernen je eines Protons pro TriazacyclononanSeitenarm. Auch hier befindet sich die Dissoziation des zusätzlich an den Pyrazolring gebundenen Protons aufgrund seiner hohen Acidität außerhalb des messbaren Bereichs, d.h. unter 2. Die Messungen lassen zusätzlich darauf schließen, dass zwei weitere Protonen in $\left[\mathrm{L}^{5} \mathrm{H}_{4}\right]^{4+}$ ebenfalls sehr acide sind, höchstwahrscheinlich jeweils ein Proton pro Makrozyklus. Diese Beobachtung kann durch die Tatsache erklärt werden, dass der Ring keine Möglichkeit bietet, dass sich zwei in einem Ring akkumulierte positive Ladungen ausweichen können. Demzufolge ist die Abspaltung eines Protons thermodynamisch bevorzugt und die bestimmten $\mathrm{pK}_{\mathrm{S}}$-Werte sind sehr niedrig. Die restlichen zwei Protonen in $\left[\mathrm{L}^{5} \mathrm{H}_{2}\right]^{2+}$, offensichtlich eines pro Makrozyklus, verbleiben bis zu einem pH von 11 in den Makrozyklen, deuten also auf sehr hohe pKs-Werte aufgrund des „makrozyklischen Effekts“ hin.

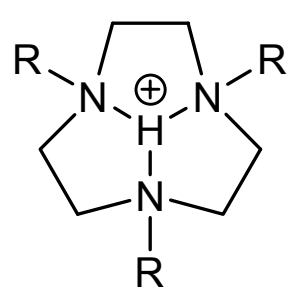

Abbildung 28: Chelatisierung eines Protons durch einen tacn-Makrozyklus. 
Die molekulare Struktur von $\left[\mathrm{L}^{5} \mathrm{H}_{2}\right]^{2+}$ mit jeweils einem Proton im tacn-Seitenarm konnte bereits durch eine Röntgenstrukturanalyse bestätigt werden. ${ }^{[64]}$

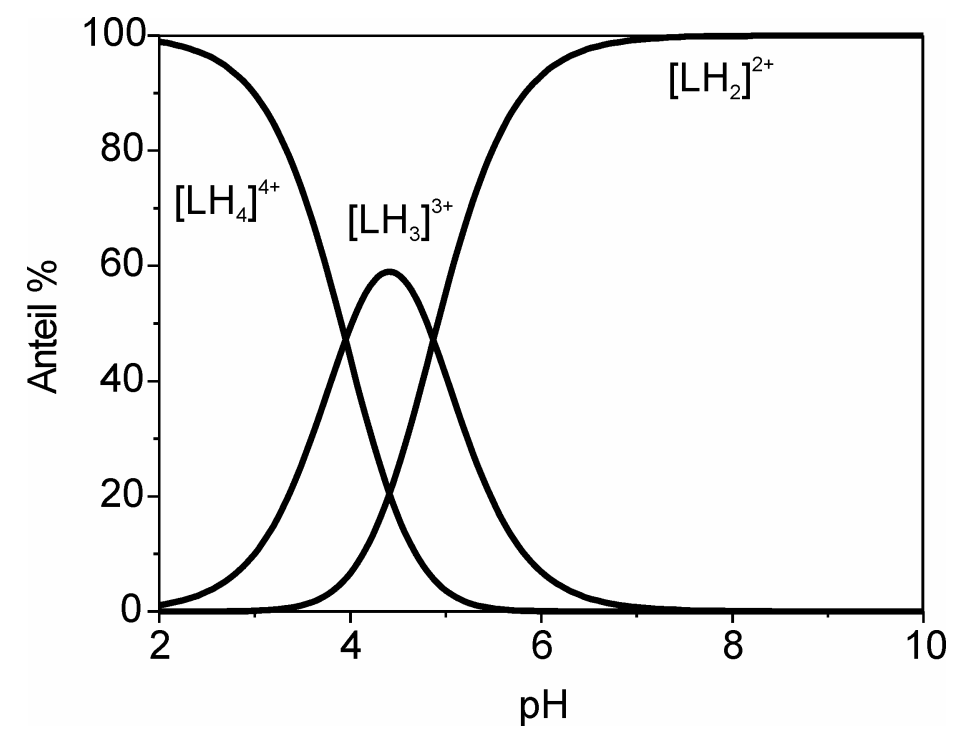

Abbildung 29: Speziesverteilung für $L^{5}$ Bereich von $p H 2$ bis 10 .

Aufgrund der veränderten Topologie in $\mathrm{L}^{3}$, also offenkettig statt makrozyklisch, sind im Einklang mit den genannten Argumenten die $\mathrm{pK}$-Werte für $\left[\mathrm{L}^{3} \mathrm{H}_{4}\right]^{4+}$ und $\left[\mathrm{L}^{3} \mathrm{H}_{3}\right]^{3+}$ signifikant höher und die pKs-Werte für $\left[\mathrm{L}^{2} \mathrm{H}_{2}\right]^{2+}$ und $\left[\mathrm{L}^{2} \mathrm{H}\right]^{+}$deutlich niedriger als die der korrespondierenden $L^{5}$-Spezies.

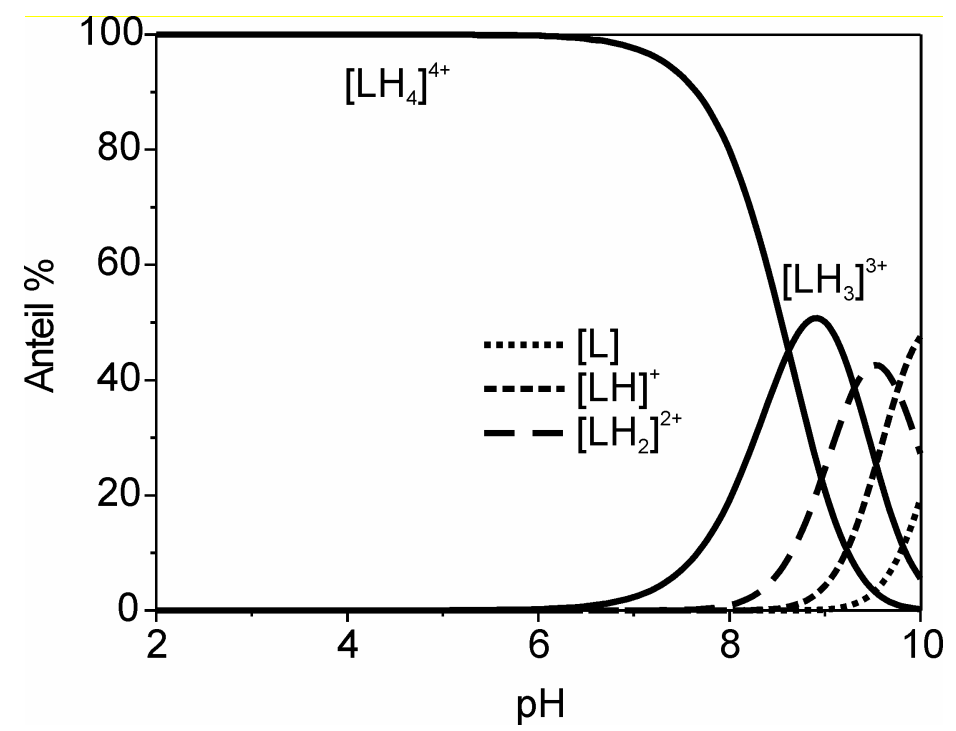

Abbildung 30: Speziesverteilung für $L^{3}$ Bereich von $p H 2$ bis 10. 
Die $p K_{s}-$ Werte für Ligand $L^{1}-$ also ein wie $L^{3}$ offenkettiges, jedoch überall um eine Methylengruppe verlängertes System - sind relativ ähnlich zu den Werten von $\mathrm{L}^{3}$. Jedoch findet man zwei zusätzliche Protonierungsschritte bei $\mathrm{pK}_{\mathrm{S}}$-Werten von 4.05 und 5.50. Wahrscheinlich ist der Grund dafür, dass $L^{1}$ bei höheren $p H-W e r t e n$ als $L^{3}$ noch zwei Protonen aufnehmen kann, die größere räumliche Separierung der Protonierungsstellen und damit der positiven Ladungen.

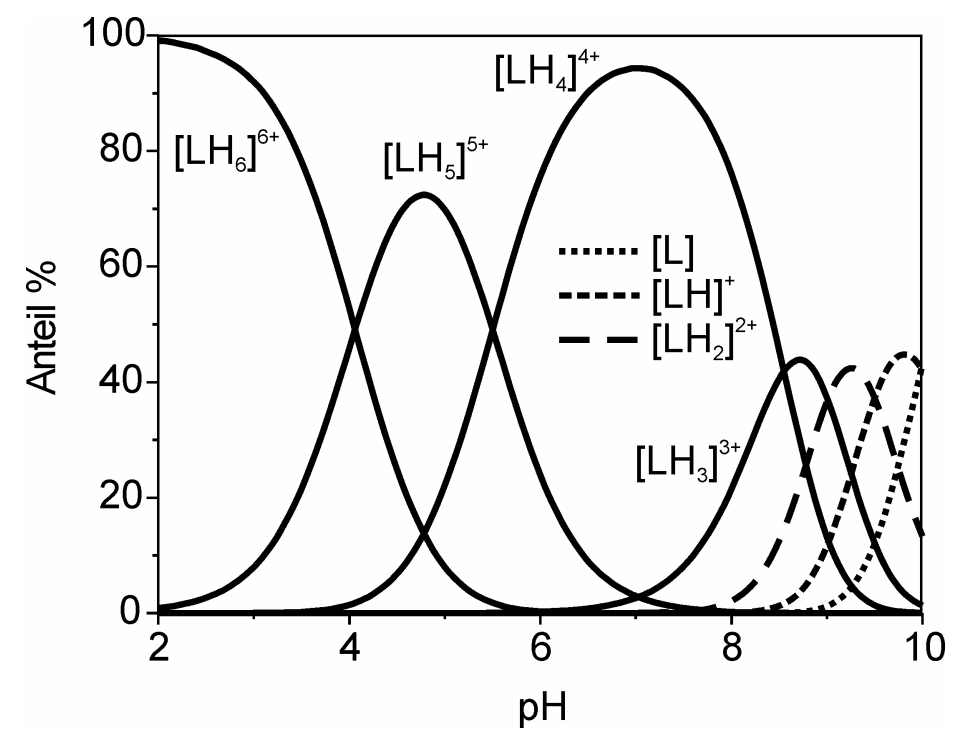

Abbildung 31: Speziesverteilung für $L^{1}$ Bereich von $\mathrm{pH} 2$ bis 10.

Als Vergleich wurden auch die Protonierungskonstanten für $L^{10}$ bestimmt. Dieser Ligand weist nur auf einer Seite einen chelatisierenden Ethylentriamin-Seitenarm auf, so dass er exakt der Hälfte von $\mathrm{L}^{3}$ entspricht (Abbildung 32). Aus den Titrationskurven erhält man zwei $\mathrm{pK}_{\mathrm{S}}$-Werte, die den Werten für einen tren-Liganden (vgl. Tabelle 2) ungefähr entsprechen. Die gemessenen Werte liegen jedoch um ungefähr 0.5 Einheiten höher (Tabelle 2), das Pyrazolsystem ist also stärker basisch. Dies lässt auf eine Beteiligung des Pyrazolheterozyklus bei den Protonierungsschritten schließen. 
<smiles>CCNCCN(CCNCC)Cc1cc(C)[nH]n1</smiles>

$\mathrm{L}^{10}$<smiles>CCNCCN(CCNCC)Cc1cc(CN(CC)CCN)[nH]n1</smiles>

$L^{3}$

Abbildung 32: Vergleich von $L^{10}$ und $L^{3}$.

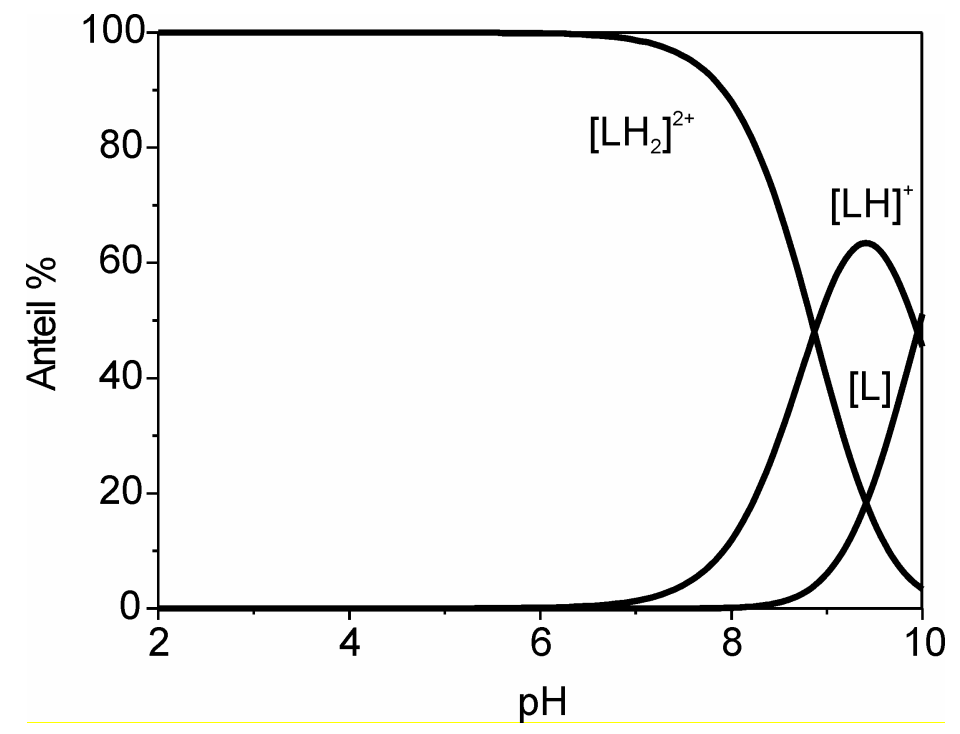

Abbildung 33: Speziesverteilung für $L^{10}$ Bereich von $p H 2$ bis 10.

Die Pyridyl-funktionalisierten Liganden zeigen aufgrund der geringeren Basizität der Pyridinringe verglichen mit den aliphatischen tertiären Aminen sehr viel niedrigere $\mathrm{pK}_{\mathrm{S}}$-Werte. Dies hat unmittelbar zur Folge, dass sich noch weniger der verfügbaren Donorfunktionen im messbaren Bereich von $\mathrm{pH} 2$ bis $\mathrm{pH} 11$ protonieren lassen. Bei $\mathrm{L}^{6}$ lassen sich fünf der sieben $\mathrm{pK}_{\mathrm{S}}$-Werte berechnen (analoger Ligand $\mathrm{L}^{1}$ sechs Werte), bei $\mathrm{L}^{8}$ wie bei $\mathrm{L}^{3}$ aufgrund der geringeren räumlichen Separierung der Protonierungsstellen nur vier von sieben und bei $L^{9}$ nur zwei der fünf Werte $\left(L^{4}\right.$ alle fünf Werte). 


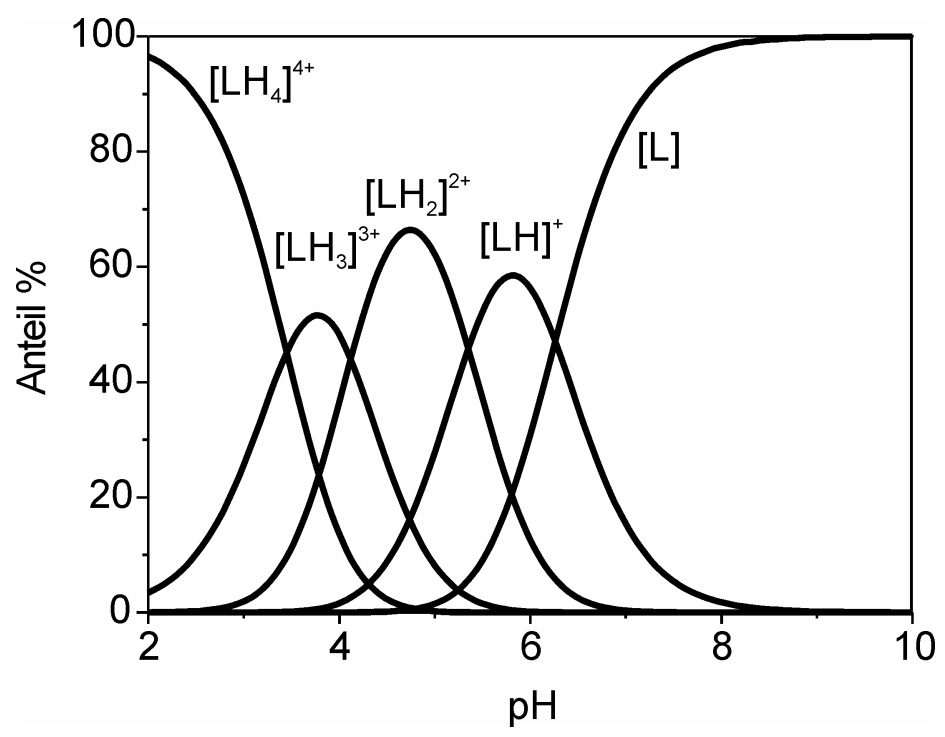

Abbildung 34: Speziesverteilung für $L^{6}$ Bereich von $p H 2$ bis 10.

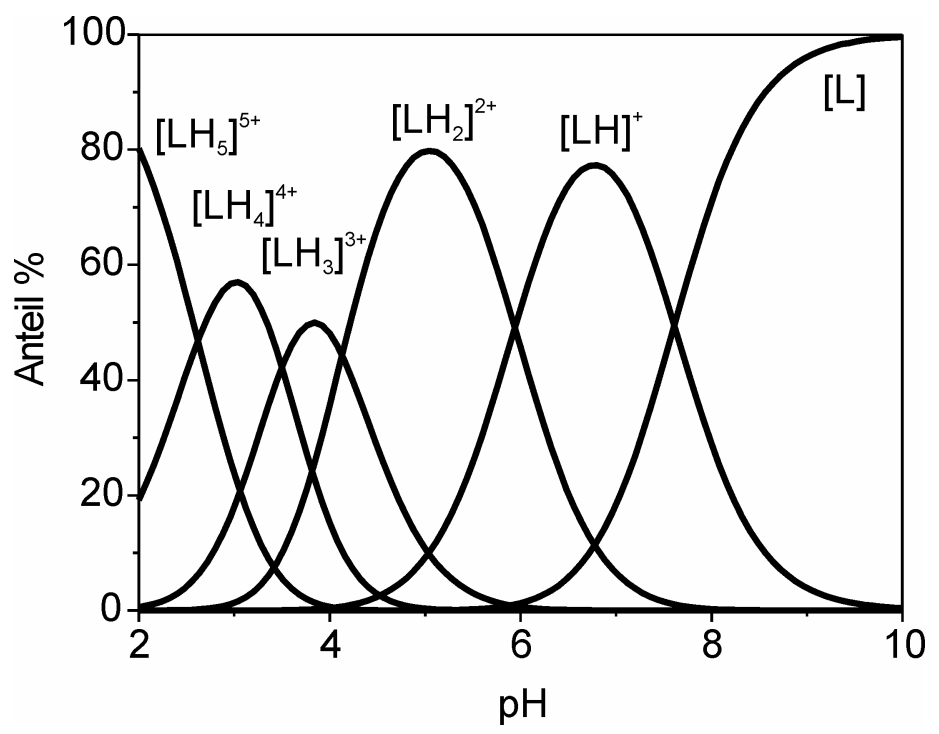

Abbildung 35: Speziesverteilung für $L^{8}$ Bereich von $p H 2$ bis 10.

Gezielte Untersuchungen der mikroskopischen Vorgänge, die üblicherweise durch NMR-Spektroskopie untersucht werden, gestalten sich für diese Liganden sehr schwierig, da es aufgrund der vier identischen Seitenarme zu sehr breiten und sich teilweise überlappenden Signalen kommt.

Für den zu $\mathrm{L}^{4}$ analogen Liganden $\mathrm{L}^{9}$ konnten zwei $\mathrm{pK}_{\mathrm{S}}$-Werte bestimmt werden. Diese entsprechen jeweils einer Protonierung eines Aminomethylpyridylseitenarms und liegen - wie erwartet - deutlich um etwa 2 bzw. 2.5 pKs-Einheiten unter den Werten des aliphatischen Analogons $L^{4}$. 


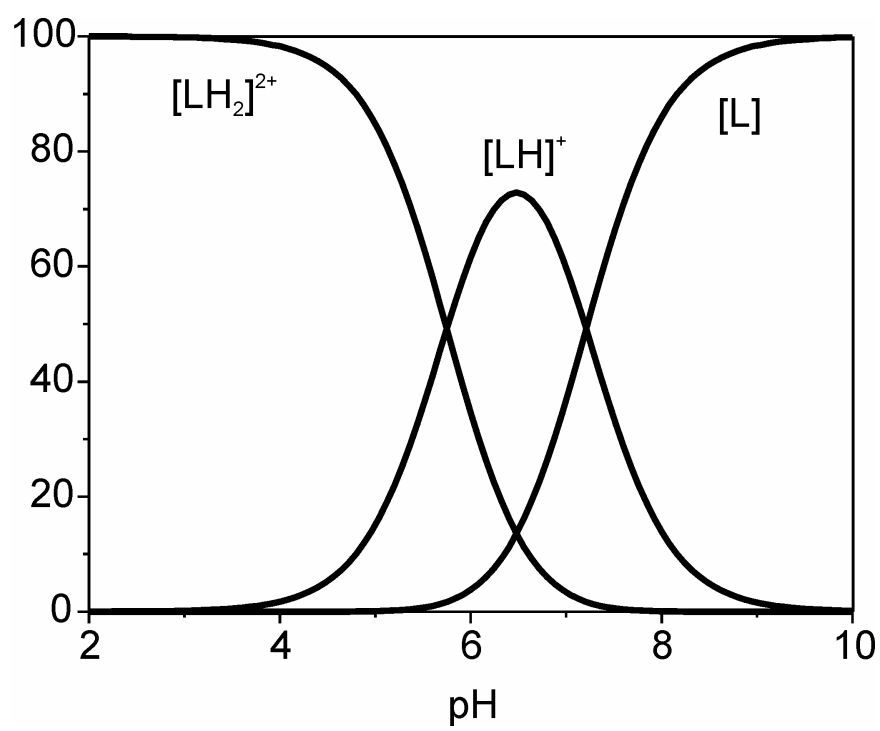

Abbildung 36: Speziesverteilung $L^{9}$ Bereich von $p H 2$ bis 10.

Potentiometrische Bestimmungen der Liganden $L^{2}$ und $L^{7}$ wurden aufgrund der niedrigen Komplexstabilitäten der entsprechenden Dizinkkomplexe nicht durchgeführt. 


\section{Darstellung und Eigenschaften von Pyrazolatodizinkkomplexen}

\subsection{Dizinkpyrazolatokomplexe - Synthesen und}

\section{Festkörperstrukturen}

Um Dizink(II)komplexe der Liganden mit einem metallgebundenen Hydroxid zu erhalten, wurde ein Äquivalent Ligand mit einer Base und zwei Äquivalenten eines Zink(II)salzes umgesetzt. Bei dieser Reaktion wurden zwei Äquivalente der Base verwendet, um zum einen den Pyrazolring zum verbrückenden Pyrazolat zu deprotonieren und zum anderen ein Hydroxid zur Ausbildung einer Zweitbrücke zur Verfügung zu stellen.

Üblicherweise wurde der Ligand mit zwei Äquivalenten Kalium-tert-butylat in Methanol und einem Zinkhexahydratsalz umgesetzt. Als Gegenionen bieten sich an: das koordinierende Nitrat, das - soweit in dieser Arbeit beobachtet - an Zink(II) nicht koordinierende Perchlorat oder das Tetraphenylboratanion. Letzteres muss jedoch durch eine Umfällung mit Natriumtetraphenylborat aus den entsprechenden Perchloratsalzen in einem zusätzlichen Syntheseschritt eingebracht werden.

Die erhaltenen Komplexe wurden schließlich in einem weiteren Schritt durch Kristallisation gereinigt.

Dinukleare Zink(II)komplexe fast aller Liganden konnten dadurch isoliert und vollständig spektroskopisch und strukturell charakterisiert werden. In allen Fällen befinden sich die beiden Zinkionen innerhalb der von den Seitenarmen vorgegebenen Kompartimente und werden durch den zentralen Pyrazolatring verbrückt. Über die Länge der Seitenarme kann dabei der im Wesentlichen durch das Ligandgerüst bestimmte Metall-Metall-Abstand gesteuert werden.

\subsubsection{Komplexe der bis-tetradentaten Liganden $L^{1}, L^{3}, L^{5}, L^{6}$ und $L^{8}$}

In $\left[\mathrm{Zn}_{2} \mathrm{~L}_{-\mathrm{H}}{ }_{\mathrm{H}}(\mathrm{OH})\right]^{2+} 1$ können die Zinkionen aufgrund der langen Propylenseitenarme sehr nahe zusammenrücken $(d(Z n \cdots Z n)=3.613 \AA)$ und erlauben ein verbrückendes Hydroxid als Koliganden in der bimetallischen Tasche (Abbildung 37). Dieser Komplex wurde anstatt nach der in der Literatur beschriebenen Methode, ${ }^{[65]}$ wie oben beschrieben durch Umsetzen des Liganden mit Kalium-tert-butylat und 
Zinkperchlorat dargestellt und durch Überschichten einer Lösung des Komplexes in Aceton mit Petrolether kristallisiert.

Der Komplex wurde als $\mathrm{BPh}_{4}$-Salz schon in vorangegangenen Arbeiten charakterisiert. $^{[65]}$ In Tabelle 3 sind einige wichtige strukturelle Parameter zum Vergleich nochmals aufgeführt.
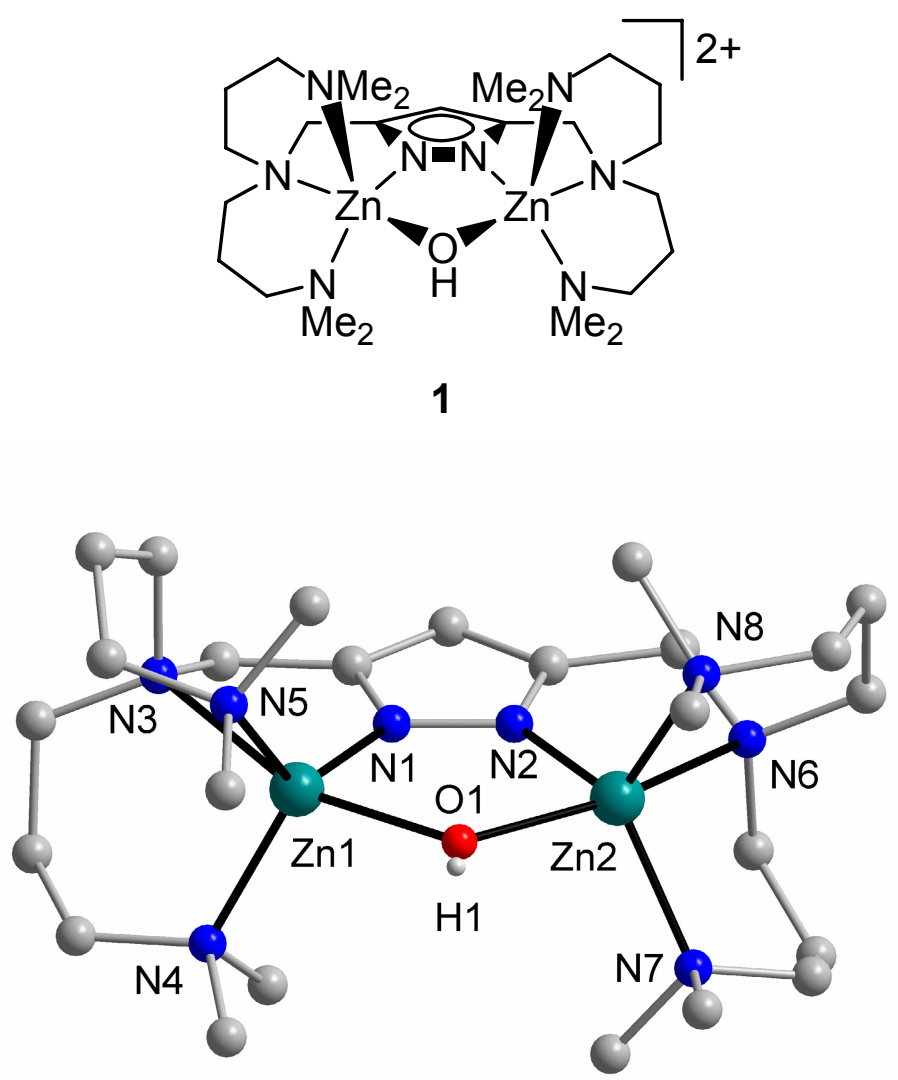

Abbildung 37: Festkörperstruktur des Kations von 1.(BPh $)_{2}{ }^{[65]}$

\begin{tabular}{|l|l|l|l|}
\hline $\mathrm{Zn}(1)-\mathrm{O}(1)$ & $2.132(2)$ & $\mathrm{Zn}(2)-\mathrm{O}(1)$ & $2.149(2)$ \\
\hline $\mathrm{Zn}(1)-\mathrm{N}(1)$ & $1.969(3)$ & $\mathrm{Zn}(2)-\mathrm{N}(2)$ & $1.959(3)$ \\
\hline $\mathrm{Zn}(1)-\mathrm{N}(3)$ & $2.480(3)$ & $\mathrm{Zn}(2)-\mathrm{N}(6)$ & $2.437(3)$ \\
\hline $\mathrm{Zn}(1)-\mathrm{N}(4)$ & $2.089(3)$ & $\mathrm{Zn}(2)-\mathrm{N}(7)$ & $2.099(3)$ \\
\hline $\mathrm{Zn}(1)-\mathrm{N}(5)$ & $2.101(3)$ & $\mathrm{Zn}(2)-\mathrm{N}(8)$ & $2.101(3)$ \\
\hline $\mathrm{Zn}(1) \cdots \mathrm{Zn}(2)$ & 3.613 & & \\
\hline $\mathrm{O}(1)-\mathrm{Zn}(1)-\mathrm{N}(3)$ & $159.2(1)$ & & \\
\hline $\mathrm{O}(2)-\mathrm{Zn}(2)-\mathrm{N}(6)$ & $160.7(1)$ & & \\
\hline $\mathrm{Zn}(1)-\mathrm{O}(1)-\mathrm{Zn}(2)$ & $115.1(1)$ & & \\
\hline
\end{tabular}

Tabelle 3: Ausgewählte Atomabstände ( $\AA$ ) und Bindungswinkel ( $\left.{ }^{\circ}\right)$. 
Im Gegensatz dazu ziehen die kürzeren Seitenarme von $L^{3}$ die zwei Zinkionen in 2a auseinander und erzwingen einen viel längeren Zink-Zink-Abstand. Der durch die beiden kurzen Chelatarme erzwungene lange Metall-Metall-Abstand ist also ungünstig für eine fest gebundene verbrückende Position kleiner Koliganden wie Hydroxid innerhalb der bimetallischen Tasche. ${ }^{[65,83]}$

Da das kleine Hydroxid die beiden Metallionen nicht mehr verbrücken kann, wird ein zusätzliches Lösungsmittelmolekül in die Brücke eingebaut. Dies führt im Fall der Aufnahme von Wasser zu einer $\mathrm{O}_{2} \mathrm{H}_{3}(\mathbf{2 a})$ und im Fall von Methanol zu einer $\mathrm{O}_{2} \mathrm{H}_{2} \mathrm{Me}-\mathrm{Brücke}$ (2b). 2b konnte durch Überschichten einer Lösung des Perchloratsalzes von $\mathbf{2 b}$ in Methanol mit Diethylether erhalten werden. Die Struktur von $\mathbf{2 b}$ ist in Abbildung 38 abgebildet, und ausgewählte interatomare Abstände und Winkel sind in Tabelle 4 aufgeführt.

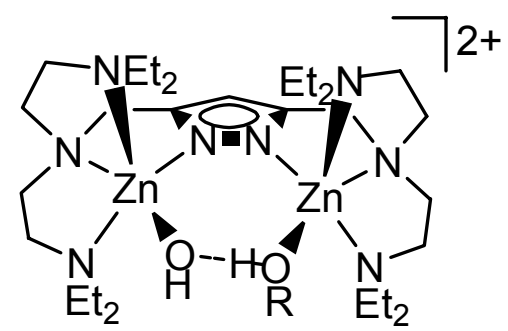

$$
2 a(R=H)
$$$$
2 b(R=M e)
$$

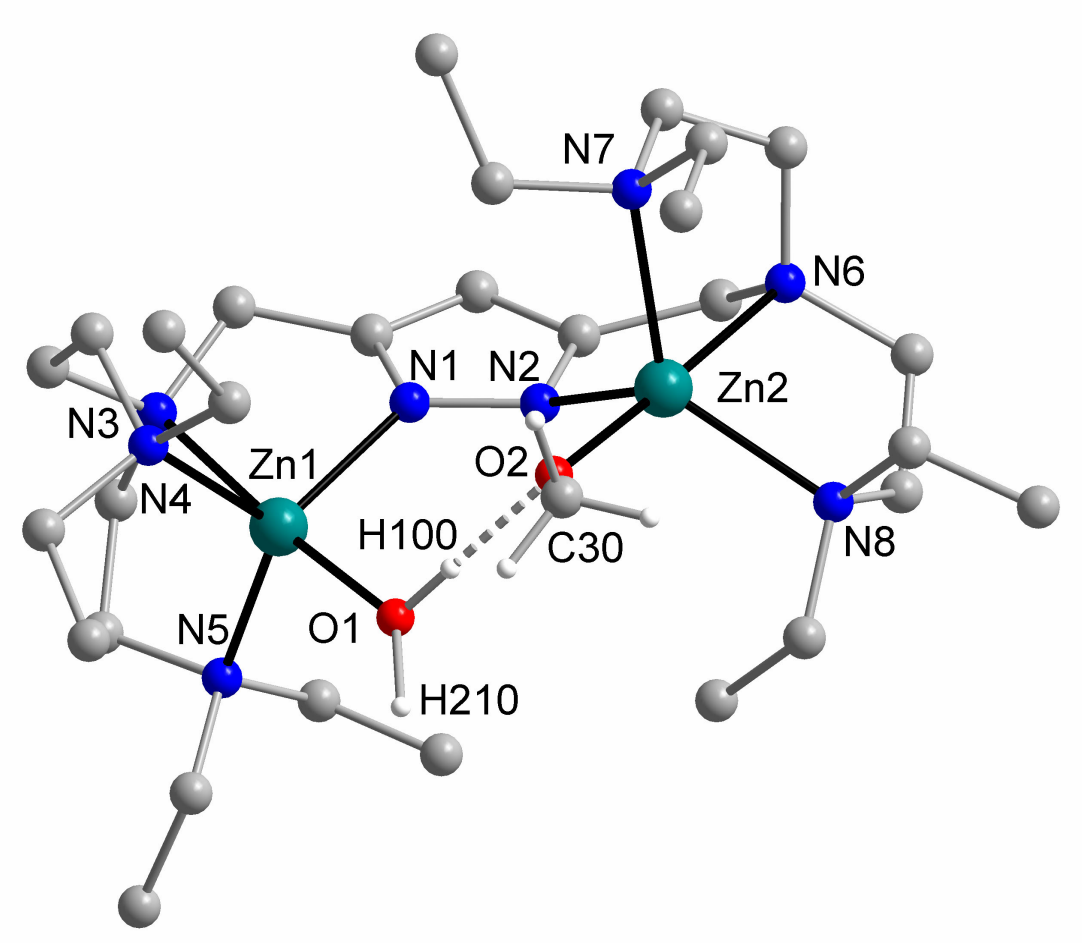

Abbildung 38: Festkörperstruktur des Kations von 2b·(C/O $)_{2}$. 


\begin{tabular}{|c|c|c|c|}
\hline $\mathrm{Zn}(1)-\mathrm{O}(1)$ & $1.982(3)[1.963(2)]$ & $\mathrm{Zn}(2)-\mathrm{O}(2)$ & $1.957(2)[1.958(3)]$ \\
\hline $\mathrm{Zn}(1)-\mathrm{N}(1)$ & $2.041(3)[2.046(3)]$ & $\mathrm{Zn}(2)-\mathrm{N}(2)$ & $2.067(3)[2.033(3)]$ \\
\hline $\mathrm{Zn}(1)-\mathrm{N}(3)$ & $2.280(3)[2.282(3)]$ & $\mathrm{Zn}(2)-\mathrm{N}(6)$ & $\begin{array}{l}2.211(9)[2.343(14)] \\
{[2.269(3)]}\end{array}$ \\
\hline $\mathrm{Zn}(1)-\mathrm{N}(4)$ & $2.169(3)[2.190(3)]$ & $\mathrm{Zn}(2)-\mathrm{N}(7)$ & $\begin{array}{l}2.261(8)[2.111(13)] \\
{[2.183(3)]}\end{array}$ \\
\hline $\mathrm{Zn}(1)-\mathrm{N}(5)$ & $2.135(3)[2.194(3)]$ & $\mathrm{Zn}(2)-\mathrm{N}(8)$ & $2.169(3)[2.148(3)]$ \\
\hline $\mathrm{O}(2)-\mathrm{C}(30)$ & $1.359(6)[1.395(4)]$ & & \\
\hline $\mathrm{O} 1 \cdots \mathrm{O} 2$ & $2.436(46)[2.425(46)]$ & & \\
\hline$N(1)-N(2)$ & $1.377(4)[1.382(4)]$ & & \\
\hline $\mathrm{Zn}(1) \cdots \mathrm{Zn}(2)$ & $4.406(7)[4.334(6)]$ & & \\
\hline $\mathrm{N}(1)-\mathrm{Zn}(1)-\mathrm{O}(1)$ & $96.19(11)[99.22(10)]$ & $\mathrm{N}(2)-\mathrm{Zn}(2)-\mathrm{O}(2)$ & $95.71(11)[100.84(11)]$ \\
\hline $\mathrm{N}(1)-\mathrm{Zn}(1)-\mathrm{N}(4)$ & $114.69(11)[120.52(10)]$ & $\mathrm{N}(2)-\mathrm{Zn}(2)-\mathrm{N}(7)$ & $\begin{array}{l}104.5(2)[104.2(3)] \\
{[109.81(11)]}\end{array}$ \\
\hline $\mathrm{O}(1)-\mathrm{Zn}(1)-\mathrm{N}(4)$ & $100.05(11)[99.86(10)]$ & $\mathrm{O}(2)-\mathrm{Zn}(2)-\mathrm{N}(7)$ & $\begin{array}{l}\text { 96.35(17) [112.3(3)] } \\
{[98.46(11)]}\end{array}$ \\
\hline $\mathrm{N}(1)-\mathrm{Zn}(1)-\mathrm{N}(5)$ & $116.50(11)[111.53(10)]$ & $\mathrm{N}(2)-\mathrm{Zn}(2)-\mathrm{N}(8)$ & $123.67(12)[111.91(10)]$ \\
\hline $\mathrm{O}(1)-\mathrm{Zn}(1)-\mathrm{N}(5)$ & $101.07(12)[100.49(10)]$ & $\mathrm{O}(2)-\mathrm{Zn}(2)-\mathrm{N}(8)$ & $99.60(11)[97.35(11)]$ \\
\hline $\mathrm{N}(4)-\mathrm{Zn}(1)-\mathrm{N}(5)$ & $121.32(11)[119.31(10)]$ & $\mathrm{N}(7)-\mathrm{Zn}(2)-\mathrm{N}(8)$ & $\begin{array}{l}\text { 126.80(19) [118.6(3)] } \\
{[131.28(11)]}\end{array}$ \\
\hline $\mathrm{N}(1)-\mathrm{Zn}(1)-\mathrm{N}(3)$ & $79.44(11)[79.13(10)]$ & $\mathrm{N}(2)-\mathrm{Zn}(2)-\mathrm{N}(6)$ & $\begin{array}{l}84.9(2)[75.1(3)] \\
{[81.17(10)]}\end{array}$ \\
\hline $\mathrm{O}(1)-\mathrm{Zn}(1)-\mathrm{N}(3)$ & $175.63(11)[178.29(9)]$ & $\mathrm{O}(2)-\mathrm{Zn}(2)-\mathrm{N}(6)$ & $\begin{array}{l}\text { 177.7(2) [166.9(3)] } \\
{[177.97(11)]}\end{array}$ \\
\hline $\mathrm{N}(4)-\mathrm{Zn}(1)-\mathrm{N}(3)$ & $81.76(11)[80.66(10)]$ & $\mathrm{N}(7)-\mathrm{Zn}(2)-\mathrm{N}(6)$ & $\begin{array}{l}81.4(2)[79.5(4)] \\
{[81.01(10)]}\end{array}$ \\
\hline $\mathrm{N}(5)-\mathrm{Zn}(1)-\mathrm{N}(3)$ & $81.20(11)[80.61(10)]$ & $\mathrm{N}(8)-\mathrm{Zn}(2)-\mathrm{N}(6)$ & $\begin{array}{l}81.8(2)[78.6(3)] \\
{[81.61(10)]}\end{array}$ \\
\hline
\end{tabular}

Tabelle 4: Ausgewählte Atomabstände $(\AA)$ und Bindungswinkel $\left({ }^{\circ}\right)$.

Beide Metallionen befinden sich in einer leicht verzerrten trigonal-bipyramidalen

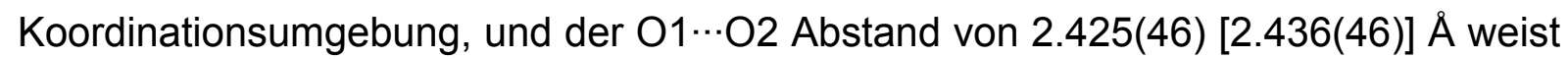
auf eine starke intramolekulare Wasserstoffbrückenbindung hin. Durch die kaum eingeschränkte Rotation der Ethylsubstituenten gibt es im Kristall auch bei $-100{ }^{\circ} \mathrm{C}$ 
keine bevorzugte Konformation, so dass die Ethylgruppen und die Seitenarme fehlgeordnet sind.

Spezies $\mathbf{2 a}$ und $\mathbf{2 b}$ können als solvatisierte Formen eines Zink-gebundenen Hydroxids in Nachbarschaft zu einer zugänglichen Koordinationsstelle eines nahen zweiten Zinkions angesehen werden, da das zusätzliche Solvensmolekül leicht durch ein Substratmolekül austauschbar sein sollte. Der experimentelle Befund, dass 2a nach und nach $\mathrm{CO}_{2}$ aus der Luft absorbiert und einen Carbonat-verbrückten Komplex bildet, wohingegen diese Reaktion bei 1 ausbleibt, ${ }^{[65]}$ untermauert diese Betrachtungsweise und liefert einen Hinweis auf die erhöhte Reaktivität einer derart maskierten terminalen Zinkhydroxidform in $\mathbf{2 a}$ und $\mathbf{2 b}$ gegenüber eines fester gebundenen verbrückenden Hydroxids wie in 1.

Da die durch Zink(II) vermittelten Hydrolysereaktionen idealerweise in gepufferten wässrigen Lösungen zu untersuchen sind, wurde Ligand $L^{5}$ mit auf Triazacyclononan basierenden Seitenarmen auch in die Arbeit aufgenommen. Komplexe des tacnLiganden zeichnen sich aufgrund des makrozyklischen Effekts durch sehr hohe Stabilitätskonstanten aus.

$\mathrm{Da}$ sich dieser Ligand topologisch von $\mathrm{L}^{3}$ durch Verbinden der äußeren $\mathrm{N}$ Donoratome ableiten lässt, wurde erwartet, dass der tacn-Ligand aufgrund der gleich kurzen Seitenarme eine ähnliche Situation wie in 2 induziert, zusätzlich aber auch dem resultierenden Dizinkkomplex eine viel höhere Stabilität in wässriger Lösung verleiht. Unerwarteterweise jedoch wurde in der Röntgenstrukturanalyse ein verbrückendes Hydroxid im Dizinkkomplex $\left[\mathrm{Zn}_{2} \mathrm{~L}^{5}{ }_{-\mathrm{H}}(\mathrm{OH})\right]\left(\mathrm{ClO}_{4}\right)_{2}$ (3) gefunden (Abbildung 39). Dies ist umso erstaunlicher, da die erwartete verbrückende $\mathrm{O}_{2} \mathrm{H}_{3}$ Einheit in den verwandten Dinickel(II)- und Dikupferkomplexen von $\mathrm{L}^{5}$ beobachtet wurde..$^{[62,64]}$

Eine genauere Betrachtung zeigt, dass der sehr kurze $\mathrm{Zn} \cdots \mathrm{Zn}$-Abstand in 3 $(3.460(3) \AA)$ durch eine signifikante Verlängerung der Bindungen zwischen den Zinkionen und den Brückenkopf-Stickstoffatomen ausgeglichen wird $(d(\mathrm{Zn}(1)-\mathrm{N}(3))=$ 2.470(2) und $d(\mathrm{Zn}(2)-\mathrm{N}(6))=2.401(2) \AA)$, so dass eine $\{4+1\}$-Koordination die Umgebung beider Zinkionen in den Hydroxy-verbrückten Komplexen wohl am zutreffendsten beschreibt. Ausgewählte Bindungslängen und Winkel für 3 finden sich in Tabelle 5. 


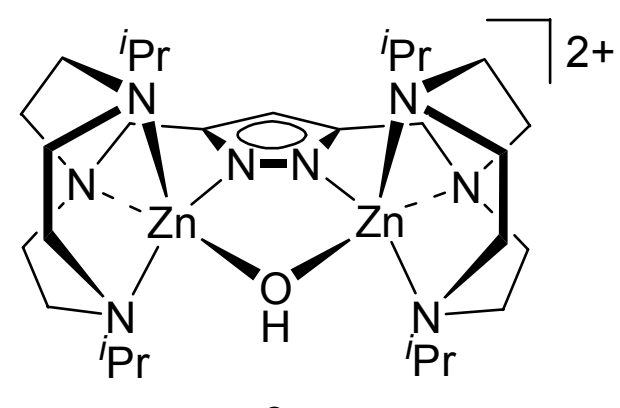

3

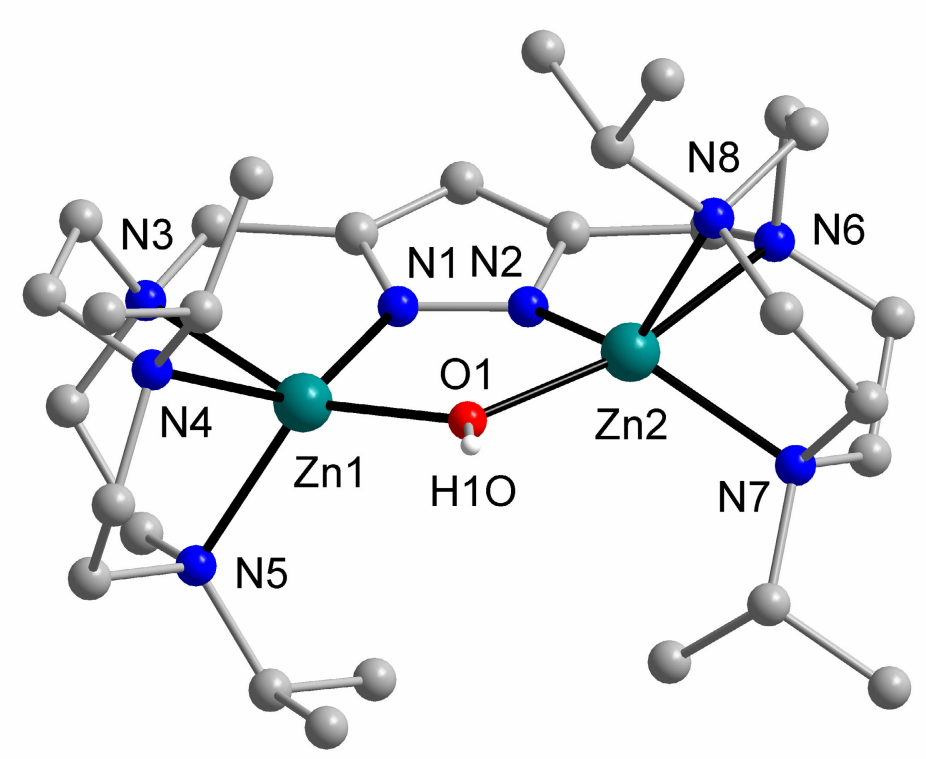

Abbildung 39: Festkörperstruktur des Kations von 3.( $\left(\mathrm{ClO}_{4}\right)_{2}$.

\begin{tabular}{|l|l|l|l|}
\hline $\mathrm{Zn}(1)-\mathrm{N}(1)$ & $1.955(2)$ & $\mathrm{Zn}(2)-\mathrm{N}(2)$ & $1.965(2)$ \\
\hline $\mathrm{Zn}(1)-\mathrm{O}(1)$ & $2.000(2)$ & $\mathrm{Zn}(2)-\mathrm{O}(1)$ & $2.0118(19)$ \\
\hline $\mathrm{Zn}(1)-\mathrm{N}(4)$ & $2.104(2)$ & $\mathrm{Zn}(2)-\mathrm{N}(7)$ & $2.104(2)$ \\
\hline $\mathrm{Zn}(1)-\mathrm{N}(5)$ & $2.137(2)$ & $\mathrm{Zn}(2)-\mathrm{N}(8)$ & $2.125(2)$ \\
\hline $\mathrm{Zn}(1)-\mathrm{N}(3)$ & $2.470(2)$ & $\mathrm{Zn}(2)-\mathrm{N}(6)$ & $2.401(2)$ \\
\hline $\mathrm{N}(1)-\mathrm{N}(2)$ & $1.353(3)$ & & \\
\hline $\mathrm{Zn}(1) \cdots \mathrm{Zn}(2)$ & $3.460(3)$ & & \\
\hline $\mathrm{N}(1)-\mathrm{Zn}(1)-\mathrm{O}(1)$ & $87.89(9)$ & $\mathrm{N}(2)-\mathrm{Zn}(2)-\mathrm{O}(1)$ & $87.13(9)$ \\
\hline $\mathrm{N}(1)-\mathrm{Zn}(1)-\mathrm{N}(4)$ & $138.03(10)$ & $\mathrm{N}(2)-\mathrm{Zn}(2)-\mathrm{N}(7)$ & $133.09(9)$ \\
\hline $\mathrm{O}(1)-\mathrm{Zn}(1)-\mathrm{N}(4)$ & $109.27(9)$ & $\mathrm{O}(1)-\mathrm{Zn}(2)-\mathrm{N}(7)$ & $116.06(8)$ \\
\hline $\mathrm{N}(1)-\mathrm{Zn}(1)-\mathrm{N}(5)$ & $117.27(10)$ & $\mathrm{N}(2)-\mathrm{Zn}(2)-\mathrm{N}(8)$ & $125.02(10)$ \\
\hline $\mathrm{O}(1)-\mathrm{Zn}(1)-\mathrm{N}(5)$ & $121.93(9)$ & $\mathrm{O}(1)-\mathrm{Zn}(2)-\mathrm{N}(8)$ & $108.83(8)$ \\
\hline $\mathrm{N}(4)-\mathrm{Zn}(1)-\mathrm{N}(5)$ & $86.41(9)$ & $\mathrm{N}(7)-\mathrm{Zn}(2)-\mathrm{N}(8)$ & $87.28(9)$ \\
\hline $\mathrm{N}(1)-\mathrm{Zn}(1)-\mathrm{N}(3)$ & $72.07(9)$ & $\mathrm{N}(2)-\mathrm{Zn}(2)-\mathrm{N}(6)$ & $74.28(9)$ \\
\hline
\end{tabular}




\begin{tabular}{|l|l|l|l|}
\hline $\mathrm{O}(1)-\mathrm{Zn}(1)-\mathrm{N}(3)$ & $156.79(8)$ & $\mathrm{O}(1)-\mathrm{Zn}(2)-\mathrm{N}(6)$ & $160.97(8)$ \\
\hline $\mathrm{N}(4)-\mathrm{Zn}(1)-\mathrm{N}(3)$ & $80.20(8)$ & $\mathrm{N}(7)-\mathrm{Zn}(2)-\mathrm{N}(6)$ & $80.65(8)$ \\
\hline $\mathrm{N}(5)-\mathrm{Zn}(1)-\mathrm{N}(3)$ & $78.84(9)$ & $\mathrm{N}(8)-\mathrm{Zn}(2)-\mathrm{N}(6)$ & $79.63(8)$ \\
\hline $\mathrm{Zn}(1)-\mathrm{O}(1)-\mathrm{Zn}(2)$ & $119.17(10)$ & & \\
\hline
\end{tabular}

Tabelle 5: Ausgewählte Atomabstände ( $\AA$ ) und Bindungswinkel ( $\left.{ }^{\circ}\right)$.

Die vorgestellten Komplexe 1 und 2 mit aliphatischen Liganden sind für Untersuchungen der Hydrolysereaktivität zwar geeignet, erweisen sich jedoch als nur beschränkt stabil in den für die Reaktivitätsuntersuchungen erwünschten wässrigen Lösungen. Ersetzt man die terminalen, tertiären Aminfunktionen dieser Liganden durch Pyrididylreste, so erhält man Liganden, die zwar topologisch den bisherigen entsprechen, in wässrigen Lösungen aber deutlich stabiler sein sollten.

Setzt man den zu $L^{1}$ analogen Liganden $L^{6}$ mit je zwei Äquivalenten Zinkperchlorat und Base um, so erhält man den zu 1 strukturell analogen Komplex $\left[\mathrm{Zn}_{2} \mathrm{~L}^{6}\right.$. $\left.{ }_{H}(\mathrm{OH})\right]\left(\mathrm{ClO}_{4}\right)_{2}$ (4). Auch hier lassen die relativ langen Ethylpyridyl-Seitenarme die Ausbildung einer stabilen Hydroxidbrücke zu. Damit einher geht ein sehr kurzer ZinkZink-Abstand von 3.479(1) [3.465(1)] $\AA$ - rund $0.13 \AA$ kürzer als der Zink-ZinkAbstand bei 1.

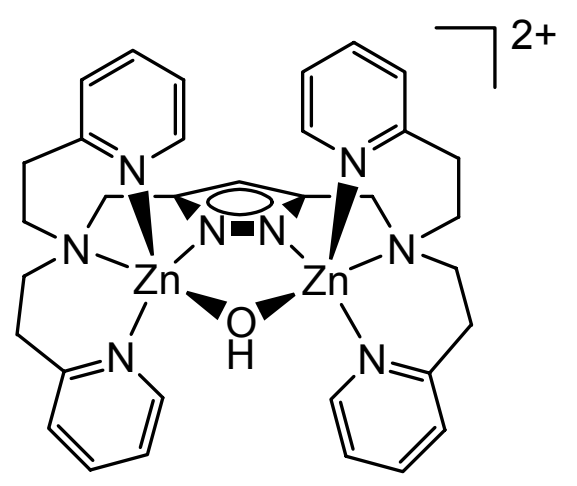

4 


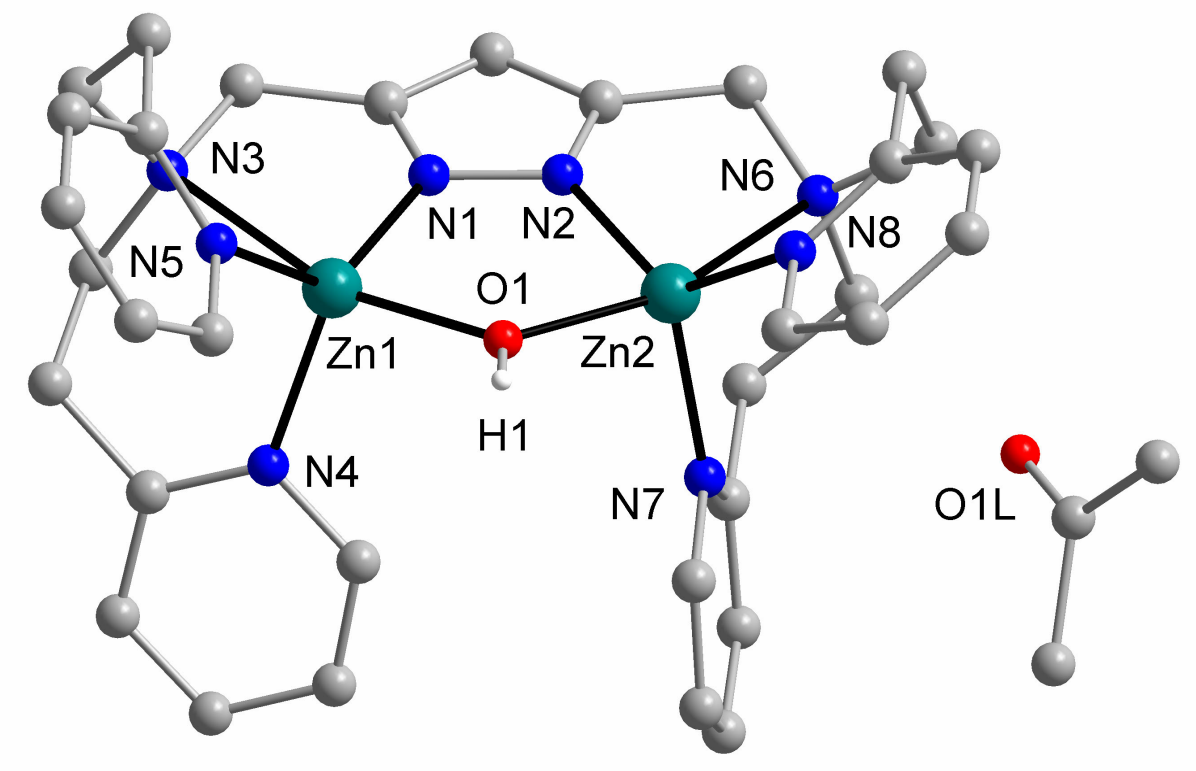

Abbildung 40: Festkörperstruktur des Kations von 4.( $\left(\mathrm{ClO}_{4}\right)_{2}$.

\begin{tabular}{|l|l|l|l|}
\hline $\mathrm{Zn}(1)-\mathrm{N}(1)$ & $1.956(2)[1.965(2)]$ & $\mathrm{Zn}(2)-\mathrm{N}(2)$ & $1.961(2)[1.966(2)]$ \\
\hline $\mathrm{Zn}(1)-\mathrm{O}(1)$ & $2.013(2)[2.020(2)]$ & $\mathrm{Zn}(2)-\mathrm{N}(8)$ & $2.054(2)[2.057(2)]$ \\
\hline $\mathrm{Zn}(1)-\mathrm{N}(5)$ & $2.047(2)[2.038(2)]$ & $\mathrm{Zn}(2)-\mathrm{O}(1)$ & $2.059(2)[2.045(2)]$ \\
\hline $\mathrm{Zn}(1)-\mathrm{N}(4)$ & $2.072(3)[2.073(2)]$ & $\mathrm{Zn}(2)-\mathrm{N}(7)$ & $2.072(2)[2.080(2)]$ \\
\hline $\mathrm{Zn}(1)-\mathrm{N}(3)$ & $2.535(2)[2.522(2)]$ & $\mathrm{Zn}(2)-\mathrm{N}(6)$ & $2.427(2)[2.453(2)]$ \\
\hline $\mathrm{N}(1)-\mathrm{N}(2)$ & $1.367(3)[1.361(3)]$ & & \\
\hline $\mathrm{Zn}(1) \cdots \mathrm{Zn}(2)$ & $3.479(1)[3.465(1)]$ & & \\
\hline $\mathrm{N}(1)-\mathrm{Zn}(1)-\mathrm{O}(1)$ & $88.37(9)[88.35(9)]$ & $\mathrm{N}(2)-\mathrm{Zn}(2)-\mathrm{N}(8)$ & $129.33(9)[126.88(9)]$ \\
\hline $\mathrm{N}(1)-\mathrm{Zn}(1)-\mathrm{N}(5)$ & $130.86(9)[134.31(9)]$ & $\mathrm{N}(2)-\mathrm{Zn}(2)-\mathrm{O}(1)$ & $86.59(8)[87.24(9)]$ \\
\hline $\mathrm{O}(1)-\mathrm{Zn}(1)-\mathrm{N}(5)$ & $103.74(8)[103.91(9)]$ & $\mathrm{N}(8)-\mathrm{Zn}(2)-\mathrm{O}(1)$ & $101.06(8)[99.58(9)]$ \\
\hline $\mathrm{N}(1)-\mathrm{Zn}(1)-\mathrm{N}(4)$ & $116.55(9)[112.75(9)]$ & $\mathrm{N}(2)-\mathrm{Zn}(2)-\mathrm{N}(7)$ & $116.64(9)[120.16(9)]$ \\
\hline $\mathrm{O}(1)-\mathrm{Zn}(1)-\mathrm{N}(4)$ & $101.44(9)[99.62(8)]$ & $\mathrm{N}(8)-\mathrm{Zn}(2)-\mathrm{N}(7)$ & $111.98(9)[110.81(8)]$ \\
\hline $\mathrm{N}(5)-\mathrm{Zn}(1)-\mathrm{N}(4)$ & $107.47(9)[108.31(9)]$ & $\mathrm{O}(1)-\mathrm{Zn}(2)-\mathrm{N}(7)$ & $97.11(8)[98.59(8)]$ \\
\hline $\mathrm{N}(1)-\mathrm{Zn}(1)-\mathrm{N}(3)$ & $72.46(8)[72.55(8)]$ & $\mathrm{N}(2)-\mathrm{Zn}(2)-\mathrm{N}(6)$ & $74.67(8)[74.36(9)]$ \\
\hline $\mathrm{O}(1)-\mathrm{Zn}(1)-\mathrm{N}(3)$ & $160.68(8)[160.67(8)]$ & $\mathrm{N}(8)-\mathrm{Zn}(2)-\mathrm{N}(6)$ & $91.70(8)[92.25(9)]$ \\
\hline $\mathrm{N}(5)-\mathrm{Zn}(1)-\mathrm{N}(3)$ & $87.67(8)[87.98(9)]$ & $\mathrm{O}(1)-\mathrm{Zn}(2)-\mathrm{N}(6)$ & $161.24(8)[161.58(8)]$ \\
\hline $\mathrm{N}(4)-\mathrm{Zn}(1)-\mathrm{N}(3)$ & $89.60(9)[90.78(8)]$ & $\mathrm{N}(7)-\mathrm{Zn}(2)-\mathrm{N}(6)$ & $90.67(8)[90.23(8)]$ \\
\hline $\begin{array}{l}\mathrm{Zn}(1)-\mathrm{O}(1)- \\
\mathrm{Zn}(2)\end{array}$ & $117.41(10)[116.93(11)]$ & & \\
\hline
\end{tabular}

Tabelle 6: Ausgewählte Atomabstände ( $\AA$ ) und Bindungswinkel ( $\left.{ }^{\circ}\right)$. 
Wie aus Tabelle 6 ersichtlich, unterscheiden sich die $\mathrm{Zn}-\mathrm{O}$ und $\mathrm{Zn}-\mathrm{N}$ Bindungslängen kaum von denen des aliphatischen Komplexes 1. Man findet wiederum $\mathrm{Zn}-\mathrm{O}-$ Bindungslängen von ungefähr 2.0 $\AA$, kurze Zn-N-Abstände (2.1 $\AA$ ) zu den insgesamt vier terminalen Pyridyl-N-Atomen sowie den beiden Pyrazolat-N-Atomen und relativ lange Zn-N-Abstände (2.4 - $2.5 \AA$ ) zu den beiden Brückenkopf-N-Atomen. Auch die Koordinationsgeometrie der beiden Komplexe unterscheidet sich kaum. Mit einem Wert von $\tau=(0.50$ [0.44], 0.53 [0.58]) liegt sie auch hier zwischen einer verzerrt trigonal-bipyramidalen und quadratisch-planaren Anordnung.

In der Struktur ist ein Acetonmolekül in enger Nachbarschaft zu einem der beiden Zinkionen zu finden $(\mathrm{d}(\mathrm{Zn}(2) \cdots \mathrm{O} 1 \mathrm{~L}=4 \AA)$. Dies führt zu einer Aufweitung der Winkel an $Z n(2)$ (z.B. N(8)-Zn(2)-N(7) 111.98(9) gegenüber N(5)-Zn(1)-N(4) 107.47(9)). Jedoch zeigt sich keine Verschiebung der Aceton-Carbonylbande im IR-Spektrum von 4, so dass eine direkte Wechselwirkung zu Zn(2) auszuschließen ist.

Auch für den zu $L^{3}$ analogen Ligand $L^{7}$ konnte durch Umsetzen des Liganden mit zwei Äquivalenten Base und Zinkperchlorat in Methanol ein zu $\mathbf{2 b}$ analoger Komplex $\left[\mathrm{Zn}_{2} \mathrm{~L}^{7}{ }_{-H}(\mathrm{OH})(\mathrm{MeOH})\right]\left(\mathrm{ClO}_{4}\right)_{2}\left(\mathbf{5} \cdot\left(\mathrm{ClO}_{4}\right)_{2}\right)$ gewonnen werden, dessen Kernstruktur der von $\mathbf{2 b}$ entspricht. Kristalle konnten sowohl durch Überschichten der Komplexlösung in Aceton mit Petrolether, als auch durch Abkühlen einer methanolischen Lösung des Komplexes erhalten werden - erstere Methode lieferte Kristalle besserer Qualität. Für die präparative Darstellung des Komplexes ist jedoch letztere Methode besser geeignet, da der Maßstab beliebig vergrößert werden kann. Die Röntgenstrukturanalyse liefert für die Bindungsparameter des Kations $\mathbf{5}$ für beide Methoden fast identische Werte, so dass hier nur die Daten der für die Reaktivitätsuntersuchungen auch verwendeten, aus Methanol erhaltenen Kristalle diskutiert werden sollen.

Wie in 2 bewirken auch in diesem System die kurzen Seitenarme einen relativ langen Zink-Zink-Abstand von 4.1518(6) $\AA$, so dass ein zusätzliches Lösungsmittelmolekül in die Zweitbrücke eingebaut wird. Der $\mathrm{O} \cdots \mathrm{O}-\mathrm{Abstand}$ in der $\mathrm{O}_{2} \mathrm{H}_{2} \mathrm{Me}$-Brücke ist verglichen mit $\mathbf{2 b}$ etwas kürzer $(d(O \cdots O)=2.390 \AA)$. Dies legt eine noch stärkere Wasserstoffbrückenbindung als in $\mathbf{2 b}$ nahe. 


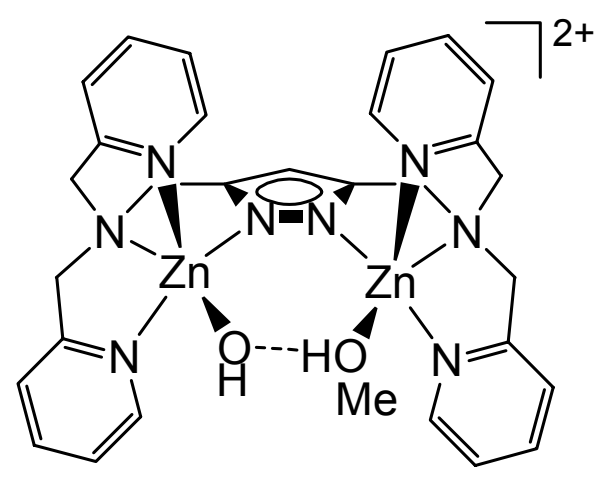

5

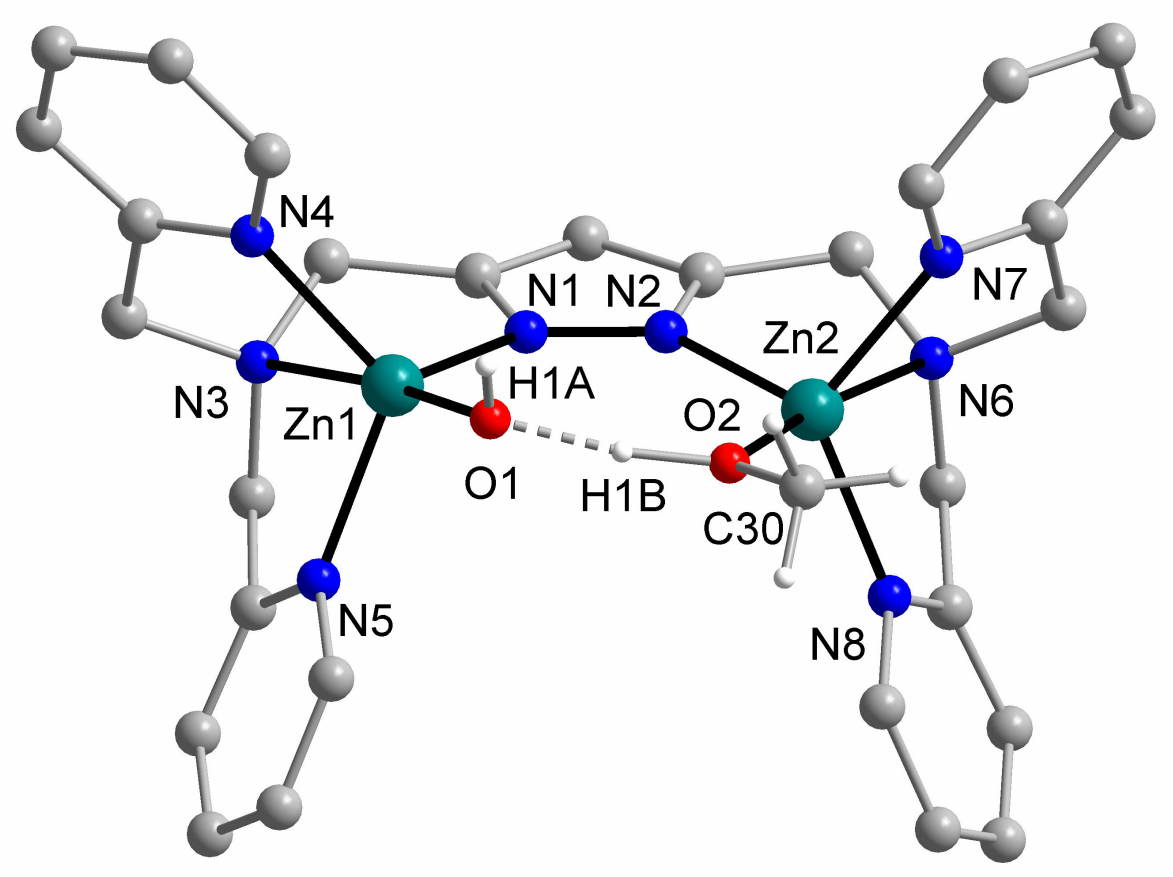

Abbildung 41: Festkörperstruktur des Kations von 5. $\left(\mathrm{ClO}_{4}\right)_{2}$.

\begin{tabular}{|l|l|l|l|}
\hline $\mathrm{Zn}(1)-\mathrm{O}(1)$ & $1.950(3)$ & $\mathrm{Zn}(2)-\mathrm{O}(2)$ & $1.950(3)$ \\
\hline $\mathrm{Zn}(1)-\mathrm{N}(1)$ & $2.023(3)$ & $\mathrm{Zn}(2)-\mathrm{N}(2)$ & $2.008(3)$ \\
\hline $\mathrm{Zn}(1)-\mathrm{N}(3)$ & $2.307(3)$ & $\mathrm{Zn}(2)-\mathrm{N}(6)$ & $2.305(3)$ \\
\hline $\mathrm{Zn}(1)-\mathrm{N}(5)$ & $2.075(3)$ & $\mathrm{Zn}(2)-\mathrm{N}(7)$ & $2.066(3)$ \\
\hline $\mathrm{Zn}(1)-\mathrm{N}(4)$ & $2.092(3)$ & $\mathrm{Zn}(2)-\mathrm{N}(8)$ & $2.076(3)$ \\
\hline $\mathrm{N}(1)-\mathrm{N}(2)$ & $1.376(4)$ & & \\
\hline $\mathrm{Zn}(1) \cdots \mathrm{Zn}(2)$ & $4.1518(6)$ & & \\
\hline $\mathrm{O}(1) \cdots \mathrm{O}(2)$ & 2.390 & & \\
\hline $\mathrm{O}(1)-\mathrm{Zn}(1)-\mathrm{N}(1)$ & $109.46(13)$ & $\mathrm{O}(2)-\mathrm{Zn}(2)-\mathrm{N}(2)$ & $107.82(12)$ \\
\hline $\mathrm{O}(1)-\mathrm{Zn}(1)-\mathrm{N}(3)$ & $173.47(12)$ & $\mathrm{O}(2)-\mathrm{Zn}(2)-\mathrm{N}(6)$ & $173.81(12)$ \\
\hline
\end{tabular}




\begin{tabular}{|l|l|l|l|}
\hline $\mathrm{O}(1)-\mathrm{Zn}(1)-\mathrm{N}(4)$ & $102.97(13)$ & $\mathrm{O}(2)-\mathrm{Zn}(2)-\mathrm{N}(7)$ & $101.23(13)$ \\
\hline $\mathrm{O}(1)-\mathrm{Zn}(1)-\mathrm{N}(5)$ & $97.86(13)$ & $\mathrm{O}(2)-\mathrm{Zn}(2)-\mathrm{N}(8)$ & $98.92(13)$ \\
\hline $\mathrm{N}(1)-\mathrm{Zn}(1)-\mathrm{N}(3)$ & $76.52(12)$ & $\mathrm{N}(2)-\mathrm{Zn}(2)-\mathrm{N}(6)$ & $78.30(12)$ \\
\hline $\mathrm{N}(1)-\mathrm{Zn}(1)-\mathrm{N}(4)$ & $115.29(13)$ & $\mathrm{N}(2)-\mathrm{Zn}(2)-\mathrm{N}(7)$ & $109.50(13)$ \\
\hline $\mathrm{N}(1)-\mathrm{Zn}(1)-\mathrm{N}(5)$ & $111.65(12)$ & $\mathrm{N}(2)-\mathrm{Zn}(2)-\mathrm{N}(8)$ & $116.14(12)$ \\
\hline $\mathrm{N}(3)-\mathrm{Zn}(1)-\mathrm{N}(4)$ & $76.08(12)$ & $\mathrm{N}(6)-\mathrm{Zn}(2)-\mathrm{N}(7)$ & $77.07(12)$ \\
\hline $\mathrm{N}(3)-\mathrm{Zn}(1)-\mathrm{N}(5)$ & $77.14(12)$ & $\mathrm{N}(6)-\mathrm{Zn}(2)-\mathrm{N}(8)$ & $77.16(12)$ \\
\hline $\mathrm{N}(4)-\mathrm{Zn}(1)-\mathrm{N}(5)$ & $117.38(13)$ & $\mathrm{N}(7)-\mathrm{Zn}(2)-\mathrm{N}(8)$ & $120.61(13)$ \\
\hline
\end{tabular}

Tabelle 7: Ausgewählte Atomabstände (Å) und Bindungswinkel ( ${ }^{\circ}$ ).

Im Unterschied zu den aliphatischen Komplexen erhält man für den Pyridylkomplex im ${ }^{1} \mathrm{H}$-NMR-Spektrum scharfe Signale für vier äquivalente Seitenarme. Es findet also in Lösung ein relativ schneller Austausch der Brücke statt.

\subsubsection{Komplexe der bis-tridentaten Liganden $L^{2}, L^{4}, L^{7}$ und $L^{9}$}

Die bis-tridentaten Liganden $L^{2}, L^{4}, L^{7}$ und $L^{9}$ stellen aufgrund ihres Aufbaus eine vielversprechende Basis für Struktur- und Reaktivitätsuntersuchungen dar. Zum Ersten sorgt die jeweils geringe Zahl von Donoratomen der beiden Kompartimente für die Gegenwart von potentiell frei zugänglichen Koordinationsstellen an beiden Metallionen, die für die Anbindung von Substraten zur Verfügung stehen sollten. Zweitens sollte es auch hier möglich sein, durch die Steuerung der Länge der Chelatarme den Zink-Zink-Abstand zu kontrollieren und damit wieder eine Differenzierung in Systeme mit $\mu$-Hydroxid-Funktionen und in solche mit pseudoterminalen Hydroxiden in einer langen $\mathrm{H}_{3} \mathrm{O}_{2}$-Brücke zu erreichen.

Um auch tatsächlich eine monomere $\left[\mathrm{LZn}_{2} \mathrm{X}_{3}\right]$-Spezies zu erhalten und die Bildung höhernuklearer Aggregate zu unterbinden, wurden die Reaktionen in Gegenwart eines Koliganden $\mathrm{X}$ durchgeführt, der alle freien Koordinationsstellen blockiert. Nitrat erwies sich in dieser Hinsicht als geeignet. Um auch den Einbau von $\mathrm{CO}_{2}$ aus der Atmosphäre als Carbonat zu verhindern, wurden diese Reaktionen unter Stickstoff durchgeführt.

Komplex 6 wurde durch Umsetzen von $\mathrm{L}^{2}$ mit zwei Äquivalenten $\mathrm{Zn}\left(\mathrm{NO}_{3}\right)_{2}$ und zwei Äquivalenten $\mathrm{KO}^{t} \mathrm{Bu}$ erhalten. Wie eine spätere Analyse ergab, lag der Ligand zu 
einem geringen Anteil als Hydrochlorid $\mathrm{L}^{2} \cdot \mathrm{xHCl}$ vor. So findet sich in den erhaltenen Kristallen ein zusätzliches Chloridanion, welches als Zweitbrücke in den Komplex eingebaut wird (Abbildung 42). Wie erwartet nimmt $L^{2}$ in beiden Kompartimenten je ein Zinkion in tridentater Koordination auf. Das Chloridion verbrückt die beiden Zinkionen und die beiden Nitratanionen sitzen als semi-bidentate terminale Liganden an jedem Zinkion. Es zeigt sich bei beiden Nitraten ein kurzer Zink-SauerstoffAbstand $(\mathrm{d}(\mathrm{Zn}(1)-\mathrm{O}(5))=2.028(2), \mathrm{d}(\mathrm{Zn}(2)-\mathrm{O}(4))=2.020(3) \AA)$ und ein signifikant längerer $(\mathrm{d}(\mathrm{Zn}(1)-\mathrm{O}(6))=2.704(2), \mathrm{d}(\mathrm{Zn}(2)-\mathrm{O}(3))=2.680(3) \AA)$. Die Metallionen sind demzufolge jeweils in einer verzerrt oktaedrischen Koordinationsumgebung eingebettet.
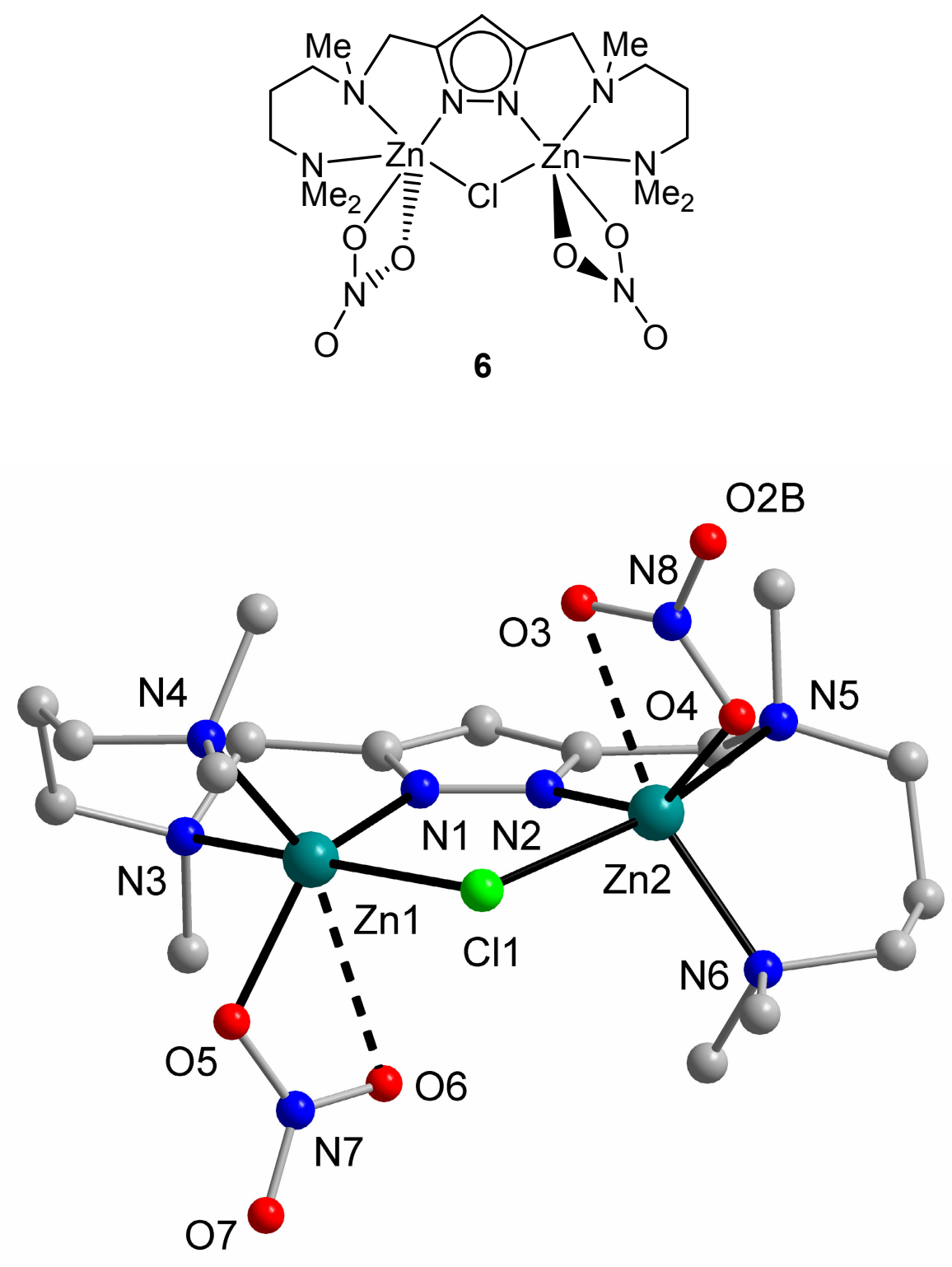

Abbildung 42: Festkörperstruktur von 6. 


\begin{tabular}{|c|c|c|c|}
\hline $\mathrm{Zn}(1)-\mathrm{N}(1)$ & $1.970(3)$ & $\mathrm{Zn}(2)-\mathrm{N}(2)$ & $1.968(3)$ \\
\hline $\mathrm{Zn}(1)-\mathrm{O}(5)$ & $2.028(2)$ & $\mathrm{Zn}(2)-\mathrm{O}(4)$ & $2.020(3)$ \\
\hline $\mathrm{Zn}(1)-\mathrm{N}(4)$ & $2.085(2)$ & $\mathrm{Zn}(2)-\mathrm{N}(6)$ & $2.089(3)$ \\
\hline $\mathrm{Zn}(1)-\mathrm{N}(3)$ & $2.379(3)$ & $\mathrm{Zn}(2)-\mathrm{N}(5)$ & $2.363(3)$ \\
\hline $\mathrm{Zn}(1)-\mathrm{Cl}(1)$ & $2.462(2)$ & $\mathrm{Zn}(2)-\mathrm{Cl}(1)$ & $2.516(1)$ \\
\hline $\mathrm{Zn}(1)-\mathrm{O}(6)$ & $2.704(2)$ & $\mathrm{Zn}(2)-\mathrm{O}(3)$ & $2.680(3)$ \\
\hline $\mathrm{N}(1)-\mathrm{N}(2)$ & $1.368(3)$ & & \\
\hline $\mathrm{Zn}(1) \cdots \mathrm{Zn}(2)$ & $3.831(2)$ & & \\
\hline $\mathrm{N}(1)-\mathrm{Zn}(1)-\mathrm{O}(5)$ & $139.14(10)$ & $\mathrm{N}(2)-\mathrm{Zn}(2)-\mathrm{O}(4)$ & $140.36(11)$ \\
\hline $\mathrm{N}(1)-\mathrm{Zn}(1)-\mathrm{N}(4)$ & $117.37(11)$ & $\mathrm{N}(2)-\mathrm{Zn}(2)-\mathrm{N}(6)$ & $120.88(11)$ \\
\hline $\mathrm{O}(5)-\mathrm{Zn}(1)-\mathrm{N}(4)$ & $101.62(10)$ & $\mathrm{O}(4)-\mathrm{Zn}(2)-\mathrm{N}(6)$ & $97.43(11)$ \\
\hline$N(1)-Z n(1)-N(3)$ & $75.78(11)$ & $\mathrm{N}(2)-\mathrm{Zn}(2)-\mathrm{N}(5)$ & $75.75(11)$ \\
\hline $\mathrm{O}(5)-\mathrm{Zn}(1)-\mathrm{N}(3)$ & $91.31(10)$ & $\mathrm{O}(4)-\mathrm{Zn}(2)-\mathrm{N}(5)$ & $92.67(11)$ \\
\hline $\mathrm{N}(4)-\mathrm{Zn}(1)-\mathrm{N}(3)$ & $92.88(11)$ & $\mathrm{N}(6)-\mathrm{Zn}(2)-\mathrm{N}(5)$ & $94.50(10)$ \\
\hline $\mathrm{N}(1)-\mathrm{Zn}(1)-\mathrm{Cl}(1)$ & $91.38(8)$ & $\mathrm{N}(2)-\mathrm{Zn}(2)-\mathrm{Cl}(1)$ & $89.94(8)$ \\
\hline $\mathrm{O}(5)-\mathrm{Zn}(1)-\mathrm{Cl}(1)$ & $93.92(8)$ & $\mathrm{O}(4)-\mathrm{Zn}(2)-\mathrm{Cl}(1)$ & $95.99(8)$ \\
\hline $\mathrm{N}(4)-\mathrm{Zn}(1)-\mathrm{Cl}(1)$ & $99.50(9)$ & $\mathrm{N}(6)-\mathrm{Zn}(2)-\mathrm{Cl}(1)$ & $96.31(9)$ \\
\hline $\mathrm{N}(3)-\mathrm{Zn}(1)-\mathrm{Cl}(1)$ & $165.29(6)$ & $\mathrm{N}(5)-\mathrm{Zn}(2)-\mathrm{Cl}(1)$ & $165.15(7)$ \\
\hline $\mathrm{N}(1)-\mathrm{Zn}(1)-\mathrm{O}(6)$ & $88.57(9)$ & $\mathrm{N}(2)-\mathrm{Zn}(2)-\mathrm{O}(3)$ & $90.20(11)$ \\
\hline $\mathrm{O}(5)-\mathrm{Zn}(1)-\mathrm{O}(6)$ & $52.72(8)$ & $\mathrm{O}(4)-\mathrm{Zn}(2)-\mathrm{O}(3)$ & $52.79(10)$ \\
\hline $\mathrm{N}(4)-\mathrm{Zn}(1)-\mathrm{O}(6)$ & $154.00(9)$ & $\mathrm{N}(6)-\mathrm{Zn}(2)-\mathrm{O}(3)$ & $148.82(11)$ \\
\hline $\mathrm{N}(3)-\mathrm{Zn}(1)-\mathrm{O}(6)$ & $91.96(9)$ & $\mathrm{N}(5)-\mathrm{Zn}(2)-\mathrm{O}(3)$ & $95.82(9)$ \\
\hline $\mathrm{Cl}(1)-\mathrm{Zn}(1)-\mathrm{O}(6)$ & $80.38(6)$ & $\mathrm{Cl}(1)-\mathrm{Zn}(2)-\mathrm{O}(3)$ & $80.03(7)$ \\
\hline
\end{tabular}

Tabelle 8: Ausgewählte Atomabstände ( $\AA$ ) und Bindungswinkel ( ${ }^{\circ}$ ).

Um Komplexe des Liganden $L^{2}$ mit Zink, aber ohne zusätzliche Chloridbrücke zu erhalten, wurden auch mehrere Kristallisationsversuche unter chloridfreien Bedingungen durchgeführt. Jedoch entstanden in keinem dieser Fälle Kristalle. Anscheinend ist die Kristallisation als reiner Nitrat- oder Perchloratkomplex nicht begünstigt. 
Die Reaktion von $\mathrm{L}^{4}$ mit zwei Äquivalenten $\mathrm{Zn}\left(\mathrm{NO}_{3}\right)_{2}$ und einem Äquivalent Base $\left(\mathrm{KO}{ }^{t} \mathrm{Bu}\right)$ führte $\mathrm{Zu}\left[\mathrm{L}^{4}{ }_{-\mathrm{H}} \mathrm{Zn}_{2}\left(\mathrm{NO}_{3}\right)_{3}\right](7)$. Die Struktur von 7 ist in Abbildung 43 gezeigt; ausgewählte interatomare Abstände und Bindungswinkel sind in Tabelle 9 zu finden.

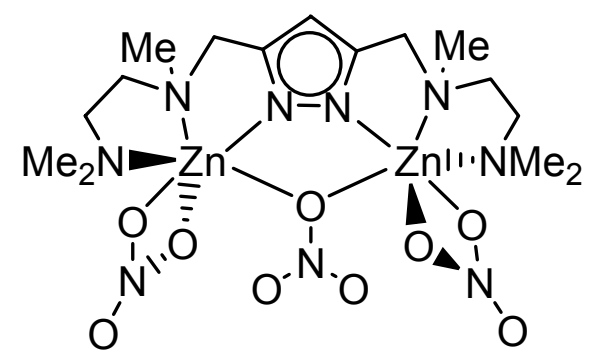

7

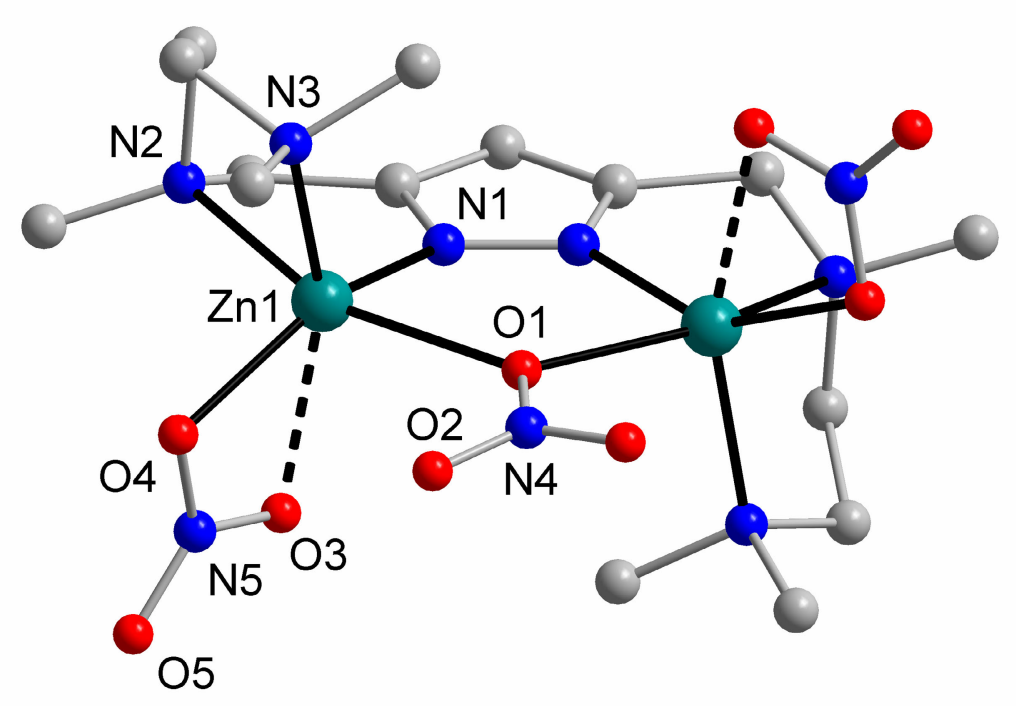

Abbildung 43: Festkörperstruktur von 7.

\begin{tabular}{|l|l|l|l|}
\hline $\mathrm{Zn}(1)-\mathrm{N}(1)$ & $1.972(2)$ & $\mathrm{Zn}(1)-\mathrm{O}(4)$ & $2.086(3)$ \\
\hline $\mathrm{Zn}(1)-\mathrm{N}(3)$ & $2.088(3)$ & $\mathrm{Zn}(1)-\mathrm{N}(2)$ & $2.286(3)$ \\
\hline $\mathrm{Zn}(1)-\mathrm{O}(1)$ & $2.316(2)$ & $\mathrm{Zn}(1)-\mathrm{O}(3)$ & $2.342(4)$ \\
\hline $\mathrm{O}(1)-\mathrm{N}(4)$ & $1.249(5)$ & $\mathrm{O}(2)-\mathrm{N}(4)$ & $1.163(5)$ \\
\hline $\mathrm{O}(3)-\mathrm{N}(5)$ & $1.216(5)$ & $\mathrm{O}(4)-\mathrm{N}(5)$ & $1.242(5)$ \\
\hline $\mathrm{O}(5)-\mathrm{N}(5)$ & $1.206(4)$ & $\mathrm{N}(1)-\mathrm{N}(1) \mathrm{A}$ & $1.351(4)$ \\
\hline $\mathrm{N}(1)-\mathrm{Zn}(1)-\mathrm{O}(4)$ & $148.71(11)$ & $\mathrm{N}(1)-\mathrm{Zn}(1)-\mathrm{N}(3)$ & $110.33(10)$ \\
\hline $\mathrm{O}(4)-\mathrm{Zn}(1)-\mathrm{N}(3)$ & $99.48(12)$ & $\mathrm{N}(1)-\mathrm{Zn}(1)-\mathrm{N}(2)$ & $77.39(10)$ \\
\hline $\mathrm{O}(4)-\mathrm{Zn}(1)-\mathrm{N}(2)$ & $96.75(12)$ & $\mathrm{N}(3)-\mathrm{Zn}(1)-\mathrm{N}(2)$ & $84.56(12)$ \\
\hline $\mathrm{N}(1)-\mathrm{Zn}(1)-\mathrm{O}(1)$ & $81.53(9)$ & $\mathrm{O}(4)-\mathrm{Zn}(1)-\mathrm{O}(1)$ & $105.49(12)$ \\
\hline
\end{tabular}




\begin{tabular}{|l|l|l|l|}
\hline $\mathrm{N}(3)-\mathrm{Zn}(1)-\mathrm{O}(1)$ & $95.37(8)$ & $\mathrm{N}(2)-\mathrm{Zn}(1)-\mathrm{O}(1)$ & $157.43(9)$ \\
\hline $\mathrm{N}(1)-\mathrm{Zn}(1)-\mathrm{O}(3)$ & $94.79(12)$ & $\mathrm{O}(4)-\mathrm{Zn}(1)-\mathrm{O}(3)$ & $55.07(13)$ \\
\hline $\mathrm{N}(3)-\mathrm{Zn}(1)-\mathrm{O}(3)$ & $154.55(13)$ & $\mathrm{N}(2)-\mathrm{Zn}(1)-\mathrm{O}(3)$ & $97.46(15)$ \\
\hline $\mathrm{O}(1)-\mathrm{Zn}(1)-\mathrm{O}(3)$ & $92.22(12)$ & $\mathrm{Zn}(1)-\mathrm{O}(1)-\mathrm{Zn}(1) \mathrm{A}$ & $115.54(13)$ \\
\hline
\end{tabular}

Tabelle 9: Ausgewählte Atomabstände ( $\AA$ ) und Bindungswinkel ( $\left.{ }^{\circ}\right)$.

Wie erwartet, nimmt wie $\mathrm{L}^{2}$ auch $\mathrm{L}^{4}$ in beiden Kompartimenten je ein Zinkion in tridentater Koordination auf. Eines der drei Nitratanionen verbrückt die beiden Zinkionen über ein Sauerstoffatom, während die anderen beiden als halbverbrückende terminale Liganden an jedem Zinkion sitzen. Man findet auch hier jeweils einen kurzen $(\mathrm{d}(\mathrm{Zn}(1)-\mathrm{O}(4))=2.086(3) \AA)$ und einen signifikant längeren ZinkSauerstoff-Abstand $(\mathrm{d}(\mathrm{Zn}(1)-\mathrm{O}(3))=2.342(4) \AA)$, so dass die Metallionen wiederum in einer verzerrt oktaedrischen Koordinationsumgebung zu finden sind.

Das Molekül besitzt $\mathrm{C}_{2}$-Symmetrie, so dass der Komplex als Racemat vorliegt. Der Kristall weist jedoch eine zentrosymmetrische Raumgruppe auf, beide Enantiomere sind also enthalten.

Aufgrund der $\mathrm{C}_{2}$-Symmetrie werden im ${ }^{1} \mathrm{H}-\mathrm{NMR}$ die Protonen der $\mathrm{CH}_{2}$-Gruppen der Verbindung diastereomer; man erhält jeweils zwei Signalsätze.

Wird $\mathrm{L}^{4}$ mit zwei Äquivalenten von Zinkperchlorat und $\mathrm{KO}^{t} \mathrm{Bu}$ umgesetzt und die Lösung der Luft ausgesetzt, so erscheint im ${ }^{13} \mathrm{C}$-NMR Spektrum des Produkts 8 ein neues Signal von gebundenem Carbonat ( $\delta=169.9$ ppm), und ESI-Massenspektren zeigen ein dominantes Signal bei $\mathrm{m} / \mathrm{z}=1039$, das in Übereinstimmung mit der Formulierung einer tetranuklearen Spezies $\left[\left\{\left(\mathrm{L}^{4}{ }_{-\mathrm{H}}\right)_{2} \mathrm{Zn}_{4}(\mathrm{OH})_{2}\left(\mathrm{CO}_{3}\right)\right\}\left(\mathrm{ClO}_{4}\right)\right]^{+}$steht. Ein weiterer Hinweis für das Vorliegen einer Carbonateinheit lässt sich aus intensiven IRBanden bei $1380 \mathrm{~cm}^{-1}$ und $1500 \mathrm{~cm}^{-1}$ ableiten. Das Carbonat hat seinen Ursprung also in der Fixierung des in der Atmosphäre enthaltenen Kohlendioxids. 8 lässt sich direkter durch Zugabe eines halben Äquivalents Kaliumcarbonat synthetisieren. Analytisch reine Kristalle konnten zwar erhalten werden, lieferten jedoch aufgrund zahlreicher Fehlordnungen nur schlechte Ergebnisse in der Röntgenstrukturanalyse. Für die kristallographische Untersuchung geeignete Kristalle konnten jedoch nach Umfällung aus dem Tetraphenylboratanalogon 8 gewonnen werden. Die Struktur des Dikations von 8 ist in Abbildung 44 und Abbildung 45 gezeigt; ausgewählte interatomare Abstände und Bindungswinkel sind in Tabelle $10 \mathrm{zu}$ finden. 


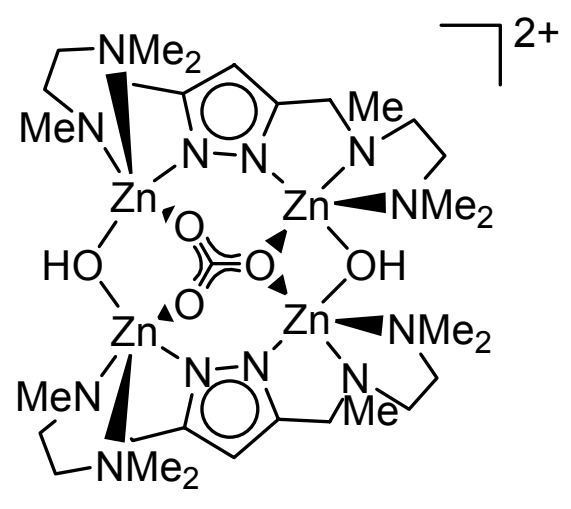

8

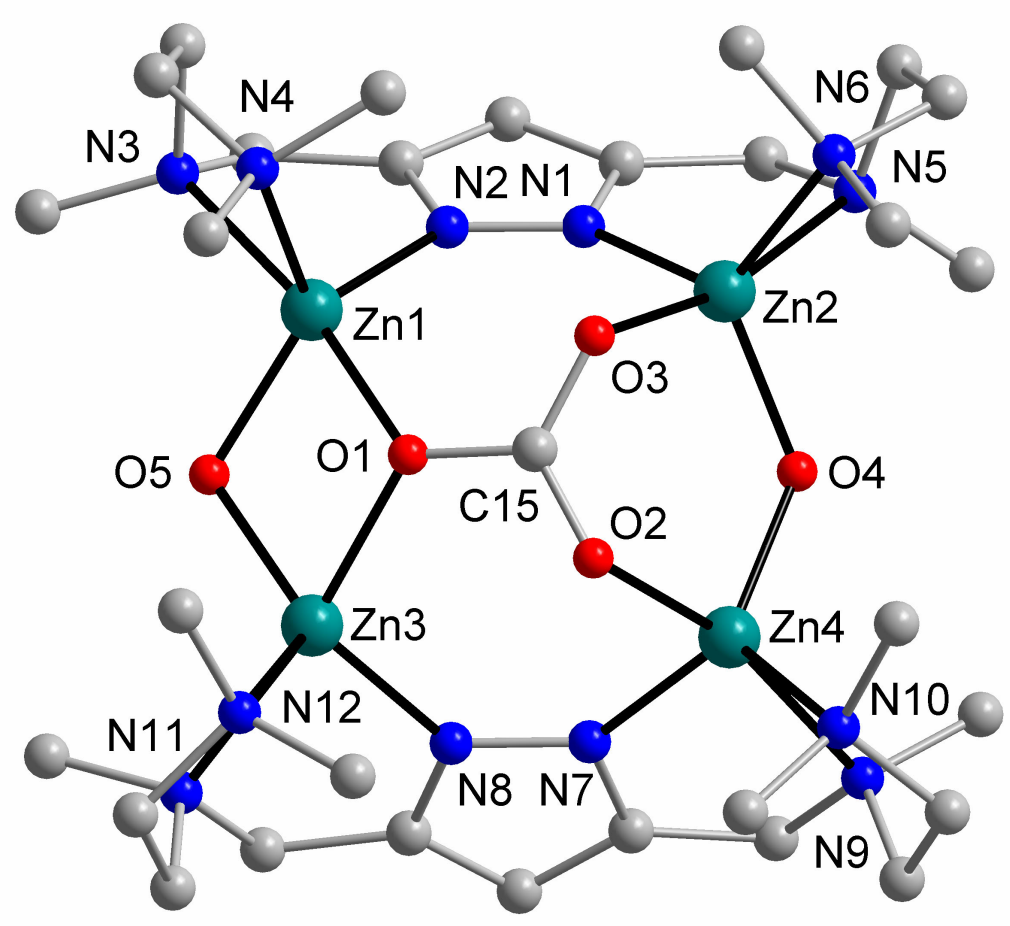

Abbildung 44: Festkörperstruktur des Kations von 8.(BPh $)_{2}$ in der Ansicht von oben. 


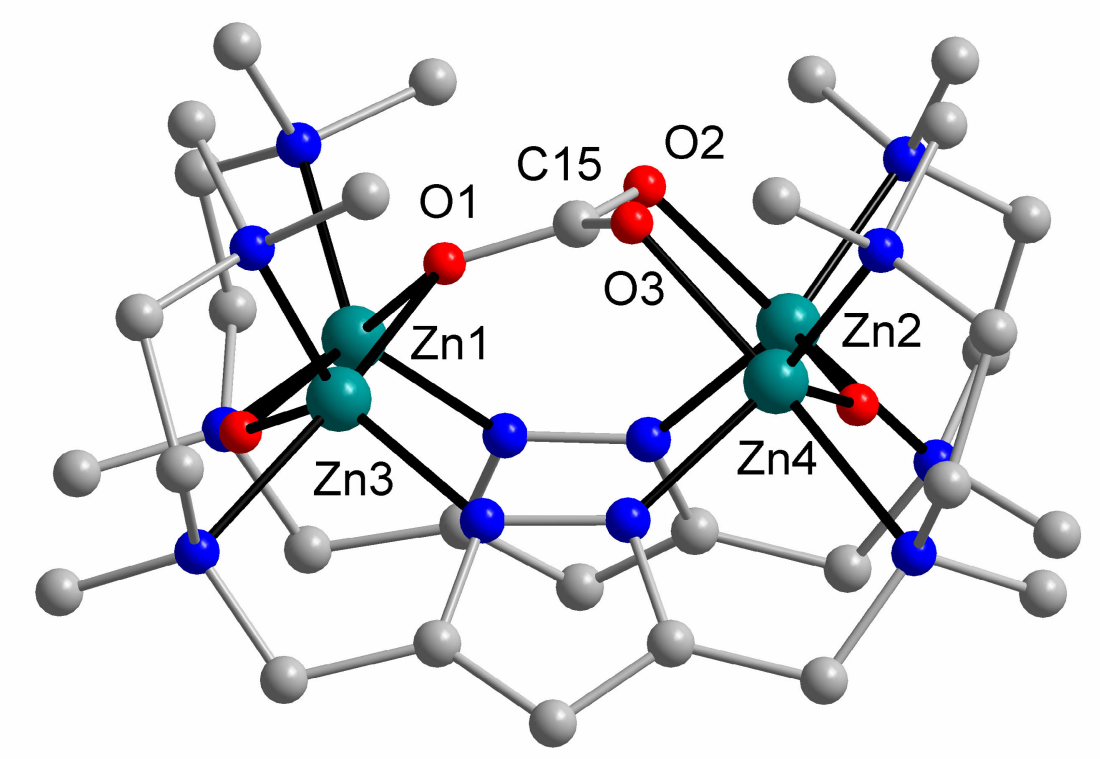

Abbildung 45: Festkörperstruktur des Kations von 8.(BPh $)_{4}$ in seitlicher Ansicht.

\begin{tabular}{|l|l|l|l|}
\hline $\mathrm{Zn}(1)-\mathrm{O}(5)$ & $1.968(3)$ & $\mathrm{Zn}(2)-\mathrm{O}(4)$ & $1.944(3)$ \\
\hline $\mathrm{Zn}(1)-\mathrm{N}(2)$ & $2.022(4)$ & $\mathrm{Zn}(2)-\mathrm{N}(1)$ & $2.026(4)$ \\
\hline $\mathrm{Zn}(1)-\mathrm{O}(1)$ & $2.099(3)$ & $\mathrm{Zn}(2)-\mathrm{O}(3)$ & $2.067(4)$ \\
\hline $\mathrm{Zn}(1)-\mathrm{N}(4)$ & $2.111(4)$ & $\mathrm{Zn}(2)-\mathrm{N}(6)$ & $2.111(4)$ \\
\hline $\mathrm{Zn}(1)-\mathrm{N}(3)$ & $2.316(4)$ & $\mathrm{Zn}(2)-\mathrm{N}(5)$ & $2.356(4)$ \\
\hline $\mathrm{Zn}(3)-\mathrm{O}(5)$ & $1.979(3)$ & $\mathrm{Zn}(4)-\mathrm{O}(4)$ & $1.942(3)$ \\
\hline $\mathrm{Zn}(3)-\mathrm{N}(8)$ & $2.008(4)$ & $\mathrm{Zn}(4)-\mathrm{N}(7)$ & $2.014(4)$ \\
\hline $\mathrm{Zn}(3)-\mathrm{O}(1)$ & $2.100(3)$ & $\mathrm{Zn}(4)-\mathrm{O}(2)$ & $2.066(3)$ \\
\hline $\mathrm{Zn}(3)-\mathrm{N}(12)$ & $2.118(4)$ & $\mathrm{Zn}(4)-\mathrm{N}(10)$ & $2.110(4)$ \\
\hline $\mathrm{Zn}(3)-\mathrm{N}(11)$ & $2.320(4)$ & $\mathrm{Zn}(4)-\mathrm{N}(9)$ & $2.317(4)$ \\
\hline $\mathrm{O}(1)-\mathrm{C}(15)$ & $1.309(6)$ & & \\
\hline $\mathrm{O}(2)-\mathrm{C}(15)$ & $1.276(5)$ & & \\
\hline $\mathrm{O}(3)-\mathrm{C}(15)$ & $1.277(5)$ & & \\
\hline $\mathrm{N}(2)-\mathrm{N}(1)$ & $1.356(6)$ & & \\
\hline $\mathrm{N}(8)-\mathrm{N}(7)$ & $1.377(6)$ & & \\
\hline $\mathrm{Zn}(1)-\mathrm{O}(5)-\mathrm{Zn}(3)$ & $106.61(17)$ & & \\
\hline $\mathrm{Zn}(4)-\mathrm{O}(4)-\mathrm{Zn}(2)$ & $127.36(19)$ & & \\
\hline $\mathrm{Zn}(1)-\mathrm{O}(1)-\mathrm{Zn}(3)$ & $97.82(14)$ & & \\
\hline
\end{tabular}

Tabelle 10: Ausgewählte Atomabstände $(\AA)$ und Bindungswinkel ( $\left.{ }^{\circ}\right)$. 
In 8 werden zwei dinukleare Einheiten über zwei Hydroxid-Brücken zusammengehalten und bilden eine tetranukleare Anordnung, die von einem $\mu_{4}$-verbrückenden Carbonat (Abbildung 45) überkappt wird.

Die Zink-Zink-Abstände innerhalb der Pyrazolat-basierten dimetallischen Bausteine sind im Vergleich zu 7 etwas aufgeweitet (4.113/4.156 A), wohingegen sich die ZinkZink-Separierung zwischen den beiden Untereinheiten der Carbonatbrücke anpasst. Man findet einen kürzeren Abstand $d(\mathrm{Zn}(1) \cdots \mathrm{Zn}(3))=3.165 \AA$ im Fall der $\mu_{1}$ Carbonatsauerstoffbrücke und einen längeren Abstand von $d(\mathrm{Zn}(2) \cdots \mathrm{Zn}(4))=3.483$ $\AA$ im Fall der dreiatomigen O-C-O-Carbonat-Bücke auf der gegenüberliegenden Seite. Auf dieser Seite sind auch alle $\mathrm{Zn}-\mathrm{O}-$ Bindungen geringfügig länger. Alle Metallionen befinden sich in einer verzerrt trigonal-bipyramidalen $\left.\mathrm{N}_{3} \mathrm{O}_{2}\right\}$ Koordinationsumgebung $(\tau=0.71-0.78)$.

Während der $\mu_{4}-\eta^{2}: \eta^{1}: \eta^{1}$-Bindungsmodus für Carbonatkomplexe anderer Metallionen gut bekannt ist, ${ }^{[84-86]}$ ist dieses Motiv in der Zinkchemie äußerst selten und wurde erst kürzlich in zwei Komplexen beobachtet. ${ }^{[87,88]}$ In diesen Verbindungen ist jedoch das Carbonation die einzige Brücke zwischen den dinuklearen bzw. mononuklearen Einheiten. Da in diesen Komplexen keine zusätzlichen Brücken vorhanden sind, befinden sich die vier Zinkionen - im Gegensatz zu der Situation in 8 - in der vom Carbonat aufgespannten Ebene.

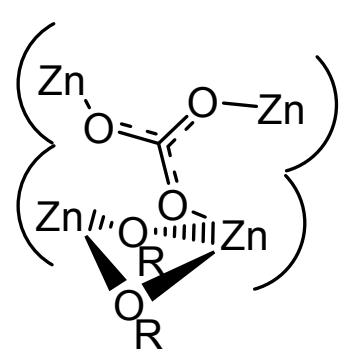

XXII

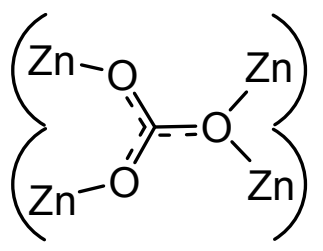

XXIII

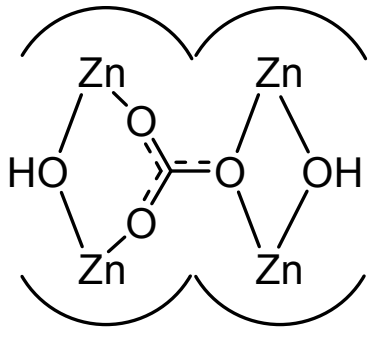

8

Abbildung 46: Vergleich strukturell charakterisierter $\mu_{4}-\eta^{2}: \eta^{1}: \eta^{1}$-Carbonato-Bindungsmodi an Zink. ${ }^{[87,88]}$

Die Anordnung des $\mu_{4}-\eta^{2}: \eta^{1}: \eta^{1}$-Carbonats in $\mathbf{8}$ ist verglichen mit XXIII in Bezug auf die dinuklearen Untereinheiten entgegengesetzt. In 8 sind in zwei jeweils gleichen Untereinheiten beide Zinkionen durch eine O-C-O-Carbonat-Brücke verbunden, in 
XXIII sind die Zinkionen in einer Einheit O-verbrückt und in der anderen O-C-Overbrückt.

Setzt man den zu $\mathrm{L}^{4}$ analogen Liganden $\mathrm{L}^{9}$ mit zwei Äquivalenten Zinknitrat und einem Äquivalent Kalium-tert-butylat um, so erhält man $\left[\mathrm{L}^{4}{ }_{-H} \mathrm{Zn}_{2}\left(\mathrm{NO}_{3}\right)_{3}\right]$ (9), der vollkommen analog zu 7 aufgebaut ist (Abbildung 47).

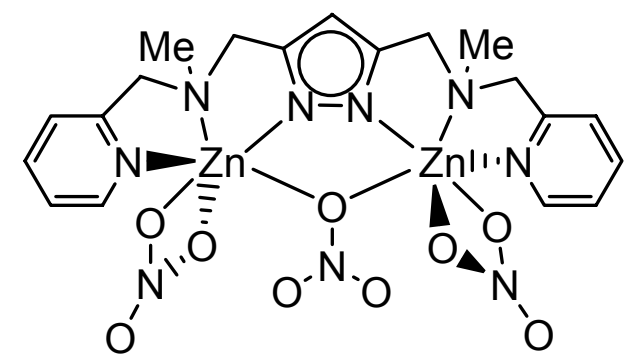

9

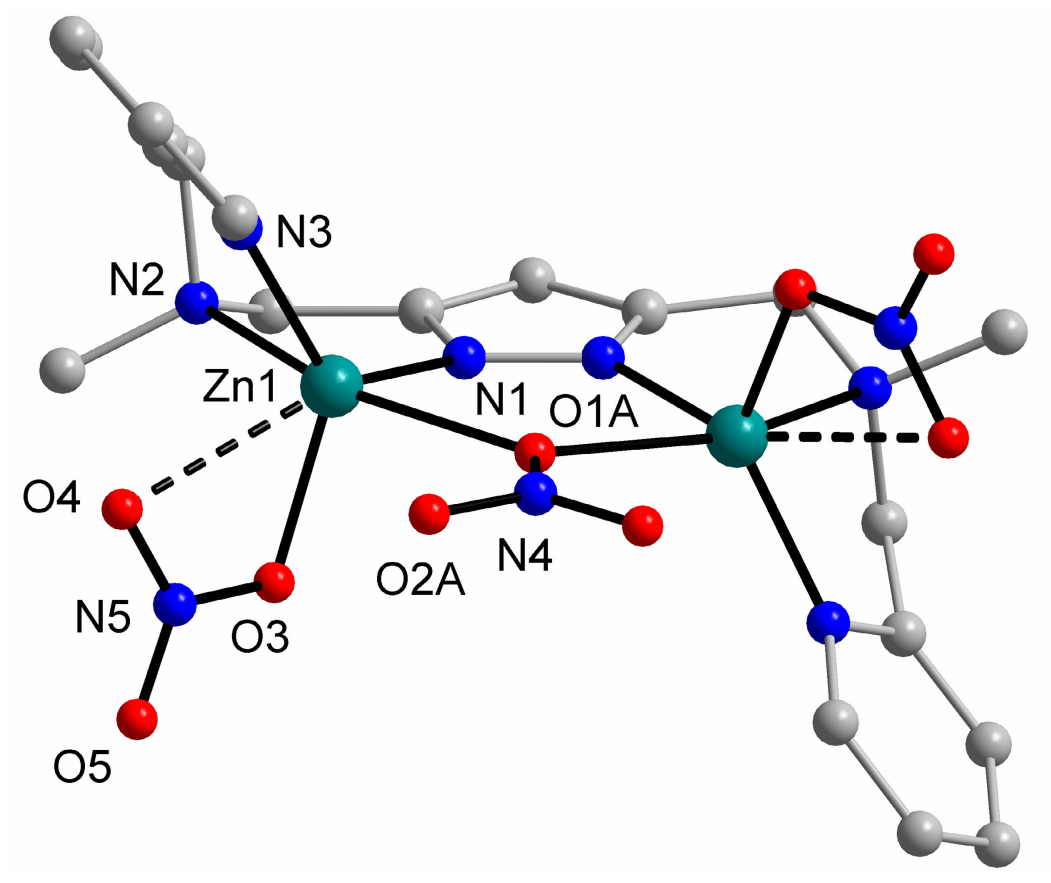

Abbildung 47: Festkörperstruktur von 9.

\begin{tabular}{|l|l|l|l|}
\hline $\mathrm{Zn}(1)-\mathrm{N}(1)$ & $1.983(2)$ & $\mathrm{N}(1)-\mathrm{Zn}(1)-\mathrm{O}(3)$ & $108.49(10)$ \\
\hline $\mathrm{Zn}(1)-\mathrm{N}(2)$ & $2.245(2)$ & $\mathrm{N}(1)-\mathrm{Zn}(1)-\mathrm{N}(3)$ & $123.15(10)$ \\
\hline $\mathrm{Zn}(1)-\mathrm{N}(3)$ & $2.059(3)$ & $\mathrm{N}(1)-\mathrm{Zn}(1)-\mathrm{O}(2 \mathrm{~B})$ & $94.8(2)$ \\
\hline $\mathrm{Zn}(1)-\mathrm{O}(1 \mathrm{~A})$ & $2.313(4)$ & $\mathrm{N}(3)-\mathrm{Zn}(1)-\mathrm{O}(2 \mathrm{~B})$ & $83.65(19)$ \\
\hline $\mathrm{Zn}(1)-\mathrm{O}(1 \mathrm{~B})$ & $2.164(8)$ & $\mathrm{N}(1)-\mathrm{Zn}(1)-\mathrm{O}(1 \mathrm{~B})$ & $109.3(2)$ \\
\hline $\mathrm{Zn}(1)-\mathrm{O}(2 \mathrm{~B})$ & $2.117(6)$ & $\mathrm{N}(3)-\mathrm{Zn}(1)-\mathrm{O}(1 \mathrm{~B})$ & $85.0(2)$ \\
\hline
\end{tabular}




\begin{tabular}{|l|l|l|l|}
\hline $\mathrm{Zn}(1)-\mathrm{O}(3)$ & $2.007(2)$ & $\mathrm{O}(3)-\mathrm{Zn}(1)-\mathrm{O}(1 \mathrm{~B})$ & $89.4(2)$ \\
\hline $\mathrm{O}(1 \mathrm{~A})-\mathrm{N}(4)$ & $1.114(8)$ & $\mathrm{O}(3)-\mathrm{Zn}(1)-\mathrm{N}(3)$ & $126.94(10)$ \\
\hline $\mathrm{O}(2 \mathrm{~A})-\mathrm{N}(4)$ & $1.276(7)$ & $\mathrm{O}(3)-\mathrm{Zn}(1)-\mathrm{O}(2 \mathrm{~B})$ & $104.6(2)$ \\
\hline $\mathrm{O}(1 \mathrm{~B})-\mathrm{N}(4)$ & $1.176(8)$ & $\mathrm{N}(1)-\mathrm{Zn}(1)-\mathrm{N}(2)$ & $78.52(10)$ \\
\hline $\mathrm{O}(2 \mathrm{~B})-\mathrm{N}(4)$ & $1.387(7)$ & $\mathrm{O}(3)-\mathrm{Zn}(1)-\mathrm{N}(2)$ & $100.44(10)$ \\
\hline $\mathrm{O}(3 \mathrm{~B})-\mathrm{N}(4)$ & $1.201(12)$ & $\mathrm{N}(3)-\mathrm{Zn}(1)-\mathrm{N}(2)$ & $80.05(10)$ \\
\hline $\mathrm{O}(3)-\mathrm{N}(5)$ & $1.289(4)$ & $\mathrm{O}(2 \mathrm{~B})-\mathrm{Zn}(1)-\mathrm{N}(2)$ & $154.9(2)$ \\
\hline $\mathrm{O}(4)-\mathrm{N}(5)$ & $1.214(4)$ & $\mathrm{O}(1 \mathrm{~B})-\mathrm{Zn}(1)-\mathrm{N}(2)$ & $165.0(2)$ \\
\hline $\mathrm{O}(5)-\mathrm{N}(5)$ & $1.197(4)$ & $\mathrm{N}(1)-\mathrm{Zn}(1)-\mathrm{O}(1 \mathrm{~A})$ & $77.68(18)$ \\
\hline $\mathrm{N}(1)-\mathrm{N}(1) \mathrm{A}$ & $1.362(5)$ & $\mathrm{O}(3)-\mathrm{Zn}(1)-\mathrm{O}(1 \mathrm{~A})$ & $88.42(10)$ \\
\hline $\mathrm{Zn}(1) \cdots \mathrm{Zn}(1) \mathrm{A}$ & $4.0122(10)$ & $\mathrm{N}(3)-\mathrm{Zn}(1)-\mathrm{O}(1 \mathrm{~A})$ & $112.24(11)$ \\
\hline & & $\mathrm{N}(2)-\mathrm{Zn}(1)-\mathrm{O}(1 \mathrm{~A})$ & $156.16(18)$ \\
\hline
\end{tabular}

Tabelle 11: Ausgewählte Atomabstände $(\AA)$ und Bindungswinkel $\left({ }^{\circ}\right)$.

$\mathrm{L}^{9}$ nimmt wie $\mathrm{L}^{4}$ in beiden Kompartimenten je ein Zinkion in tridentater Koordination auf. Eines der drei Nitratanionen verbrückt die beiden Zinkionen, während die anderen beiden als halbverbrückende terminale Liganden an jedem Zinkion sitzen. Man findet auch hier jeweils einen kurzen $(\mathrm{d}(\mathrm{Zn}(1)-\mathrm{O}(3))=2.007(2) \AA)$ und einen signifikant längeren Zink-Sauerstoff-Abstand $(\mathrm{d}(\mathrm{Zn}(1)-\mathrm{O}(4))=2.758 \AA)$, so dass die Metallionen wiederum in einer verzerrt oktaedrischen Koordinationsumgebung zu finden sind. Im Gegensatz zu 7, bei dem bei $25^{\circ} \mathrm{C}$ röntgenspektroskopisch eindeutig ein monodentat verbrückendes Nitrat gefunden wurde, ist in Komplex 9 das verbrückende Nitration bei einer Temperatur von $-140^{\circ} \mathrm{C}$ fehlgeordnet (Abbildung 48). 


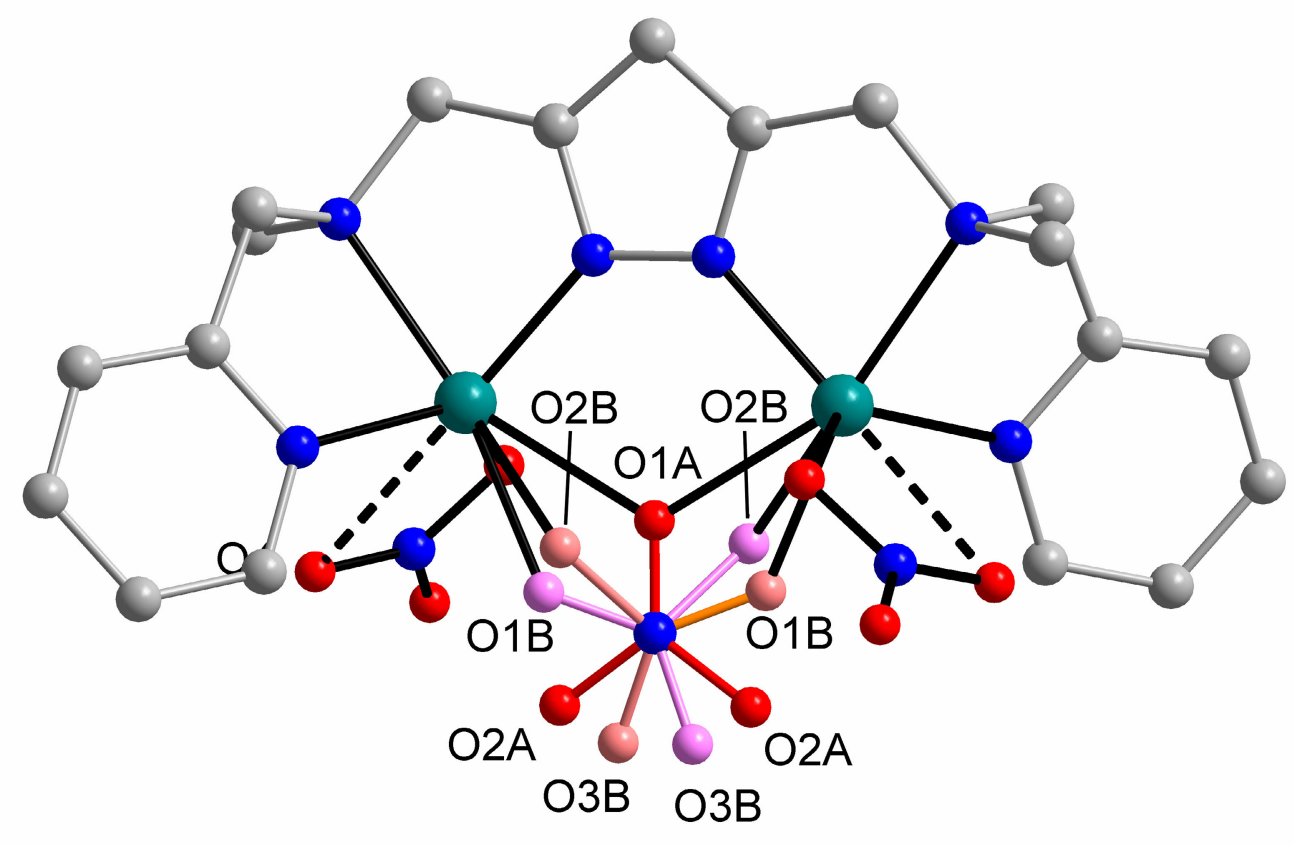

Abbildung 48: Fehlordnung des verbrückenden Nitrations in 9.

Jedoch kann aufgrund der erhaltenen Ergebnisse keine Aussage getroffen werden, ob der Komplex als Isomerengemisch vorliegt oder ob noch eine freie Rotation des Nitrats möglich ist (Abbildung 49).
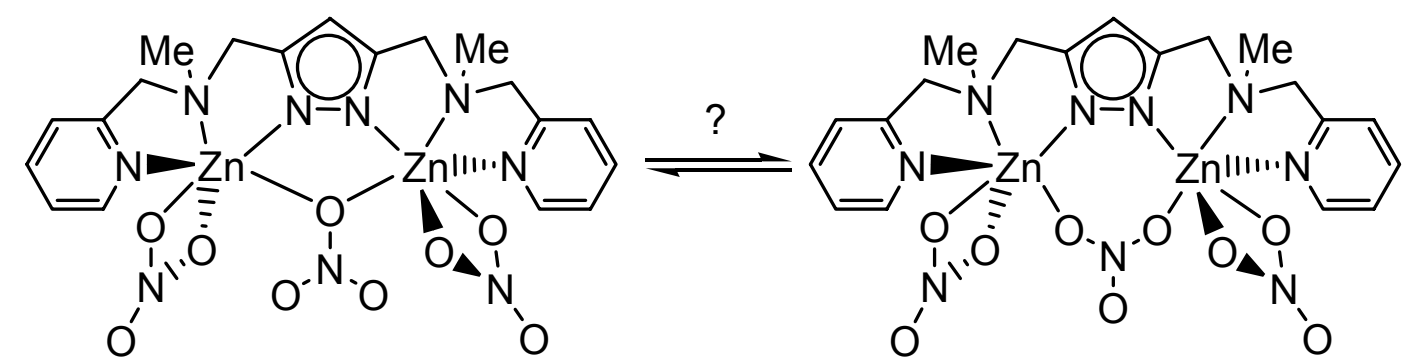

Abbildung 49: Freie Rotation des verbrückenden Nitrats in 9.

Nach einer CSD-Suche ${ }^{[89]}$ sind dies die einzigen anderen kristallographisch charakterisierten Dizinkkomplexe neben $\mathbf{X X V I}^{\left[{ }^{[0]}\right.}$ (Abbildung 50), die eine Nitratbrücke über nur ein Sauerstoffatom $(\mu-\kappa \mathrm{K}, \kappa \mathrm{O})$ aufweisen. Auch ein $\mu-\kappa \mathrm{O}, \kappa \mathrm{O}$-Bindungsmodus von Nitrat war für Dizinkkomplexe bisher noch nicht bekannt. 

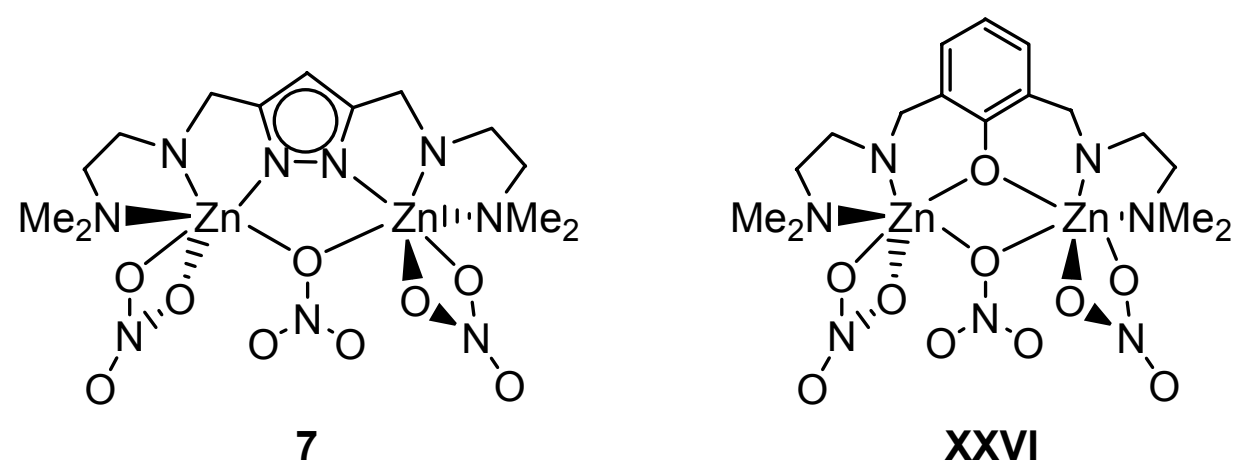

Abbildung 50: $\mu$-Nitratverbrückte Dizinkkomplexe.

Komplexe des Liganden $\mathrm{L}^{6}$ konnten nicht in kristalliner Form erhalten werden.

\subsubsection{Komplexe bisfacialer Liganden}

Kristalle von Komplexen der bisfacialen Liganden XXIVa und XXIVb konnten während dieser Arbeit nicht erhalten werden, so dass eine röntgenstrukturanalytische Charakterisierung nicht möglich war. Im Fall des Methylimidazolyl-substituierten Liganden XXIVa stellt sich die Frage, ob dieser Komplex überhaupt in der Lage ist, zwei Zinkionen zu binden, da weder im FAB- noch im ESI-MS Signale für eine Dizinkspezies zu detektieren waren. Möglicherweise können die Methylimidazolylgruppen aufgrund der insgesamt drei Methylgruppen auf jeder Seite nicht frei rotieren, so dass sich aus sterischen Gründen kein Dizinkkomplex ausbilden kann (Abbildung 51).

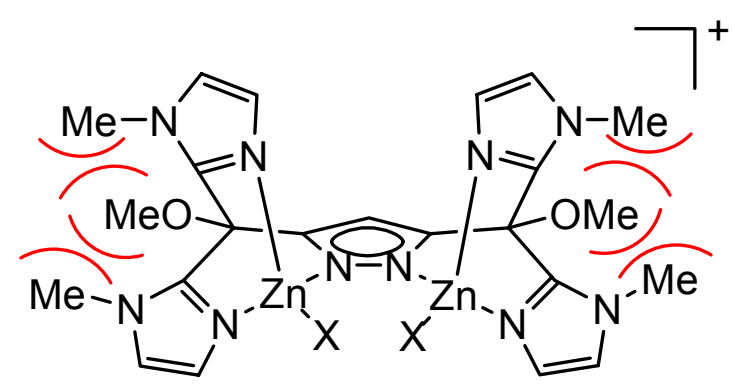

Abbildung 51: Sterische Wechselwirkung in Dizinkkomplexen von XXIVa.

Dagegen beobachtet man nach Zugabe von Zinkchlorid zu Ligand XXIVb im ESI-MS eine dominante $\left[\mathrm{Zn}_{2} \mathrm{~L}_{-H} \mathrm{Cl}_{2}\right]^{+}$-Spezies (Abbildung 52). Jedoch konnten bisher keine Kristalle eines Komplexes von $\mathbf{X X I V b}$ erhalten werden. 

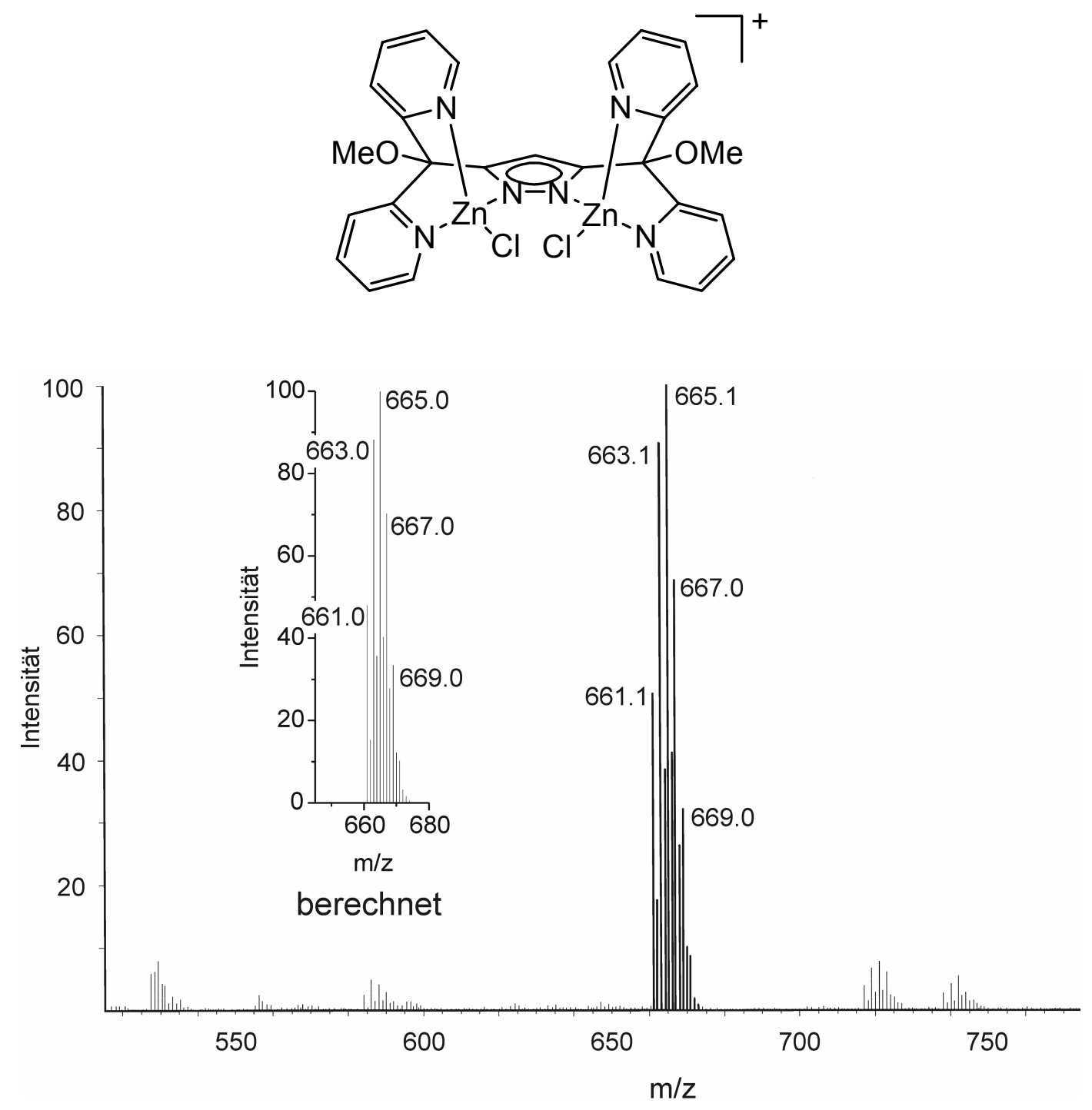

Abbildung 52: Aufgenommenes ESI-Spektrum und berechnete Isotopenverteilung von $\left[\mathrm{C}_{27} \mathrm{H}_{23} \mathrm{~N}_{6} \mathrm{O}_{2} \mathrm{Zn}_{2} \mathrm{Cl}_{2}\right]^{+}$. 


\subsection{Potentiometrische Charakterisierung von Zink(II)- Pyrazolatokomplexen}

Titrationen des jeweiligen Liganden in Anwesenheit unterschiedlicher Äquivalente von $\mathrm{Zn}^{2+}$ wurden durch Batch-Berechnungen mit dem Programm PSEQUAD ${ }^{[77]}$ analysiert, in denen alle Titrationskurven gleichzeitig durch ein Modellsystem ersetzt wurden. Die Ergebnisse der potentiometrischen Bestimmungen sind in Tabelle 12 dargestellt. Die Titrationskurven und experimentellen Details sind in Kapitel 8 aufgeführt.

Die erhaltenen Stabilitätskonstanten und $\mathrm{pK}_{\mathrm{s}}$-Werte sind in Tabelle 12 zusammengefasst. Zum Vergleich wurden die von Kaden et al. bestimmten Stabilitätskonstanten der Liganden $\mathrm{L}^{3}{ }^{[78]}$ und der $\mathrm{pK}_{\mathrm{S}}$-Wert für die Bildung von $\left[\mathrm{Zn}_{2}(\mathbf{X X V}) \mathrm{H}_{-2}\right]^{2+}{ }^{[91]}$ mit aufgenommen. 


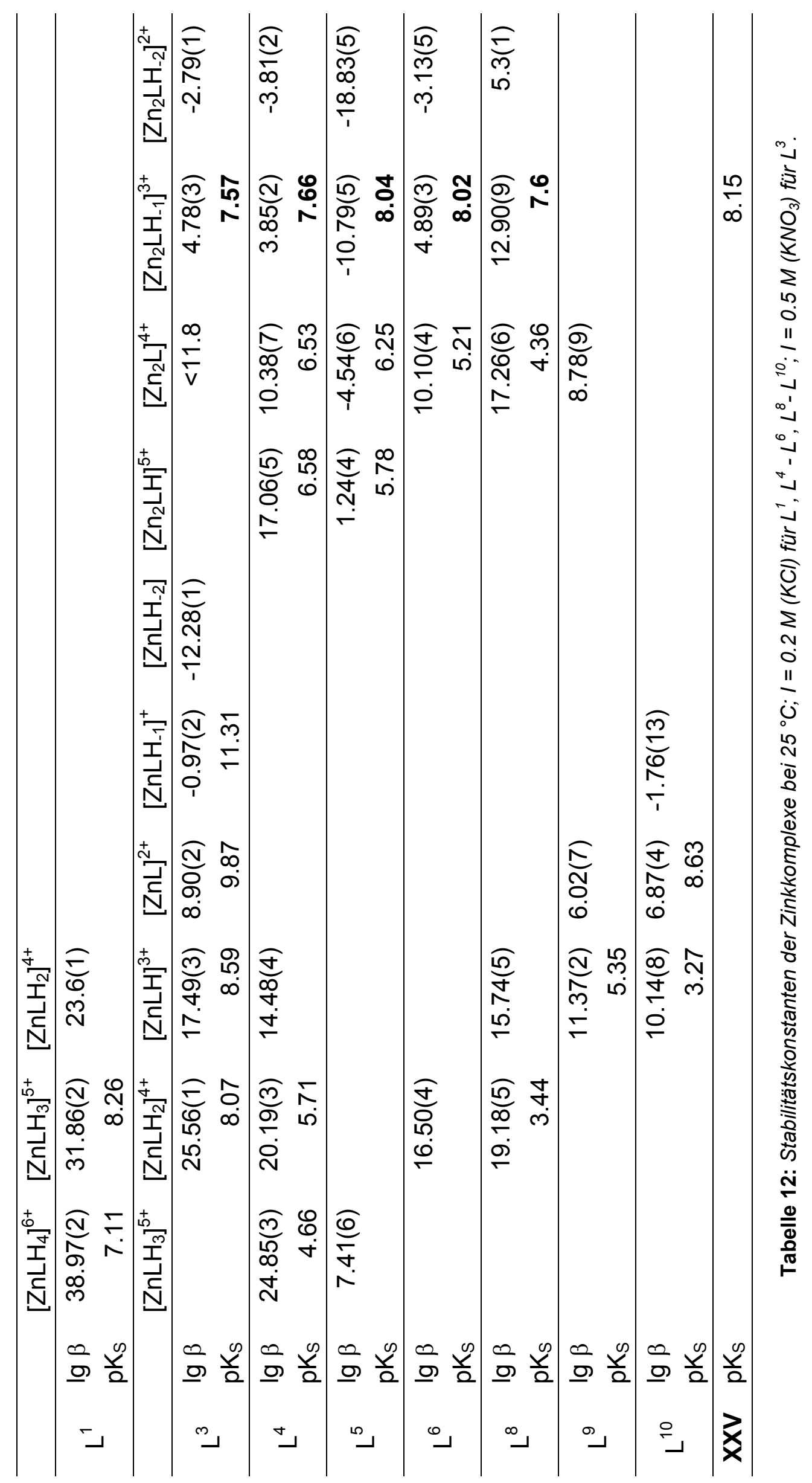




\section{$\mathrm{L}^{1} / \mathrm{Zn}^{2+}$}

Die Auswertung der Titrationskurven des Systems $\mathrm{Zn}^{2+} / \mathrm{L}^{1}$ zeigt, dass dieser Ligand in wässriger Lösung $\mathrm{Zn}^{2+}$ nur relativ schwach binden kann. Es treten nur die mononuklearen Spezies $\left[\mathrm{ZnL}^{1} \mathrm{H}_{4}\right]^{6+},\left[\mathrm{ZnL}^{1} \mathrm{H}_{3}\right]^{5+}$ und $\left[\mathrm{ZnL}^{1} \mathrm{H}_{2}\right]^{4+}$ im Bereich von pH 4 bis $\mathrm{pH} 7$ auf. Bei höheren $\mathrm{pH}-$ Werten wurde die Bildung von Zinkhydroxid beobachtet, dinukleare Spezies konnten nicht berechnet werden. Diese geringe Affinität zu $\mathrm{Zn}^{2+}$ entspringt der Größe der Chelatringe - sechsgliedrige Ringe weisen eine geringere Stabilität als fünfgliedrige Ringe auf.

\section{$\mathrm{L}^{4} / \mathrm{Zn}^{2+}$}

Im Fall von $\mathrm{L}^{4}$ erlauben die Resultate der Modellrechnungen die Identifikation der mononuklearen Spezies $\left[\mathrm{ZnL}^{4} \mathrm{H}_{3}\right]^{5+},\left[\mathrm{ZnL}^{4} \mathrm{H}_{2}\right]^{4+}$ und $\left[\mathrm{ZnL}^{4} \mathrm{H}\right]^{3+}$ wie auch der dinuklearen Spezies $\left[\mathrm{Zn}_{2} \mathrm{~L}^{4} \mathrm{H}\right]^{5+},\left[\mathrm{Zn}_{2} \mathrm{~L}^{4}\right]^{4+},\left[\mathrm{Zn}_{2} \mathrm{~L}^{4} \mathrm{H}_{-1}\right]^{3+}$ und $\left[\mathrm{Zn}_{2} \mathrm{~L}^{4} \mathrm{H}_{-2}\right]^{2+}$.

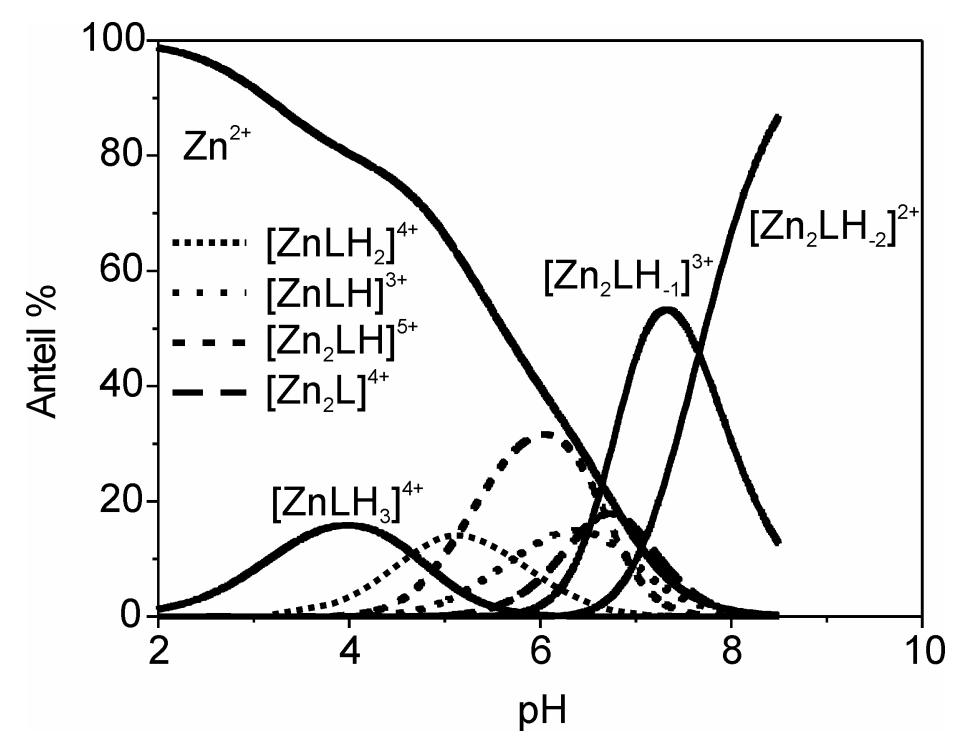

Abbildung 53: Speziesverteilung des Systems $L^{4} / Z n^{2+}\left(I=0.2 M(K C l), T=25^{\circ} \mathrm{C}, c(L)=4 m M, c\left(Z n^{2+}\right)\right.$ $=8 \mathrm{mM})$.

Die Berechnungen wurden auf den pH-Bereich zwischen 2 und 8.5 beschränkt, da ein Niederschlag von Zinkhydroxid bei höherem pH-Wert beobachtet wurde. Anscheinend ist $\mathrm{L}^{4}$ mit zwei tridentaten Koordinationskompartimenten nicht geeignet, um zwei Zinkionen in wässrigen Lösungen im basischen $\mathrm{pH}$-Bereich fest zu binden. $\left[\mathrm{Zn}_{2} \mathrm{~L}^{4} \mathrm{H}_{-1}\right]^{3+}$ und $\left[\mathrm{Zn}_{2} \mathrm{~L}^{4} \mathrm{H}_{-2}\right]^{2+}$ sind höchstwahrscheinlich Pyrazolat-verbrückte Spezies, wobei der berechnete $\mathrm{pK}_{\mathrm{S}}$-Wert für $\left[\mathrm{Zn}_{2} \mathrm{~L}^{4} \mathrm{H}_{-1}\right]^{3+}$ von 7.66 demzufolge den 
$\mathrm{pK}_{\mathrm{S}}$-Wert der Deprotonierung eines Zink-gebundenen Wassermoleküls zu einer Hydroxidfunktion in $\left[\mathrm{Zn}_{2} \mathrm{~L}^{4} \mathrm{H}_{-2}\right]^{2+}$ darstellt.

\section{$\mathrm{L}^{5} / \mathrm{Zn}^{2+}$}

Im Fall von $\mathrm{L}^{5}$ erlauben die Titrationskurven die Berechnung der Stabilitätskonstanten der mononuklearen Spezies $\left[\mathrm{ZnL}^{5} \mathrm{H}_{3}\right]^{5+}$ und der dinuklearen Spezies $\left[\mathrm{Zn}_{2} \mathrm{~L}^{5} \mathrm{H}\right]^{5+}$, $\left[\mathrm{Zn}_{2} \mathrm{~L}^{5}\right]^{4+},\left[\mathrm{Zn}_{2} \mathrm{~L}^{5} \mathrm{H}_{-1}\right]^{3+}$ und $\left[\mathrm{Zn}_{2} \mathrm{~L}^{5} \mathrm{H}_{-2}\right]^{2+}$ im gesamten gemessenen $\mathrm{pH}-$ Bereich von 2 bis 10. Kein Niederschlag von Zinkhydroxid wurde beobachtet und auch die Bildung dinuklearer Komplexe findet im Einklang mit der hohen Stabilität Triazacyclononanbasierter Komplexe bereits bei relativ niedrigen $\mathrm{pH}-$ Werten statt.

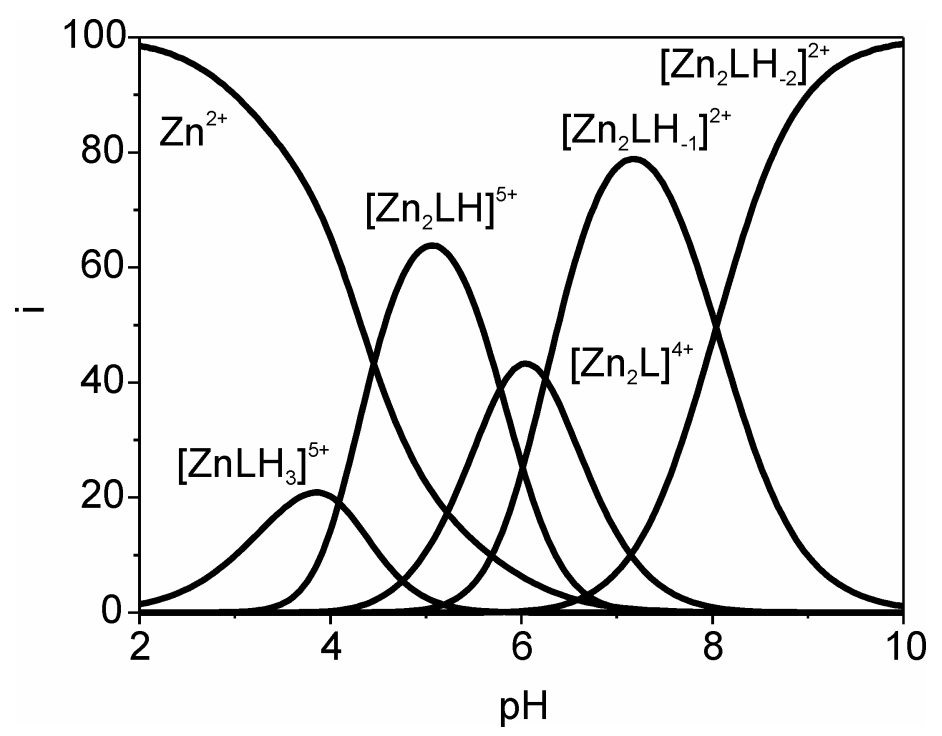

Abbildung 54: Speziesverteilung des Systems $L^{5} / Z n^{2+}\left(I=0.2 M(K C l), T=25^{\circ} \mathrm{C}, c(L)=4 \mathrm{mM}\right.$, $\left.c\left(Z n^{2+}\right)=8 m M\right)$.

Das zusätzliche Proton in der Spezies $\left[\mathrm{Zn}_{2} \mathrm{~L}^{5} \mathrm{H}\right]^{5+}$ ist entweder an einem Triazacyclononanring oder an dem Pyrazolring positioniert. Jedoch lässt sich gegen erstere Möglichkeit einwenden, dass der Makrozyklus wohl stets als tridentater Ligand fungieren sollte; gegen eine Positionierung am Pyrazolring aber spricht, dass ein protonierter Pyrazolring in diesen Systemen einen sehr niedrigen $\mathrm{pK}_{\mathrm{S}}-\mathrm{Wert}_{\mathrm{w}}$ von etwa 2 aufweist - die Maximalkonzentration der Spezies $\left[\mathrm{Zn}_{2} \mathrm{~L}^{5} \mathrm{H}\right]^{5+}$ wird bei einem $\mathrm{pH}-$ Wert von etwa 5 erreicht.

In $\left[\mathrm{Zn}_{2} \mathrm{~L}^{5}\right]^{4+}$ bindet vermutlich jeweils ein Zinkion an eine Triazacyclononaneinheit, während der Pyrazolring unkoordiniert bleibt. Diese Spezies kann in zwei aufeinanderfolgenden Schritten zu den Pyrazolat-verbrückten Spezies $\left[\mathrm{Zn}_{2} \mathrm{~L}^{5} \mathrm{H}_{-1}\right]^{3+}$ 
und $\left[\mathrm{Zn}_{2} \mathrm{~L}^{5} \mathrm{H}_{-2}\right]^{2+}$ deprotoniert werden, wobei die letztere der kristallographisch bestimmten Festkörperstruktur 3 entsprechen sollte. Jedoch kann potentiometrisch eine $\mathrm{OH}$ - nicht von einer eventuellen $\mathrm{H}_{3} \mathrm{O}_{2}$-Brücke unterschieden werden.
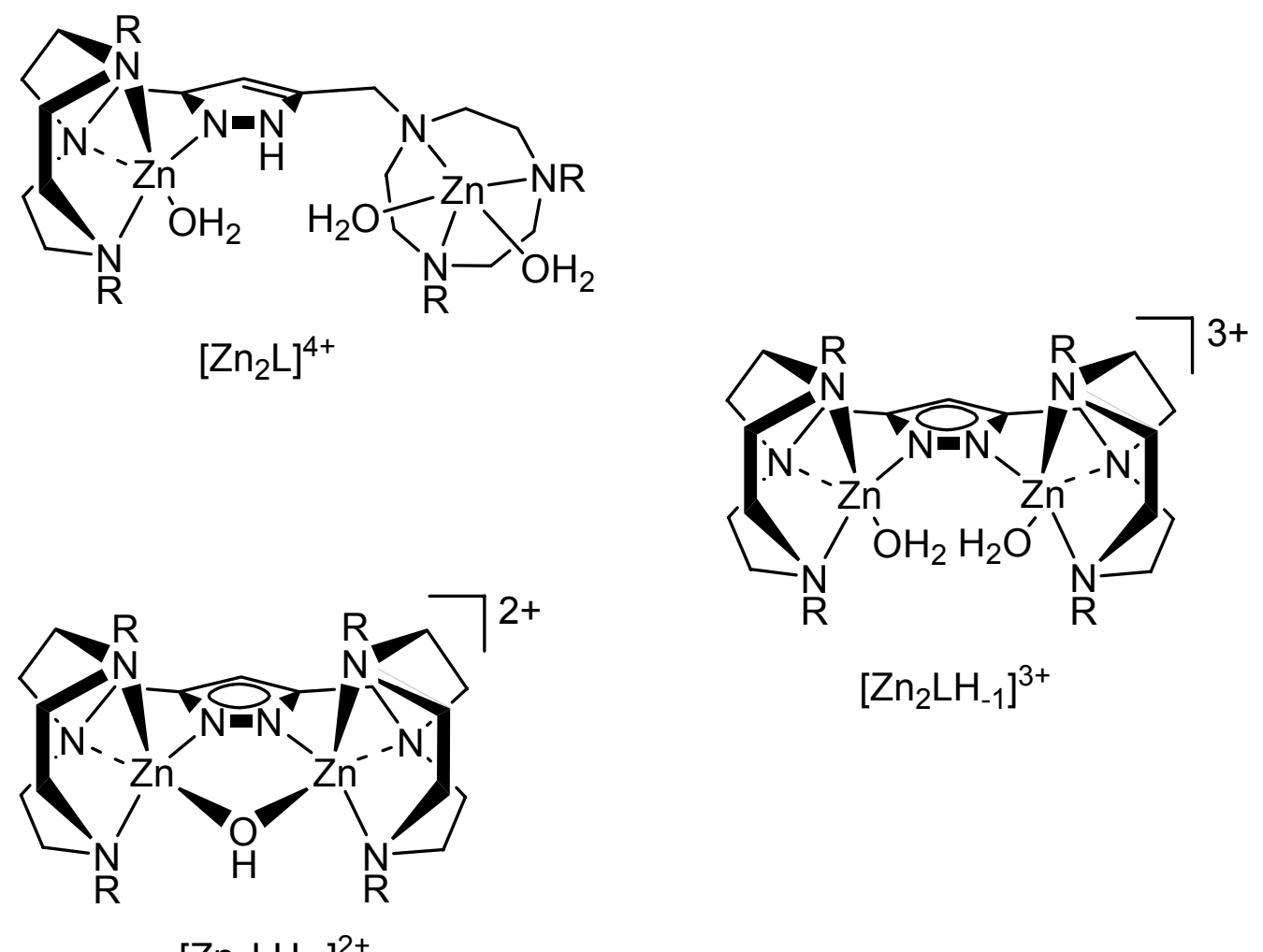

$\left[\mathrm{Zn}_{2} \mathrm{LH}_{-1}\right]^{3+}$

$$
\left[\mathrm{Zn}_{2} \mathrm{LH}_{-2}\right]^{2+}
$$

Abbildung 55: Strukturvorschläge für die dinuklearen Spezies $\left[\mathrm{Zn}_{2} \mathrm{~L}^{5}\right]^{4+},\left[\mathrm{Zn} \mathrm{L}^{5} \mathrm{H}_{-1}\right]^{3+}$ und $\left[\mathrm{Zn_{2 }} \mathrm{L}^{5} \mathrm{H}_{-2}\right]^{2+}$.

Der pKs-Wert von 8.04 für $\left[\mathrm{Zn}_{2} \mathrm{~L}^{5} \mathrm{H}_{-1}\right]^{3+}$ also stellt den pK $\mathrm{K}_{\mathrm{S}}$-Wert eines Zinkgebundenen Wassers dar. Obschon er geringer ist als der $\mathrm{pK}_{\mathrm{s}}$-Wert von 8.96 des Hexaquazinkkomplexes $\left[\mathrm{Zn}\left(\mathrm{H}_{2} \mathrm{O}\right)_{6}\right]^{2+}{ }^{2}{ }^{92]}$ ist dieser Wert jedoch etwas höher als erwartet, wenn man die verbrückende Position des Hydroxids in $\mathbf{3}$ in Betracht zieht. Für ein verbrückendes Wassermolekül in dinuklearen Zink(II)komplexen findet man oft pKs-Werte unter 8. Andererseits steht der pKs-Wert von 8.04 für $\left[\mathrm{Zn}_{2} \mathrm{~L}^{5} \mathrm{H}_{-1}\right]^{3+}$ gut im Einklang mit dem $\mathrm{pK}_{\mathrm{s}}$-Wert von 8.15, der für ein koordiniertes Wasser eines Dizinkkomplexes in einem verwandten Pyrazolat-verbrückten Ligand mit tacnSeitenarmen bestimmt wurde. ${ }^{[91]}$

$\mathrm{L}^{3} / \mathrm{Zn}^{2+}$ 
Der niedrige pK $\mathrm{K}_{\mathrm{S}}$ Wert von 7.57 für $\left[\mathrm{Zn}_{2} \mathrm{~L}^{3} \mathrm{H}_{-1}\right]^{3+}$ bestätigt die zusätzliche Stabilisierung des Hydroxids in $\left[\mathrm{Zn}_{2} \mathrm{~L}^{3} \mathrm{H}_{-2}\right]^{2+}(2 \mathrm{a})$, die aus der Aufnahme in die energetisch begünstigte intramolekulare $\mathrm{H}_{3} \mathrm{O}_{2}$-Brücke resultiert. $\left[\mathrm{Zn}_{2} \mathrm{~L}^{3} \mathrm{H}_{-2}\right]^{2+}$ ist die einzige Spezies von $\mathrm{L}^{2}$, die in einem nennenswerten Anteil entsteht. ${ }^{[78]}$

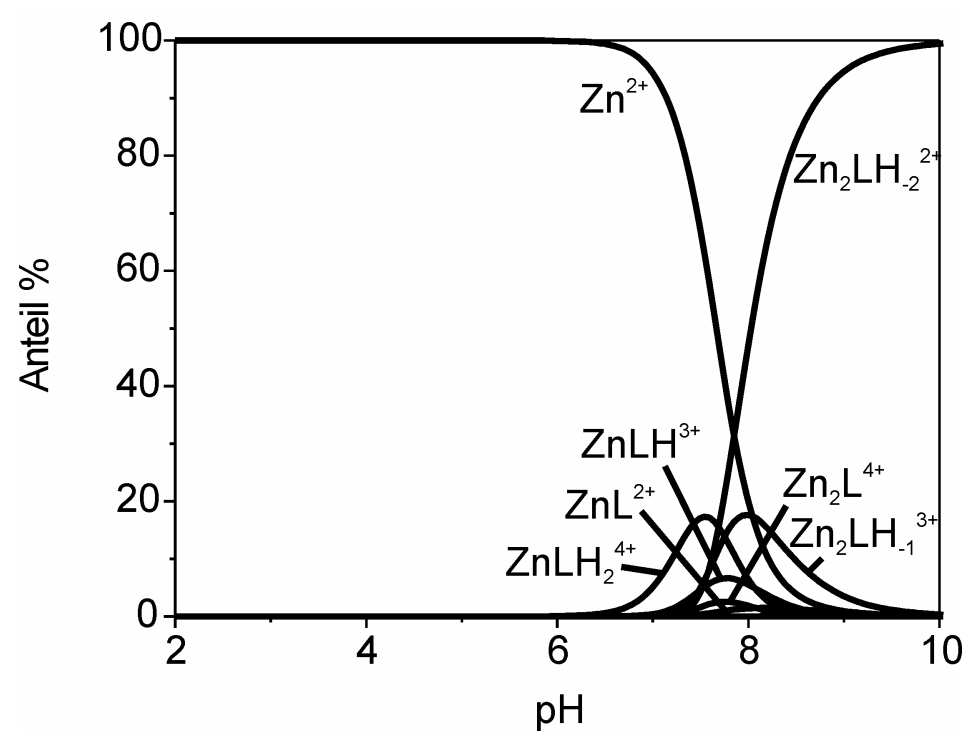

Abbildung 56: Speziesverteilung des Systems $L^{3} / \mathrm{Zn}^{2+}\left(I=0.5 \mathrm{M}\left(\mathrm{KNO}_{3}\right), T=25^{\circ} \mathrm{C}, c(L)=4 \mathrm{mM}\right.$, $\left.c\left(Z n^{2+}\right)=8 m M\right)$.

\section{$\mathrm{L}^{2} / \mathrm{Zn}^{2+}$}

Aufgrund der schwachen Komplexierung von $\mathrm{Zn}^{2+}$ schon durch $\mathrm{L}^{1}$ wurde auf eine potentiometrische Untersuchung von $\mathrm{L}^{2} / \mathrm{Zn}^{2+}$-Systemen verzichtet. Die Stabilität dieser Systeme sollte in wässriger Lösung noch geringer sein als die von $\mathrm{L}^{1} / \mathrm{Zn}^{2+}$, da die Propylendiaminseitenarme in $L^{2}$ wie $L^{1}$ nur die relativ instabilen Sechsringchelate ausbilden. Zusätzlich erniedrigt wird hier die Stabilität dadurch, dass auf jeder Koordinationsseite nur ein Chelatarm vorhanden ist. Eine Bestätigung dieser These liefern auch die NMR-Spektren der Verbindung 6. Sowohl im ${ }^{1} \mathrm{H}$ - als auch im ${ }^{13} \mathrm{C}$ NMR-Spektrum in $d_{6}$-DMSO findet man durch Austauschvorgänge mit dem in Spuren vorhandenen Wasser stark verbreiterte Signale.

\section{$\mathrm{L}^{6} / \mathrm{Zn}^{2+}$}

Die Stabilitäten der Komplexe der Pyridylliganden $L^{6}, L^{7}, L^{8}$ und $L^{9}$ mit Zink sollten aufgrund der linearen Abhängigkeit zwischen dem $\mathrm{pK}_{\mathrm{S}}$-Wert und $\lg \beta$ deutlich höher sein als die der entsprechenden aliphatischen Liganden. 
So bildet $L^{6}$ anders als der analoge aliphatische Ligand $L^{1}$ nicht nur eine stabile mononukleare Spezies $\left[\mathrm{ZnL}^{6} \mathrm{H}_{2}\right]^{4+}$, sondern auch die stabilen dinuklearen Spezies $\left[\mathrm{Zn}_{2} \mathrm{~L}^{6}\right]^{4+},\left[\mathrm{Zn}_{2} \mathrm{~L}^{6} \mathrm{H}_{-1}\right]^{3+}$ und $\left[\mathrm{Zn}_{2} \mathrm{~L}^{6} \mathrm{H}_{-2}\right]^{2+}$.

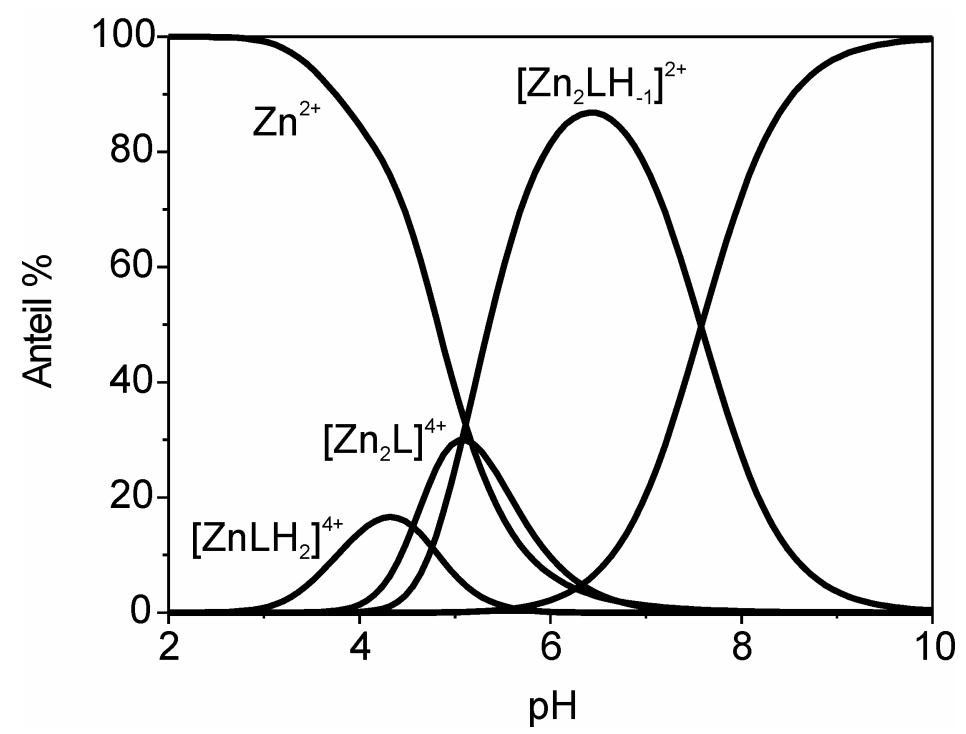

Abbildung 57: Speziesverteilung des Systems $L^{6} / Z n^{2+} ; I=0.2 M(K C l), T=25^{\circ} \mathrm{C}, c(L)=1 \mathrm{mM}$, $c\left(Z n^{2+}\right)=2 m M$.

Aufgrund der ermittelten pK $\mathrm{s}-$ Werte stellt $\left[\mathrm{Zn}_{2} \mathrm{~L}^{6}\right]^{4+}$ ähnlich wie $\left[\mathrm{Zn}_{2} \mathrm{~L}^{5}\right]^{4+}$ wohl eine Spezies dar, in der der Pyrazolring die beiden Metallionen nicht verbrückt. Die hohe Übereinstimmung des pKs-Werts des letzten Schritts der Deprotonierung von $\left[\mathrm{Zn}_{2} \mathrm{~L}^{6} \mathrm{H}_{-1}\right]^{3+} \mathrm{zu}\left[\mathrm{Zn}_{2} \mathrm{~L}^{6} \mathrm{H}_{-2}\right]^{2+}$ mit den entsprechenden Werten des $\mathrm{L}^{5} / \mathrm{Zn}^{2+}$-Systems legt nahe, dass der Schritt analog verläuft und eine Deprotonierung eines Zinkgebundenen Wassermoleküls zu der Hydroxy-verbrückten Spezies $\left[\mathrm{Zn}_{2} \mathrm{~L}^{6} \mathrm{H}_{-2}\right]^{2+}$ darstellt. 


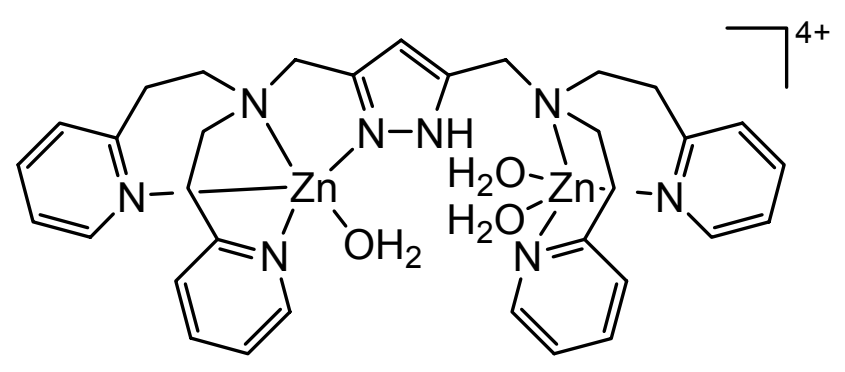

$\left[\mathrm{Zn}_{2} \mathrm{~L}^{6}\right]^{4+}$
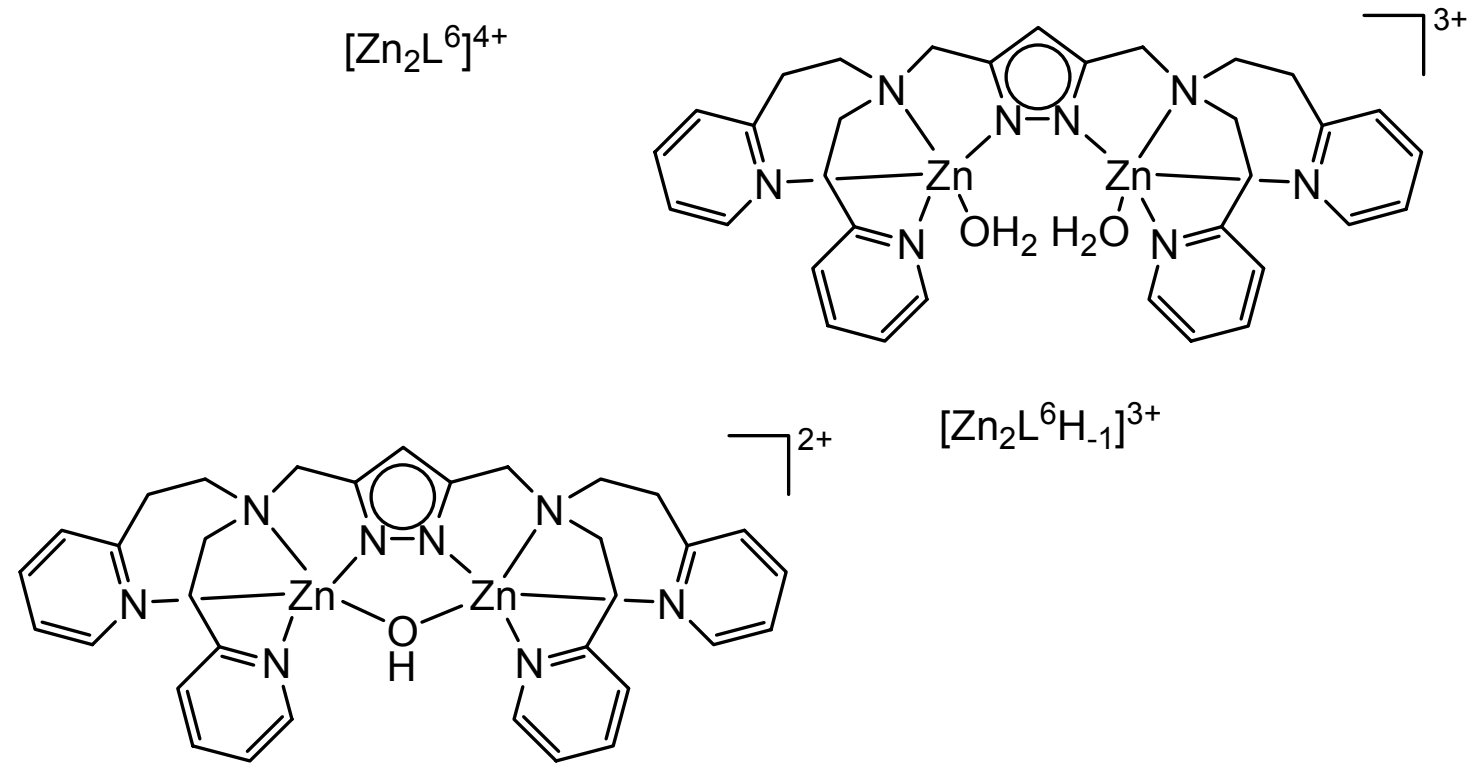

$$
\left[\mathrm{Zn}_{2} \mathrm{~L}^{6} \mathrm{H}_{-2}\right]^{2+}
$$

Abbildung 58: Strukturvorschläge für die dinuklearen Spezies $\left[\mathrm{Zn}_{2} L^{6}\right]^{4+},\left[\mathrm{Zn} \mathrm{n}_{2}{ }^{6} \mathrm{H}_{-1}\right]^{3+}$ und $\left[\mathrm{Zn} \mathrm{n}_{2} \mathrm{~L}^{6} \mathrm{H}_{-2}\right]^{2+}$.

Die potentiometrische Untersuchung dieses Systems konnte nur bis zu einem $\mathrm{pH}-$ Wert von etwa 8.5 durchgeführt werden, da sich bei höheren $\mathrm{pH}-$ Werten ein weißer Niederschlag bildete. Um auszuschließen, dass es sich bei dem Niederschlag um Zinkhydroxid handelt, wurde dieser abfiltriert und mittels ESI-MS als LiganddizinkSpezies charakterisiert.

\section{$\mathrm{L}^{7} / \mathrm{Zn}^{2+}$}

Die potentiometrische Untersuchung des Systems $\mathrm{L}^{7} / \mathrm{Zn}^{2+}$ wurde aufgrund der erwarteten geringen Stabilitätskonstanten nicht durchgeführt.

\section{$\mathrm{L}^{8} / \mathrm{Zn}^{2+}$}

Aus den Titrationskurven des zu $\mathrm{L}^{3}$ analogen Ligand $\mathrm{L}^{8}$ mit zwei Bis(pyridylmethyl)aminoseitenarmen in Gegenwart von $\mathrm{Zn}^{2+}$ konnten die mononuklearen Spezies 
$\left[\mathrm{ZnLH}_{2}\right]^{4+}$ und $[\mathrm{ZnLH}]^{3+}$ sowie die dinuklearen Spezies $\left[\mathrm{Zn}_{2} \mathrm{~L}^{6}\right]^{4+},\left[\mathrm{Zn}_{2} \mathrm{~L}^{6} \mathrm{H}_{-1}\right]^{3+}$ und $\left[\mathrm{Zn}_{2} \mathrm{~L}^{6} \mathrm{H}_{-2}\right]^{2+}$ errechnet werden.

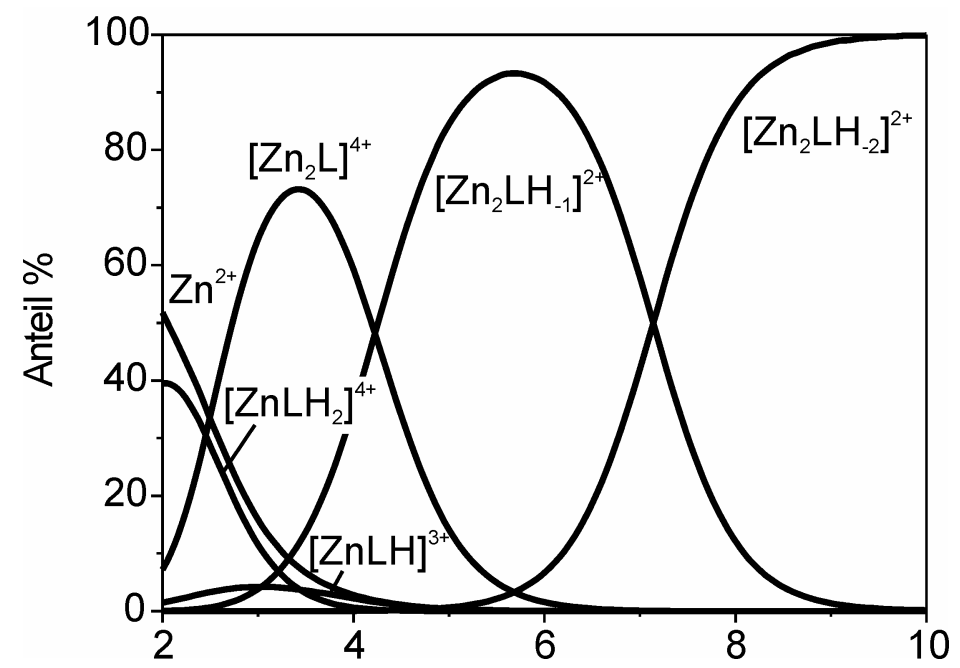

Abbildung 59: Speziesverteilung des Systems $L^{8} / Z n^{2+}\left(I=0.2 \mathrm{M}(K C l), T=25^{\circ} \mathrm{C}, c(L)=1 \mathrm{mM}\right.$, $\left.c\left(\mathrm{Zn}^{2+}\right)=2 \mathrm{mM}\right)$.

In der Speziesverteilung ist zu erkennen, dass $\mathrm{L}^{8}$ eine viel höhere Affinität $z u \mathrm{Zn}^{2+}$ hat als $\mathrm{L}^{6}$. Man findet schon bei $\mathrm{pH}-$ Werten $>4$ kein freies $\mathrm{Zn}^{2+}$ mehr in der Lösung, wohingegen dies im System $\mathrm{L}^{6}$ erst bei $\mathrm{pH}-$ Werten $>6$ der Fall ist. Außerdem ist zu erkennen, dass $\mathrm{L}^{8}$ bevorzugt dinukleare Systeme ausbildet; das Maximum der dinuklearen Spezies $\left[\mathrm{Zn}_{2} \mathrm{~L}^{6}\right]^{4+}$ wird unter den gemessenen Bedingungen bereits bei pH 3.5 erreicht.

Vergleicht man die Stabilitätskonstanten dieses Systems mit dem homologen System von $L^{6}$ und mit dem topologisch identischen System $L^{3}$, so fällt auf, dass man für $\mathrm{L}^{8} / \mathrm{Zn}^{2+}$ die höchsten Stabilitätskonstanten lg $\beta$ erhält. Die Bildungskonstante der $\left[\mathrm{Zn}_{2} \mathrm{~L}^{8} \mathrm{H}_{-2}\right]^{2+}$-Spezies ist um etwa acht Größenordnungen höher, als die der vergleichbaren $\left[\mathrm{Zn}_{2} \mathrm{~L}^{6} \mathrm{H}_{-2}\right]^{2+}$-Spezies.

Da auch die Löslichkeit der $\left[\mathrm{Zn}_{2} \mathrm{~L}^{8} \mathrm{H}_{-2}\right]^{2+}$-Spezies verglichen mit jener der analogen $\mathrm{L}^{6}$-Spezies in Wasser sehr viel höher ist, konnten die Titrationen bis zu einem $\mathrm{pH}$ von 11 durchgeführt werden; es wurde keine Zinkhydroxidbildung beobachtet.

\section{$\mathrm{L}^{9} / \mathrm{Zn}^{2+}$}

Die Auswertung des Systems $\mathrm{L}^{9} / \mathrm{Zn}^{2+}$ musste bei einem $\mathrm{pH}$-Wert von 5 abgebrochen werden, da sich während der Messung ein farbloser Niederschlag bildete. Dieser wurde durch Filtration isoliert und im ESI-MS als Chlorid-haltige Liganddizinkspezies 
charakterisiert. Auch die Ergebnisse der durchgeführten Rechnungen über $\mathrm{pH} 5$ deuteten auf die Entstehung höhernukleare Spezies hin.

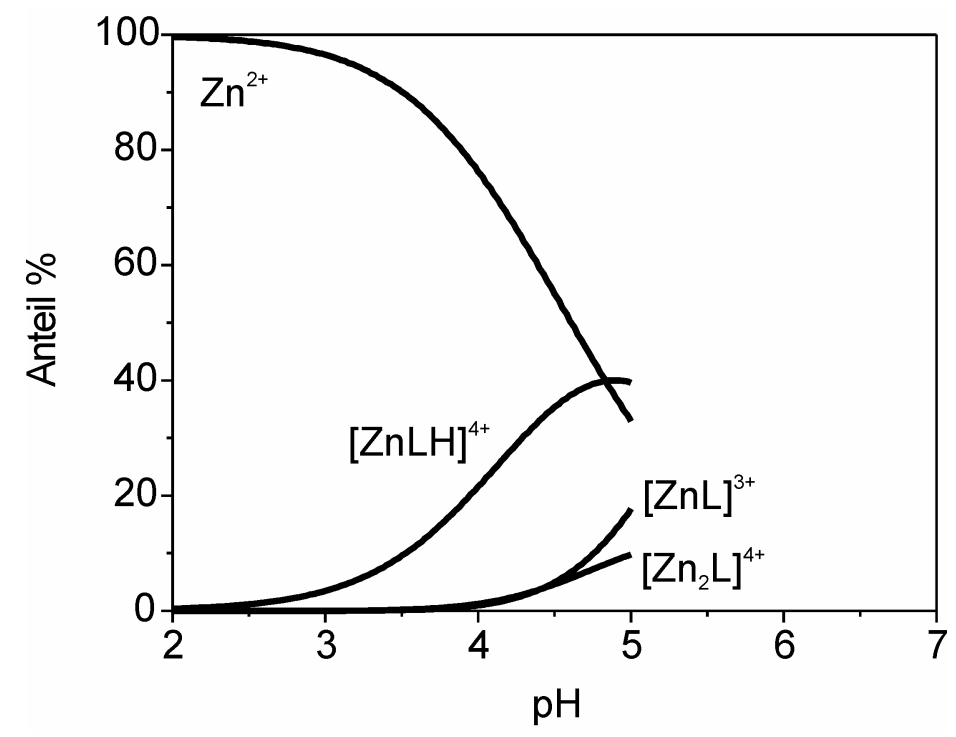

Abbildung 60: Speziesverteilung des Systems $L^{9} / \mathrm{Zn}^{2+}\left(I=0.2 \mathrm{M}(\mathrm{KCl}), T=25^{\circ} \mathrm{C}, \mathrm{c}(L)=4 \mathrm{mM}\right.$, $\left.c\left(\mathrm{Zn}^{2+}\right)=8 \mathrm{mM}\right)$.

Aus den durch die Titrationen erhaltenen Werten konnten im $\mathrm{pH}-$ Bereich zwischen 2 und 5 die beiden mononuklearen Spezies $\left[\mathrm{ZnL}^{9} \mathrm{H}\right]^{3+}$ und $\left[\mathrm{ZnL}^{9}\right]^{2+}$ und die dinukleare Spezies $\left[\mathrm{Zn}_{2} \mathrm{~L}^{9}\right]^{4+}$ errechnet werden.

Um die Bildung von höhermolekularen Ligand/Zink/Chlorid-Spezies auszuschließen, wurde eine zusätzliche Titration des Liganden und Zink(II) in Anwesenheit von Kaliumnitrat zur Einstellung der lonenstärke durchgeführt. Jedoch musste die Titration bei einem $\mathrm{pH}$ von 8.5 wegen der Bildung von Zinkhydroxid abgebrochen werden. Dieses Ligandsystem bildet also entgegen der Erwartung keine stabileren Dizinkkomplexe als das analoge $L^{4}$-System.

Die Messungen zeigen, dass in den Dizinkkomplexen eine gegenüber mononuklearen Komplexen deutlich stärkere Aktivierung des Zink-gebundenen Wassers erfolgt. Terminal gebundene Wassermoleküle vergleichbarer, einkerniger Zinkkomplexe tripodaler Liganden (Abbildung 61) besitzen $\mathrm{pK}_{\mathrm{s}}$-Werte um 10. ${ }^{\text {[9-95] }}$ 


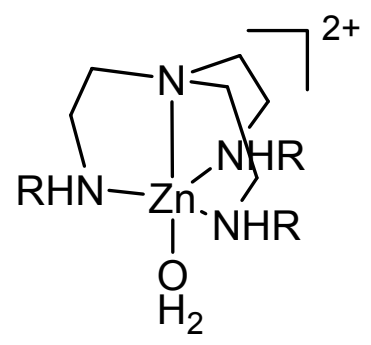

XXVII

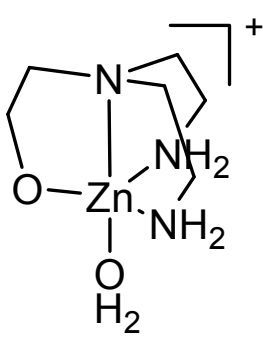

XXVIII<smiles>N[N+]1(N)CC[CH+]CN1c1ccccc1O</smiles>

XXIX
a $\mathrm{R}=\mathrm{H} \quad \mathrm{pK}_{\mathrm{S}}=10.7$
$\mathrm{R}=\mathrm{H}$
$\mathrm{R}=\mathrm{H}$
b $\mathrm{R}=\mathrm{Bn} \mathrm{pK}_{\mathrm{S}}=9.61$
$\mathrm{pK}_{\mathrm{S}}=9.8$
$\mathrm{pK}_{\mathrm{S}}=10.7$

Abbildung 61: pKs-Werte tripodaler Monozinkkomplexe.

Unklar ist im Fall des mononuklearen Systems $\mathrm{L}^{10} / \mathrm{Zn}^{2+}$, ob der gemessene $\mathrm{pK}$-Wert von 8.63 der Deprotonierung der [ZnL]-Spezies auch einer Deprotonierung eines Zink-gebundenen Wassers entspricht, da der Wert, vergleicht man inn mit den Literaturwerten aus Abbildung 61, relativ niedrig ist.

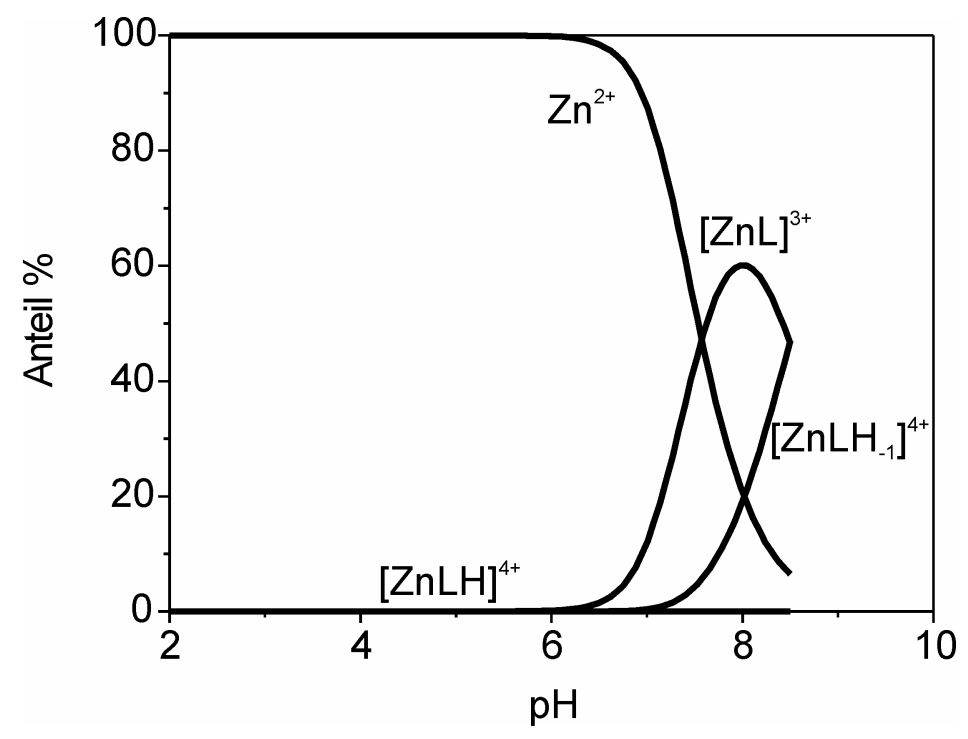

Abbildung 62: Speziesverteilung des Systems $L^{10} / \mathrm{Zn}^{2+}\left(I=0.2 \mathrm{M}(K C l), T=25^{\circ} \mathrm{C}, \mathrm{c}(\mathrm{L})=4 \mathrm{mM}\right.$, $\left.c\left(\mathrm{Zn}^{2+}\right)=4 \mathrm{mM}\right)$.

Auffallend bei einem Vergleich der $\mathrm{pK}_{\mathrm{s}}$-Werte für den jeweils letzten Schritt der Deprotonierung eines Zink-gebundenen Wassermoleküls ist die starke Übereinstimmung von Struktur und $\mathrm{pK}_{\mathrm{S}}$-Wert der Bildung der $\left[\mathrm{Zn}_{2} \mathrm{LH}_{-2}\right]$-Spezies der bis(tetradentaten) Liganden. So findet man für die Bildung der hydroxyverbrückten Dizinkkomplexe von $L^{5}$ und $L^{6}$ einen $p K_{S}$-Wert von etwa $8.0\left(L^{5} 8.04, L^{6} 8.02\right)$ und für 
die Bildung der $\mathrm{H}_{3} \mathrm{O}_{2}$-Brücken von $\mathrm{L}^{3}$ und $\mathrm{L}^{8}$ einen pKs-Wert von etwa $7.6\left(\mathrm{~L}^{5} 7.57\right.$, $\mathrm{L}^{6}$ 7.60). Die Bildung einer $\mathrm{H}_{3} \mathrm{O}_{2}$-Brücke ist also thermodynamisch günstiger als die Bildung einer Hydroxidbrücke. Am leichtesten lässt sich dieses Ergebnis durch eine Analyse der Produkte erklären. Im Fall der $\mathrm{H}_{3} \mathrm{O}_{2}$-Brücke kann sich die negative Ladung durch die flexible Wasserstoffbrückenbindung in der Brücke über einen größeren Bereich verteilen; zudem macht die lange Brücke optimale, ausgeglichene $\mathrm{Zn}-\mathrm{N}-$ Abstände möglich. Demgegenüber ist in den $\mu-\mathrm{OH}$-Dizinkkomplexen jeweils ein $\mathrm{Zn}-\mathrm{N}$-Abstand stark verlängert, so dass die dadurch verzerrte Koordinationsumgebung thermodynamisch insgesamt ungünstiger ist.

Als Konsequenz scheint das dinukleare Gerüst in 1, 3 und 4 gespannt zu sein, und das verbrückende Hydroxid kann sich möglicherweise unter geeigneten Reaktionsbedingungen relativ einfach in eine halbverbrückende oder sogar nicht-verbrückende Position verschieben.

In allen vollständig potentiometrisch bestimmten Systemen ist die Bildung eines dinuklearen Komplexes gegenüber zwei mononuklearen Spezies bevorzugt. Setzt man Ligand und $\mathrm{Zn}^{2+}$ nicht im Verhältnis 1:2, sondern äquimolar miteinander um, so entstehen bei höherem $\mathrm{pH}$ nicht mononukleare Spezies; stattdessen liegt die eine Hälfte des Liganden als Dizinkkomplex vor, die andere Hälfte als freier Ligand (Abbildung 63). Diese Kooperativität bereits in der Bindung der Zinkionen unterstreicht die hervorragende Eignung dieser Liganden zur Untersuchung dinuklearer Zinkkomplexe.

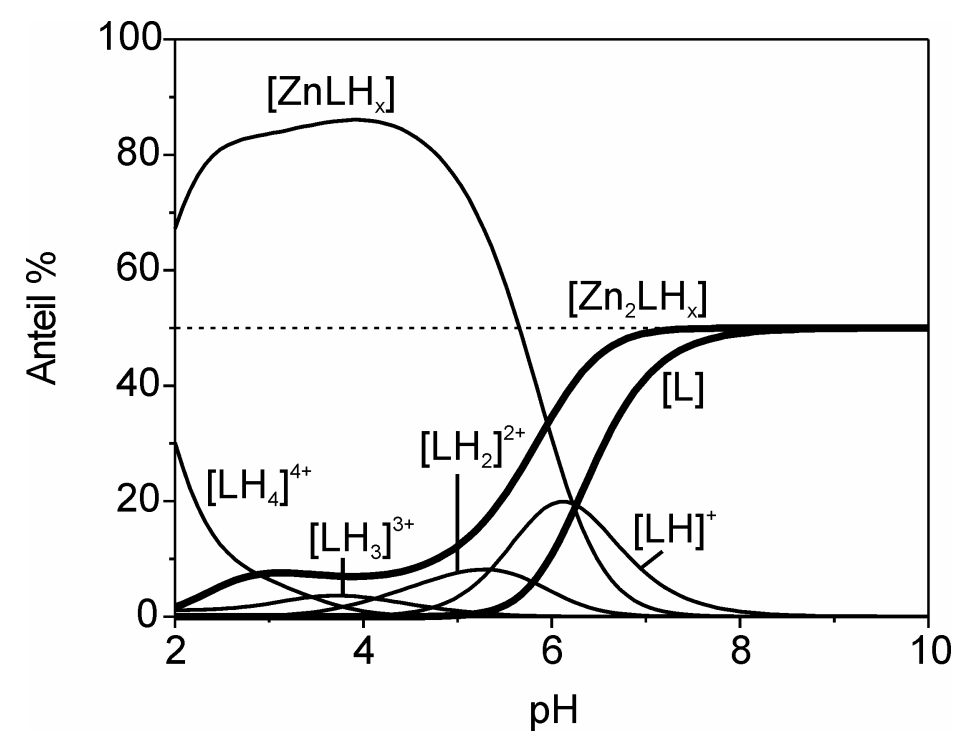


Abbildung 63: Speziesanteile bezogen auf den Liganden für eine äquimolare Lösung von $L^{8}$ und $Z n^{2+}$ $\left(c\left(L^{8}\right)=c\left(Z n^{2+}\right)=1 m M\right)$. 


\section{Aktivität der Pyrazolatodizinkkomplexe in der Phosphatesterhydrolyse}

\subsection{Modellsubstrate für die Spaltung von Phosphatestern}

Zur Messung von Aktivitäten werden oft Modellsubstrate verwendet, die leichter als natürliche Phosphatester, wie z.B. DNA, hydrolysiert werden können. Dabei haben sich vor allem 4-Nitrophenylphosphate aufgrund ihrer erhöhten Reaktionsgeschwindigkeit als Substrate durchgesetzt. ${ }^{[96,97]}$ Die Spaltung der Phosphatesterbindung und die damit verbundene Freisetzung von 4-Nitrophenolat kann sehr einfach anhand der starken Absorption bei ca. $410 \mathrm{~nm}$ verfolgt werden. Aufgrund der stark abnehmenden Reaktionsgeschwindigkeiten ${ }^{[93]}$ der drei Hydrolyseschritte $k_{1}>$ $k_{2} \gg k_{3}$ (Abbildung 64) können Tris(4-nitrophenyl)phosphat (TNPP), Bis(4-nitrophenyl)phosphat (BNPP) und Mono(4-nitrophenyl)phosphat (MNPP) als Modellverbindungen für die Spaltung von Phosphattriestern, -diestern bzw. -monoestern eingesetzt werden.
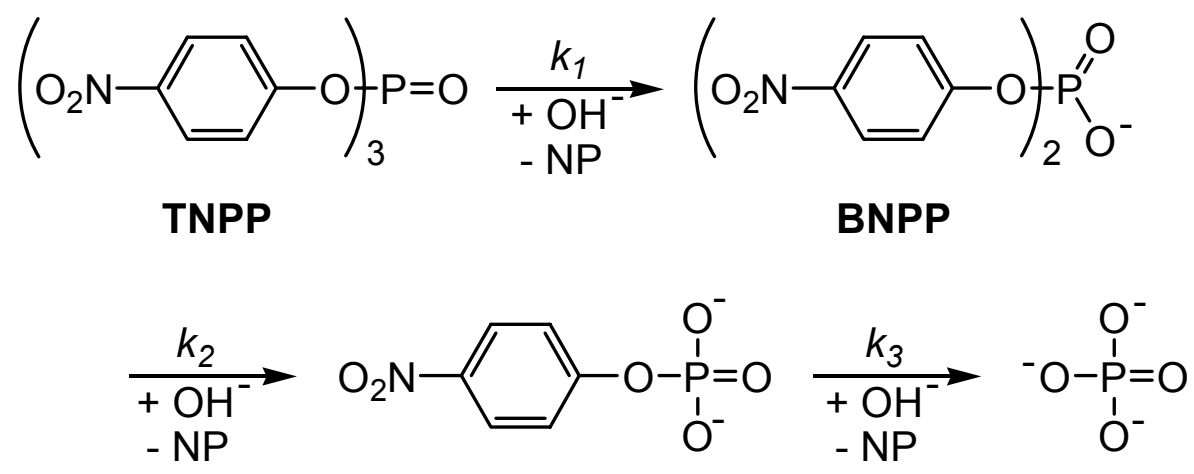

MNPP

Abbildung 64: Spaltung der Modellsubstrate TNPP, BNPP und MNPP.

Im Fall der RNA erfolgt die Spaltung nicht durch den intermolekularen Angriff eines freien oder metallgebunden Hydroxidions, sondern durch den intramolekularen Angriff der 2'-Hydroxyfunktion. Um diese Reaktion zu modellieren, wird oft 2Hydroxyproyl-4-nitrophenylphosphat (HPNPP) als Modellsubstrat eingesetzt. Dieses ist in Abwesenheit von Metallkatalysatoren relativ stabil. ${ }^{[98]}$ 


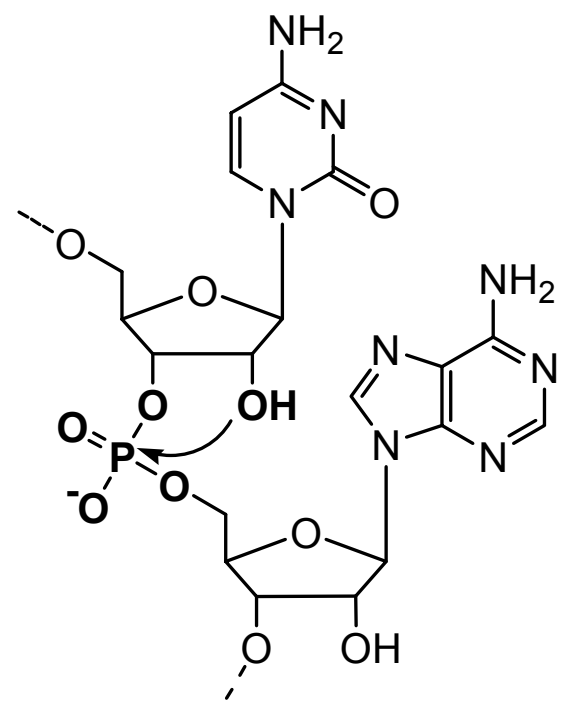

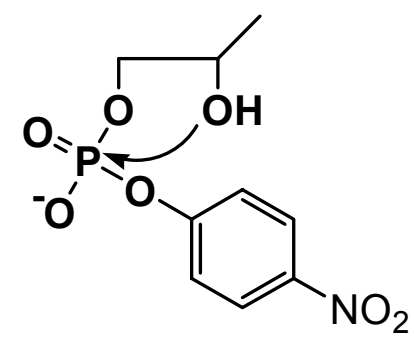

HPNPP

Abbildung 65: Spaltung von RNA (links) und dem Modellsubstrat HPNPP (rechts).

\subsection{Zweikernige Zinkkomplexe als Modelle für Hydrolasen}

Ein erstes Ziel der Untersuchung von Modellverbindungen für bimetallische Enzymzentren ist der Aufbau und die strukturelle Charakterisierung zweikerniger Systeme, um Vergleiche mit den Enzymstrukturen zu ermöglichen. Ein zweites ist, die Kooperativität zwischen den beiden Metallzentren durch die höhere Aktivität dinuklearer gegenüber mononuklearen Komplexen zu belegen, und ein drittens ist, Einblicke in Mechanismen der ablaufenden Hydrolysen zu gewinnen.

So sind bereits zahlreiche Modellverbindungen synthetisiert worden, um zwei Metallionen zu binden und Phosphatester zu hydrolysieren. ${ }^{[4,6,99]}$ Einige Aspekte neuerer Arbeiten seien hier kurz vorgestellt.

Um den Einfluss kooperativer Effekte in einem Dizinkzentrum gegenüber einem mononuklearen Zinkkomplex zu untersuchen, wurden von Iranzo et al. zwei tacnbasierte Komplexe dargestellt und charakterisiert (Abbildung 66). ${ }^{[100]}$ Dabei zeigt das Bis(triazacyclononan)system XXXI eine deutlich höhere Aktivität gegenüber HPNPP als der entsprechende mononukleare Baustein XXX. Dies wird auf eine Aktivierung des Substrats durch den Komplex und die Stabilisierung des Übergangszustands durch XXXI um $9.3 \mathrm{kcal} / \mathrm{mol}$ (bei $\mathrm{pH}$ 7.6) aufgrund elektrostatischer Wechselwirkungen zurückgeführt. 


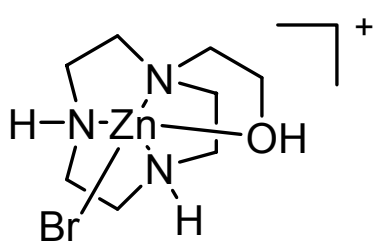

$\mathbf{X X X}$

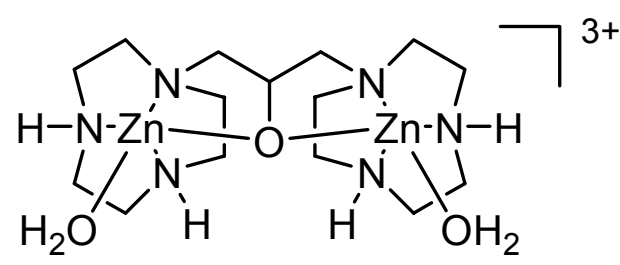

$\mathbf{X X X I}$

Abbildung 66: Mono- und dinuklearer Zinkkomplex von Iranzo et al.

Die Beteiligung eines Zink-gebundenen Hydroxids konnte bisher durch mehrere Untersuchungen belegt werden:

So sind die Dizinkspezies $\left[\mathrm{Zn}_{2} \mathrm{~L}\right]$ und $\left[\mathrm{Zn}_{2} \mathrm{~L}(\mathrm{OH})\right]^{2+}$ - eine Dizinkhydroxidspezies des von Martell et al. verwendeten makrozyklischen Liganden in Abbildung 67 an der hohen Aktivität in der Hydrolyse von TNPP beteiligt. ${ }^{[101]}$

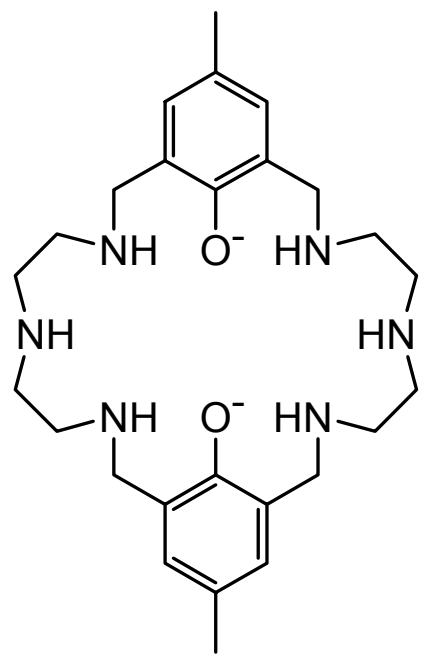

Abbildung 67: Makrozyklischer Ligand BDBPH von Martell et al.

Auch die von Krebs et al. eingesetzten Dizinkkomplexe der Liganden in Abbildung 68 zeigen nur in dem pH-Bereich eine Aktivität gegenüber HPNPP, in dem Zinkhydroxid-haltige $\left[\mathrm{Zn}_{2} \mathrm{~L}(\mathrm{OH})\right]^{2+}$-Spezies vorliegen. ${ }^{[102]}$ 
<smiles>[O-]C(CN(Cc1ccccn1)Cc1ccccn1)CN(Cc1ccccn1)Cc1ccccn1</smiles><smiles>Cc1cc2nc(CN(Cc3nc4cc(C)c(C)cc4[nH]3)CC([O-])CN(Cc3ccccc3)Cc3ccccc3)[nH]c2cc1C</smiles>

Abbildung 68: Von Krebs et al. verwendete Liganden.

Ein weiterer Schritt bei der Modellierung von Metalloenzymen besteht in der Nachbildung hydrophober Enzymbereiche, die oft einen deutlichen Einfluss auf Vorgänge wie der Substratanbindung besitzen.

Reinhoudt et al. entwickelten beispielsweise Calix[4]arene mit zwei (Abbildung 69)

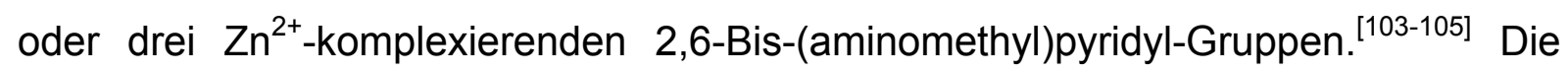
Ergebnisse der Hydrolyse von HPNPP lassen auf eine Kooperativität zwischen den Zn(II)-Zentren der Komplexe schließen. Eine Erhöhung der Reaktionsrate um Faktoren von 23000 bis 32000 mit diesen di- und trinuklearen Zinkspezies gegenüber der unkatalysierten Reaktion werden durch hydrophobe Effekte und auch durch die Flexibilität der einzelnen Zinkeinheiten hervorgerufen.

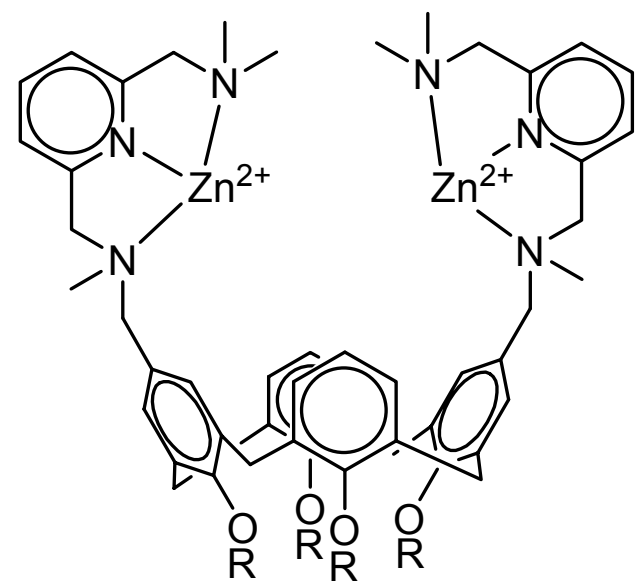

Abbildung 69: Calix[4]aren Dizinkkomplex.

Scrimin et al. ${ }^{[106,107]}$ synthetisierten naturähnliche, auf Aminosäuren basierte Dizinksysteme und konnten zeigen, dass auch kurze Peptidsequenzen - sofern man in der Lage ist, die Sekundärstruktur zu kontrollieren - dazu geeignet sind, als Gerüst für effektive Transphosphorylierungskatalysatoren zu dienen. 
Durch das Design eines Heptapeptids mit zwei Triazacyclononan-funktionalisierten $\alpha$-Aminosäuren an den $\mathrm{i} /(\mathrm{i}+3)$-Positionen des Peptids können sie eine $33_{10}$-helikale Konformation in neutraler wässriger Lösung stabilisieren. Dieses Heptapeptid bildet einen dinuklearen $\mathrm{Zn}(\mathrm{II})-K o m p l e x$, der die intramolekulare Transesterifizierung von HPNPP katalysiert und auch in der Lage ist, Plasmid-DNA zu spalten.

Um dem Ziel einer sequenzspezifischen Nuklease näher zu kommen, wurde von Komiyama et al. ein Dizinkkomplex mit DNA verknüpft. Durch die so erreichte sequenzspezifische Positionierung des Komplexes zur RNA konnte eine selektive Spaltung einer komplementären RNA erreicht werden. Reaktionen mit einem analogen mononuklearen Zinkkomplex führten hingegen nicht zur RNA-Spaltung. ${ }^{[108]}$

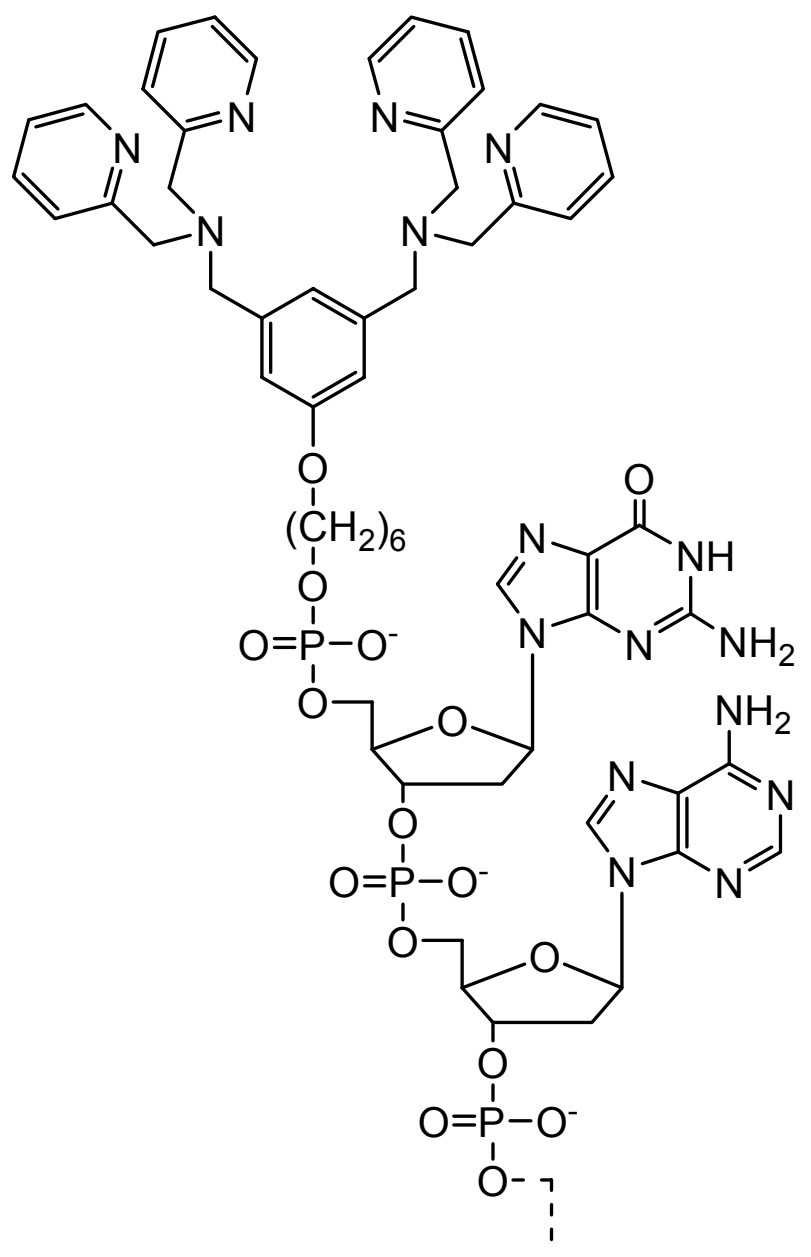

Abbildung 70: Ligand-DNA Konjugat.

Vergleiche der Aktivität der einzelnen Modellkomplexe miteinander sind jedoch kaum möglich, da Löslichkeit und Reaktivität sich über einen weiten Bereich erstrecken und 
so für die einzelnen Systeme sehr unterschiedliche Messbedingungen wie Temperatur, Lösungsmittel, lonenstärke oder Pufferzusammensetzung und -konzentration gewählt werden.

\subsection{Kinetische Untersuchung der Hydrolyse von BNPP}

In dieser Arbeit wurde die Hydrolyse von BNPP durch die beschriebenen Komplexe als Modellreaktion für die Phosphatdiesterspaltung eingehend untersucht. Dazu wurde der Reaktionsverlauf in Abhängigkeit der Komplexkonzentration, der Substratkonzentration und des $\mathrm{pH}-$ Werts durchgeführt.

Für die Untersuchungen wurde ein 1:1 (v:v)-Lösungsmittelgemisch aus Dimethylsulfoxid und Wasser verwendet. Dies gewährleistet eine aufgrund des hohen Wasseranteils quasi wassergleiche Lösung, so dass die Speziesverteilungen, die in reinem Wasser ermittelt wurden, auf die Reaktivitätsuntersuchungen übertragen werden können. Gleichzeitig sorgt der DMSO-Anteil für eine gute Löslichkeit der Komplexe. Der relativ hohe Wasseranteil ermöglicht auch eine Fixierung der lonenstärke durch Zugabe von Natriumperchlorat. Dies besitzt eine ausreichende Löslichkeit in der verwendeten Lösungsmittelmischung.

Als Puffer wurden Sulfonatpuffer (Abbildung 71) eingesetzt, da sich durch sie ein weiter pH-Bereich abdecken lässt, und sie im Gegensatz etwa zu Acetat- oder Phosphatpuffer im Allgemeinen nicht an Metallzentren koordinieren. Zudem sind sich die einzelnen Puffer chemisch sehr ähnlich; ein aufgrund des pH-Werts nötiger Wechsel des Puffers sollte deshalb nicht zu Änderungen der Art und Stärke ihrer Wechselwirkungen mit Komplexen und Substrat führen. Die Puffer wurden jeweils mit Perchlorsäure oder Natronlauge eingestellt. 


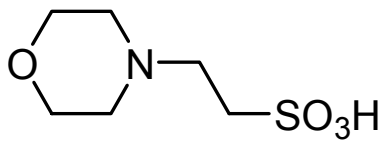

MES

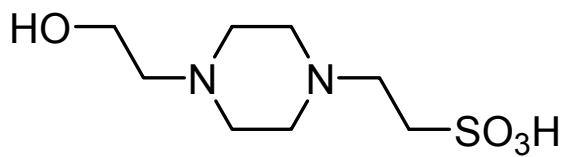

HEPES

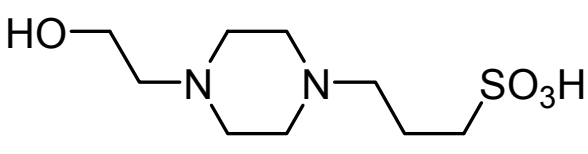

HEPPS

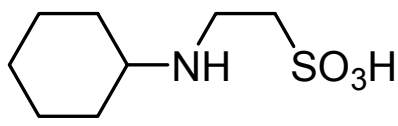

CHES

Abbildung 71: Verwendete Sulfonatpuffer.

Die Reaktionsgeschwindigkeit bei $50{ }^{\circ} \mathrm{C}$ wurde durch den Anstieg der Nitrophenolatkonzentration anhand der Absorption bei $414.5 \mathrm{~nm}$ verfolgt, nach dem Prinzip der Zweiwellenlängenmethode bei $500 \mathrm{~nm}$ referenziert und nur die Anfangsgeschwindigkeit zur weiteren Auswertung herangezogen („method of initial rates"). ${ }^{[109]}$

Die Extinktionswerte wurden mit dem Lambert-Beerschen Gesetz in Konzentrationswerte umgerechnet. Die Extinktionswerte des Nitrophenolats wurden dazu durch Messung der Extinktion von Nitrophenolat unter den gleichen Messbedingungen bestimmt. $\mathrm{Zu}$ dem Zweck wurden pro pH-Wert vier Extinktionswerte in Abhängigkeit der Nitrophenolatkonzentration gemessen und durch lineare Regression der Kalibriergerade die pH-abhängigen effektiven Extinktionskoeffizienten $\varepsilon_{\text {eff }}$ nach dem Lambert-Beerschen Gesetz bestimmt.

Die Hintergrundhydrolyse von BNPP kann vernachlässigt werden; unter den gewählten Reaktionsbedingungen konnte während des Zeitraums einer Messung keine Hydrolyse von BNPP allein detektiert werden.

\subsection{1 pH-Abhängigkeit der BNPP-Hydrolyse}

Um die aktive Spezies zu identifizieren, wurde die Anfangsgeschwindigkeit in Abhängigkeit vom pH-Wert bestimmt und mit den ermittelten Speziesverteilungen verglichen.

Wie bereits beschrieben, ist Komplex 1 in wässrigen Lösungen nicht stabil. Die pHabhängigen Geschwindigkeitskonstanten pseudo-erster Ordnung für die Hydrolyse von BNPP zeigen eine glockenförmige Abhängigkeit aufgrund des Niederschlags von 
Zinkhydroxid $\mathrm{Zn}(\mathrm{OH})_{2}$ bei höherem $\mathrm{pH}$. Über die Ursache der beobachteten Aktivität bis zu einem pH von etwa 8 lässt sich jedoch nur spekulieren. Möglicherweise sind Dizinkspezies dieses Liganden im verwendeten Lösungsmittelgemisch im neutralen pH-Bereich im Gegensatz zur reinen wässrigen Lösung noch stabil. Dagegen spricht jedoch, dass in ESI-MS-Spektren von 1 in einer 1:1 (v:v) Mischung von Wasser und Methanol (1:1) keine Dizinkspezies wie $\left[\mathrm{L}^{1}{ }_{-} \mathrm{Zn}_{2}(\mathrm{OH})\left(\mathrm{ClO}_{4}\right)\right]^{+} \mathrm{Zu}$ finden waren und auch im ${ }^{1} \mathrm{H}$-NMR-Spektrum von 1 schon in reinem $\mathrm{d}_{6}$-DMSO sehr breite Signale zu beobachten sind. Genauere Aussagen über die Stabilität des Komplexes in Lösungsmittelgemischen und über die aktiven Spezies in der BNPP-Hydrolyse lassen sich aus den vorliegenden Ergebnissen daher nicht treffen.

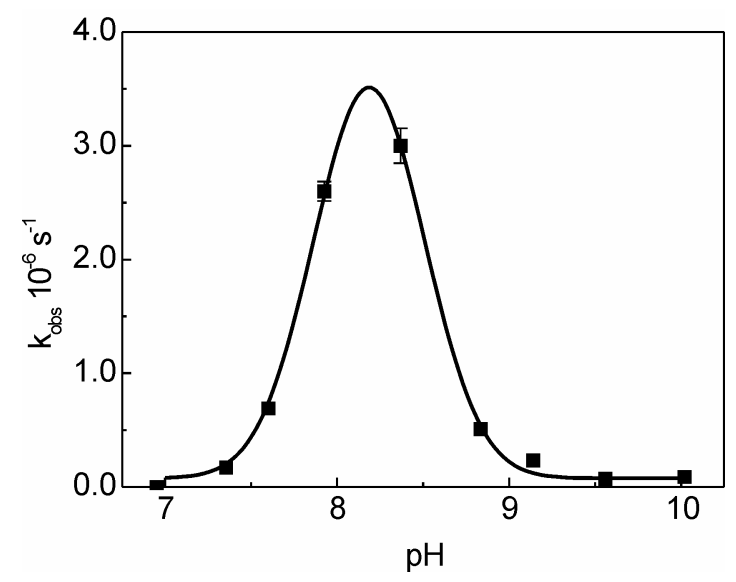

Abbildung 72: $p H$-Abhängigkeit der BNPP Hydrolyse durch 1. $[1]_{0}=0.8 \mathrm{mM},[B N P P]_{0}=4 \mathrm{mM}, 50^{\circ} \mathrm{C}$, in DMSO/Pufferlösung (1:1).

$\mathrm{Da}$ in den Messungen stets die Perchlorat-Salze der Komplexe eingesetzt wurden und um den eventuellen Einfluss eines anderen, stärker koordinierenden Anions auszuschließen, wurde anstatt Komplex 7 für $L^{4}$ eine Komplexlösung aus einem Äquivalent $L^{4}$ und zwei Äquivalenten Zinkperchlorat hergestellt (8'). 
Für 3, 4, 5 und 8' zeigt die Auftragung von $k_{\text {obs }}$ gegen den pH-Wert sigmoide Kurven mit Wendepunkten, die mit den $\mathrm{pK}_{\mathrm{S}}$-Werten der zugehörigen $\left[\mathrm{Zn}_{2} \mathrm{LH}_{-1}\right]^{3+}$-Spezies übereinstimmen. Dies gibt einen klaren Hinweis darauf, dass $\left[\mathrm{Zn}_{2} \mathrm{LH}_{-2}\right]^{2+}$ die aktive Spezies sein muss, und dass ein Zink-gebundenes Hydroxid für die Reaktivität notwendig ist.

$$
v_{0}=k_{\mathrm{obs}} \cdot[\text { Komplex }]_{0}
$$

\section{Gleichung 1}

Für 4 zeigt sich ein starker Abfall der Aktivität von pH $8 \mathrm{zu}$ pH 8.6 durch den Pufferwechsel von HEPES zu CHES (Abbildung 74). Um zu überprüfen, ob dies durch den Puffer verursacht wird oder durch andere Ursachen hervorgerufen wird, wurde die Hydrolyse auch mit HEPPS-Puffer durchgeführt, der im Übergangsbereich beider Puffer verwendet werden kann. Hier zeigt sich nur ein geringer Aktivitätsunterschied im Bereich des Pufferwechsels, so dass man annehmen muss, dass der CHES-Puffer die Reaktion beeinflusst. ESI-MS-Messungen von 4 mit CHES zeigten jedoch keine Adduktbildung, so dass die Ursache des Abfalls nicht klar ist.

Bei einem pH-Wert > 8.5 fällt die Aktivität von 8' aufgrund der Instabilität dieses Komplexes im basischen Milieu stark ab; jedoch konnte im Unterschied zu Systemen von $\mathrm{L}^{1}$ und $\mathrm{L}^{2}$ kein Niederschlag von Zinkhydroxid beobachtet werden.

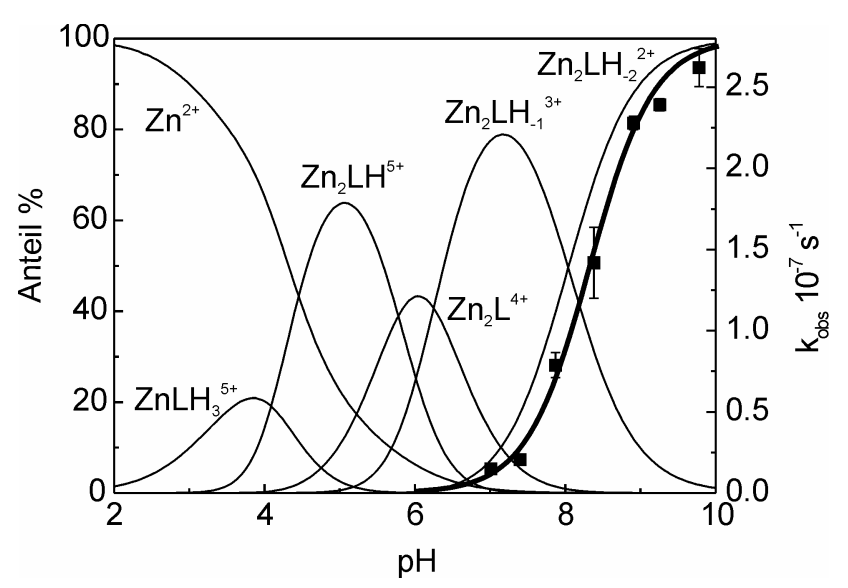

Abbildung 73: Speziesverteilung und pH-Abhängigkeit der BNPP Hydrolyse durch 3; $[3]_{0}=0.8 \mathrm{mM}$, $[B N P P]_{0}=4 \mathrm{mM}, 50^{\circ} \mathrm{C}$, in DMSO/Pufferlösung (1:1). 


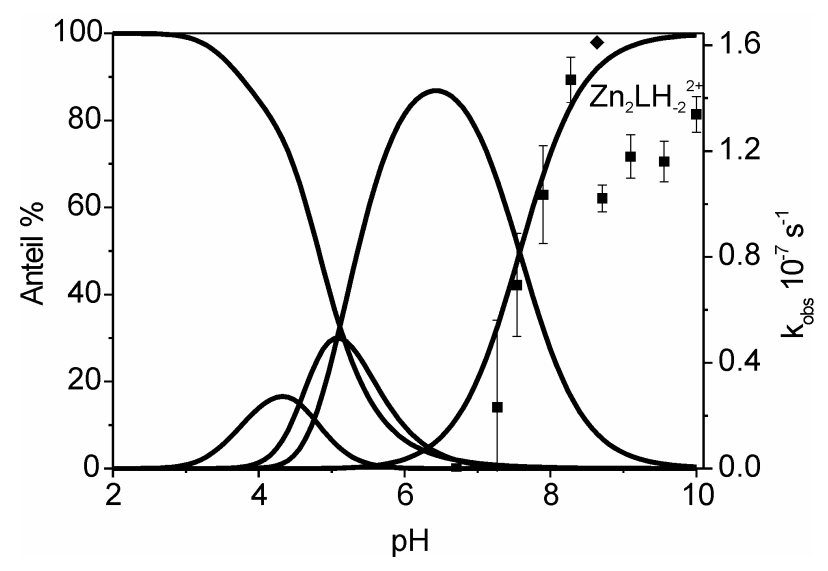

Abbildung 74: Speziesverteilung und pH-Abhängigkeit der BNPP Hydrolyse durch 4; $[4]_{0}=0.8 \mathrm{mM}$, $[B N P P]_{0}=2 \mathrm{mM}, 50^{\circ} \mathrm{C}$, in DMSO/Pufferlösung (1:1) ( HEPPS-Puffer).

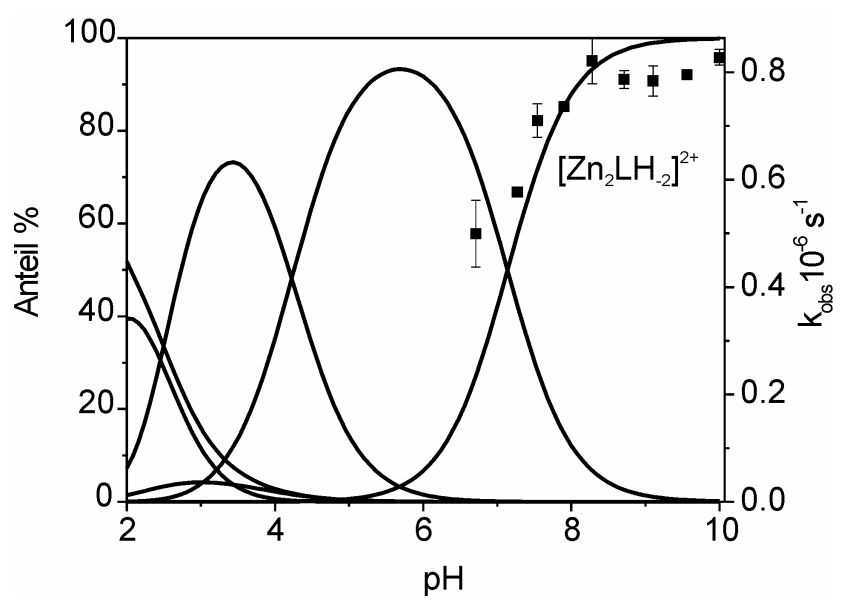

Abbildung 75: Speziesverteilung und pH-Abhängigkeit der BNPP Hydrolyse durch 5; $[5]_{0}=0.8 \mathrm{mM}$, $[B N P P]_{0}=2 \mathrm{mM}, 50^{\circ} \mathrm{C}$, in DMSO/Pufferlösung (1:1).

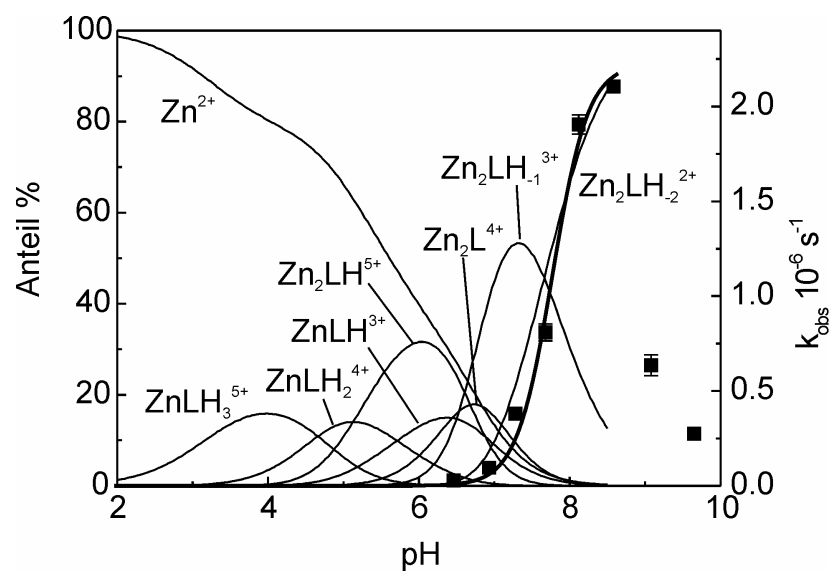

Abbildung 76: Speziesverteilung und pH-Abhängigkeit der BNPP-Hydrolyse durch 8; $\left[L^{4}\right]_{0}=0.8 \mathrm{mM}$, $\left[\mathrm{Zn}^{2+}\right]_{0}=1.6 \mathrm{mM},[\mathrm{BNPP}]_{0}=2 \mathrm{mM}, 50^{\circ} \mathrm{C}$, in DMSO/Pufferlösung (1:1). 
Auch Komplex 2b wurde für die kinetischen Studien einsetzt. Das in der $\mathrm{O}_{2} \mathrm{H}_{2} \mathrm{Me}-$ Brücke enthaltene Methanol sollte schnell mit Wasser austauschen und in Lösung die gleiche Spezies wie in $\mathbf{2 a}$ bilden.

Jedoch zeigt sich im Gegensatz zu den anderen Komplexen, dass für 2 die Spezies $\left[\mathrm{Zn}_{2} \mathrm{~L}^{2} \mathrm{H}_{-2}\right]$, die den Typ der $\mathrm{O}_{2} \mathrm{H}_{3}$-Brücke repräsentiert, nicht aktiv ist (Abbildung 77 ). Die relativ geringe Aktivität von 2 beruht anscheinend auf geringfügigen Anteilen mono- und/oder dinuklearer Spezies, die um pH 8 anwesend sind.

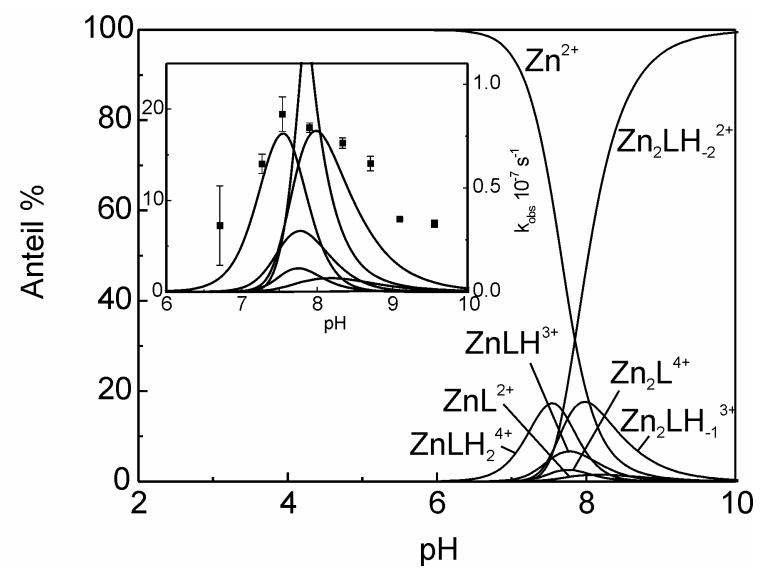

Abbildung 77: Speziesverteilung von $\mathrm{L}^{3}$ mit zwei Äquivalenten $\mathrm{Zn}^{2+}$; der Ausschnitt zeigt eine Überlagerung der Speziesverteilung und die $\mathrm{pH}$-Abhängigkeit der BNPP-Hydrolyse durch 2b; $[\mathbf{2 b}]_{0}=0.8 \mathrm{mM},[B N P P]_{0}=2 \mathrm{mM}, 50{ }^{\circ} \mathrm{C}$, in DMSO/Pufferlösung (1:1).

\subsubsection{Abhängigkeit der BNPP-Hydrolyse von der Komplexkonzentration}

Um die hydrolytische Aktivität der dargestellten Komplexe zu untersuchen, wurden diese bei einem pH-Wert verglichen, bei dem alle diese Komplexe in wässriger Lösung stabil sind. Ein pH-Wert von etwa 8.3 erwies sich dabei als optimal, da hier die Komplexe schon zum größten Teil als hydroxidhaltige Dizinkspezies vorliegen, und auch das zu detektierende 4-Nitrophenol $\left(\mathrm{pK}_{\mathrm{S}}=7.15\right.$ in $\left.\mathrm{H}_{2} \mathrm{O}\right)$ zu einem großen Teil in seiner deprotonierten Form vorliegt, demnach bei $415 \mathrm{~nm}$ sehr gut zu detektieren ist. Der protonierte Anteil des 4-Nitrophenolats kann aufgrund der Überlagerungen mit Absorptionen des Pyrazolatrings und der Pyridylringe der aromatischen Liganden nur mit geringer Genauigkeit erfasst werden.

Die kinetischen Daten (Abbildung 78) zeigen, dass die Hydrolyse von BNPP linear von der Komplexkonzentration abhängt. Dies steht im Einklang mit der Formulierung von dinuklearen aktiven Spezies in allen Fällen. Die Geschwindigkeitskonstanten $k_{\text {obs }}$ 
(Gleichung 1) erster Ordnung sind in Tabelle 13 aufgelistet; sie steigen in der Reihenfolge $3<2<4<5<8$ '.

\begin{tabular}{l|lll} 
Komplex & $k_{\mathrm{obs}}\left(\mathrm{s}^{-1}\right)$ & $k_{\mathrm{cat}}\left(\mathrm{s}^{-1}\right)$ & $K_{\mathrm{M}}(\mathrm{mM})$ \\
\hline $\mathbf{2}$ & $(9.1 \pm 0.2) \cdot 10^{-8}$ & $(1.9 \pm 0.3) \cdot 10^{-6}$ & $42 \pm 4$ \\
$\mathbf{3}$ & $(4.3 \pm 0.1) \cdot 10^{-8}$ & - & - \\
$\mathbf{4}$ & $(2.6 \pm 0.1) \cdot 10^{-7}$ & $(4.9 \pm 0.4) \cdot 10^{-6}$ & $51 \pm 5$ \\
$\mathbf{5}$ & $(8.5 \pm 0.1) \cdot 10^{-7}$ & $(2.3 \pm 0.1) \cdot 10^{-5}$ & $56 \pm 4$ \\
$\mathbf{8}^{\prime}$ & $(1.8 \pm 0.1) \cdot 10^{-6}$ & $(4.2 \pm 0.2) \cdot 10^{-5}$ & $55 \pm 1$
\end{tabular}

Tabelle 13: Kinetische Daten für die Hydrolyse von BNPP;

$50^{\circ} \mathrm{C}, \mathrm{pH} 8.28$ in DMSO/Pufferlösung (1:1).

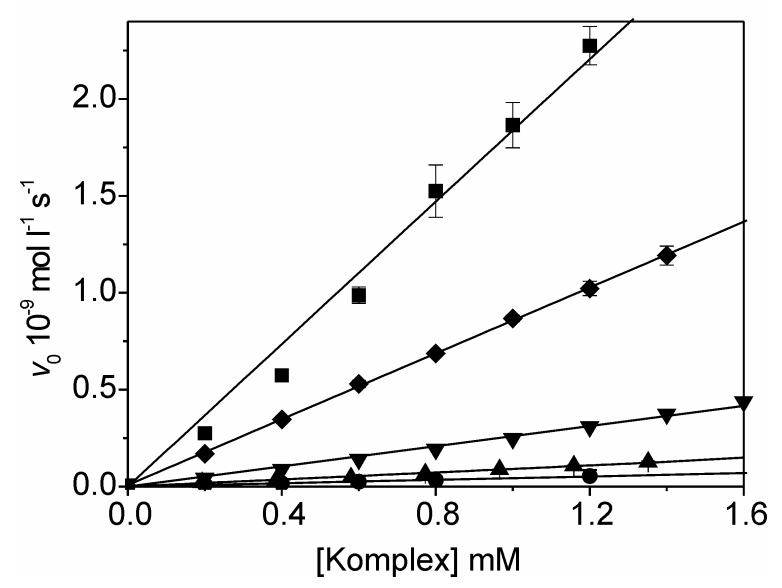

Abbildung 78: Anfangsgeschwindigkeit der BNPP-Hydrolyse in Abhängigkeit von der Komplexkonzentration von $\mathbf{2}(\mathbf{\Delta}), \mathbf{3}(\bullet), \mathbf{4}(\boldsymbol{\nabla}), \mathbf{5}(\bullet)$ und $\mathbf{8}^{\prime}(\boldsymbol{\bullet}) ;[B N P P]_{0}=2 \mathrm{mM}, 50{ }^{\circ} \mathrm{C}, \mathrm{pH}=8.28$, in DMSO/Pufferlösung (1:1).

\subsubsection{Abhängigkeit der BNPP-Hydrolyse von der Substratkonzentration}

Messungen der Abhängigkeit der Anfangsgeschwindigkeit der Hydrolyse von der Substratkonzentration ergeben, dass die Reaktion für $\mathbf{3}$ - und auch für die anderen Komplexe 2, 4, 5 und 8', wenn diese in kleiner Konzentration vorliegen - erster Ordnung bezüglich BNPP ist. Bei höheren BNPP-Konzentrationen jedoch zeigt die geringere Zunahme der Reaktionsgeschwindigkeit für 2, 4, 5 und 8' Sättigungsverhalten an (Abbildung 79, Abbildung 81, Abbildung 82, Abbildung 83), das durch ein der Reaktion vorgelagertes Gleichgewicht erklärt werden kann. Die kinetischen 
Daten wurden durch das in Gleichung 2 gezeigte Geschwindigkeitsgesetz modelliert, das an eine enzymtypische Michaelis-Menten-Kinetik erinnert. Die aus der Modellierung erhaltenen Werte für $K_{\mathrm{M}}$ und $k_{\text {cat }}$ wurden bereits in Tabelle 13 mit aufgeführt.

$$
\begin{gathered}
\text { Komplex }+ \text { BNPP } \frac{k_{1}}{k_{-1}}\left\{\text { Komplex-BNPP } \stackrel{k_{\text {cat }}}{\longrightarrow}\right. \text { Produkte } \\
\qquad v_{0}=\frac{k_{\text {cat }} \cdot[\text { Komplex }]_{0} \cdot[\mathrm{BNPP}]_{0}}{K_{\mathrm{M}}+[\mathrm{BNPP}]_{0}} \\
\text { mit } K_{\mathrm{M}}=\frac{k_{-1}+k_{\mathrm{cat}}}{k_{1}}
\end{gathered}
$$

\section{Gleichung 2}

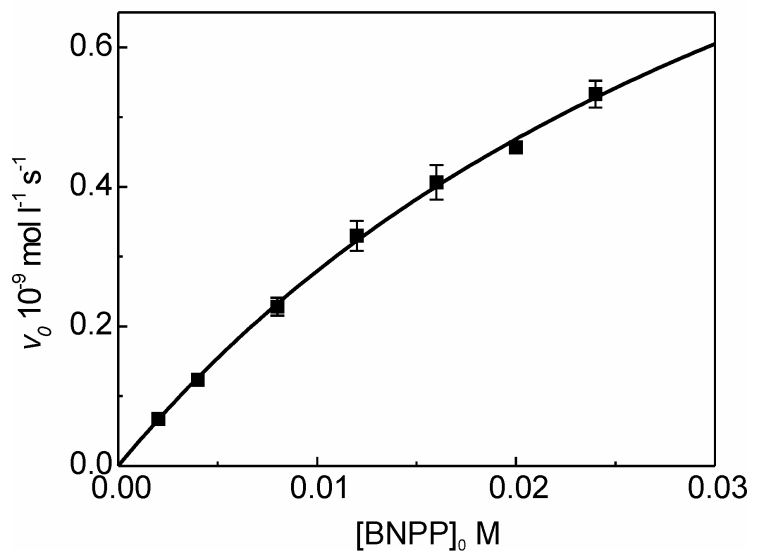

Abbildung 79: Einfluss der BNPP-Konzentration auf die Anfangsgeschwindigkeit der Hydrolyse durch 2b; $[\mathbf{2 b}]_{0}=0.8 \mathrm{mM}, 50^{\circ} \mathrm{C}, \mathrm{pH}=8.28$, in DMSO/Pufferlösung (1:1). 


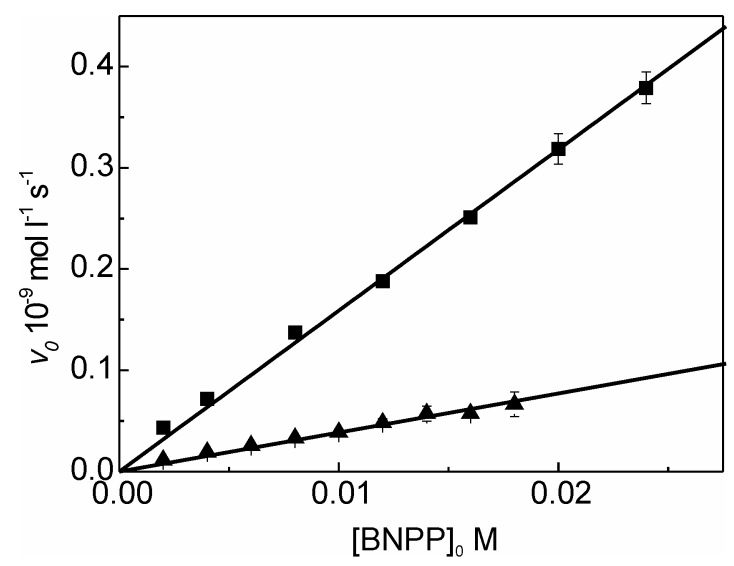

Abbildung 80: Einfluss der BNPP-Konzentration auf die Anfangsgeschwindigkeit der Hydrolyse durch 3; $[3]_{0}=0.8 \mathrm{mM}(\mathbf{-}) ;[3]_{0}=0.2 \mathrm{mM}(\mathbf{\Delta}), 50^{\circ} \mathrm{C}, \mathrm{pH}=8.28$, in DMSO/Pufferlösung (1:1).

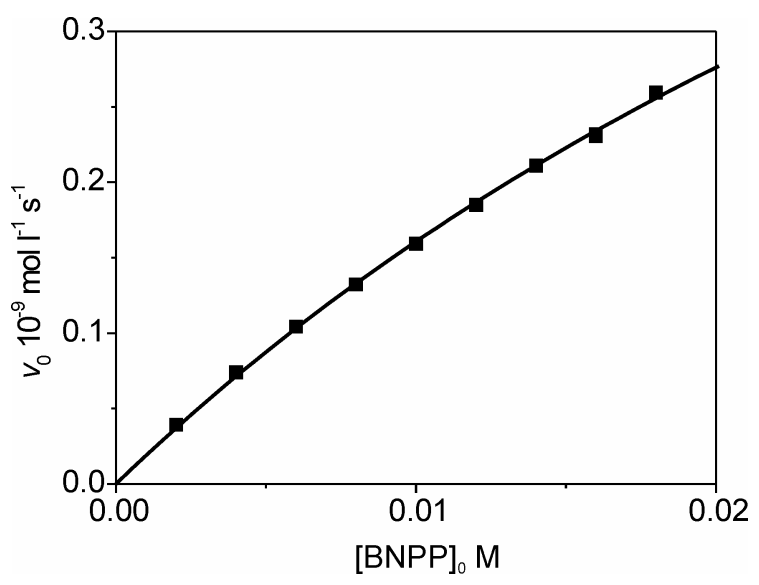

Abbildung 81: Einfluss der BNPP-Konzentration auf die Anfangsgeschwindigkeit der Hydrolyse durch 4; $[4]_{0}=0.2 \mathrm{mM}, 50^{\circ} \mathrm{C}, \mathrm{pH}=8.28$, in DMSO/Pufferlösung (1:1).

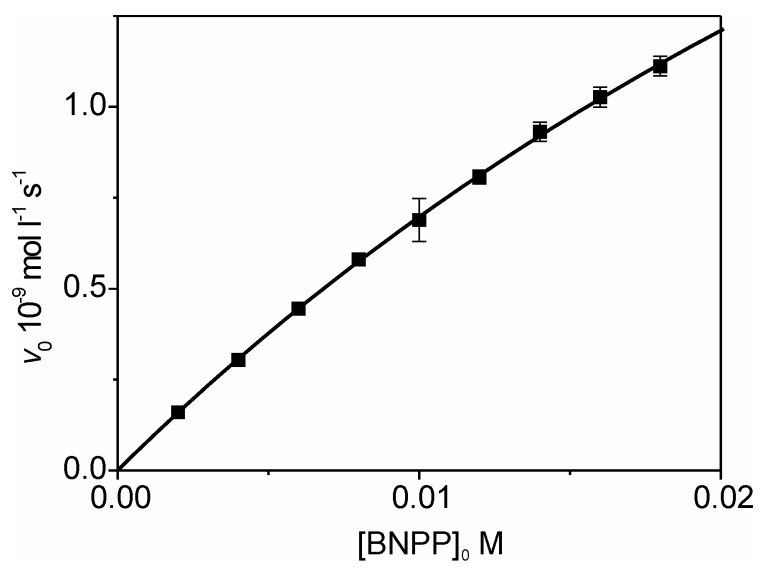

Abbildung 82: Einfluss der BNPP-Konzentration auf die Anfangsgeschwindigkeit der Hydrolyse durch 5; $[5]_{0}=0.2 \mathrm{mM}, 50^{\circ} \mathrm{C}, \mathrm{pH}=8.28$, in DMSO/Pufferlösung (1:1). 


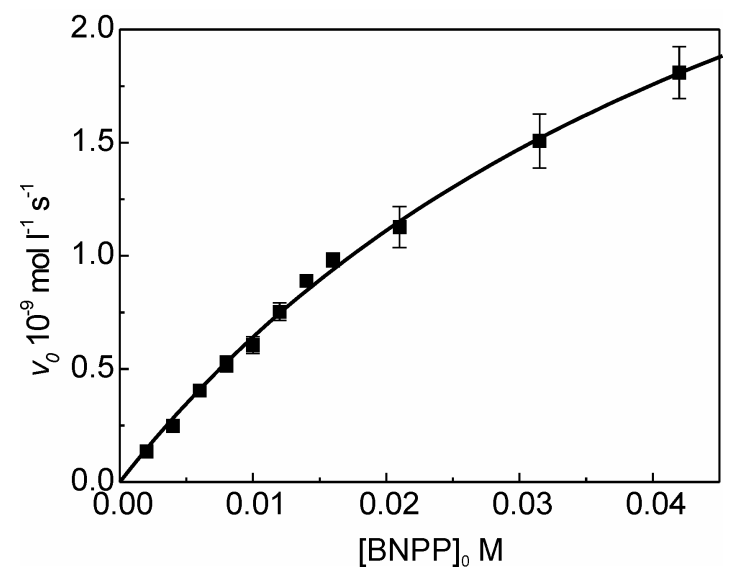

Abbildung 83: Einfluss der BNPP-Konzentration auf die Anfangsgeschwindigkeit der Hydrolyse durch 8'; $\left[\mathrm{L}^{4}\right]_{0}=0.1 \mathrm{mM},\left[\mathrm{Zn}^{2+}\right]_{0}=0.2 \mathrm{mM}, 50{ }^{\circ} \mathrm{C}, \mathrm{pH}=8.28$, in DMSO/Pufferlösung (1:1).

Die Substratbindungskonstanten $K$ lassen sich unter der Voraussetzung $k_{\text {cat }}<<k_{-1}$ aus der Michaelis-Menten-Konstante $K_{M}$ berechnen.

$$
K=k_{1} / k_{-1}=1 / K_{\mathrm{M}}
$$

\section{Gleichung 3}

Augenscheinlich sind die Bindungskonstanten mit $24 \pm 3$ (2), $20 \pm 3$ (4), $18 \pm 3$ (5) und $18 \pm 1$ ( $\left.\mathbf{8}^{\prime}\right)$ relativ klein. Dies stimmt überein mit früheren Beobachtungen, dass BNPP nur ein schwach koordinierender Ligand ist. ${ }^{[1,110,111]}$ Die Abwesenheit von Sättigungseffekten bei $\mathbf{3}$ - selbst bei hohem Substratüberschuss - lässt Raum für Vermutungen, dass entweder ein direkter Angriff eines Zink-gebundenen Hydroxids auf BNPP erfolgt, d.h. ohne vorherige Koordination des Substrats, oder die Substratbindungskonstante noch sehr viel kleiner ist.

Die scheinbaren Geschwindigkeitskonstanten zweiter Ordnung $k_{\text {app }}$ für die Gesamtreaktion der Hydrolyse von BNPP mit Zinkkomplexen sind in Tabelle 14 aufgeführt. Unter der Annahme, dass die einzig aktive Spezies im Fall der Komplexe 3, 4, 5 und 8' die [ $\left.\mathrm{Zn}_{2} \mathrm{LH}_{-2}\right]$-Spezies sind, können die wahren Geschwindigkeitskonstanten für die Reaktion zweiter Ordnung $k_{\text {bim }}$ erhalten werden, indem man $k_{\text {app }}$ durch den Anteil der $\left[\mathrm{Zn}_{2} \mathrm{LH}_{-2}\right]$-Spezies beim zugehörigen $\mathrm{pH}$-Wert dividiert. Diese können aus den gemessenen Speziesverteilungen entnommen werden. 


$$
v_{0}=k_{\text {app }} \cdot[\text { Komplex }]_{0} \cdot[\mathrm{BNPP}]_{0}=k_{\mathrm{bim}} \cdot\left[\mathrm{Zn}_{2} \mathrm{LH}_{-2}\right] \cdot[\mathrm{BNPP}]_{0}
$$

\section{Gleichung 4}

\begin{tabular}{l|lll} 
Komplex & $k_{\mathrm{app}}\left(\mathrm{M}^{-1} \mathrm{~s}^{-1}\right)$ & $k_{\mathrm{bim}}\left(\mathrm{M}^{-1} \mathrm{~s}^{-1}\right)$ & $\mathrm{pK}_{\mathrm{S}}\left(\left[\mathrm{LZn}_{2} \mathrm{H}_{-1}\right]^{3+}\right)$ \\
\hline $\mathbf{2}$ & $(4.5 \pm 0.2) \cdot 10^{-5}$ & - & 7.57 \\
$\mathbf{3}$ & $(2.1 \pm 0.2) \cdot 10^{-5}$ & $(3.3 \pm 0.3) \cdot 10^{-5}$ & 8.04 \\
$\mathbf{4}$ & $(1.3 \pm 0.1) \cdot 10^{-4}$ & $(1.6 \pm 0.1) \cdot 10^{-4}$ & 8.02 \\
$\mathbf{5}$ & $(4.3 \pm 0.1) \cdot 10^{-4}$ & $(4.6 \pm 0.1) \cdot 10^{-4}$ & 7.60 \\
$\mathbf{8}$ & $(9.0 \pm 0.5) \cdot 10^{-4}$ & $(1.1 \pm 0.1) \cdot 10^{-3}$ & 7.66
\end{tabular}

Tabelle 14: Geschwindigkeitskonstanten zweiter Ordnung für die Hydrolyse von BNPP durch Zink(II)komplexe bei $50^{\circ} \mathrm{C}, \mathrm{pH} 8.2$ in DMSO/Pufferlösung (1:1).

\subsection{Untersuchungen zur Anbindung von Phosphaten an Dizinkzentren}

Die Bindung von BNPP an die unterschiedlichen Zinkzentren wurde in Lösung durch ${ }^{31}$ P-NMR-Spektroskopie und ESI-Massenspektrometrie untersucht und durch röntgenographische Analysen von Phosphataddukten ergänzt. Zusätzlich wurde die Anbindung des hydrolytisch inerten Dimethylphosphats (DMP) studiert, da in diesem Fall jegliche Phosphatesterspaltung auf der NMR- und MS-Zeitskala ausgeschlossen werden kann.

\subsection{1 ${ }^{31}$ P-NMR-Spektroskopie}

Titrationen der Dizinkkomplexe 2a, 3, 4 und 5 mit Phosphaten wurden mittels ${ }^{31} \mathrm{P}$ NMR-Spektroskopie verfolgt, um den Einfluss der Stärke der Anbindung des Phosphats an das Dizinkzentrum zu bestimmen.

Anstelle des für die kinetischen Untersuchungen verwendeten BNPP wurde das gegen Hydrolyse sehr viel stabilere Dimethylphosphat DMP verwendet, um eine Spaltung während der Dauer des Experiments auszuschließen. Aliquote von Dimethylphosphorsäure wurden zu einer Lösung der Dizinkkomplexe $\mathbf{2 b}$ und $\mathbf{3}$ in einer $\mathrm{d}_{6}$-DMSO/Pufferlösung bei $\mathrm{pH} 8$ gegeben. Die Komplexe binden DMP rasch, und die Anbindung verursacht eine Hochfeldverschiebung des ${ }^{31} \mathrm{P}-\mathrm{NMR}$-Signals um etwa 4 ppm. Der Zusatz eines Überschusses an DMP zeigt, dass nur ein Äquivalent 
des Phosphats an den Komplex koordiniert. Die Analyse der Titrationskurven (Abbildung 84) durch Anpassung der Gleichgewichtskonstante $K_{\mathrm{B}}$ des in Gleichung 5 dargestellten Gleichgewichts an die experimentellen Daten ergibt Bindungskonstanten $K_{\mathrm{B}}=(9291 \pm 4400) \mathrm{M}^{-1}$ für 2 und $(2722 \pm 913) \mathrm{M}^{-1}$ für 3.

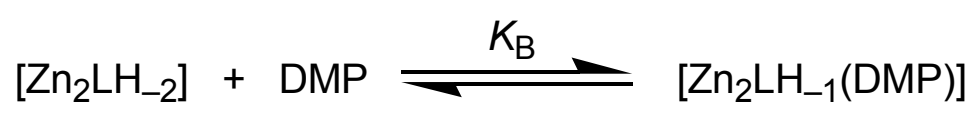

\section{Gleichung 5}

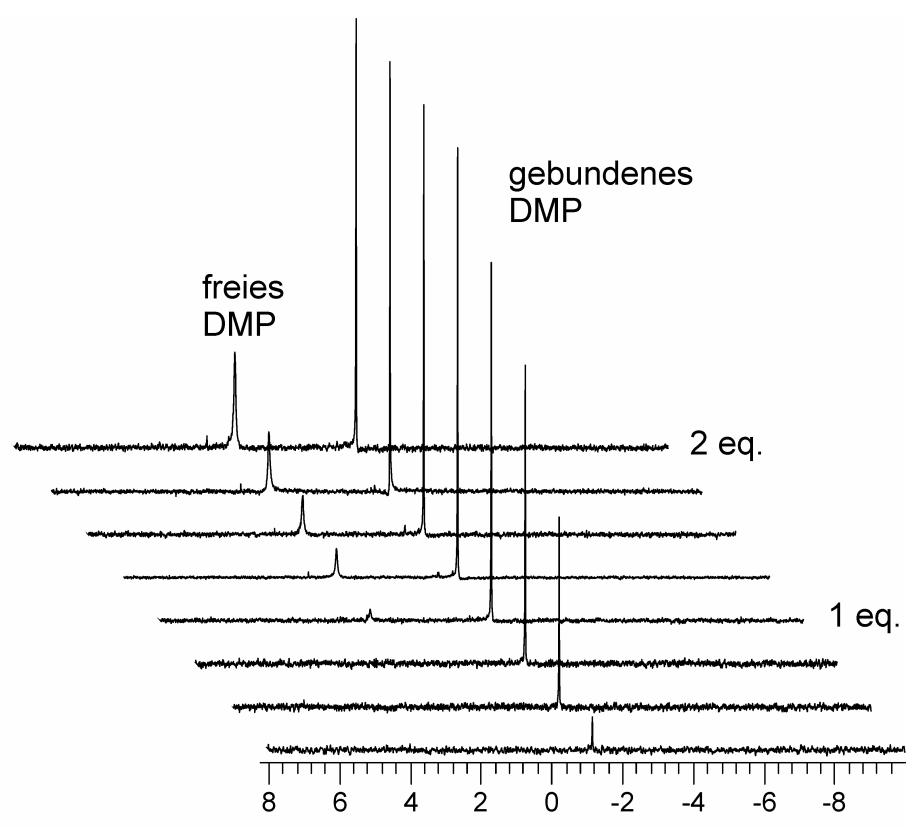

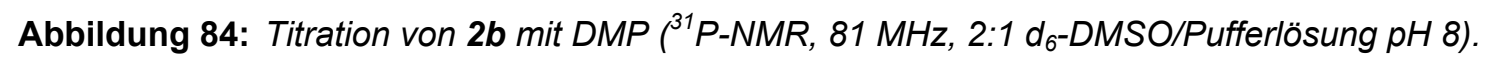

Die höhere Bindungskonstante für 2 spiegelt die höhere Stabilität der OH-Brücke in 3 verglichen mit der $\mathrm{H}_{3} \mathrm{O}_{2}$-Brücke in 2 wider. Sie könnte jedoch genauso darauf hinweisen, dass der längere Zink-Zink-Abstand in $\mathbf{2}$ besser dazu geeignet ist, das Phosphat in einem O,O'-verbrückenden Modus zu binden.

Titrationen der Komplexe $\mathbf{4}$ und $\mathbf{5}$ mit DMP erwiesen sich als wenig nützlich, da die Phosphataddukte von $\mathbf{4}$ und $\mathbf{5}$ chemische Verschiebungen besitzen, die sich kaum von der des Phosphats unterscheidet.

Eine Bestimmung der Anbindung von Phosphaten an 8' wurde nicht durchgeführt, da dieses System aufgrund der vorhandenen freien Koordinationsstellen auch in der Lage ist, zwei Phosphate aufzunehmen (wie in Komplex 14 s.u.) oder höher aggregierte Spezies auszubilden. 


\subsubsection{ESI-Massenspektrometrie}

Nach der Zugabe eines Äquivalents NaBNPP zu dem jeweiligen in Methanol gelösten Komplex zeigen die ESI-Spektren sowohl ein dominantes Signal des ursprünglichen Komplexdikations zusammen mit nur einem Perchloratanion als auch weitere Spezies mit gebundenem BNPP.

Für $\mathbf{2 b}$ wird im Einklang mit dem leichten Austausch und Ausstoß des zusätzlich gebundenen Solvensmoleküls das lon $\left[\mathrm{Zn}_{2} \mathrm{~L}^{2} \mathrm{H}_{-1}(\mathrm{OMe})\left(\mathrm{ClO}_{4}\right)\right]^{+}\left(\mathbf{2} \mathbf{b}^{\prime}\right)$ mit einer einfachen OMe-Brücke beobachtet. Nur im Fall von 1 wird ein deutliches Signal für den freien Liganden $\left[\mathrm{L}^{1} \mathrm{H}\right]^{+}$detektiert. Dies belegt die sehr viel geringere Stabilität dieses Systems in Lösung. Die Assoziation mit BNPP durch Austausch mit einem Perchlorat findet man in $\mathbf{1}, \mathbf{3}$ und $\mathbf{8 a}$ als $\left[\mathrm{Zn}_{2} \mathrm{~L}^{1} \mathrm{H}_{-1}(\mathrm{OH})(\mathrm{BNPP})\right]^{+}, \quad\left[\mathrm{Zn}_{2} \mathrm{~L}^{3} \mathrm{H}_{-}\right.$ $\left.{ }_{1}(\mathrm{OH})(\mathrm{BNPP})\right]^{+}$bzw. $\left[\mathrm{Zn}_{4}\left(\mathrm{~L}^{4} \mathrm{H}_{-1}\right)_{2}\left(\mathrm{CO}_{3}\right)(\mathrm{OH})_{2}(\mathrm{BNPP})\right]$. Bei 2b' jedoch wird die Methanolatbrücke substituiert und führt zu einer Spezies $\left[\mathrm{Zn}_{2} \mathrm{~L}^{2} \mathrm{H}_{-1}(\mathrm{BNPP})\left(\mathrm{ClO}_{4}\right)\right]^{+}$ (Abbildung 85). Dies zeigt deutlich, dass die $\mathrm{O}_{2} \mathrm{H}_{3}$ - und $\mathrm{O}_{2} \mathrm{H}_{2} \mathrm{Me}$-Gruppen (und die im ESI-MS daraus resultierende OMe-Einheit) im letzteren Komplex relativ labil sind, hingegen das verbrückende Hydroxid in $\mathbf{1}$ und $\mathbf{3}$ fester gebunden ist.

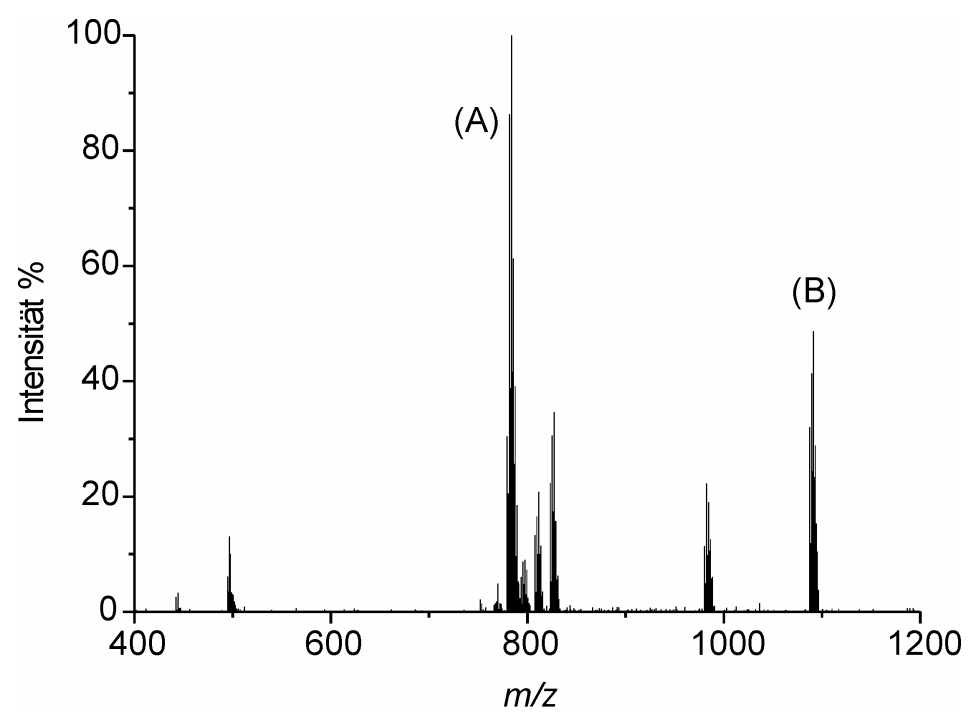

Abbildung 85: ESI-MS Spektrum von $\mathbf{2 b}$ in Gegenwart von BNPP.
(A) $\left[\mathrm{Zn}_{2} \mathrm{~L}^{2} \mathrm{H}_{-1}(\mathrm{OMe})\left(\mathrm{ClO} \mathrm{O}_{4}\right)\right]^{+}$
(B) $\left[\mathrm{Zn}_{2} \mathrm{~L}^{1} \mathrm{H}_{-1}(\mathrm{OH})(\mathrm{BNPP})\right]^{+}$

Um die $\mathrm{Zn}-\mathrm{OH}-\mathrm{Zn}$-Funktionalität $\mathrm{zu}$ labilisieren und das Verhalten in weniger basischen $\mathrm{pH}$ Bereichen zu modellieren, wurde eine zweite Reihe von Experimenten in der Gegenwart von einem Äquivalent Perchlorsäure durchgeführt. Eine Protonierung und Abspaltung von Metall-gebundenen Hydroxidgruppen sollte die 
Anbindung von BNPP erleichtern. Dies wird durch die nun im Vergleich zur ersten Reihe höheren Intensitäten jener Signale bestätigt, die BNPP-gebundenen Spezies entsprechen. Darüber hinaus ersetzt in Anwesenheit einer Säure das Substrat BNPP vorzugsweise das metallgebundene Hydroxid anstelle des Perchlorats: für Komplex 3 beispielsweise bildet sich neben dem $\left[\mathrm{Zn}_{2} \mathrm{~L}^{3} \mathrm{H}_{-1}(\mathrm{OH})\left(\mathrm{ClO}_{4}\right)\right]^{+}$lon auch die Spezies $\left[\mathrm{Zn}_{2} \mathrm{~L}^{3} \mathrm{H}_{-1}(\mathrm{BNPP})\left(\mathrm{ClO}_{4}\right)\right]^{+}$. Im Fall von 8a führt die Zugabe von Säure zu einem Zerfall des tetranuklearen Komplexes, und es finden sich Signale zweikerniger Spezies mit einem oder zwei koordinierten BNPP-Molekülen.

In den Komplexen $\mathbf{4}$ und $\mathbf{5}$ findet man in den Spektren schon bei Zugabe von bloßem BNPP nur noch wenig intensive Signale für die Spezies $\left[\mathrm{Zn}_{2} \mathrm{LH}_{-1}(\mathrm{OH})\left(\mathrm{ClO}_{4}\right)\right]^{+}$, sondern hauptsächlich Phosphatester-enthaltende Spezies. Überraschenderweise ist BNPP in Anwesenheit von 4 und 5 nicht stabil, sondern wird durch Methanol umgeestert. So finden sich in den Spezies $\left[\mathrm{Zn}_{2} \mathrm{LH}_{-1}\left(\mathrm{PO}_{4} \mathrm{R}_{2}\right)\left(\mathrm{ClO}_{4}\right)\right]^{+}$und $\left[\mathrm{Zn}_{2} \mathrm{LH}_{-}\right.$ $\left.{ }_{1}\left(\mathrm{PO}_{4} \mathrm{R}_{2}\right)_{2}\right]^{+}$die Phosphate BNPP, DMP und der gemischte Ester Nitrophenylmethylphosphat NPMP (Abbildung 86).

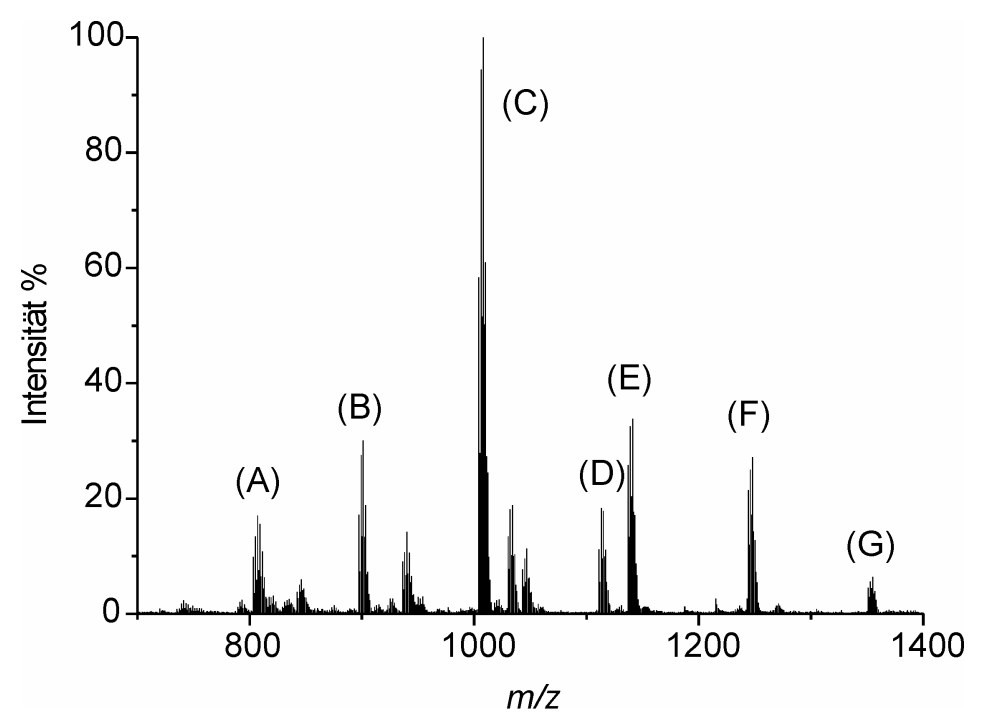

Abbildung 86: ESI-MS Spektrum von 4 in Gegenwart von BNPP.
(A) $\left[\mathrm{Zn}_{2} \mathrm{~L}^{6}{ }_{-\mathrm{H}}(\mathrm{OMe})\left(\mathrm{ClO}_{4}\right)\right]^{+}$
(B) $\left[\mathrm{Zn}_{2} \mathrm{~L}_{-\mathrm{H}}{ }_{-\mathrm{H}}(\mathrm{DMP})\left(\mathrm{ClO}_{4}\right)\right]^{+}$
(C) $\left[\mathrm{Zn}_{2} \mathrm{~L}^{6}{ }_{-H}(\mathrm{NPMP})\left(\mathrm{ClO} \mathrm{O}_{4}\right)\right]^{+}$
(D) $\left[\mathrm{Zn}{ }_{2} L^{6}{ }_{-H}(B N P P)\left(C / O_{4}\right)\right]^{+}$
(E) $\left[\mathrm{Zn} \mathrm{L}_{2}{ }^{6}{ }_{-H}(\mathrm{NPMP})_{2}\right]^{+}$
(F) $\left[Z n_{2} L^{6}{ }_{-H}(N P M P)(B N P P)\right]^{+}$
(G) $\left[Z n_{2} L^{6}{ }_{-H}(B N P P)_{2}\right]^{+}$ 
Aus den Intensitäten der ESI-Massenspektren zeigt sich, dass DMP sehr viel stärker als BNPP an die Zinkkomplexe bindet; dies führt zu Spezies mit einem und zwei gebundenen DMP-Anionen, wobei letztere in allen Fällen außer $\mathbf{2 b}$ die dominante Spezies in den ESI-MS-Spektren darstellt. Nur für $\mathbf{1}$ und $\mathbf{3}$ werden noch Spezies gefunden, die noch OMe- oder $\mathrm{OH}-G r u p p e n$ beinhalten. Es ist also anzunehmen, dass die labile Brücke in der bimetallischen Tasche von $\mathbf{2 b}$ und $\mathbf{5}$, aber auch in $\mathbf{4}$ vollständig durch DMP ersetzt wird.

Um die angenommene bidentat verbrückende Koordination des DMP an das $Z \mathrm{n}_{2} \mathrm{~L}^{2}$ $\mathrm{H}_{-1}$-Gerüst und den Ersatz der intramolekularen $\mathrm{O}_{2} \mathrm{H}_{3} / \mathrm{O}_{2} \mathrm{H}_{2} \mathrm{Me}-\mathrm{Brücke}$ in $\mathbf{2 a}$ /2b unter Bildung von 10b zu überprüfen, wurde der Komplex $\left[\mathrm{Zn}_{2} \mathrm{~L}^{2} \mathrm{H}_{-1}(\mathrm{DMP})\right]\left(\mathrm{ClO}_{4}\right)_{2} 10 \mathrm{a}$ synthetisiert (Abbildung 87) und kristallographisch charakterisiert (Abbildung 88).

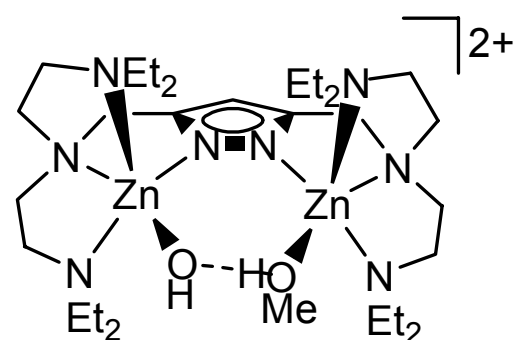

2b
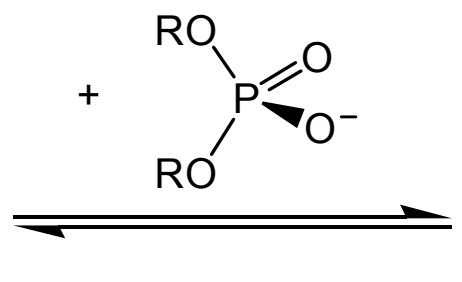

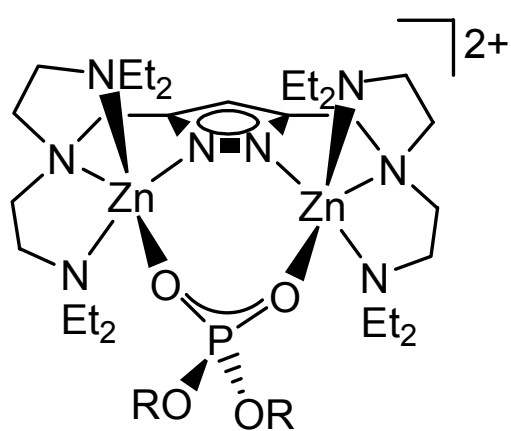

$10 \mathbf{a}(\mathrm{R}=\mathrm{Me})$

$10 \mathrm{~b}\left(\mathrm{R}=\longrightarrow \mathrm{NO}_{2}\right)$

Abbildung 87: Anbindung von Phosphatdiestern an $\mathbf{2 b .}$ 


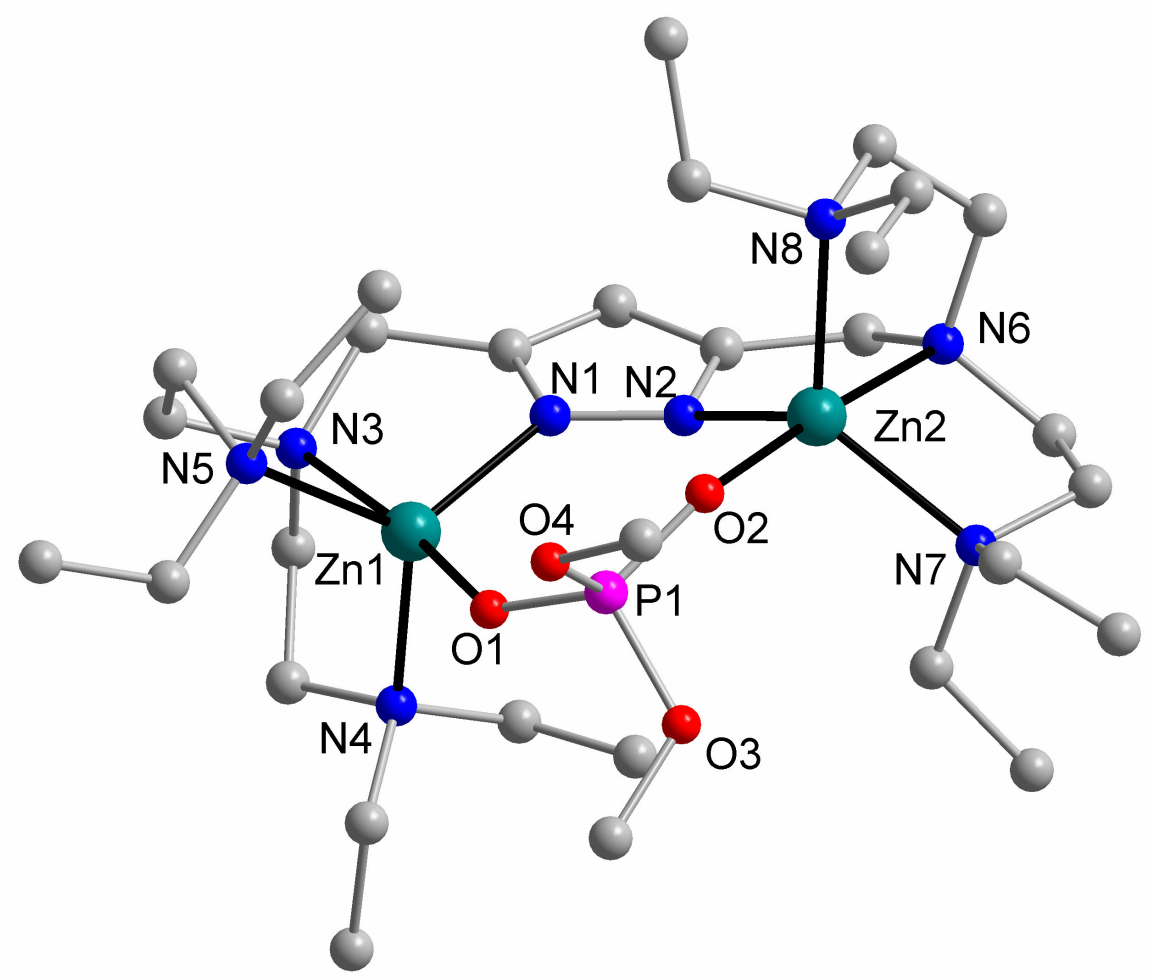

Abbildung 88: Festkörperstruktur des Kations von 10a $\cdot\left(\mathrm{ClO}_{4}\right)_{2}$.

\begin{tabular}{|l|l|l|l|}
\hline $\mathrm{Zn}(1)-\mathrm{N}(1)$ & $2.047(2)[2.035(2)]$ & $\mathrm{Zn}(2)-\mathrm{N}(2)$ & $2.047(2)[2.043(2)]$ \\
\hline $\mathrm{Zn}(1)-\mathrm{N}(3)$ & $\begin{array}{l}2.221(2)[2.207(22), \\
2.32(2)]\end{array}$ & $\mathrm{Zn}(2)-\mathrm{N}(6)$ & $2.242(2)[2.233(2)]$ \\
\hline $\mathrm{Zn}(1)-\mathrm{N}(4)$ & $\begin{array}{l}2.151(2)[2.138(3), \\
2.21(1)]\end{array}$ & $\mathrm{Zn}(2)-\mathrm{N}(7)$ & $2.134(2)[2.157(2)]$ \\
\hline $\mathrm{Zn}(1)-\mathrm{N}(5)$ & $\begin{array}{l}2.164(2)[2.153(4), \\
2.15(2)]\end{array}$ & $\mathrm{Zn}(2)-\mathrm{N}(8)$ & $2.141(2)[2.159(2)]$ \\
\hline $\mathrm{Zn}(1)-\mathrm{O}(1)$ & $1.993(2)[1.997(2)]$ & $\mathrm{Zn}(2)-\mathrm{O}(2)$ & $2.012(2)[1.980(2)]$ \\
\hline $\mathrm{O}(2)-\mathrm{P}(1)$ & $1.490(2)[1.490(2)]$ & & \\
\hline $\mathrm{O}(1)-\mathrm{P}(1)$ & $1.493(2)[1.487(2)]$ & & \\
\hline $\mathrm{P}(1)-\mathrm{O}(4)$ & $1.587(2)[1.583(2)]$ & & \\
\hline $\mathrm{P}(1)-\mathrm{O}(3)$ & $1.577(2)[1.579(2)]$ & $\mathrm{O}(2)-\mathrm{Zn}(2)-\mathrm{N}(7)$ & $96.26(7)[92.47(6)]$ \\
\hline $\mathrm{N}(1)-\mathrm{N}(2)$ & $1.381(2)[1.377(2)]$ & & \\
\hline $\mathrm{Zn}(1) \cdots \mathrm{Zn}(2)$ & $4.406(1)[4.365(1)]$ & & \\
\hline $\mathrm{O}(1)-\mathrm{Zn}(1)-\mathrm{N}(1)$ & $106.52(7)[100.83(7)]$ & & \\
\hline $\mathrm{O}(1)-\mathrm{Zn}(1)-\mathrm{N}(4)$ & $\begin{array}{l}98.06(6)[92.55(8), \\
107.5(3)]\end{array}$ & & \\
\hline
\end{tabular}




\begin{tabular}{|l|l|l|l|}
\hline $\mathrm{N}(1)-\mathrm{Zn}(1)-\mathrm{N}(4)$ & $\begin{array}{l}108.39(7)[117.03(8), \\
104.0(3)]\end{array}$ & $\mathrm{N}(2)-\mathrm{Zn}(2)-\mathrm{N}(7)$ & $127.63(7)[125.61(7)]$ \\
\hline $\mathrm{O}(1)-\mathrm{Zn}(1)-\mathrm{N}(5)$ & $\begin{array}{l}91.02(6)[99.72(9), \\
94.6(4)]\end{array}$ & $\mathrm{O}(2)-\mathrm{Zn}(2)-\mathrm{N}(8)$ & $100.28(7)[96.69(6)]$ \\
\hline $\mathrm{N}(1)-\mathrm{Zn}(1)-\mathrm{N}(5)$ & $125.13(7)[116.3(1)$, & $\mathrm{N}(2)-\mathrm{Zn}(2)-\mathrm{N}(8)$ & $104.70(7)[100.80(7)]$ \\
& $121.1(5)]$ & & \\
\hline $\mathrm{N}(4)-\mathrm{Zn}(1)-\mathrm{N}(5)$ & $120.24(7)[121.4(1)$, & $\mathrm{N}(7)-\mathrm{Zn}(2)-\mathrm{N}(8)$ & $121.50(7)[127.36(7)]$ \\
& $124.5(6)]$ & & \\
\hline $\mathrm{O}(1)-\mathrm{Zn}(1)-\mathrm{N}(3)$ & $171.63(6)[176.17(8)$, & $\mathrm{O}(2)-\mathrm{Zn}(2)-\mathrm{N}(6)$ & $176.17(7)[171.48(6)]$ \\
\hline $\mathrm{N}(1)-\mathrm{Zn}(1)-\mathrm{N}(3)$ & $80.97(7)[79.91(8)$, & $\mathrm{N}(2)-\mathrm{Zn}(2)-\mathrm{N}(6)$ & $80.67(7)[81.56(7)]$ \\
\hline $\mathrm{N}(4)-\mathrm{Zn}(1)-\mathrm{N}(3)$ & $82.75(7)[83.80(9)$, & $\mathrm{N}(7)-\mathrm{Zn}(2)-\mathrm{N}(6)$ & $81.37(7)[81.29(7)]$ \\
& $78.8(4)]$ & & \\
\hline $\mathrm{N}(5)-\mathrm{Zn}(1)-\mathrm{N}(3)$ & $81.45(6)[83.2(1)$, & $\mathrm{N}(8)-\mathrm{Zn}(2)-\mathrm{N}(6)$ & $83.53(7)[82.69(7)]$ \\
\hline $\mathrm{O}(2)-\mathrm{P}(1)-\mathrm{O}(1)$ & $118.96(8)[118.38(9)]$ & & \\
\hline $\mathrm{O}(2)-\mathrm{P}(1)-\mathrm{O}(3)$ & $106.32(8)[105.45(8)]$ & & \\
\hline $\mathrm{O}(1)-\mathrm{P}(1)-\mathrm{O}(3)$ & $110.36(8)[110.41(9)]$ & & \\
\hline $\mathrm{O}(2)-\mathrm{P}(1)-\mathrm{O}(4)$ & $110.20(8)[110.07(8)]$ & & \\
\hline $\mathrm{O}(1)-\mathrm{P}(1)-\mathrm{O}(4)$ & $104.78(8)[106.24(9)]$ & & \\
\hline $\mathrm{O}(3)-\mathrm{P}(1)-\mathrm{O}(4)$ & $105.48(8)[105.65(8)]$ & & \\
\hline
\end{tabular}

Tabelle 15: Ausgewählte Atomabstände $(\AA)$ und Bindungswinkel ( $\left.{ }^{\circ}\right)$.

In Komplex 10a wird das Substratanalogon DMP, wie erwartet, zwischen den beiden Zinkionen koordiniert $(d(\mathrm{Zn}(1) \cdots \mathrm{Zn}(2))=4.406(4)[4.365(3)] \AA$; zwei unabhängige, aber nahezu identische Moleküle werden in der asymmetrischen Einheit gefunden). Die Struktur ähnelt den Dizink-Phosphatzentren, die für viele Metallohydrolasen vorgeschlagen werden. Die beiden Zinkionen in 10a ragen deutlich aus der Ebene des Pyrazolatheterozyklus heraus (um 0.569 - 0.794 [0.580 - 0.615] Å). Obwohl alle Bindungslängen im normalen Bereich sind, könnte diese Verschiebung eine leicht gespannte Bindungssituation andeuten. In Anbetracht der gleichen Zusammensetzung mit den mittels ESI-MS erhaltenen $\left[\mathrm{Zn}_{2} \mathrm{~L}^{2} \mathrm{H}_{-1}(\mathrm{BNPP})\left(\mathrm{ClO}_{4}\right)\right]^{+}-$und $\left[\mathrm{Zn}_{2} \mathrm{~L}^{2}\right.$ $\left.\mathrm{H}_{-1}(\mathrm{DMP})\left(\mathrm{ClO}_{4}\right)\right]^{+}$-Spezies ist anzunehmen, dass die kristallographisch bestimmte Struktur von 10a ein sehr gutes Modell für die Anbindung von BNPP in 10b ist. 
Eine analoge Substratanbindung wird auch für 4 (11a) (Abbildung 89) und 5 (12a) gefunden (Abbildung 90).

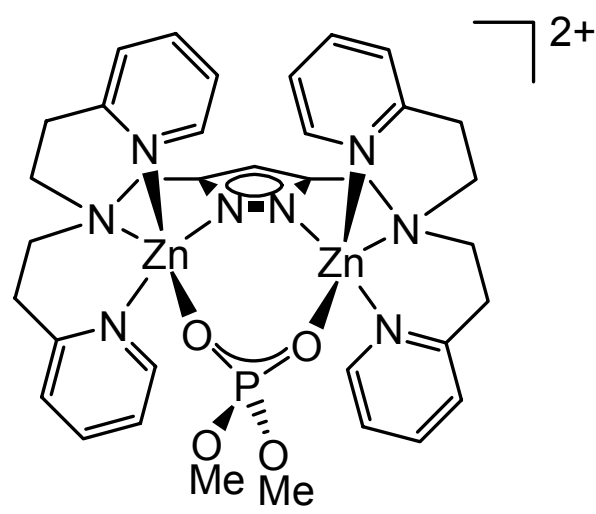

$11 \mathrm{a}$

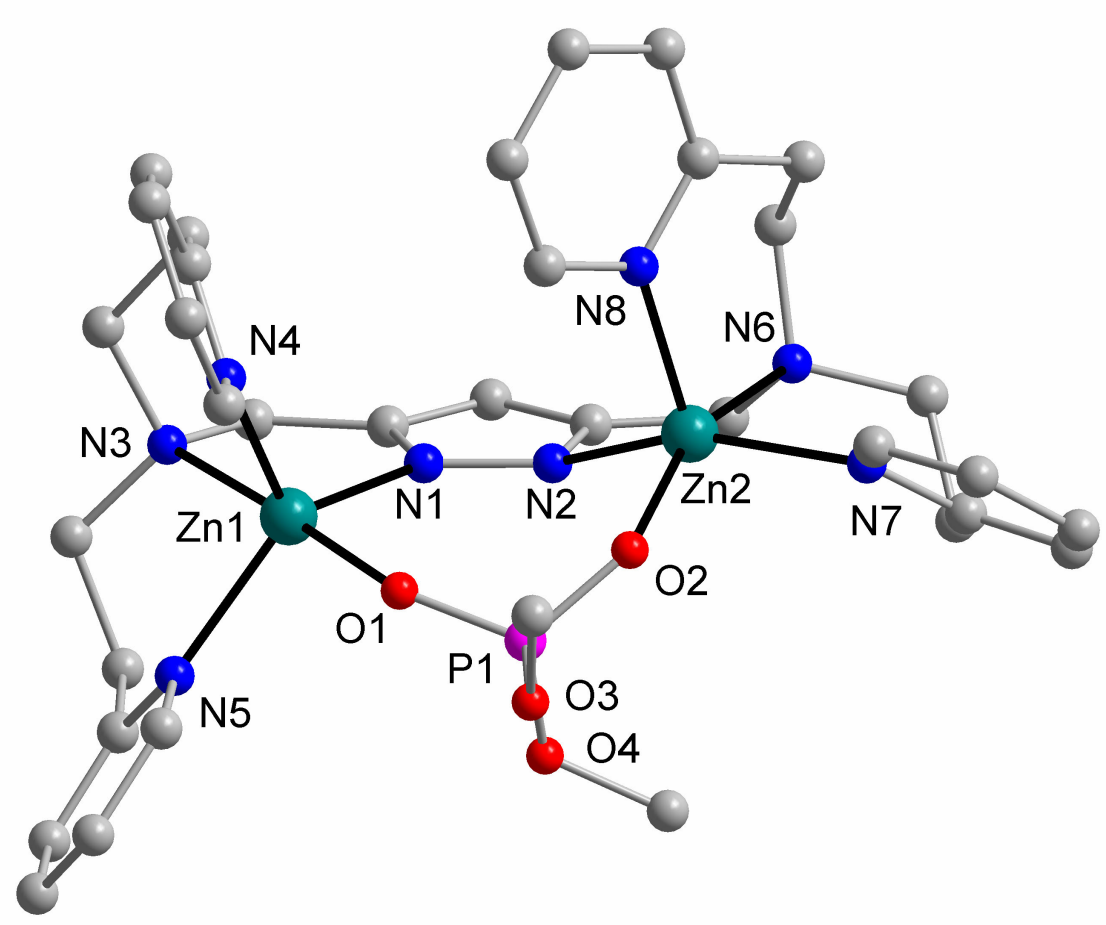

Abbildung 89: Festkörperstruktur des Kations von 11a $\left(\mathrm{ClO}_{4}\right)_{2}$.

\begin{tabular}{|l|l|l|l|}
\hline $\mathrm{Zn}(1)-\mathrm{N}(1)$ & $2.018(2)$ & $\mathrm{Zn}(2)-\mathrm{O}(2)$ & $1.983(2)$ \\
\hline $\mathrm{Zn}(1)-\mathrm{O}(1)$ & $2.039(2)$ & $\mathrm{Zn}(2)-\mathrm{N}(8)$ & $2.054(3)$ \\
\hline $\mathrm{Zn}(1)-\mathrm{N}(4)$ & $2.091(3)$ & $\mathrm{Zn}(2)-\mathrm{N}(2)$ & $2.091(2)$ \\
\hline $\mathrm{Zn}(1)-\mathrm{N}(5)$ & $2.106(3)$ & $\mathrm{Zn}(2)-\mathrm{N}(7)$ & $2.157(3)$ \\
\hline $\mathrm{Zn}(1)-\mathrm{N}(3)$ & $2.290(3)$ & $\mathrm{Zn}(2)-\mathrm{N}(6)$ & $2.181(2)$ \\
\hline $\mathrm{N}(1)-\mathrm{N}(2)$ & $1.382(3)$ & & \\
\hline
\end{tabular}




\begin{tabular}{|l|l|l|l|}
\hline $\mathrm{Zn}(1)-\mathrm{Zn}(2)$ & $4.402(1)$ & & \\
\hline $\mathrm{N}(1)-\mathrm{Zn}(1)-\mathrm{O}(1)$ & $98.21(9)$ & $\mathrm{O}(2)-\mathrm{Zn}(2)-\mathrm{N}(8)$ & $99.64(10)$ \\
\hline $\mathrm{N}(1)-\mathrm{Zn}(1)-\mathrm{N}(4)$ & $115.53(10)$ & $\mathrm{O}(2)-\mathrm{Zn}(2)-\mathrm{N}(2)$ & $99.59(9)$ \\
\hline $\mathrm{O}(1)-\mathrm{Zn}(1)-\mathrm{N}(4)$ & $90.87(10)$ & $\mathrm{N}(8)-\mathrm{Zn}(2)-\mathrm{N}(2)$ & $103.20(10)$ \\
\hline $\mathrm{N}(1)-\mathrm{Zn}(1)-\mathrm{N}(5)$ & $134.13(9)$ & $\mathrm{O}(2)-\mathrm{Zn}(2)-\mathrm{N}(7)$ & $82.87(9)$ \\
\hline $\mathrm{O}(1)-\mathrm{Zn}(1)-\mathrm{N}(5)$ & $88.13(10)$ & $\mathrm{N}(8)-\mathrm{Zn}(2)-\mathrm{N}(7)$ & $100.33(10)$ \\
\hline $\mathrm{N}(4)-\mathrm{Zn}(1)-\mathrm{N}(5)$ & $109.70(9)$ & $\mathrm{N}(2)-\mathrm{Zn}(2)-\mathrm{N}(7)$ & $155.53(9)$ \\
\hline $\mathrm{N}(1)-\mathrm{Zn}(1)-\mathrm{N}(3)$ & $80.70(9)$ & $\mathrm{O}(2)-\mathrm{Zn}(2)-\mathrm{N}(6)$ & $160.13(10)$ \\
\hline $\mathrm{O}(1)-\mathrm{Zn}(1)-\mathrm{N}(3)$ & $176.05(10)$ & $\mathrm{N}(8)-\mathrm{Zn}(2)-\mathrm{N}(6)$ & $99.28(10)$ \\
\hline $\mathrm{N}(4)-\mathrm{Zn}(1)-\mathrm{N}(3)$ & $93.02(10)$ & $\mathrm{N}(2)-\mathrm{Zn}(2)-\mathrm{N}(6)$ & $81.91(9)$ \\
\hline $\mathrm{N}(5)-\mathrm{Zn}(1)-\mathrm{N}(3)$ & $89.93(10)$ & $\mathrm{N}(7)-\mathrm{Zn}(2)-\mathrm{N}(6)$ & $87.81(9)$ \\
\hline $\mathrm{O}(1)-\mathrm{P}(1)-\mathrm{O}(2)$ & $116.39(15)$ & & \\
\hline $\mathrm{O}(1)-\mathrm{P}(1)-\mathrm{O}(4)$ & $107.43(14)$ & & \\
\hline $\mathrm{O}(2)-\mathrm{P}(1)-\mathrm{O}(4)$ & $111.29(13)$ & & \\
\hline $\mathrm{O}(1)-\mathrm{P}(1)-\mathrm{O}(3)$ & $109.95(12)$ & & \\
\hline $\mathrm{O}(2)-\mathrm{P}(1)-\mathrm{O}(3)$ & $108.51(12)$ & & \\
\hline $\mathrm{O}(4)-\mathrm{P}(1)-\mathrm{O}(3)$ & $102.36(14)$ & & \\
\hline
\end{tabular}

Tabelle 16: Ausgewählte Atomabstände $(\AA)$ und Bindungswinkel ( $\left.{ }^{\circ}\right)$.

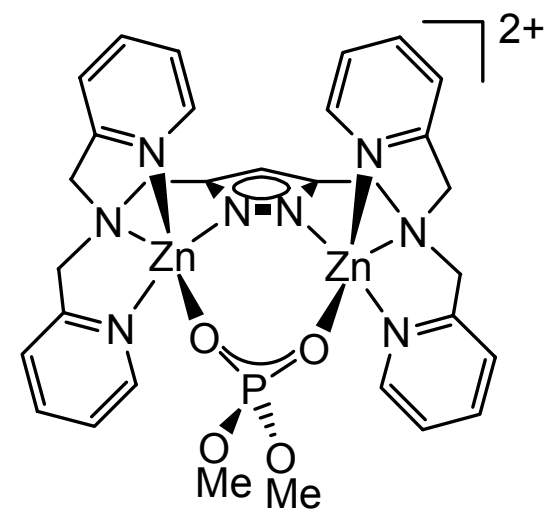

$12 a$ 


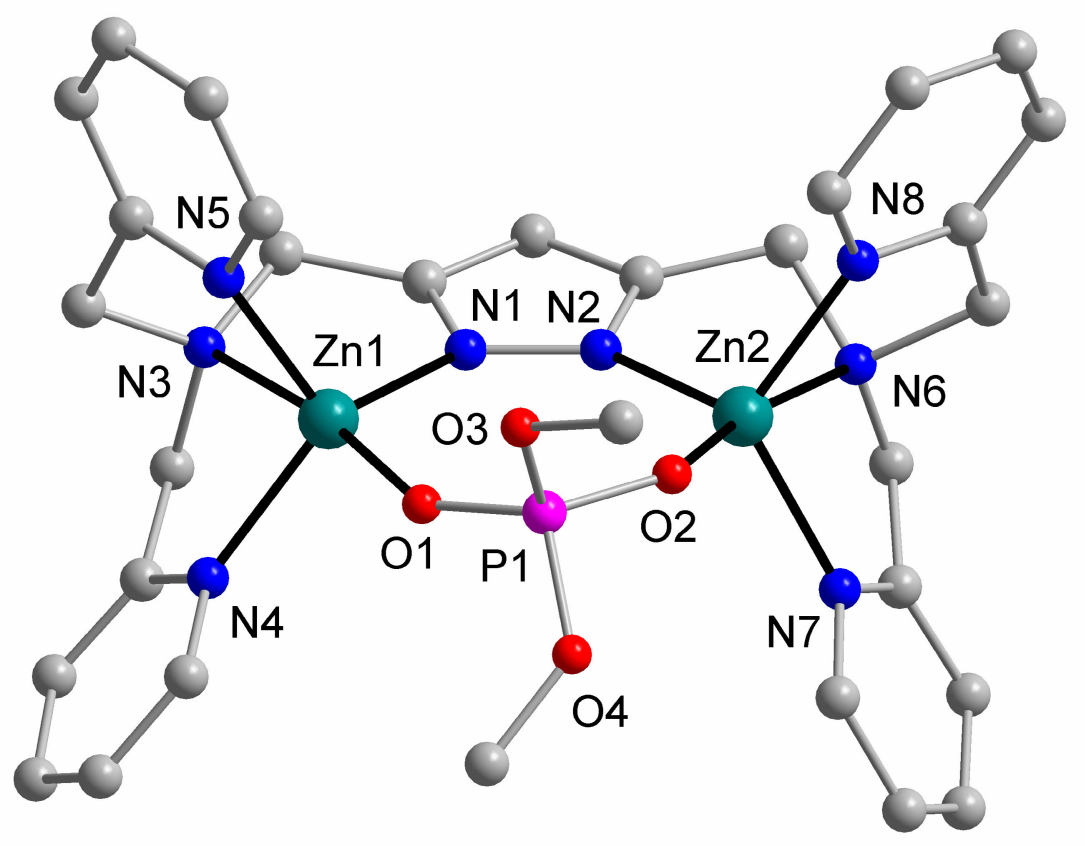

Abbildung 90: Festkörperstruktur des Kations von 12a $\cdot\left(\mathrm{ClO}_{4}\right)_{2}$.

\begin{tabular}{|l|l|l|l|}
\hline $\mathrm{Zn}(1)-\mathrm{O}(1)$ & $1.947(5)$ & $\mathrm{Zn}(2)-\mathrm{O}(2)$ & $1.973(4)$ \\
\hline $\mathrm{Zn}(1)-\mathrm{N}(1)$ & $2.006(5)$ & $\mathrm{Zn}(2)-\mathrm{N}(2)$ & $2.020(5)$ \\
\hline $\mathrm{Zn}(1)-\mathrm{N}(4)$ & $2.075(6)$ & $\mathrm{Zn}(2)-\mathrm{N}(6)$ & $2.257(6)$ \\
\hline $\mathrm{Zn}(1)-\mathrm{N}(3)$ & $2.296(14)[2.222(13)]$ & $\mathrm{Zn}(2)-\mathrm{N}(7)$ & $2.069(5)$ \\
\hline $\mathrm{Zn}(1)-\mathrm{N}(5)$ & $2.091(10)[2.074(13)]$ & $\mathrm{Zn}(2)-\mathrm{N}(8)$ & $2.114(15)[1.972(14)]$ \\
\hline $\mathrm{P}(1)-\mathrm{O}(1)$ & $1.497(5)$ & & \\
\hline $\mathrm{P}(1)-\mathrm{O}(2)$ & $1.487(5)$ & & \\
\hline $\mathrm{P}(1)-\mathrm{O}(3)$ & $1.577(5)$ & & \\
\hline $\mathrm{P}(1)-\mathrm{O}(4)$ & $1.540(6)$ & & \\
\hline $\mathrm{N}(1)-\mathrm{N}(2)$ & $1.360(7)$ & & \\
\hline $\mathrm{Zn}(1)-\mathrm{Zn}(2)$ & $4.212(1)$ & & \\
\hline $\mathrm{O}(1)-\mathrm{Zn}(1)-\mathrm{N}(1)$ & $111.3(2)$ & $\mathrm{O}(2)-\mathrm{Zn}(2)-\mathrm{N}(2)$ & $107.1(2)$ \\
\hline $\mathrm{O}(1)-\mathrm{Zn}(1)-\mathrm{N}(4)$ & $98.2(2)$ & $\mathrm{O}(2)-\mathrm{Zn}(2)-\mathrm{N}(7)$ & $93.9(2)$ \\
\hline $\mathrm{N}(1)-\mathrm{Zn}(1)-\mathrm{N}(4)$ & $118.5(2)$ & $\mathrm{N}(2)-\mathrm{Zn}(2)-\mathrm{N}(7)$ & $125.4(2)$ \\
\hline $\mathrm{O}(1)-\mathrm{Zn}(1)-\mathrm{N}(5)$ & $99.9(4)[87.8(5)]$ & $\mathrm{O}(2)-\mathrm{Zn}(2)-\mathrm{N}(8)$ & $100.8(5)[107.7(5)]$ \\
\hline $\mathrm{N}(1)-\mathrm{Zn}(1)-\mathrm{N}(5)$ & $109.3(4)[110.6(5)]$ & $\mathrm{N}(2)-\mathrm{Zn}(2)-\mathrm{N}(8)$ & $110.4(4)[110.9(5)]$ \\
\hline $\mathrm{N}(4)-\mathrm{Zn}(1)-\mathrm{N}(5)$ & $117.2(4)[123.6(5)]$ & $\mathrm{N}(7)-\mathrm{Zn}(2)-\mathrm{N}(8)$ & $114.3(5)[109.4(5)]$ \\
\hline $\mathrm{O}(1)-\mathrm{Zn}(1)-\mathrm{N}(3)$ & $170.3(3)[164.2(4)]$ & $\mathrm{O}(2)-\mathrm{Zn}(2)-\mathrm{N}(6)$ & $171.7(2)$ \\
\hline $\mathrm{N}(1)-\mathrm{Zn}(1)-\mathrm{N}(3)$ & $78.3(3)[82.1(3)]$ & $\mathrm{N}(2)-\mathrm{Zn}(2)-\mathrm{N}(6)$ & $78.3(2)$ \\
\hline
\end{tabular}




\begin{tabular}{|l|l|l|l|}
\hline $\mathrm{N}(4)-\mathrm{Zn}(1)-\mathrm{N}(3)$ & $75.8(4)[81.6(4)]$ & $\mathrm{N}(7)-\mathrm{Zn}(2)-\mathrm{N}(6)$ & $77.8(2)$ \\
\hline $\mathrm{N}(5)-\mathrm{Zn}(1)-\mathrm{N}(3)$ & $76.8(5)[79.3(6)]$ & $\mathrm{N}(8)-\mathrm{Zn}(2)-\mathrm{N}(6)$ & $82.7(5)[75.3(5)]$ \\
\hline $\mathrm{O}(2)-\mathrm{P}(1)-\mathrm{O}(1)$ & $118.3(3)$ & & \\
\hline $\mathrm{O}(2)-\mathrm{P}(1)-\mathrm{O}(4)$ & $112.9(3)$ & & \\
\hline $\mathrm{O}(1)-\mathrm{P}(1)-\mathrm{O}(4)$ & $101.9(4)$ & & \\
\hline $\mathrm{O}(2)-\mathrm{P}(1)-\mathrm{O}(3)$ & $105.6(3)$ & & \\
\hline $\mathrm{O}(1)-\mathrm{P}(1)-\mathrm{O}(3)$ & $109.7(3)$ & & \\
\hline $\mathrm{O}(4)-\mathrm{P}(1)-\mathrm{O}(3)$ & $108.1(3)$ & & \\
\hline
\end{tabular}

Tabelle 17: Ausgewählte Atomabstände $(\AA)$ und Bindungswinkel $\left({ }^{\circ}\right)$.

Eine ähnliche Phosphatanbindung wie in den Komplexen 10a, 11a und 12a kann auch für 8' angenommen werden. Dies wird durch die Isolierung und kristallographische Charakterisierung von $\left[\mathrm{Zn}_{2} \mathrm{~L}^{4} \mathrm{H}_{-1}(\mathrm{DMP})\left(\mathrm{NO}_{3}\right)_{2}\right]$ (13a) bestätigt. In 13a bindet der Phophatdiester in dem erwarteten bidentaten Modus an die Dizinkeinheit (Abbildung 91). Aufgrund der geringeren Zahl an Donoren in $L^{4}$ verglichen mit den bistetradentaten $\mathrm{L}^{3}, \mathrm{~L}^{6}$ und $\mathrm{L}^{8}$ müssen die verbleibenden Koordinationsstellen durch zusätzliche Liganden, wie beispielsweise hier durch die beiden Nitratanionen, besetzt werden. Jedes der beiden Nitrate bindet an eines der beiden Zinkionen bidentat mit zwei vergleichsweise langen $\mathrm{Zn}-\mathrm{O}$ Bindungsabständen $(d(Z n-O)=2.215(3)-2.291(3) \AA)$. Die Zinkionen weisen somit eine verzerrt oktaedrische Koordinationsumgebung auf. In Gegenwart schwächer koordinierender Gegenionen wie $\mathrm{ClO}_{4}^{-}$anstelle der Nitrate ist es wahrscheinlich, dass Lösungsmittelmoleküle, in den Reaktivitätsuntersuchungen also Wasser, die exo-ständigen Koordinationsstellen der Zinkionen besetzen.

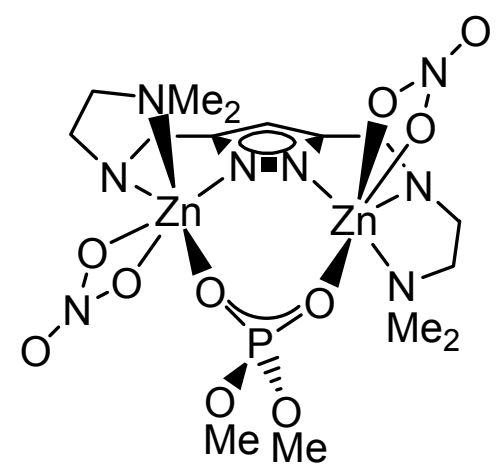

$13 a$ 


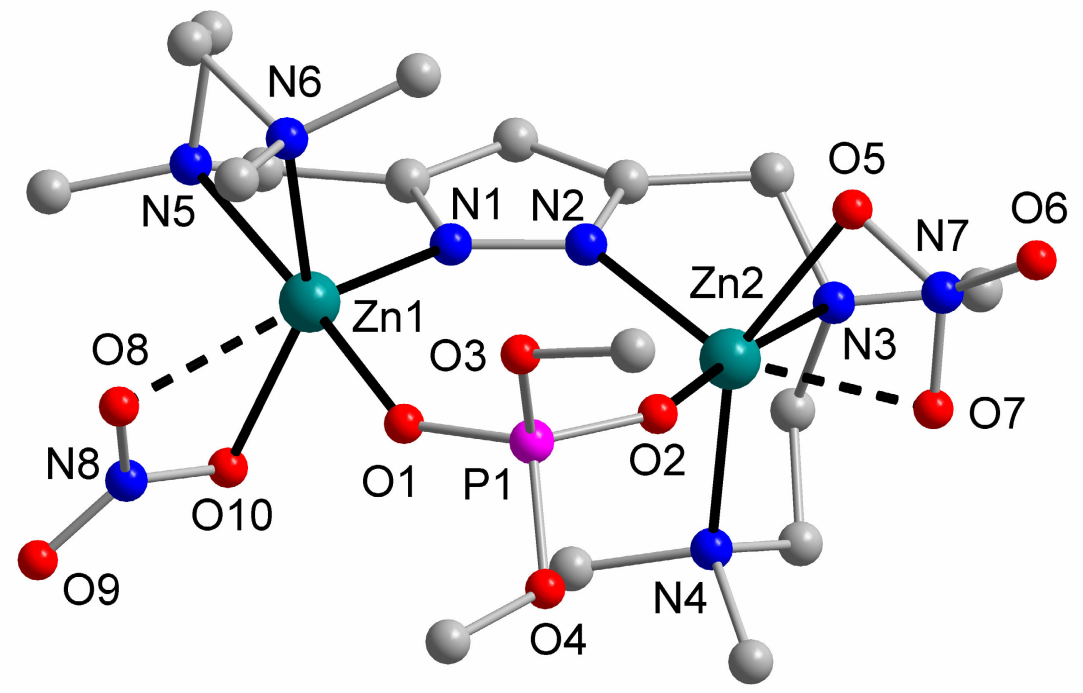

Abbildung 91: Festkörperstruktur von 13a.

\begin{tabular}{|l|l|l|l|}
\hline $\mathrm{Zn}(1)-\mathrm{O}(1)$ & $2.009(3)$ & $\mathrm{Zn}(2)-\mathrm{O}(2)$ & $1.995(3)$ \\
\hline $\mathrm{Zn}(1)-\mathrm{N}(1)$ & $2.056(4)$ & $\mathrm{Zn}(2)-\mathrm{N}(2)$ & $2.058(3)$ \\
\hline $\mathrm{Zn}(1)-\mathrm{N}(6)$ & $2.131(4)$ & $\mathrm{Zn}(2)-\mathrm{N}(4)$ & $2.159(4)$ \\
\hline $\mathrm{Zn}(1)-\mathrm{O}(10)$ & $2.227(3)$ & $\mathrm{Zn}(2)-\mathrm{O}(5)$ & $2.215(3)$ \\
\hline $\mathrm{Zn}(1)-\mathrm{O}(8)$ & $2.261(3)$ & $\mathrm{Zn}(2)-\mathrm{N}(3)$ & $2.254(3)$ \\
\hline $\mathrm{Zn}(1)-\mathrm{N}(5)$ & $2.306(3)$ & $\mathrm{Zn}(2)-\mathrm{O}(7)$ & $2.291(3)$ \\
\hline $\mathrm{N}(2)-\mathrm{N}(1)$ & $1.362(5)$ & & \\
\hline $\mathrm{Zn} 1 \cdots \mathrm{Zn} 2$ & $4.307(2)$ & & $\mathrm{O}(2)-\mathrm{Zn}(2)-\mathrm{N}(2)$ \\
\hline $\mathrm{O}(1)-\mathrm{Zn}(1)-\mathrm{N}(1)$ & $105.37(13)$ & $106.27(13)$ \\
\hline $\mathrm{O}(1)-\mathrm{Zn}(1)-\mathrm{N}(6)$ & $97.17(13)$ & $\mathrm{O}(2)-\mathrm{Zn}(2)-\mathrm{N}(4)$ & $97.26(14)$ \\
\hline $\mathrm{N}(1)-\mathrm{Zn}(1)-\mathrm{N}(6)$ & $105.85(14)$ & $\mathrm{N}(2)-\mathrm{Zn}(2)-\mathrm{N}(4)$ & $103.79(13)$ \\
\hline $\mathrm{O}(1)-\mathrm{Zn}(1)-\mathrm{O}(10)$ & $93.70(12)$ & $\mathrm{O}(2)-\mathrm{Zn}(2)-\mathrm{O}(5)$ & $88.21(13)$ \\
\hline $\mathrm{N}(1)-\mathrm{Zn}(1)-\mathrm{O}(10)$ & $95.97(13)$ & $\mathrm{N}(2)-\mathrm{Zn}(2)-\mathrm{O}(5)$ & $102.61(13)$ \\
\hline $\mathrm{N}(6)-\mathrm{Zn}(1)-\mathrm{O}(10)$ & $151.96(14)$ & $\mathrm{N}(4)-\mathrm{Zn}(2)-\mathrm{O}(5)$ & $150.28(13)$ \\
\hline $\mathrm{O}(1)-\mathrm{Zn}(1)-\mathrm{O}(8)$ & $87.83(12)$ & $\mathrm{O}(2)-\mathrm{Zn}(2)-\mathrm{N}(3)$ & $173.79(13)$ \\
\hline $\mathrm{N}(1)-\mathrm{Zn}(1)-\mathrm{O}(8)$ & $152.00(12)$ & $\mathrm{N}(2)-\mathrm{Zn}(2)-\mathrm{N}(3)$ & $79.64(13)$ \\
\hline $\mathrm{N}(6)-\mathrm{Zn}(1)-\mathrm{O}(8)$ & $96.60(13)$ & $\mathrm{N}(4)-\mathrm{Zn}(2)-\mathrm{N}(3)$ & $82.93(13)$ \\
\hline $\mathrm{O}(10)-\mathrm{Zn}(1)-\mathrm{O}(8)$ & $57.98(11)$ & $\mathrm{O}(5)-\mathrm{Zn}(2)-\mathrm{N}(3)$ & $88.62(12)$ \\
\hline $\mathrm{O}(1)-\mathrm{Zn}(1)-\mathrm{N}(5)$ & $176.86(14)$ & $\mathrm{O}(2)-\mathrm{Zn}(2)-\mathrm{O}(7)$ & $85.47(12)$ \\
\hline $\mathrm{N}(1)-\mathrm{Zn}(1)-\mathrm{N}(5)$ & $77.77(13)$ & $\mathrm{N}(2)-\mathrm{Zn}(2)-\mathrm{O}(7)$ & $157.43(13)$ \\
\hline $\mathrm{N}(6)-\mathrm{Zn}(1)-\mathrm{N}(5)$ & $81.94(13)$ & $\mathrm{N}(4)-\mathrm{Zn}(2)-\mathrm{O}(7)$ & $93.48(12)$ \\
\hline $\mathrm{O}(10)-\mathrm{Zn}(1)-\mathrm{N}(5)$ & $85.80(12)$ & $\mathrm{O}(5)-\mathrm{Zn}(2)-\mathrm{O}(7)$ & $57.70(11)$ \\
\hline
\end{tabular}




\begin{tabular}{|l|l|l|l|}
\hline $\mathrm{O}(8)-\mathrm{Zn}(1)-\mathrm{N}(5)$ & $89.28(12)$ & $\mathrm{N}(3)-\mathrm{Zn}(2)-\mathrm{O}(7)$ & $88.33(12)$ \\
\hline $\mathrm{O}(2)-\mathrm{P}(1)-\mathrm{O}(1)$ & $118.84(18)$ & & \\
\hline $\mathrm{O}(2)-\mathrm{P}(1)-\mathrm{O}(4)$ & $105.07(19)$ & & \\
\hline $\mathrm{O}(1)-\mathrm{P}(1)-\mathrm{O}(4)$ & $110.20(18)$ & & \\
\hline $\mathrm{O}(2)-\mathrm{P}(1)-\mathrm{O}(3)$ & $111.07(19)$ & & \\
\hline $\mathrm{O}(1)-\mathrm{P}(1)-\mathrm{O}(3)$ & $105.98(18)$ & & \\
\hline $\mathrm{O}(4)-\mathrm{P}(1)-\mathrm{O}(3)$ & $104.90(17)$ & & \\
\hline
\end{tabular}

Tabelle 18: Ausgewählte Atomabstände $(\AA)$ und Bindungswinkel $\left({ }^{\circ}\right)$.

Der Versuch, den Carbonat-verbrückten vierkernigen Zinkkomplex 8a mit Phosphaten umzusetzen, lieferte im ${ }^{31}$ P-NMR kein Signal für ein gebundenes Phosphat. Der Austausch von Carbonat durch Phosphat oder die Anbindung an das tetranukleare Zentrum erfolgt also nicht oder nur sehr langsam. Dennoch konnten durch Umsetzen einer Lösung des Carbonat-Komplexes mit einer äquimolaren Menge Diphenylphosphinsäure im Laufe von Monaten neben einer großen Menge von nicht umgesetzten $\mathbf{8 a}$ einige wenige Kristalle des Komplexes $\left[\mathrm{Zn}_{2} \mathrm{~L}^{4}{ }_{-H}\right.$ $\left.\left(\mathrm{O}_{2} \mathrm{PPh}_{2}\right)_{2}\right]\left(\mathrm{ClO}_{4}\right) 14$ erhalten werden.

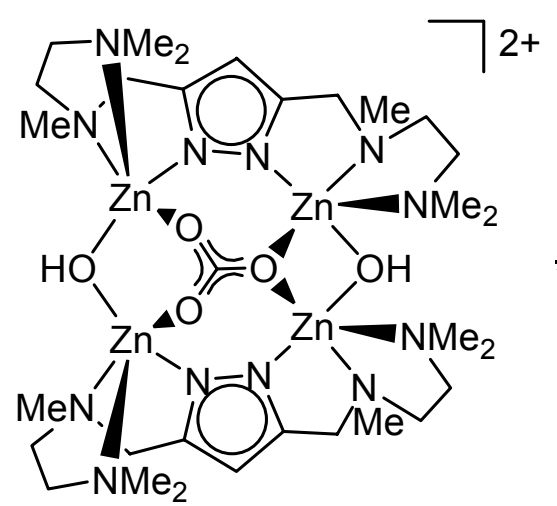

$+2 \mathrm{Ph}_{\mathrm{Ph}}^{\mathrm{Ph}}+2 \mathrm{ClO}_{4}^{-}$

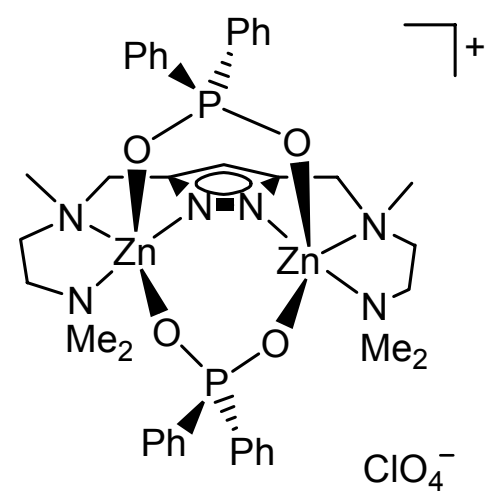

14

+ andere Produkte 


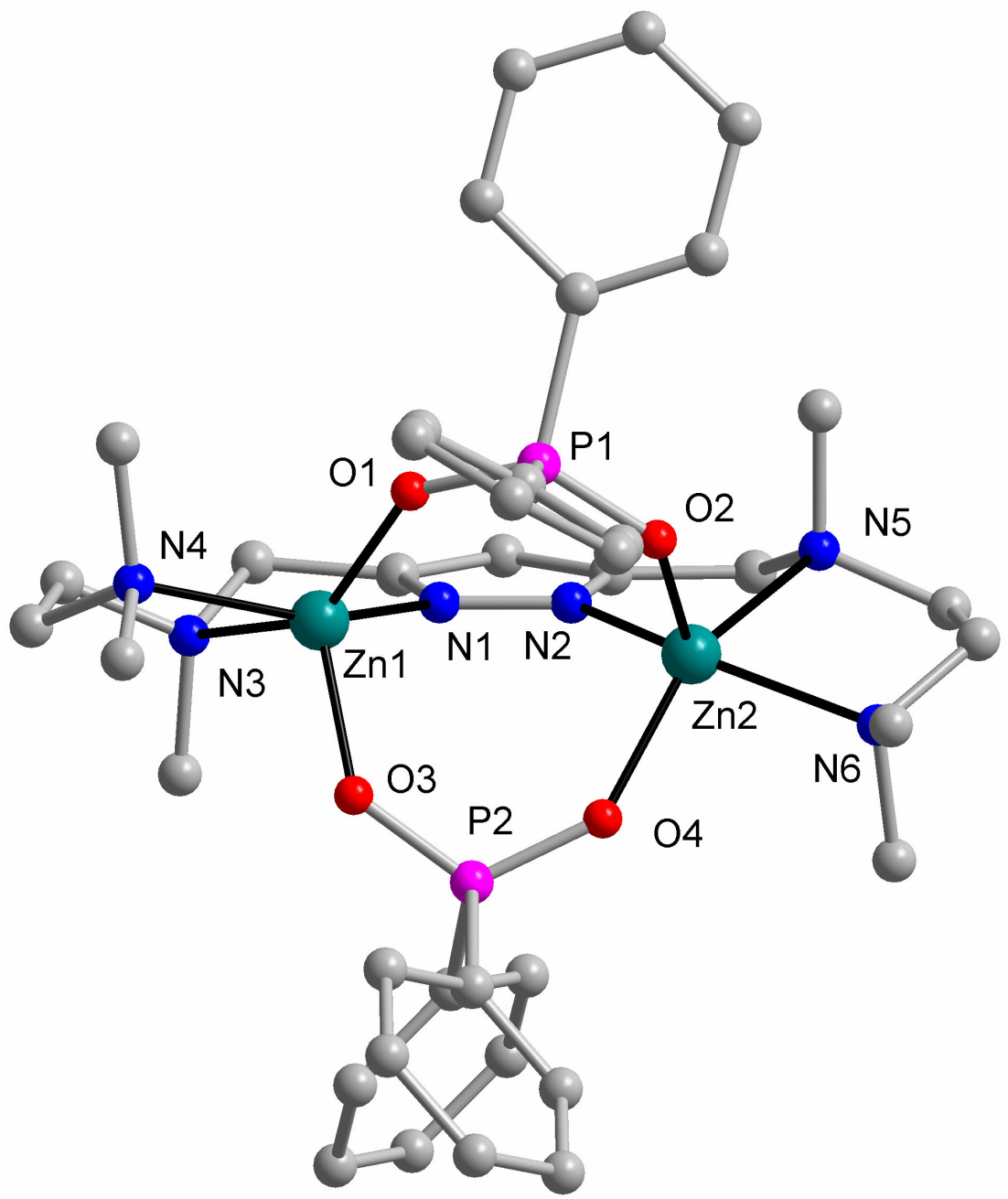

Abbildung 92: Festkörperstruktur des Kations von $14 \cdot\left(\mathrm{ClO}_{4}\right)$.

\begin{tabular}{|l|l|l|l|}
\hline $\mathrm{Zn}(1)-\mathrm{O}(1)$ & $2.0331(16)$ & $\mathrm{Zn}(2)-\mathrm{O}(2)$ & $1.9598(17)$ \\
\hline $\mathrm{Zn}(1)-\mathrm{O}(3)$ & $1.9675(16)$ & $\mathrm{Zn}(2)-\mathrm{O}(4)$ & $2.0075(16)$ \\
\hline $\mathrm{Zn}(1)-\mathrm{N}(1)$ & $2.0003(19)$ & $\mathrm{Zn}(2)-\mathrm{N}(2)$ & $1.9986(19)$ \\
\hline $\mathrm{Zn}(1)-\mathrm{N}(3)$ & $2.3119(19)$ & $\mathrm{Zn}(2)-\mathrm{N}(5)$ & $2.3610(19)$ \\
\hline $\mathrm{Zn}(1)-\mathrm{N}(4)$ & $2.1435(19)$ & $\mathrm{Zn}(2)-\mathrm{N}(6)$ & $2.1160(18)$ \\
\hline $\mathrm{P}(1)-\mathrm{O}(1)$ & $1.5056(16)$ & $\mathrm{Zn}(1) \cdots \mathrm{Zn}(2)$ & $3.8529(4)$ \\
\hline $\mathrm{P}(1)-\mathrm{O}(2)$ & $1.5140(17)$ & $\mathrm{N}(1)-\mathrm{N}(2)$ & $1.359(3)$ \\
\hline $\mathrm{P}(2)-\mathrm{O}(4)$ & $1.5045(17)$ & & \\
\hline $\mathrm{P}(2)-\mathrm{O}(3)$ & $1.5160(17)$ & & \\
\hline $\mathrm{O}(1)-\mathrm{Zn}(1)-\mathrm{N}(1)$ & $97.11(8)$ & $\mathrm{O}(2)-\mathrm{Zn}(2)-\mathrm{N}(2)$ & $107.68(8)$ \\
\hline $\mathrm{O}(1)-\mathrm{Zn}(1)-\mathrm{N}(3)$ & $143.06(7)$ & $\mathrm{O}(2)-\mathrm{Zn}(2)-\mathrm{N}(5)$ & $111.52(7)$ \\
\hline $\mathrm{O}(1)-\mathrm{Zn}(1)-\mathrm{N}(4)$ & $92.74(7)$ & $\mathrm{O}(2)-\mathrm{Zn}(2)-\mathrm{N}(6)$ & $100.46(7)$ \\
\hline $\mathrm{O}(1)-\mathrm{Zn}(1)-\mathrm{O}(3)$ & $107.23(7)$ & $\mathrm{O}(2)-\mathrm{Zn}(2)-\mathrm{O}(4)$ & $101.66(7)$ \\
\hline $\mathrm{O}(3)-\mathrm{Zn}(1)-\mathrm{N}(1)$ & $104.86(8)$ & $\mathrm{O}(4)-\mathrm{Zn}(2)-\mathrm{N}(2)$ & $98.81(7)$ \\
\hline
\end{tabular}




\begin{tabular}{|l|l|l|l|}
\hline $\mathrm{O}(3)-\mathrm{Zn}(1)-\mathrm{N}(3)$ & $109.70(7)$ & $\mathrm{O}(4)-\mathrm{Zn}(2)-\mathrm{N}(5)$ & $146.77(7)$ \\
\hline $\mathrm{O}(3)-\mathrm{Zn}(1)-\mathrm{N}(4)$ & $99.91(7)$ & $\mathrm{O}(4)-\mathrm{Zn}(2)-\mathrm{N}(6)$ & $93.09(7)$ \\
\hline $\mathrm{N}(1)-\mathrm{Zn}(1)-\mathrm{N}(4)$ & $149.18(8)$ & $\mathrm{N}(2)-\mathrm{Zn}(2)-\mathrm{N}(5)$ & $73.37(7)$ \\
\hline $\mathrm{N}(1)-\mathrm{Zn}(1)-\mathrm{N}(3)$ & $74.51(7)$ & $\mathrm{N}(2)-\mathrm{Zn}(2)-\mathrm{N}(6)$ & $146.34(8)$ \\
\hline $\mathrm{N}(3)-\mathrm{Zn}(1)-\mathrm{N}(4)$ & $80.09(7)$ & $\mathrm{N}(5)-\mathrm{Zn}(2)-\mathrm{N}(6)$ & $79.45(7)$ \\
\hline $\mathrm{O}(1)-\mathrm{P}(1)-\mathrm{O}(2)$ & $119.16(10)$ & & \\
\hline $\mathrm{O}(3)-\mathrm{P}(2)-\mathrm{O}(4)$ & $118.11(10)$ & & \\
\hline
\end{tabular}

Tabelle 19: Ausgewählte Atomabstände $(\AA)$ und Bindungswinkel $\left({ }^{\circ}\right)$.

Dabei handelt es sich nicht um ein Addukt von Phosphat an das tetranukleare Zentrum, sondern um einen zweikernigen Komplex mit zwei gebundenen Phosphatanaloga. Die beiden Phosphinate sitzen jeweils bidentat verbrückend oberhalb und unterhalb der durch die beiden Zinkionen und den Pyrazolatzyklus aufgespannten Ebene, so dass eine verzerrt trigonal-bipyramidale Koordinationsgeometrie für die beiden Zinkionen resultiert. Durch die beiden bidentat verbrückenden Phosphinate werden die beiden Zinkatome verglichen mit anderen Zinkkomplexen von $\mathrm{L}^{4}$ vergleichsweise nahe zueinander $(\mathrm{d}(\mathrm{Zn}(1) \cdots \mathrm{Zn}(2))=3.8529(4) \AA)$ gezogen.

Während man für diesen Komplex bei Raumtemperatur im ${ }^{1} \mathrm{H}-\mathrm{NMR}$ sehr breite Signale erhält, liegen bei $-60^{\circ} \mathrm{C}$ zwei Spezies vor. Unklar ist jedoch, welche Spezies neben 14 gebildet wird oder ob nur eine andere Konformation vorliegt; auch das ${ }^{31} \mathrm{P}$ NMR-Spektrum zeigt nur ein breites Signal bei 26.4 ppm. Möglicherweise ist der Phosphinatligand in $\mathbf{1 4}$ labil und kann so dissoziieren oder nur an ein Zinkion binden. 


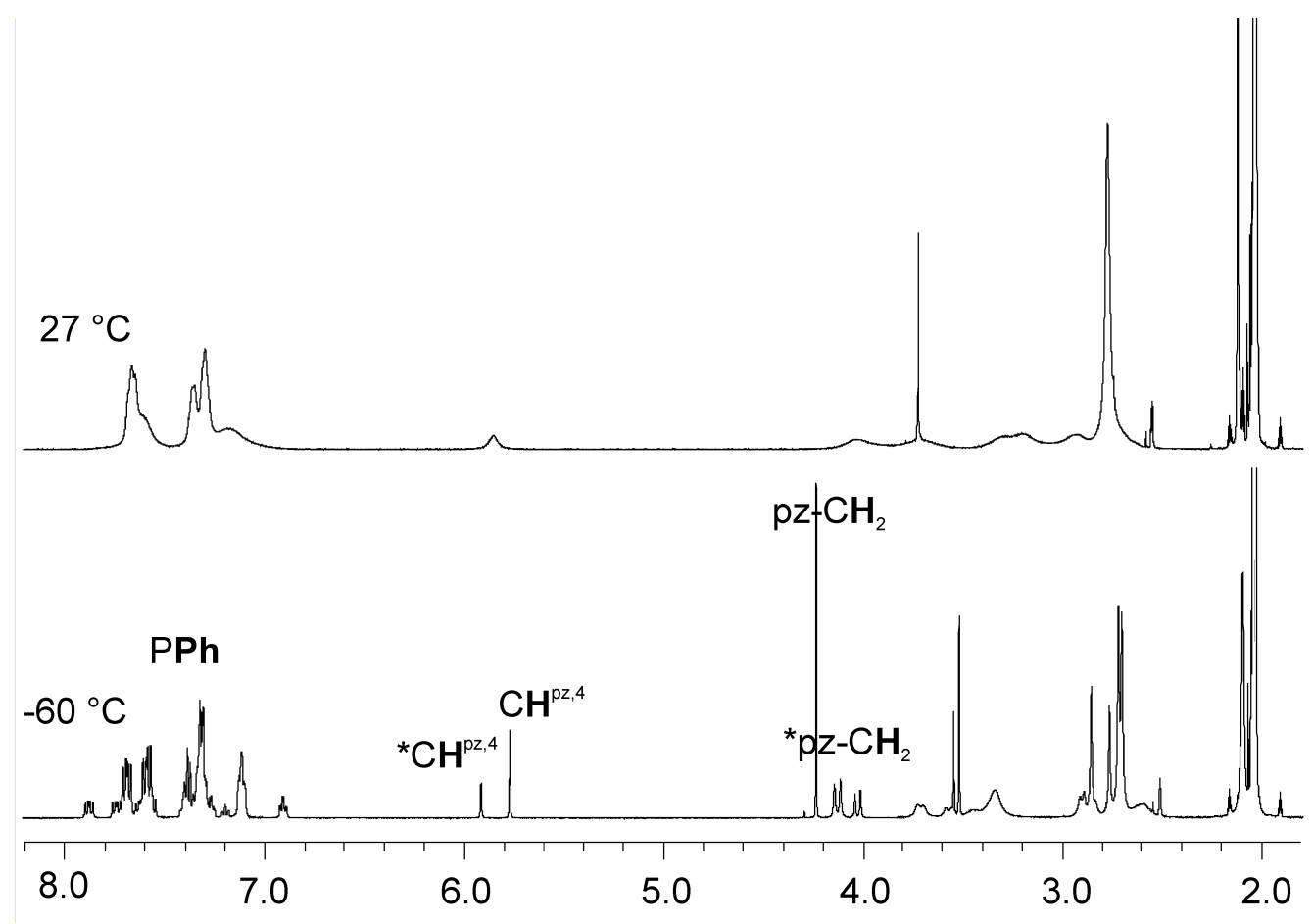

Abbildung 93: ${ }^{1} \mathrm{H}-N M R$ Spektrum von 14 bei $27^{\circ} \mathrm{C}$ und bei $-60{ }^{\circ} \mathrm{C}\left(500.13 \mathrm{MHz}, d_{6}\right.$-Aceton).

\subsection{Vergleichende Diskussion der Phosphatesterhydrolysen}

Da eine Erniedrigung des pKs-Werts metallgebundenen Wassers allgemein die Nukleophilie des entstehenden Hydroxids verringern sollte, würde man aus dieser simplen Betrachtung eine Reaktivitätsreihe von $3 \approx 4>8^{\prime}>\mathbf{2} \approx 5$ erwarten; diese widerspricht jedoch der gemessenen Reaktivitätsreihe bei pH 8.3 von $\mathbf{3}<\mathbf{2}<\mathbf{4}<\mathbf{5}<$ 8' (Tabelle 14).

Offenbar müssen die Unterschiede der einzelnen Systeme etwas genauer betrachtet werden. Speziell die Reaktivitätsreihe $\mathbf{3}<\mathbf{2}$ und $\mathbf{4}<\mathbf{5}$ erlaubt einige Rückschlüsse auf die hydrolytische Wirksamkeit des Zink-gebundenen Hydroxids bezüglich seiner unterschiedlichen Bindungsmodi: Ein verbrückend gebundenes Hydroxid, wie es im Fall des am wenigsten aktiven Komplexes $\mathbf{3}$ und des verglichen mit 5 sehr viel weniger aktiven Komplexes 4 auftritt, ist aufgrund seiner verringerten Nukleophilie - ungeachtet seines pKs-Werts - anscheinend ungünstig.

Da von dem maskierten terminalen Hydroxid in $\mathbf{2}$ eine deutlich höhere Nukleophilie erwartet wurde, müssen andere Effekte für die immer noch vergleichsweise geringe Aktivität von 2 verantwortlich sein. Aufgrund der Untersuchung zur Bindung von DMP, den ESI-Experimenten und der vorgestellten Struktur 10a ist anzunehmen, dass das BNPP-Substrat an 2 in bidentater Art in die bimetallische Tasche 
koordiniert (Abbildung 87) und die $\mathrm{H}_{3} \mathrm{O}_{2}$-Brücke vollständig ersetzt. Obwohl dies zu einer Aktivierung des Substrats führen könnte, sind alle Koordinationsstellen im resultierenden Addukt 10b nun blockiert, und die Aktivierung eines Wassermoleküls zu einem nukleophilen, Zink-gebundenen Hydroxid ist nicht länger möglich. Dies erscheint umso wahrscheinlicher, als das es bisher keine Hinweise darauf gibt, dass Komplexe mit der Koordinationszahl sechs vorliegen. Auch eine CSD-Recherche mononuklearer Zinkkomplexe tripodaler Liganden zeigt, dass die sechsfache Koordination nur durch zusätzliche bidentat-verbrückende Liganden erreicht werden kann.

Auch an dem verwandten Dinickel(II)komplex $\left[\mathrm{Ni}_{2} \mathrm{~L}^{3}{ }_{-\mathrm{H}}\left(\mathrm{O}_{2} \mathrm{H}_{3}\right)\right]^{2+}$ wurde gezeigt, dass die $\mathrm{H}_{3} \mathrm{O}_{2}$-Einheit eher als Base denn als Nukleophil gegenüber bidentat verbrückenden Substraten wie Harnstoff wirkt und zu einem relativ stabilen Einbau von anionischem Ureat führt. ${ }^{[112,113]}$

Desgleichen ist die Spezies $\left[\mathrm{Zn}_{2} \mathrm{~L}^{3} \mathrm{H}_{-2}\right]$ selbst hydrolytisch inaktiv gegenüber bidentaten Substraten wie BNPP. Die beobachtete niedrige Restaktivität im mittleren $\mathrm{pH}$-Bereich kann auf die Anwesenheit verschiedener anderer Spezies in Lösung zurückgeführt werden, wie es auch der Vergleich in Abbildung 77 der beobachteten Hydrolysegeschwindigkeit in Abhängigkeit vom pH-Wert mit der Speziesverteilung nahe legt.

Im Gegensatz dazu wird im Fall von 3 das Zink-gebundene Hydroxid in der Spezies $\left[\mathrm{Zn}_{2} \mathrm{~L}^{5} \mathrm{H}_{-2}\right]$ nicht oder nur zu einem geringen Teil durch ein BNPP-Molekül ersetzt und ist aufgrund der pH-Abhängigkeit der Reaktionsgeschwindigkeit eindeutig für die hydrolytische Spaltung des Substrats verantwortlich. Die geringe Aktivität von $\mathbf{3}$ ist vermutlich auf zwei Ursachen zurückzuführen. Zum Ersten ist die Bindungskonstante für die Koordination des Substrats sehr klein. Dies kann der Abschirmung der beiden Metallionen durch die relativ starren und sterisch anspruchsvollen tacn-Seitenarme und den Mangel an einfach zugänglichen Koordinationsstellen am Dizinkzentrum zugeschrieben werden. Zum Zweiten ist das verbrückende Hydroxid in $\mathbf{3}$ zwischen den beiden Zinkionen fest gebunden und zeigt nur geringe Nukleophilie. Eine Substratanbindung könnte jedoch das Hydroxid in eine halbverbrückende oder sogar terminale Position verschieben und so einen nukleophilen Angriff ermöglichen.

Die Aktivitäten der Pyridyldizinkkomplexe $\mathbf{4}$ und $\mathbf{5}$ erweisen sich als deutlich höher als die der aliphatischen Komplexe. Da die strukturellen Unterschiede des Dizinkkerns zwischen $\mathbf{4}$ und $\mathbf{3}$ und zwischen $\mathbf{5}$ und $\mathbf{2 b}$ sehr klein sind, kann der 
beobachtete Reaktivitätsunterschied nur durch die unterschiedlichen sterischen und möglicherweise elektronischen Verhältnisse erklärt werden. Die synthetisierten Phosphataddukte zeigen, dass das dimetallische Zentrum durch die bidentate Koordination des Phosphats völlig blockiert wird; so muss für das angreifende Hydroxid eine freie Koordinationsstelle geschaffen werden. Eine Dissoziation der Seitenarme scheint aufgrund der scharfen Signale der NMR-Spektren von 10a, 11a und 12a, die dynamische Vorgänge wie eine solche Dissoziation an den Seitenarmen unwahrscheinlich machen, jedoch nicht zu erfolgen. Es muss also, wie auch schon für andere Systeme vorgeschlagen, ${ }^{[102]}$ ein zusätzliches Hydroxid an ein Zink gebunden werden. So sollte die Zugänglichkeit der Zinkionen für einen weiteren Liganden für die Reaktivität eine Rolle spielen. Aufgrund der starken Koordination des Zinks durch den tacn-Makrozyklus und der dadurch vollständigen Abschirmung der Zinkionen ist die niedrige Reaktivität von 3 offensichtlich. Vermutlich führt auch die freie Beweglichkeit der terminalen Ethylgruppen in 2 bzw. 10a dazu, dass das Zinkzentrum nach außen relativ abgeschirmt ist. Im Fall der Pyridinringe zeigt das CPK-Modell von 10a und 11a in Abbildung 94, dass die Zinkionen von außen noch zugänglich sind und so ein zusätzliches Hydroxid an das Zentrum binden kann.
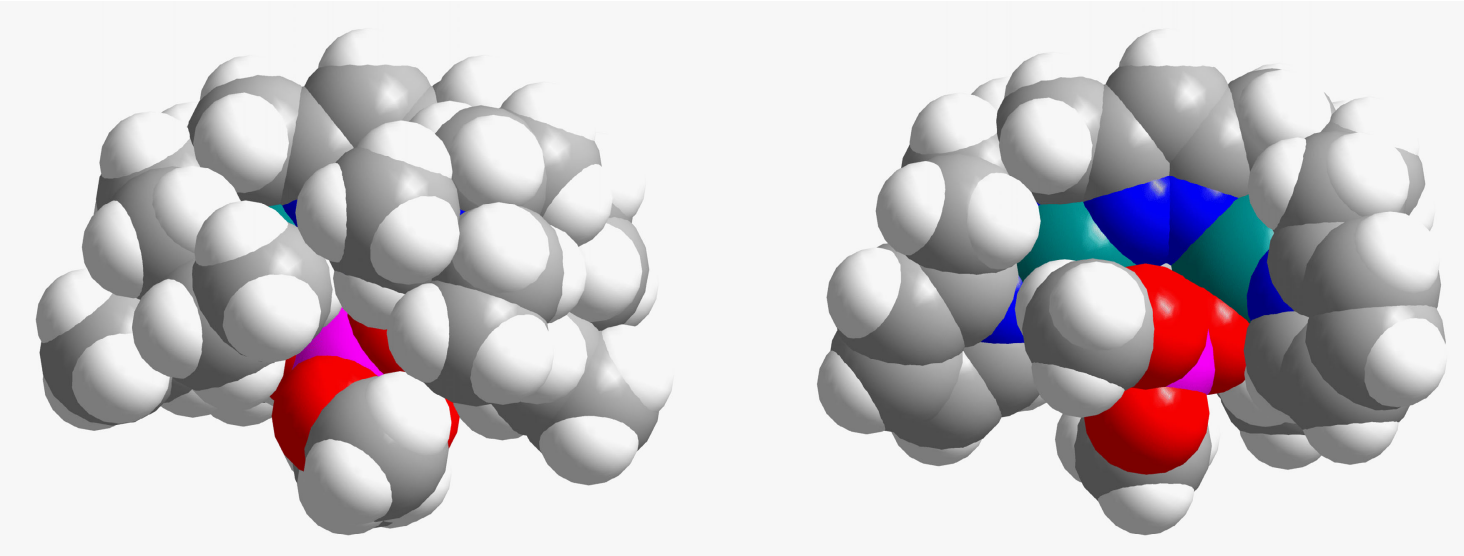

Abbildung 94: CPK-Modelle der DMP-Addukte 10a und 11a.

Die röntgenkristallographischen Ergebnisse für 10a, 11a, 12a und 13a legen nahe, dass eine analoge bidentate Substratkoordination für 2, 4, 5 und 8' erfolgt. Dies steht auch im Einklang mit den sehr ähnlichen $K_{\mathrm{M}}$-Werten, die man aus der MichaelisMenten-Analyse der beiden Systeme erhält. Der entscheidende Vorteil von 8' jedoch ist die freie Verfügbarkeit zusätzlicher Koordinationsstellen an den Metallionen. Diese machen zum einen eine bidentate Substratbindung möglich, erlauben aber 
gleichzeitig die Bildung eines aktiven Zink-gebundenen Hydroxids aus Wasser, das nicht in einer intramolekularen verbrückenden Position wie in $\mathbf{3}$ oder $\mathbf{4}$ abgefangen werden kann. Daher wird $\left[\mathrm{Zn}_{2} \mathrm{~L}^{4} \mathrm{H}_{-2}\right]^{2+}$ als aktive Spezies identifiziert, und es wird, verglichen mit 2, ein 20-fach höherer Wert für $k_{\text {cat }}$ und auch für die Geschwindigkeitskonstanten zweiter Ordnung beobachtet.

Um zu überprüfen, ob die verwendeten Dizinkkomplexe als Katalysator in der Spaltung von BNPP wirken, wurde die Reaktion nach Zufügen eines 10-fachen Überschusses an BNPP mittels ${ }^{31} \mathrm{P}$-NMR-Spektroskopie verfolgt. Für $\mathbf{5}$ zeigt sich ein Maximalumsatz von einem Äquivalent. Dies kann durch eine Inhibierung des Produkts erklärt werden. Das entstehende MNPP ist aufgrund seiner erhöhten Ladung von -2 nukleophiler als das ursprüngliche einfach negative BNPP-Substrat und verbleibt deshalb am Dizinkzentrum. Aufgrund der höheren Stabilität des MNPP wird dieses nicht weiter zu Phosphat hydrolysiert. Solch eine Produktinhibierung ist ein generelles Phänomen in der Phosphatdiester-Hydrolyse durch biomimetische Komplexe und erinnert an ähnliches Verhalten in Metalloenzymen.

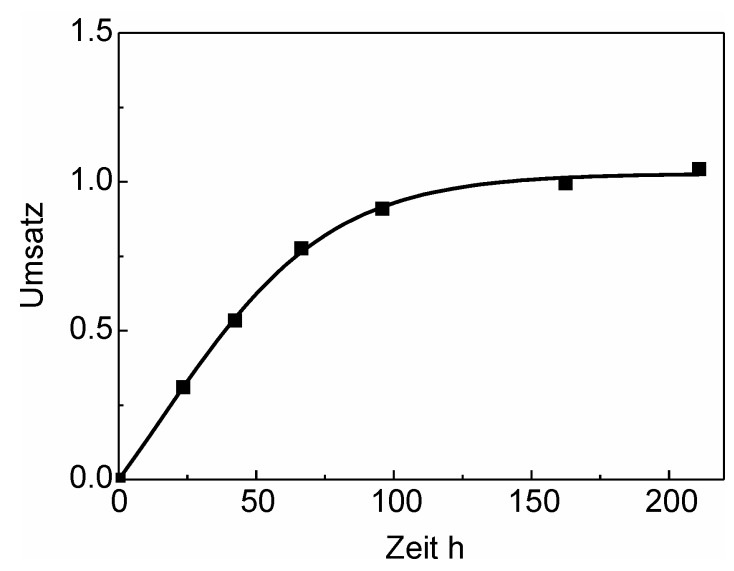

Abbildung 95: Im ${ }^{31} \mathrm{P}-\mathrm{NMR}$ beobachtete Hydrolyse von BNPP durch 5 bei $\mathrm{pH} 8,50^{\circ} \mathrm{C}$, in DMSO/Pufferlösung $\mathrm{H}_{2} \mathrm{O}$ (1:1).

Die Umsetzung von 8' mit einem Äquivalent BNPP zeigt eine rasch abnehmende Reaktionsgeschwindigkeit und einen Maximalumsatz, der sich bei etwas unterhalb von 0.5 Äquivalenten Substrat einpendelt (Abbildung 96). Dies offenbart eine sehr effiziente Produktinhibierung des aktiven Zentrums, wobei jedes durch die Hydrolyse gebildete Mono(4-nitrophenyl)phosphat (MNPP) mehr als eine $\left[\mathrm{Zn}_{2} \mathrm{~L}^{4} \mathrm{H}_{-2}\right]$-Spezies blockiert. 


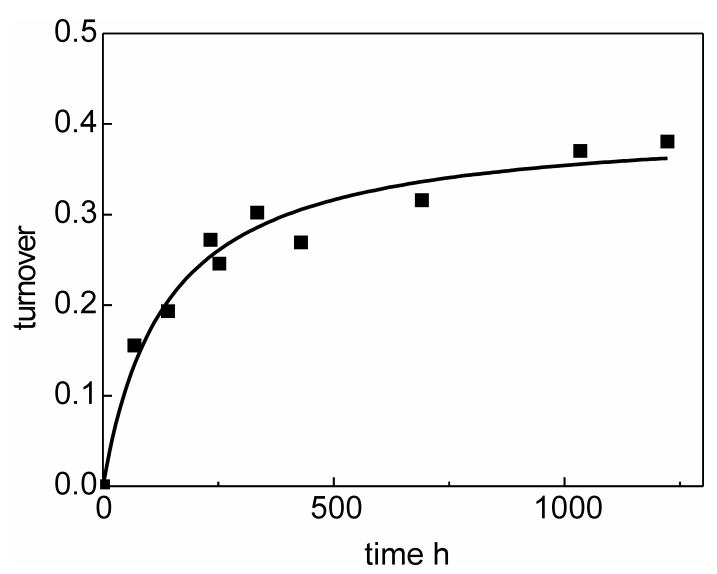

Abbildung 96: Im ${ }^{31}$ P-NMR beobachtete Hydrolyse von BNPP durch 8' bei $\mathrm{pH} 8,50^{\circ} \mathrm{C}$, in DMSO/Pufferlösung $\mathrm{H}_{2} \mathrm{O}$ (1:1).

Kristalle des produktinhibierten Komplexes 15 konnten direkt aus einer Reaktionsmischung erhalten und durch Röntgenkristallographie bestimmt werden. Die molekulare Struktur von 15 ist in Abbildung 98 abgebildet, ausgewählte Atomabständen und Bindungswinkel sind in Tabelle 20 zusammengestellt.
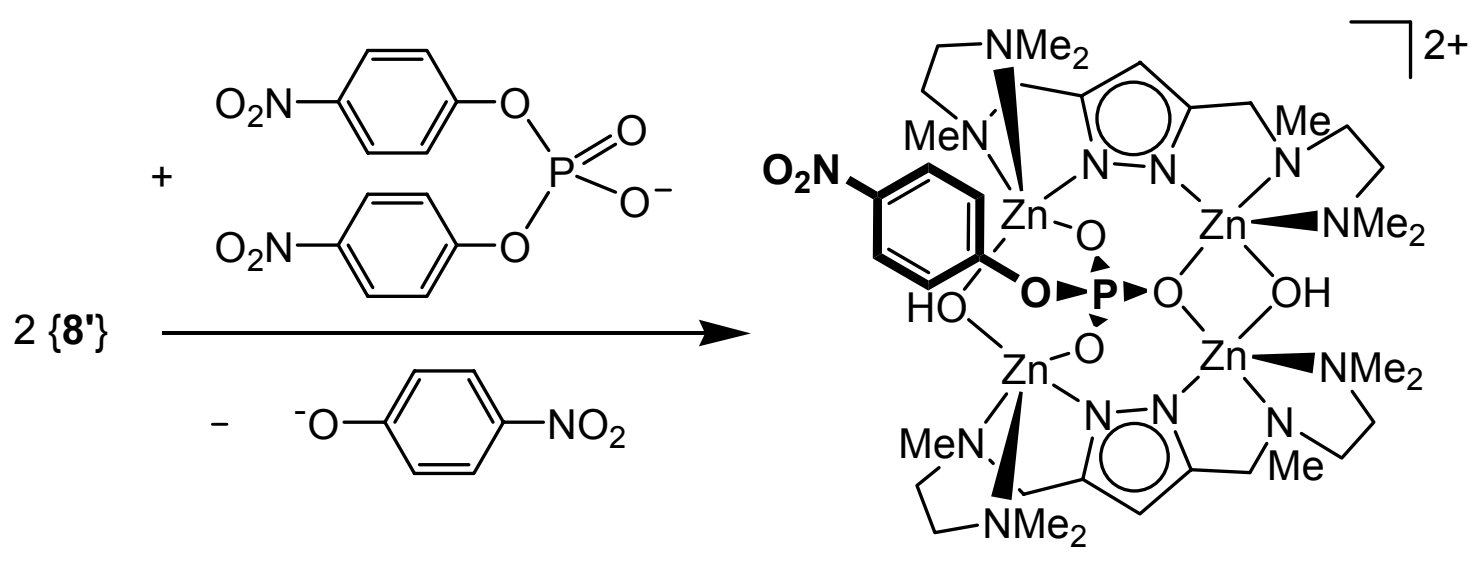

15

Abbildung 97: Bildung des produktinhibierten Komplexes 15. 


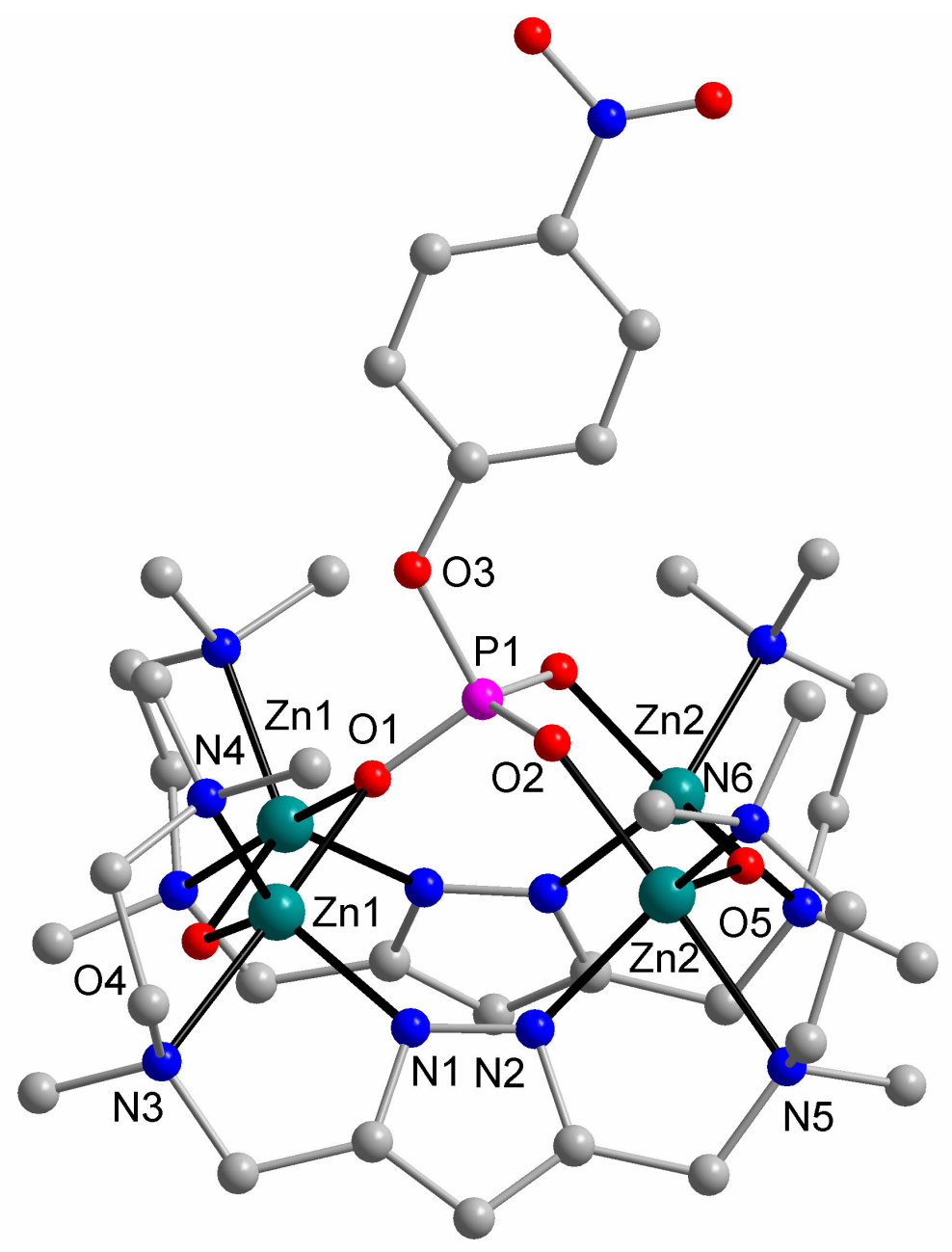

Abbildung 98: Festkörperstruktur des Kations von $15 \cdot\left(\mathrm{ClO}_{4}\right)_{2}$.

\begin{tabular}{|l|l|l|l|}
\hline $\mathrm{Zn}(1)-\mathrm{O}(1)$ & $2.122(5)$ & $\mathrm{Zn}(2)-\mathrm{O}(5)$ & $1.957(4)$ \\
\hline $\mathrm{Zn}(1)-\mathrm{O}(4)$ & $1.985(6)$ & $\mathrm{Zn}(2)-\mathrm{N}(2)$ & $2.049(6)$ \\
\hline $\mathrm{Zn}(1)-\mathrm{N}(1)$ & $2.021(7)$ & $\mathrm{Zn}(2)-\mathrm{N}(6)$ & $2.131(7)$ \\
\hline $\mathrm{Zn}(1)-\mathrm{N}(4)$ & $2.111(8)$ & $\mathrm{Zn}(2)-\mathrm{N}(5)$ & $2.338(7)$ \\
\hline $\mathrm{Zn}(1)-\mathrm{N}(3)$ & $2.279(7)$ & $\mathrm{Zn}(2)-\mathrm{O}(2)$ & $2.061(6)$ \\
\hline $\mathrm{P}(1)-\mathrm{O}(1)$ & $1.505(8)$ & $\mathrm{Zn} 1 \cdots \mathrm{Zn} 1 \mathrm{~A}$ & $3.146(2)$ \\
\hline $\mathrm{P}(1)-\mathrm{O}(2)$ & $1.510(5)$ & $\mathrm{Zn} 1 \cdots \mathrm{Zn} 2$ & 4.257 \\
\hline $\mathrm{P}(1)-\mathrm{O}(3)$ & $1.641(10)$ & $\mathrm{Zn} 2 \cdots \mathrm{Zn} 2 \mathrm{~A}$ & 3.545 \\
\hline $\mathrm{O}(4)-\mathrm{Zn}(1)-\mathrm{N}(1)$ & $118.9(4)$ & $\mathrm{O}(5)-\mathrm{Zn}(2)-\mathrm{N}(2)$ & $120.0(3)$ \\
\hline $\mathrm{O}(4)-\mathrm{Zn}(1)-\mathrm{N}(4)$ & $126.5(4)$ & $\mathrm{O}(5)-\mathrm{Zn}(2)-\mathrm{O}(2)$ & $96.9(3)$ \\
\hline $\mathrm{N}(1)-\mathrm{Zn}(1)-\mathrm{N}(4)$ & $112.9(3)$ & $\mathrm{N}(2)-\mathrm{Zn}(2)-\mathrm{O}(2)$ & $98.2(2)$ \\
\hline $\mathrm{O}(4)-\mathrm{Zn}(1)-\mathrm{O}(1)$ & $79.7(3)$ & $\mathrm{O}(5)-\mathrm{Zn}(2)-\mathrm{N}(6)$ & $119.8(3)$ \\
\hline $\mathrm{N}(1)-\mathrm{Zn}(1)-\mathrm{O}(1)$ & $103.9(3)$ & $\mathrm{N}(2)-\mathrm{Zn}(2)-\mathrm{N}(6)$ & $118.3(3)$ \\
\hline
\end{tabular}




\begin{tabular}{|l|l|l|l|}
\hline $\mathrm{N}(4)-\mathrm{Zn}(1)-\mathrm{O}(1)$ & $100.4(3)$ & $\mathrm{O}(2)-\mathrm{Zn}(2)-\mathrm{N}(6)$ & $88.8(3)$ \\
\hline $\mathrm{O}(4)-\mathrm{Zn}(1)-\mathrm{N}(3)$ & $93.3(3)$ & $\mathrm{O}(5)-\mathrm{Zn}(2)-\mathrm{N}(5)$ & $96.0(3)$ \\
\hline $\mathrm{N}(1)-\mathrm{Zn}(1)-\mathrm{N}(3)$ & $79.2(3)$ & $\mathrm{N}(2)-\mathrm{Zn}(2)-\mathrm{N}(5)$ & $78.7(3)$ \\
\hline $\mathrm{N}(4)-\mathrm{Zn}(1)-\mathrm{N}(3)$ & $83.8(3)$ & $\mathrm{O}(2)-\mathrm{Zn}(2)-\mathrm{N}(5)$ & $166.4(2)$ \\
\hline $\mathrm{O}(1)-\mathrm{Zn}(1)-\mathrm{N}(3)$ & $173.0(3)$ & $\mathrm{N}(6)-\mathrm{Zn}(2)-\mathrm{N}(5)$ & $81.2(3)$ \\
\hline $\mathrm{O}(1)-\mathrm{P}(1)-\mathrm{O}(2)$ & $112.5(3)$ & $\mathrm{Zn}(1)-\mathrm{O}(1)-\mathrm{Zn}(1)$ & $95.7(3)$ \\
\hline $\mathrm{O}(1)-\mathrm{P}(1)-\mathrm{O}(2)$ & $112.5(3)$ & $\mathrm{Zn}(2)-\mathrm{O}(5)-\mathrm{Zn}(2)$ & $129.8(5)$ \\
\hline $\mathrm{O}(2)-\mathrm{P}(1)-\mathrm{O}(2)$ & $116.1(4)$ & $\mathrm{Zn}(1)-\mathrm{O}(4)-\mathrm{Zn}(1)$ & $104.8(4)$ \\
\hline $\mathrm{O}(1)-\mathrm{P}(1)-\mathrm{O}(3)$ & $100.0(4)$ & & \\
\hline $\mathrm{O}(2)-\mathrm{P}(1)-\mathrm{O}(3)$ & $107.0(3)$ & & \\
\hline $\mathrm{O}(2 \mathrm{a})-\mathrm{P}(1)-\mathrm{O}(3)$ & $107.0(3)$ & & \\
\hline
\end{tabular}

Tabelle 20: Ausgewählte Atomabstände $(\AA)$ und Bindungswinkel ( $\left.{ }^{\circ}\right)$.

In 15 werden zwei $\left\{\mathrm{Zn}_{2} \mathrm{~L}^{4} \mathrm{H}_{-1}\right\}$-Bausteine durch zwei Hydroxidbrücken verbunden und formen eine tetranukleare Anordnung von vier Zinkionen, die durch ein $\mu_{4}-\eta^{2}: \eta^{1}: \eta^{1}-$ verbrückendes MNPP überkappt wird. 15 kann beschrieben werden als ein Komplex, in dem zwei $\left[\mathrm{Zn}_{2} \mathrm{~L}^{4} \mathrm{H}_{-2}\right]$-Spezies durch ein einziges Produktmolekül aus der Hydrolyse von BNPP inhibiert werden. Das MNPP blockiert alle vier Koordinationsstellen an den Metallionen und eine weitergehende Nukleophilie und damit Reaktivität der noch vorhandenen metallgebundenen Hydroxide in 15 ist durch ihre fest gebundene Brückenposition stark erniedrigt.

Erst durch die Wahl anderer Substrate ist es möglich, eine katalytische Spaltung von Phosphatestern zu bewirken. Die katalytische Umesterung des RNA-Modellsubstrats HPNPP führt zum zyklischen Methylethylenphosphat CycP (Abbildung 99). Dieses ist ebenfalls ein Phosphatester mit ähnlicher Nukleophilie wie das Edukt, so dass Substrat und Produkt gleich fest angebunden werden und keine Produktinhibierung, wie im Fall der Hydrolyse von BNPP in MNPP, auftritt. 


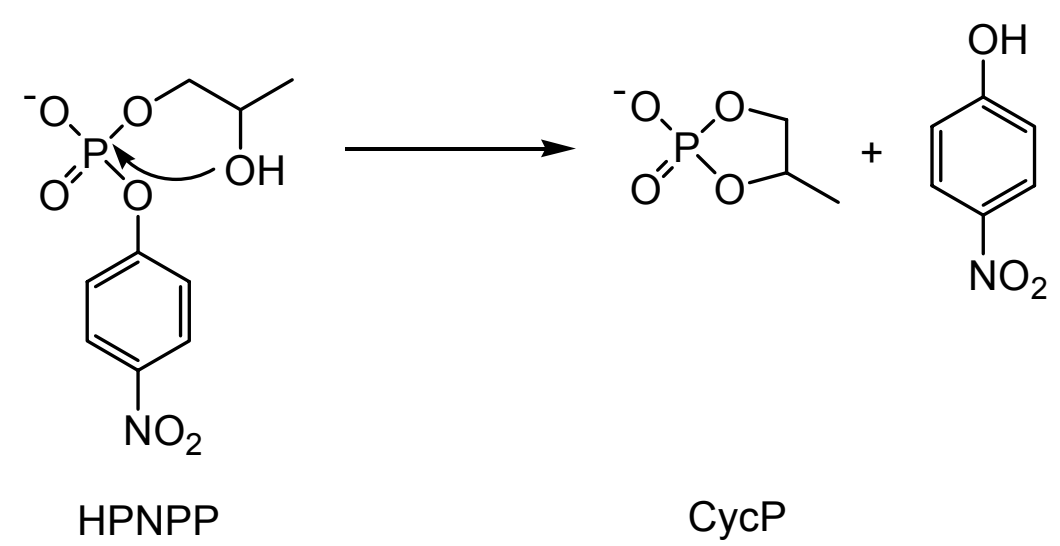

Abbildung 99: Intramolekulare Umesterung von HPNPP.

Der Verlauf der katalytischen Umsetzung von zehn Äquivalenten HPNPP mit $\mathbf{5}$ wurde mittels ${ }^{31} \mathrm{P}$-NMR-Spektroskopie bis zur Umsetzung von acht Äquivalenten verfolgt (Abbildung 100).

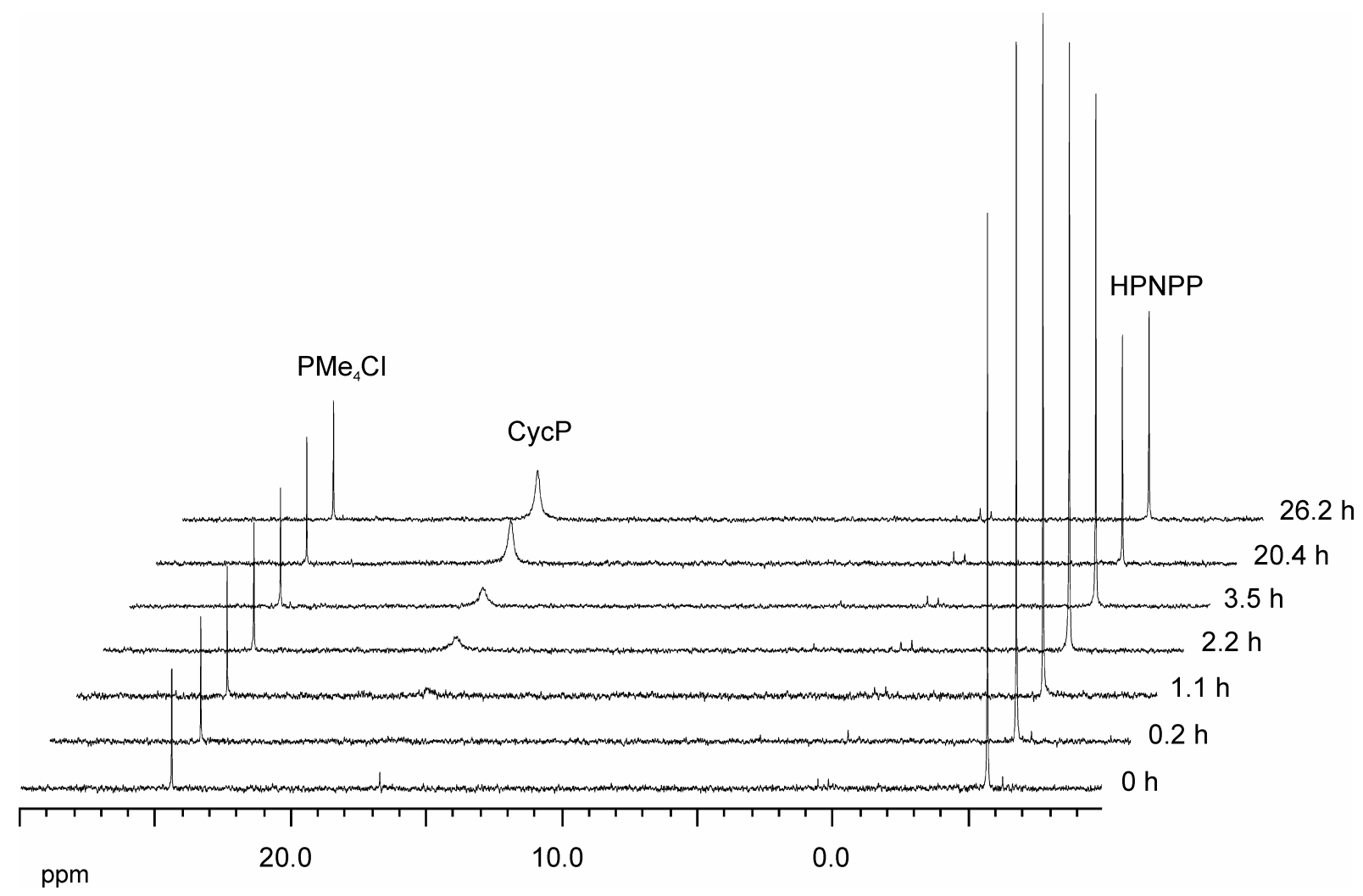

Abbildung 100: Beobachtete katalytische Umesterung von HPNPP durch 5 mittels ${ }^{31} P$-NMR-

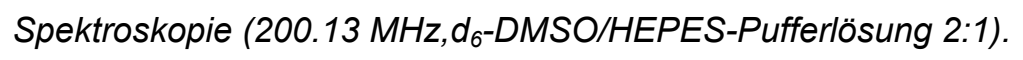

Da in dieser Reaktion das Nukleophil im Substrat selbst vorhanden ist und ein intramolekularer Angriff erfolgt, ist die Bildung eines metallgebundenen Hydroxids 
nicht mehr zwingend notwendig. Anhand dieses Substrats kann deshalb lediglich der Einfluss der Substrataktivierung ermittelt werden. Auf weitergehende, detaillierte kinetische Studien wurde deshalb in dieser Arbeit verzichtet. 


\section{6. $\beta$-Lactamase-Aktivität}

\subsection{Modellkomplexe für $\beta$-Lactam-Metallkomplexe und Dizink- Metallolactamasen}

Es ist bereits eine Reihe von Komplexen beschrieben, in denen ein $\beta$-Lactam an ein Metall koordiniert. Das einfachste $\beta$-Lactam, 2-Azetidinon ( $\beta$-Propiolactam) stellt dabei einen Sonderfall dar, da es bevorzugt Amidkomplexe bildet. So finden sich in Abbildung 101 Beispiele für kristallographisch charakterisierte, an Metallionen koordinierende Azetidinoneinheiten. ${ }^{[14-118]}$<smiles>O=C1CCN1N(CI)N1CCC1=O</smiles>

$$
M=P d, P t
$$$$
L=C O D, P R_{3}, C N R
$$

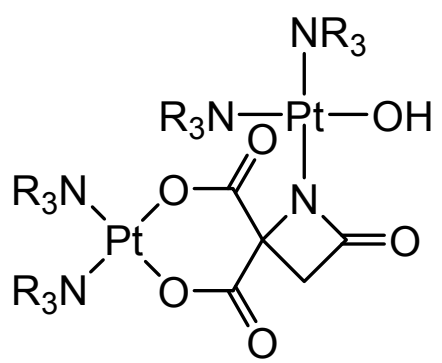

$\mathbf{X X X I}$<smiles>C[SiH3]C(=CC(=O)N1CCC1=O)[Ge](C)(C)C</smiles>

$\mathbf{X X X}$

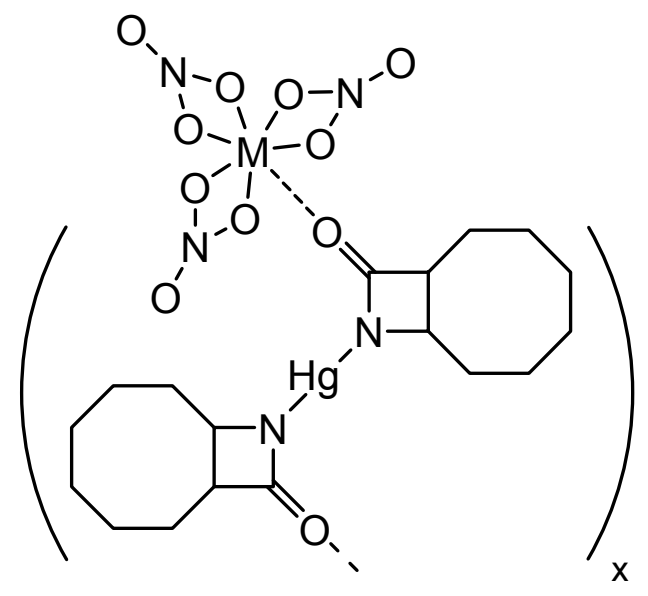

XXXIII $M=N d, x=4$

$\mathbf{X X X I V} M=E r, x=3$
XXXII

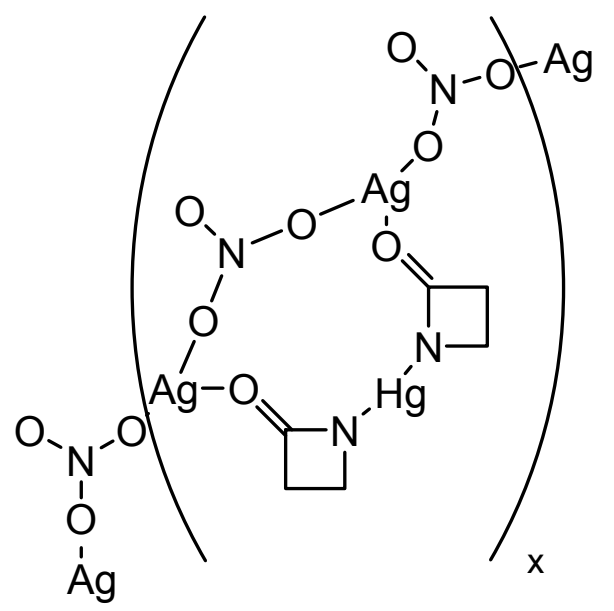

XXXV

Abbildung 101: Kristallographisch charakterisierte Metallkomplexe und Festkörperstrukturen von 2Azetidinon-Metallaggregaten. 
Höher substituierte oder bizyklische $\beta$-Lactam-Koordinationsverbindungen konnten bisher nicht durch Röntgenstrukturanalyse aufgeklärt werden. Die Komplexierung einfacher Metallsalze lassen aufgrund der geringen Änderungen der NMR- und IRspektroskopischen Daten selten Rückschlüsse auf die Koordinationsmodi zu. ${ }^{\text {[119-122] }}$ Im Fall der Komplexierung von Penicillin G und Penicillin V durch Platin(II) wurde anhand von IR- und NMR-spektroskopischen Daten die Bildung eines fünfgliedrigen Rings durch Koordination an die Amid- und an die Thioetherfunktion der Penicilline vorgeschlagen (XXXVI; Abbildung 102). ${ }^{[123]}$

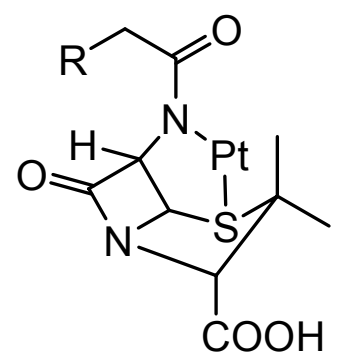

$$
\begin{aligned}
& \text { XXXVla } \mathrm{R}=\mathrm{C}_{6} \mathrm{H}_{5} \\
& \text { XXXVIb } \mathrm{R}=\mathrm{OC}_{6} \mathrm{H}_{5}
\end{aligned}
$$

Abbildung 102: Vorgeschlagene Koordination von Pt(II) an Penicillin G (XXXVla) und V (XXXVIb).

Die Geschwindigkeit der Hydrolyse von $\beta$-Lactamen wird durch eine Reihe von Übergangsmetallionen wie $\mathrm{Cu}^{2+}, \mathrm{Zn}^{2+}, \mathrm{Co}^{2+}$ und $\mathrm{Ni}^{2+}$ gesteigert. Der Mechanismus der Hydrolyse von Benzylpenicillin durch Metallionen wurde von Page et al. untersucht. ${ }^{[124-126]}$ Danach binden die Metallionen erst an die Carboxylatgruppe und den $\beta$ Lactamstickstoff unter Ausbildung der aktiven Spezies (Abbildung 103; XXXVII) und beeinflussen die Spaltung der C-N-Bindung durch eine Stabilisierung des tetraedrischen Übergangszustands (Abbildung 103; XXXVIII) für einen nukleophilen Angriff durch ein externes Hydroxid. So wurden auch eine Steigerung der Hydrolysegeschwindigkeit durch $\mathrm{OH}^{-}$und $\mathrm{Zn}^{2+}$ oder $\mathrm{Cu}^{2+}$ um $4 \cdot 10^{4}$ bzw. $8 \cdot 10^{7}$ beobachtet. ${ }^{[124,125]}$ Detailliertere kinetische Studien von Hay et al. führten zu dem Vorschlag, dass $\mathrm{Cu}^{2+}$ an das deprotonierte Amid von Benzylpenicillin (Abbildung 103; XXXIX) bindet, und die nachfolgende Hydrolyse über einen intramolekularen nukleophilen Angriff eines Metall-gebundenen Hydroxids auf die Carbonylfunktion verläuft. $^{[127]}$ Einen ähnlichen intramolekularen Angriff durch ein Metall-gebundenes Hydroxid schlagen Fife et al. für die Hydrolyse von N-(8-Chinolyl)-azetidin-2-on durch 
$\mathrm{Zn}^{2+}$ und $\mathrm{Ni}^{2+}$-lonen vor (Abbildung 103; XL). ${ }^{[128]}$ In all diesen Studien jedoch konnten eindeutige kinetische Ergebnisse nur schwierig erhalten werden, da in wässriger Lösung stets noch andere Spezies wie freies $\mathrm{M}^{2+}, \mathrm{M}^{2+}$-Lactam, $\mathrm{M}^{2+}-\mathrm{OH}$ etc. koexistieren.

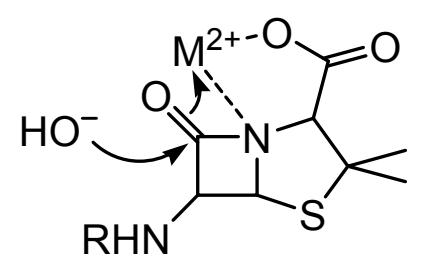

XXXVII

$(\mathrm{M}=\mathrm{Cu}, \mathrm{Zn}, \mathrm{Ni}, \mathrm{Co})$

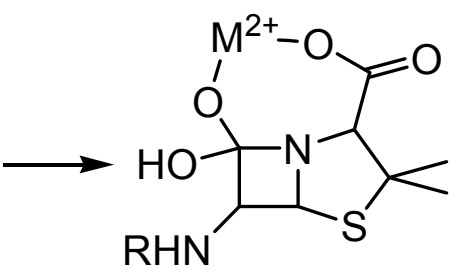

XXXVIII

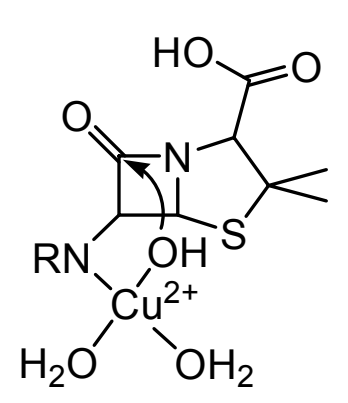

XXXIX

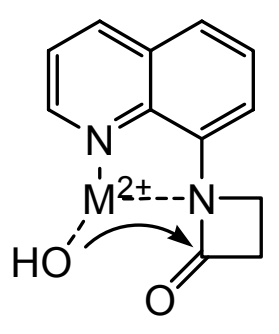

$\mathbf{X L}$

$$
\mathrm{M}=\mathrm{Ni}, \mathrm{Zn}
$$

Abbildung 103: Koordination und Spaltung von bizyklischen $\beta$-Lactamen (schematisch) durch Metallionen.

Um eindeutigere Ergebnisse zu erhalten, wurden deshalb mehrere Studien mit einkernigen Metallkomplexen chelatisierender Liganden durchgeführt. So konnten Ichikawa et al. die Spaltung von 2-Azetidinon zu $\beta$-Alanin durch die mononuklearen Zinkkomplexe XLI und XLII (Abbildung 104) beobachten. ${ }^{[129]}$ Die Spaltung des $\beta$ Lactamrings von Benzylpenicillin durch einen $\mathrm{Zn}$ (cyclen)(OH)-Komplex (XLIII; Abbildung 104) wurde von Kimura et al. untersucht. ${ }^{[130]}$ Die pH-Ahängigkeit der katalytisch ablaufenden Hydrolyse zeigt, dass vermutlich ein Zink-gebundenes Hydroxid die aktive Spezies in der Reaktion ist. 
<smiles></smiles>

XLI

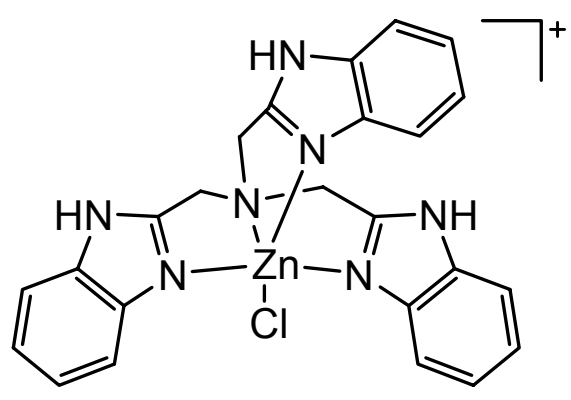

XLII

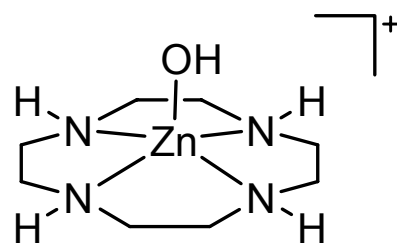

XLIII

Abbildung 104: Zur Spaltung von 2-Azetidinon eingesetzte mononukleare Zinkkomplexe.

Als Modellsysteme für dinukleare $\beta$-Lactamasen wurden bisher nur Dizinkkomplexe der in Abbildung 105 abgebildeten Liganden von Lippard et al. verwendet. ${ }^{[90,110,131]}$<smiles>c1ccc(CNCc2ccc3ccc(CNCc4ccccn4)nc3n2)nc1</smiles>

XLIV<smiles>CN(C)CCN(C)Cc1cccc(CN(C)C)c1[O-]</smiles>

XLV<smiles>CN(C)CCN(C)Cc1cccc(CN(C)C)c1[O-]</smiles>

XLVI

Abbildung 105: Von Lippard et al. verwendete Liganden für Dizinkkomplexe.

Diese Komplexe sind in der Lage, die Hydrolyse von Penicillin G und Nitrocefin zu katalysieren. Eine Analyse der Substratbindung an das Zinkzentrum durch ${ }^{13} \mathrm{C}-\mathrm{NMR}$ und IR-Spekroskopie zeigt, dass die Anbindung sowohl von Penicillin, als auch von Cephalothin anscheinend über die Carboxylatgruppe des Substrats erfolgt. Ein Vergleich der Hydrolysegeschwindigkeit der dinuklearen Komplexe mit den mononuklearen Zinkkomplexen $\mathrm{Zn}$ (cyclen) $\left(\mathrm{NO}_{3}\right)_{2}$ und $\mathrm{Zn}(\mathrm{bpta})\left(\mathrm{NO}_{3}\right)_{2}$ zeigt jedoch keinen Aktivitätsgewinn durch das zweite Zinkion. Jedoch konnte durch stoppedflow-Messungen der Hydrolyse von Nitrocefin die Bildung eines zinkgebundenen $\mathrm{N}$ deprotonierten hydrolysierten Nitrocefins beobachtet werden, ein Intermediat, das in ähnlicher Form beim Abbau von Nitrocefin durch $\beta$-Lactamasen entsteht. ${ }^{[57]}$ Die für dieses Intermediat vorgeschlagene Struktur ist in Abbildung 106 gezeigt. $^{[131]}$ 


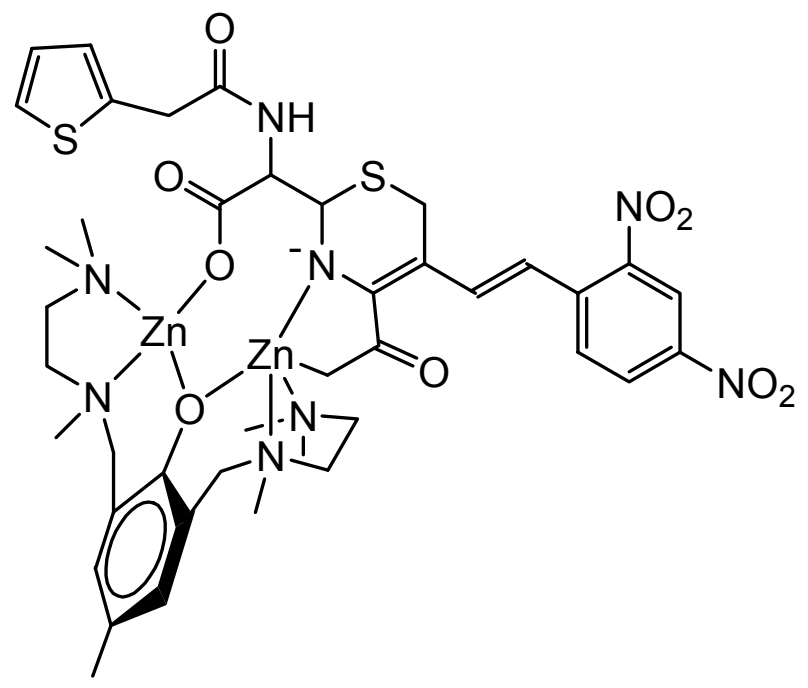

Abbildung 106: Postulierte Struktur des Intermediats der Hydrolyse von Nitrocefin.

\subsection{Verwendete $\beta$-Lactamsubstrate}

Zur Untersuchung der Hydrolyse von $\beta$-Lactamsubstraten durch die in Kapitel 4 vorgestellten Pyrazolatodizinkkomplexe wurde auf kommerziell erhältliche $\beta$-Lactame zurückgegriffen. Ausgewählt wurden chemisch möglichst einfach aufgebaute Substrate aus verschiedenen $\beta$-Lactamklassen (Abbildung 107): das $\beta$ Lactamantibiotikum Penicillin G (Pen), das Cephalosporin Cephalothin (Ceph) und das für Penicillinasen als Suizidinhibitor wirkende Sulbactam (Sul).<smiles>CC1(C)S[C@@H]2[C@H](NC(=O)Cc3ccccc3)C(=O)N2[C@H]1C(=O)[O-]</smiles>

Pen<smiles>CC(=O)OCC1=C([O-])[N+]2C(=O)[C@@H](NC(=O)Cc3cccs3)[C@H]2SC1</smiles>

Ceph<smiles>CC1(C)[C@H](C(=O)[O-])N2C(=O)C[C@H]2S1(=O)=O</smiles>

Sul

Abbildung 107: Bizyklische $\beta$-Lactam-Antibiotika: Penicillin G, Cephalothin, Sulbactam.

$\mathrm{Zu}$ einer gezielten Untersuchung der Wechselwirkungen zwischen den Dizinkkomplexen und $\beta$-Lactamen wurden zusätzlich die beiden einfachen $\beta$-Lactame N-Benzylazetidinon XLVII und Oxazetidinylacetat XLVIII synthetisiert. XLVIII beinhaltet das wesentliche Strukturelement bizyklischer $\beta$-Lactam-Antibiotika, besitzt aufgrund der monozyklischen Struktur aber eine höhere Flexibilität (Abbildung 108). 
<smiles>O=C1CCN1Cc1ccccc1</smiles>

XLVII<smiles>O=C([O-])CN1CCC1=O</smiles>

XLVIII<smiles>CC1(C)SC2C(NC(=O)Cc3ccccc3)C(=O)N2C1C(=O)[O-]</smiles>

Pen

Abbildung 108: Verwendete monozyklische $\beta$-Lactame im Vergleich mit Penicillin (rechts).

XLVII ist durch Umsetzen von N-Benzyl-3-aminopropionsäuremethylester mit $\mathrm{Sn}\left[\mathrm{N}\left(\mathrm{SiMe}_{3}\right)_{2}\right]_{2}{ }^{[132]}$ leicht zugänglich (Abbildung 109). ${ }^{[133]}$

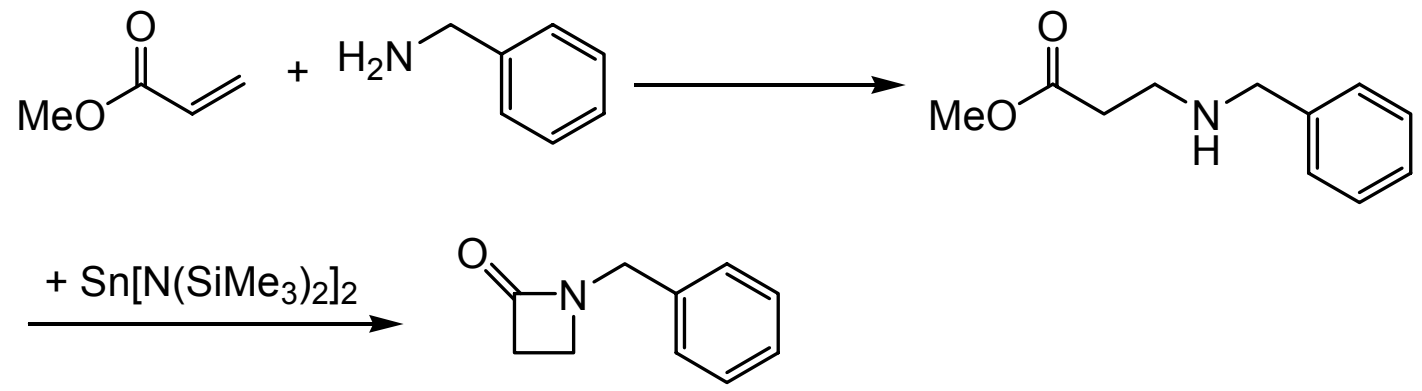

Abbildung 109: Synthese von N-Benzyl-azetidinon XLVII.

Nach literaturbekannter Vorschrift kann auch Natriumoxazetidinylacetat XLVIII in drei Stufen aus kommerziell erhältlichen Edukten synthetisiert werden. ${ }^{[134]}$ Der durch Umsetzen des 3-Brompropionsäurechlorids mit Glycinbenzylester erhaltene N-(3Bromopropionyl)glycinbenzylester wird in einer Phasentransferreaktion zyklisiert und die Benzylschutzgruppe im erhaltenen 2-(2-Oxoazetidin-1-yl)essigsäurebenzylester anschließend reduktiv abgespalten (Abbildung 110). Eine Synthese durch Entschützen des analogen, leicht zugänglichen 2-(2-Oxoazetidin-1-yl)essigsäure-tertbutylesters unter sauren Bedingungen führte stets zur Ringöffnung. 
<smiles>NCC(=O)OCc1ccccc1</smiles><smiles>O=C(CCBr)NCC(=O)OCc1cccc(O)c1</smiles><smiles>CCOCCNC(=O)CC1CCN1CC(=O)O</smiles>

Abbildung 110: Synthese von Natrium-2-oxoazetidin-1-ylacetat XLVIII.

\subsection{Kinetische Untersuchungen der $\beta$-Lactamhydrolyse durch IR- Spektroskopie}

Schwingungsspektroskopisch ist die Position der intensiven $\mathrm{C}=\mathrm{O}-$ Valenzschwingung in $\beta$-Lactamen gegenüber den Banden anderer Carbonyle wie Estern, Amiden und Säuren stark nach höheren Wellenzahlen hin verschoben. Ihre isolierte Lage macht eine quantitative Auswertung von IR-Spektren möglich. Die quantitative Auswertung der Intensität der $\beta$-Lactambande und damit der Konzentration des $\beta$-Lactams kann sowohl über die Höhe als auch über die Fläche der Bande bei der Auftragung von Extinktion gegen Wellenzahl erfolgen, da sich beide im verwendeten Konzentrationsbereich linear zur Konzentration des Substrats verhalten. Aufgrund der geringen absoluten Intensitäten zum Ende der Reaktion und den dadurch resultierenden großen Halbwertsbreiten ist aber der integralen Auswertung der Vorzug zu geben, da auftretendes Rauschen durch die Integration gemittelt wird.

\section{In ungepufferten wässrigen Lösungen}

Die synthetisierten Dizinkkomplexe 1, 2b, 3, 4 und 5 wurden in DMSO/Wasser 9:1 (v/v) mit vier Äquivalenten Penicillin $G$ bei Raumtemperatur umgesetzt. In Kontrollversuchen wurden anstelle der Dizinkkomplexe sowohl ein Äquivalent Base $\left(\mathrm{KO}{ }^{t} \mathrm{Bu}\right)$ als auch ein Äquivalent eines $\mathrm{Zn}(\mathrm{II})$-Salzes $\left(\mathrm{Zn}\left(\mathrm{NO}_{3}\right)_{2} \cdot 6 \mathrm{H}_{2} \mathrm{O}\right.$ oder $\left.\mathrm{Zn}\left(\mathrm{ClO}_{4}\right)_{2} \cdot 6 \mathrm{H}_{2} \mathrm{O}\right)$ eingesetzt. Anhand der Abnahme der Intensität der $\beta$-Lactambande bei $1768 \mathrm{~cm}^{-1}$ konnte die Reaktion mittels IR-Spektroskopie verfolgt werden. 


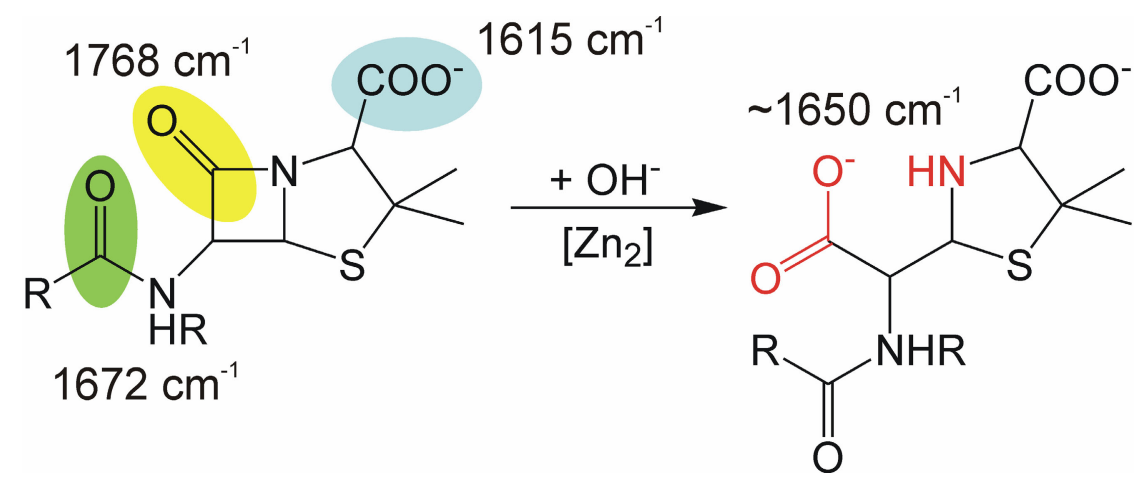

Abbildung 111: IR-Banden der Carbonylgruppen bei der Hydrolyse von Penicillin G.

Eine wesentliche Änderung der Lage der Amidbande bei $1672 \mathrm{~cm}^{-1}$ sowie der Lage der Carboxylatbande bei $1615 \mathrm{~cm}^{-1}$ konnte im Verlauf der Reaktion nicht beobachtet werden. Letztere wird jedoch durch das Auftreten einer neuen Bande bei etwa 1650 $\mathrm{cm}^{-1}$ teilweise überlagert. Diese neu auftretende Bande geht einher mit der Abnahme der Intensität der $\beta$-Lactambande bei $1768 \mathrm{~cm}^{-1}$, also der Spaltung des Lactamrings. Es handelt sich demzufolge um die Bande der aus der Hydrolyse resultierenden Carboxylatgruppe.

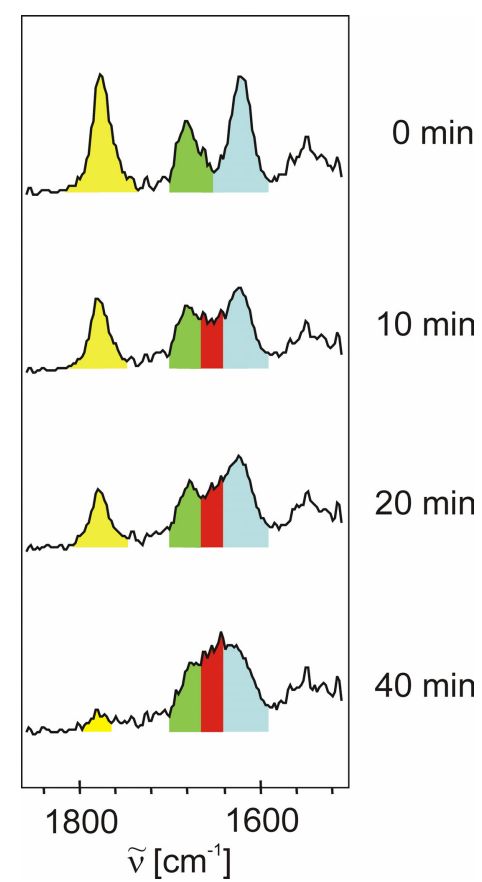

Abbildung 112: Ausschnitt aus den IR-Spektren während der Hydrolyse von Penicillin G durch 1.

Die zeitlichen Verläufe der relativen Konzentrationen an Penicillin gegenüber den eingesetzten Komplexen sind in Abbildung 113 dargestellt. 


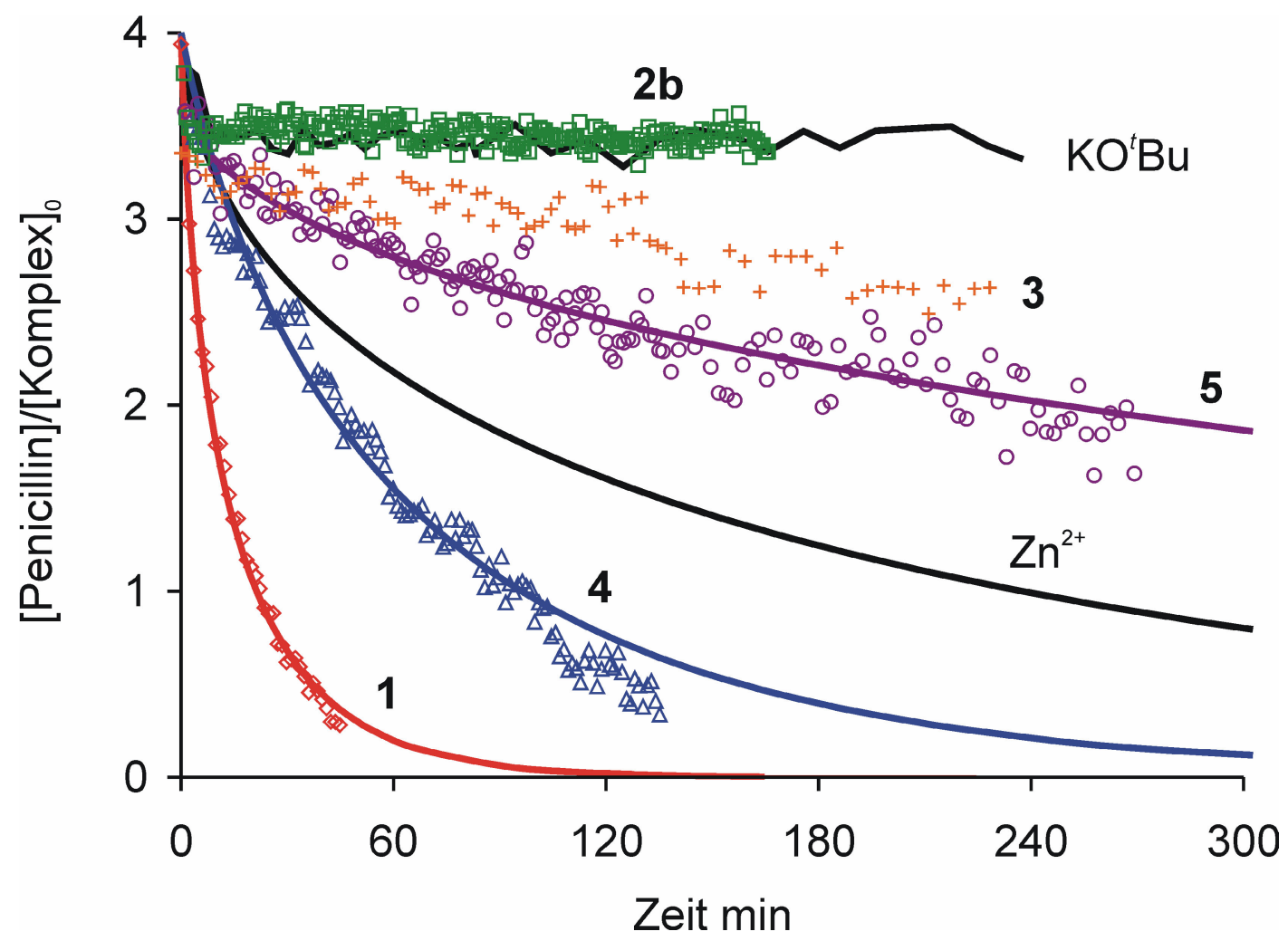

Abbildung 113: Hydrolyse von Penicillin $G$ durch 1, 2b, 3, 4 und 5, sowie durch $\mathrm{Zn}\left(\mathrm{ClO}_{4}\right)_{2}$ und $\mathrm{KO} \mathrm{B}^{t} \mathrm{Bu}$ in $D M S O / H_{2} \mathrm{O}$ 9:1 (v/V) bei RT; $[\text { Komplex }]_{0}$ bzw. $\left[\mathrm{Zn}^{2+}\right]_{0}=10.7 \mathrm{mM}$, $[\text { Penicillin }]_{0}=42.8 \mathrm{mM}$.

Aus diesem Diagramm lassen sich folgende Schlüsse ziehen:

1) Die Geschwindigkeit der Hydrolyse folgt der Reihe $\mathrm{KO}^{t} \mathrm{Bu}<2 \mathrm{~b}<3<5<\mathrm{Zn}^{2+}<4<1$.

2) Alle Dizinkkomplexe hydrolysieren Penicillin $G$ schneller als ein Äquivalent einer Base. Ein Mechanismus, in dem als einziger Faktor die HydroxidionenKonzentration von Bedeutung ist, kann demnach ausgeschlossen werden.

3) Im Fall von 5, 4 und $\mathbf{1}$ wird Penicillin katalytisch abgebaut; im Fall von $\mathbf{2 b}$ und $\mathbf{3}$ verläuft die Spaltung des $\beta$-Lactamrings relativ langsam, eine katalytische Umsetzung wurde im untersuchten Zeitfenster nicht beobachtet.

4) Einfache $\mathrm{Zn}(\mathrm{II})$-Salze weisen bereits eine relativ hohe Aktivität auf, jedoch wird Penicillin $\mathbf{G}$ durch 1 und $\mathbf{4}$ noch deutlich schneller hydrolysiert.

Der zeitliche Verlauf des katalytischen Abbaus von Penicillin G durch 1, 4 und 5 kann durch eine Michaelis-Menten-Kinetik beschrieben werden. Dazu wurde die integrierte Form des Michaelis-Menten-Gesetzes verwendet (Gleichung 6). ${ }^{[109]}$ 


$$
\begin{gathered}
\frac{[\mathrm{S}]_{0}-[\mathrm{S}]}{\mathrm{t}}=v-K_{\mathrm{M}} \frac{\ln [\mathrm{S}]_{0}-\ln [\mathrm{S}]}{\mathrm{t}} \\
\quad \mathrm{mit} \\
v=k_{\text {cat }} \cdot[\text { Komplex }]
\end{gathered}
$$

\section{Gleichung 6}

Die erhaltenen Werte sind in Tabelle 21 gezeigt.

\begin{tabular}{c|lc} 
Komplex & \multicolumn{1}{|c}{$K_{\mathrm{M}} \mathrm{mM}$} & $k_{\mathrm{cat}} 10^{-3} \mathrm{~min}^{-1}$ \\
\hline $\mathbf{1}$ & 54.4 & 191 \\
$\mathbf{2 b}$ & - & - \\
$\mathbf{4}$ & 74.7 & 69.8 \\
$\mathbf{5}$ & 40.3 & 2.49
\end{tabular}

Tabelle 21: Kinetische Parameter nach der Michaelis-Menten-Beziehung für die Hydrolyse von Penicillin $\mathrm{G}$ in $\mathrm{DMSO} / \mathrm{H}_{2} \mathrm{O}$ 9:1.

Versuche, Cephalothin mit den in dieser Arbeit dargestellten Dizinkkomplexen zu hydrolysieren zeigten, dass dieses Substrat über Stunden hinweg unverändert bleibt und nicht gespalten wird. Dies entspricht der auch medizinisch beobachteten, höheren Stabilität von Cephalothinen verglichen mit Penicillinen gegenüber $\beta$ Lactamasen.

\section{In gepufferten wässrigen Lösungen}

Für die Untersuchungen der pH-Abhängigkeit der Hydrolyse wurden TRISPufferlösungen in einem Lösungsmittelgemisch DMSO/Wasser 8:2 (v/v) bei $\mathrm{pH}$ Werten von 7.32, 7.83 und 8.47 verwendet. Im Fall der Komplexe 1 und $\mathbf{4}$ beobachtet man im IR-Bereich jedoch innerhalb weniger Minuten nach Zugabe des Komplexes zur Penicillin-Lösung eine starke Abnahme der $\beta$-Lactambande bei $1770 \mathrm{~cm}^{-1}$ und das Auftreten einer neuen, breiten Bande bei $1749 \mathrm{~cm}^{-1}$, deren Intensität sich im weiteren Verlauf verringert. 


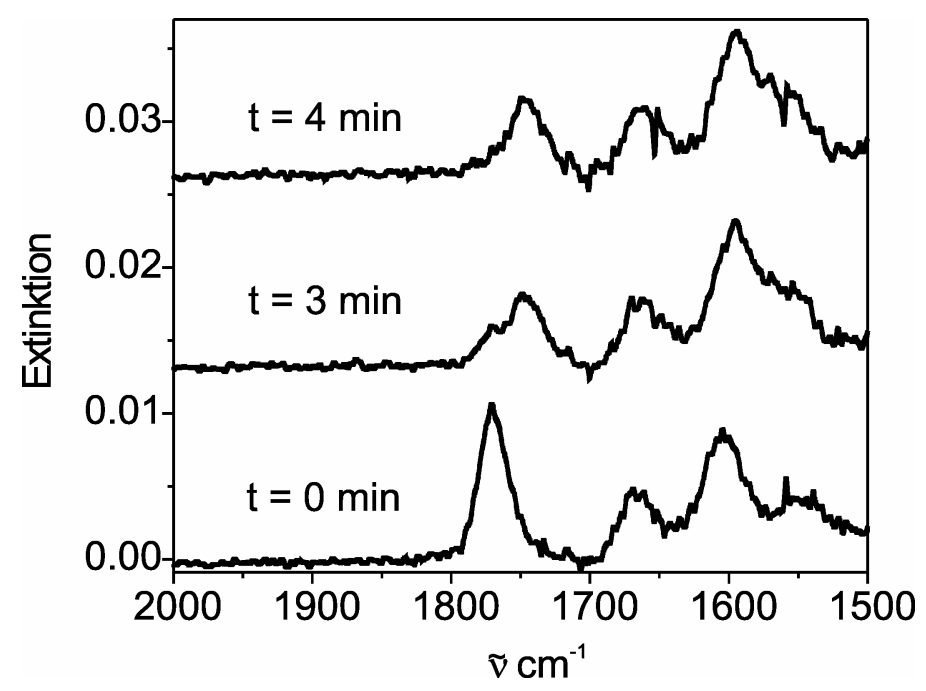

Abbildung 114: Carbonylbereich der IR-Spektren nach Zugabe von 4 zu vier Äquivalenten Penicillin in $\mathrm{DMSO} / \mathrm{H}_{2} \mathrm{O}$ 8:2 (v:v) bei $\mathrm{pH} 7.32$.

Dies lässt vermuten, dass die neu auftretende Bande einem koordinierten $\beta$-Lactam zuzuordnen ist. Dies würde man eigentlich nicht erwarten, da die erhöhte Wasserkonzentration im Vergleich zum ungepufferten System der Koordination der Lactamcabonylfunktion entgegenwirkt. Ein Vergleich mit Literaturwerten (Tabelle 22) für an Metalle koordinierte $\beta$-Lactame zeigt jedoch, dass eine Verschiebung zu niedrigeren Wellenzahlen um ca. $30-50 \mathrm{~cm}^{-1}$ typisch für eine solche Koordination ist. So wurde in einem Penicillin-Eisen(II)-Komplex eine IR-Bande von $1739 \mathrm{~cm}^{-1}$ beobachtet.

\begin{tabular}{l|llll} 
& $\widetilde{v}(\mathrm{CO})$ Lactam & $\widetilde{v}_{\text {as }}(\mathrm{COO})$ & $\widetilde{v}_{s}(\mathrm{COO})$ & Lit. \\
\hline PenNa & 1768 & 1622 & 1419 & \\
$\mathrm{Fe}(\mathrm{Pen})_{2}$ & 1739 & 1625 & 1411 & ${ }^{[121]}$ \\
CephNa & 1730 & 1620 & 1400 & \\
$\mathrm{Zn}($ Ceph)(Imid)Cl & 1680 & 1580 & 1380 & [120]
\end{tabular}

Tabelle 22: IR-Absorptionen für einige mononukleare Penicillin und Cephalothin-Metallkomplexe.

Die ungleichmäßige Abnahme der $\beta$-Lactambande zu Anfang der Hydrolyse lässt eine kinetische Auswertung der gemessenen Daten nicht zu, so dass nur ein qualitativer Vergleich zwischen den Komplexen erfolgen kann. 
Messungen der Hintergrundhydrolyse ergaben, dass sich Penicillin $G$ allein in gepufferter Lösung bei pH 7.32 und 7.83 nicht zersetzt, bei $\mathrm{pH} 8.47$ jedoch eine leichte Hydrolyse festzustellen ist.

Setzt man die Komplexe zu, so bewirkt im Fall von Komplex 1 eine Erhöhung des $\mathrm{pH}-$ Werts eine Zunahme der Reaktionsgeschwindigkeit.

Dies steht im Einklang mit der Formulierung eines metallgebundenen oder freien Hydroxids als Nukleophil. Aufgrund der starken Erhöhung gegenüber der unkatalysierten Reaktion auch bei den niedrigen $\mathrm{pH}$-Werten ist anzunehmen, dass ein metallgebundenes Hydroxid vorliegt, da die Konzentration von freiem Hydroxid in der Lösung relativ gering ist und auch der Zusatz einer Base fast keine Auswirkung auf die Hydrolysegeschwindigkeit zeigt. Es ist jedoch nicht auszuschließen, dass die Hydrolyse nur durch eine starke Aktivierung des Substrats und durch einen anschließenden intermolekularen Angriff eines Hydroxids verursacht wird.

Außerdem beobachtet man in gepufferter Lösung die gleiche Reaktivitätsreihe nach der anfänglichen Hydrolyse wie in ungepufferter Lösung, d. h. eine Abnahme der Komplexstabilität bewirkt eine Zunahme der Reaktivität. Dies kann damit erklärt werden, dass es für die Reaktion notwendig ist, durch die Dissoziation eines Donors eine freie Koordinationsstelle zu schaffen. Diese Dissoziation sollte aufgrund der thermodynamischen Stabilitäten in der Reihenfolge 6-Ring-Chelat > 5-Ring-Chelat, sowie Amin-Donor > Pyridin-Donor erfolgen: Eine Reihenfolge, wie sie auch experimentell bestätigt werden konnte.

Betrachtet man die Art der Abnahme der Bande in gepufferter Lösung, so fällt auf, dass bei der Hydrolyse durch 4 eine lineare Abnahme der Substratkonzentration zu beobachten ist, wohingegen sie bei 1 exponentiell erfolgt (Abbildung 115). 

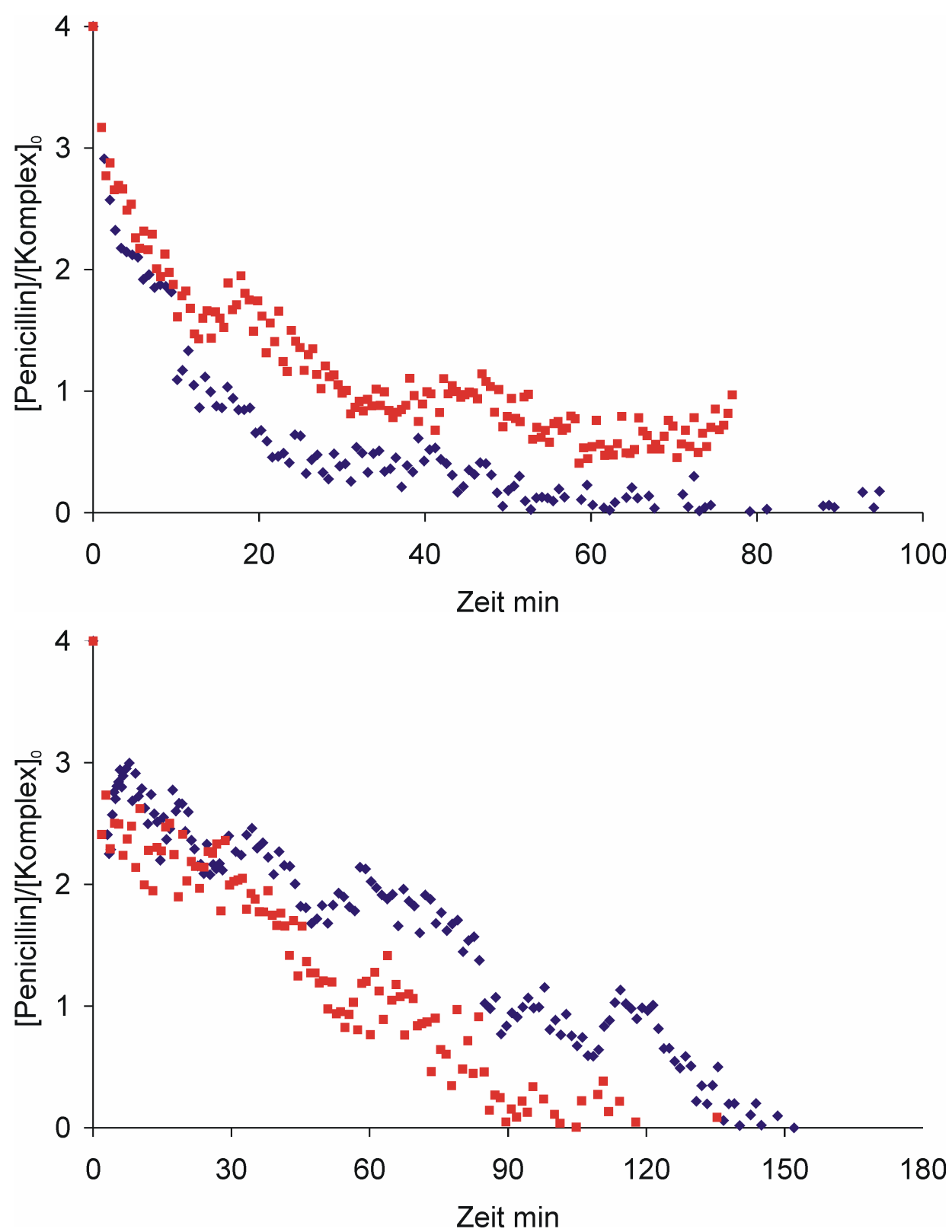

Abbildung 115: Hydrolyse von Penicillin G durch 1 und 4 in $D M S O / H_{2} \mathrm{O}$ 8:2 (v:v) bei pH 7.32 (blau; $\bullet$ ) oder pH 7.83 (rot, घ); [Komplex $]_{0}=10.7 \mathrm{mM}$, [Penicillin $]_{0}=42.8 \mathrm{mM}$.

Bei 4 ist demnach die Spaltung oder Dissoziation des Produkts geschwindigkeitsbestimmend. Das Substrat wird in einem vorgelagerten Gleichgewicht stark gebunden, so dass eine Sättigung des reaktiven Zentrums auftritt. Das gebundene $\beta$ Lactamsubstrat wird dann in einer geschwindigkeitsbestimmenden intramolekularen Reaktion nullter Ordnung bezüglich des Substrats gespalten, so dass eine lineare Abnahme der Lactambande auch zu erwarten ist. 
Die für die Hydrolyse durch 1 gefundene exponentielle Abnahme der Reaktionsgeschwindigkeit zeigt, dass die Bindung des $\beta$-Lactam-Substrats der geschwindigkeitsbestimmende Faktor ist.

\subsection{Untersuchungen zur Bindungssituation von $\beta$-Lactamen an Dizinkkomplexe}

\subsubsection{ESI-Massenspektrometrie}

Um weitergehende Aussagen zum Mechanismus der Reaktion und über die Ursache der Reaktivitätsunterschiede zu treffen, wurde die Anbindung von $\beta$-Lactamsubstraten an die Pyrazolatodizinkkomplexe näher untersucht.

Eine Anbindung der Substrate an die Dizinkzentren sollte sich am einfachsten durch die massenspektrometrische Beobachtung von Addukten bestätigen lassen. $\mathrm{Zu}$ diesem Zweck wurde eine Reihe von Experimenten durchgeführt, in denen ein Äquivalent eines $\beta$-Lactams (Lac) zu einer Lösung des jeweiligen Komplexes in Methanol gegeben wird und im ESI-MS gemessen wird. Man erhält dabei dominante Signale für [L.HZn2(Lac)(OMe)] ${ }^{+}$(Abbildung 116). Signale dieser Zusammensetzung konnten trotz stark schwankender Intensitäten für jede beliebige Kombination von Komplex und Lactam erhalten werden; Beispiele sind in Tabelle 23 aufgeführt.

\begin{tabular}{|l|c|c|c|c|c|c|c|c|c|}
\hline Ligand & $\mathrm{L}^{1}$ & $\mathrm{~L}^{1}$ & $\mathrm{~L}^{3}$ & $\mathrm{~L}^{4}$ & $\mathrm{~L}^{5}$ & $\mathrm{~L}^{6}$ & $\mathrm{~L}^{6}$ & $\mathrm{~L}^{8}$ & $\mathrm{~L}^{6}$ \\
\hline Lactam & Sul & Ceph & Ceph & Sul & Pen & Pen & Ceph & Ceph & Sul \\
\hline $\begin{array}{l}m / z\left(\left[\mathrm{~L}_{-H} \mathrm{Zn}_{2}\right.\right. \\
\left.(\mathrm{Lac})(\mathrm{OMe})]^{+}\right)\end{array}$ & 856 & 1019 & 1075 & 686 & 981 & 1037 & 1099 & 1043 & 936 \\
\hline
\end{tabular}

Tabelle 23: Beispiele einiger gefundener Signale der Zusammensetzung ([L $\left.\left.L_{-H} Z n_{2}(\operatorname{Lac})(\mathrm{OMe})\right]^{+}\right)$. 


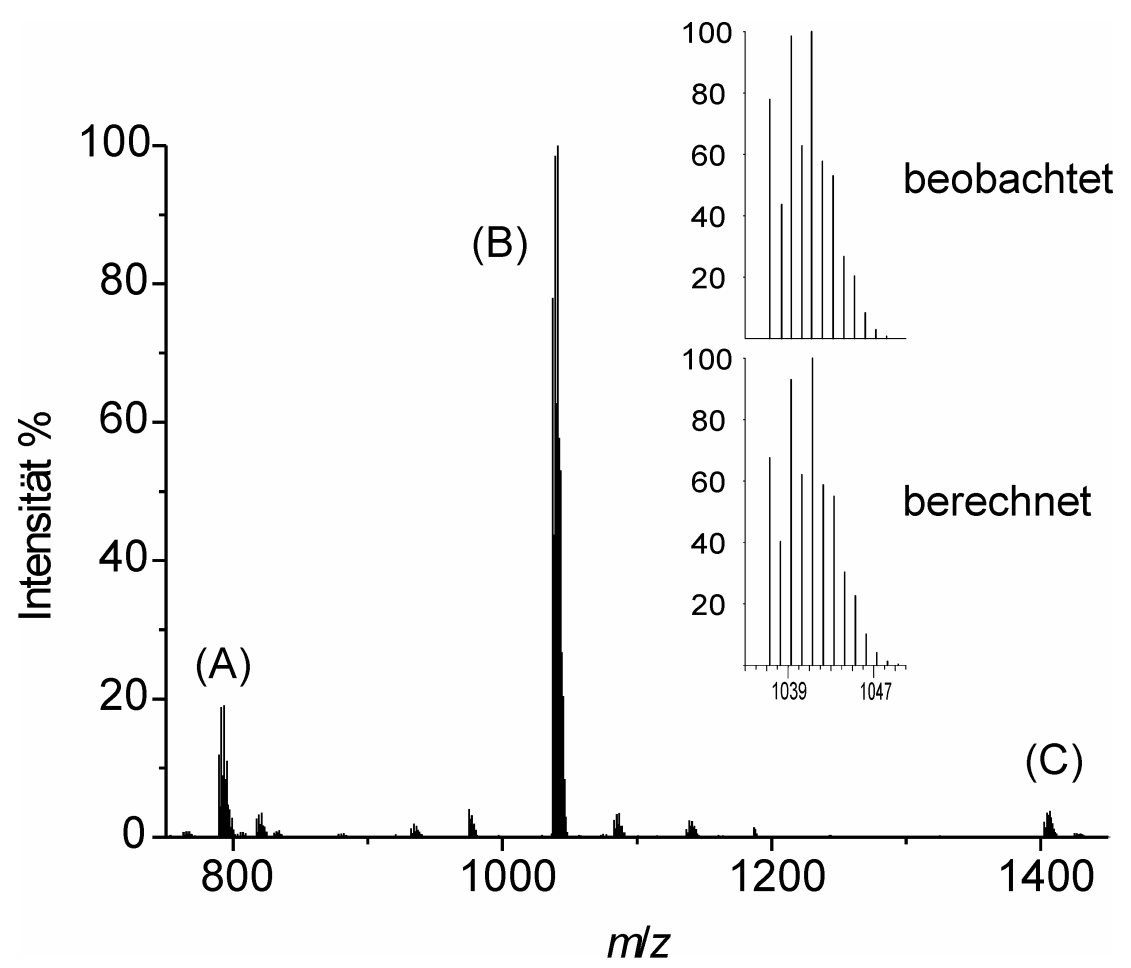

Abbildung 116: Im ESI-MS beobachtete Signale.
(A) $\left[\mathrm{L}^{6}{ }_{-\mathrm{H}} \mathrm{Zn} \mathrm{n}_{2}(\mathrm{OH})\left(\mathrm{ClO}{ }_{4}\right)\right]^{+}$
(B) $\left[L^{6}{ }_{-H} Z n_{2} \operatorname{Pen}(\mathrm{OMe})\right]^{+}$
(C) $\left[L^{6}{ }_{-H} \mathrm{Zn} n_{2}(\mathrm{Pen})_{2}(\mathrm{MeOH})_{2}\right]^{+}$

Außer diesem meist dominanten Signal finden sich oft noch weitere Signale, die sich Spezies $\left[\mathrm{L}_{-H} \mathrm{Zn}_{2}(\mathrm{Lac}) \mathrm{X}\right]^{+}$zuordnen lassen, wobei $\mathrm{X}$ dem verwendeten Anion wie Perchlorat oder einem weiteren Lactammolekül entspricht.

Über die Struktur der Spezies, speziell der Bindung des OMe-Fragments, lassen sich jedoch nur Vermutungen anstellen. So ist die Bindung an ein Zinkion unter Dissoziation eines Donorarms (Abbildung 117 oben rechts), eine verbrückende Position (Abbildung 117 oben links) oder eine rein elektrostatische Anziehung (Abbildung 117 unten) denkbar. 

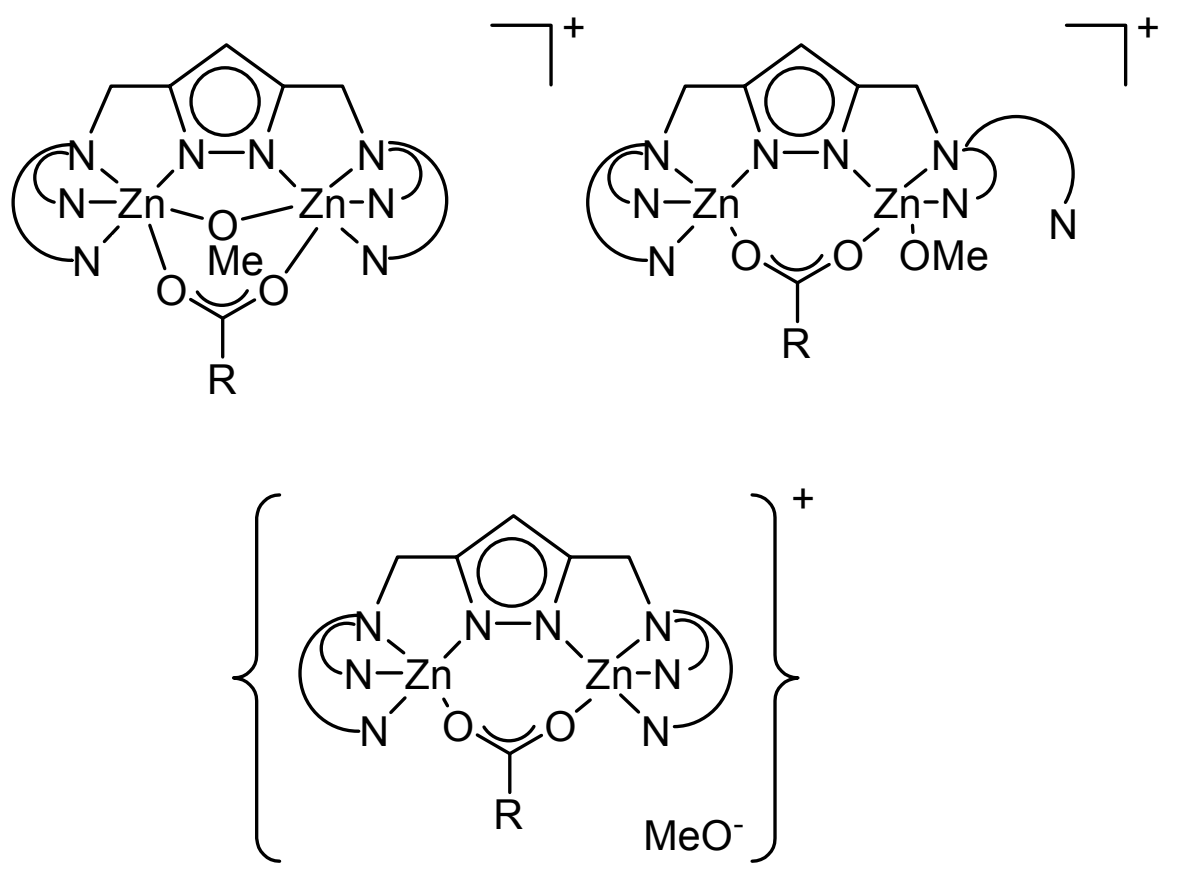

Abbildung 117: Möglichkeiten der Assoziation des OMe-Fragments an ein Carboxylat-verbrücktes Dizinkzentrum.

Die Möglichkeit der Methanolyse des Lactams unter Bildung eines Methylesters ist, obwohl man sie durch massenspektroskopische Methoden nicht ausschließen kann, relativ unwahrscheinlich, denn die Zeitspanne von der Zugabe der Komplexe zu den Antibiotika bis zur Messung beträgt höchstens eine Minute. Eine derart schnelle Hydrolyse des Lactamrings ist jedoch aufgrund der beobachteten Geschwindigkeiten in Abbildung 113 nicht anzunehmen. Zusätzlich beobachtet man auch beim schwer zu hydrolysierenden Cephalothin analoge Signale. Cephalothin konnte, wie bereits beschrieben, nicht durch die Dizinkkomplexe hydrolysiert werden.

Schließlich sind in den meisten Spektren weitere Spezies der Zusammensetzung $\left[\mathrm{L}_{-} \mathrm{Z} \mathrm{n}_{2}(\mathrm{Lac})_{2}\right]^{+} \mathrm{zu}$ beobachten: Spezies, in denen aufgrund der Zusammensetzung und Ladung unhydrolysierte Lactamringe vorliegen müssen, jedoch auch die gesamte Zweitbrücke des ursprünglich eingesetzten Dizinkkomplexes durch ein $\beta$ Lactam ausgetauscht wurde.

All diese Argumente sprechen für einen schnellen Austausch der Zweitbrücke durch die verwendeten $\beta$-Lactame; am plausibelsten erscheint deshalb die Koordination über die Carboxylatgruppe der eingesetzten $\beta$-Lactame, da durch die elektrostatische Anziehung zwischen negativ geladenem Substrat und positiv geladenem Dizinkzentrum eine starke Wechselwirkung zu erwarten ist. 


\subsubsection{NMR-Spektroskopie}

Die Hydrolyse von Penicillin $G$ mit den Komplexen 1, 3, 4 und 5 wurde auch mittels ${ }^{1} \mathrm{H}-\mathrm{NMR}$ - und ${ }^{13} \mathrm{C}-\mathrm{NMR}$-Spektroskopie verfolgt. Dabei lässt sich anhand der Abnahme der Signale des $\beta$-Lactams von Penicillin $G$ der Abbau des Edukts verfolgen. Im Verlauf der Hydrolyse von Penicillin $G$ entstehen neben dem primären Abbauprodukt stets auch einige weitere Produkte. ${ }^{[135-138]}$

Als besonders geeignet für die NMR-spektroskopische Beobachtung von Vorgängen an der Dizinkeinheit erwiesen sich die Pyridylkomplexe $\mathbf{4}$ und 5. Die isolierte Position der Pyridinsignale im ${ }^{1} \mathrm{H}-\mathrm{NMR}$ - und im ${ }^{13} \mathrm{C}$-NMR-Spektrum, vor allem die durch die Komplexbildung stark beeinflussten Signale in o- und p-Stellung zum Stickstoffatom, machen sie zu geeigneten Sonden für die Beobachtung von Prozessen am Metallzentrum.

In Abbildung 118 ist der Tieffeldbereich, in dem die Pyridylsignale zu finden sind, für den freien Liganden $L^{6}$ (oben), für den Dizinkkomplex 4 (unten) und für eine äquimolare Mischung von $\mathbf{4}$ und Penicillin $\mathrm{G}$ (Mitte) dargestellt. Man erkennt während der Reaktion deutlich eine Hochfeldverschiebung des sich zum Donor-N-Atom in ortho-Position befindenden $\mathrm{H}^{6}$-Pyridinsignals von $9.1 \mathrm{ppm}$ auf 8.8/8.5 ppm und eine starke Verbreiterung der Signale. Auch die zum N-Donoratom para-ständigen H-Atome zeigen ein analoges Verhalten (von 8.1 ppm auf 8.0 - 7.6 ppm). Dies lässt auf einen stark dynamischen Prozess schließen, in dem ein oder mehrere Seitenarme vom Zink abdissoziieren und so in ihren chemischen Verschiebungen dem freien Liganden ähneln sollten. Eine auf diese Weise geschaffene freie Koordinationsstelle kann zur weiteren Aktivierung des Substrats durch Koordination der Lactamcarbonylgruppe oder zur Anbindung eines Nukleophils genutzt werden. So ist parallel zur Anbindung auch die Abnahme der $\beta$-Lactamsignale, also die Ringöffnung zu beobachten. 


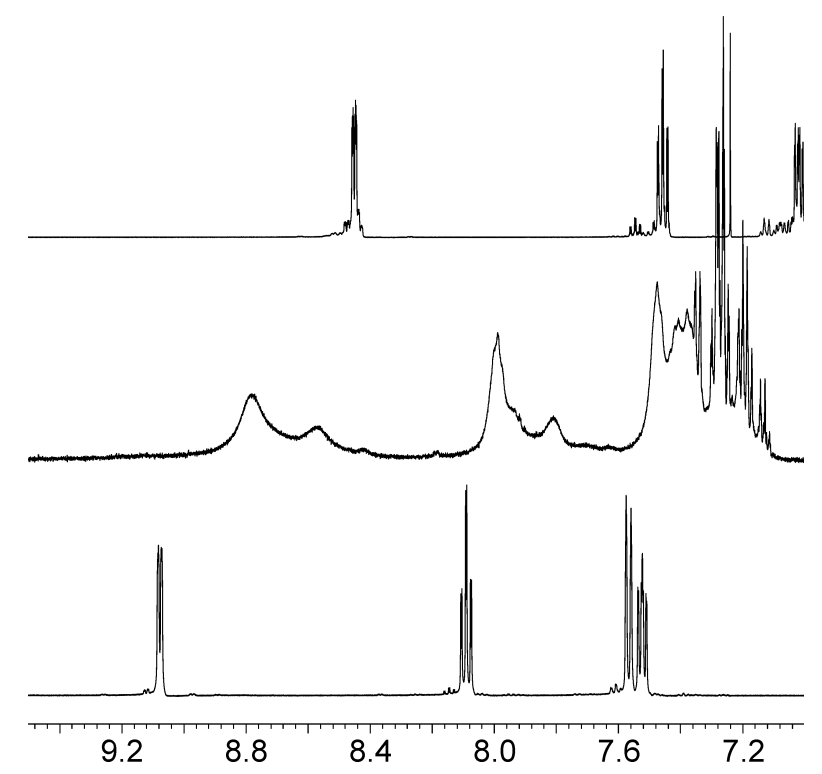

Abbildung 118: Ausschnitt aus den ${ }^{1} H$-NMR-Spektren von $L^{6}$ (oben), 4 (unten) und für eine äquimolare Mischung von 4 und Penicillin $\mathrm{G}$ (Mitte) $\left(500.13 \mathrm{MHz}, 300 \mathrm{~K}, d_{6}\right.$-Aceton/ $\mathrm{D}_{2} \mathrm{O}$ 8:1).

Eine derartige Dissoziation sollte für ein auf $L^{8}$ basierendes System nicht auftreten. Der Verlauf der analogen Reaktion von $\mathbf{5}$ mit Penicillin $\mathbf{G}$ ist in Abbildung 119 dargestellt.

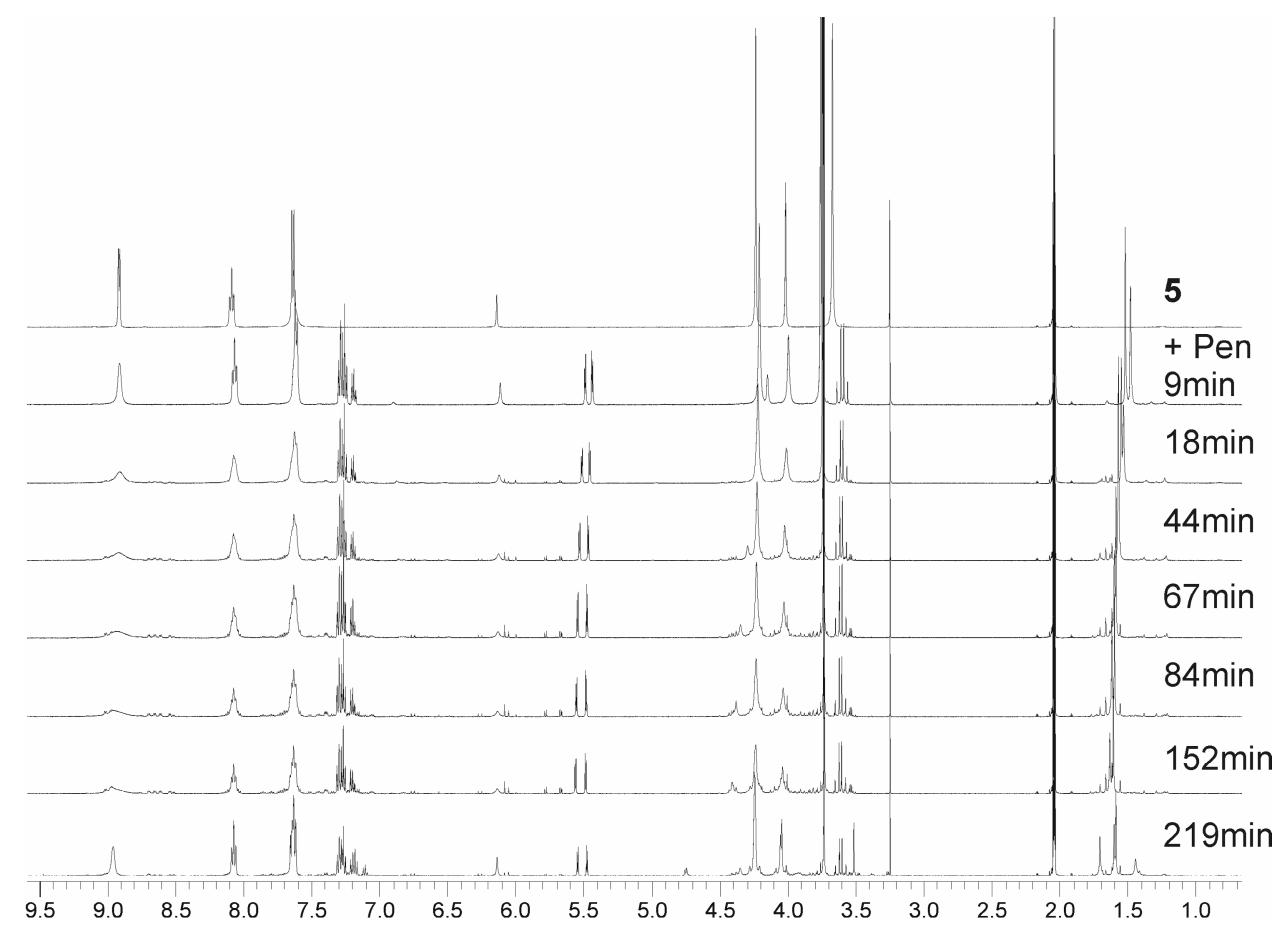

Abbildung 119: Umsetzung von 5 mit Penicillin $G\left({ }^{1} H-N M R, 500.13 \mathrm{MHz}, 300 \mathrm{~K}, d_{6}-\right.$ Aceton/ $_{2} \mathrm{O}$ 8:1). 
Im Unterschied zu Abbildung 118 zeigen sich hier keine drastischen Veränderungen im Tieffeldbereich des ${ }^{1} \mathrm{H}-\mathrm{NMR}$-Spektrums. Die Positionen der Pyridylsignale zeigen fast keine Veränderung nach der Zugabe von Penicillin G. Die im Verlauf der Reaktion breiter und wieder scharf werdenden Linien deuten aber auf dynamische Prozesse während der Reaktion hin. Aufgrund der geringen Veränderungen der chemischen Verschiebungen ist anzunehmen, dass die Pyridylliganden in diesem Komplex während der Reaktion überwiegend gebunden bleiben. Bei genauerer Betrachtung zeigen sich zusätzlich noch mehrere vergleichsweise kleine Signale mit identischer Intensität im Bereich um die Hauptsignale der Pyridinringe (Abbildung 120; z.B. 8.7 - 8.5 ppm). Dies lässt sich durch die Anbindung des chiralen Substrats erklären, da nun alle vier Pyridylringe nicht mehr äquivalent sind.

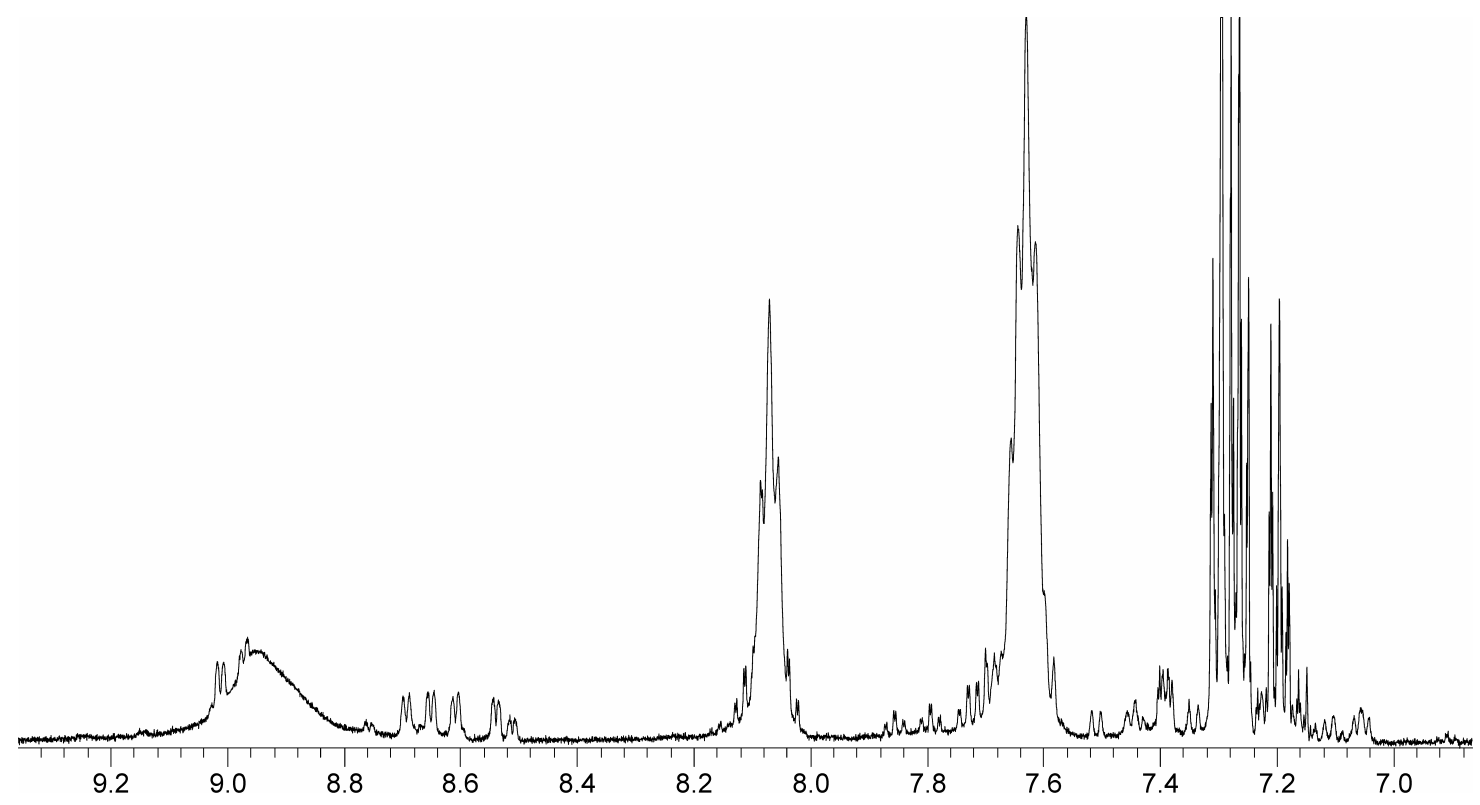

Abbildung 120: Ausschnitt aus einem Spektrum während der Umsetzung von 5 mit Penicillin $G$ (Bedingungen wie in Abbildung 119).

Es erfolgt also auch hier die Anbindung eines Substrats, so dass das Ausbleiben einer signifikanten Hydrolyse durch die Abwesenheit eines Nukleophils oder durch das Fehlen einer Substrataktivierung erklärt werden kann. Änderungen anderer Signale des Lactams beruhen vermutlich auf Epimerisierungen vor allem an den Carbonyl-C $\alpha$-Atomen des Penicillinrings.

Für die Untersuchungen mittels ${ }^{13} \mathrm{C}-\mathrm{NMR}$-Spektroskopie konnte nur Cephalothin als Substrat verwendet werden, da Penicillin während des Experiments auch durch 5 
etwas hydrolysiert wird, und nur die Anbindung des Substrats untersucht werden sollte.

Um die Bindungsweise zu analysieren, wurden ${ }^{13} \mathrm{C}-\mathrm{NMR}-S p e k t r e n$ von 4 bzw. 5 mit einer äquimolaren Menge an Cephalothin aufgenommen. Für die Anbindung an das Dizinkzentrum stehen bei Cephalothin sechs Donoren zur Verfügung:

(1) Carboxylatfunktion

(2) $\beta$-Lactamcarbonylgruppe

(3) Estergruppe

(4) Amid

(5) Thiophen-Schwefelatom

(6) Cephalosporin-Schwefelatom

Ein Vergleich mit dem ${ }^{13} \mathrm{C}-\mathrm{NMR}$-Spektren von freiem Cephalothin (als Natriumsalz) zeigt, dass sich alle Signale des bizyklischen Gerüsts verschieben, nicht jedoch die des Thiophenrings, die der Estergruppe und die des Amids. So scheint eine Koordination über die Carboxylatgruppe und möglicherweise die Lactamcarbonylfunktion plausibel.

\subsubsection{Darstellung von $\beta$-Lactamaddukten}

Addukte der verwendeten $\beta$-Lactame an Dizinkkomplexe wurden je nach gewünschtem Anion nach zwei verschiedenen Methoden dargestellt. Für die Perchloratkomplexe, die bessere Eigenschaften für die massenspektrometrischen Untersuchungen aufweisen, kann die schlechte Löslichkeit der Addukte in Wasser ausgenutzt werden. Dazu werden äquimolare Mengen Komplex und $\beta$-Lactam jeweils in wässriger Lösung zusammengegeben und der sich sofort bildende, voluminöse Niederschlag abfiltriert. Für die Gewinnung von Tetraphenylboratkomplexen, die für Kristallisationsansätze verwendet wurden, die aber aufgrund der geringen Flüchtigkeit der Tetraphenylboratzinkkomplexe im ESI-Massenspektrometer nicht gut zu detektieren sind, wird der Komplex mit einem $\beta$-Lactam in Methanol umgesetzt. Nach Zufügen von Natriumtetraphenylborat bildet sich ebenfalls sofort ein Niederschlag des $\beta$-Lactamdizinkkomplexes.

Sämtliche Kristallisationsansätze erbrachten jedoch bisher keine für die Röntgenstrukturanalyse geeigneten Einkristalle von Addukten der bizyklischen $\beta$-Lactame an ein Dizinkzentrum. Dies ist mit auf die leichte und durch die Komplexe katalysierte 
Zersetzung des $\beta$-Lactamrings zurückzuführen. Erschwert wird auch die Kristallisation durch entstehende Abbauprodukte, da die Zersetzung zu einer ganzen Reihe von Produkten führt. Auch die Racemisierung von Edukt und Produkten ist sehr leicht möglich. Lediglich im Fall der Umsetzung von 8' mit Sulbactam konnten nach mehreren Monaten Einkristalle isoliert werden. Die röntgenographische Bestimmung allerdings ergab, dass es sich um einen Sulfat-verbrückten tetranuklearen Komplex handelt. Es liegt nahe, dass das Sulfat durch eine langsame Oxidation des Substrats entsteht. Die Struktur des Komplexes $\left[\mathrm{L}^{4}{ }_{-\mathrm{H}} \mathrm{Zn}_{2}\left(\mathrm{NO}_{3}\right)_{2}\right]_{2}\left(\mathrm{SO}_{4}\right) 16$ ist in Abbildung 121 gezeigt.

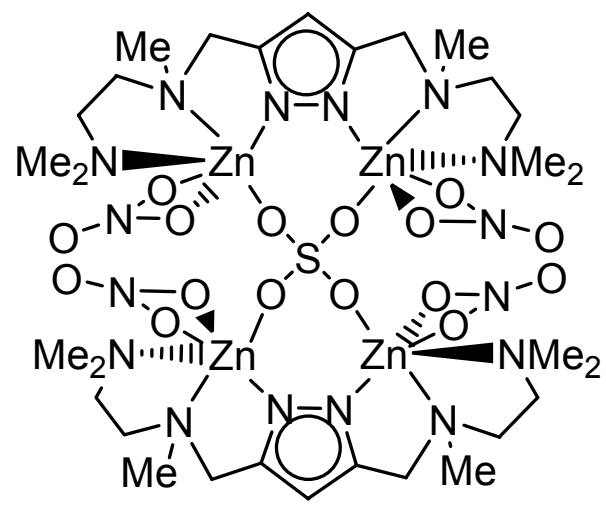

16 

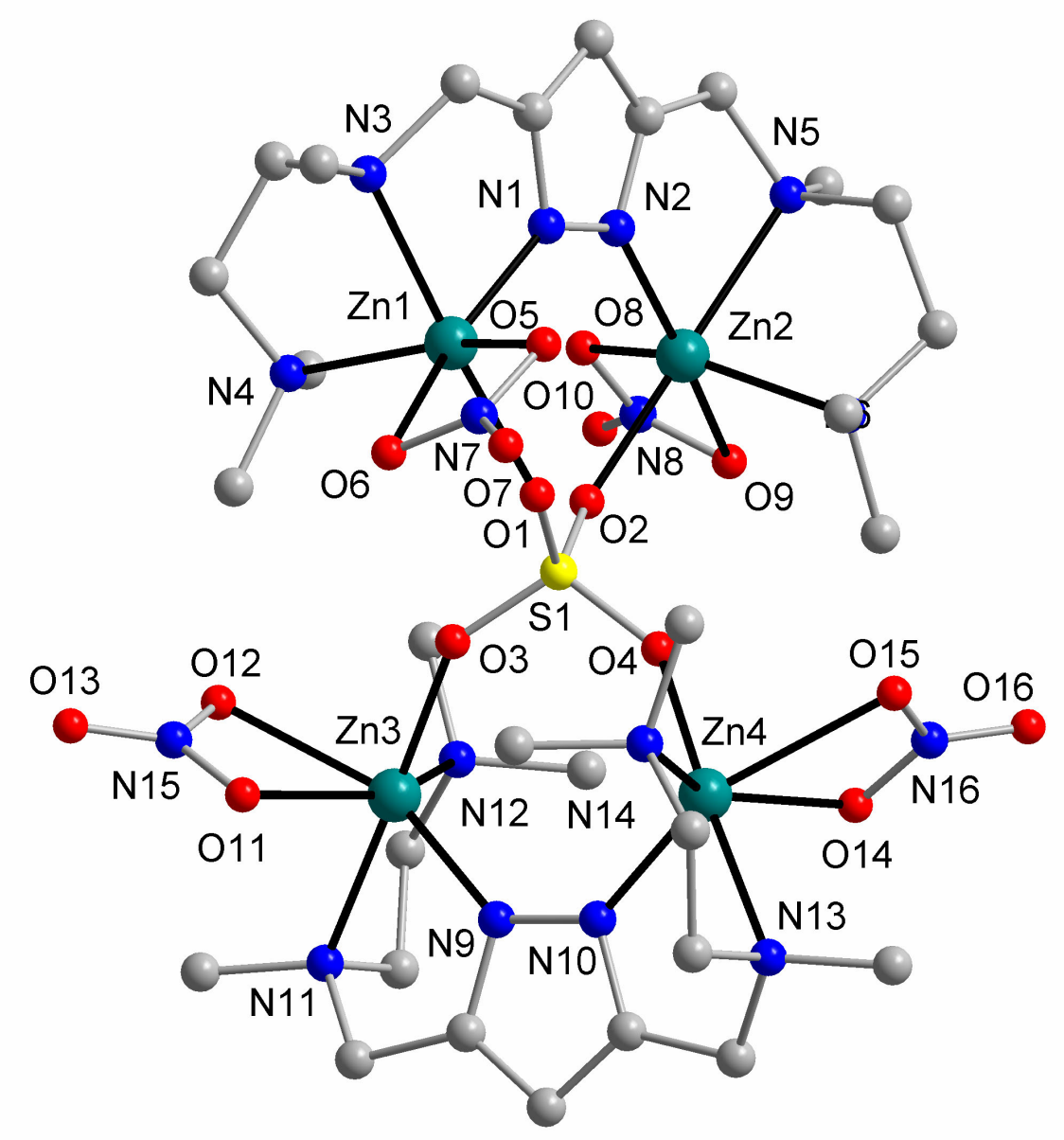

Abbildung 121: Festkörperstruktur von 16.

\begin{tabular}{|l|l|l|l|}
\hline $\mathrm{Zn}(1)-\mathrm{N}(1)$ & $2.0471(16)$ & $\mathrm{Zn}(3)-\mathrm{N}(9)$ & $2.0545(17)$ \\
\hline $\mathrm{Zn}(1)-\mathrm{N}(3)$ & $2.2592(16)$ & $\mathrm{Zn}(3)-\mathrm{N}(11)$ & $2.2955(17)$ \\
\hline $\mathrm{Zn}(1)-\mathrm{N}(4)$ & $2.1234(16)$ & $\mathrm{Zn}(3)-\mathrm{N}(12)$ & $2.1317(17)$ \\
\hline $\mathrm{Zn}(1)-\mathrm{O}(1)$ & $2.0573(13)$ & $\mathrm{Zn}(3)-\mathrm{O}(3)$ & $2.0555(13)$ \\
\hline $\mathrm{Zn}(1)-\mathrm{O}(5)$ & $2.1582(14)$ & $\mathrm{Zn}(3)-\mathrm{O}(11)$ & $2.1391(15)$ \\
\hline $\mathrm{Zn}(1)-\mathrm{O}(6)$ & $2.4303(16)$ & $\mathrm{Zn}(3)-\mathrm{O}(12)$ & $2.3644(16)$ \\
\hline $\mathrm{Zn}(2)-\mathrm{N}(2)$ & $2.0440(15)$ & $\mathrm{Zn}(4)-\mathrm{N}(10)$ & $2.0446(17)$ \\
\hline $\mathrm{Zn}(2)-\mathrm{N}(5)$ & $2.3074(16)$ & $\mathrm{Zn}(4)-\mathrm{N}(13)$ & $2.2821(17)$ \\
\hline $\mathrm{Zn}(2)-\mathrm{N}(6)$ & $2.1168(15)$ & $\mathrm{Zn}(4)-\mathrm{N}(14)$ & $2.1084(17)$ \\
\hline $\mathrm{Zn}(2)-\mathrm{O}(2)$ & $2.0766(13)$ & $\mathrm{Zn}(4)-\mathrm{O}(4)$ & $2.0480(13)$ \\
\hline $\mathrm{Zn}(2)-\mathrm{O}(8)$ & $2.0895(13)$ & $\mathrm{Zn}(4)-\mathrm{O}(14)$ & $2.0679(14)$ \\
\hline $\mathrm{Zn}(2)-\mathrm{O}(9)$ & $2.5440(17)$ & $\mathrm{Zn}(4)-\mathrm{O}(15)$ & $2.5212(16)$ \\
\hline $\mathrm{S}(1)-\mathrm{O}(1)$ & $1.4791(14)$ & $\mathrm{N}(1)-\mathrm{N}(2)$ & $1.376(2)$ \\
\hline $\mathrm{S}(1)-\mathrm{O}(2)$ & $1.4801(13)$ & $\mathrm{N}(9)-\mathrm{N}(10)$ & $1.380(2)$ \\
\hline
\end{tabular}




\begin{tabular}{|l|l|l|l|}
\hline $\mathrm{S}(1)-\mathrm{O}(3)$ & $1.4812(13)$ & $\mathrm{Zn}(1) \cdots \mathrm{Zn}(2)$ & $4.3270(3)$ \\
\hline $\mathrm{S}(1)-\mathrm{O}(4)$ & $1.4784(13)$ & $\mathrm{Zn}(3) \cdots \mathrm{Zn}(4)$ & $4.3222(3)$ \\
\hline
\end{tabular}

Tabelle 24: Ausgewählte Atomabstände $(\AA)$.

Das Molekül besitzt annähernd eine $S_{4}$-Symmetrie, in der das zentrale Sulfat zweimal bidentat eine $\left\{L Z \mathrm{n}_{2}\right\}$-Einheit verbrückt. Die offenen Koordinationsstellen werden jeweils bidentat durch insgesamt vier Nitrate besetzt, so dass für jedes Zinkion eine verzerrt oktaedrische Koordinationsumgebung resultiert.

Dieses Bindungsmotiv für Sulfat konnte bereits für eine Reihe anderer Metallionen gefunden werden, nach einer CSD-Suche ${ }^{[89]}$ ist dieser Bindungsmodus für Zink(II) jedoch bisher unbekannt.

Hingegen konnte aus dem als Modellsubstrat eingesetzten Oxazetidinylacetat und 8' für die Röntgenstrukturanalyse geeignete Kristalle erhalten werden. Es zeigt sich für 17 eine dimere Struktur, in der die beiden Dizinkeinheiten durch zwei Oxazetidinylacetatmoleküle verbrückt werden. Die Carboxylateinheit findet sich dabei bidentat verbrückend in beiden Einheiten und die Lactamcarbonylgruppe koordiniert über das Sauerstoffatom an ein Zinkion $\mathrm{Zn}(1)$ der anderen bimetallischen Einheit. An Zn(2) ist ein zusätzliches Wassermolekül gebunden, so dass für beide Zinkionen eine Koordinationszahl von fünf resultiert.

Das IR-Spektrum des von festem 17 zeigt für die $\beta$-Lactamcarbonylfunktion eine Bande bei $1712 \mathrm{~cm}^{-1}$, dies entspricht einer Verschiebung von $23 \mathrm{~cm}^{-1}$ gegenüber der Bande des Oxazetidinylacetats XLVIII bei $1735 \mathrm{~cm}^{-1}$. Das Dimer ist in Lösung jedoch nicht stabil, sondern zerfällt in Methanol zu einem großen Teil unter Dissoziation der $\beta$-Lactamcarbonylfunktion vom Zink in zwei monomere dinukleare Einheiten. In Lösung erhält man ein intensives Signal bei $1735 \mathrm{~cm}^{-1}$ für die freie $\beta$-Lactamcarbonylfunktion und nur eine wenig ausgeprägte Schulter bei etwa $1714 \mathrm{~cm}^{-1}$. 


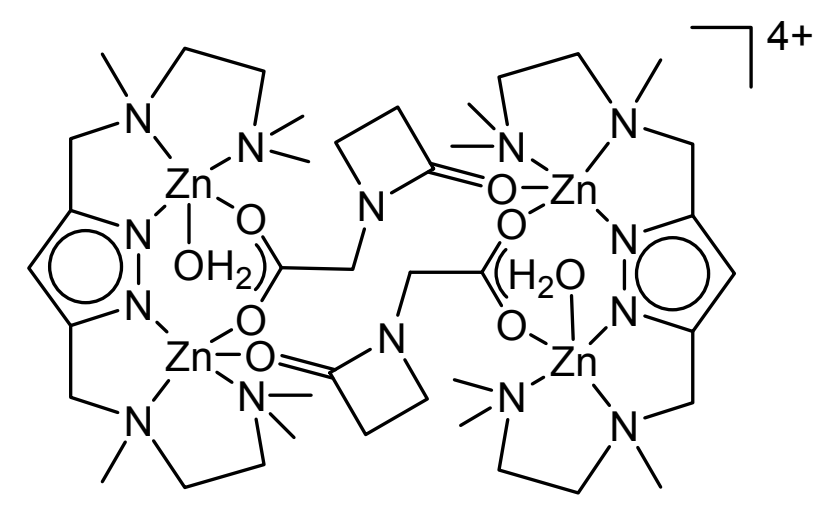

17

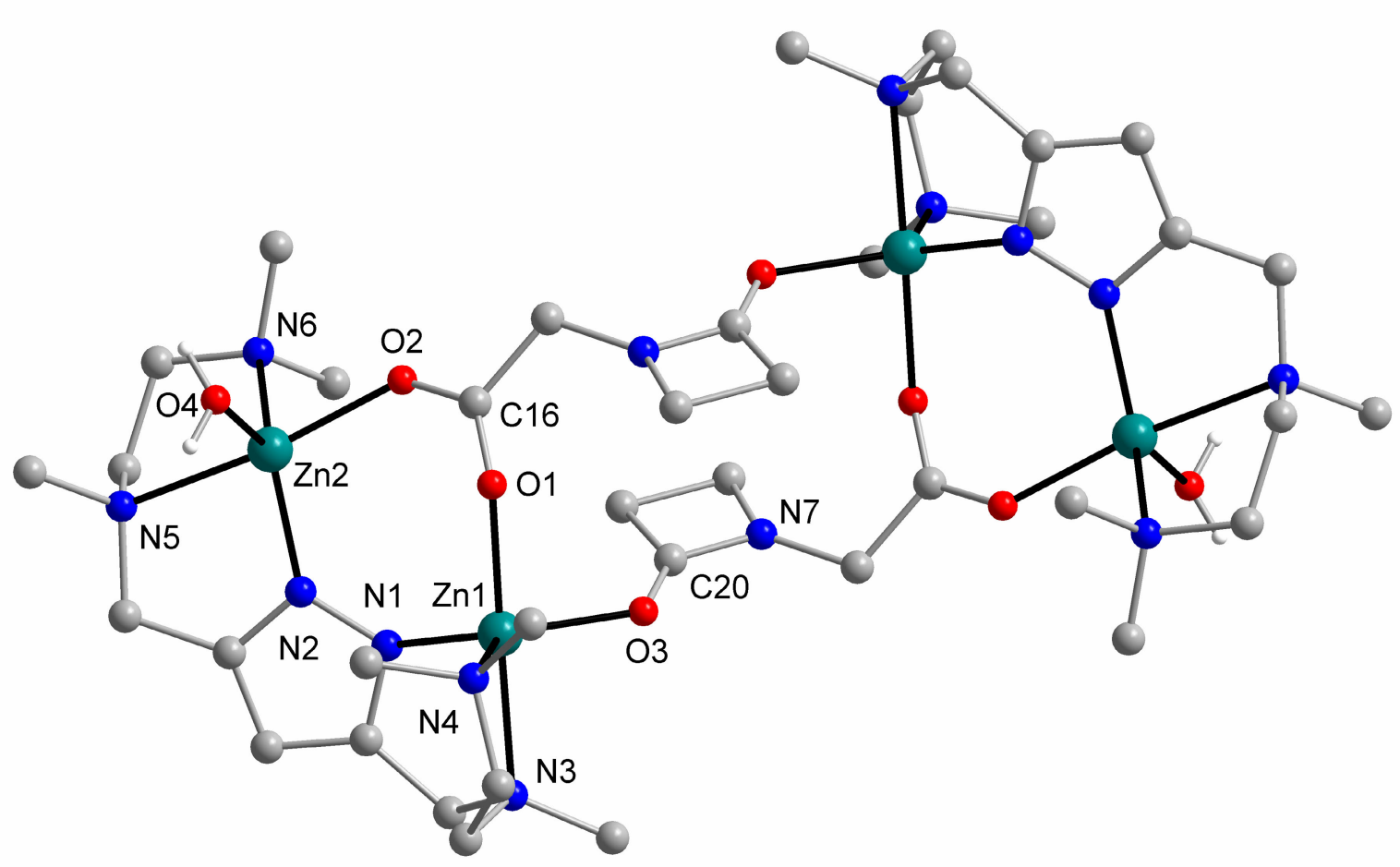

Abbildung 122: Festkörperstruktur des Kations von $17 \cdot\left(\mathrm{ClO}_{4}\right)_{4}$.

\begin{tabular}{|l|l|l|l|}
\hline $\mathrm{Zn}(1)-\mathrm{N}(1)$ & $1.989(3)$ & $\mathrm{Zn}(2)-\mathrm{N}(2)$ & $1.984(3)$ \\
\hline $\mathrm{Zn}(1)-\mathrm{N}(3)$ & $2.232(3)$ & $\mathrm{Zn}(1) \cdots \mathrm{Zn}(2)$ & $4.1359(6)$ \\
\hline $\mathrm{Zn}(1)-\mathrm{N}(4)$ & $2.060(3)$ & $\mathrm{Zn}(2)-\mathrm{N}(5)$ & $2.300(3)$ \\
\hline $\mathrm{Zn}(1)-\mathrm{O}(1)$ & $2.093(3)$ & $\mathrm{Zn}(2)-\mathrm{N}(6)$ & $2.121(3)$ \\
\hline $\mathrm{Zn}(1)-\mathrm{O}(3 \mathrm{~A})$ & $2.005(2)$ & $\mathrm{Zn}(2)-\mathrm{O}(2)$ & $2.060(2)$ \\
\hline $\mathrm{O}(1)-\mathrm{C}(16)$ & $1.248(4)$ & $\mathrm{Zn}(2)-\mathrm{O}(4)$ & $2.031(3)$ \\
\hline $\mathrm{O}(2)-\mathrm{C}(16)$ & $1.254(4)$ & & \\
\hline $\mathrm{O}(3)-\mathrm{C}(20)$ & $1.242(4)$ & & \\
\hline $\mathrm{N}(1)-\mathrm{N}(2)$ & $1.383(4)$ & & \\
\hline
\end{tabular}




\begin{tabular}{|l|l|l|l|}
\hline $\mathrm{N}(1)-\mathrm{Zn}(1)-\mathrm{N}(3)$ & $81.58(11)$ & $\mathrm{N}(2)-\mathrm{Zn}(2)-\mathrm{N}(5)$ & $79.26(11)$ \\
\hline $\mathrm{N}(1)-\mathrm{Zn}(1)-\mathrm{N}(4)$ & $115.60(11)$ & $\mathrm{N}(2)-\mathrm{Zn}(2)-\mathrm{N}(6)$ & $120.93(11)$ \\
\hline $\mathrm{N}(1)-\mathrm{Zn}(1)-\mathrm{O}(1)$ & $101.69(11)$ & $\mathrm{N}(2)-\mathrm{Zn}(2)-\mathrm{O}(2)$ & $106.66(11)$ \\
\hline $\mathrm{N}(1)-\mathrm{Zn}(1)-\mathrm{O}(3 \mathrm{~A})$ & $128.98(11)$ & $\mathrm{N}(2)-\mathrm{Zn}(2)-\mathrm{O}(4)$ & $120.68(13)$ \\
\hline $\mathrm{N}(3)-\mathrm{Zn}(1)-\mathrm{N}(4)$ & $84.83(11)$ & $\mathrm{N}(5)-\mathrm{Zn}(2)-\mathrm{N}(6)$ & $82.57(11)$ \\
\hline $\mathrm{N}(3)-\mathrm{Zn}(1)-\mathrm{O}(1)$ & $175.86(11)$ & $\mathrm{N}(5)-\mathrm{Zn}(2)-\mathrm{O}(2)$ & $168.83(11)$ \\
\hline $\mathrm{N}(3)-\mathrm{Zn}(1)-\mathrm{O}(3 \mathrm{~A})$ & $92.12(10)$ & $\mathrm{N}(5)-\mathrm{Zn}(2)-\mathrm{O}(4)$ & $95.26(11)$ \\
\hline $\mathrm{N}(4)-\mathrm{Zn}(1)-\mathrm{O}(1)$ & $91.43(11)$ & $\mathrm{N}(6)-\mathrm{Zn}(2)-\mathrm{O}(2)$ & $86.26(11)$ \\
\hline $\mathrm{N}(4)-\mathrm{Zn}(1)-\mathrm{O}(3 \mathrm{~A})$ & $114.10(11)$ & $\mathrm{N}(6)-\mathrm{Zn}(2)-\mathrm{O}(4)$ & $116.61(13)$ \\
\hline $\mathrm{O}(1)-\mathrm{Zn}(1)-\mathrm{O}(3 \mathrm{~A})$ & $87.78(10)$ & $\mathrm{O}(2)-\mathrm{Zn}(2)-\mathrm{O}(4)$ & $89.81(11)$ \\
\hline $\mathrm{O}(1)-\mathrm{C}(16)-\mathrm{O}(2)$ & $126.7(3)$ & & \\
\hline
\end{tabular}

Tabelle 25: Ausgewählte Atomabstände $(\AA)$ und Bindungswinkel ( $\left.{ }^{\circ}\right)$. 


\subsection{Diskussion}

Die erhaltenen Ergebnisse stützen insgesamt den für das natürliche Dizinkzentrum in $\operatorname{der} B$. fragilis Metallo- $\beta$-Lactamase postulierten Mechanismus. Dieser kann prinzipiell auf die hier untersuchten Dizinkmodellkomplexe übertragen werden (Abbildung 123).

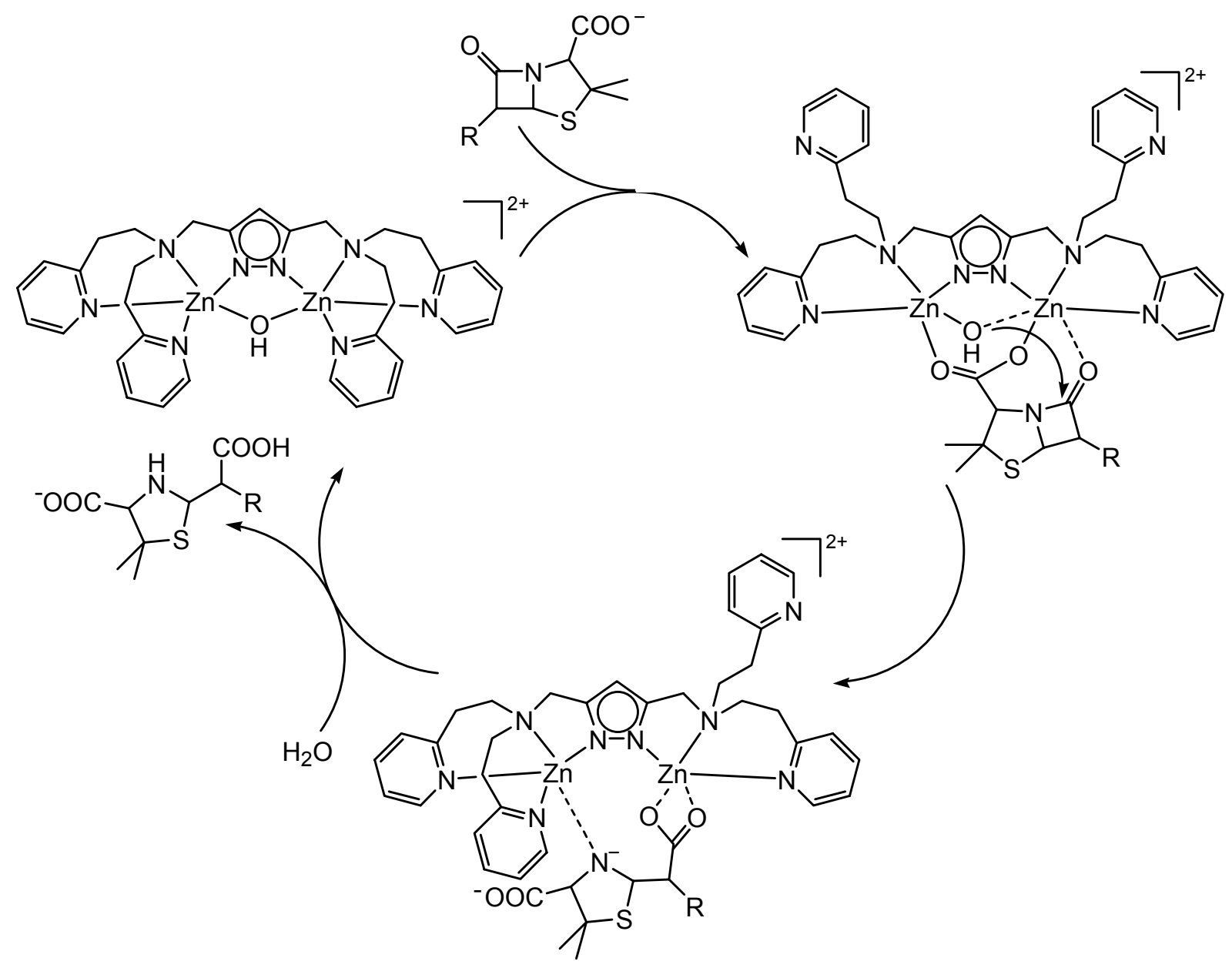

Abbildung 123: Postulierter Mechanismus für die Hydrolyse von Penicillin durch 1 und 4.

Aufgrund der Ergebnisse der ESI-Massenspektroskopie und der ${ }^{13} \mathrm{C}-\mathrm{NMR}$ spektroskopischen Daten kann für alle Komplexe als erster Schritt die Substratanbindung über die Carboxylatfunktion des Penicillins an das dinukleare Zentrum angenommen werden. Wie anhand der kinetischen Untersuchungen festgestellt wurde, ist die Labilität von Seitenarmen eine unabdingbare Voraussetzung für die Hydrolyse von Penicillin. Mögliche Gründe dafür können zum Ersten sein, dass für eine schnelle Hydrolyse die Aktivierung der Carbonylfunktion durch Koordination an das Lewis-saure Metallzentrum nötig ist. Dass eine solche Koordination erfolgen kann, zeigt der vorgestellte Modellkomplex 17, in dem sich einige Details des 
vorgeschlagenen Bindungsmotivs wiederfinden. So bindet eine Carboxylatgruppe bidentat verbrückend an das Dizinkzentrum, und noch offene Koordinationsstellen werden durch eine Carbonylfunktion eines $\beta$-Lactams und durch ein Wassermolekül besetzt.

Zum Zweiten kann die Konkurrenz um die freie Koordinationsstelle zwischen Liganddonoratom, Substrat und Produkt entscheidend für den schnellen katalytischen Abbau von Penicillin durch $\mathbf{1}$ und $\mathbf{4}$ sein, da so das Substrat nicht nur angebunden werden kann, sondern auch die Ablösung des Produkts nach der Reaktion möglich ist. Die katalytische Hydrolyse scheint demnach auf derart hemistabile Systeme angewiesen zu sein.

Über den weiteren Mechanismus nach Anbindung des Substrats können auf der Basis der vorliegenden Ergebnisse keine Angaben gemacht werden. Ausgehend von kristallographischen Studien wird vorgeschlagen, dass im nächsten Schritt des Mechanismus' ein Zink-gebundenes Hydroxid als Nukleophil beim Angriff auf das Substrat fungiert. ${ }^{[5]}$ Jedoch gibt es bis jetzt keinen eindeutigen Beleg über die exakte Position des Nukleophils während der Reaktion, d. h. ob es in verbrückender oder in terminaler Position im Dizinkzentrum vorliegt. 


\section{Zusammenfassung}

Die vorliegende Arbeit befasst sich mit dem Aufbau und der Reaktivität dinuklearer Zinkkomplexe mit präorganisierenden Pyrazolat-Dikompartimentliganden. Die im Anbringen der Seitenarme flexible Ligandsynthese ermöglicht es in Anlehnung an bestehende Literaturvorschriften, einen Satz von neun verschiedenen Bis(aminomethyl)pyrazolliganden darzustellen, der eine systematische Korrelation von Struktur und Reaktivität der entsprechenden Dizinkkomplexe erlaubt. Die dabei einstellbaren Parameter sind erstens die Länge der Seitenarme und damit die Größe der entstehenden Chelatringe und den Zn $\cdots$ Zn-Abstand, zweitens die elektronischen Eigenschaften der terminalen Donoren, die die Stärke der Zink-Donor-Wechselwirkung beeinflussen, drittens die Zahl der Donoratome, die sich auf die Stabilität der Komplexe und die Anzahl freier Koordinationsstellen auswirkt, und schließlich die Topologie der chelatisierenden Seitenarme, die im Fall makrozyklischer Seitenarme in $L^{5}$ einen enormen Stabilitätsgewinn der Komplexe mit sich bringt.<smiles>CNCCCN(CCCN(C)C)Cc1cc(CN(CCCNC)CCCNC)[nH]n1</smiles>

$L^{1}$<smiles>CCCN(CCNCC)Cc1cc(CN(CCNCC)CCNCC)n[nH]1</smiles><smiles>CNCCCN(C)Cc1cc(CN(C)CCCN(C)C)[nH]n1</smiles>

$L^{2}$<smiles>CN(C)CCN(C)Cc1cc(CN(C)C)[nH]n1</smiles><smiles>CCCN1CCN(CC)CCN(Cc2cc(CN3CCN(CCC)CCN(CCC)CC3)[nH]n2)CC1</smiles> 
<smiles>c1ccc(CCN(CCc2ccccn2)Cc2cc(CN(CCc3ccccn3)CCc3ccccn3)[nH]n2)nc1</smiles>

$\mathrm{L}^{6}$<smiles>c1ccc(CN(Cc2ccccn2)Cc2cc(CN(Cc3ccccn3)Cc3ccccn3)[nH]n2)nc1</smiles>

$\mathrm{L}^{8}$<smiles>CN(C)Cc1cc(CN(C)CCc2ccccn2)[nH]n1</smiles>

$\mathrm{L}^{7}$<smiles>CN(Cc1ccccn1)Cc1cc(CN(C)Cc2ccccn2)[nH]n1</smiles>

$\mathrm{L}^{9}$

Bei der Komplexierung der verwendeten Liganden zeigt sich ein unmittelbarer Zusammenhang zwischen der Struktur des Liganden und des gebildeten Komplexes. So erhält man im Fall bis(tetradentater) Pyrazolatliganden dinukleare Zinkkomplexe, die je nach Länge der Seitenarme eine $\mathrm{H}_{3} \mathrm{O}_{2}$ - bzw. eine $\mathrm{HOHOMe-}(\mathbf{2 b}, \mathbf{5})$ oder eine Hydroxid-Brücke $(1,3,4)$ ausbilden.

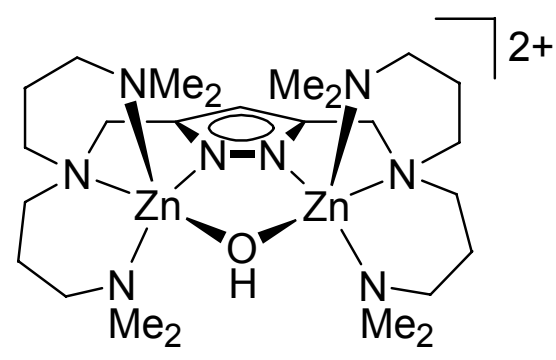

1

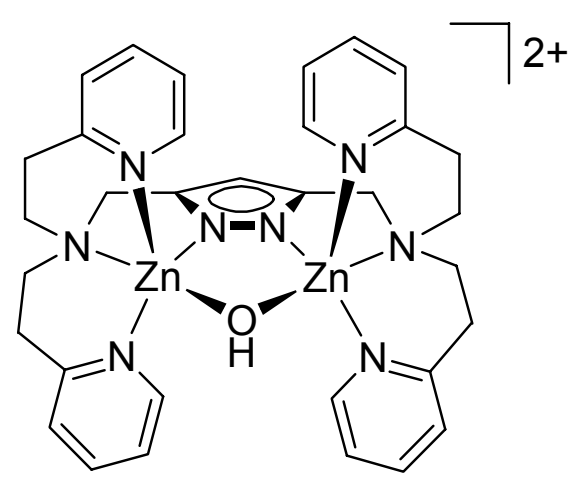

4

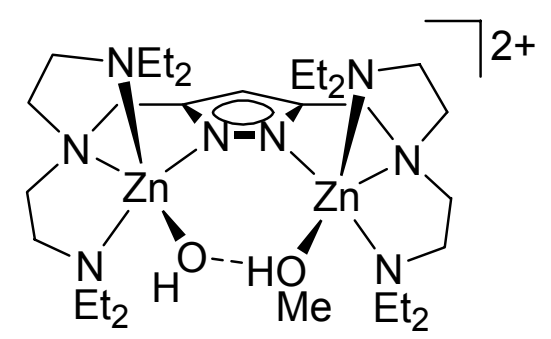

2b

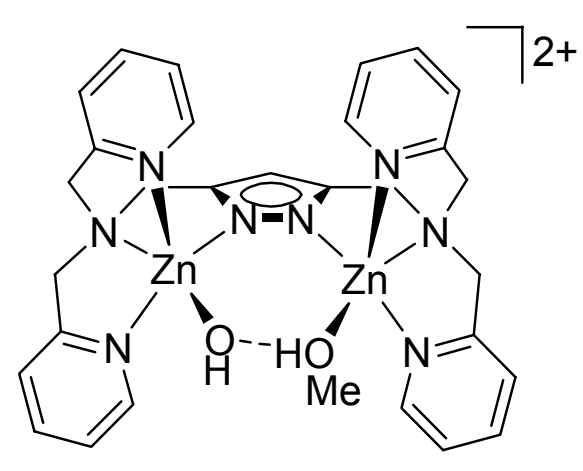

5 
Während die langen Seitenarme in $L^{1}$ und $L^{6}$ durch einen relativ kurzen Zink-ZinkAbstand die Ausbildung der thermodynamisch günstigeren Hydroxidbrücke erlauben, ziehen die kurzen Seitenarme in $L^{3}$ und $L^{8}$ die beiden Zinkionen auseinander, und es resultiert ein langer Zink-Zink-Abstand. Eine kleine, monoatomare Einheit kann diesen Abstand nun nicht mehr überbrücken, und ein zusätzliches Lösungsmittelmolekül wird in die Brücke eingebaut.

Einen Sonderfall bilden die Dizinkkomplexe des Liganden $L^{5}$. Trotz der kurzen Seitenarme bildet sich hier eine kurze Hydroxidbrücke anstatt der erwarteten und bei analogen Nickel- und Kupferkomplexen auch gefundenen $\mathrm{H}_{3} \mathrm{O}_{2}$-Brücke aus.

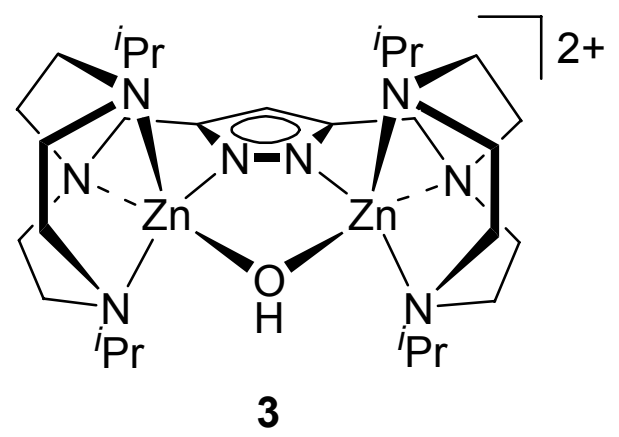

Die bis(tridentaten) Liganden besitzen in jedem Kompartiment jeweils eine Donorfunktion weniger. Die dadurch geringeren Stabilitätskonstanten reichen im Fall der energetisch ungünstigeren Sechsringchelate, wie sie mit $L^{2}$ und $L^{7}$ entstehen, nicht mehr aus, um stabile dinukleare Komplexe zu bilden. Lediglich im Fall von $\mathrm{L}^{2}$ konnte ein Chlorid-verbrückter Komplex isoliert werden, der jedoch in Lösung wenig beständig ist. Komplexe der Liganden $\mathrm{L}^{4}$ und $\mathrm{L}^{9}$ lassen sich erhalten, wenn die freien Koordinationsstellen durch geeignete, koordinierende Nitratanionen stabilisiert werden $(7,9)$. Ansonsten entstehen äußerst reaktive Dizinkspezies, die rasch Kohlendioxid aus der Luft binden und dieses unter Ausbildung des tetranuklearen Komplexes 8 als Carbonat fixieren. 


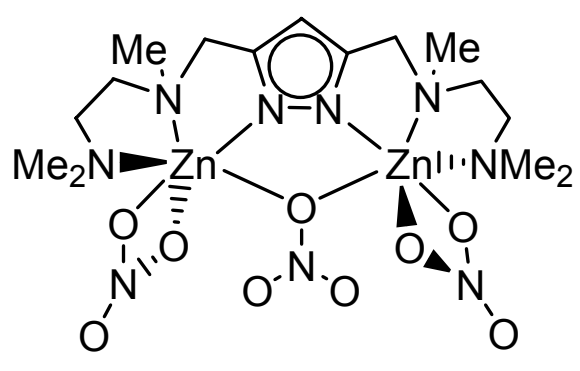

7

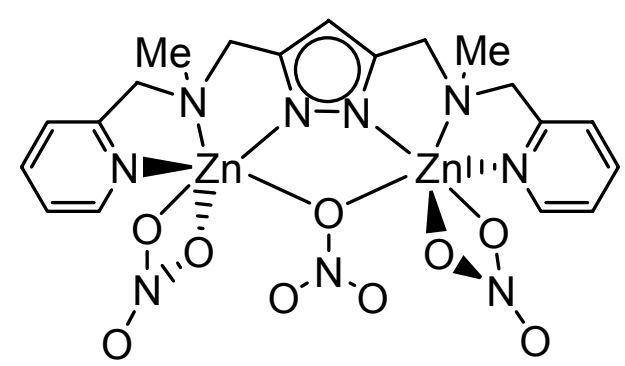

9

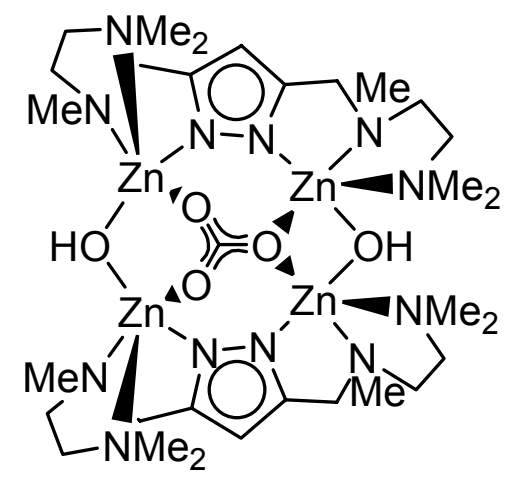

8

Um die Reaktivität der Komplexe auch in Lösung zu untersuchen, wurden die aus der Röntgenstrukturanalyse der Festkörper erhaltenen Strukturen durch potentiometrische Titrationen ergänzt. Aus diesen erhält man eine relativ genaue Vorstellung über das Verhalten und die Zusammensetzung der Komplexe in Lösung. Vier wichtige Ergebnisse seien hier kurz zusammengefasst:

(I) Alle untersuchten Dikompartimentliganden bilden auch in wässriger Lösung dinukleare Zinkkomplexe.

(II) Oberhalb eines pH-Werts von etwa 8 bilden sich in Lösung Spezies der gleichen Zusammensetzung wie im Festkörper unter Ausbildung metallgebundener Hydroxideinheiten.

(III) Die ermittelten $\mathrm{pK}_{\mathrm{S}}$-Werte der Deprotonierung eines Metall-gebundenen Wassermoleküls korrelieren mit der Länge der Seitenarme und lassen sich klar der Entstehung einer Hydroxidbrücke $\left(\mathrm{pK}_{\mathrm{S}} \approx 8.0\right.$ ) oder einer $\mathrm{H}_{3} \mathrm{O}_{2}$-Brücke $\left(\mathrm{pK}_{\mathrm{S}} \approx 7.6\right.$ ) zuordnen.

(IV) Der Energiegewinn durch die Ausbildung einer Zweitbrücke führt bei Vorliegen einer äquimolaren Lösung von Ligand und Zink(II) oberhalb eines pH-Werts von 8 dazu, dass sich aus je zwei Ligandmolekülen und zwei Zinkionen nicht zwei mononukleare Spezies, sondern eine dinukleare Spezies und ein freier Ligand bilden. 
Durch die Variabilität dieser Dizinkzentren eignen sich die dargestellten Zinkkomplexe hervorragend als Modellverbindungen für die aktiven Zentren in dinuklearen Metallohydrolasen. Insbesondere die Reaktivitätsunterschiede zwischen einem terminalen und einem verbrückenden Hydroxid können mit diesen Komplexen untersucht werden. Dabei entspricht die Hydroxidbrücke in den Komplexen 1, 3 und 4 einem verbrückenden Hydroxid in dinuklearen Enzymzentren, und die $\mathrm{H}_{3} \mathrm{O}_{2}$-Brücke in $\mathbf{2}$ und $\mathbf{5}$ kann aufgrund der leichten Austauschbarkeit des zusätzlich eingebauten Lösungsmittels als terminale Hydroxidbrücke in einem dinuklearen Zentrum betrachtet werden.

Als erstes zu modellierendes System wurde in dieser Arbeit die Hydrolyse von Phosphatestern durch dinukleare Phosphatasen untersucht. Denn obwohl Phosphatesterbindungen thermodynamisch instabil sind, weisen sie hohe kinetische Barrieren auf. Phosphatester-spaltende Enzyme spielen an vielen Stellen des Aufbaus und der Funktion lebenswichtiger Zellprozesse und der Signalübertragung eine unentbehrliche Rolle und sind auch das Ziel medizinischer Anwendung. So sind ihre oft noch nicht vollständig aufgeklärten Reaktionsmechanismen Ziel umfangreicher Forschungsarbeiten.

In diesem Sinne wurden die hier dargestellten Dizinkkomplexe auf ihre Aktivität in der Phosphatesterspaltung von Bis(4-nitrophenyl)phosphat BNPP zu Mono(4-nitrophenyl)phosphat MNPP getestet und miteinander verglichen.

Analysen der Anbindung von Phosphatdiestern durch NMR-Spektroskopie, ESIMassenspektrometrie und Röntgenstrukturanalyse lieferten ein genaues Bild über die thermodynamischen und kinetischen Aspekte dieser Hydrolyse. Deshalb können aus dieser Arbeit einige Punkte für die Wirkungsweise biologischer Phosphatesterspaltungen und für das Design neuer biomimetischer Phosphatasen abgeleitet werden.

So ist ein zwei Metallionen verbrückendes Hydroxid wie in $\mathbf{3}$ und $\mathbf{4}$ ein vergleichsweise schwaches Nukleophil, wohingegen eine nichtverbrückende Position des Hydroxids wie in $\mathbf{2}$ und $\mathbf{5}$ für eine hydrolytische Aktivität günstiger ist. 


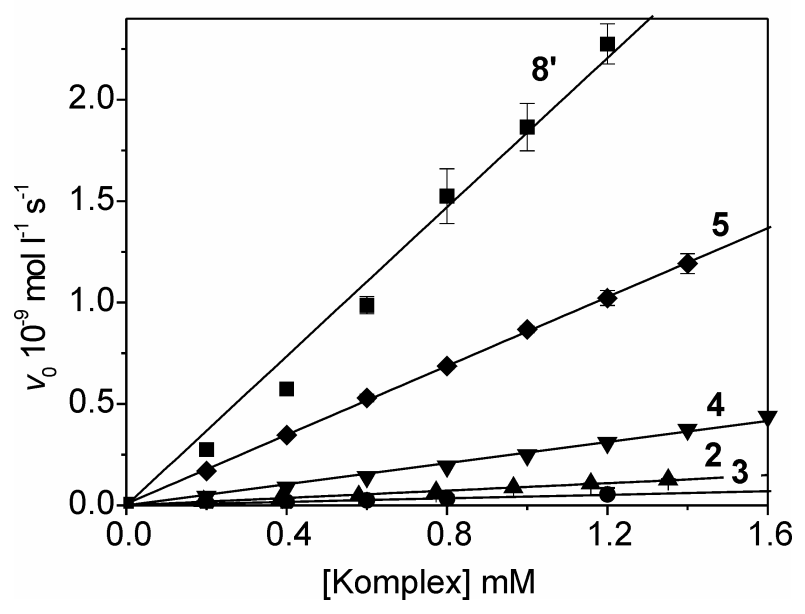

Die Notwendigkeit des metallgebundenen Hydroxids für die Hydrolyse ist aus der starken Korrelation zwischen den beobachteten pH-Abhängigkeiten der Hydrolysegeschwindigkeit und der potentiometrisch ermittelten Bildung von Zink-gebundenen Hydroxidspezies offensichtlich. Darüber hinaus muss das Dimetallzentrum eine ausreichende Zahl von freien Koordinationsstellen aufweisen, um sowohl das Substrat als auch das Nukleophil zu aktivieren. Im Fall der Phosphatdiester wie BNPP wird das Substrat bevorzugt bidentat verbrückend angebunden, wie die gefundenen Strukturen für 10a, 11a, 12a und 13a belegen, so dass eine weitere freie Koordinationsstelle für die Anbindung eines zu aktivierenden Wassermoleküls zur Verfügung stehen muss.

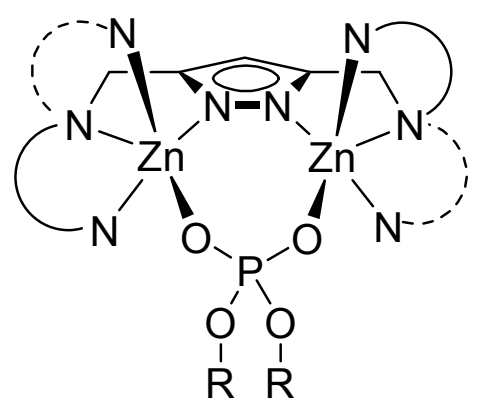

Dies kann entweder durch ein trinukleares Zentrum, wie im aktiven Zentrum des Enzyms P1 Nuklease, oder durch koordinativ ungesättigte, dinukleare Systeme wie in 8' erreicht werden, die in diesen Untersuchungen mit Abstand die höchste Aktivität aufwiesen. Ist beides aufgrund struktureller Gründe nicht möglich, so beeinflusst die Zugänglichkeit zum fünffach koordinierten Zinkzentrum die Reaktivität. 
Die durch die Hydrolyse entstehenden Phosphatmonoester koordinieren für gewöhnlich stärker an Metallzentren als Phosphatdiester und führen zu Verbindungen wie 15, in denen ein Phosphatmonoester zwei reaktive Zentren blockiert. Diese Verbindung stellt eine der wenigen Beispiele für eine vollständig identifizierte und charakterisierte produktinhibierte Spezies dar.

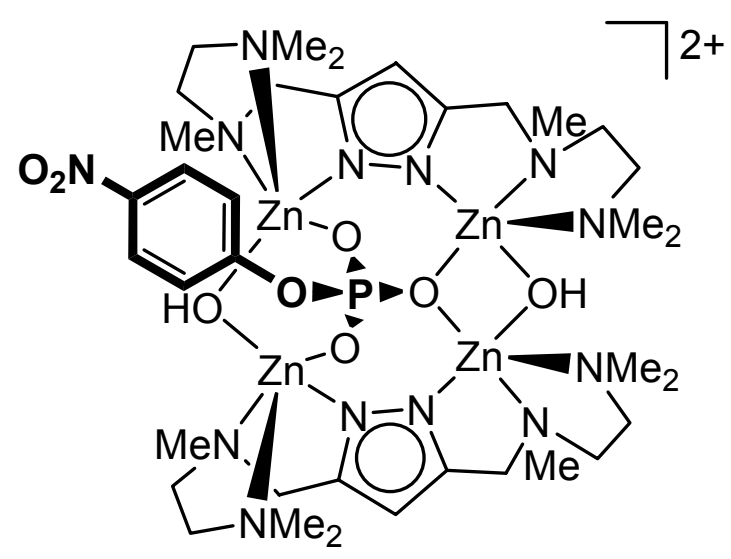

15

Um einen mehrfachen Umsatz an einem Metallzentrum zu ermöglichen, müssen deshalb Systeme entwickelt werden, die auch bei geringen Bindungskonstanten von Substrat und Produkt noch reaktiv sind. Bis dahin kann eine katalytische Umsetzung nur erreicht werden - auch dies wurde in dieser Arbeit am Beispiel des Substrats Hydroxypropyl(4-nitrophenyl)phosphat HPNPP gezeigt -, wenn Substrat und Produkt die gleiche oder zumindest eine sehr ähnliche Affinität aufweisen.

Auch in den bislang vorgeschlagenen Mechanismen der Hydrolyse von $\beta$-Lactamen durch dinukleare Metallo- $\beta$-Lactamasen spielt ein Zink-gebundenes Hydroxid die Rolle des angreifenden Nukleophils. Diese durch Bakterien produzierten Enzyme stellen eine immer ernster zu nehmende Gefahr für die Menschheit dar, da sie in der Lage sind, fast alle $\beta$-Lactamantibiotika, angefangen von den lange bekannten Penicillinen bis zu neuesten Entwicklungen wie Cephalosporinen oder Carbapenemen, zu hydrolysieren und damit unwirksam zu machen. Da bisher keine medizinisch brauchbaren Inhibitoren gefunden wurden und die Verbreitung dieser Enzyme durch den starken Selektionsdruck immer weiter zunimmt, stellen Beiträge zur Aufklärung dieser Mechanismen wichtige Schritte für die pharmazeutische Forschung dar. 
Die synthetisierten Modellverbindungen wurden deshalb auf ihre Aktivität in der Spaltung des $\beta$-Lactamrings von Penicillin G untersucht. Zu diesem Zweck wurde die IR-Spektroskopie als Methode etabliert, mittels der Abnahme des Signals der $\beta$ Lactamcarbonylfunktion die Spaltung des $\beta$-Lactamrings quantitativ zu verfolgen. Ein Vergleich der beobachteten Reaktionsgeschwindigkeiten zeigt, dass nur für die Komplexe 1 und 5, nicht jedoch für 2, 3 und 4 eine deutliche katalytische Aktivität zu nachzuweisen ist. Für diese katalytische Aktivität scheint jedoch nicht die Anbindungsweise des Hydroxids die Rolle zu spielen, sondern wichtiger ist eine gewisse Labilität der Koordinationsumgebung.

So konnte ein Mechanismus aufgestellt und untermauert werden, in dem Ligandseitenarme, Edukt und Produkt um freie Koordinationsstellen konkurrieren und dadurch, anders als im Fall der Phosphatdiester, eine katalytische Reaktion ermöglicht wird.

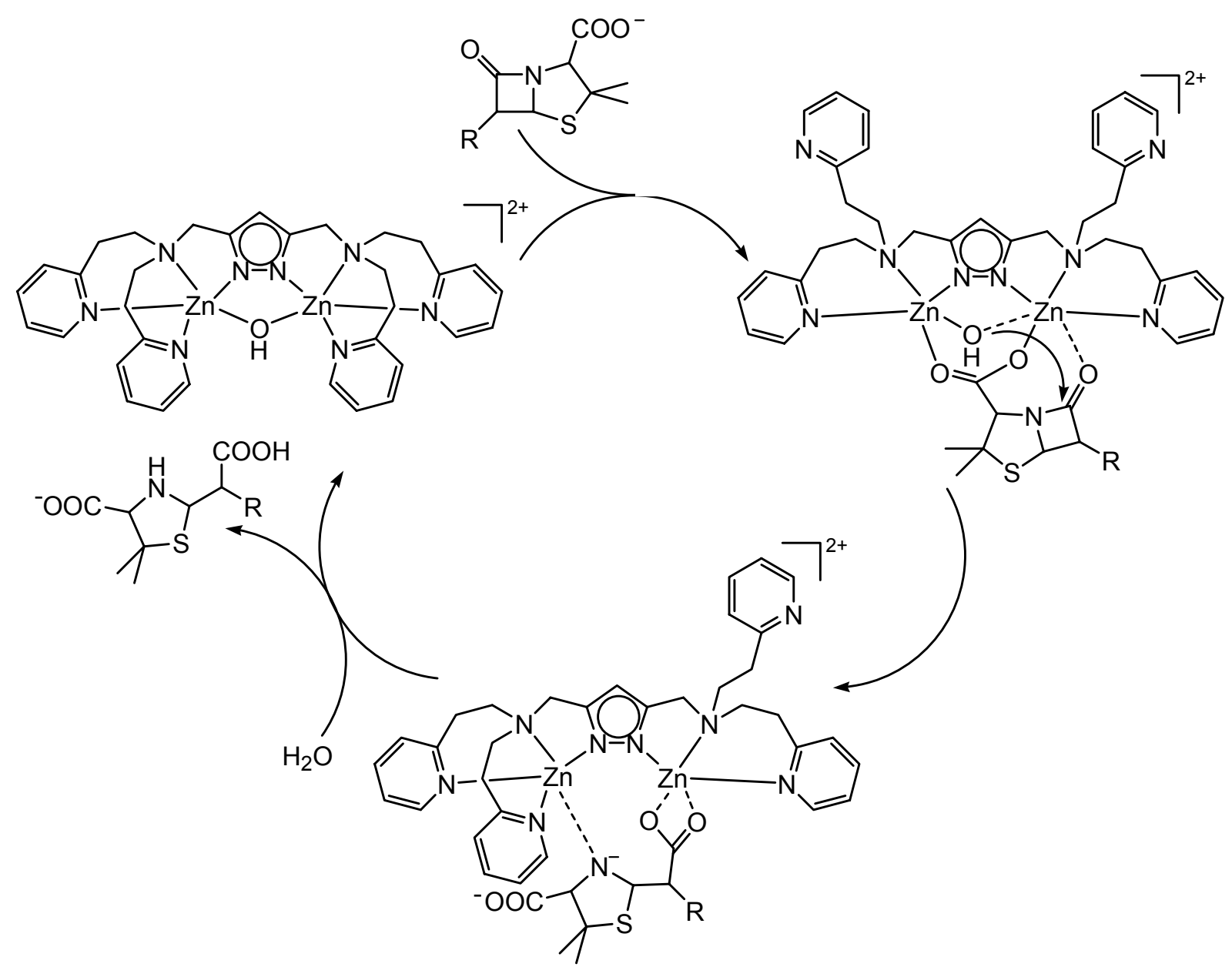

Um diesen Mechanismus zu belegen, wurden umfassende Untersuchungen durchgeführt. Die durch ESI-Massenspektrometrie und NMR-Spektroskopie erhaltenen Ergebnisse lassen darauf schließen, dass die Anbindung der Substrate über 
ihre Carboxylatfunktion erfolgt. Des Weiteren zeigte sich je nach eingesetztem Komplex ein bei den aktiven Komplexen mehr, bei den inaktiven Komplexen weniger ausgeprägtes dynamisches Verhalten der Ligandseitenarme, - Beobachtungen, die dem aufgestellten Mechanismus entsprechen.

Da eine eindeutige Bestimmung der Substratkoordination an das Dizinkzentrum jedoch nur über die Röntgenstrukturanalyse möglich ist, wurden viele Versuche unternommen, Addukte bizyklischer $\beta$-Lactamantibiotika an Dizinkzentren zu kristallisieren. Erfolgreich erwies sich letztendlich nur der Einsatz des monozyklischen Oxazetidinylacetats, das ein gutes Modell für die Struktur der Bindungsstelle der meisten $\beta$-Lactamantibiotika darstellt.<smiles>O=C([O-])CN1CCC1=O</smiles><smiles>CC1(C)SC2C(NC(=O)Cc3ccccc3)C(=O)N2C1C(=O)[O-]</smiles>

Penicillin

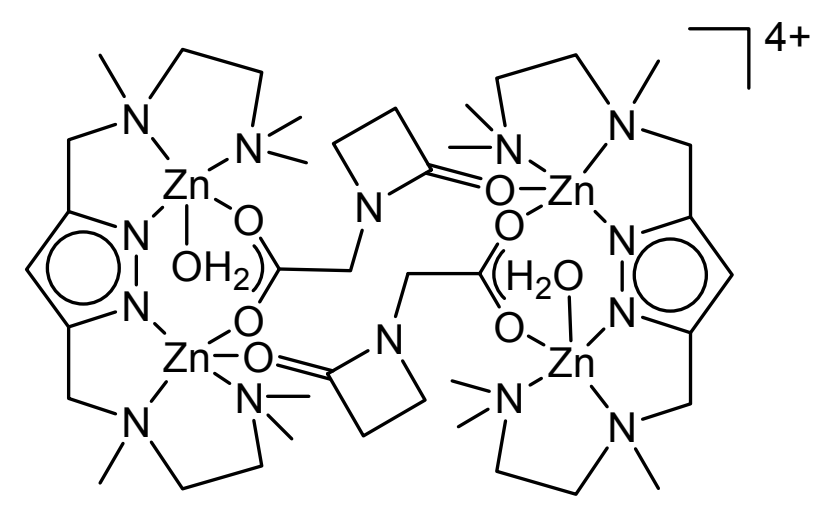

17

Anhand dieses Komplexes konnte gezeigt werden, dass die Carboxylatfunktion genutzt wird, um das Substrat an das Dizinkzentrum zu binden, und zusätzlich eine Koordination und damit eine Aktivierung der $\beta$-Lactam-Carbonylgruppe möglich ist. 


\section{Experimenteller Teil}

\subsection{Allgemeines}

\section{Warnung}

Perchloratsalze von Übergangsmetallkomplexen können explosiv sein! Obwohl während dieser Arbeit keine Probleme auftraten, sollten diese mit entsprechender Vorsicht gehandhabt werden!

\section{Apparaturen}

Es wurden konventionelle Glasapparaturen oder Schlenkkolben verwendet. Sofern nötig, wurden die Apparaturen unter Verwendung eines Heißluftföns ausgeheizt, mehrmals im Ölpumpenvakuum evakuiert und mit Stickstoff (Trocknung durch Sicapent der Firma Merck) gespült.

\section{Lösungsmittel}

Alle Reaktionen wurden sofern notwendig in frisch absolutierten, desoxygenierten und destillierten Lösungsmitteln durchgeführt. Folgende Trocknungsmittel fanden Verwendung:

Aceton: Calciumchlorid Petrolether 40/60: Calciumhydrid

Acetonitril: Phosphorpentoxid Tetrahydrofuran: Natrium/Kalium

Ethanol: Magnesium

Für die kinetischen Messungen mittels UV/Vis-Spektroskopie und IR- Spektroskopie wurde Dimethylsulfoxid SPECTRANAL der Firma Fluka und entgastes bidestilliertes Wasser des Mikroanalytischen Labors der Universität Göttingen verwendet.

\section{NMR-Spektroskopie}

Die NMR-Spektren wurden mit Geräten des Typs Bruker Avance $500\left({ }^{1} \mathrm{H}: 500.13\right.$ $\left.\mathrm{MHz},{ }^{13} \mathrm{C}: 125.77 \mathrm{MHz}\right), 300\left({ }^{1} \mathrm{H}: 300.13 \mathrm{MHz},{ }^{13} \mathrm{C}: 75.47 \mathrm{MHz}\right)$ und $200\left({ }^{1} \mathrm{H}: 200.13\right.$ $\mathrm{MHz},{ }^{13} \mathrm{C}: 50.32 \mathrm{MHz}$ ) aufgenommen. Die angegebenen Werte der Verschiebung $\delta$ beziehen sich auf den internen Standard durch die Lösungsmittel $\mathrm{CDCl}_{3}(\delta=7.24$ für ${ }^{1} \mathrm{H}$-NMR bzw. $\delta=77.0$ für $\left.{ }^{13} \mathrm{C}-\mathrm{NMR}\right), \mathrm{d}_{6}$-Dimethylsulfoxid $\left(\delta=2.49\right.$ für ${ }^{1} \mathrm{H}-\mathrm{NMR}$ bzw. $\delta=39.5$ für $\left.{ }^{13} \mathrm{C}-\mathrm{NMR}\right)$ und $\mathrm{d}_{6}$-Aceton $\left(\delta=2.04\right.$ für ${ }^{1} \mathrm{H}-\mathrm{NMR}$ bzw. $\delta=29.8$ für ${ }^{13} \mathrm{C}$ - 
NMR) relativ zu externem Tetramethylsilan (jeweils $\delta=0$ ). Die ${ }^{13} \mathrm{C}$ - und ${ }^{31} \mathrm{P}-\mathrm{NMR}$ Spektren wurden ${ }^{1} \mathrm{H}$-entkoppelt aufgenommen. ${ }^{31} \mathrm{P}$-NMR Spektren wurden durch $85 \% \mathrm{H}_{3} \mathrm{PO}_{4}$ in $\mathrm{D}_{2} \mathrm{O}$ als externem Standard oder durch Zugabe von Tetramethylphosphoniumchlorid referenziert $(\delta=24.4)$. Wenn nicht anders angegeben, wurden die Spektren bei $300 \mathrm{~K}$ aufgenommen.

Abkürzungen: $\mathrm{s}=$ Singulett, $\mathrm{d}=$ Dublett, $\mathrm{t}=$ Triplett, $\mathrm{m}=$ Multiplett .

Zur Zuordnung der Signale wurden - wenn nötig - zusätzlich DEPT, H-H-COSY und $\mathrm{H}$-C-COSY Experimente durchgeführt. Die Kopplungskonstanten und chemischen Verschiebungen der Pyridylsignale findet sich zusammengefasst in Kapitel 8.7.

\section{Massenspektrometrie}

Die Massenspektren wurden auf den Geräten Finnigan MAT 8200 für El-Spektren (70 eV), Finnigan MAT 95 für FAB-Spektren (Matrix: 4-Nitrobenzylalkohol) und Finnigan MAT LCQ für ESI-Spektren (Lösungsmittel Methanol, wenn nicht anders angegeben) aufgenommen.

\section{Elementaranalytik}

Die CHN-Elementaranalysen erfolgten durch das Mikroanalytische Labor des Instituts für Anorganische Chemie der Universität Göttingen mit einem CHN-O-Rapid der Firma Elementar.

\section{Infrarotspektroskopie}

Die Infrarotspektren wurden auf einem Digilab Excalibur Spektrometer aufgenommen. Feststoffe wurden als KBr-Pressling gemessen. Die Lage der Banden ist in Wellenzahlen angegeben $\left(\tilde{v}\right.$ in $\left.\mathrm{cm}^{-1}\right)$. Die Intensitäten sind wie folgt abgekürzt: $\mathrm{w}=$ schwach, $\mathrm{m}=$ mittel, $\mathrm{s}=$ stark, $\mathrm{vs}=$ sehr stark

\section{Reagentien}

Die nicht aufgeführten Verbindungen waren durch die Chemikalienausgabe des Anorganisch-chemischen Institutes der Universität Heidelberg bzw. der Universität Göttingen erhältlich. Folgende Reagentien wurden über die angegebenen Firmen bezogen oder nach Literaturvorschriften hergestellt: 
2-Azetidinon

Aldrich

Benzylpenicillosäure

Aldrich

2-Brompyridin

Aldrich

tert-Butyl-2-bromacetat

Aldrich

Cephalothin Natriumsalz

Sigma

CHES (Cyclohexylaminethansulfonsäure) Sigma

Dimethylphosphorsäure

Acros

Diphenylphosphinsäure

Aldrich

HEPES

Sigma

(Hydroxyethylpiperazinethansulfonsäure)

HEPPS

Sigma

(Hydroxyethylpiperazinpropansulfonsäure)

Kalium-tert-butylat

Aldrich

Kaliumcarbonat

Merck

Lithiumhydroxid Monohydrat

Fluka

MES (Morpholinethansulfonsäure)

Sigma

$\mathrm{N}$-Methylimidazol

Aldrich

Methanol CHROMASOLV

Fluka

Natriumcyanoborhydrid

Aldrich

Natrium-bis(4-nitrophenyl)phosphat

Sigma

(BNPP)

Penicillin G Natriumsalz

Sigma

Pyridin-2-aldehyd

Aldrich

Sulbactam

Pfizer

Tetrabutylammoniumbromid

Aldrich

Tris(hydroxymethyl)methylamin (TRIS)

Aldrich

Zinkchlorid

Aldrich

Zinkperchlorat Hexahydrat

Aldrich

Zinknitrat Hexahydrat

Aldrich

N-Benzylazetidin-2-on XLVII ${ }^{133]}$

3,5-Bis(chlormethyl)-1-(tetrahydropyran-2-yl)pyrazol $\mathbf{X}^{\text {[69-71] }}$

Dimethyl-1-(tetrahydropyran-2yl)-1H-pyrazol-3,5-dicarboxylat XXI ${ }^{[76]}$

Natrium(2-oxoazetidin-1-yl)acetat XXXLVIII ${ }^{[134]}$ 
N,N'-Diisopropyltriazacyclononan $\mathbf{X V I}^{[72]}$

Bis(2-pyridyl-2-ethyl)amin XVIII ${ }^{\text {[73] }}$

2-Hydroxypropyl-4-nitrophenylphosphat (HPNPP) ${ }^{[139]}$

$\mathrm{Sn}\left[\mathrm{N}\left(\mathrm{SiMe}_{3}\right)_{2}\right]^{[132]}$

Die Synthesen der Liganden $\mathrm{L}^{1}, \mathrm{~L}^{2}, \mathrm{~L}^{3}, \mathrm{~L}^{4}, \mathrm{~L}^{5}, \mathrm{~L}^{6}, \mathrm{~L}^{7}$ und $\mathrm{L}^{8}$ sind in der Literatur beschrieben. ${ }^{[62,63,140,141]}$ Sämtliche Liganden und Ligandvorstufen wurden in dieser Arbeit selbst synthetisiert.

\subsection{Ligandsynthesen}

\subsubsection{Synthese von N-(2-Picolyl)methylamin XIV}

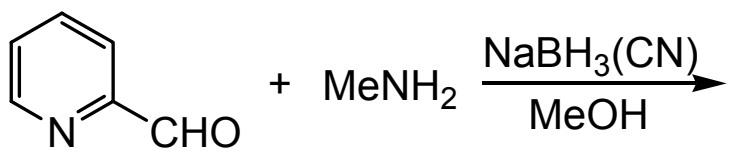

XIII<smiles>CNCc1ccccn1</smiles>

XIV

Zu Pyridin-2-aldehyd $(5.6 \mathrm{ml}, 58.8 \mathrm{mmol})$ in Methanol $(300 \mathrm{ml})$ wird wasserfreies Methylammoniumchlorid (20.0 g, $294 \mathrm{mmol})$ gegeben. Unter Rühren wird Natriumcyanoborhydrid $(3.70 \mathrm{~g}, 58.8 \mathrm{mmol})$ portionsweise zugefügt. Nach drei Tagen Rühren bei Raumtemperatur wird das Reaktionsgemisch mit $3 \mathrm{M} \mathrm{HCl}$ versetzt, bis keine $\mathrm{HCN}$-Entwicklung mehr zu beobachten ist $(\mathrm{pH} 2)$. Das Lösungsmittel wird im Hochvakuum entfernt und der Rückstand mit Dichlormethan (80 ml) extrahiert. Die wässrige Phase wird mit festem $\mathrm{KOH}$ auf $\mathrm{pH} 12$ eingestellt, mit festem $\mathrm{NaCl}$ gesättigt und mit Dichlormethan $(300 \mathrm{ml})$ extrahiert. Die organische Phase wird über Magnesiumsulfat getrocknet und das Lösungsmittel destillativ entfernt. Nach weiterer Destillation im Vakuum erhält man eine farblose Flüssigkeit.

Ausbeute $3.3 \mathrm{~g},(27 \mathrm{mmol}), 46 \%$.

Summenformel Molekulargewicht
$\mathrm{C}_{7} \mathrm{H}_{10} \mathrm{~N}_{2}$

$122.17 \mathrm{~g} / \mathrm{mol}$. 


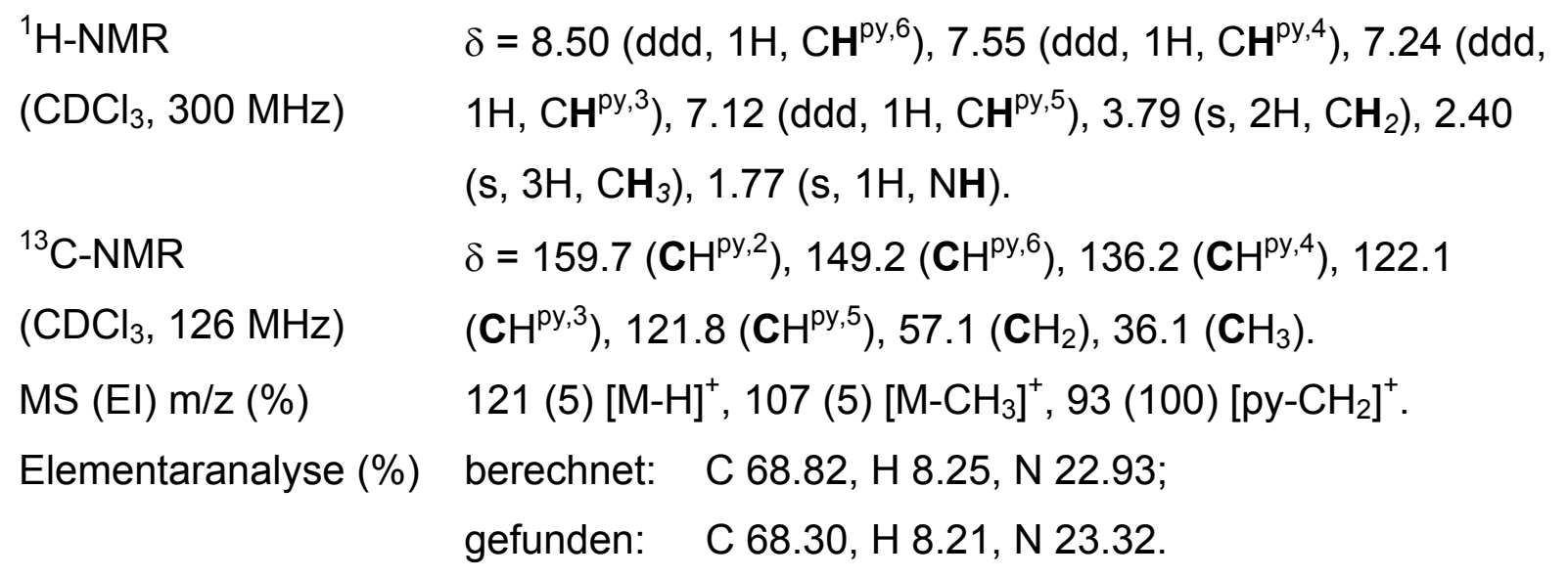

\subsubsection{Synthese von $L^{9}$}

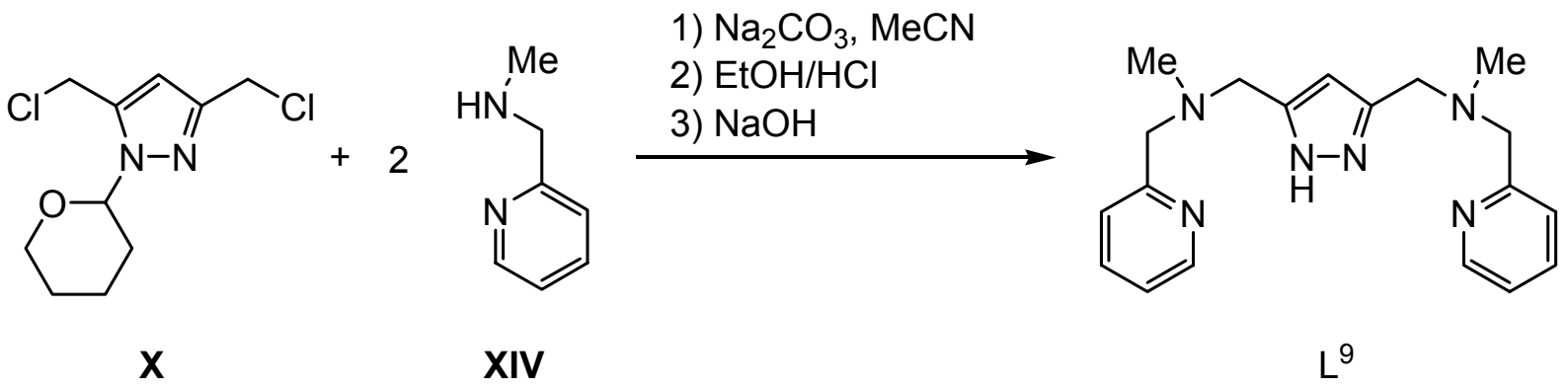

Natriumcarbonat $(5.47 \mathrm{~g}, 51.6 \mathrm{mmol})$ wird bei $100{ }^{\circ} \mathrm{C}$ unter Hochvakuum 2 Stunden erhitzt. Zum wasserfreien Natriumcarbonat werden Acetonitril (225 ml), 3,5Bis(chlormethyl)-1-(tetrahydropyran-2-yl)pyrazol X (3.00 g, $12.0 \mathrm{mmol})$ und N-(2Picolyl)methylamin XIV (3.02 g, $24.7 \mathrm{mmol}$ ) gegeben und die Reaktionsmischung 24 Stunden unter Rückfluss erhitzt. Der unlösliche Rückstand wird anschließend abfiltriert und das Lösungsmittel im Hochvakuum entfernt. Die erhaltene bräunliche ölige Substanz wird in Ethanol $(15 \mathrm{ml})$ gelöst und anschließend mit ethanolischer $\mathrm{HCl}$ (50 ml) versetzt. Nach $1 \mathrm{~h}$ Rühren wird bis zur vollständigen Fällung Diethylether (90 ml) zugegeben. Der Niederschlag wird abfiltriert, in konz. NaOH-Lösung $(75 \mathrm{ml})$ gelöst und mit Dichlormethan $(4 \times 75 \mathrm{ml})$ extrahiert. Die vereinigten organischen Phasen werden mit Magnesiumsulfat getrocknet und das Lösungsmittel im Hochvakuum entfernt. Das Produkt $\mathbf{L}^{9}$ erhält man als bräunliches Öl. 


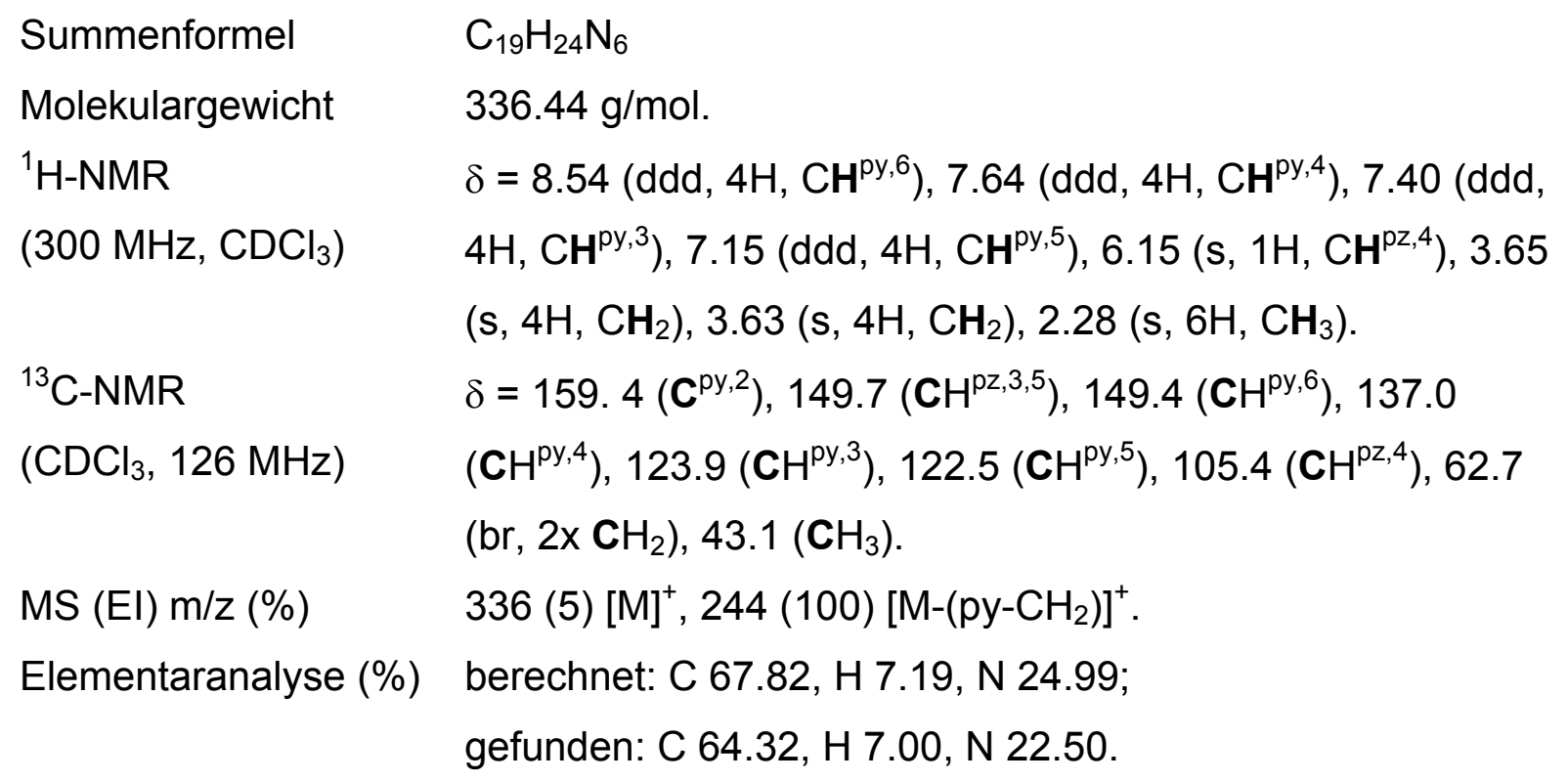

\subsubsection{Synthese von 3,5-Bis(bis(1-methyl)imidazol-2-yl-hydroxymethyl)-1- tetrahydropyran-2-yl-1H-pyrazol XXIla}

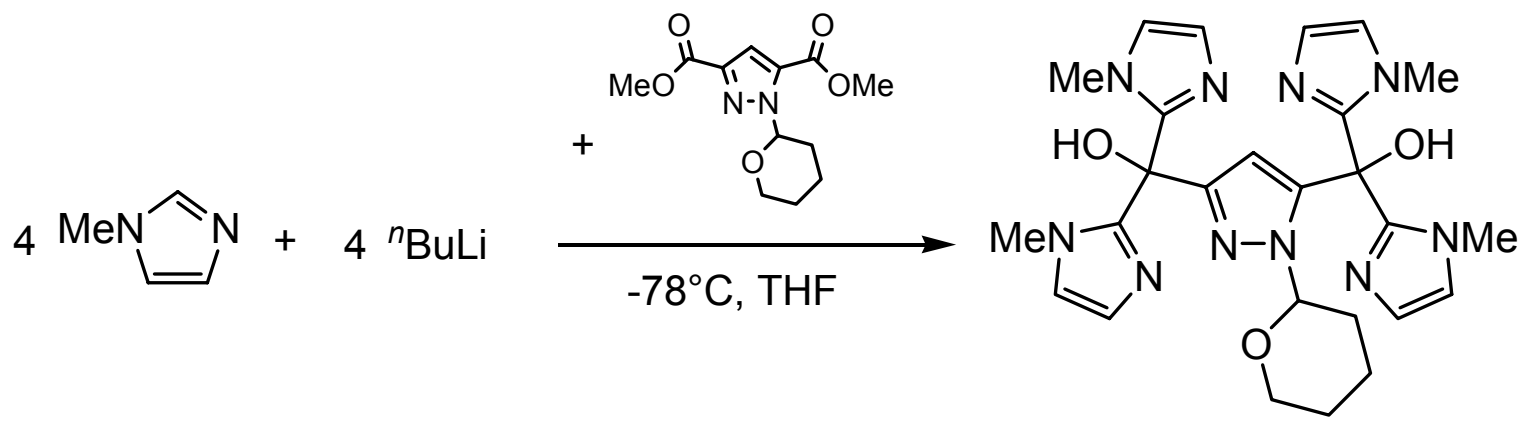

$\mathrm{Zu}$ einer auf $-78{ }^{\circ} \mathrm{C}$ gekühlten Lösung von 1-Methylimidazol $(4.5 \mathrm{ml}, 57 \mathrm{mmol})$ in Tetrahydrofuran $(275 \mathrm{ml})$ wird eine Lösung $(1.6 \mathrm{M}, 39 \mathrm{~mL}, 62 \mathrm{mmol})$ von ${ }^{n} \mathrm{BuLi}$ in Hexan innerhalb von 10 min unter Stickstoff zugetropft. Nach 30 min Rühren bei $-78{ }^{\circ} \mathrm{C}$ wird tropfenweise $\mathbf{X X I}(3.41 \mathrm{~g}, 12.7 \mathrm{mmol})$ in Tetrahydrofuran (30 ml) hinzugefügt und lässt über Nacht unter Rühren auftauen. 5\%-ige Salzsäure wird anschließend bis zu pH 1 zugegeben, die organische Phase verworfen, die wässrige Phase mit verdünnter $\mathrm{NaOH}$ bis $\mathrm{zu} \mathrm{pH} 13$ alkalisch gemacht und mit Ethylacetat $(2 \mathrm{x}$ $100 \mathrm{ml}$ ) extrahiert. Nach dem Trocknen mit Magnesiumsulfat wird das Lösungsmittel im Hochvakuum entfernt. Man erhält ein gelbliches Öl. 
Summenformel

Molekulargewicht

${ }^{1} \mathrm{H}-\mathrm{NMR}$

$\left(\mathrm{CDCl}_{3}, 500 \mathrm{MHz}\right)$

${ }^{13} \mathrm{C}-\mathrm{NMR}$

$\left(\mathrm{CDCl}_{3}, 126 \mathrm{MHz}\right)$

MS (FAB) $\mathrm{m} / \mathrm{z}(\%)$
$\mathrm{C}_{26} \mathrm{H}_{32} \mathrm{~N}_{10} \mathrm{O}_{3}$

$532.60 \mathrm{~g} / \mathrm{mol}$.

$\delta=6.84\left(\mathrm{~m}, 8 \mathrm{H}, \mathrm{CH}^{\mathrm{imid}, 4,5}\right), 5.86\left(\mathrm{~s}, 1 \mathrm{H}, \mathrm{CH}^{\mathrm{pz}, 4}\right), 5.39(\mathrm{~m}, 1 \mathrm{H}$, $\left.\mathrm{CH}^{\text {thp, } 2}\right), 3.61,3.45,3.42,3.34\left(\mathrm{~s}, 12 \mathrm{H}, \mathrm{CH}_{3}\right), 3.29-3.66(\mathrm{~m}$, $\left.2 \mathrm{H}, \mathrm{CH}^{\text {thp }, 6,6^{\prime}}\right), 2.17\left(\mathrm{~m}, 1 \mathrm{H}, \mathrm{CH}^{\mathrm{thp}, 3^{\prime}}\right), 1.86\left(\mathrm{~m}, 1 \mathrm{H}, \mathrm{CH}^{\mathrm{thp}, 3}\right)$, $1.77\left(\mathrm{~m}, 1 \mathrm{H}, \mathrm{CH}^{\mathrm{thp}, 4}\right), 1.47\left(\mathrm{~m}, 2 \mathrm{H}, \mathrm{CH}^{\mathrm{thp} 5,4}\right), 1.35(\mathrm{~m}, 1 \mathrm{H}$, $\left.\mathrm{CH}^{\text {thp }, 5^{\prime}}\right)$.

$\delta=152.03\left(\mathbf{C}^{\mathrm{pz}, 5}\right), 147.29,147.16,146.66,146.26\left(\mathbf{C H}^{\mathrm{imid}, 2}\right)$, $142.82\left(\mathbf{C}^{\mathrm{pz}, 3}\right), 126.48,125.69,125.60,125.38\left(\mathbf{C H}^{\mathrm{imid}, 5}\right)$, 123.71, 123.40, 123.39, $123.30\left(\mathbf{C H}^{\mathrm{imid}, 4}\right), 107.25\left(\mathbf{C H}^{\mathrm{pz}, 4}\right)$, $85.00\left(\mathrm{CH}^{\text {thp, }, 2}\right), 71.38,70.10(\mathrm{C}-\mathrm{OMe}), 67.68\left(\mathrm{CH}_{2}^{\text {thp }, 6}\right)$, $34.44,34.38,34.35,34.27\left(\mathrm{NCH}_{3}\right), 29.16\left(\mathrm{CH}_{2}^{\text {thp }, 3}\right), 24.66$ $\left(\mathrm{CH}_{2}^{\text {thp, } 5}\right), 22.64\left(\mathrm{CH}_{2}^{\text {thp, }, 4}\right)$.

$555(7)[\mathrm{M}+\mathrm{Na}]^{+}, 533(12)[\mathrm{M}+\mathrm{H}]^{+}$.

\subsubsection{Synthese von 3,5-Bis(bis(1-methyl)imidazol-2-yl-methoxymethyl)-1- tetrahydropyran-2-yl-1H-pyrazol XXIIla}
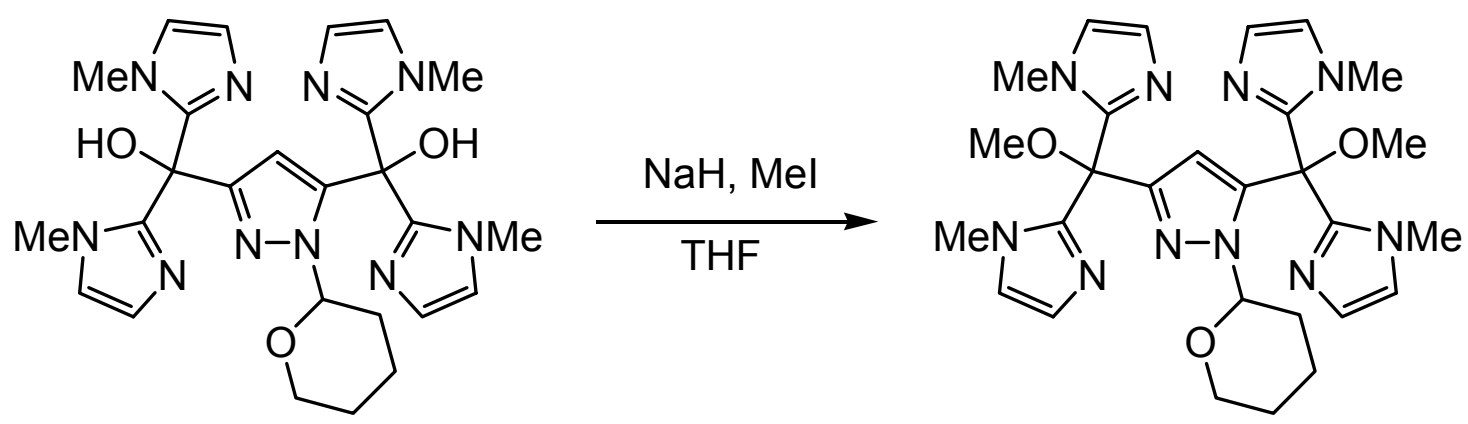

Zu einer Lösung von $\mathrm{NaH}(0.19 \mathrm{mg}, 7.92 \mathrm{mmol})$ in THF $(50 \mathrm{ml})$ unter Stickstoff wird bei Raumtemperatur XXIla $(2.00 \mathrm{~g}, 3.75 \mathrm{mmol})$ unter Rühren zugegeben und $1 \mathrm{~h}$ gerührt. Anschließend wird Mel (1.06 g, $7.47 \mathrm{mmol})$ zugefügt und über Nacht gerührt. Nach der Zugabe von ges. NaCl-Lösung $(25 \mathrm{ml}$ ) wird die Mischung mit Chloroform (2x $30 \mathrm{ml}$ ) extrahiert, die Chloroformphase mit Magnesiumsulfat getrocknet und das Lösungsmittel im Vakuum entfernt. Durch Säulenchromatographie (Kieselgel, Methanol/Ethylacetat 1:2) wird ein schwach gelblicher Feststoff erhalten. 
Ausbeute

Summenformel

Molekulargewicht

${ }^{1} \mathrm{H}-\mathrm{NMR}$

$\left(\mathrm{CDCl}_{3}, 500 \mathrm{MHz}\right)$

${ }^{13} \mathrm{C}-\mathrm{NMR}$

$\left(\mathrm{CDCl}_{3}, 126 \mathrm{MHz}\right)$

MS (FAB) $\mathrm{m} / \mathrm{z}(\%)$
$0.721 \mathrm{~g},(1.3 \mathrm{mmol}), 35 \%$.

$\mathrm{C}_{28} \mathrm{H}_{36} \mathrm{~N}_{10} \mathrm{O}_{3}$

$560.65 \mathrm{~g} / \mathrm{mol}$.

$\delta=6.95\left(\mathrm{~m}, 4 \mathrm{H}, \mathrm{CH}^{\mathrm{imid}, 5}\right), 6.83\left(\mathrm{~m}, 4 \mathrm{H}, \mathrm{CH}^{\mathrm{imid}, 4}\right), 6.43(\mathrm{~s}, 1 \mathrm{H}$,

$\left.\mathrm{CH}^{\mathrm{pz}, 4}\right), 5.49\left(\mathrm{~d},{ }^{3} \mathrm{~J}=8.06 \mathrm{~Hz}, 1 \mathrm{H}, \mathrm{CH}^{\mathrm{thp}, 2}\right), 3.62(\mathrm{~m}, 1 \mathrm{H}$,

$\left.\mathrm{CH}^{\text {thp,6}}\right), 3.54,3.48,3.39,3.37$ (s, $\left.12 \mathrm{H}, \mathrm{Me}\right), 3.38,3.35$ (s,

$\left.6 \mathrm{H}, \mathrm{OCH}_{3}\right), 3.29\left(\mathrm{~m}, 1 \mathrm{H}, \mathrm{CH}^{\text {thp, } 6^{\prime}}\right), 2.16\left(\mathrm{~m}, 1 \mathrm{H}, \mathrm{CH}^{\text {thp, } 3^{\prime}}\right), 1.81$

$\left(\mathrm{m}, 1 \mathrm{H}, \mathrm{CH}^{\mathrm{thp}, 3}\right), 1.62\left(\mathrm{~m}, 1 \mathrm{H}, \mathrm{CH}^{\text {thp, } 4}\right), 1.48\left(\mathrm{~m}, 2 \mathrm{H}, \mathrm{CH}^{\text {thp5,4'}}\right)$,

$1.32\left(\mathrm{~m}, 1 \mathrm{H}, \mathrm{CH}^{\text {thp, } 5^{\prime}}\right)$.

$\delta=149.54\left(\mathbf{C}^{\mathrm{pz}, 5}\right), 145.81,145.64,144.16,144.10,\left(\mathbf{C H}^{\mathrm{imid}, 2}\right)$, $140.02\left(\mathbf{C}^{\mathrm{pz}, 3}\right), 126.77,126.70,126.62,126.55,\left(\mathbf{C H}^{\mathrm{imid}, 5}\right)$, 123.77, 123.76, 123.08, $123.04\left(\mathrm{CH}^{\mathrm{imid}, 4}\right), 108.81\left(\mathrm{CH}^{\mathrm{pz}, 4}\right)$, $85.31\left(\mathrm{CH}^{\text {thp }, 2}\right), 78.46,77.20(\mathrm{COMe}), 67.83\left(\mathrm{CH}_{2}^{\text {thp }, 6}\right)$, 54.22, 53.77, $\left(\mathrm{OCH}_{3}\right), 34.67,34.57,34.55,34.49\left(\mathrm{NCH}_{3}\right)$, $29.59\left(\mathrm{CH}_{2}^{\text {thp, }, 3}\right), 24.80\left(\mathrm{CH}_{2}^{\text {thp, }, 5}\right), 22.88\left(\mathrm{CH}_{2}^{\text {thp }, 4}\right)$.

$583(50)[\mathrm{M}+\mathrm{Na}]^{+}, 561(100)[\mathrm{M}+\mathrm{H}]^{+}$

\subsubsection{Synthese von 3,5-Bis(bis(1-methyl)imidazol-2-yl-methoxymethyl)- 1H-pyrazol XXIVa}
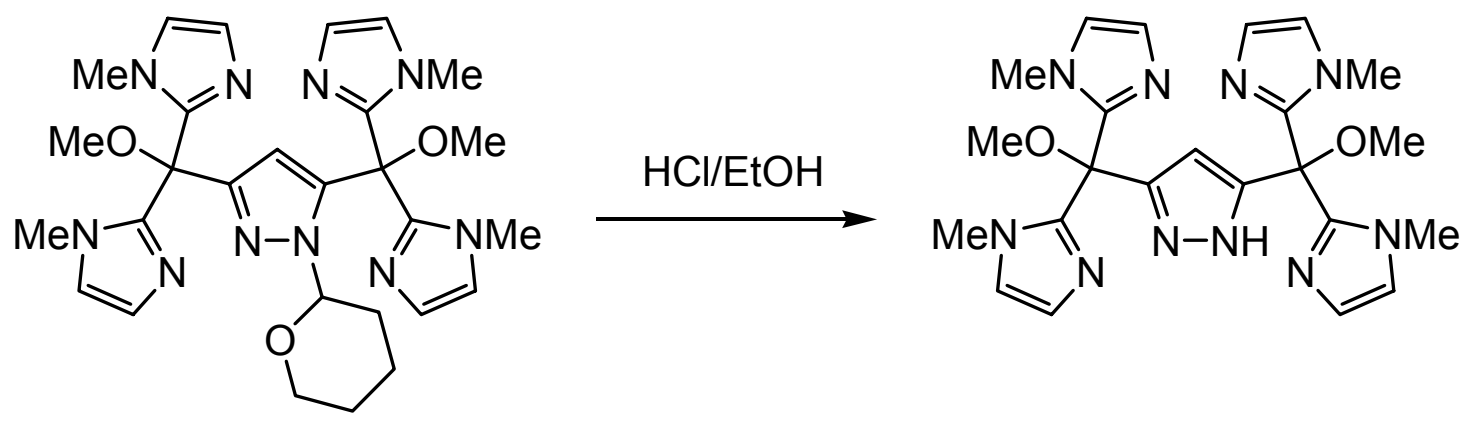

Zu XXIlla (0.7 g, $1.25 \mathrm{mmol})$ wird gesättigten Chlorwasserstofflösung in Ethanol (75 ml) gegeben und über Nacht gerührt. Anschließend wird zur vollständigen Fällung Diethylether $(150 \mathrm{ml})$ zugegeben und der weiße Niederschlag abfiltriert. Nach Trocknen im Vakuum wird der weiße Feststoff in verdünnter $\mathrm{NaOH}(50 \mathrm{ml})$ gelöst, mit Dichlormethan $(2 \times 100 \mathrm{ml})$ extrahiert, mit Magnesiumsulfat getrocknet und das Lösungsmittel im Vakuum entfernt. Man erhält das Produkt als farbloses Öl. 
Ausbeute

Summenformel

Molekulargewicht

${ }^{1} \mathrm{H}-\mathrm{NMR}$

$\left(\mathrm{CDCl}_{3}, 500 \mathrm{MHz}\right)$

${ }^{13} \mathrm{C}-\mathrm{NMR}$

$\left(\mathrm{CDCl}_{3}, 126 \mathrm{MHz}\right)$

MS (FAB) $\mathrm{m} / \mathrm{z}(\%)$
$0.31 \mathrm{~g},(0.65 \mathrm{mmol}), 52 \%$.

$\mathrm{C}_{23} \mathrm{H}_{28} \mathrm{~N}_{10} \mathrm{O}_{2}$

$476.24 \mathrm{~g} / \mathrm{mol}$.

$\delta=13.3(\mathrm{~s}, \mathrm{br}, 1 \mathrm{H}, \mathrm{NH}), 6.95\left(\mathrm{~m}, 4 \mathrm{H}, \mathrm{CH}^{\mathrm{imid}, 5}\right), 6.83(\mathrm{~m}, 4 \mathrm{H}$,

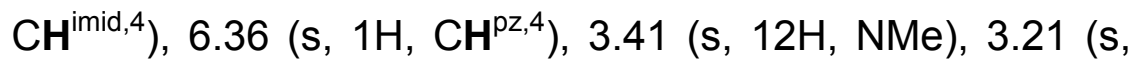
$\left.6 \mathrm{H}, \mathrm{OCH}_{3}\right)$.

$\delta=145.1\left(\mathbf{C H}^{\text {imid,2}}\right), 126.5\left(\mathbf{C H}^{\text {imid,5}}\right), 123.1\left(\mathbf{C H}^{\text {imid,4 }}\right), 106.4$ $\left(\mathrm{CH}^{\mathrm{pz}, 4}\right), 52.5\left(\mathrm{OCH}_{3}\right), 34.1\left(\mathrm{NCH}_{3}\right)$.

$477.4(100)[\mathrm{M}+\mathrm{H}]^{+}$.

\subsubsection{Synthese von 3,5-Bis(dipyridin-2-yl-hydroxymethyl)-1- tetrahydropyran-2-yl-1H-pyrazol XXIlb}

4

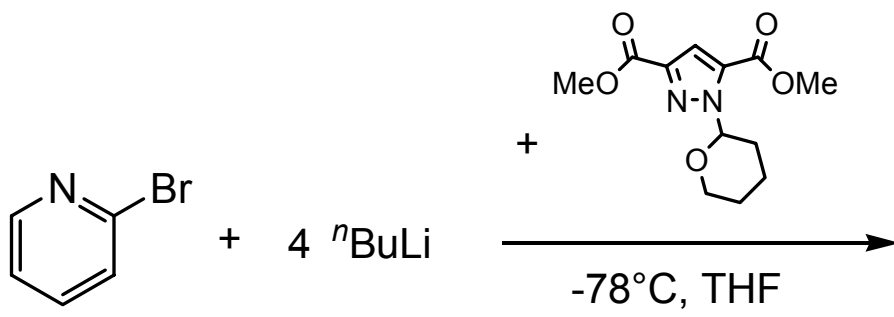

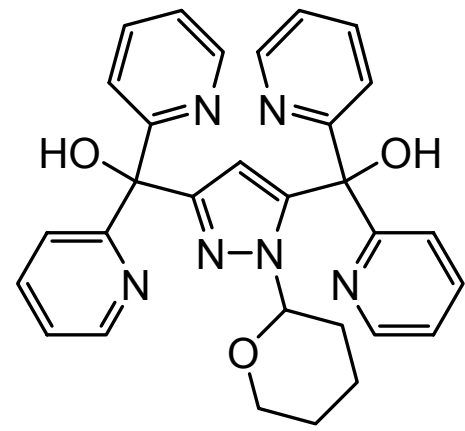

Unter Stickstoffatmosphäre wird zu einer Lösung von 2-Brompyridin (5.9 ml, $9.5 \mathrm{~g}, 60$ mmol, $4.5 \mathrm{eq})$ in trockenem THF $(300 \mathrm{ml})$ bei $-78^{\circ} \mathrm{C}{ }^{n} \mathrm{BuLi}$ in Hexan $(1.6 \mathrm{M}, 41 \mathrm{ml}, 66$ mmol, 4.9 eq) zugetropft. Nach $1 \mathrm{~h}$ wird XXI (3.58 g, $13.3 \mathrm{mmol}, 1 \mathrm{eq})$ in trockenem THF $(20 \mathrm{ml})$ zugegeben und lässt die Reaktion über Nacht auftauen. Die Reaktionsmischung wird mit Ammoniumchloridlösung $(30 \mathrm{ml})$ hydrolysiert und die entstandenen Salze werden abfiltriert. Die organische Phase wird abgetrennt und das Lösungsmittel im Vakuum entfernt. Das Produkt wird in Dichlormethan aufgenommen und mit Wasser gewaschen. Die gesammelten organischen Phasen werden über Magnesiumsulfat getrocknet und das Lösungsmittel am Vakuum entfernt. Das Produkt wird ohne weitere Reinigung direkt weiterverwendet. 
Ausbeute

Summenformel

Molekulargewicht

${ }^{1} \mathrm{H}-\mathrm{NMR}$

$\left(\mathrm{CDCl}_{3}, 200 \mathrm{MHz}\right)$ : nicht bestimmt

$\mathrm{C}_{30} \mathrm{H}_{28} \mathrm{~N}_{6} \mathrm{O}_{2}$

$504.23 \mathrm{~g} / \mathrm{mol}$.

$\delta=7.10-6.20(\mathrm{~m}, 16 \mathrm{H}, \mathrm{py}), 5.80(\mathrm{~s}, 1 \mathrm{H}$, pyrazol) $), 4.20$ -

$3.90(\mathrm{~m}, 1 \mathrm{H}$, thp), $2.90-0.70(\mathrm{~m}, 8 \mathrm{H}$, thp).

\subsubsection{Synthese von 3,5-Bis(dipyridin-2-yl-methoxymethyl)-1- tetrahydropyran-2-yl-1H-pyrazol XXIIIb}
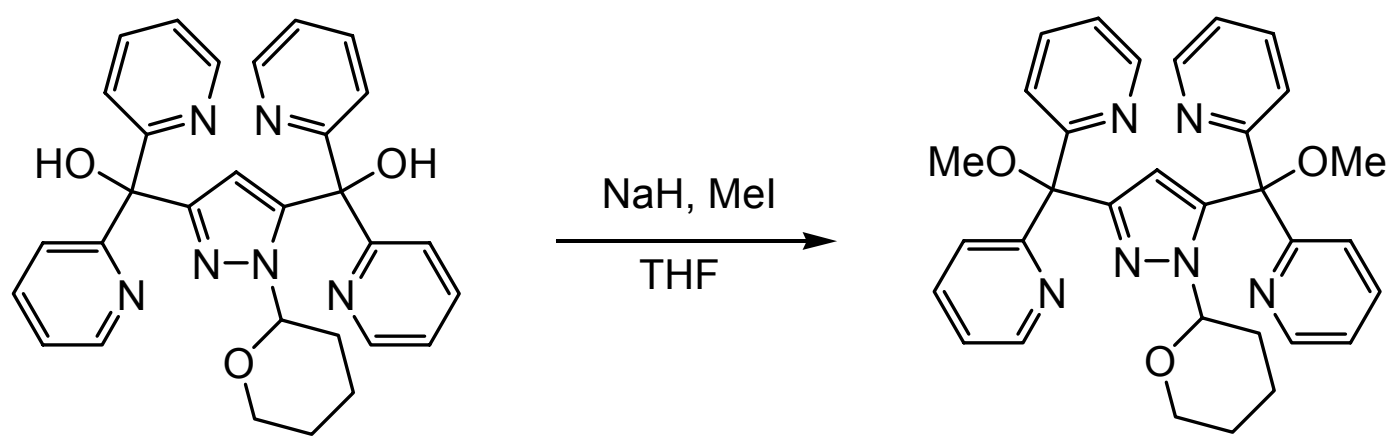

Unter Stickstoffatmosphäre wird XXIIb $(13.3 \mathrm{mmol})$ in THF $(100 \mathrm{ml})$ gelöst und mit $(0.800 \mathrm{~g}, 26.6 \mathrm{mmol})$ Natriumhydrid versetzt. Nach $10 \mathrm{~min}$ wird $(2.5 \mathrm{ml}, 5.7 \mathrm{~g}, 40$ mmol) Methyliodid zugetropft. Nach weiteren 2 Stunden wird der Niederschlag abfiltriert und das Lösungsmittel im Vakuum entfernt. Das Produkt wird säulenchromatographisch (Kieselgel, Ethylacetat mit 1\% Triethylamin) gereinigt. Man erhält das Produkt als gelbes Öl.

Ausbeute

Summenformel

Molekulargewicht

${ }^{1} \mathrm{H}-\mathrm{NMR}$

$\left(\mathrm{CDCl}_{3}, 500 \mathrm{MHz}\right)$ :
$0.779 \mathrm{~g},(1.46 \mathrm{mmol}), 11 \%$ (über zwei Stufen).

$$
\mathrm{C}_{32} \mathrm{H}_{32} \mathrm{~N}_{6} \mathrm{O}_{2}
$$

$532.26 \mathrm{~g} / \mathrm{mol}$.

$\delta=8.50-8.53\left(\mathrm{~m}, 4 \mathrm{H}, \mathrm{CH}^{\mathrm{py}, 6}\right), 7.60-7.69\left(\mathrm{~m}, 8 \mathrm{H}, \mathrm{CH}^{\mathrm{py}, 3,4}\right)$, 7.09-7.12 (m, 4H, $\left.\mathrm{CH}^{\mathrm{py}, 5}\right), 6.46\left(\mathrm{~s}, 1 \mathrm{H}, \mathrm{CH}^{\mathrm{pz}, 4}\right), 5.12(\mathrm{~d}, J=$ $\left.8.5 \mathrm{~Hz}, 1 \mathrm{H}, \mathrm{CH}^{\text {thp, } 2}\right), 4.10\left(\mathrm{~d}, J=7.0 \mathrm{~Hz}, 2 \mathrm{H}, \mathrm{CH}_{2}^{\text {thp, } 6}\right), 3.33$ (s, $\left.3 \mathrm{H}, \mathrm{OCH}_{3}\right), 3.27$ (s, 3H, $\left.\mathrm{OCH}_{3}\right), 1.75-1.85(\mathrm{~m}, 2 \mathrm{H}$, $\left.\mathrm{CH}_{2}{ }^{\text {thp }, 3}\right), 1.40-1.55\left(\mathrm{~m}, 2 \mathrm{H}, \mathrm{CH}_{2}{ }^{\text {thp }, 5}\right), 1.26-1.29(\mathrm{~m}, 2 \mathrm{H}$, 


$$
\left.\mathrm{CH}_{2}{ }^{\text {thp }, 5}\right) \text {. }
$$

${ }^{13} \mathrm{C}-\mathrm{NMR}$

$\left(\mathrm{CDCl}_{3}, 126 \mathrm{MHz}\right)$ :
$\delta=161.81\left(\mathbf{C}^{\mathrm{py}, 2}\right), 149.57,149.64,149.67,149.69\left(\mathbf{C} \mathrm{H}^{\mathrm{py}, 6}\right)$, 137.30, 137.45, 137.59, $137.66\left(\mathrm{CH}^{\mathrm{py}, 4}\right), 124.44,125.12$ $\left(\mathbf{C}^{\mathrm{pz}, 3,5}\right), 123.97,123.15,123.19,123.25123 .39,123.55$ $\left(\mathbf{C H} \mathrm{H}^{\mathrm{py}, 3,5}\right), 113.15\left(\mathbf{C H}^{\mathrm{pz}, 4}\right), 86.00\left(\mathbf{C H}^{\mathrm{thp}, 2}\right), 68.73\left(\mathrm{CH}_{2}^{\text {thp }, 6}\right)$, 54.10, $54.39\left(\mathrm{OCH}_{3}\right), 30.95\left(\mathrm{CH}_{2}{ }^{\text {thp }, 3}\right), 25.63\left(\mathrm{CH}_{2}{ }^{\text {thp }, 5}\right), 23.87$ $\left(\mathrm{CH}_{2}^{\text {thp,4}}\right)$.

\subsubsection{Synthese von 3,5-Bis(dipyridin-2-yl-methoxymethyl)-1-H-pyrazol XXIVb}

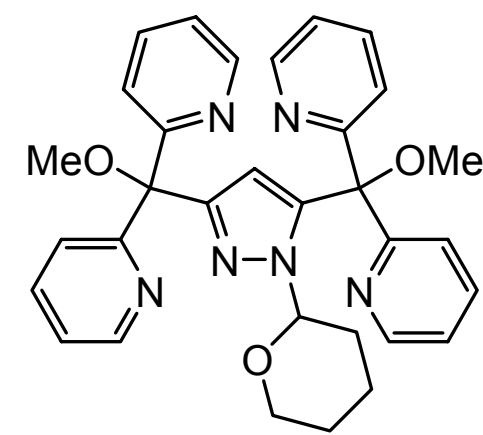

$\mathrm{HCl} / \mathrm{EtOH}$

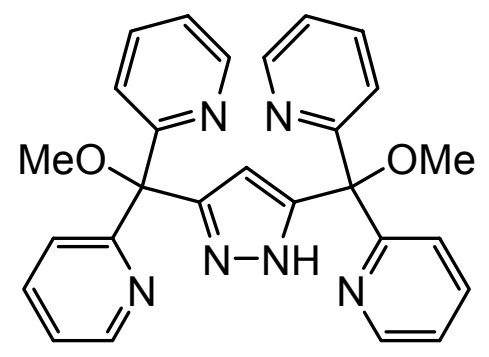

Zur Entschützung wird XXIIlb $0.779 \mathrm{~g}$ ) in ethanolischer $\mathrm{HCl}$ aufgenommen. Nach zweistündigem Rühren wird das Hydrochlorid mit Diethylether ausgefällt, der Niederschlag mit wässriger $\mathrm{NaOH}$-Lösung neutralisiert, mit Diethylether extrahiert und die organische Phase mit $\mathrm{MgSO}_{4}$ getrocknet. Nach Entfernen des Lösungsmittels im Vakuum erhält man das Produkt als gelblichen Feststoff.

Ausbeute

Summenformel

Molekulargewicht

${ }^{1} \mathrm{H}-\mathrm{NMR}$

$\left(\mathrm{CDCl}_{3}, 500 \mathrm{MHz}\right)$ :

${ }^{13} \mathrm{C}-\mathrm{NMR}$

$\left(\mathrm{CDCl}_{3}, 126 \mathrm{MHz}\right)$ :
$332 \mathrm{mg},(0.715 \mathrm{mmol}), 49 \%$.

$\mathrm{C}_{27} \mathrm{H}_{24} \mathrm{~N}_{6} \mathrm{O}_{2}$

$464.20 \mathrm{~g} / \mathrm{mol}$.

$\delta=8.48-8.51\left(\mathrm{~m}, 4 \mathrm{H}, \mathrm{CH}^{\mathrm{py}, 6}\right), 7.53-7.84\left(\mathrm{~m}, 8 \mathrm{H}, \mathrm{CH}^{\mathrm{py}, 4,3}\right)$, 7.06-7.14 (m, 4H, $\left.\mathrm{CH}^{\mathrm{py}, 5}\right), 6.17\left(\mathrm{~s}, 1 \mathrm{H}, \mathrm{CH}^{\mathrm{pz}, 4}\right), 3.24(\mathrm{~s}, 3 \mathrm{H}$, $\left.\mathrm{OCH}_{3}\right), 3.17\left(\mathrm{~s}, 3 \mathrm{H}, \mathrm{OCH}_{3}\right)$. $\delta=162.13\left(\mathbf{C}^{\mathrm{py}, 2}\right), 148.48\left(\mathbf{C H}^{\mathrm{py}, 6}\right), 148.00,148.37,\left(\mathbf{C}^{\mathrm{pz}, 3,5}\right)$, 136.49, $136.63\left(\mathrm{CH}^{\mathrm{py}, 4}\right), 121.80,122.16,122.43\left(\mathbf{C H}^{\mathrm{py}, 3,5}\right)$, 
MS (EI) $\mathrm{m} / \mathrm{z}(\%)$

$107.25\left(\mathrm{CH}^{\mathrm{pz}, 4}\right), 52.82\left(\mathrm{OCH}_{3}\right)$.

$464(8)[\mathrm{M}]^{+}, 434(70)[\mathrm{M}-2 \mathrm{Me}]^{+}, 402(55)[\mathrm{M}-2 \mathrm{OMe}]^{+}, 386$

(16) $\left[\mathrm{M}-\mathrm{C}_{5} \mathrm{H}_{4} \mathrm{~N}\right]^{+}$. 


\subsection{Substratsynthesen}

\subsubsection{Synthese von tert-Butyl-2-(2-Oxoazetidin-1-yl)acetat}

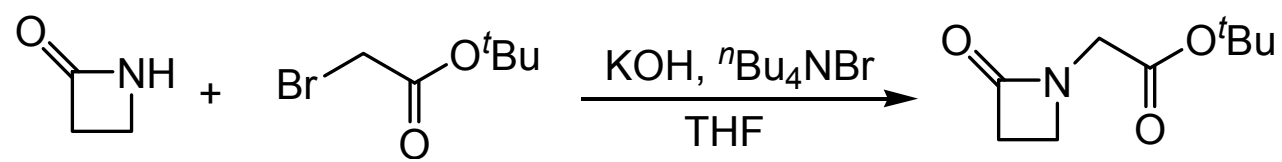

tert-Butyl-2-bromacetat $(3.05 \mathrm{~g}, 17.8 \mathrm{mmol})$ und pulverisiertes Kaliumhydroxid $(1.16 \mathrm{~g}, 17.6 \mathrm{mmol})$ werden zu einer Suspension von 2-Azetidinon $(1.11 \mathrm{~g}, 15.6$ $\mathrm{mmol}$ ) und Tetrabutylammoniumbromid (51 $\mathrm{mg}, 0.16 \mathrm{mmol}$ ) in Tetrahydrofuran (100 $\mathrm{ml}$ ) gegeben. Die Mischung wird $18 \mathrm{~h}$ gerührt, filtriert und das Filtrat im Vakuum eingeengt. Der Rückstand wird durch Säulenchromatographie (Kieselgel, Ethylacetat/Hexan 2:1) gereinigt. Man erhält das Produkt als weißen Feststoff.

Ausbeute

Summenformel

Molekulargewicht

${ }^{1} \mathrm{H}-\mathrm{NMR}$

$\left(\mathrm{CDCl}_{3}, 500 \mathrm{MHz}\right)$

${ }^{13} \mathrm{C}-\mathrm{NMR}$

$\left(\mathrm{CDCl}_{3}, 126 \mathrm{MHz}\right)$

IR (KBr)

MS (El) m/z (\%)

Elementaranalyse
$1.61 \mathrm{~g},(6.26 \mathrm{mmol}), 40 \%$.

$\mathrm{C}_{9} \mathrm{H}_{15} \mathrm{NO}_{3}$

$185.22 \mathrm{~g} / \mathrm{mol}$.

$3.84\left(\mathrm{~s}, 2 \mathrm{H}, \mathrm{CH}_{2}{ }^{\mathrm{az}, 4}\right), 3.38\left(\mathrm{t},{ }^{3} \mathrm{~J}_{\mathrm{HH}}=4.2 \mathrm{~Hz}, 2 \mathrm{H}, \mathrm{CH}_{2}\right), 2.97$

$\left(\mathrm{t},{ }^{3} \mathrm{~J}_{\mathrm{HH}}=4.2 \mathrm{~Hz}, 2 \mathrm{H}, \mathrm{CH}_{2}^{\mathrm{az}, 3}\right), 1.42\left(\mathrm{~s}, 9 \mathrm{H}, \mathrm{C}\left(\mathrm{CH}_{3}\right)_{3}\right)$.

$167.9(\mathrm{CO}), 167.3(\mathrm{CO}), 82.3\left(\mathbf{C}\left(\mathrm{CH}_{3}\right)_{3}\right), 43.8\left(\mathrm{~N}-\mathrm{CH}_{2}-\mathrm{CO}\right)$,

$39.9\left(\mathrm{CH}_{2}^{\mathrm{az}, 4}\right), 37.5\left(\mathrm{CH}_{2}^{\mathrm{az}, 3}\right), 28.0\left(\mathrm{C}\left(\mathrm{CH}_{3}\right)_{3}\right)$.

$\widetilde{v}=2981(w), 2921(w), 1747(s), 1730(s), 1458(w), 1413$

(m), $1368(\mathrm{~m}), 1305$ (w), $1234(\mathrm{~m}), 1160$ (m), 1059 (w), 933

$(w), 861(w), 798(w), 739(w), 581(w), 451(w) \mathrm{cm}^{-1}$.

$185(5)[\mathrm{M}]^{+}, 129(5)\left[\mathrm{M}-{ }^{t} \mathrm{Bu}\right]^{+}, 112(10)\left[\mathrm{M}-\mathrm{O}^{t} \mathrm{Bu}\right]^{+}, 101(5)$

$\left[\mathrm{COO}^{t} \mathrm{Bu}\right]^{+}, 84(70)\left[\mathrm{M}-\mathrm{COO}^{t} \mathrm{Bu}\right]^{+}, 57\left(100,\left[{ }^{t} \mathrm{Bu}\right]^{+}\right.$.

berechnet: C 58.36, H 8.16, N 7.56;

gefunden: C 58.36, H 8.13, N 7.54. 


\subsection{Komplexsynthesen}

\subsubsection{Synthese von $\left[\mathrm{L}^{1}{ }_{\mathrm{H}} \mathrm{Zn}_{2}(\mathrm{OH})\right]\left(\mathrm{ClO}_{4}\right)_{2} 1 \cdot\left(\mathrm{ClO}_{4}\right)_{2}$}

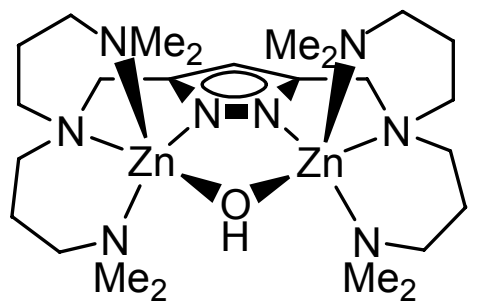

$\left(\mathrm{ClO}_{4}\right)_{2}$

$\mathrm{Zu}$ einer Lösung von $\mathrm{L}^{1}(629 \mathrm{mg}, 1.35 \mathrm{mmol})$ in $\mathrm{MeOH}(150 \mathrm{~mL})$ werden zwei Äquivalente $\mathrm{KO}^{t} \mathrm{Bu}\left(303 \mathrm{mg}, 2.70 \mathrm{mmol}\right.$ ) und zwei Äquivalente $\mathrm{Zn}\left(\mathrm{ClO}_{4}\right)_{2}-6 \mathrm{H}_{2} \mathrm{O}$ (1004 mg, $2.70 \mathrm{mmol}$ ) zugefügt und bei Raumtemperatur für $12 \mathrm{~h}$ gerührt. Nach Entfernung aller flüchtigen Bestandteile im Vakuum wird der Rückstand in Aceton (70 $\mathrm{mL}$ ) aufgenommen, abfiltriert und mit Petrolether überschichtet. Die nach Kristallisation erhaltenen farblosen Kristalle sind analysenrein.

Ausbeute

Summenformel

Molekulargewicht

${ }^{1} \mathrm{H}-\mathrm{NMR}$

( $d_{6}$-Aceton, $500 \mathrm{MHz}$ )

${ }^{13} \mathrm{C}-\mathrm{NMR}$

( $\mathrm{d}_{6}$-Aceton, $126 \mathrm{MHz}$ )

$\mathrm{IR}(\mathrm{KBr})$

$592 \mathrm{mg},(0.729 \mathrm{mmol}), 54 \%$.

$\mathrm{C}_{25} \mathrm{H}_{54} \mathrm{Cl}_{2} \mathrm{~N}_{8} \mathrm{O}_{9} \mathrm{Zn}_{2}$

$812.43 \mathrm{~g} / \mathrm{mol}$.

$\delta=6.09\left(\mathrm{~s}, 1 \mathrm{H}, \mathrm{pz}-\mathrm{H}^{4}\right), 3.76\left(\mathrm{~s}, 4 \mathrm{H}, \mathrm{pz}-\mathrm{CH}_{2}\right), 3.29(\mathrm{br}, 4 \mathrm{H}$, $\mathrm{CH}_{2}$ ), 2.80 (br, 36H, $\mathrm{CH}_{2}, \mathrm{CH}_{3}$ ), 2.55 (br, 8H, $\mathrm{CH}_{2}$ ), 2.15 (br, $8 \mathrm{H}, \mathrm{CH}_{2}$ ).

$\delta=150.3\left(\mathrm{pz}-\mathrm{C}^{3,5}\right), 100.1\left(\mathrm{pz}-\mathrm{C}^{4}\right)$, 62.6, 54.8, 54.0, 50.8, 48.4, $23.0\left(\mathrm{CH}_{2}\right)$.

$\widetilde{v}=3614(\mathrm{~m}), 3115(\mathrm{w}), 2932(\mathrm{br}, \mathrm{m}), 2851(\mathrm{~m}), 1712(\mathrm{~m})$, 1465 (m), 1183 (m), 1098 (vs), $1014(\mathrm{~m}), 973$ (m), 804 (m), $623(\mathrm{~s}) \mathrm{cm}^{-1}$.

MS (ESI) $\mathrm{m} / \mathrm{z}(\%)$ $709.5(100)\left[\mathrm{L}_{-\mathrm{H}} \mathrm{Zn}_{2}(\mathrm{OH})\left(\mathrm{ClO}_{4}\right)\right]^{+}$.

Elementaranalyse (\%) berechnet C 36.96 H 6.70 N 13.79;

gefunden C 36.94 H 6.79 N 12.55 . 


\subsubsection{Synthese von $\left[\mathrm{L}^{3}{ }_{\mathrm{H}} \mathrm{Zn}_{2}(\mathrm{MeOH})(\mathrm{OH})\right]\left(\mathrm{ClO}_{4}\right)_{2} 2 \mathrm{~b} \cdot\left(\mathrm{ClO}_{4}\right)_{2}$}

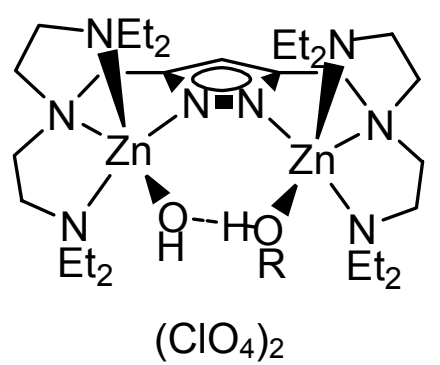

Zu einer Lösung von $\mathrm{L}^{3}(300 \mathrm{mg}, 0.57 \mathrm{mmol})$ in Methanol $(70 \mathrm{~mL})$ werden zwei Äquivalente $\mathrm{KO}^{t} \mathrm{Bu}(129 \mathrm{mg}, 1.15 \mathrm{mmol})$ und zwei Äquivalenten $\mathrm{Zn}\left(\mathrm{ClO}_{4}\right)_{2} \cdot 6 \mathrm{H}_{2} \mathrm{O}(427$ $\mathrm{mg}, 1.15 \mathrm{mmol}$ ) zugefügt und bei Raumtemperatur für $12 \mathrm{~h}$ gerührt. Nach Entfernung aller flüchtigen Bestandteile im Vakuum wird der Rückstand in Methanol (50 mL) aufgenommen, abfiltriert und mit Diethylether überschichtet. Die nach Kristallisation erhaltenen farblosen Kristalle sind für die Röntgenstrukturanalyse geeignet.

Ausbeute

Summenformel

Molekulargewicht

${ }^{1} \mathrm{H}-\mathrm{NMR}$

$\left(\mathrm{d}_{6}-\mathrm{DMSO}, 500 \mathrm{MHz}\right)$

${ }^{13} \mathrm{C}-\mathrm{NMR}$

( $\left.\mathrm{d}_{6}-\mathrm{DMSO}, 126 \mathrm{MHz}\right)$

$\mathrm{IR}(\mathrm{KBr})$

MS (ESI) $\mathrm{m} / \mathrm{z}(\%)$

Elementaranalyse (\%)
$401 \mathrm{mg},(0.430 \mathrm{mmol}), 75 \%$.

$\mathrm{C}_{30} \mathrm{H}_{66} \mathrm{Cl}_{2} \mathrm{~N}_{8} \mathrm{O}_{10} \mathrm{Zn}_{2} \cdot \mathrm{CH}_{3} \mathrm{OH}$

$932.62 \mathrm{~g} / \mathrm{mol}$.

$\delta=5.93\left(\mathrm{~s}, 1 \mathrm{H}, \mathrm{pz}-\mathrm{H}^{4}\right), 3.86\left(\mathrm{~s}, 4 \mathrm{H}, \mathrm{CH}_{2}\right), 2.77-2.67(\mathrm{~m}, \mathrm{br}$,

$\left.32 \mathrm{H}, \mathrm{CH}_{2}\right), 1.01\left(\mathrm{~s}, \mathrm{br}, 24 \mathrm{H}, \mathrm{CH}_{3}\right.$ ).

$\delta=151.1\left(\mathrm{pz}-\mathrm{C}^{3,5}\right), 97.1\left(\mathrm{pz}-\mathrm{C}^{4}\right), 53.1\left(\mathrm{CH}_{2}\right), 51.1\left(\mathrm{CH}_{2}\right)$,

$50.9\left(\mathrm{CH}_{2}\right), 50.0\left(\mathrm{CH}_{2}\right), 46.6\left(\mathrm{CH}_{2}\right), 11.5\left(\mathrm{CH}_{3}\right), 8.9\left(\mathrm{CH}_{3}\right)$.

$\widetilde{v}=2979(\mathrm{~s}), 2944(\mathrm{~s}), 2881$ (s), $1473(\mathrm{~s}), 1386(\mathrm{~m}), 1263$

(m), $1096(\mathrm{vs}), 983(\mathrm{~m}), 736(\mathrm{~m}), 623(\mathrm{~s}) \mathrm{cm}^{-1}$.

$779.4(60)\left[\mathrm{L}_{-} \mathrm{Zn}_{2}(\mathrm{OMe})\left(\mathrm{ClO}_{4}\right)\right]^{+}$,

$765.5(20)\left[\mathrm{L}_{-H} \mathrm{Zn}_{2}(\mathrm{OH})\left(\mathrm{ClO}_{4}\right)\right]^{+}$.

gefunden C 39.77, H 7.56, N 12.20. 


\subsubsection{Synthese von $\left[\mathrm{L}^{5} \cdot \mathrm{Zn}_{2}(\mathrm{OH})\right]\left(\mathrm{ClO}_{4}\right)_{2} 3 \cdot\left(\mathrm{ClO}_{4}\right)_{2}$}

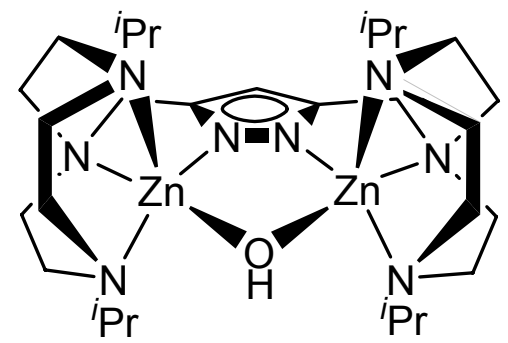

$\left(\mathrm{ClO}_{4}\right)_{2}$

$\mathrm{Zu}$ einer Lösung von $\mathrm{L}^{5}(428 \mathrm{mg}, 0.83 \mathrm{mmol})$ in $\mathrm{MeOH}(100 \mathrm{~mL})$ werden zwei Äquivalenten $\mathrm{KO}$ B Bu $\left(185 \mathrm{mg}\right.$ ) und zwei Äquivalenten $\mathrm{Zn}\left(\mathrm{ClO}_{4}\right)_{2} \cdot 6 \mathrm{H}_{2} \mathrm{O}(614 \mathrm{mg})$ zugefügt und bei Raumtemperatur für $12 \mathrm{~h}$ gerührt. Nach Entfernung aller flüchtigen Bestandteile im Vakuum wird der Rückstand in Aceton $(50 \mathrm{~mL})$ aufgenommen, abfiltriert und mit Petrolether überschichtet. Die nach Kristallisation erhaltenen farblosen Kristalle sind für die Röntgenstrukturanalyse geeignet.

Ausbeute

Summenformel

Molekulargewicht

${ }^{1} \mathrm{H}-\mathrm{NMR}$

$\left(d_{6}\right.$-Aceton, $\left.200 \mathrm{MHz}\right)$

${ }^{13} \mathrm{C}-\mathrm{NMR}$

( $d_{6}$-Aceton, $\left.75 \mathrm{MHz}\right)$

IR ( $\mathrm{KBr})$

MS (FAB) $\mathrm{m} / \mathrm{z}(\%)$

Elementaranalyse (\%)
$571 \mathrm{mg},(0.660 \mathrm{mmol}), 80 \%$

$\mathrm{C}_{29} \mathrm{H}_{58} \mathrm{Cl}_{2} \mathrm{~N}_{8} \mathrm{O}_{9} \mathrm{Zn}_{2}$

864.50

$\delta=6.02\left(\mathrm{~s}, 1 \mathrm{H}, \mathrm{pz}-\mathrm{H}^{4}\right), 4.11\left(\mathrm{~s}, 4 \mathrm{H}, \mathrm{CH}_{2}\right), 3.64$ (sept, ${ }^{3} \mathrm{~J}_{\mathrm{HH}}=$ $6.6 \mathrm{~Hz}, 4 \mathrm{H}, \mathrm{CH}), 3.13\left(\mathrm{~m}, 16 \mathrm{H}, \mathrm{CH}_{2}\right), 2.81\left(\mathrm{~m}, 8 \mathrm{H}, \mathrm{CH}_{2}\right)$, $1.17\left(\mathrm{~d},{ }^{3} \mathrm{~J}_{\mathrm{HH}}=6.6 \mathrm{~Hz}, 24 \mathrm{H}, \mathrm{CH}_{3}\right)$.

$\delta=148.5\left(\mathbf{C}^{\mathrm{pz}, 3,5}\right), 99.4\left(\mathbf{C}^{\mathrm{pz}, 4}\right), 58.1(\mathbf{C H}), 55.7\left(\mathrm{pz}-\mathrm{CH}_{2}\right)$, $53.0\left(2 x \mathrm{CH}_{2}\right), 52.0\left(\mathrm{CH}_{2}\right), 22.9\left(\mathrm{CH}_{3}\right), 16.1\left(\mathrm{CH}_{3}\right)$. $\widetilde{v}=2969(\mathrm{~s}), 2931(\mathrm{~m}), 2873(\mathrm{~m}), 1998(\mathrm{w}), 1694(\mathrm{~m}), 1489$ (m), $1370(\mathrm{~m}), 1285(\mathrm{~m}), 1088$ (vs), $622(\mathrm{~s}) \mathrm{cm}^{-1}$.

$\left.762(80)\left[\mathrm{L}_{-\mathrm{H}} \mathrm{Zn}_{2}(\mathrm{OH})\left(\mathrm{ClO}_{4}\right)\right]^{+}\right)$.

berechnet C 40.29, H 6.76, N 12.96;

gefunden C 40.16, H 6.73, N 12.41. 


\subsubsection{Synthese von $\left[\mathrm{L}^{6}{ }_{-\mathrm{H}} \mathrm{Zn}_{2}(\mathrm{OH})\right]\left(\mathrm{ClO}_{4}\right)_{2} 4 \cdot\left(\mathrm{ClO}_{4}\right)_{2}$}

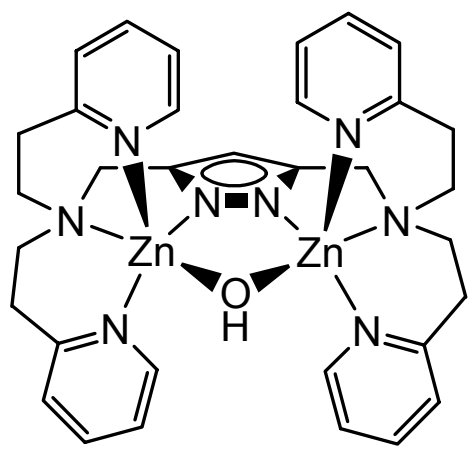

$\left(\mathrm{ClO}_{4}\right)_{2}$

$\mathrm{L}^{6}(1.02 \mathrm{~g}, 1.74 \mathrm{mmol})$ wird mit $\mathrm{H}_{2} \mathrm{O}(20 \mathrm{ml})$ versetzt und Lithiumhydroxidmonohydrat (145.8 mg, $3.48 \mathrm{mmol})$ und $\mathrm{Zn}\left(\mathrm{ClO}_{4}\right)_{2} \cdot 6 \mathrm{H}_{2} \mathrm{O}(1.29 \mathrm{~g}, 3.48 \mathrm{mmol})$ dazugegeben. Das Reaktionsgemisch wird über Nacht gerührt. Der sich bildende Niederschlag wird abfiltriert und im Vakuum getrocknet. Zur Kristallisation wird der weiße Feststoff in Aceton $(70 \mathrm{ml})$ gelöst, die Lösung abfiltriert und mit Petrolether überschichtet. Die nach Kristallisation erhaltenen farblosen Kristalle sind für die Röntgenstrukturanalyse geeignet.

Ausbeute

Summenformel

Molekulargewicht

${ }^{1} \mathrm{H}-\mathrm{NMR}$

$\left(\mathrm{d}_{6}-\mathrm{DMSO}, 500 \mathrm{MHz}\right)$ :

${ }^{1} \mathrm{H}-\mathrm{NMR}$

$\left(\mathrm{d}_{6}\right.$-Aceton, $\left.500 \mathrm{MHz}\right)$

${ }^{13} \mathrm{C}-\mathrm{NMR}$

$\left(d_{6}-\mathrm{DMSO}, 126 \mathrm{MHz}\right)$
$650 \mathrm{mg},(0.728 \mathrm{mmol}), 42 \%$.

$\mathrm{C}_{33} \mathrm{H}_{38} \mathrm{~N}_{8} \mathrm{OZn}{ }_{2} \mathrm{Cl}_{2} \mathrm{O}_{8}$

$892.39 \mathrm{~g} / \mathrm{mol}$.

$\delta=8.82-8.81\left(\mathrm{~m}, 4 \mathrm{H}, \mathrm{CH}^{\mathrm{py}, 6}\right), 8.06\left(\mathrm{~m}, 4 \mathrm{H}, \mathrm{CH}^{\mathrm{py}, 4}\right), 7.51-$

$7.54\left(\mathrm{~m}, 8 \mathrm{H}, \mathrm{CH}^{\mathrm{py}, 3,5}\right), 6.02\left(\mathrm{~s}, 1 \mathrm{H}, \mathrm{CH}^{\mathrm{pz}, 4}\right), 3.98(\mathrm{~s}, 4 \mathrm{H}, \mathrm{pz}-$

$\mathrm{CH}_{2}$ ), 2.75-2.74 (m, 8H, py- $\left.\mathrm{CH}_{2}-\mathrm{CH}_{2}\right), 2.49-2.48$ (m, 8H, py$\left.\mathrm{CH}_{2}-\mathrm{CH}_{2}\right)$.

$\delta=9.08\left(\mathrm{~m}, 4 \mathrm{H}, \mathrm{CH}^{\mathrm{py}, 6}\right), 8.09\left(\mathrm{~m}, 4 \mathrm{H}, \mathrm{CH}^{\mathrm{py}, 4}\right), 7.57(\mathrm{~m}, 4 \mathrm{H}$, $\left.\mathrm{CH}^{\mathrm{py}, 3}\right), 7.52\left(\mathrm{~m}, 4 \mathrm{H}, \mathrm{CH}^{\mathrm{py}, 5}\right), 6.05\left(\mathrm{~s}, 1 \mathrm{H}, \mathrm{CH}^{\mathrm{pz}, 4}\right), 4.13$ (s, $\left.4 \mathrm{H}, \mathrm{pz}-\mathrm{CH}_{2}\right), 3.20\left(\mathrm{~m}, 8 \mathrm{H}, \mathrm{py}-\mathrm{CH}_{2}-\mathrm{CH}_{2}\right), 2.93$ (m, 8H, py$\left.\mathrm{CH}_{2}-\mathrm{CH}_{2}\right)$.

$\delta=161.5\left(\mathbf{C}^{\mathrm{py}, 2}\right), 149.2\left(\mathbf{C H}^{\mathrm{py}, 6}\right), 141.3\left(\mathbf{C H}^{\mathrm{py}, 4}\right), 126.2$

$\left(\mathbf{C H}^{\mathrm{py}, 3}\right), 123.4\left(\mathrm{CH}^{\mathrm{py}, 5}\right), 97.3\left(\mathrm{CH}^{\mathrm{pz}, 4}\right), 57.4\left(\mathrm{pz}-\mathrm{CH}_{2}\right), 55.7$

(py- $\mathrm{CH}_{2}-\mathrm{CH}_{2}$ ), 33.8 (py- $\mathrm{CH}_{2}-\mathrm{CH}_{2}$ ). 
$\mathrm{IR}(\mathrm{KBr})$

$\widetilde{v}=3118(w), 3073(w), 3043(w), 2961(w), 2919(w), 2859$

$(w), 1610(s), 1570(w), 1491(m), 1447(m), 1314(w), 1262$

(w), 1094 (vs), $1026(\mathrm{~m}), 769(\mathrm{~m}), 623(\mathrm{~s}), 419(\mathrm{w}) \mathrm{cm}^{-1}$.

MS (ESI) m/z (\%) $789(100)\left[\mathrm{L}_{-} \mathrm{Zn}_{2}(\mathrm{OH})\left(\mathrm{ClO}_{4}\right)\right]^{+}$,

803 (15) [ $\left[\mathrm{L}_{-} \mathrm{Zn}_{2}(\mathrm{OMe})\left(\mathrm{ClO}_{4}\right)\right]^{+}$.

Elementaranalyse (\%) berechnet C 44.42, H 4.29, N 12.56;

gefunden $\mathrm{C} 44.12 \mathrm{H} 4.26 \mathrm{~N} 12.45$.

\subsubsection{Synthese von $\left[\mathrm{L}^{5}{ }_{\mathrm{H}} \mathrm{Zn}_{2}(\mathrm{OH})(\mathrm{MeOH})\right]\left(\mathrm{ClO}_{4}\right)_{2} 5 \cdot\left(\mathrm{ClO}_{4}\right)_{2}$}

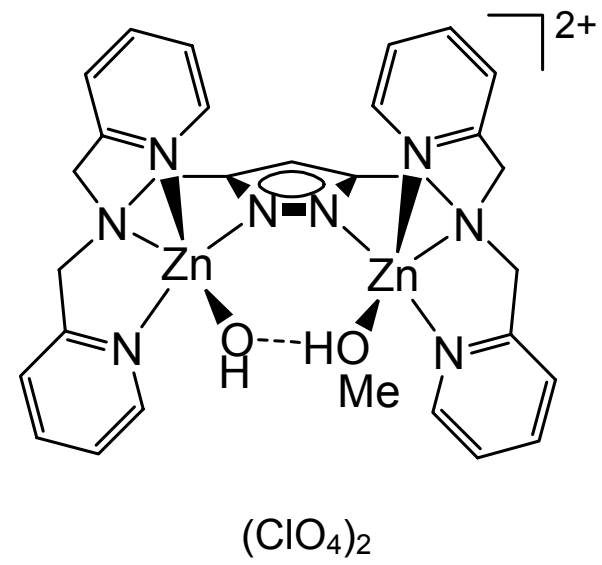

Ein Äquivalent $(460 \mathrm{mg}, 0.938 \mathrm{mmol}) \mathrm{L}^{8}$ wird mit $20 \mathrm{ml} \mathrm{H} \mathrm{H}_{2} \mathrm{O}$ versetzt, zwei Äquivalente $\mathrm{LiOH} \cdot \mathrm{H}_{2} \mathrm{O}(78.7 \mathrm{mg}, 1.88 \mathrm{mmol})$ und zwei Äquivalente $\mathrm{Zn}\left(\mathrm{ClO}_{4}\right)_{2} \cdot 6 \mathrm{H}_{2} \mathrm{O}$ (698 mg, $1.88 \mathrm{mmol}$ ) zugegeben, die Reaktionsmischung kurz erhitzt und über Nacht gerührt. Der Niederschlag wird abfiltriert und im Vakuum getrocknet. Zur Kristallisation wird der Komplex in siedendem Methanol $(80 \mathrm{ml})$ gelöst und von unlöslichen Rückständen abfiltriert. Durch langsames Abkühlen der Lösung auf Raumtemperatur erhält man das Produkt in Form farbloser Nadeln. Die erhaltenen Kristalle sind für die Röntgenstrukturanalyse geeignet.

Ausbeute

Summenformel

Molekulargewicht $1 \mathrm{H}-\mathrm{NMR}$
$374 \mathrm{mg}$ (0.430 mmol), 51\%.

$\mathrm{C}_{30} \mathrm{H}_{34} \mathrm{Cl}_{2} \mathrm{~N}_{8} \mathrm{O}_{10} \mathrm{Zn}_{2}$

$868.32 \mathrm{~g} / \mathrm{mol}$.

$\delta=8.81\left(\mathrm{~m}, 4 \mathrm{H}, \mathrm{CH}^{\mathrm{py}, 6}\right), 8.10\left(\mathrm{~m}, 4 \mathrm{H}, \mathrm{CH}^{\mathrm{py}, 4}\right), 7.66(\mathrm{~m}, 4 \mathrm{H}$, 


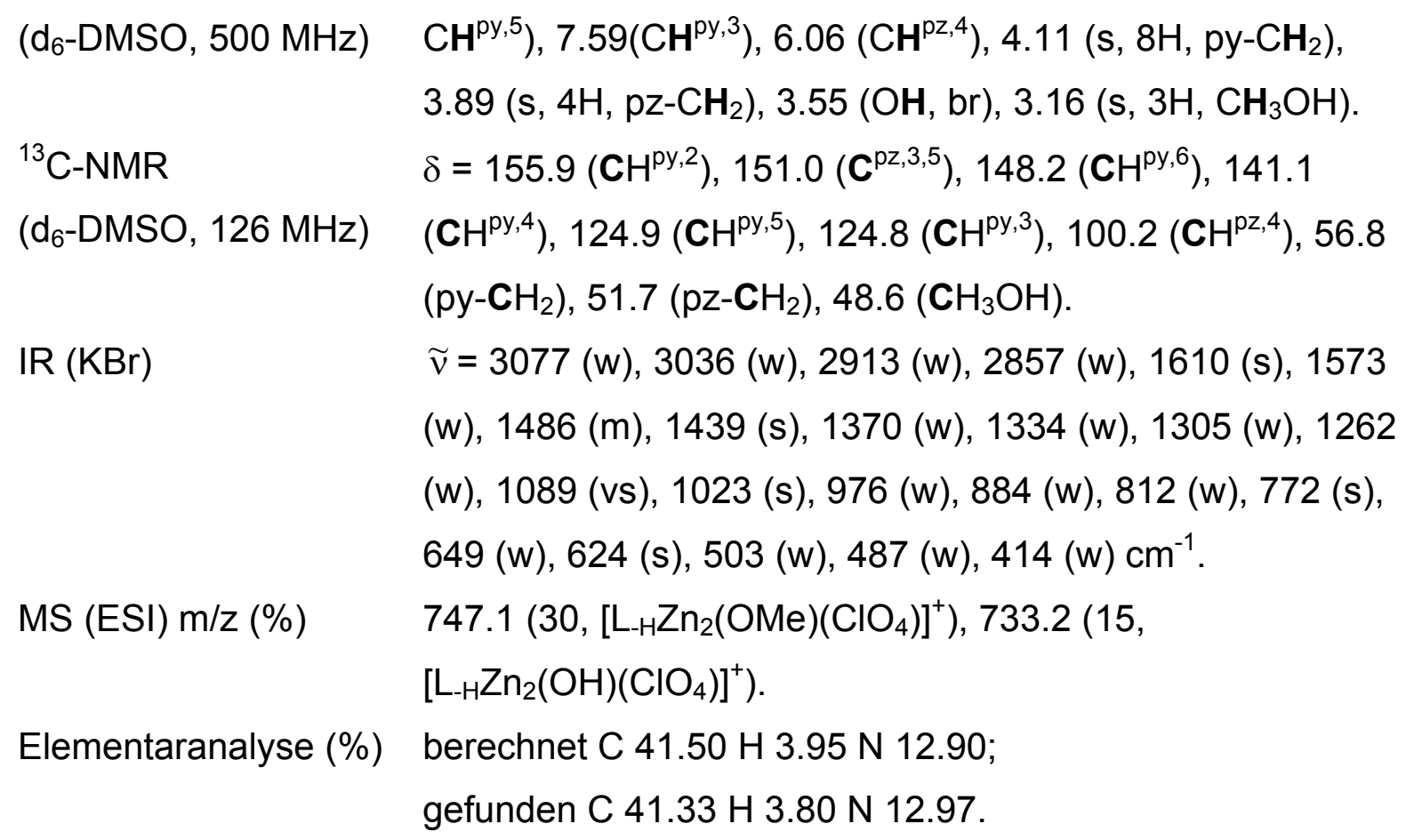

\subsubsection{Synthese von $\mathrm{L}^{2}{ }_{-\mathrm{H}} \mathrm{Zn}_{2} \mathrm{Cl}\left(\mathrm{NO}_{3}\right)_{2} 6$}

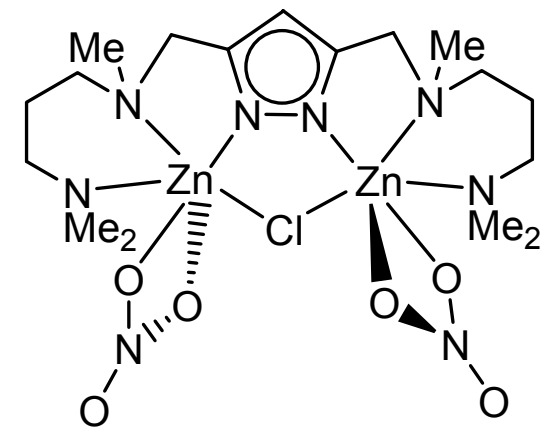

In $70 \mathrm{ml} \mathrm{MeOH}$ wird L ${ }^{2}$ (188 mg, $\left.0.580 \mathrm{mmol}\right)^{*}$ unter $\mathrm{N}_{2}$-Atmosphäre gelöst und zwei Äquivalente $\mathrm{KO}{ }^{t} \mathrm{Bu}(130 \mathrm{mg}, 1.16 \mathrm{mmol})$ und zwei Äquivalente $\mathrm{Zn}\left(\mathrm{NO}_{3}\right)_{2} \cdot 6 \mathrm{H}_{2} \mathrm{O}(345$ $\mathrm{mg}, 1.16 \mathrm{mmol}$ ) zugegeben und bei Raumtemperatur über Nacht gerührt.

Das Lösungsmittel wurde über Hochvakuum entfernt und der gelbliche Feststoff in Aceton $(50 \mathrm{ml})$ gelöst. Nach dem Abfiltrieren wurde das Filtrat zur Kristallisation mit Petrolether überschichtet. Die erhaltenen farblosen Kristalle sind für die Röntgenstrukturanalyse geeignet. 
Summenformel

Molekulargewicht

${ }^{1} \mathrm{H}-\mathrm{NMR}$

$\left(\mathrm{d}_{6}-\mathrm{DMSO} / \mathrm{d}_{6}-\right.$ Aceton

$1: 1,500 \mathrm{MHz})$

IR $(\mathrm{KBr})$

MS (ESI) $\mathrm{m} / \mathrm{z}(\%)$
$\mathrm{C}_{17} \mathrm{H}_{35} \mathrm{ClN}_{8} \mathrm{O}_{6} \mathrm{Zn}_{2}$

$613.74 \mathrm{~g} / \mathrm{mol}$.

sehr breite Signale

$6.15\left(\mathrm{~s}, 1 \mathrm{H}, \mathrm{CH}^{\mathrm{pz}, 4}\right), 3.8-3.5(6 \mathrm{H}), 2.9-2.7(10 \mathrm{H}), 2.35,2.4-$

$2.2(10 \mathrm{H}), 2.0-1.8(4 \mathrm{H})$.

$\widetilde{v}=$ 2964(w), $2919(w), 2850(w), 1480(w), 1385(s)$,

1294(w), $1262(m), 1096(m), 1039(m), 1021(m), 803(m)$ $\mathrm{cm}^{-1}$.

$521.6(100)\left[\mathrm{L}_{-H} \mathrm{Zn}_{2} \mathrm{Cl}_{2}\right]^{+} 548.5$ (95) [L-H $\left.\mathrm{Zn}_{2} \mathrm{Cl}\left(\mathrm{NO}_{3}\right)\right]^{+}, 575.3$

(25) $\left[\mathrm{L}-\mathrm{H} \mathrm{Zn} \mathrm{n}_{2}\left(\mathrm{NO}_{3}\right)_{2}\right]^{+}$.

* Der in dieser Synthese verwendete Ligand enthielt Spuren des Ligandhydrochlorids.

\subsubsection{Synthese von $\mathrm{L}^{4}{ }_{-\mathrm{H}} \mathrm{Zn}_{2}\left(\mathrm{NO}_{3}\right)_{3} 7$}

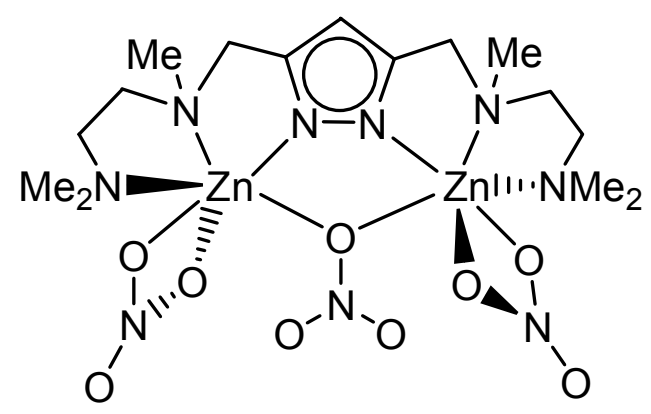

Zu einer Lösung von $\mathrm{L}^{4}(378 \mathrm{mg}, 1.27 \mathrm{mmol})$ in Methanol $(70 \mathrm{ml})$ werden ein Äquivalent $\mathrm{KOtBu}\left(143 \mathrm{mg}, 1.27 \mathrm{mmol}\right.$ ) und zwei Äquivalente $\mathrm{Zn}\left(\mathrm{NO}_{3}\right)_{2} \cdot 6 \mathrm{H}_{2} \mathrm{O}(759$ $\mathrm{mg}, 2.55 \mathrm{mmol}$ ) zugefügt und bei Raumtemperatur für $12 \mathrm{~h}$ gerührt. Nach Entfernung aller flüchtigen Bestandteile im Vakuum wird der Rückstand wieder in Methanol (50 ml) aufgenommen, abfiltriert und mit Diethylether überschichtet. Die nach Kristallisation erhaltenen farblosen Kristalle von 7 sind für die Röntgenstrukturanalyse geeignet. Das Produkt kann jedoch nicht vollständig von gleichzeitig kristallisierendem $\mathrm{KNO}_{3}$ befreit werden. 
Summenformel

Molekulargewicht

${ }^{1} \mathrm{H}-\mathrm{NMR}$

( $\mathrm{d}_{6}$-Aceton, $\left.500 \mathrm{MHz}\right)$ :

${ }^{13} \mathrm{C}-\mathrm{NMR}$

( $\mathrm{d}_{6}$-Aceton, $126 \mathrm{MHz}$ )

$\mathrm{IR}(\mathrm{KBr})$

MS (FAB) $\mathrm{m} / \mathrm{z}(\%)$

Elementaranalyse
$\mathrm{C}_{15} \mathrm{H}_{31} \mathrm{~N}_{9} \mathrm{O}_{9} \mathrm{Zn}_{2}$

$612.24 \mathrm{~g} / \mathrm{mol}$.

$\delta=6.08\left(\mathrm{~s}, 1 \mathrm{H}, \mathrm{CH}^{\mathrm{pz}, 4}\right), 3.89\left(\mathrm{~d},{ }^{2} \mathrm{~J}_{\mathrm{HH}}=15.1 \mathrm{~Hz}, 2 \mathrm{H}, \mathrm{pz}-\right.$

$\left.\mathrm{CH}_{2}\right), 3.80\left(\mathrm{~d},{ }^{2} \mathrm{~J}_{\mathrm{HH}}=15.1 \mathrm{~Hz}, 2 \mathrm{H}, \mathrm{CH}_{2}\right), 3.02\left(\mathrm{~m}, 2 \mathrm{H}, \mathrm{CH}_{2}\right)$,

$2.83\left(\mathrm{~m}, 2 \mathrm{H}, \mathrm{CH}_{2}\right), 2.63\left(\mathrm{~s}, 6 \mathrm{H}, \mathrm{CH}_{3}\right), 2.62\left(\mathrm{~m}, 2 \mathrm{H}, \mathrm{CH}_{2}\right)$,

$2.60\left(\mathrm{~s}, 6 \mathrm{H}, \mathrm{CH}_{3}\right), 2.45\left(\mathrm{~m}, 2 \mathrm{H}, \mathrm{CH}_{2}\right), 2.15\left(\mathrm{~s}, 6 \mathrm{H}, \mathrm{CH}_{3}\right)$.

$\delta=151.1\left(\mathrm{~s}, \mathrm{C}^{\mathrm{pz}, 3,5}\right), 100.2\left(\mathrm{~s}, \mathrm{CH}^{4}\right), 58.6\left(\mathrm{~s}, \mathrm{CH}_{2}\right), 56.4(\mathrm{~s}$,

$\left.\mathrm{CH}_{2}\right), 54.5\left(\mathrm{~s}, \mathrm{CH}_{2}\right), 48.5\left(\mathrm{~s}, \mathrm{CH}_{3}\right), 45.6\left(\mathrm{~s}, \mathrm{CH}_{3}\right), 43.9$ (s,

$\mathrm{CH}_{3}$ ).

$\widetilde{v}=2965(w), 2924(w), 2856(w), 1493(m), 1385(s), 1290$

$(\mathrm{m}), 1262(\mathrm{~m}), 1101(\mathrm{~m}), 1019(\mathrm{~m}), 964(\mathrm{w}), 806(\mathrm{~m}) \mathrm{cm}^{-1}$.

$\mathrm{m} / \mathrm{z}(\%)=547\left(70,\left[\mathrm{LZn}_{2}\left(\mathrm{NO}_{3}\right)_{2}\right]^{+}\right)$.

aufgrund von kokristallisiertem $\mathrm{KNO}_{3}$ konnte keine passende Elementaranalyse erhalten werden.

\subsubsection{Synthese von $\left[\left(\mathrm{L}^{4}{ }_{-\mathrm{H}}\right)_{2} \mathrm{Zn}_{4}(\mathrm{OH})_{2}\left(\mathrm{CO}_{3}\right)\right]\left(\mathrm{ClO}_{4}\right)_{2} 8 \cdot\left(\mathrm{ClO}_{4}\right)_{2}$}

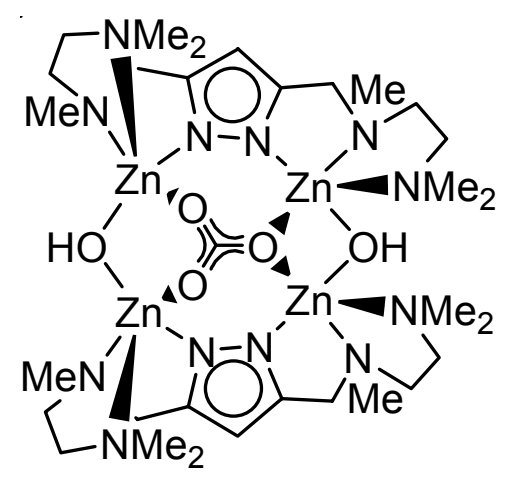

$\left(\mathrm{ClO}_{4}\right)_{2}$

Methode 1: Zu einer Lösung von einem Äquivalent $L^{4}$ (302 mg, $\left.1.02 \mathrm{mmol}\right)$ in Methanol $(100 \mathrm{ml})$ werden zwei Äquivalente $\mathrm{KO}^{\mathrm{t}} \mathrm{Bu}(229 \mathrm{mg}, 2.04 \mathrm{mmol})$ und zwei Äquivalente $\mathrm{Zn}\left(\mathrm{ClO}_{4}\right)_{2} \cdot 6 \mathrm{H}_{2} \mathrm{O}$ (759 mg, $2.04 \mathrm{mmol}$ ) zugefügt und bei Raumtemperatur für $12 \mathrm{~h}$ gerührt. Alle flüchtigen Bestandteile werden im Vakuum entfernt, der Rückstand in Methanol $(100 \mathrm{ml})$ aufgenommen und filtriert. Durch langsame Verdunstung des Lösungsmittels an der Luft erhält man farblose Kristalle des Produkts. 
Methode 2: $\mathrm{Zu}$ einer Lösung von einem Äquivalent $L^{4}(575 \mathrm{mg}, 1.94 \mathrm{mmol})$ in Methanol $(100 \mathrm{ml})$ werden zwei Äquivalente $\mathrm{KO}{ }^{t} \mathrm{Bu}(435 \mathrm{mg}, 3.88 \mathrm{mmol})$, zwei Äquivalente $\mathrm{Zn}\left(\mathrm{ClO}_{4}\right)_{2} 6 \mathrm{H}_{2} \mathrm{O}(1444 \mathrm{mg}, 3.88 \mathrm{mmol})$ und ein halbes Äquivalent Kaliumcarbonat (134 mg, $0.97 \mathrm{mmol}$ ) zugefügt und bei Raumtemperatur für $12 \mathrm{~h}$ gerührt. Alle flüchtigen Bestandteile werden im Vakuum entfernt, der Rückstand in Methanol $(100 \mathrm{ml})$ aufgenommen und filtriert. Durch langsame Verdunstung des Lösungsmittels an der Luft erhält man farblose Kristalle des Produkts, die nach analytischen und spektroskopischen Daten identisch mit dem nach erster Methode erhaltenen Produkt sind.

Ausbeute

Summenformel

Molekulargewicht

${ }^{1} \mathrm{H}-\mathrm{NMR}$

( $\mathrm{d}_{6}$-Aceton, $\left.500 \mathrm{MHz}\right)$

${ }^{13}$ C-NMR

( $\mathrm{d}_{6}$-Aceton, $\left.500 \mathrm{MHz}\right)$

IR ( $\mathrm{KBr})$

MS (ESI) $\mathrm{m} / \mathrm{z}(\%)$

Elementaranalyse (\%)
Methode 1: $221 \mathrm{mg},(0.193 \mathrm{mmol}), 38 \%$

Methode 2: $800 \mathrm{mg},(0.698 \mathrm{mmol}), 72 \%$.

$\mathrm{C}_{31} \mathrm{H}_{64} \mathrm{Cl}_{2} \mathrm{~N}_{12} \mathrm{O}_{13} \mathrm{Zn}_{4}$

$1145.38 \mathrm{~g} / \mathrm{mol}$.

$\delta=5.90\left(\mathrm{~s}, 2 \mathrm{H} ; \mathrm{CH}^{\mathrm{pz}, 4}\right), 3.79\left(\mathrm{~d},{ }^{2} \mathrm{~J}_{\mathrm{HH}}=15.8 \mathrm{~Hz}, 4 \mathrm{H}, \mathrm{pz}-\right.$

$\left.\mathrm{CH}_{2}\right), 3.72\left(\mathrm{~d},{ }^{2} \mathrm{~J}_{\mathrm{HH}}=15.8 \mathrm{~Hz}, 4 \mathrm{H}, \mathrm{CH}_{2}\right), 3.12\left(\mathrm{~m}, 4 \mathrm{H}, \mathrm{CH}_{2}\right)$,

$2.85\left(\mathrm{~m}, 4 \mathrm{H}, \mathrm{CH}_{2}\right), 2.80$ (s, 12H, $\left.\mathrm{CH}_{3}\right), 2.77$ (br, $\left.2 \mathrm{H}, \mathrm{OH}\right)$,

$2.64\left(\mathrm{~s}, 12 \mathrm{H}, \mathrm{CH}_{3}\right), 2.60\left(\mathrm{~m}, 4 \mathrm{H}, \mathrm{CH}_{2}\right), 2.57\left(\mathrm{~m}, 4 \mathrm{H}, \mathrm{CH}_{2}\right)$,

$2.50\left(\mathrm{~s}, 12 \mathrm{H}, \mathrm{CH}_{3}\right)$.

$\delta=169.9\left(\mathrm{CO}_{3}\right), 151.7\left(\mathbf{C}^{\mathrm{pz}, 3,5}\right), 99.4\left(\mathrm{pz}-\mathbf{C}^{\mathrm{pz}, 4}\right), 58.3\left(\mathrm{CH}_{2}\right)$,

$56.0\left(\mathrm{CH}_{2}\right), 54.5\left(\mathrm{CH}_{2}\right), 49.0\left(\mathrm{CH}_{3}\right), 46.8\left(\mathrm{CH}_{3}\right), 44.3\left(\mathrm{CH}_{3}\right)$.

$\widetilde{v}=3440(\mathrm{br}, \mathrm{m}), 2963(\mathrm{~m}), 2857(\mathrm{~m}), 1628(\mathrm{br}, \mathrm{m}), 1507$

(m), $1475(\mathrm{~m}), 1463(\mathrm{~m}), 1437(\mathrm{w}), 1389(\mathrm{~m}), 1376(\mathrm{~m})$,

$1324(w), 1301(w), 1262(w), 1098(s), 1033(m), 1022(m)$,

$967(\mathrm{~m}), 942(\mathrm{w}), 810(\mathrm{~m}), 779(\mathrm{w}), 766(\mathrm{w}), 625(\mathrm{~m}) \mathrm{cm}^{-1}$.

$\mathrm{m} / \mathrm{z}(\%)=1039\left(100,\left[\mathrm{~L}_{2} \mathrm{Zn}_{4}(\mathrm{OH})_{2}\left(\mathrm{CO}_{3}\right)\left(\mathrm{ClO}_{4}\right)\right]^{+}\right)$.

berechnet C 32.51, H 5.63, N 14.67;

gefunden C 32.43, H 5.78, N 14.31. 


\subsubsection{Synthese von $\left[\left(\mathrm{L}^{4}{ }_{\mathrm{H}}\right)_{2} \mathrm{Zn}_{4}(\mathrm{OH})_{2}\left(\mathrm{CO}_{3}\right)\right]\left(\mathrm{BPh}_{4}\right)_{2} 8 \cdot\left(\mathrm{BPh}_{4}\right)_{2}$}

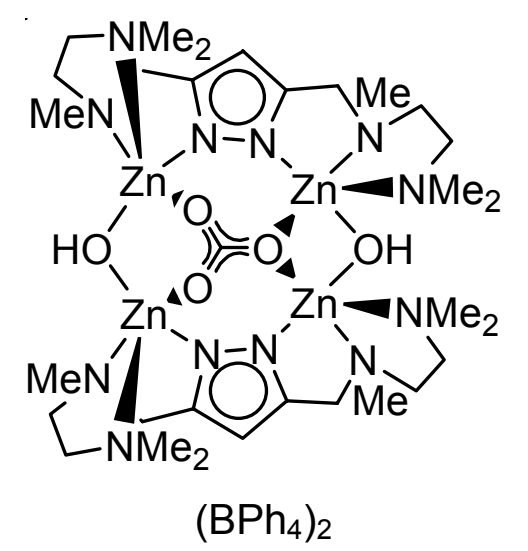

Zu einer Lösung von einem Äquivalent $L^{4}(302 \mathrm{mg}, 1.02 \mathrm{mmol})$ in Methanol (100 ml) werden zwei Äquivalente $\mathrm{KO}^{t} \mathrm{Bu}\left(229 \mathrm{mg}, 2.04 \mathrm{mmol}\right.$ ), zwei Äquivalente $\mathrm{Zn}\left(\mathrm{ClO}_{4}\right)_{2}$ $6 \mathrm{H}_{2} \mathrm{O}(759 \mathrm{mg}, 2.04 \mathrm{mmol})$ und ein halbes Äquivalent Kaliumcarbonat $(71 \mathrm{mg}, 0.51$ $\mathrm{mmol}$ ) zugefügt und bei Raumtemperatur für $12 \mathrm{~h}$ gerührt. Alle flüchtigen Bestandteile werden im Vakuum entfernt, der Rückstand in Ethanol $(100 \mathrm{ml})$ aufgenommen und zwei Äquivalente Natriumtetraphenylborat (697 mg, $2.04 \mathrm{mmol}$ ) zugefügt. Der entstehende Niederschlag wird abfiltriert, im Vakuum getrocknet und in Aceton (50 $\mathrm{ml}$ ) aufgenommen. Die Lösung wird filtriert und mit Petrolether überschichtet. Die erhaltenen, farblosen Kristalle des Produkts $\mathbf{8 b}$ sind für die Röntgenstrukturanalyse geeignet. Das Produkt kann jedoch nicht vollständig von gleichzeitig kristallisierendem $\mathrm{KNO}_{3}$ befreit werden.

Ausbeute

Summenformel

${ }^{1} \mathrm{H}-\mathrm{NMR}$

( $\mathrm{d}_{6}$-Aceton, $500 \mathrm{MHz}$ )
Molekulargewicht

$512 \mathrm{mg},(0.312 \mathrm{mmol}), 61 \%$.

$\mathrm{C}_{79} \mathrm{H}_{104} \mathrm{~B}_{2} \mathrm{~N}_{12} \mathrm{O}_{5} \mathrm{Zn}_{4} \cdot \mathrm{C}_{3} \mathrm{H}_{6} \mathrm{O}$

$1643.01 \mathrm{~g} / \mathrm{mol}$.

$\delta=7.33\left(\mathrm{~m}, 16 \mathrm{H}, \mathrm{CH}^{\mathrm{ph}, 2}\right), 6.91\left(\mathrm{~m}, 16 \mathrm{H}, \mathrm{CH}^{\mathrm{ph}, 3}\right), 6.76(\mathrm{~m}$,

$\left.8 \mathrm{H}, \mathrm{CH}^{\mathrm{ph}, 4}\right), 5.91\left(\mathrm{~s}, 2 \mathrm{H}, \mathrm{CH}^{\mathrm{pz}, 4}\right), 3.79\left(\mathrm{~d},{ }^{2} \mathrm{~J}_{\mathrm{HH}}=15.8 \mathrm{~Hz}, 4 \mathrm{H}\right.$, $\left.\mathrm{CH}_{2}\right), 3.69\left(\mathrm{~d},{ }^{2} \mathrm{~J}_{\mathrm{HH}}=15.8 \mathrm{~Hz}, 4 \mathrm{H}, \mathrm{CH}_{2}\right), 3.06\left(\mathrm{~m}, 4 \mathrm{H}, \mathrm{CH}_{2}\right)$,

$2.85\left(\mathrm{~m}, 4 \mathrm{H}, \mathrm{CH}_{2}\right), 2.78\left(\mathrm{~s}, 12 \mathrm{H}, \mathrm{CH}_{3}\right), 2.75(\mathrm{br}, 2 \mathrm{H}, \mathrm{OH})$, $2.62\left(\mathrm{~s}, 12 \mathrm{H}, \mathrm{CH}_{3}\right), 2.53-2.60\left(\mathrm{~m}, 8 \mathrm{H}, \mathrm{CH}_{2}\right), 2.50(\mathrm{~s}, 12 \mathrm{H}$, $\mathrm{CH}_{3}$ ).

${ }^{13} \mathrm{C}-\mathrm{NMR}$ $\delta=169.9\left(\mathrm{~s}, \mathrm{CO}_{3}\right), 165.0\left(\mathrm{q},{ }^{1} \mathrm{~J}_{\mathrm{BC}}=49.4 \mathrm{~Hz}, \mathrm{C}^{\mathrm{ph}, 1}\right), 151.7(\mathrm{~s}$, 


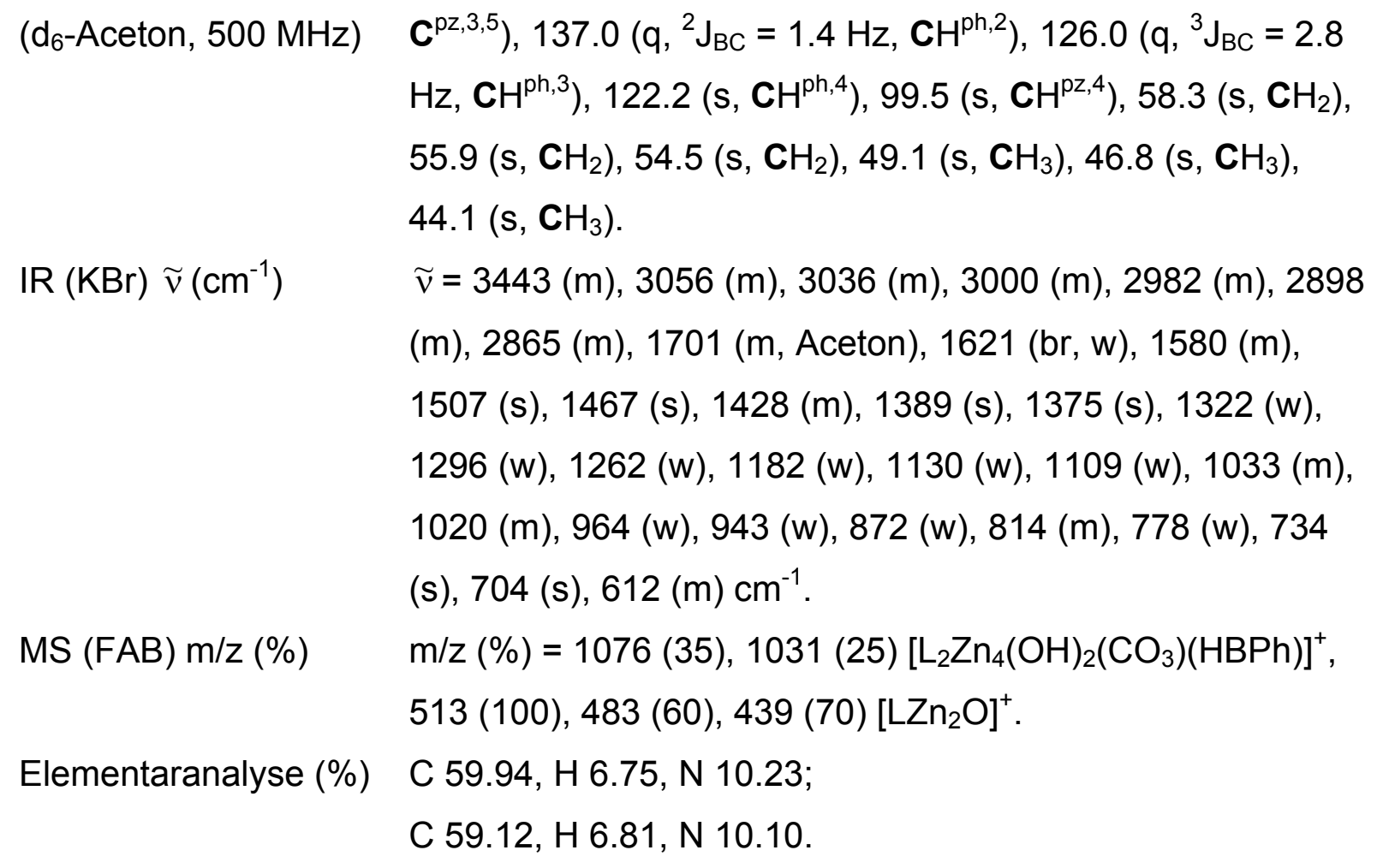

\subsubsection{Synthese von $\mathrm{L}^{9}{ }_{-\mathrm{H}} \mathrm{Zn}_{2}\left(\mathrm{NO}_{3}\right)_{3} 9$}

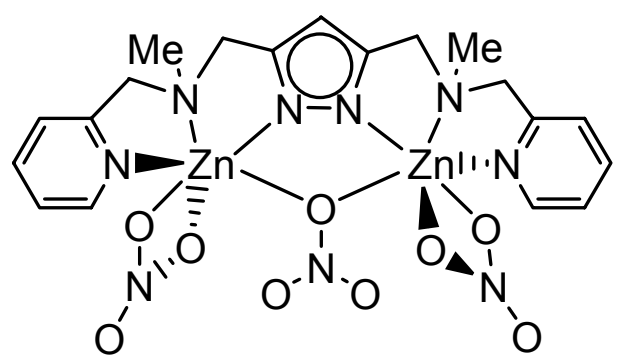

Unter $\mathrm{N}_{2}$-Atmosphäre wird $\mathrm{L}^{9}(430 \mathrm{mg}, 1.33 \mathrm{mmol})$ in Methanol $(70 \mathrm{ml})$ gelöst. Hierzu werden ein Äquivalent $\mathrm{KO}^{\mathrm{t}} \mathrm{Bu}(149 \mathrm{mg}, 1.33 \mathrm{mmol}$ ) und zwei Äquivalente $\mathrm{Zn}\left(\mathrm{NO}_{3}\right)_{2} \cdot 6 \mathrm{H}_{2} \mathrm{O}(788 \mathrm{mg}, 2.65 \mathrm{mmol})$ gegeben und bei Raumtemperatur für $12 \mathrm{~h}$ gerührt. Alle flüchtigen Bestandteile werden im Hochvakuum entfernt und der Feststoff in Aceton $(70 \mathrm{ml})$ gelöst. Nach dem Abfiltrieren wird das Filtrat mit Petrolether überschichtet. Die erhaltenen, farblosen Kristalle des Produkts $\mathbf{9}$ sind für die Röntgenstrukturanalyse geeignet.

Ausbeute

Summenformel
$235 \mathrm{mg},(0.360 \mathrm{mmol}), 27 \%$.

$\mathrm{C}_{19} \mathrm{H}_{23} \mathrm{~N}_{9} \mathrm{O}_{9} \mathrm{Zn}_{2}$ 
Molekulargewicht

${ }^{1} \mathrm{H}-\mathrm{NMR}$

(d -Aceton, $500 \mathrm{MHz}$ )

${ }^{13} \mathrm{C}-\mathrm{NMR}$

$\left(d_{6}-\right.$ DMSO, $\left.126 \mathrm{MHz}\right)$

$\mathrm{IR}(\mathrm{KBr})$
$652.22 \mathrm{~g} / \mathrm{mol}$.

$8.91\left(\mathrm{~m}, 2 \mathrm{H}, \mathrm{CH}^{\mathrm{py}, 6}\right), 8.15\left(\mathrm{~m}, 2 \mathrm{H}, \mathrm{CH}^{\mathrm{py}, 4}\right), 7.72,7.65(\mathrm{~m}, 4 \mathrm{H}$, $\left.\mathrm{CH}^{\mathrm{py}, 3,5}\right), 6.13\left(\mathrm{~m}, 2 \mathrm{H}, \mathrm{CH}^{\mathrm{pz}, 4}\right), 4.1-3.9(\mathrm{~m}, \mathrm{br}, 8 \mathrm{H}), 2.78$, 2.75, 2.48, 2.44 .

(in $\mathrm{d}_{6}$-DMSO sehr breite Signale)

$155.7\left(\mathbf{C H}^{\mathrm{py}, 6}\right), 149.3,142.5\left(\mathbf{C}^{\mathrm{py}, 2}, \mathbf{C}^{\mathrm{pz}, 3,5}\right), 140.2\left(\mathbf{C H}^{\mathrm{py}, 4}\right)$,

$124.4,124.2\left(\mathrm{CH}^{\mathrm{py}, 3,5}\right), 99.0\left(\mathrm{CH}^{\mathrm{pz}, 4}\right), 61.7\left(\mathrm{br}, \mathbf{C H}_{2}\right), 56.0$ (br, $\left.\mathrm{CH}_{2}\right), 46.0\left(\mathrm{br}, \mathrm{CH}_{3}\right)$.

$\widetilde{v}=2969(w), 2907(w), 2825(w), 2427(w), 2397(w), 1608$

(m), 1573 (w), 1483 (s), 1445 (s), 1424 (m), 1385 (vs), 1325

(m), 1300 (s), 1255 (m), 1156 (w), 1107 (w), 1053 (w), 1025

(m), $1015(\mathrm{~m}), 978(\mathrm{w}), 963(\mathrm{w}), 876(\mathrm{w}), 825(\mathrm{w}), 792(\mathrm{w})$,

$772(\mathrm{~m}), 734(\mathrm{w}), 650(\mathrm{w}), 490(\mathrm{w}), 417(\mathrm{w}) \mathrm{cm}^{-1}$.

MS (ESI) $\mathrm{m} / \mathrm{z}(\%)$

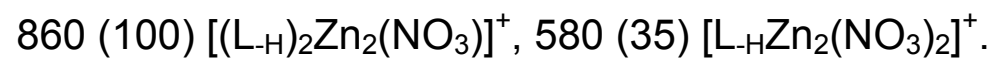

aufgrund von kokristallisiertem $\mathrm{KNO}_{3}$ konnte keine passende Elementaranalyse erhalten werden. 


\subsection{Komplexsynthesen - Phosphatesterkomplexe}

\subsubsection{Synthese von $\left[\mathrm{L}^{3}{ }_{\mathrm{H}} \mathrm{Zn}_{2}\left\{\mathrm{O}_{2} \mathrm{P}(\mathrm{OMe})_{2}\right\}\right]\left(\mathrm{ClO}_{4}\right)_{2} 10 \mathrm{a} \cdot\left(\mathrm{ClO}_{4}\right)_{2}$}

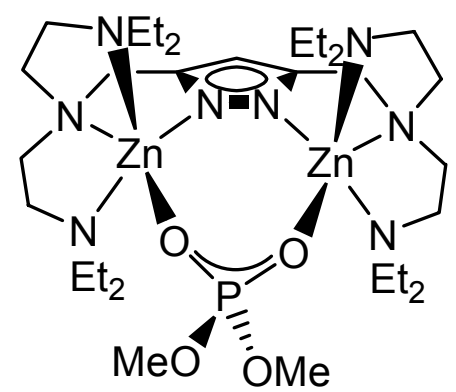

$\left(\mathrm{ClO}_{4}\right)_{2}$

Zu einer Lösung von $L^{2}(140 \mathrm{mg}, 0.27 \mathrm{mmol})$ in Methanol $(50 \mathrm{ml})$ werden zwei Äquivalente $\mathrm{KO}^{\mathrm{t}} \mathrm{Bu}\left(60 \mathrm{mg}, 0.535 \mathrm{mmol}\right.$ ), zwei Äquivalente $\mathrm{Zn}\left(\mathrm{ClO}_{4}\right)_{2} \cdot 6 \mathrm{H}_{2} \mathrm{O}(199 \mathrm{mg}$, $0.535 \mathrm{mmol}$ ) und ein Äquivalent Dimethylphosphorsäure (34 mg, $0.535 \mathrm{mmol}$ ) zugefügt und bei Raumtemperatur für $12 \mathrm{~h}$ gerührt. Alle flüchtigen Bestandteile werden im Vakuum entfernt, der Rückstand in Aceton $(50 \mathrm{ml})$ aufgenommen, filtriert und mit Petrolether überschichtet. Die erhaltenen, farblosen Kristalle des Produkts 10a sind für die Röntgenstrukturanalyse geeignet.

Ausbeute

Summenformel

Molekulargewicht

${ }^{1} \mathrm{H}-\mathrm{NMR}$

$\left(d_{6}-\mathrm{DMSO}, 500 \mathrm{MHz}\right)$

${ }^{13} \mathrm{C}-\mathrm{NMR}$

$\left(d_{6}-\mathrm{DMSO}, 126 \mathrm{MHz}\right)$

${ }^{31} \mathrm{P}-\mathrm{NMR}$

$\left(d_{6}-\right.$ DMSO, $\left.202 \mathrm{MHz}\right)$

$\mathrm{IR}(\mathrm{KBr})$
$193 \mathrm{mg},(0.198 \mathrm{mmol}), 74 \%$.

$\mathrm{C}_{31} \mathrm{H}_{67} \mathrm{Cl}_{2} \mathrm{~N}_{8} \mathrm{O}_{12} \mathrm{PZn}_{2}$

$976.57 \mathrm{~g} / \mathrm{mol}$.

$\delta=6.09\left(\mathrm{~s}, 1 \mathrm{H}, \mathrm{CH}^{\mathrm{pz}, 4}\right), 3.98\left(\mathrm{~s}, 4 \mathrm{H}, \mathrm{pz}-\mathrm{CH}_{2}\right), 3.66\left(\mathrm{~d},{ }^{3} \mathrm{~J}_{\mathrm{PH}}=\right.$ $\left.11.1 \mathrm{~Hz}, 6 \mathrm{H}, \mathrm{OCH}_{3}\right), 3.05\left(\mathrm{~m}, 4 \mathrm{H}, \mathrm{CH}_{2}\right), 2.78\left(\mathrm{~m}, 24 \mathrm{H}, \mathrm{CH}_{2}\right)$, $2.67\left(\mathrm{~m}, 4 \mathrm{H}, \mathrm{CH}_{2}\right), 1.03\left(\mathrm{~s}, 24 \mathrm{H}, \mathrm{CH}_{3}\right)$.

$\delta=154.0\left(\mathbf{C}^{\mathrm{pz}, 3,5}\right), 99.8\left(\mathbf{C H}^{\mathrm{pz}, 4}\right), 53.8\left(\mathrm{~d},{ }^{2} \mathrm{~J}_{\mathrm{CP}}=5.3 \mathrm{~Hz}\right.$, $\left.\mathrm{OCH}_{3}\right), 53.3\left(\mathrm{pz}-\mathrm{CH}_{2}\right), 51.9\left(\mathrm{CH}_{2}\right), 50.0\left(\mathrm{CH}_{2}\right), 47.5\left(\mathrm{CH}_{2}\right)$, $9.3\left(\mathrm{CH}_{3}\right)$.

$\delta=-1.40(\mathrm{~s})$.

$\widetilde{v}=2981(\mathrm{~m}), 2952(\mathrm{~m}), 2882(\mathrm{~m}), 1474(\mathrm{~m}), 1387(\mathrm{~m}), 1263$ (m), 1223 (m), 1097 (vs), 1052 (s), 1030 (s), 831 (m), 804 
(m), $624(\mathrm{~s}) \mathrm{cm}^{-1}$.

MS (ESI) m/z (\%)

$874(100)\left[\mathrm{L}^{2}{ }_{-\mathrm{H}} \mathrm{Zn}_{2}\left\{\mathrm{O}_{2} \mathrm{P}(\mathrm{OMe})_{2}\right\}\left(\mathrm{ClO}_{4}\right)\right]^{+}$.

Elementaranalyse (\%) berechnet C 38.13, H 6.92, N 11.47;

gefunden C 37.55, H 6.78, N 10.86.

\subsubsection{Synthese von $\left[\mathrm{L}^{6}{ }_{-\mathrm{H}} \mathrm{Zn}_{2}\left\{\mathrm{O}_{2} \mathrm{P}(\mathrm{OMe})_{2}\right\}\right]\left(\mathrm{ClO}_{4}\right)_{2} 11 \mathrm{a} \cdot\left(\mathrm{ClO}_{4}\right)_{2}$}

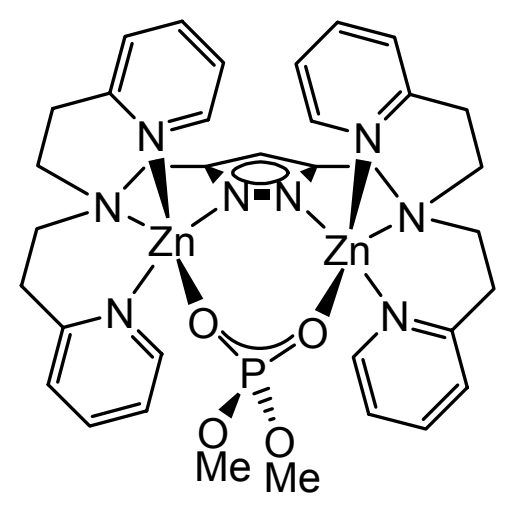

$\left(\mathrm{ClO}_{4}\right)_{2}$

Zu einer Lösung von $L^{6}(170 \mathrm{mg}, 0.31 \mathrm{mmol})$ in Methanol $(70 \mathrm{ml})$ werden zwei Äquivalente $\mathrm{KO}{ }^{t} \mathrm{Bu}(69.8 \mathrm{mg}, 0.62 \mathrm{mmol})$, zwei Äquivalente $\mathrm{Zn}\left(\mathrm{ClO}_{4}\right)_{2}-6 \mathrm{H}_{2} \mathrm{O}(232$ $\mathrm{mg}, 0.62 \mathrm{mmol}$ ) und ein Äquivalent Dimethylphosphorsäure (39.2 mg, $0.31 \mathrm{mmol}$ ) zugefügt und bei Raumtemperatur für $12 \mathrm{~h}$ gerührt. Alle flüchtigen Bestandteile werden im Vakuum entfernt, der Rückstand in Aceton (50 ml) aufgenommen, filtriert und mit Petrolether überschichtet. Die erhaltenen, farblosen Kristalle des Produkts 11a sind für die Röntgenstrukturanalyse geeignet.

Ausbeute

Summenformel

Molekulargewicht

${ }^{1} \mathrm{H}-\mathrm{NMR}$

( $\mathrm{d}_{6}$-Aceton, $500 \mathrm{MHz}$ )
$137 \mathrm{mg},(0.134 \mathrm{mmol}), 43 \%$.

$\mathrm{C}_{35} \mathrm{H}_{43} \mathrm{Cl}_{2} \mathrm{~N}_{8} \mathrm{O}_{12} \mathrm{PZn}_{2} \cdot 0.5 \mathrm{C}_{3} \mathrm{H}_{6} \mathrm{O}$

$1029.46 \mathrm{~g} / \mathrm{mol}$.

$\delta=8.80\left(\mathrm{ddd}, 4 \mathrm{H}, \mathrm{CH}^{\mathrm{py}, 6}\right), 8.17\left(\mathrm{ddd}, 4 \mathrm{H}, \mathrm{CH}^{\mathrm{py}, 4}\right), 7.76(\mathrm{ddd}$, $\left.4 \mathrm{H}, \mathrm{CH}^{\mathrm{py}, 3}\right), 7.71\left(\mathrm{ddd}, 4 \mathrm{H}, \mathrm{CH}^{\mathrm{py}, 5}\right), 6.23\left(\mathrm{~s}, 1 \mathrm{H}, \mathrm{CH}^{\mathrm{pz}, 4}\right), 4.08$ (s, $\left.4 \mathrm{H}, \mathrm{pz}-\mathrm{CH}_{2}\right), 3.56\left(\mathrm{~d},{ }^{3} \mathrm{~J}_{\mathrm{HP}}=11.1 \mathrm{~Hz}, 6 \mathrm{H}, \mathrm{OMe}\right), 3.21$ (ddd, $4 \mathrm{H}$, py- $\mathrm{CH}_{2}-\mathrm{CH}_{2}-\mathrm{N}$ ), 3.14 (ddd, $4 \mathrm{H}$, py- $\mathrm{CH}_{2}-\mathrm{CH}_{2}-\mathrm{N}$ ), 2.95 (ddd, $4 \mathrm{H}$, py- $\mathrm{CH}_{2}-\mathrm{CH}_{2}-\mathrm{N}$ ), 2.92 (ddd, 4H, py- $\mathrm{CH}_{2}-\mathrm{CH}_{2-}$ 
N).

${ }^{13} \mathrm{C}-\mathrm{NMR}$

( $\mathrm{d}_{6}$-Aceton, $\left.126 \mathrm{MHz}\right)$

${ }^{31}$ P-NMR

( $\mathrm{d}_{6}$-Aceton, $121 \mathrm{MHz}$ )

IR (KBr)

MS (ESI) $\mathrm{m} / \mathrm{z}(\%)$

Elementaranalyse (\%)
$\delta=162.1\left(\mathbf{C}^{\mathrm{py}, 3}\right), 153.1\left(\mathbf{C}^{\mathrm{pz}, 3,5}\right), 150.4\left(\mathbf{C H}^{\mathrm{py}, 6}\right), 142.4$

$\left(\mathbf{C H}^{\text {py,4 }}\right), 126.9\left(\mathbf{C H}^{\text {py,3}}\right), 124.6\left(\mathbf{C H}^{\text {py,5}}\right), 101.9\left(\mathbf{C H}^{\text {pz,4 }}\right), 58.5$

(py-CH $\left.\mathrm{CH}_{2}-\mathrm{CH}_{2}-\mathrm{N}\right), 56.7\left(\mathrm{pz}-\mathrm{CH}_{2}\right), 53.9\left(\mathrm{OCH}_{3}, \mathrm{~d},{ }^{2} \mathrm{~J}_{\mathrm{CP}}=6.0\right.$

$\mathrm{Hz}), 34.2\left(\right.$ py- $\left.\mathrm{CH}_{2}-\mathrm{CH}_{2}-\mathrm{N}\right)$.

$\delta=3.12(\mathrm{~s})$.

$\widetilde{v}=3118(w), 3085(w), 2953(w), 2879(w), 2856(w) 1611$

(m, Aceton), 1571 (w), $1489(\mathrm{w}), 1446(\mathrm{~m}), 1366(\mathrm{w}), 1332$

m), 1308 (m), 1208 (m), $1184(\mathrm{~m}), 1160$ (m), 1105 (vs),

1092 (vs), 1021 (s), 977 (w), $829(\mathrm{~m}), 787(\mathrm{~m}), 773(\mathrm{~m}), 623$

(m), $597(\mathrm{w}) \mathrm{cm}^{-1}$.

$897.5\left(30,\left[\mathrm{~L}_{-H} \mathrm{Zn}_{2}\left(\mathrm{PO}_{4} \mathrm{Me}_{2}\right)\left(\mathrm{ClO}_{4}\right)\right]^{+}\right), 923.5(100$,

$\left.\left[\mathrm{L}_{-} \mathrm{Zn}_{2}\left(\mathrm{PO}_{4} \mathrm{Me}_{2}\right)_{2}\right]^{+}\right)$.

berechnet C 42.58 H 4.50 N 10.88;

gefunden C 42.33 H 4.50 N 10.88 .

\subsubsection{Synthese von $\left[\mathrm{L}^{8}{ }_{-\mathrm{H}} \mathrm{Zn}_{2}\left\{\mathrm{O}_{2} \mathrm{P}(\mathrm{OMe})_{2}\right\}\right]\left(\mathrm{ClO}_{4}\right)_{2} 12 \mathrm{a} \cdot\left(\mathrm{ClO}_{4}\right)_{2}$}

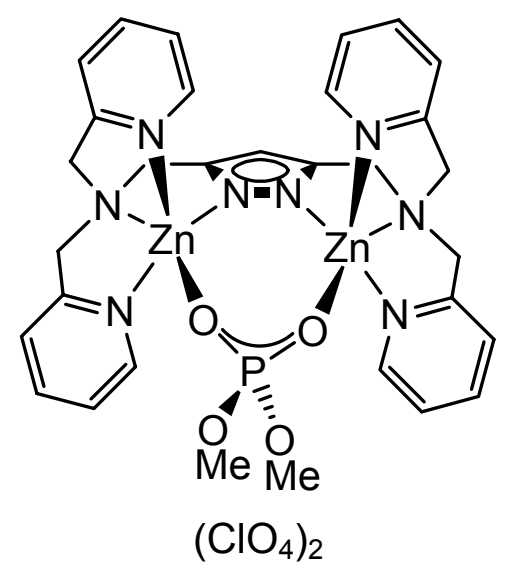

$\mathrm{Zu}$ einer Lösung von $\mathrm{L}^{8}(150 \mathrm{mg}, 0.30 \mathrm{mmol})$ in Methanol $(70 \mathrm{ml})$ werden zwei Äquivalente $\mathrm{KO}^{t} \mathrm{Bu}(66.3 \mathrm{mg}, 0.59 \mathrm{mmol})$, zwei Äquivalente $\mathrm{Zn}\left(\mathrm{ClO}_{4}\right)_{2}=6 \mathrm{H}_{2} \mathrm{O}(220$ $\mathrm{mg}, 0.59 \mathrm{mmol})$ und ein Äquivalent Dimethylphosphorsäure (37.2 mg, $0.30 \mathrm{mmol})$ zugefügt und bei Raumtemperatur für $12 \mathrm{~h}$ gerührt. Alle flüchtigen Bestandteile werden im Vakuum entfernt, der Rückstand in Aceton $(50 \mathrm{ml})$ aufgenommen, filtriert 
und mit Petrolether überschichtet. Die erhaltenen, farblosen Kristalle des Produkts 12a sind für die Röntgenstrukturanalyse geeignet.

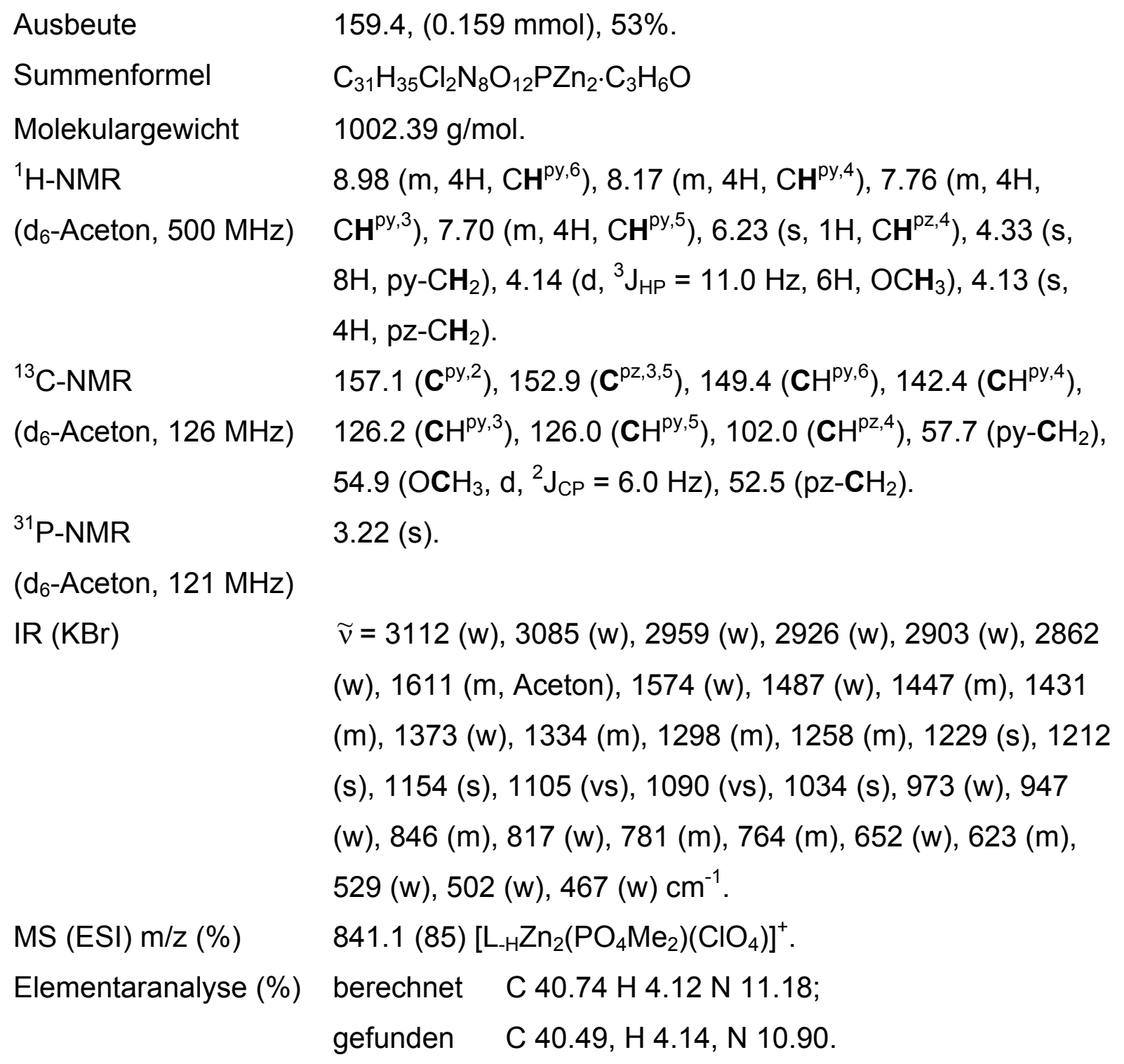




\subsubsection{Synthese von $\mathrm{L}^{4}{ }_{-\mathrm{H}} \mathrm{Zn}_{2}\left\{\mathrm{O}_{2} \mathrm{P}(\mathrm{OMe})_{2}\right\}\left(\mathrm{NO}_{3}\right)_{2} 13 \mathrm{a}$}

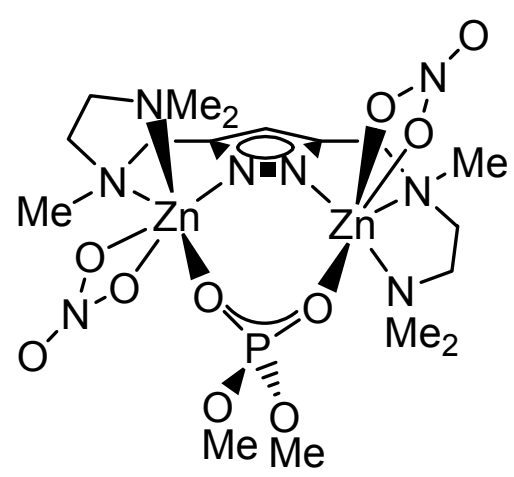

Zu einer Lösung von $L^{4}(125 \mathrm{mg}, 0.421 \mathrm{mmol})$ in Methanol $(70 \mathrm{ml})$ werden zwei Äquivalente $\mathrm{KO}{ }^{t} \mathrm{Bu}\left(95 \mathrm{mg}, 0.847 \mathrm{mmol}\right.$ ), zwei Äquivalente $\mathrm{Zn}\left(\mathrm{NO}_{3}\right)_{2} \cdot 6 \mathrm{H}_{2} \mathrm{O}(251 \mathrm{mg}$, $0.844 \mathrm{mmol}$ ) und ein Äquivalent Dimethylphosphorsäure $(53 \mathrm{mg}, 0.420 \mathrm{mmol}$ ) zugefügt und bei Raumtemperatur für $12 \mathrm{~h}$ gerührt. Alle flüchtigen Bestandteile werden im Vakuum entfernt, der Rückstand in Aceton (50 ml) aufgenommen, filtriert und mit Petrolether überschichtet. Die erhaltenen, farblosen Kristalle des Produkts 13a sind für die Röntgenstrukturanalyse geeignet.

Ausbeute

Summenformel

Molekulargewicht

${ }^{1} \mathrm{H}-\mathrm{NMR}$

( $\mathrm{d}_{6}$-Aceton, $500 \mathrm{MHz}$ )

${ }^{13} \mathrm{C}-\mathrm{NMR}$

( $\mathrm{d}_{6}$-Aceton, $126 \mathrm{MHz}$ )

${ }^{31}$ P-NMR

( $\mathrm{d}_{6}$-Aceton, $202 \mathrm{MHz}$ )

$\mathrm{IR}(\mathrm{KBr})$
$211 \mathrm{mg},(0.312 \mathrm{mmol}), 74 \%$.

$\mathrm{C}_{17} \mathrm{H}_{37} \mathrm{~N}_{8} \mathrm{O}_{10} \mathrm{PZn}$

$675.28 \mathrm{~g} / \mathrm{mol}$.

$\delta=6.08\left(\mathrm{~s}, 1 \mathrm{H}, \mathrm{CH}^{\mathrm{pz}, 4}\right), 3.81\left(\mathrm{~d},{ }^{2} \mathrm{~J}_{\mathrm{HH}}=14.1 \mathrm{~Hz}, 2 \mathrm{H}, \mathrm{pz}-\right.$

$\left.\mathrm{CH}_{2}\right), 3.64\left(\mathrm{~d},{ }^{2} \mathrm{~J}_{\mathrm{HH}}=14.1 \mathrm{~Hz}, 2 \mathrm{H}, \mathrm{pz}-\mathrm{CH}_{2}\right), 3.60\left(\mathrm{~d},{ }^{3} \mathrm{~J}_{\mathrm{PH}}=\right.$ $\left.10.9 \mathrm{~Hz}, 6 \mathrm{H}, \mathrm{OCH}_{3}\right), 2.87\left(\mathrm{~m}, 2 \mathrm{H}, \mathrm{CH}_{2}\right), 2.71\left(\mathrm{~m}, 2 \mathrm{H}, \mathrm{CH}_{2}\right)$, $2.51\left(\mathrm{~s}, 6 \mathrm{H}, \mathrm{CH}_{3}\right), 2.50\left(\mathrm{~s}, 6 \mathrm{H}, \mathrm{CH}_{3}\right), 1.96\left(\mathrm{~s}, 6 \mathrm{H}, \mathrm{CH}_{3}\right)$. $\delta=151.7\left(\mathbf{C}^{\mathrm{pz}, 3,5}\right), 102.0\left(\mathbf{C H}^{\mathrm{pz}, 4}\right), 57.2\left(\mathrm{pz}-\mathrm{CH}_{2}\right), 56.0\left(\mathrm{CH}_{2}\right)$, $53.4\left(\mathrm{CH}_{2}\right), 53.2\left(\mathrm{~d},{ }^{2} \mathrm{~J}_{\mathrm{CP}}=5.8 \mathrm{~Hz}, \mathrm{OCH}_{3}\right), 48.0\left(\mathrm{CH}_{3}\right), 44.5$ $\left(\mathrm{CH}_{3}\right), 43.6\left(\mathrm{CH}_{3}\right)$.

$\delta=1.11(\mathrm{~s})$.

$\widetilde{v}=2959(\mathrm{~m}), 2909(\mathrm{~m}), 2852(\mathrm{~m}), 2816(\mathrm{w}), 1482(\mathrm{~s}), 1458$

(s), 1444 (s), 1385 (s), $1328(\mathrm{~m}), 1296$ (s), 1237 (s), 1127

(s), 1069 (s), 1047 (s), 1036 (s), 1030 (s), 1016 (m), 967 
(m), $831(\mathrm{~m}), 809(\mathrm{~m}) \mathrm{cm}^{-1}$.

MS (ESI) $\left.\mathrm{m} / \mathrm{z}(\%) \quad 611(40)\left[\mathrm{L}^{4} \cdot \mathrm{H}_{\mathrm{Z}} \mathrm{Zn}_{2}\left\{\mathrm{O}_{2} \mathrm{P}(\mathrm{OMe})_{2}\right\}\left(\mathrm{NO}_{3}\right)\right]^{+}\right)$,

$673(100)\left[\mathrm{L}^{4} \cdot \mathrm{H}_{2} \mathrm{Zn}_{2}\left\{\mathrm{O}_{2} \mathrm{P}(\mathrm{OMe})_{2}\right\}_{2}\right]^{+}$.

Elementaranalyse (\%) berechnet C 30.24 H 5.52 N 16.59;

gefunden C 30.17, H 5.46, N 16.72.

\subsubsection{Synthese von $\left[\mathrm{L}^{4}{ }_{-\mathrm{H}} \mathrm{Zn}_{2}\left(\mathrm{O}_{2} \mathrm{PPh}_{2}\right)_{2}\right]\left(\mathrm{ClO}_{4}\right)$ 14. $\left(\mathrm{ClO}_{4}\right)$}

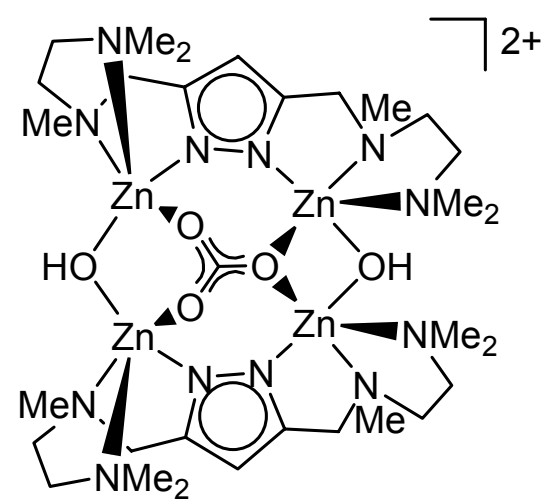

$+2{ }_{\mathrm{Ph}}^{\mathrm{Ph}}=\mathrm{O}=\mathrm{O}^{-}+2 \mathrm{ClO}_{4}^{-}$
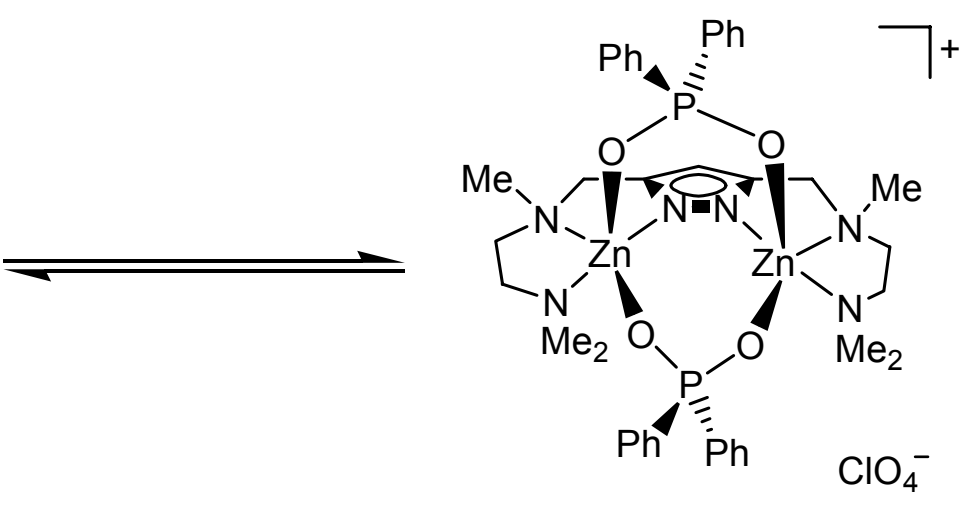

+ andere Produkte

Zu einer Lösung von $\mathrm{L}^{4}(110 \mathrm{mg}, 0.371 \mathrm{mmol})$ in Methanol $(100 \mathrm{ml})$ werden zwei Äquivalente $\mathrm{KO}^{t} \mathrm{Bu}(83.4 \mathrm{mg}, 0.743 \mathrm{mmol})$, zwei Äquivalente $\mathrm{Zn}\left(\mathrm{ClO}_{4}\right)_{2}=6 \mathrm{H}_{2} \mathrm{O}(277$ $\mathrm{mg}, 0.744 \mathrm{mmol}$ ) und ein halbes Äquivalent Kaliumcarbonat (26 mg, $0.188 \mathrm{mg}$ ) unter Rühren zugegeben. Nach 10 min wird ein halbes Äquivalent Diphenylphosphinsäure (40.5 mg, $0.187 \mathrm{mmol}$ ) zugefügt und bei Raumtemperatur für $4 \mathrm{~h}$ gerührt. Alle flüchtigen Bestandteile werden im Vakuum entfernt, Aceton $(50 \mathrm{ml})$ zugefügt, filtriert und mit Petrolether überschichtet. Neben einigen farblosen Kristallen des Produkts 14 bildet sich überwiegend $8 \mathbf{a}$.

Ausbeute

Summenformel

Molekulargewicht

${ }^{1} \mathrm{H}-\mathrm{NMR}(213 \mathrm{~K}$, $\mathrm{d}_{6}$-Aceton, $500 \mathrm{MHz}$ )
$20 \mathrm{mg},(0.021 \mathrm{mmol}), 11 \%$.

$\mathrm{C}_{39} \mathrm{H}_{51} \mathrm{CIN}_{6} \mathrm{O}_{8} \mathrm{P}_{2} \mathrm{Zn}_{2}$

$960.04 \mathrm{~g} / \mathrm{mol}$.

$\delta=7.90-6.86\left(20 \mathrm{H}, \mathrm{CH}^{\mathrm{Ph}}\right), 5.91,5.76\left(1 \mathrm{H}, \mathrm{CH}^{\mathrm{pz}, 4}\right), 4.23-$

$4.01\left(4 \mathrm{H}, \mathrm{pz}-\mathrm{CH}_{2}\right), 3.75-2.50\left(\mathrm{CH}_{2}, \mathrm{CH}_{3}\right), 2.10\left(6 \mathrm{H}, \mathrm{CH}_{3}\right)$. 
${ }^{13} \mathrm{C}-\mathrm{NMR}$

(d6-Aceton, $126 \mathrm{MHz}$ )

$\mathrm{IR}(\mathrm{KBr})$

$\mathrm{MS}(\mathrm{ESI}) \mathrm{m} / \mathrm{z}(\%)$

Elementaranalyse (\%) berechnet C 48.79, H 5.35, N 8.75;

gefunden C 48.84, H 5.37, N 8.65.
$\delta=150.7\left(\mathbf{C}^{\mathrm{pz}, 3,5}\right), 131.8(\mathrm{Ph}), 131.2(\mathrm{Ph}), 128.8(\mathrm{Ph}), 128.7$

$(\mathrm{Ph}), 58.5\left(\mathrm{CH}_{2}\right), 57.6\left(\mathrm{CH}_{2}\right), 54.4\left(\mathrm{CH}_{2}\right), 48.7\left(\mathrm{CH}_{3}\right), 45.6$ $\left(\mathrm{CH}_{3}\right), 42.7\left(\mathrm{CH}_{3}\right)$.

$\widetilde{v}=3073(w), 2963(w), 2915(w), 2892(w), 2851(w), 1484$

(w), $1464(\mathrm{~m}), 1438(\mathrm{~m}), 1319(\mathrm{~m}), 1296(\mathrm{w}), 1245(\mathrm{~m}), 1211$

(m), 1184 (s), 1154 (s), 1129 (s), 1096 (s), 1086 (s), 1045

(s), $1021(\mathrm{~s}), 998(\mathrm{~m}), 942(\mathrm{w}), 832(\mathrm{w}), 806(\mathrm{w}), 776(\mathrm{w})$,

$756(\mathrm{~m}), 724(\mathrm{~m}), 698(\mathrm{~m}), 623(\mathrm{~m}), 555(\mathrm{~s}), 536(\mathrm{~m}), 504$

(w) $\mathrm{cm}^{-1}$.

$857.6(100)\left[\mathrm{L}^{4}{ }_{-\mathrm{H}} \mathrm{Zn}_{2}\left(\mathrm{O}_{2} \mathrm{PPh}_{2}\right)\right]^{+}$.

\subsubsection{Synthese von $\left[\left(\mathrm{L}^{4}{ }_{-\mathrm{H}}\right)_{2} \mathrm{Zn}_{4}(\mathrm{OH})_{2}(\mathrm{MNPP})\right]\left(\mathrm{ClO}_{4}\right)_{2} 15 \cdot\left(\mathrm{ClO}_{4}\right)_{2}$}

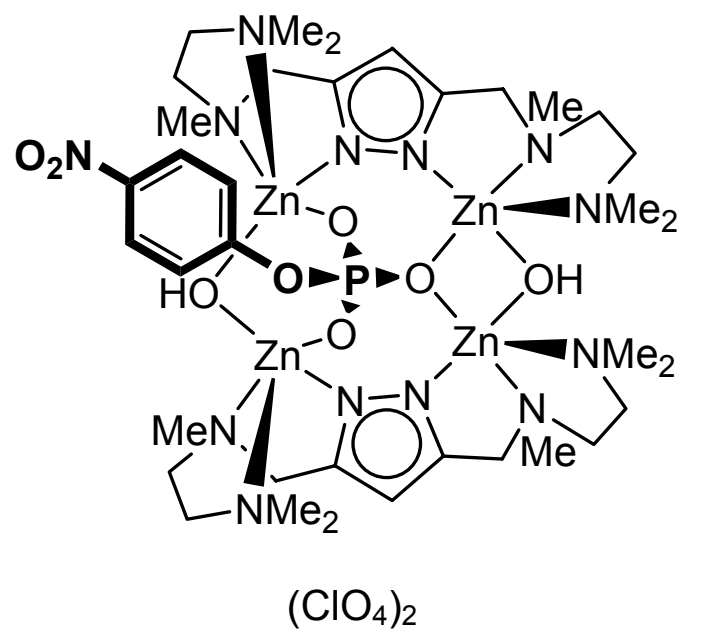

Zu einer Lösung von $\mathrm{L}^{4}(53 \mathrm{mg}, 0.179 \mathrm{mmol})$ in Methanol werden ein Äquivalent $\mathrm{KO}{ }^{t} \mathrm{Bu}\left(20 \mathrm{mg}, 0.178 \mathrm{mmol}\right.$ ), zwei Äquivalente $\mathrm{Zn}\left(\mathrm{ClO}_{4}\right)_{2}-6 \mathrm{H}_{2} \mathrm{O}(134 \mathrm{mg}, 0.360$ $\mathrm{mmol}$ ) und ein Äquivalent Natriumbis(4-nitrophenyl)phosphat (65 mg, $0.179 \mathrm{mmol}$ ) zugefügt, für $12 \mathrm{~h}$ gerührt und abfiltriert. Aus der gelben Lösung bilden sich farblose Kristalle von 15. 


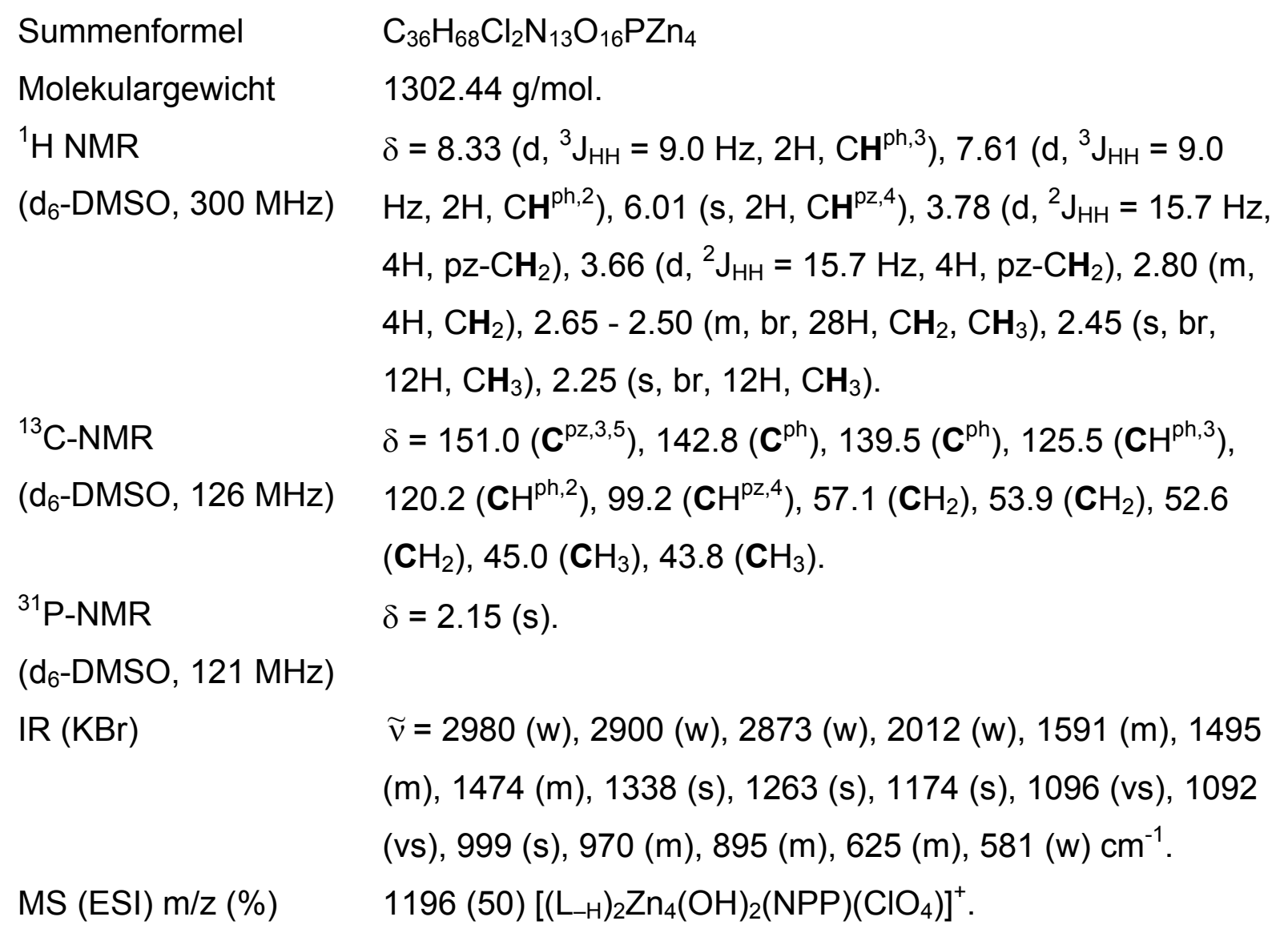




\subsection{Komplexsynthesen - $\beta$-Lactamaddukte}

\subsubsection{Synthese von $\left(\mathrm{L}^{4}{ }_{\mathrm{H}}\right)_{2} \mathrm{Zn}_{4}\left(\mathrm{SO}_{4}\right)\left(\mathrm{NO}_{3}\right)_{4} 16$}

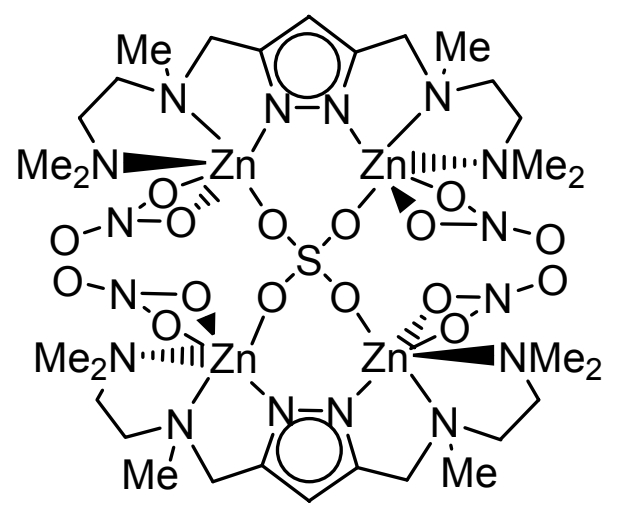

Zu einer Lösung von $\mathrm{L}^{4}(104 \mathrm{mg}, 0.351 \mathrm{mmol})$ in Methanol $(70 \mathrm{ml})$ werden ein Äquivalent $\mathrm{KO}{ }^{t} \mathrm{Bu}(39.4 \mathrm{mg}, 0.351 \mathrm{mmol})$, zwei Äquivalente $\mathrm{Zn}\left(\mathrm{NO}_{3}\right)_{2}-6 \mathrm{H}_{2} \mathrm{O}(209 \mathrm{mg}$, $0.703 \mathrm{mmol}$ ) und ein Äquivalent Sulbactam-Natriumsalz (89.5 mg, $0.351 \mathrm{mmol}$ ) zugefügt und bei Raumtemperatur für $12 \mathrm{~h}$ gerührt. Alle flüchtigen Bestandteile werden im Vakuum entfernt, der Rückstand in Aceton (50 ml) aufgenommen, filtriert und mit Petrolether überschichtet. Es bilden sich im Laufe mehrerer Monate einige farblose Kristalle des Produkts 16, die für die Röntgenstrukturanalyse geeignet sind.

Ausbeute

Summenformel

Molekulargewicht

${ }^{1} \mathrm{H}$ NMR

( $\mathrm{d}_{6}$-Aceton, $\left.500 \mathrm{MHz}\right)$

${ }^{13} \mathrm{C}-\mathrm{NMR}$

( $\mathrm{d}_{6}$ - Aceton, $\left.126 \mathrm{MHz}\right)$

$\mathrm{IR}(\mathrm{KBr})$
$9 \mathrm{mg},(8.1 \mu \mathrm{mol}), 5 \%$.

$\mathrm{C}_{30} \mathrm{H}_{62} \mathrm{~N}_{10} \mathrm{O}_{16} \mathrm{SZn}_{4}$

$1112.50 \mathrm{~g} / \mathrm{mol}$.

$\delta=6.03\left(\mathrm{~s}, 2 \mathrm{H}, \mathrm{CH}^{\mathrm{pz}, 4}\right), 3.85\left(\mathrm{~d},{ }^{2} \mathrm{~J}_{\mathrm{HH}}=14.6 \mathrm{~Hz}, 4 \mathrm{H}, \mathrm{pz}-\right.$

$\left.\mathrm{CH}_{2}\right), 3.70\left(\mathrm{~d},{ }^{2} \mathrm{~J}_{\mathrm{HH}}=14.6 \mathrm{~Hz}, 4 \mathrm{H}, \mathrm{pz}-\mathrm{CH}_{2}\right), 3.00(\mathrm{~m}, 4 \mathrm{H}$,

$\left.\mathrm{CH}_{2}\right), 2.78\left(\mathrm{~m}, 4 \mathrm{H}, \mathrm{CH}_{2}\right), 2.70\left(\mathrm{~s}, 12 \mathrm{H}, 2.45, \mathrm{CH}_{3}\right) 2.50$ (s,

$\left.12 \mathrm{H}, \mathrm{CH}_{3}\right), 2.36\left(\mathrm{~m}, 8 \mathrm{H}, \mathrm{CH}_{2}\right), 2.34\left(\mathrm{~s}, 12 \mathrm{H}, \mathrm{CH}_{3}\right)$.

$\delta=150.3\left(\mathbf{C}^{\mathrm{pz}, 3,5}\right), 100.4\left(\mathbf{C H}^{\mathrm{pz}, 4}\right), 58.2\left(\mathbf{C H}_{2}\right), 55.6\left(\mathbf{C H}_{2}\right)$,

$54.2\left(\mathrm{CH}_{2}\right), 47.2\left(\mathrm{CH}_{3}\right), 44.3\left(\mathrm{CH}_{3}\right), 42.4\left(\mathrm{CH}_{3}\right)$.

$\widetilde{v}=2965(\mathrm{w}), 2923(\mathrm{~m}), 2856 / \mathrm{m}), 2813(\mathrm{w}), 1711(\mathrm{~m}), 1467$

(s), $1384(\mathrm{~s}), 1293$ (s), 1262 (m), 1143 (s), 1134 (s), 1030

(s), $968(\mathrm{~m}), 813(\mathrm{~m}), 633(\mathrm{w}), 604(\mathrm{w}) \mathrm{cm}^{-1}$. 
MS (FAB) m/z (\%) $519(15)\left[\mathrm{L}_{-H} \mathrm{Zn}_{2}\left(\mathrm{SO}_{4}\right)\right]^{+}$.

\subsubsection{Synthese von $\left[\mathrm{L}^{4}{ }_{-\mathrm{H}} \mathrm{Zn}_{2}\left(\mathrm{OH}_{2}\right)\left(\mathrm{C}_{5} \mathrm{H}_{6} \mathrm{NO}_{3}\right)\right]_{2}\left(\mathrm{ClO}_{4}\right)_{4}$ 17.($\left(\mathrm{ClO}_{4}\right)_{4}$}

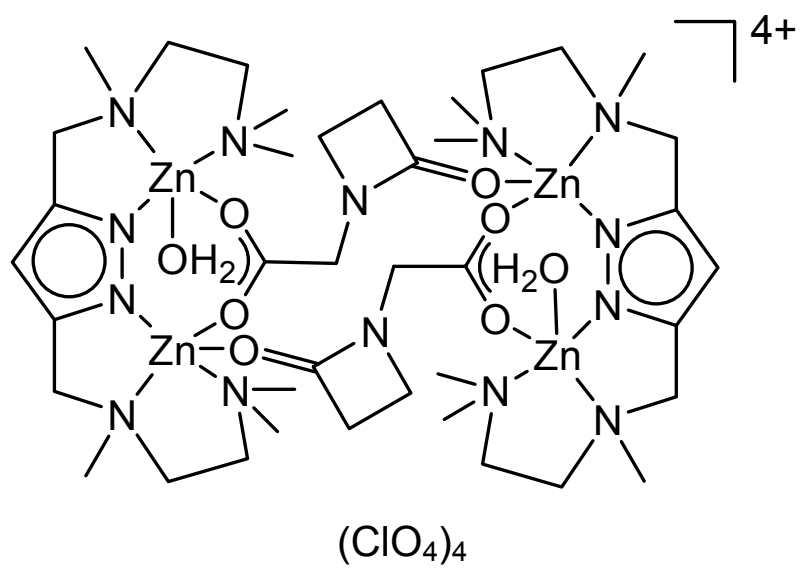

$\mathrm{Zu}$ einer Lösung von $\mathrm{L}^{4}(130 \mathrm{mg}, 0.438 \mathrm{mmol})$ in Methanol $(75 \mathrm{ml})$ werden ein Äquivalent $\mathrm{KO}{ }^{t} \mathrm{Bu}(49.2 \mathrm{mg}, 0.438 \mathrm{mmol})$, zwei Äquivalente $\mathrm{Zn}\left(\mathrm{ClO}_{4}\right)_{2} \cdot 6 \mathrm{H}_{2} \mathrm{O}(327$ $\mathrm{mg}, 0.876 \mathrm{mmol}$ ) und ein Äquivalent XXXLVIII $(66.3 \mathrm{mg}, 0.438 \mathrm{mmol})$ zugefügt und bei Raumtemperatur für $4 \mathrm{~h}$ gerührt. Alle flüchtigen Bestandteile werden im Vakuum entfernt, der Rückstand in Aceton $(50 \mathrm{ml}$ ) aufgenommen, filtriert und mit Petrolether überschichtet. Es bilden sich farblose Kristalle des Produkts 17, die für die Röntgenstrukturanalyse geeignet sind.

Ausbeute

Summenformel

Molekulargewicht $\mathrm{IR}(\mathrm{KBr})$

IR (in $\mathrm{MeOH})$
$102 \mathrm{mg},(0.066 \mathrm{mmol}), 30 \%$.

$\mathrm{C}_{40} \mathrm{H}_{78} \mathrm{Cl}_{4} \mathrm{~N}_{14} \mathrm{O}_{24} \mathrm{Zn}_{4}$

$1542.50 \mathrm{~g} / \mathrm{mol}$.

$\widetilde{v}=3016(w), 2968(w), 2920(w), 2826(w), 1712(s), 1618$

$(\mathrm{s}), 1523(\mathrm{w}), 1460(\mathrm{~m}), 1434(\mathrm{~m}), 1412(\mathrm{~m}), 1359(\mathrm{w}), 1321$

(m), $1247(\mathrm{~m}), 1222(\mathrm{~m}), 1154(\mathrm{~s}), 1107$ (s), 1055 (s), 963

$(\mathrm{m}), 934(\mathrm{w}), 806(\mathrm{~m}), 695(\mathrm{w}), 623(\mathrm{~m}), 503(\mathrm{w}) \mathrm{cm}^{-1}$.

$\widetilde{v}=1735(\mathrm{~m}), 1714(\mathrm{w}), 1613(\mathrm{~m}), 1429(\mathrm{~s}), 1106(\mathrm{~s}), 1005$ (vs), $877(\mathrm{w}) \mathrm{cm}^{-1}$. 


\subsubsection{Synthese von Penicillin G-Addukten}

Ein Äquivalent des entsprechenden Liganden wird in $\mathrm{MeOH}$ gelöst, zwei Äquivalente $\mathrm{Zn}\left(\mathrm{ClO}_{4}\right)_{2} \cdot 6 \mathrm{H}_{2} \mathrm{O}$ und zwei Äquivalente einer Natriummethanolatlösung in Methanol (1M) zugegeben und für $1 \mathrm{~h}$ gerührt. Anschließend wird ein Äquivalent Penicillin G Natriumsalz zugegeben, 1 min gerührt und 2 Äquivalente Natriumtetraphenylborat unter starkem Rühren zugegeben. Der sich bildende, weiße Niederschlag wird schnell abfiltriert und im Vakuum getrocknet.

\section{7 ${ }^{1}$ H-NMR-Parameter für die Pyridylliganden und -komplexe}

Für die Pyridinringe wurden - soweit aufgrund der Spektrenqualität möglich - die Kopplungskonstanten und chemischen Verschiebungen in den ${ }^{1} \mathrm{H}-\mathrm{NMR}$ Spektren des ABCD-Spinsystems durch Simulation der Signale mit dem Programm SpinWorks ${ }^{[142]}$ unter Verwendung des NUMARIT ${ }^{[143]}$ Algorithmusses ermittelt.

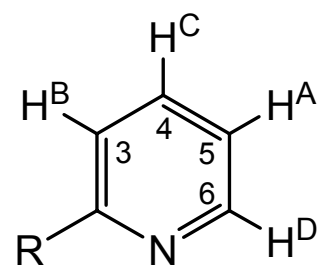

\begin{tabular}{l|llll}
\hline & $\mathbf{1 2 a}$ & $\mathrm{L}^{6}$ & $\mathbf{4}$ & $\mathrm{L}^{9}$ \\
\hline Lösungsmittel & $\mathrm{d}_{6}$-Aceton & $\mathrm{d}_{6}$-Aceton & $\mathrm{d}_{6}$-Aceton & $\mathrm{CDCl}_{3}$ \\
Messfrequenz $(\mathrm{MHz})$ & 500.13 & 500.13 & 500.13 & 300.13 \\
\hline$\delta_{\mathrm{A}}(\mathrm{ppm})$ & 7.76 & 7.14 & 7.52 & 7.15 \\
$\delta_{\mathrm{B}}(\mathrm{ppm})$ & 7.71 & 7.19 & 7.57 & 7.40 \\
$\delta_{\mathrm{C}}(\mathrm{ppm})$ & 8.17 & 7.61 & 8.09 & 7.64 \\
$\delta_{\mathrm{D}}(\mathrm{ppm})$ & 8.99 & 8.40 & 9.08 & 8.54 \\
${ }^{4} \mathrm{~J}_{\mathrm{AB}}(\mathrm{Hz})$ & 1.0 & 1.2 & 1.3 & 1.1 \\
${ }^{3} \mathrm{~J}_{\mathrm{AC}}(\mathrm{Hz})$ & 7.6 & 7.7 & 7.6 & 7.6 \\
${ }^{3} \mathrm{~J}_{\mathrm{AD}}(\mathrm{Hz})$ & 5.4 & 5.0 & 5.6 & 4.9 \\
${ }^{3} \mathrm{~J}_{\mathrm{BC}}(\mathrm{Hz})$ & 7.8 & 7.7 & 7.9 & 7.7 \\
${ }^{5} \mathrm{~J}_{\mathrm{BD}}(\mathrm{Hz})$ & 0.9 & 1.0 & 0.8 & 0.9 \\
${ }^{4} \mathrm{~J}_{\mathrm{CD}}(\mathrm{Hz})$ & 1.7 & 2.0 & 1.7 & 1.8 \\
\hline
\end{tabular}




\subsection{Potentiometrische Messungen}

Die pH-potentiometrischen Messungen wurden bei $25.0(1){ }^{\circ} \mathrm{C}$ bei einer lonenstärke von $0.2 \mathrm{M}(\mathrm{KCl})$ durchgeführt. Zur Titration stand ein Radiometer PHM 84 pH-Meter mit einer Metrohm 6.0234.100 Glaselektrode und ein Metrohm Dosimat 715 zur Verfügung. Unter Verwendung eines Phtalsäuremonokaliumsalz-Puffers bei $\mathrm{pH}$ 4.008 wurden die Kalibrierung der Elektroden und des pH-Meters durchgeführt, die Konzentrationen der $\mathrm{HCl}$ und $\mathrm{KOH}$-Lösungen überprüft sowie der $\mathrm{pK}_{\mathrm{w}}$ und der IrvingFaktor unter Verwendung der Methode nach $\operatorname{Gran}^{[144]}$ ermittelt $\left(\mathrm{pK}_{\mathrm{W}}=13.765\right.$, IrvingFaktor $=0.082$ ).

Die Titrationen der freien Liganden wurden zwischen $\mathrm{pH} 2$ und $\mathrm{pH} 11.5$ und bei Zusatz von Zinksalzen zwischen pH 2 und $\mathrm{pH} 11$ unter Verwendung der $\mathrm{KOH}-$ Lösung durchgeführt. Die pH-metrischen Ergebnisse wurden verwendet um die Zusammensetzung der Spezies zu ermitteln und die Stabilitätskonstanten zu errechnen. Die Berechnungen erfolgten mit Hilfe der Programme SUPERQUAD und PSEQUAD ${ }^{[77]}$ und die Diagramme der Speziesverteilungen wurden mit Hilfe des MEDUSA Programms erstellt. ${ }^{[145]}$

Die angeführten Säurekonstanten und Stabilitätskonstanten sind wie folgt definiert:

$$
\begin{gathered}
\mathrm{pK}_{\mathrm{s}}=-\lg \frac{\mathrm{a}\left(\mathrm{H}^{+}\right) \cdot \mathrm{a}\left(\mathrm{A}^{-}\right)}{\mathrm{a}(\mathrm{HA})} \\
\lg \beta=\lg \frac{\left[\mathrm{H}_{\mathrm{b}} \mathrm{A}_{\mathrm{c}} \mathrm{Zn}_{\mathrm{d}}\right]}{\mathrm{a}^{\mathrm{b}}\left(\mathrm{H}^{+}\right) \cdot[\mathrm{A}]^{\mathrm{c}} \cdot\left[\mathrm{Zn}^{2+}\right]^{d}}
\end{gathered}
$$

Für die Bestimmungen wurden folgende Standardlösungen verwendet:

$\mathrm{HCl} \quad 0.2073 \mathrm{M}$

$\mathrm{KOH} \quad 0.1986 \mathrm{M}$ für $L^{1}, L^{4}$ und $L^{5} ; 0.1850 \mathrm{M}$ für $L^{6}, L^{8}, L^{9}, L^{10}$

$\mathrm{L}^{1} \quad 7.6 \mathrm{mM} ; 0.033 \mathrm{M} \mathrm{HCl}$

$\mathrm{L}^{4} \quad 7.6 \mathrm{mM} ; 0.031 \mathrm{M} \mathrm{HCl}$

$\mathrm{L}^{5} \quad 8.0 \mathrm{mM} ; 0.042 \mathrm{M} \mathrm{HCl}$

$\mathrm{L}^{6} \quad 1.9 \mathrm{mM} ; 0.031 \mathrm{M} \mathrm{HCl}$

$\mathrm{L}^{8} \quad 2.1 \mathrm{mM} ; 0.032 \mathrm{M} \mathrm{HCl}$

$\mathrm{L}^{9} \quad 7.2 \mathrm{mM} ; 0.047 \mathrm{M} \mathrm{HCl}$

$\mathrm{L}^{10} \quad 5.3 \mathrm{mM} ; 0.033 \mathrm{M} \mathrm{HCl}$

$\mathrm{KCl} \quad 2 \mathrm{M}$

$\mathrm{ZnCl}_{2} \quad 0.0979 \mathrm{M}$ mit $0.0163 \mathrm{M} \mathrm{HCl}$ 
Es ergaben sich die folgenden, mit dem Programm SUPERQUAD für $[\mathrm{L}]_{0}$ und $\left[\mathrm{H}^{+}\right]_{0}$ berechneten Anfangskonzentrationen $\left(V_{0}=10 \mathrm{ml}\right)$ :

\begin{tabular}{|c|c|c|c|c|c|c|c|c|}
\hline & & $L^{1}$ & $\mathrm{~L}^{4}$ & $L^{5}$ & $L^{6}$ & $\mathrm{~L}^{8}$ & $\mathrm{~L}^{9}$ & $L^{10}$ \\
\hline \multirow{2}{*}{ Ligand } & {$[\mathrm{L}]_{0} \mathrm{mM}$} & 3.828 & 3.822 & 3.974 & 0.956 & 1.050 & 3.635 & 2.653 \\
\hline & {$\left[\mathrm{H}^{+}\right]_{0} \mathrm{mM}$} & 32.24 & 31.10 & 28.80 & 15.60 & 15.99 & 23.66 & 16.57 \\
\hline \multirow{2}{*}{$\begin{array}{l}\mathrm{L} / \mathrm{Zn}^{2+} \\
\text { (A) }\end{array}$} & {$\left[\mathrm{Zn}^{2+}\right]_{0, \mathrm{~A}} \mathrm{mM}$} & 2.937 & 2.937 & 2.937 & 0.979 & 0.979 & 3.916 & 13.71 \\
\hline & {$\left[\mathrm{H}^{+}\right]_{0, \mathrm{~A}} \mathrm{mM}$} & 32.49 & 31.58 & 33.58 & 15.78 & 16.15 & 24.03 & 16.62 \\
\hline \multirow{2}{*}{$\begin{array}{l}\mathrm{L} / \mathrm{Zn}^{2+} \\
\text { (B) }\end{array}$} & {$\left[\mathrm{Zn}^{2+}\right]_{0, \mathrm{~B}} \mathrm{mM}$} & 3.720 & 4.895 & 4.895 & 1.469 & 1.469 & 5.874 & 2.127 \\
\hline & {$\left[\mathrm{H}^{+}\right]_{0, \mathrm{~B}} \mathrm{mM}$} & 32.74 & 31.91 & 33.91 & 15.87 & 16.24 & 24.35 & 13.09 \\
\hline \multirow{3}{*}{$\begin{array}{l}\mathrm{L} / \mathrm{Zn}^{2+} \\
\text { (C) }\end{array}$} & {$\left[\mathrm{Zn}^{2+}\right]_{0, \mathrm{c}} \mathrm{mM}$} & - & 6.853 & 6.853 & 1.860 & 1.860 & 6.853 & - \\
\hline & {$\left[\mathrm{H}^{+}\right]_{0, \mathrm{c}} \mathrm{mM}$} & - & 32.23 & 34.24 & 15.94 & 16.30 & 24.51 & - \\
\hline & $\begin{array}{l}\text { fitting } \\
\text { Parameter } 10^{-3}\end{array}$ & & 6.4 & 19.7 & 3.5 & 7.8 & 9.7 & 11 \\
\hline
\end{tabular}
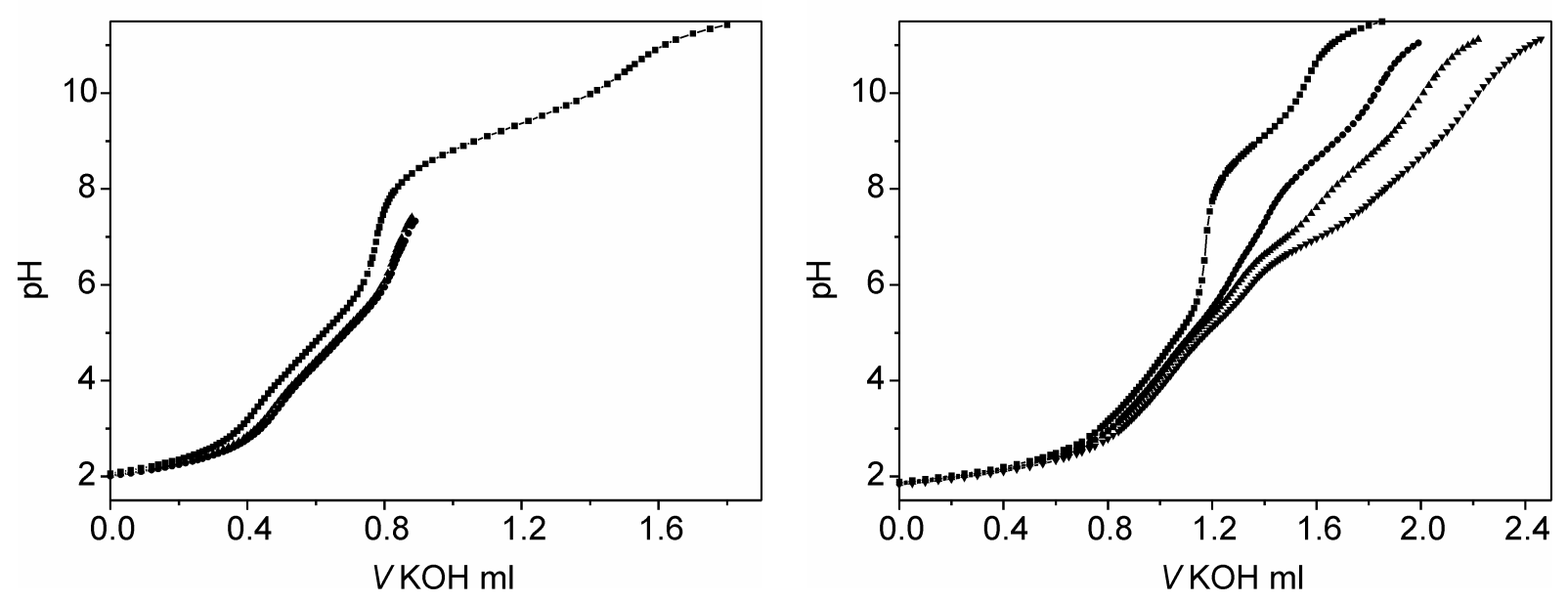

Abbildung 124: Links: Titrationskurven von $L^{1}$; 1 eq. Ligand $\triangleq 0.191 \mathrm{ml} \mathrm{KOH;}$

Rechts: Titrationskurven von $\mathrm{L}^{4} ; 1$ eq. Ligand $\hat{=} 0.192 \mathrm{ml} \mathrm{KOH}$;

von links nach rechts: jeweils Titration von $L$ ohne $\mathrm{Zn}^{2+}$, mit $\mathrm{Zn}^{2+}(\mathrm{A}),(\mathrm{B}),(\mathrm{C})$. 

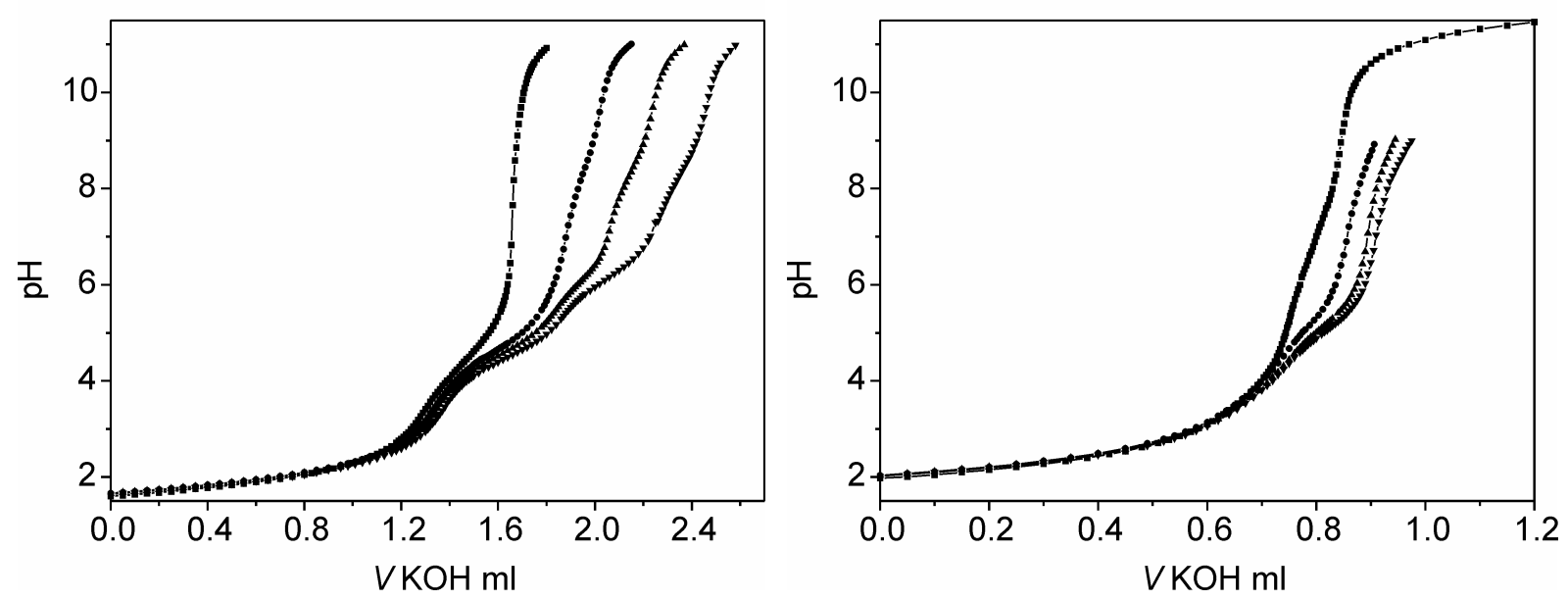

Abbildung 125: Links: Titrationskurven von $\mathrm{L}^{5} ; 1$ eq. Ligand $\hat{=} 0.200 \mathrm{ml} \mathrm{KOH}$;

Rechts: Titrationskurven von $\mathrm{L}^{6} ; 1$ eq. Ligand $\hat{=} 0.052 \mathrm{ml} \mathrm{KOH}$.
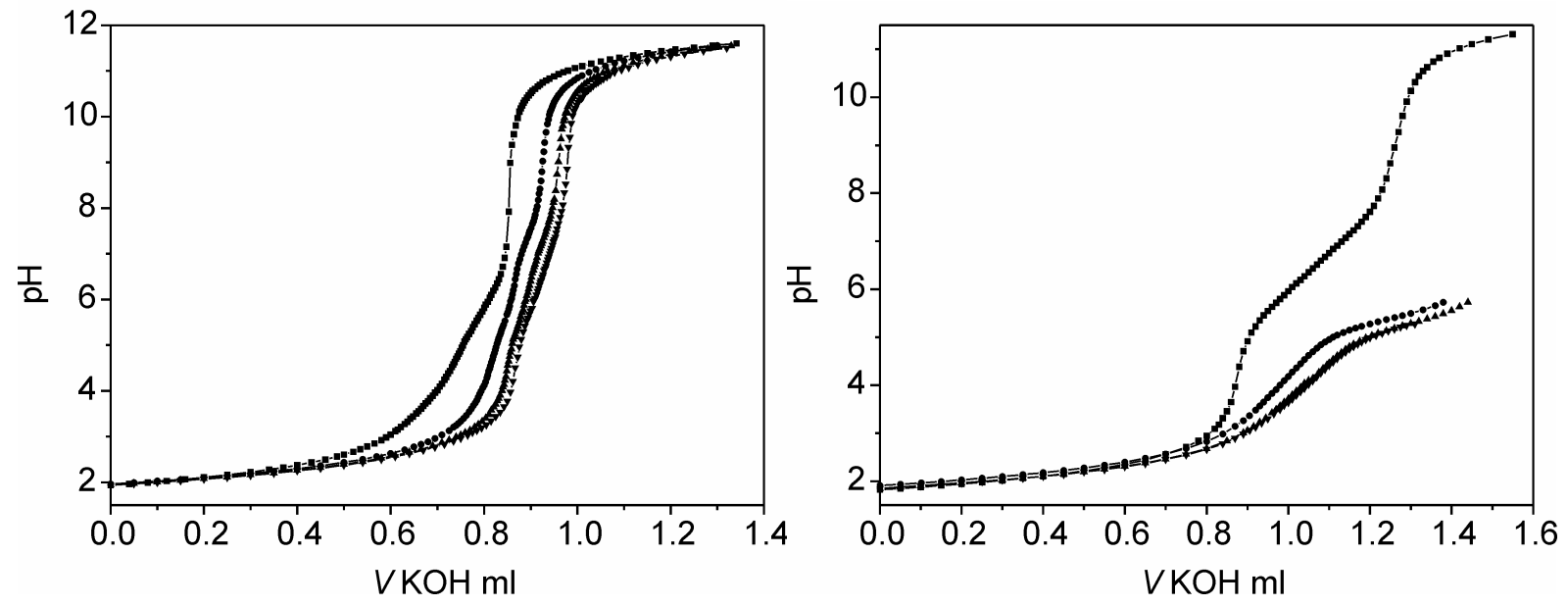

Abbildung 126: Links: Titrationskurven von $\mathrm{L}^{8} ; 1$ eq. Ligand $\hat{=} 0.057 \mathrm{ml} \mathrm{KOH}$;

Rechts: Titrationskurven von $\mathrm{L}^{9} ; 1$ eq. Ligand $\hat{=} 0.196 \mathrm{ml} \mathrm{KOH}$. 


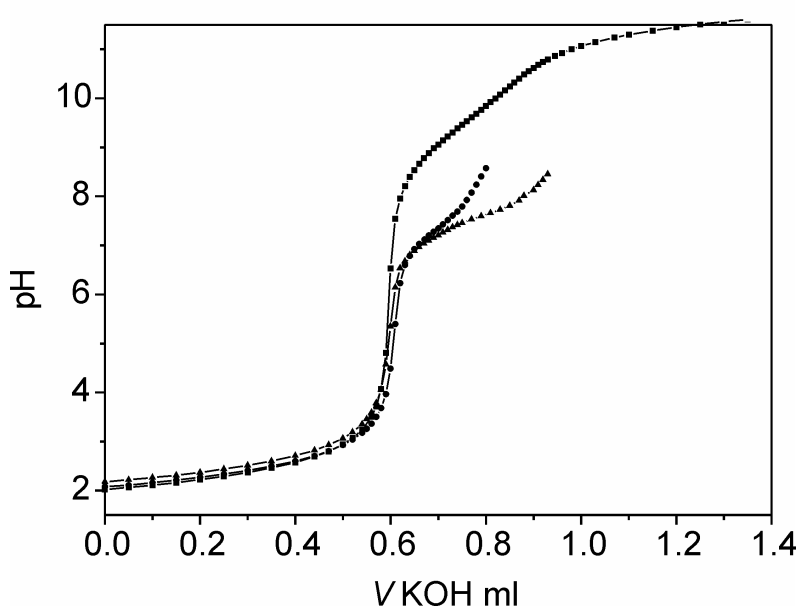

Abbildung 127: $\quad$ Titrationskurven von $\mathrm{L}^{10} ; 1$ eq. Ligand $\hat{=} 0.143 \mathrm{ml} \mathrm{KOH}$. 


\subsection{Untersuchungen zur BNPP-Hydrolyse}

\subsubsection{Kinetische Messungen der BNPP-Hydrolyse mittels UV/Vis- Spektroskopie}

Die kinetischen Messungen wurden auf einem Specord S 100 Spektrometer der Firma Analytik Jena unter Verwendung eines thermostatisierten 10-fach Küvettenwechslers für Quarzküvetten der Dicke $1.0 \mathrm{~cm}$ (Suprasil QS Firma Hellma) aufgenommen.

Die kinetischen Messungen zur BNPP-Hydrolyse wurden bei $50^{\circ} \mathrm{C}$ in gepufferten Lösungen von DMSO/Wasser (1:1) durchgeführt. MES (2-(N-Morpholin)ethansulfonsäure), HEPES (N-(2-Hydroxyethyl)piperazin-N'-2-propansulfonsäure), HEPPS (N-(2hydroxyethyl)piperazin-N'-2-ethansulfonsäure) und CHES (2-(N-Cyclohexylamino)ethansulfonsäure) der Konzentration 0.05 M wurden als Puffer benutzt. Die Konzentrationen der Standardlösungen der Komplexe in DMSO betrugen stets $4.8 \mathrm{mM}$, BNPP-Lösungen in DMSO wurden in den Konzentrationen $12 \mathrm{mM}, 48 \mathrm{mM}$ und 96 $\mathrm{mM}$ eingesetzt.

Die lonenstärke wurde mit Natriumperchlorat auf $0.1 \mathrm{M}$ eingestellt. In einem typischen Experiment wurden $1.5 \mathrm{ml}$ der wässrigen Pufferlösung zu $0.5 \mathrm{ml}$ der Komplexlösung und $0.5 \mathrm{ml}$ DMSO in einer thermostatisierten Küvette gegeben. Nach $10 \mathrm{~min}$ Äquilibrierung wurden $0.5 \mathrm{ml}$ der BNPP-Lösung zugefügt und die Datensammlung sofort gestartet. Die Hydrolyse von BNPP wurde durch den Anstieg der Extinktion von 4-Nitrophenolat bei $414.5 \mathrm{~nm}$ verfolgt und durch Subtraktion der Extinktion bei $500 \mathrm{~nm}$ referenziert. Die Umrechnung von Extinktion in Konzentration wurde mit Hilfe des Lambert-Beerschen Gesetzes $A=\varepsilon_{\text {eff }} C$ vorgenommen. Zur Bestimmung der $\mathrm{pH}$ Abhängigkeit von $\varepsilon_{\text {eff }}$ wurden für jede Pufferlösung unter identischen Bedingungen die Extinktionen von 4-Nitrophenolat bestimmt, indem für jede Pufferlösung eine Eichgerade mit jeweils vier Messpunkten aufgestellt wurde. Die Aktivität der Komplexe wurde nach der Methode der Anfangsgeschwindigkeiten bestimmt. Für jeden Messpunkt wurden mindestens zwei unabhängige Messungen durchgeführt.

Die Geschwindigkeitskonstanten erster Ordnung

$$
v_{0}=k_{\mathrm{obs}} \cdot[\text { Komplex }]_{0}
$$


wurden durch lineare Regression aus den [Komplex] $]_{0} / v_{0}$-Kurven ermittelt. Die Michaelis-Menten-Parameter $K_{\mathrm{M}}$ und $k_{\text {cat }}$ wurden direkt durch Anpassung der $[B N P P]_{0} / v_{0}-K u r v e n$ an die Michaelis-Menten Beziehung

$$
\begin{gathered}
v_{0}=v_{\max } \cdot[\mathrm{BNPP}]_{0} /\left(K_{\mathrm{M}}+[\mathrm{BNPP}]_{0}\right) \\
\text { mit } \\
v_{\max }=k_{\text {cat }} \cdot[\text { Komplex }]_{0}
\end{gathered}
$$

erhalten. Die erhaltenen Parameter stimmen auch gut mit den Ergebnissen der nachfolgend abgebildeten Lineweaver-Burk-Auftragungen überein.

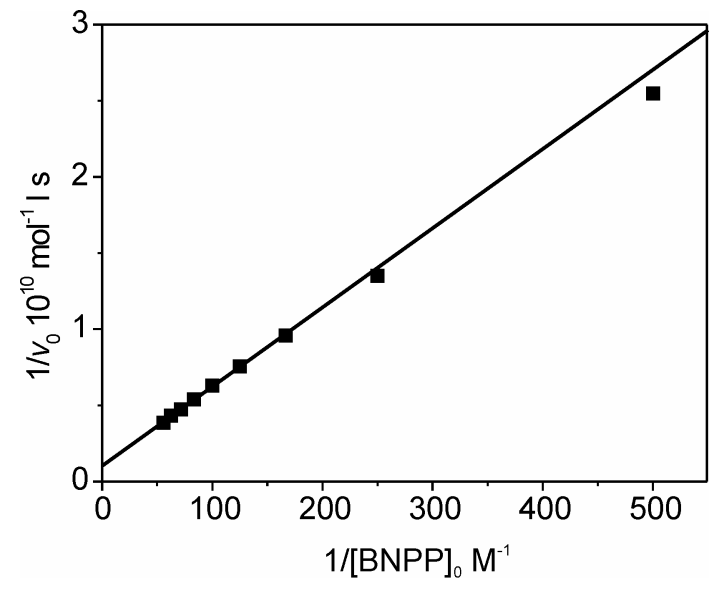

Abbildung 128: Lineweaver-Burk-Plot für 4.

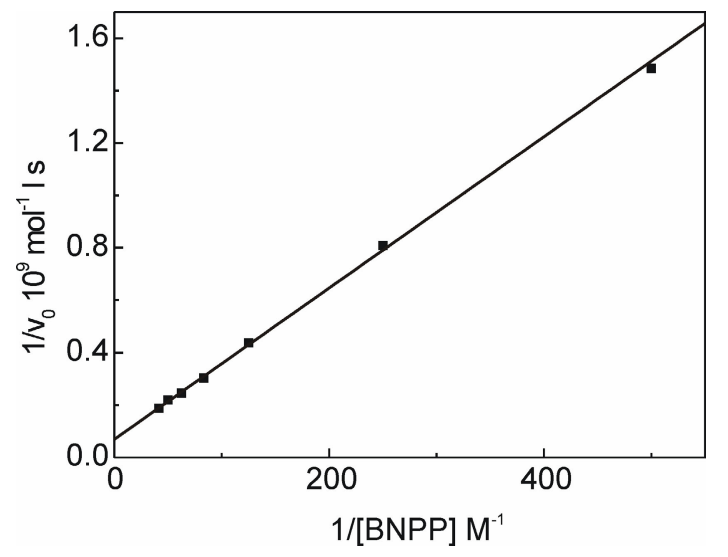

Abbildung 130: Lineweaver-Burk-Plot für 2b.

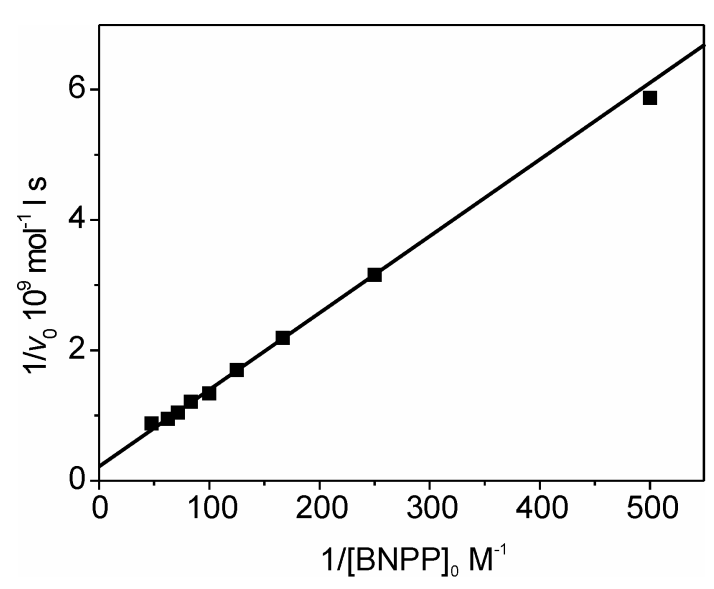

Abbildung 129: Lineweaver-Burk-Plot für 5.

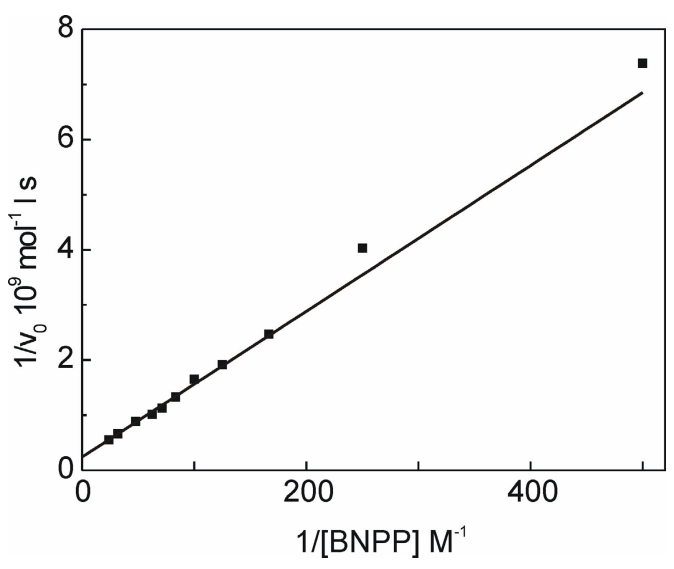

Abbildung 131: Lineweaver-Burk-Plot für 8'. 


\subsection{2 ${ }^{31} \mathrm{P}$-NMR-spektroskopische Untersuchung der BNPP-Hydrolyse}

$\mathrm{Zu}$ einer Lösung von $1.2 \mu \mathrm{mol} \mathrm{L}{ }^{4}$ und $2.4 \mu \mathrm{mol} \mathrm{Zn}\left(\mathrm{ClO}_{4}\right)_{2} \cdot 6 \mathrm{H}_{2} \mathrm{O}$ in $0.25 \mathrm{ml}$ DMSO

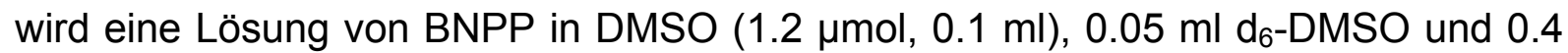
$\mathrm{ml}$ einer wässrigen Pufferlösung (HEPES, pH 8) in ein NMR-Röhrchen gegeben und in einem Wasserbad bei $50^{\circ} \mathrm{C}$ erwärmt. Die Reaktion wird durch ${ }^{31} \mathrm{P}-\mathrm{NMR}$ Spektroskopie verfolgt; zur Auswertung wird das Integralverhältnis des BNPPSignals bei -12.5 ppm und des Signals von 15 bei 1.7 ppm benutzt.

\subsubsection{ESI-MS Untersuchungen}

$\mathrm{Zu}$ einer Lösung des entsprechenden Komplexes in $0.5 \mathrm{ml}$ Methanol wurde ein Äquivalent Dimethylphosphorsäure, Natrium-bis(4-nitrophenyl)phosphat oder eine Mischung von einem Äquivalent Natrium-bis(4-nitrophenyl)phosphat und ein Äquivalent konz. Perchlorsäure hinzugefügt. Die Lösungen wurden für $15 \mathrm{~min}$ in einem Wasserbad bei $45^{\circ} \mathrm{C}$ erwärmt und anschließend im ESI-Massenspektrometer gemessen.

NPMP: (4-Nitrophenyl)(methyl)phosphat

\begin{tabular}{|c|c|c|c|}
\hline \multirow{2}{*}{$\begin{array}{l}\text { Kom- } \\
\text { plex }\end{array}$} & \multicolumn{3}{|c|}{ Phosphatester } \\
\hline & NaBNPP & $\mathrm{NaBNPP} / \mathrm{HClO}_{4}$ & DMP \\
\hline \multirow{5}{*}{1} & {$[\mathrm{LH}]^{+}(100)$} & {$[\mathrm{LH}]^{+}(27)$} & - \\
\hline & {$\left[\mathrm{L}^{1} \mathrm{Zn}_{2}(\mathrm{OH})\left(\mathrm{ClO}_{4}\right)\right]^{+}(60)$} & {$\left[\mathrm{L}^{1} \mathrm{Zn}_{2}(\mathrm{OH})\left(\mathrm{ClO}_{4}\right)\right]^{+}(100)$} & - \\
\hline & {$\left[\mathrm{L}^{1} \mathrm{Zn} \mathrm{n}_{2}(\mathrm{OH})(\mathrm{BNPP})\right]^{+}(70)$} & {$\left[\mathrm{L}^{1} \mathrm{Zn} \mathrm{n}_{2}(\mathrm{OH})(\mathrm{BNPP})\right]^{+}(50)$} & {$\left[\mathrm{L}^{1} \mathrm{Zn} \mathrm{n}_{2}(\mathrm{OH})(\mathrm{DMP})\right]^{+}(12)$} \\
\hline & - & {$\left[\mathrm{L}^{1} \mathrm{Zn} 2(\mathrm{BNPP})\left(\mathrm{ClO}_{4}\right)\right]^{+}(15)$} & {$\left[\mathrm{L}^{1} \mathrm{Zn} \mathrm{n}_{2}(\mathrm{DMP})\left(\mathrm{ClO}_{4}\right)\right]^{+}(12)$} \\
\hline & - & - & {$\left[\mathrm{L}^{1} \mathrm{Zn} \mathrm{n}_{2}(\mathrm{DMP})_{2}\right]^{+}(100)$} \\
\hline \multirow{3}{*}{2} & {$\left[\mathrm{~L}^{2} \mathrm{Zn} 2(\mathrm{OMe})\left(\mathrm{ClO}_{4}\right)\right]^{+}(100)$} & {$\left[\mathrm{L}^{2} \mathrm{Zn} \mathrm{n}_{2}(\mathrm{OMe})\left(\mathrm{ClO}_{4}\right)\right]^{+}(32)$} & - \\
\hline & {$\left[\mathrm{L}^{2} \mathrm{Zn} \text { (BNPP) }\left(\mathrm{ClO}_{4}\right)\right]^{+}(50)$} & {$\left[\mathrm{L}^{2} \mathrm{Zn} \mathrm{n}_{2}(\mathrm{BNPP})\left(\mathrm{ClO}_{4}\right)\right]^{+}(100)$} & {$\left[\mathrm{L}^{2} \mathrm{Zn} 2(\mathrm{DMP})\left(\mathrm{ClO}_{4}\right)\right]^{+}(100)$} \\
\hline & - & - & {$\left[\mathrm{L}^{2} \mathrm{Zn} \mathrm{n}_{2}(\mathrm{DMP})_{2}\right]^{+}(75)$} \\
\hline \multirow{4}{*}{3} & {$\left[\mathrm{~L}^{3} \mathrm{Zn}{ }_{2}(\mathrm{OH})\left(\mathrm{ClO}_{4}\right)\right]^{+}(100)$} & {$\left[\mathrm{L}^{3} \mathrm{Zn}_{2}(\mathrm{OH})\left(\mathrm{ClO}_{4}\right)\right]^{+}(60)$} & - \\
\hline & {$\left[\mathrm{L}^{3} \mathrm{Zn}_{2}(\mathrm{OH})(\mathrm{BNPP})\right]^{+}(15)$} & - & {$\left[\mathrm{L}^{3} \mathrm{Zn}{ }_{2}(\mathrm{OH})(\mathrm{DMP})\right]^{+}(5)$} \\
\hline & - & {$\left[\mathrm{L}^{3} \mathrm{Zn}{ }_{2}(\mathrm{BNPP})\left(\mathrm{ClO}_{4}\right)\right]^{+}(40)$} & {$\left[\mathrm{L}^{3} \mathrm{Zn}_{2}(\mathrm{DMP})\left(\mathrm{ClO}_{4}\right)\right]^{+}(35)$} \\
\hline & - & - & {$\left[\mathrm{L}^{3} \mathrm{Zn} \mathrm{n}_{2}(\mathrm{DMP})_{2}\right]^{+}(100)$} \\
\hline \multirow{2}{*}{4} & {$\left[\mathrm{~L}^{6} \mathrm{Zn}_{2}(\mathrm{OMe})\left(\mathrm{ClO}_{4}\right)\right]^{+}(17)$} & - & - \\
\hline & {$\left[\mathrm{L}^{6} \mathrm{Zn}_{2}(\mathrm{DMP})\left(\mathrm{ClO}_{4}\right)\right]^{+}(30)$} & - & - \\
\hline
\end{tabular}




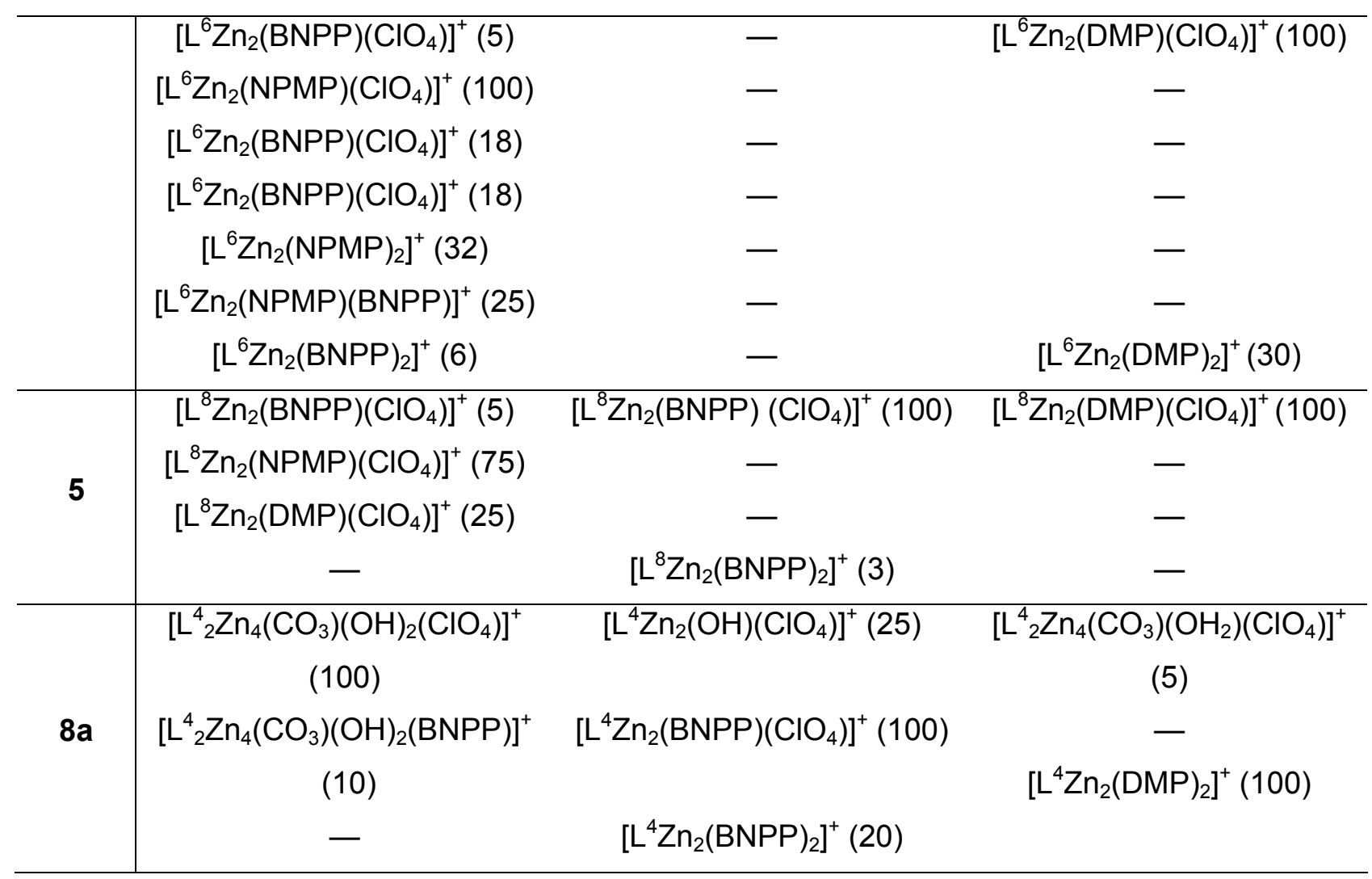

\subsection{4 ${ }^{31}$ P-NMR-Titrationen}

Für die Titrationen wurde eine Lösung von DMP (139 mM) in einer 2:1 $\mathrm{d}_{6^{-}}$ DMSO/Pufferlösung (HEPES, $\mathrm{pH}$ 8) sukzessive zu einer Lösung von $\mathbf{2 b}\left(\mathrm{c}_{0}=9.0\right.$ $\mathrm{mM})$ oder $3\left(\mathrm{c}_{0}=8.7 \mathrm{mM}\right), 4\left(\mathrm{c}_{0}=9.6 \mathrm{mM}\right)$ oder $5\left(\mathrm{c}_{0}=9.6 \mathrm{mM}\right)$ in $0.6 \mathrm{ml}$ der obigen Lösung gegeben. Die Proben wurden mindestens $6 \mathrm{~h}$ äquilibriert, bevor die ${ }^{31} \mathrm{P}$ NMR-Spektren aufgenommen wurden.

\subsection{Untersuchungen zur $\beta$-Lactam Hydrolyse}

\subsubsection{IR-Spektroskopie}

Die IR-Spektren wurden auf einem Digilab Excalibur Spektrometer mit einem Axiom Analytical DPR-210 Dipper und einem MCT-Detektor aufgenommen. Für die Messungen wurden folgende Lösungsmittelmischungen verwendet (v/v): DMSO/Wasser 9:1, DMSO/Wasser 8:2, DMSO/Wasser gepuffert $\mathrm{pH} 7.32,7.83$ oder 8.47 .

In einem typischen Experiment wurde Penicillin in dem entsprechenden Lösungsmittelgemisch gelöst. Anschließend wurde der Komplex zugegeben und die 
Messung gestartet. Die Umrechnung in Konzentrationen erfolgte durch Integration der Fläche des $\beta$-Lactamsignals.

Die Parameter $K_{\mathrm{M}}$ und $k_{\mathrm{cat}}$ wurden durch eine Anpassung der erhaltenen Daten an die Gleichung

$$
\begin{gathered}
\frac{[\mathrm{S}]_{0}-[\mathrm{S}]}{\mathrm{t}}=v-K_{\mathrm{M}} \frac{\ln [\mathrm{S}]_{0}-\ln [\mathrm{S}]}{\mathrm{t}} \\
\quad \mathrm{mit} \\
v=k_{\text {cat }} \cdot[\text { Komplex }]
\end{gathered}
$$

erhalten.

\subsubsection{NMR-Spektroskopie}

4 (53.5 mg, mmol) bzw. 5 (52.1 mg) wurden zusammen mit Cephalothin (25.1 mg) in $\mathrm{d}_{6}$-DMSO (0.6 ml, bei 5 zusätzlich $\left.50 \mu \mathrm{l} \mathrm{D}_{2} \mathrm{O}\right)$ gelöst.

${ }^{13} \mathrm{C}-N M R-D a t e n$ für freies Cephalothin Natriumsalz (500 MHz, $\mathrm{d}_{6}$-DMSO): $\delta=170.4$ (CO, Ester, C15), 169.9 (CO, Amid, C6), 163.8 (COO, C13), 162.8 (CO, Lactam, C8), 136.9, 135.1 (C4, C12), $126.5,126.1,124.8$ (C1, C2, C3), 111.7 (C11)), 64.5 (C14), 58.5 (C7), 57.1 (C9), 35.7 (C5), 25.1 (C10), 20.6 (C16).

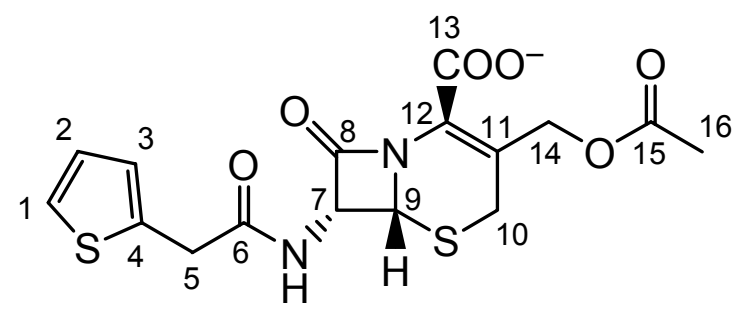

${ }^{13} \mathrm{C}-N M R-D a t e n$ für Cephalothin in Gegenwart von 4 (500 MHz, $\mathrm{d}_{6}$-DMSO): $\delta=170.1$ (C15), 169.9 (C6), 166.7, 164.1, 150.8 (py), 149.1, 148.8, 148.7, 140.4, 126.5, 126.2, $125.3,124.9,124.7,124.1,123.0,100.5,63.2,58.7,57.2,56.3,56.0,54.9,35.7$, $32.7,30.6,25.1,20.4$.

${ }^{13}$ C-NMR-Daten für Cephalothin in Gegenwart von 5 (500 MHz, $\mathrm{d}_{6}$-DMSO): $\delta=171.2$ (C15), 170.7 (C6), 164.2, 137.2, 156.3, 151.3, 148.6, 141.6, 137.2, 127.2, 126.9, $125.4,125.3,125.2,100.7,64.5,59.1,57.7,52.5,49.0,36.2,25.6,21.1$. 


\subsection{Röntgenstrukturanalyse}

\subsubsection{Allgemeines}

Die Bestimmung der Elementarzelle und die Sammlung der Messdaten erfolgte an der Universität Heidelberg auf einem Bruker-AXS CCD Diffraktometer mit graphitmonochromatisierter Mo- $\mathrm{K}_{\alpha}$-Strahlung $(\lambda=0.71073 \AA)$. An der Universität Göttingen erfolgten die Messungen an einem STOE IPDS II Gerät, einem Vierkreisdiffraktometer mit Offset-Eulerwiege der Firma Huber mit einem Bruker SMART 6K CCDZähler mit graphitmonochromatisierte Mo- $K_{\alpha}-$ Strahlung $(\lambda=0.711 \AA$ ), sowie an einem SMART 6000 CCD Diffraktometer mit Cu-K $K_{\alpha}$-Strahlung $(\lambda=1.542 \AA)$. Die Messwerte wurden bezüglich Lorentz- und Polarisationseffekten korrigiert. Zusätzlich erfolgten Absorptionskorrekturen. Die Lösung und Verfeinerung wurde mit den Programmen SHELXS-97 ${ }^{[146]}$ und SHELXL-97 ${ }^{[147]}$ durchgeführt. Bei den angegebenen Kristalldaten und den folgenden Gitterkonstanten sind in Klammern hinter den jeweiligen Werten die Standardabweichungen in Einheiten der letzten Dezimale angegeben.

Für die Struktur des Komplexes 15 wurden Daten eines nicht merohedral verzwillingten Kristalls gesammelt. Das Zwillingsgesetz $\begin{array}{lllllllllll}1 & 0 & 0 & 0 & -1 & 0 & -0.5025 & 0 & -1\end{array}$ (zweizählige Drehung um die a Achse) zeigt, dass die Reflexe $h=2 \mathrm{n}$ an der zweiten Zwillingsdomäne beteiligt sind. Eine zusätzliche racemische Verzwilligung wurde gefunden und die anteiligen Beiträge zu 0.16(2), 0.09(2) und 0.02(2) verfeinert.

Die Röntgenstrukturanalysen wurden durchgeführt von Dr. Sebastian Dechert, Dr. Regine Herbst-Irmer, Dr. Hans Pritzkow, José Antonio Cuesta Seijo und Denis Vidovic. 


\subsubsection{Kristalldaten und Angaben zur Struktur im Festkörper}

\begin{tabular}{|c|c|c|}
\hline Verbindung & $2 b$ & 3 \\
\hline Formel & $\begin{array}{l}\mathrm{C}_{31} \mathrm{H}_{70} \mathrm{Cl}_{2} \mathrm{~N}_{8} \mathrm{O}_{11} \mathrm{Zn}_{2} \\
\cdot\left(\mathrm{CH}_{3} \mathrm{OH}\right)\end{array}$ & $\mathrm{C}_{29} \mathrm{H}_{58} \mathrm{Cl}_{2} \mathrm{~N}_{8} \mathrm{O}_{9} \mathrm{Zn}_{2}$ \\
\hline$M\left[\mathrm{~g} \mathrm{~mol}^{-1}\right]$ & 932.59 & 864.47 \\
\hline Kristallgröße [mm] & $0.40 \times 0.30 \times 0.24$ & $0.31 \times 0.21 \times 0.15$ \\
\hline Kristallsystem & orthorhombisch & monoklin \\
\hline Raumgruppe & Pbca & $P 2_{1}$ \\
\hline$a[\AA]$ & $19.1705(10)$ & $9.5207(4)$ \\
\hline$b[\AA]$ & $18.2978(10)$ & $12.1629(5)$ \\
\hline$c[\AA]$ & $48.448(3)$ & $16.0064(7)$ \\
\hline$\alpha\left[^{\circ}\right]$ & 90 & 90 \\
\hline$\beta\left[^{\circ}\right]$ & 90 & $99.780(1)$ \\
\hline$\gamma\left[^{\circ}\right]$ & 90 & 90 \\
\hline$V\left[\AA^{3}\right]$ & $16995(2)$ & $1827(1)$ \\
\hline$\rho_{\text {ber. }}\left[\mathrm{g} \mathrm{cm}^{-3}\right]$ & 1.458 & 1.572 \\
\hline Z & 16 & 2 \\
\hline$F(000)$ & 7904 & 908 \\
\hline$T[\mathrm{~K}]$ & $173(2)$ & $190(2)$ \\
\hline$\lambda[\AA]$ & 0.71073 & 0.71073 \\
\hline \multirow[t]{2}{*}{$h k l$ Bereich } & \multicolumn{2}{|c|}{0 bis 23,0 bis 22,0 bis $\pm 13,-17$ bis 18,0 bis } \\
\hline & 60 & 23 \\
\hline$\theta$ Bereich $\left[{ }^{\circ}\right]$ & 1.35 bis 26.37 & 1.29 bis 32.03 \\
\hline Gemessene Reflexe & 103779 & 19758 \\
\hline Unabhängige Reflexe $\left(R_{\text {int }}\right)$ & $17372(0.0604)$ & $10986(0.0324)$ \\
\hline Verfeinerte Parameter & 1552 & 662 \\
\hline Restelektronendichte $\left[\mathrm{e} \AA^{-3}\right]$ & $0.821 /-0.582$ & $0.897 /-0.300$ \\
\hline$R 1(I>2 \sigma(I))$ & 0.047 & 0.035 \\
\hline wR2 (alle Daten) & 0.113 & 0.086 \\
\hline Goodness-of-fit & 1.040 & 1.022 \\
\hline Gerät & Bruker AXS CCD & Bruker AXS CCD \\
\hline
\end{tabular}




\begin{tabular}{|c|c|c|}
\hline & 4 & 5 aus Aceton \\
\hline Formel & $\begin{array}{l}\mathrm{C}_{72} \mathrm{H}_{88} \mathrm{Cl}_{4} \mathrm{~N}_{16} \mathrm{O}_{20} \mathrm{Zn}_{4} \\
\cdot\left(\mathrm{H}_{2} \mathrm{O}\right)_{0.2}\end{array}$ & $\begin{array}{l}\mathrm{C}_{30} \mathrm{H}_{34} \mathrm{Cl}_{2} \mathrm{~N}_{8} \mathrm{O}_{10} \mathrm{Zn}_{2} \\
\cdot\left(\mathrm{C}_{3} \mathrm{H}_{6} \mathrm{O}\right)\end{array}$ \\
\hline$M\left[\mathrm{~g} \mathrm{~mol}^{-1}\right]$ & 1904.47 & 926.37 \\
\hline Kristallgröße [mm] & nicht bestimmt & $0.38 \times 0.30 \times 0.28$ \\
\hline Kristallsystem & triklin & monoklin \\
\hline Raumgruppe & $P-1$ & $P 2_{1} / c$ \\
\hline$a[\AA]$ & $14.746(3)$ & $17.3382(4)$ \\
\hline$b[\AA]]$ & $15.465(3)$ & $15.4608(3)$ \\
\hline$c[\AA]]$ & $18.638(4)$ & $15.0678(3)$ \\
\hline$\alpha\left[^{\circ}\right]$ & $92.47(3)$ & 90 \\
\hline$\beta\left[^{\circ}\right]$ & $100.47(3)$ & $106.048(1)$ \\
\hline$\gamma\left[{ }^{\circ}\right]$ & $102.43(3)$ & 90 \\
\hline$V\left[\AA^{3}\right]$ & $4066.8(14)$ & $3881.71(14)$ \\
\hline$\rho_{\text {ber. }}\left[\mathrm{g} \mathrm{cm}^{-3}\right]$ & 1.555 & 1.585 \\
\hline Z & 2 & 4 \\
\hline$F(000)$ & 1964 & 1904 \\
\hline$T[\mathrm{~K}]$ & $133(2)$ & $173(2)$ \\
\hline$\lambda[\AA]$ & 0.71073 & 0.71073 \\
\hline$h k l$ Bereich & \multicolumn{2}{|c|}{$\begin{array}{c}-16 \text { bis } 17, \pm 18, \pm 21-23 \text { bis } 22,0 \text { bis } 20,0 \\
\text { bis } 19\end{array}$} \\
\hline$\theta$ Bereich $\left[{ }^{\circ}\right]$ & 1.44 bis 24.71 & 1.22 bis 28.31 \\
\hline Gemessene Reflexe & 51122 & 30981 \\
\hline Unabhängige Reflexe $\left(R_{\text {int }}\right)$ & $13862(0.0795)$ & $9382(0.028)$ \\
\hline Verfeinerte Parameter & 1062 & 684 \\
\hline Restelektronendichte $\left[\mathrm{e}^{-3}\right]$ & 0.558 und -0.465 & 1.047 und -0.515 \\
\hline$R 1(I>2 \sigma(I))$ & 0.030 & 0.0384 \\
\hline wR2 (alle Daten) & 0.070 & 0.1152 \\
\hline Goodness-of-fit & 0.881 & 1.075 \\
\hline Gerät & STOE IPDS II & Bruker AXS CCD \\
\hline
\end{tabular}




\begin{tabular}{|c|c|c|}
\hline & 5 aus Methanol & 6 \\
\hline Formel & $\begin{array}{l}\mathrm{C}_{30} \mathrm{H}_{34} \mathrm{Cl}_{2} \mathrm{~N}_{8} \mathrm{O}_{10} \mathrm{Zn}_{2} \\
\cdot\left(\mathrm{CH}_{3} \mathrm{OH}\right)_{2}\end{array}$ & $\mathrm{C}_{17} \mathrm{H}_{35} \mathrm{CIN}_{8} \mathrm{O}_{6} \mathrm{Zn}_{2}$ \\
\hline$M\left[\mathrm{~g} \mathrm{~mol}^{-1}\right]$ & 932.38 & 613.72 \\
\hline Kristallgröße [mm] & $0.62 \times 0.23 \times 0.21$ & nicht bestimmt \\
\hline Kristallsystem & monoklin & triklin \\
\hline Raumgruppe & $P 2_{1} / c$ & $P-1$ \\
\hline$a[\AA]]$ & $17.1822(11)$ & $8.6183(17)$ \\
\hline$b[\AA]$ & $15.0256(6)$ & $12.228(2)$ \\
\hline$c[\AA]$ & $15.2378(9)$ & $14.217(3)$ \\
\hline$\alpha\left[^{\circ}\right]$ & 90 & $115.00(3)$ \\
\hline$\beta\left[^{\circ}\right]$ & $104.450(5)$ & $95.18(3)$ \\
\hline$\gamma\left[{ }^{\circ}\right]$ & 90 & $103.07(3)$ \\
\hline$V\left[\AA^{3}\right]$ & $3809.5(4)$ & $1293.1(4)$ \\
\hline$\rho_{\text {ber. }}\left[\mathrm{g} \mathrm{cm}^{-3}\right]$ & 1.626 & 1.576 \\
\hline$Z$ & 4 & 2 \\
\hline$F(000)$ & 1920 & 636 \\
\hline$T[\mathrm{~K}]$ & $133(2)$ & $133(2)$ \\
\hline$\lambda[\AA]$ & 0.71073 & 0.71073 \\
\hline$h k l$ Bereich & $\pm 20, \pm 17, \pm 17$ & -10 bis $7, \pm 14, \pm 16$ \\
\hline$\theta$ Bereich $\left[{ }^{\circ}\right]$ & 1.83 bis 24.67 & 1.92 bis 24.71 \\
\hline Gemessene Reflexe & 27264 & 9721 \\
\hline Unabhängige Reflexe $\left(R_{\text {int }}\right)$ & $6379(0.0553)$ & $4209(0.0775)$ \\
\hline Verfeinerte Parameter & 510 & 319 \\
\hline Restelektronendichte $\left[\mathrm{e} \AA^{-3}\right]$ & $0.975 /-0.693$ & $0.815 /-0.677$ \\
\hline$R 1(I>2 \sigma(I))$ & 0.048 & 0.034 \\
\hline wR2 (alle Daten) & 0.121 & 0.085 \\
\hline Goodness-of-fit & 1.059 & 0.951 \\
\hline Gerät & STOE IPDS II & STOE IPDS II \\
\hline
\end{tabular}




\begin{tabular}{|c|c|c|}
\hline & 7 & $8 b$ \\
\hline Formel & $\mathrm{C}_{15} \mathrm{H}_{31} \mathrm{~N}_{9} \mathrm{O}_{9} \mathrm{Zn}_{2}$ & $\mathrm{C}_{90} \mathrm{H}_{126} \mathrm{~B}_{2} \mathrm{~N}_{12} \mathrm{O}_{7} \mathrm{Zn}_{4}$ \\
\hline$M\left[\mathrm{~g} \mathrm{~mol}^{-1}\right]$ & 612.23 & 1771.13 \\
\hline Kristallgröße [mm] & $0.46 \times 0.25 \times 0.24$ & $0.20 \times 0.20 \times 0.15$ \\
\hline Kristallsystem & monoklin & triklin \\
\hline Raumgruppe & $C 2 / c$ & $P-1$ \\
\hline$a[\AA]$ & $13.366(1)$ & $15.317(3)$ \\
\hline$b[\AA]]$ & $10.161(1)$ & $16.974(3)$ \\
\hline$c[\AA]$ & $19.837(2)$ & $20.313(4)$ \\
\hline$\alpha\left[^{\circ}\right]$ & 90 & $102.64(3)$ \\
\hline$\beta\left[^{\circ}\right]$ & $107.342(2)$ & $110.10(3)$ \\
\hline$\gamma\left[^{\circ}\right]$ & 90 & $103.40(3)$ \\
\hline$V\left[\AA^{3}\right]$ & $2571.7(4)$ & $4559.7(16)$ \\
\hline$\rho_{\text {ber. }}\left[\mathrm{g} \mathrm{cm}^{-3}\right]$ & 1.581 & 1.290 \\
\hline$Z$ & 4 & 2 \\
\hline$F(000)$ & 1264 & 1872 \\
\hline$T[\mathrm{~K}]$ & $298(2)$ & $133(2)$ \\
\hline$\lambda[\AA]$ & 0.71073 & 0.71073 \\
\hline$h k l$ Bereich & \multicolumn{2}{|c|}{$\begin{array}{l}-19 \text { bis } 18,0 \text { bis } 14,0 \pm 18, \pm 19,-23 \text { bis } 21 \\
\text { bis } 28\end{array}$} \\
\hline$\theta$ Bereich $\left[{ }^{\circ}\right]$ & 2.51 bis 30.51 & 1.50 bis 24.71 \\
\hline Gemessene Reflexe & 11285 & 38591 \\
\hline Unabhängige Reflexe $\left(R_{\text {int }}\right)$ & $3915(0.024)$ & $15274(0.080)$ \\
\hline Verfeinerte Parameter & 222 & 1005 \\
\hline Restelektronendichte $\left[\mathrm{e}^{-3}\right]$ & $0.815 /-0.500$ & $1.467 /-0.774$ \\
\hline$R 1(I>2 \sigma(I))$ & 0.046 & 0.054 \\
\hline wR2 (alle Daten) & 0.132 & 0.132 \\
\hline Goodness-of-fit & 1.030 & 1.010 \\
\hline Gerät & Bruker AXS CCD & STOE IPDS II \\
\hline
\end{tabular}




\begin{tabular}{|c|c|c|}
\hline & 9 & $10 a$ \\
\hline \multirow[t]{2}{*}{ Formel } & $\mathrm{C}_{19} \mathrm{H}_{24} \mathrm{~N}_{9} \mathrm{O}_{9} \mathrm{Zn}_{2}$ & $\mathrm{C}_{31} \mathrm{H}_{67} \mathrm{Cl}_{2} \mathrm{~N}_{8} \mathrm{O}_{12} \mathrm{Zn}_{2}$ \\
\hline & $\cdot \mathrm{CH}_{3} \mathrm{OH}$ & \\
\hline$M\left[\mathrm{~g} \mathrm{~mol}^{-1}\right]$ & 684.25 & 976.54 \\
\hline Kristallgröße [mm] & $0.30 \times 0.20 \times 0.20$ & $0.50 \times 0.30 \times 0.20$ \\
\hline Kristallsystem & monoklin & monoklin \\
\hline Raumgruppe & $C 2 / c$ & $P 2_{1} / c$ \\
\hline$a[\AA]$ & $12.241(2)$ & $13.064(3)$ \\
\hline$b[\AA]]$ & $15.814(3)$ & $13.314(3)$ \\
\hline$c[\AA]$ & $13.724(3)$ & $49.814(10)$ \\
\hline$\alpha\left[^{\circ}\right]$ & 90 & 90 \\
\hline$\beta\left[^{\circ}\right]$ & $95.19(3)$ & $93.19(3)$ \\
\hline$\gamma\left[{ }^{\circ}\right]$ & 90 & 90 \\
\hline$V\left[\AA^{3}\right]$ & $2645.8(9)$ & $8651(3)$ \\
\hline$\rho_{\text {ber. }}\left[\mathrm{g} \mathrm{cm}^{-3}\right]$ & 1.718 & 1.500 \\
\hline$Z$ & 4 & 8 \\
\hline$F(000)$ & 1400 & 4112 \\
\hline$T[\mathrm{~K}]$ & $133(2)$ & $100(2)$ \\
\hline$\lambda[\AA]]$ & 0.71073 & 1.54178 \\
\hline \multirow[t]{2}{*}{ hkl Bereich } & $\pm 14, \pm 18, \pm 16$ & $\pm 14, \pm 14$ \\
\hline & & -55 bis 53 \\
\hline$\theta$ Bereich $\left[{ }^{\circ}\right]$ & 2.49 bis 24.81 & 1.78 bis 60.23 \\
\hline Gemessene Reflexe & 14116 & 64317 \\
\hline Unabhängige Reflexe $\left(R_{\text {int }}\right)$ & $2265(0.0438)$ & $12679(0.0221)$ \\
\hline Verfeinerte Parameter & 203 & 1350 \\
\hline Restelektronendichte $\left[\mathrm{e} \AA^{-3}\right]$ & $0.632 /-0.477$ & $0.571 /-0.394$ \\
\hline$R 1(I>2 \sigma(I))$ & 0.035 & 0.028 \\
\hline wR2 (alle Daten) & 0.088 & 0.069 \\
\hline Goodness-of-fit & 1.023 & 1.054 \\
\hline Gerät & STOE IPDS II & SMART 6K CCD \\
\hline
\end{tabular}




\begin{tabular}{|c|c|c|}
\hline & $11 a$ & $12 a$ \\
\hline Formel & $\mathrm{C}_{73} \mathrm{H}_{92} \mathrm{C}_{14} \mathrm{~N}_{16} \mathrm{O}_{25} \mathrm{P}_{2} \mathrm{Zn}_{4}$ & $\begin{array}{l}\mathrm{C}_{31} \mathrm{H}_{35} \mathrm{Cl}_{2} \mathrm{~N}_{8} \mathrm{O}_{12} \mathrm{PZn}_{2} \\
\cdot \mathrm{C}_{3} \mathrm{H}_{6} \mathrm{O}\end{array}$ \\
\hline$M\left[\mathrm{~g} \mathrm{~mol}^{-1}\right]$ & 2058.85 & 1002.36 \\
\hline Kristallgröße [mm] & nicht bestimmt & $0.32 \times 0.26 \times 0.24$ \\
\hline Kristallsystem & monoklin & monoklin \\
\hline Raumgruppe & $C 2 / c$ & $P 2_{1} / n$ \\
\hline$a[\AA]]$ & $40.401(8)$ & $14.1816(8)$ \\
\hline$b[\AA]]$ & $8.7426(17)$ & $12.7628(7)$ \\
\hline$c[\AA]]$ & $25.624(5)$ & $23.0335(13)$ \\
\hline$\alpha\left[^{\circ}\right]$ & 90 & 90 \\
\hline$\beta\left[^{\circ}\right]$ & $107.27(3)$ & $94.307(5)$ \\
\hline$\gamma\left[^{\circ}\right]$ & 90 & 90 \\
\hline$V\left[\AA^{3}\right]$ & $8643(3)$ & $4157.2(4)$ \\
\hline$\rho_{\text {ber. }}\left[\mathrm{g} \mathrm{cm}^{-3}\right]$ & 1.582 & 1.602 \\
\hline Z & 4 & 4 \\
\hline$F(000)$ & 4240 & 2056 \\
\hline$T[\mathrm{~K}]$ & $133(2)$ & $133(2)$ \\
\hline$\lambda[\AA]$ & 0.71073 & 0.71073 \\
\hline$h k l$ Bereich & -47 bis $44, \pm 10, \pm 30$ & $\pm 15, \pm 14,-25$ bis 26 \\
\hline$\theta$ Bereich $\left[{ }^{\circ}\right]$ & 1.66 bis 24.83 & 1.63 bis 24.50 \\
\hline Gemessene Reflexe & 32367 & 18763 \\
\hline Unabhängige Reflexe $\left(R_{\text {int }}\right)$ & $7429(0.0672)$ & $6511(0.0391)$ \\
\hline Verfeinerte Parameter & 563 & 521 \\
\hline Restelektronendichte $\left[\mathrm{e} \AA^{-3}\right]$ & $0.627 /-0.309$ & $1.082 /-1.026$ \\
\hline$R 1(I>2 \sigma(I))$ & 0.032 & 0.070 \\
\hline wR2 (alle Daten) & 0.064 & 0.175 \\
\hline Goodness-of-fit & 0.950 & 1.037 \\
\hline Gerät & STOE IPDS II & STOE IPDS II \\
\hline
\end{tabular}




\begin{tabular}{|c|c|c|}
\hline & $13 a$ & 14 \\
\hline Formel & $\mathrm{C}_{17} \mathrm{H}_{37} \mathrm{~N}_{8} \mathrm{O}_{10} \mathrm{PZn}_{2}$ & $\mathrm{C}_{39} \mathrm{H}_{51} \mathrm{CIN}_{6} \mathrm{O}_{8} \mathrm{P}_{2} \mathrm{Zn}_{2}$ \\
\hline$M\left[\mathrm{~g} \mathrm{~mol}^{-1}\right]$ & 675.26 & 959.99 \\
\hline Kristallgröße [mm] & nicht bestimmt & $0.50 \times 0.30 \times 0.20$ \\
\hline Kristallsystem & monoklin & monoklin \\
\hline Raumgruppe & $P 2_{1} / n$ & $P 2_{1} / n$ \\
\hline$a[\AA]]$ & $11.127(2)$ & $13.9568(6)$ \\
\hline$b[\AA]]$ & $7.9082(16)$ & $15.1926(8)$ \\
\hline$c[\AA]$ & $31.715(6)$ & 21.5761(9) \\
\hline$\alpha\left[^{\circ}\right]$ & 90 & 90 \\
\hline$\beta\left[^{\circ}\right]$ & $95.89(3)$ & $106.526(3)$ \\
\hline$\gamma\left[^{\circ}\right]$ & 90 & 90 \\
\hline$V\left[\AA^{3}\right]$ & $2776(1)$ & $4386.0(3)$ \\
\hline$\rho_{\text {ber. }}\left[\mathrm{g} \mathrm{cm}^{-3}\right]$ & 1.616 & 1.454 \\
\hline$Z$ & 4 & 4 \\
\hline$F(000)$ & 1400 & 1992 \\
\hline$T[\mathrm{~K}]$ & $133(2)$ & $133(2)$ \\
\hline$\lambda[\AA]$ & 0.71073 & 0.71073 \\
\hline$h k l$ Bereich & $\begin{array}{l}-13 \text { bis } 12,-9 \text { bis } \\
-37 \text { bis } 36\end{array}$ & $\begin{array}{l}-16 \text { bis } 15, \pm 17,-25 \text { bis } \\
24\end{array}$ \\
\hline$\theta$ Bereich $\left[{ }^{\circ}\right]$ & 1.89 bis 24.65 & 1.66 bis 24.50 \\
\hline Gemessene Reflexe & 12301 & 25162 \\
\hline Unabhängige Reflexe $\left(R_{\text {int }}\right)$ & $2952(0.0609)$ & $6887(0.0350)$ \\
\hline Verfeinerte Parameter & 351 & 529 \\
\hline Restelektronendichte $\left[\mathrm{e} \AA^{-3}\right]$ & $0.516 /-0.563$ & $0.423 /-0.321$ \\
\hline$R 1(I>2 \sigma(I))$ & 0.039 & 0.027 \\
\hline wR2 (alle Daten) & 0.073 & 0.068 \\
\hline Goodness-of-fit & 1.007 & 1.025 \\
\hline Gerät & STOE IPDS II & STOE IPDS II \\
\hline
\end{tabular}




\begin{tabular}{|c|c|c|c|}
\hline & 15 & 16 & \\
\hline Formel & $\mathrm{C}_{39} \mathrm{H}_{80} \mathrm{Cl}_{2} \mathrm{~N}_{13} \mathrm{O}_{20} \mathrm{PZn}_{4}$ & $\begin{array}{l}\mathrm{C}_{30} \mathrm{H}_{62} \mathrm{~N}_{16} \mathrm{O}_{16} \mathrm{SZn}_{4} \\
\cdot\left(\mathrm{C}_{3} \mathrm{H}_{6} \mathrm{O}\right)_{2}\end{array}$ & \\
\hline$M\left[\mathrm{~g} \mathrm{~mol}^{-1}\right]$ & 1414.51 & 1312.65 & \\
\hline Kristallgröße [mm] & nicht bestimmt & $0.42 \times 0.34 \times 0.28$ & \\
\hline Kristallsystem & monoklin & monoklin & \\
\hline Raumgruppe & $\mathrm{Cm}$ & $P 2_{1} / n$ & \\
\hline$a[\AA]]$ & $17.586(4)$ & $18.0341(2)$ & \\
\hline$b[\AA]$ & $14.211(3)$ & $12.7609(2)$ & \\
\hline$c[\AA]$ & $12.943(3)$ & $25.9329(3)$ & \\
\hline$\alpha\left[^{\circ}\right]$ & 90 & 90 & \\
\hline$\beta\left[^{\circ}\right]$ & $109.96(3)$ & $109.458(1)$ & \\
\hline$\gamma\left[^{\circ}\right]$ & 90 & 90 & \\
\hline$V\left[\AA^{3}\right]$ & $3040(1)$ & $5627.12(13)$ & \\
\hline$\rho_{\text {ber. }}\left[\mathrm{g} \mathrm{cm}^{-3}\right]$ & 1.545 & 1.549 & \\
\hline$Z$ & 2 & 4 & \\
\hline$F(000)$ & 1468 & 2728 & \\
\hline$T[\mathrm{~K}]$ & $133(2)$ & $133(2)$ & \\
\hline$\lambda[\AA]$ & 0.71073 & 0.71073 & \\
\hline$h k /$ Bereich & $\pm 20, \pm 16, \pm 15$ & $\begin{array}{l}-20 \text { bis } 23, \pm 16 \\
-33 \text { bis } 30\end{array}$ & \\
\hline$\theta$ Bereich $\left[{ }^{\circ}\right]$ & 1.89 bis 24.69 & 1.67 bis 27.50 & \\
\hline Gemessene Reflexe & 14092 & 84140 & \\
\hline Unabhängige Reflexe $\left(R_{\text {int }}\right)$ & 4786 & $12885(0.0393)$ & \\
\hline Verfeinerte Parameter & 516 & 692 & \\
\hline Restelektronendichte $\left[\mathrm{e}^{-3}\right]$ & $1.155 /-0.865$ & $0.401 /-0.416$ & \\
\hline$R 1(I>2 \sigma(I))$ & 0.062 & 0.026 & \\
\hline wR2 (alle Daten) & 0.149 & 0.068 & \\
\hline Goodness-of-fit & 1.034 & 1.026 & \\
\hline Gerät & STOE IPDS II & $\begin{array}{l}\text { Bruker SMART } \\
\text { CCD }\end{array}$ & $4 \mathrm{~K}$ \\
\hline
\end{tabular}




\begin{tabular}{|c|c|}
\hline & 17 \\
\hline Formel & $\begin{array}{l}\mathrm{C}_{40} \mathrm{H}_{78} \mathrm{Cl}_{4} \mathrm{~N}_{14} \mathrm{O}_{24} \mathrm{Zn}_{4} \\
\cdot\left(\mathrm{C}_{3} \mathrm{H}_{6} \mathrm{O}\right)\end{array}$ \\
\hline$M\left[\mathrm{~g} \mathrm{~mol}^{-1}\right]$ & 1600.52 \\
\hline Kristallgröße [mm] & $0.40 \times 0.13 \times 0.12$ \\
\hline Kristallsystem & monoklin \\
\hline Raumgruppe & $C 2 / c$ \\
\hline$a[\AA]$ & $35.014(2)$ \\
\hline$b[\AA]$ & $7.9311(4)$ \\
\hline$c[\AA]$ & $25.1307(16)$ \\
\hline$\alpha\left[^{\circ}\right]$ & 90 \\
\hline$\beta\left[^{\circ}\right]$ & $111.856(5)$ \\
\hline$\gamma\left[^{\circ}\right]$ & 90 \\
\hline$V\left[\AA^{3}\right]$ & $6477.2(7)$ \\
\hline$\rho_{\text {ber. }}\left[\mathrm{g} \mathrm{cm}^{-3}\right]$ & 1.641 \\
\hline$Z$ & 4 \\
\hline$F(000)$ & 3312 \\
\hline$T[\mathrm{~K}]$ & $133(2)$ \\
\hline$\lambda[\AA]$ & 0.71073 \\
\hline \multirow[t]{2}{*}{$h k l$ Bereich } & $\pm 40,-8$ bis $9,-28$ bis \\
\hline & 29 \\
\hline$\theta$ Bereich $\left[{ }^{\circ}\right]$ & 1.25 to 24.64 \\
\hline Gemessene Reflexe & 17508 \\
\hline Unabhängige Reflexe $\left(R_{\text {int }}\right)$ & $5455(0.0540)$ \\
\hline Verfeinerte Parameter & 422 \\
\hline Restelektronendichte $\left[\mathrm{e}^{-3}\right]$ & $0.446 /-0.396$ \\
\hline$R 1(I>2 \sigma(I))$ & 0.037 \\
\hline wR2 (alle Daten) & 0.089 \\
\hline Goodness-of-fit & 1.036 \\
\hline Gerät & STOE IPDS II \\
\hline
\end{tabular}




\section{Literaturverzeichnis}

[1] H. M. Berman, J. Westbrook, Z. Feng, G. Gilliland, T. N. Bhat, H. Weissig, I. N. Shindyalov, P. E. Bourne, Nucl. Acids Res. 2000, 28, 235-242.

[2] D. E. Wilcox, Chem. Rev. 1996, 96, 2435-2458.

[3] N. Sträter, W. N. Libscomb, T. Klabunde, B. Krebs, Angew. Chem. 1996, 108, 2158-2191.

[4] G. Parkin, Chem. Rev. 2004, 104, 699-767.

[5] N. H. Williams, B. Takasaki, M. Wall, J. Chin, Acc. Chem. Res. 1999, 32, 485493.

[6] E. Kimura, Curr. Opin. Chem. Biol. 2000, 4, 207-213.

[7] N. O. Concha, B. A. Rasmussen, K. Bush, O. Herzberg, Structure 1996, 4, 823-836.

[8] M. Komiyama, Biochem. 1995, 118, 665-670.

[9] A. D. Mesmaeker, R. Häner, P. Martin, H. E. Moser, Acc. Chem. Res. 1995, 28, 366.

[10] R. Häner, J. Hall, Antisense Nucleic Acid Drug Dev. 1997, 7, 423-430.

[11] M. J. Jedrzejas, P. Setlow, Chem. Rev. 2001, 101, 607-618.

[12] S. C. Burdette, S. J. Lippard, PNAS 2003, 100, 3605-3610.

[13] E. H. Cox, G. L. McLendon, Curr. Opin. Chem. Biol. 2000, 4, 162-165.

[14] M. Shibasaki, H. Sasai, T. Arai, Angew. Chem. 1997, 109, 1290-1310.

[15] B. M. Trost, H. Ito, E. R. Silcoff, J. Am. Chem. Soc. 2001, 123, 3367-3368.

[16] B. M. Trost, V. S. C. Yeh, Angew. Chem. 2002, 114, 889-891.

[17] C. K. Williams, N. R. Brooks, M. A. Hillmyer, W. B. Tolman, Chem. Commun. 2002, 2132-2133.

[18] D. M. Perreault, E. V. Anslyn, Angew. Chem. 1997, 109, 470-490.

[19] R. Wolfenden, C. Ridgeway, G. Young, J. Am. Chem. Soc. 1998, 120, 833834.

[20] K. M. Holtz, E. R. Kantrowitz, FEBS Letters 1999, 462, 7-11.

[21] T. W. Reid, I. B. Wilson, in The Enzymes, Vol. 4 (Ed.: B. D. Boyer), Academic Press, New York, 1971, S. 373-415.

[22] P. Gettins, J. E. Coleman, J. Biol. Chem. 1983, 258, 396-407.

[23] J. E. Coleman, K. Nakamura, J. F. Chlebowski, J. Biol. Chem. 1983, 258, 386395.

[24] T. T. Simopoulos, W. P. Jencks, Biochem. 1994, 33, 10375-10380.

[25] B. Stec, K. M. Holtz, E. R. Kantrowitz, J. Mol. Biol. 2000, 299, 1323-1331.

[26] Y. Kaneko, N. Hayashi, A. Toh-e, I. Banno, Y. Oshima, Gene 1987, 58, $137-$ 148.

[27] W. Kam, E. Clauser, Y. S. Kim, Y. W. Kan, W. J. Rutter, PNAS 1985, 82, 8715-8719.

[28] J. Berger, E. Garattini, J. C. Hua, S. Udenfried, PNAS 1987, 84, 695-698.

[29] M. J. Weiss, P. S. Henthorn, M. A. Lafferty, C. Slaughter, M. Raducha, H. Harris, PNAS 1986, 83, 7182-7186.

[30] E. E. Kim, H. W. Wyckoff, Clin. Chim. Acta 1989, 186, 175-188.

[31] J. E. Murphy, T. T. Tibbitts, E. R. Kantrowitz, J. Mol. Biol. 1995, 253, 604-617.

[32] E. Hough, e. al., Nature 1989, 338, 357-360.

[33] A. Volbeda, A. Lahm, F. Sakiyama, D. Suck, EMBO J. 1991, 10, 1607-1618.

[34] E. E. Kim, H. W. Wyckoff, J. Mol. Biol. 1991, 218, 449-464.

[35] J. E. Murphy, B. Stec, L. Ma, E. R. Kantrowitz, Nature Struct. Biol. 1997, 4, 618-622. 
[36] M. Fujimoto, A. Kuninaka, H. Yoshino, Agr. Biol. Chem. 1974, 38, 785-790.

[37] M. Fujimoto, A. Kuninaka, H. Yoshino, Agr. Biol. Chem. 1974, 38, 1555-1561.

[38] M. Fujimoto, K. Fujiyama, A. Kuninaka, H. Yoshino, Agr. Biol. Chem. 1974, 38, 2141-2147.

[39] M. Fujimoto, A. Kuninaka, H. Yoshino, Agr. Biol. Chem. 1975, 39, 1991-1997.

[40] B. V. L. Potter, B. A. Conolly, F. Eckstein, Biochemistry 1983, 22, 1369-1377.

[41] C. Romier, R. Dominguez, A. Lahm, O. Dahl, PROTEINS: Structure, Function and Genetics 1998, 32, 414-424.

[42] B. C. Antanaitis, P. Aisen, Adv. Inorg. Biochem. 1983, 5, 111-136.

[43] K. Doi, B. C. Antanaitis, P. Aisen, Struct. Bonding 1988, 70, 1-26.

[44] L. Que, Jr., A. E. True, Prog. Inorg. Chem. 1990, 38, 97-200.

[45] J. B. Vincent, G. L. Olivier-Lilley, B. A. Averill, Chem. Rev. 1990, 90, 14471467.

[46] P. R. Nuttleman, R. M. Roberts, J. Biol. Chem. 1990, 265, 12192-12199.

[47] N. Sträter, T. Klabunde, P. Tucker, H. Witzel, B. Krebs, Science 1995, 268, $1489-1492$.

[48] T. Klabunde, N. Sträter, R. Fröhlich, H. Witzel, B. Krebs, J. Mol. Biol. 1996, 259, 737-748.

[49] E. G. Mueller, M. W. Crowder, B. A. Averill, J. R. Knowles, J. Am. Chem. Soc. 1993, 115, 2974-2975.

[50] C. J. Wynne, S. E. Hamilton, D. A. Dionysius, J. L. Beck, J. de Jersey, Arch. Biochem. Biophys. 1995, 319, 133-141.

[51] J. H. Ullah, T. R. Walsh, I. A. Taylor, D. C. Emery, C. S. Verma, S. J. Gamblin, J. Spencer, J. Mol. Biol. 1998, 284, 125-136.

[52] S. M. Fabiane, M. K. Sohi, T. Wan, D. J. Payne, J. H. Bateson, T. Mitchell, B. J. Sutton, Biochemistry 1998, 37, 12404-12411.

[53] J. A. Cricco, E. G. Orellano, R. M. Rasia, E. A. Ceccarelli, A. J. Vila, Coord. Chem. Rev. 1999, 190-192, 519-535.

[54] Z. Wang, W. Fast, A. M. Valentine, S. J. Benkovic, Curr. Opin. Chem. Biol. 1999, 3, 614-622.

[55] Z. Wang, W. Fast, S. J. Benkovic, Biochemistry 1999, 38, 10013-10023.

[56] Z. Wang, S. J. Benkovic, J. Biol. Chem. 1998, 273, 22402-22408.

[57] Z. Wang, W. Fast, S. J. Benkovic, J. Am. Chem. Soc. 1998, 120, 1078810789.

[58] G. C. Dismukes, Chem. Rev. 1996, 96, 2909-2926.

[59] N. Diaz, D. Suárez, K. M. Merz, Jr., J. Am. Chem. Soc. 2000, 122, 4197-4208.

[60] D. Suárez, E. N. Brothers, K. M. Merz, Jr., Biochemistry 2002, 41, 6615-6630.

[61] J. Ackermann, F. Meyer, E. Kaifer, H. Pritzkow, Chem. Eur. J 2002, 8, 247258.

[62] S. Buchler, F. Meyer, E. Kaifer, H. Pritzkow, Inorg. Chim. Acta 2002, 337, 371386.

[63] J. Ackermann, Dissertation, Universität Göttingen 2003.

[64] S. Buchler, Dissertation, Universität Heidelberg 2002.

[65] F. Meyer, P. Rutsch, Chem. Commun. 1998, 1037-1038.

[66] C. Acerete, Tetrahedron 1994, 50, 4765-4774.

[67] F. Meyer, U. Ruschewitz, P. Schober, B. Antelmann, L. Zsolnai, J. Chem. Soc., Dalton Trans. 1998, 1181-1186.

[68] F. Meyer, S. Beyreuther, K. Heinze, L. Zsolnai, Chem. Ber./Recueil 1997, 130, 605-613.

[69] J. C. Röder, F. Meyer, H. Pritzkow, Organomet. 2001, 20, 811-817. 
[70] T. G. Schenck, J. M. Downes, C. R. C. Milne, P. B. MacKenzie, H. Boucher, J. Whelan, B. Bosnich, Inorg. Chem. 1985, 24, 2334-2337.

[71] J. S. Bradshaw, R. B. Nielsen, P. Tse, G. Arena, B. E. Wilson, N. K. Dalley, J. D. Lamb, J. C. Christensen, R. M. Izatt, J. Heterocyclic. Chem. 1986, 23, 361368.

[72] J. A. Halfen, W. B. Tolman, Inorg. Synth. 1998, 32, 75-81.

[73] H. J. Hoorn, P. de Joode, W. L. Driessen, J. Reedijk, Recl. Trav. Chim. PaysBas 1996, 115, 191-197.

[74] U. Holzgrabe, Arch. Pharm. 1987, 320, 647-654.

[75] S. Kondo, K. Shinbo, T. Yamaguchi, K. Yoshida, Y. Yano, J. Chem. Soc., Perkin Trans. 2 2001, 128-131.

[76] J. C. Röder, F. Meyer, M. Konrad, S. Sandhöfner, E. Kaifer, H. Pritzkow, Eur. J. Org. Chem. 2001, 4479-4487.

[77] P. Gans, A. Sabatini, A. Vacca, J. Chem. Soc., Dalton Trans. 1985, 11951200.

[78] L. Siegfried, T. A. Kaden, F. Meyer, P. Kircher, J. Chem. Soc., Dalton Trans. 2001, 2310-2315.

[79] T. A. Kaden, Coord. Chem. Rev. 1999, 190-192, 371-389.

[80] R. Rometsch, A. Marxer, K. Miescher, Helv. Chim. Acta 1951, 34, 1611-1619.

[81] R. Nakon, P. R. Rechani, R. J. Angelici, J. Am. Chem. Soc. 1974, 96, 21172120.

[82] J. K. Romary, J. E. Bunds, J. D. Barger, J. Chem. Eng. Data 1967, 12, 224226.

[83] F. Meyer, K. Heinze, B. Nuber, L. Zsolnai, J. Chem. Soc., Dalton Trans. 1998, 207-213.

[84] F. W. B. Einstein, A. C. Willis, Inorg. Chem. 1981, 20, 609-614.

[85] M. Rodríguez, A. Llobet, M. Corbella, M. P., M. A. Uson, A. E. Martell, J. Reibenspies, J. Chem. Soc., Dalton Trans. 2002, 2900-2906.

[86] A. Escuer, E. Penalba, R. Vicente, X. Solans, M. Font-Bardia, J. Chem. Soc., Dalton Trans. 1997, 2315-2319.

[87] M. Fondo, A. M. García-Deibe, M. R. Bermejo, J. Sanmartín, A. L. LlamasSaiz, J. Chem. Soc., Dalton Trans. 2002, 4746-4750.

[88] M. Döring, M. Ciesielski, O. Walter, H. Görls, Eur. J. Inorg. Chem. 2002, 16151621.

[89] F. H. Allen, J. E. Davis, J. J. Galloy, O. Johnson, O. Kennard, C. F. Macrae, E. M. Mitchell, G. F. Mitchell, J. M. Smith, D. G. Watson, J. Chem. Inf. Comput. Sci. 1991, 31, 187-204.

[90] N. V. Kaminskaia, B. Spiegler, S. J. Lippard, J. Am. Chem. Soc. 2000, 122, 6411-6422.

[91] C. Vichard, T. A. Kaden, Inorg. Chim. Acta 2002, 337, 173-180.

[92] L. H. Gade, Koordinationschemie, Wiley-VCH, Weinheim, 1998, S. 436.

[93] M. M. Ibrahim, N. Shimomura, K. Ichikawa, M. Shiro, Inorg. Chim. Acta 2001, 313, 125-136.

[94] J. Xia, Y. Xu, S. Li, W. Sun, K. Yu, W. Tang, Inorg. Chem. 2001, 40, 23942401.

[95] J. W. Canary, J. Xu, J. M. Castagnetto, D. Rentzeperis, L. A. Marky, 1995, 117, 11545-11547.

[96] F. M. Menger, M. Ladika, J. Am. Chem. Soc. 1987, 109, 3145-3146.

[97] B. N. Trawick, A. T. Daniher, J. K. Bashkin, Chem. Rev. 1998, 98, 939-960.

[98] P. Molenveld, S. Kapsabelis, J. F. J. Engbersen, D. N. Reinhoudt, J. Am. Chem. Soc. 1997, 120, 6726-6737. 
[99] J. K. Bashkin, Curr. Opin. Chem. Biol. 1999, 3, 752-758.

[100] O. Iranzo, A. Y. Kovalevsky, J. R. Morrow, J. P. Richard, J. Am. Chem. Soc. 2003, 125, 1988-1993.

[101] D. Kong, A. E. Martell, J. Reibenspies, Inorg. Chim. Acta 2002, 333, 7-14.

[102] S. Albedyhl, D. Schnieders, A. Jancsó, T. Gajda, B. Krebs, Eur. J. Inorg. Chem. 2002, 1400-1409.

[103] P. Molenveld, J. F. J. Engbersen, D. N. Reinhoudt, Eur. J. Org. Chem. 1999, 3269-3275.

[104] P. Molenveld, J. F. J. Engbersen, D. N. Reinhoudt, J. Org. Chem. 1999, 64, 6337-6341.

[105] P. Molenveld, W. M. G. Stikvoort, H. Kooijman, A. L. Spek, J. F. J. Engbersen, D. N. Reinhoudt, J. Org. Chem. 1999, 64, 3896-3906.

[106] C. Sissi, P. Rossi, F. Felluga, F. Formaggio, M. Palumbo, P. Tecilla, C. Toniolo, P. Scrimin, J. Am. Chem. Soc. 2001, 123, 3169-3170.

[107] P. Rossi, F. Felluga, P. Tecilla, F. Formaggio, M. Crisma, C. Toniolo, P. Scrimin, J. Am. Chem. Soc. 1999, 121, 6948-6949.

[108] S. Matsuda, A. Ishikubo, A. Kuzuya, M. Yashiro, M. Komiyama, Angew. Chem. 1998, 110, 3477-3479.

[109] H. Bisswanger, in Enzymkinetik: Theorie und Methoden, VCH, Weinheim; New York; Basel, 1994.

[110] N. V. Kaminskaia, C. He, S. J. Lippard, Inorg. Chem. 2000, 39, 3365-3373.

[111] C. He, S. J. Lippard, J. Am. Chem. Soc. 2000, 122, 184-185.

[112] F. Meyer, H. Pritzkow, Chem. Commun. 1998, 1555-1556.

[113] S. V. Kryatov, E. V. Rybak-Akimova, F. Meyer, H. Pritzkow, Eur. J. Inorg. Chem. 2003, 1581-1590.

[114] W. Henderson, M. Sabat, Polyhedron 1997, 16, 1663-1677.

[115] S. Masao, Y. Ichihara, T. Kobayashi, Japanese Patent 1991, JP 03, 271, 297.

[116] P. Arndt, C. Lefeber, R. Kempe, A. Tillack, U. Rosenthal, Chem. Ber. 1996, $129,1281-1285$.

[117] D. M. L. Goodgame, S. P. W. Hill, R. Lincoln, M. Quiros, D. J. Williams, Polyhedron 1993, 12, 2753-2762.

[118] D. M. L. Goodgame, S. P. W. Hill, D. J. Williams, Polyhedron 1992, 11, 1841 1847.

[119] T. Kupka, Spectochim. Acta Part A 1997, 53, 2649-2658.

[120] J. R. Anacona, J. Serrano, J. Coord. Chem. 2003, 56, 313-320.

[121] M. Asso, R. Panossian, M. Guiliano, Spectrosc. Lett. 1984, 17, 271-278.

[122] P. J. Montoya-Pelaez, R. S. Brown, Inorg. Chem. 2002, 41, 309-316.

[123] T. Grochowski, K. Samochocka, Polyhedron 1991, 10, 1471-1477.

[124] N. P. Gensmantel, P. Proctor, M. I. Page, J. Chem. Soc., Perkin Trans. 2 1980, 1725-1732.

[125] P. Proctor, N. P. Gensmantel, M. I. Page, J. Chem. Soc., Perkin Trans. 2 1982, 1185-1192.

[126] M. I. Page, Acc. Chem. Res. 1984, 17, 144-151.

[127] R. W. Hay, A. K. Basak, M. P. Pujari, A. Perotti, J. Chem. Soc., Dalton Trans. 1989, 197-201.

[128] T. J. Przystas, T. H. Fife, J. Chem. Soc., Perkin Trans. 2 1990, 393-399.

[129] K. Ogawa, K. Nakata, K. Ichikawa, Chem. Lett. 1998, 797-798.

[130] T. Koike, M. Takamura, E. Kimura, J. Am. Chem. Soc. 1994, 116, 8443-8449.

[131] N. V. Kaminskaia, B. Spingler, S. J. Lippard, J. Am. Chem. Soc. 2001, 123, 6555-6563.

[132] C. D. Schaeffer, Jr., L. K. Myers, J. Chem. Educ. 1990, 6, 347-349. 
[133] W. Wang, E. J. Roskamp, J. Am. Chem. Soc. 1993, 115, 9417-9420.

[134] K. H. Baggaley, S. W. Elson, N. H. Nicholson, J. T. Sime, J. Chem. Soc., Perkin Trans. 1 1990, 1513-1520.

[135] J. P. Degelaen, S. L. Loukas, J. Feeney, G. C. K. Roberts, A. S. V. Burgen, J. Chem. Soc., Perkin Trans. 2 1978, 86-90.

[136] A. Llinás, B. Vilanova, F. Muñoz, J. Donoso, J. Molec. Cat. A 2001, 175, 3-16.

[137] I. Ghebre-Sellassie, S. I. Hem, A. M. Knevel, J. Pharm. Sc. 1984, 73, 125-128.

[138] E. R. Lewis, K. M. Winterberg, A. L. Fink, Biochem. 1997, 94, 443-447.

[139] D. M. Brown, D. A. Usher, J. Chem. Soc. 1965, 6558-6564.

[140] F. Meyer, S. Beyreuther, K. Heinze, L. Zsolnai, Chem. Ber./Recueil 1997, 130, 605-613.

[141] F. Meyer, U. Ruschewitz, P. Schober, B. Antelmann, L. Zsolnai, J. Chem. Soc., Dalton Trans. 1998, 1181-1186.

[142] K. Marat, SpinWorks V. 1.3,

[143] A. R. Quirt, J. S. Martin, J. Magn. Reson. 1971, 5, 318-327.

[144] G. Gran, Analyst 1952, 77, 661-671.

[145] I. Puigdomenech, MEDUSA and Hydra software for chemical equilibrium calculations, Royal Institute of Technology (KTH), Stockholm, Sweden,

[146] G. M. Sheldrick, SHELXS-97, "Program for Crystal Structure Solution", Universität Göttingen, 1997.

[147] G. M. Sheldrick, SHELXL-97, "Program for Crystal Structure Refinement", Universität Göttingen, 1997. 


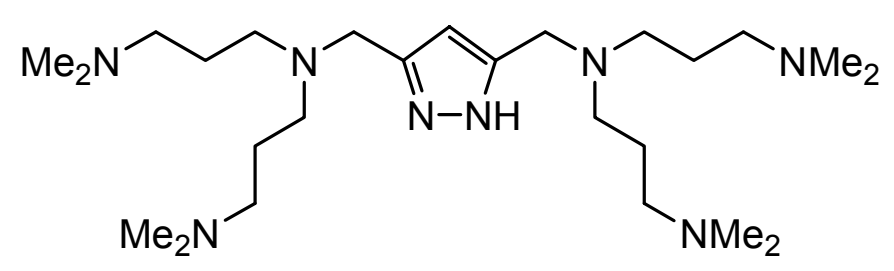

$L^{1}$<smiles>CCNCCN(CCNCC)Cc1cc(CN(CCNCC)CCNCC)[nH]n1</smiles>

$L^{3}$

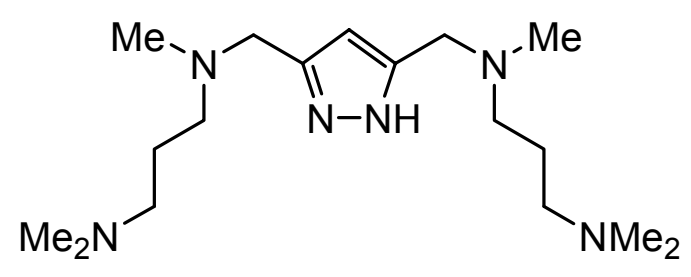

$\mathrm{L}^{2}$<smiles>CN(C)CCN(C)Cc1cc(CN(C)CCNC(C)(C)C)[nH]n1</smiles>

$L^{4}$<smiles>CCCN1CCN(Cc2cc(CN3CCN(CCC)CCN(CCC)N(C(C)C)CC3)n[nH]2)CCN(C(C)C)CC1</smiles>

$\mathrm{L}^{5}$<smiles>c1ccc(CCN(CCc2ccccn2)Cc2cc(CN(CCc3ccccn3)CCc3ccccn3)[nH]n2)nc1</smiles>

$L^{6}$<smiles>c1ccc(CN(Cc2ccccn2)Cc2cc(CN(Cc3ccccn3)Cc3ccccn3)[nH]n2)nc1</smiles>

$L^{8}$<smiles>CN(C)Cc1cc(CN(C)CCc2ccccn2)n[nH]1</smiles>

$\mathrm{L}^{7}$<smiles>CN(Cc1ccccn1)Cc1cc(CN(C)Cc2ccccn2)[nH]n1</smiles>

$L^{9}$<smiles>CCNCCN(CCN(CC)CC)Cc1cc(C)[nH]n1</smiles> 

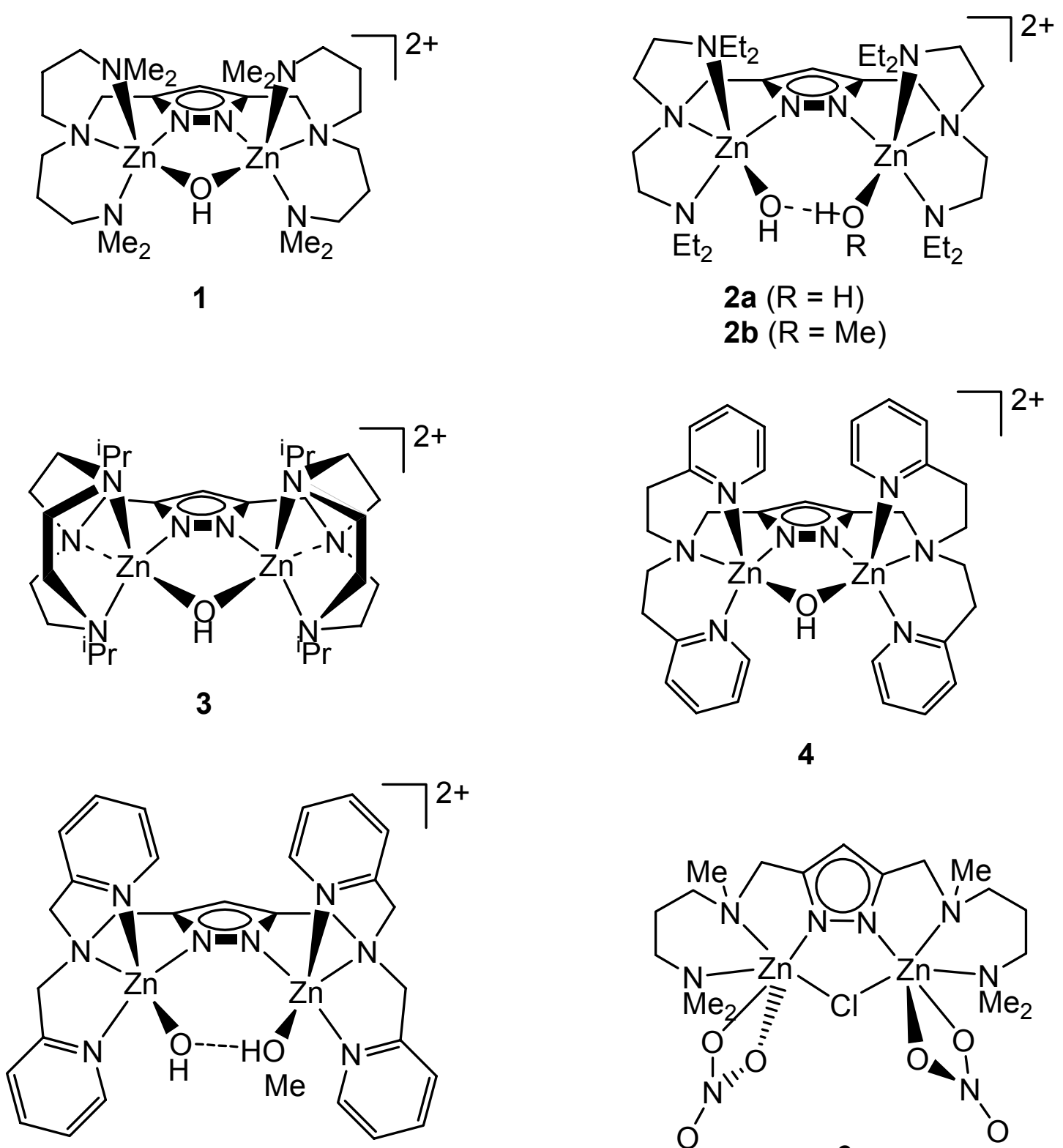

5

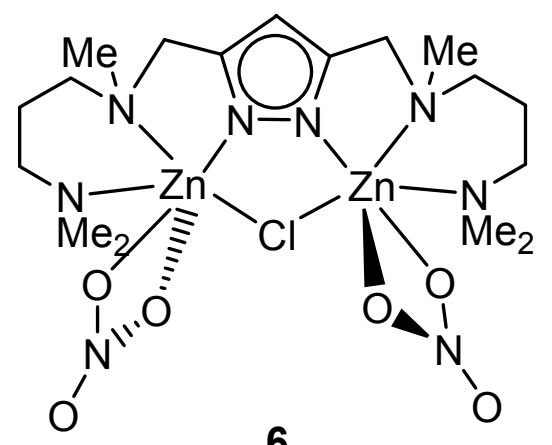

6

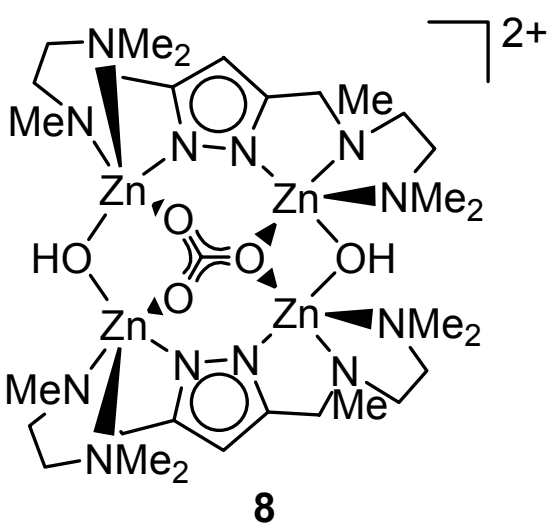




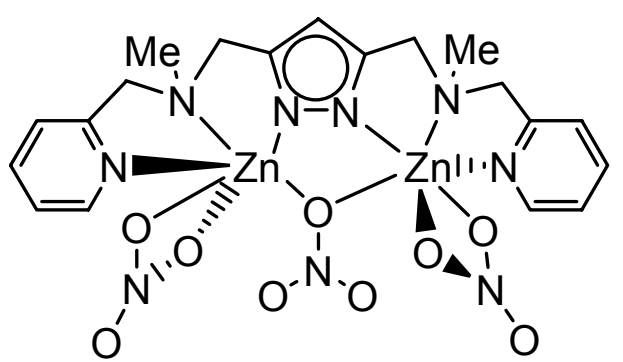

9

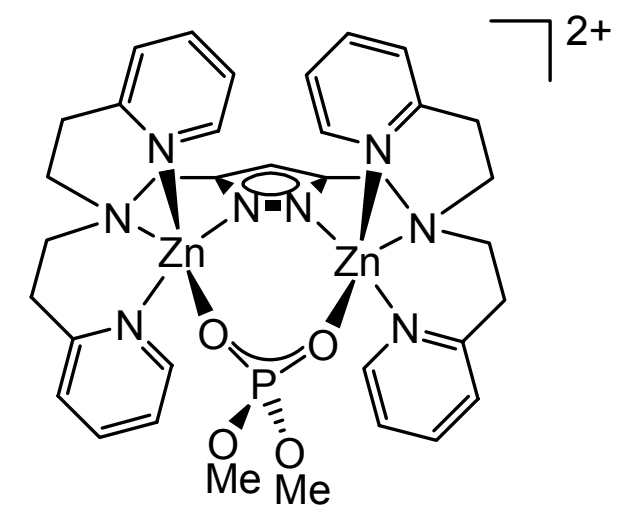

11a

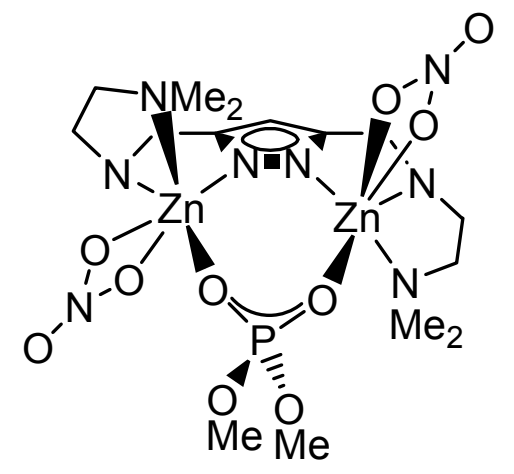

$13 a$

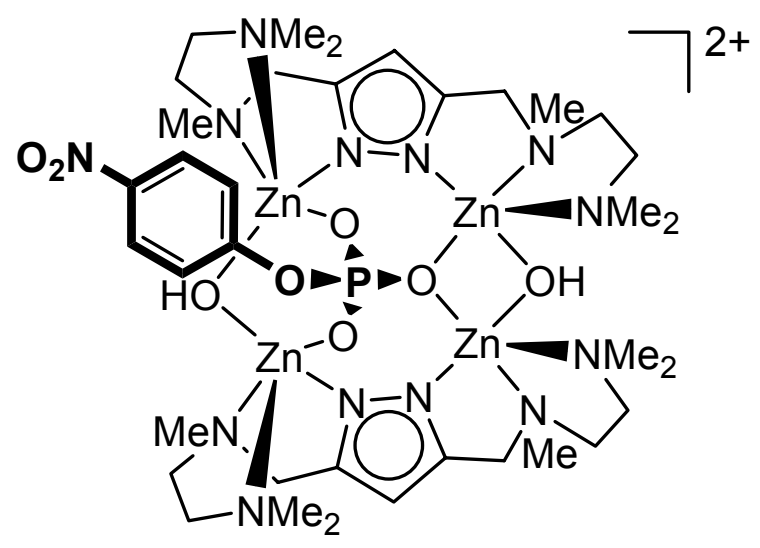

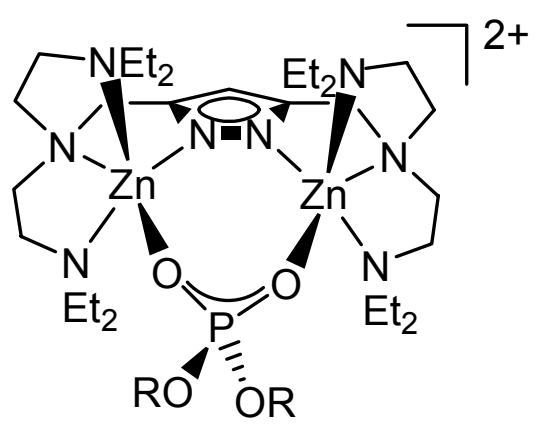

$10 \mathbf{a}(\mathrm{R}=\mathrm{Me})$
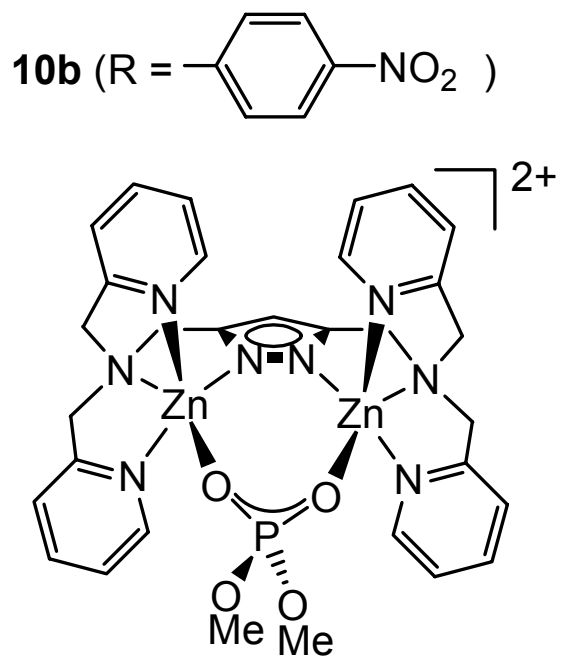

$12 a$

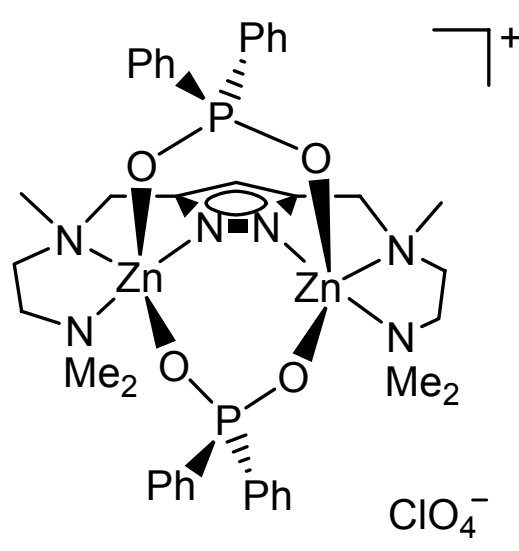

14 


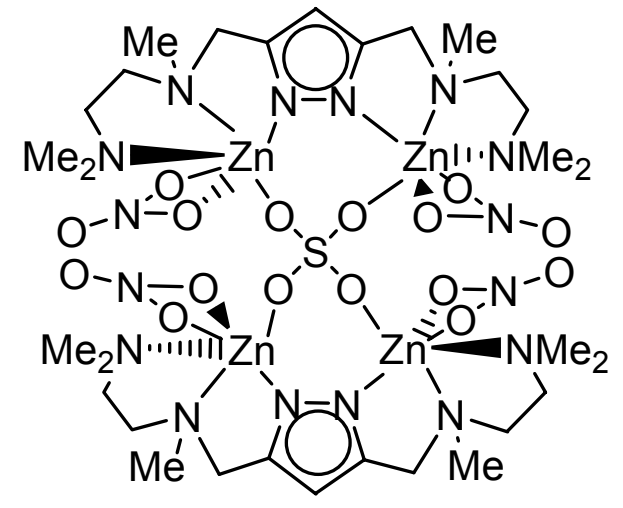

16

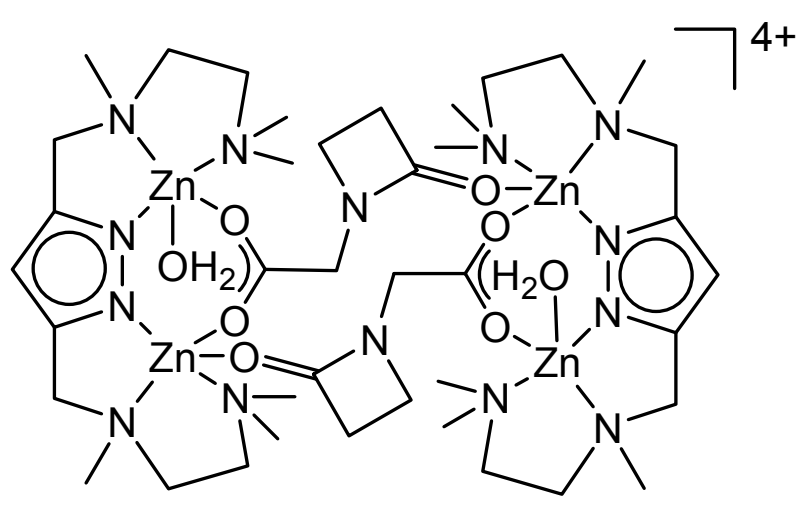

17 


\section{Publikationen}

Teile dieser Arbeit wurden bereits veröffentlicht:

B. Bauer-Siebenlist, F. Meyer, D. Vidovic, H. Pritzkow, Z. Allg. Anorg. Chem. 2003, $629,2152-2156$.

"A new tetranuclear zinc complex with a $\mu_{4}$-bridging carbonate cap"

B. Bauer-Siebenlist, F. Meyer, E. Farkas, D. Vidovic, J. A. Cuesta Seijo, R. HerbstIrmer, H. Pritzkow, Inorg. Chem. 2004, 43, 4189-4202.

"Correlation of Structure and Function in Oligonuclear Zinc(II) Model Phosphatases"

B. Bauer-Siebenlist, F. Meyer, E. Farkas, D. Vidovic, S. Dechert, Chem. Eur. J., eingereicht.

"Enhanced activity of a pseudo-terminal over a bridging hydroxide in model dizinc phosphodiesterases"

B. Bauer-Siebenlist, F. Meyer, S. Dechert, in Vorbereitung. "Biomimetic hydrolysis of Penicillin G catalyzed by dinuclear zinc(II) complexes: structure-activity correlations in $\beta$-lactamase model systems"

Andere Publikationen:

N. W. Mitzel, U. Losehand, B. Bauer, Inorg. Chem. 2000, 39, 1998-2000.

"SiONB Unit as Reference for Blocked Si $\cdots N$ Interactions in SiON Compounds"

G. Leibeling, S. Demeshko, B. Bauer-Siebenlist, F. Meyer, H. Pritzkow, Eur. J. Inorg. Chem. 2004, 2413-2420.

„Polynuclear and extended coordination compounds from preorganized bimetallic components: tuning the magnetic properties of dinickel(II) building blocks"

\section{Poster}

International Conference on Coordination Chemistry ICCC 35, Heidelberg 2002 „Dizinc complexes mimicking natural hydrolases - Reactivity of dizinc centers in model studies".

GDCh-Tagung München 2003

"Dinuclear zinc complexes mimicking natural metallo- $\beta$-lactamases".

\section{Vorträge}

Weihnachtskolloquium der Fakultät für Chemie, Göttingen 2002 „Präorganisierte, zweikernige Zinkkomplexe als Enzymmodelle“. 


\section{Dank}

Mein großer Dank gilt zuallererst Herrn Professor Dr. Franc Meyer für die freundliche Aufnahme in den Arbeitskreis, die persönliche, anregende Betreuung meiner Arbeit durch sein stetes Interesse und die Gewährung der wissenschaftlichen Freiheit mit allen zur Forschung erforderlichen Möglichkeiten.

Danken möchte ich auch allen, die zum Gelingen der Arbeit beigetragen haben:

Den Kristallographen Denis Vidovic, Dr. Sebastian Dechert, Dr. Hans Pritzkow, José Antonio Cuesta Seijo und Dr. Regine Herbst-Irmer für die Mühe mit den Kristallen; der NMR-, der MS-Abteilung und dem Mikroanalytischen Labor des Instituts für Anorganische Chemie, speziell Wolfgang Zoltke und Anke Rehbein für die Erfüllung meiner zahlreichen Sonderwünsche;

Frau Professor Dr. Etelka Farkas für die hervorragende Betreuung in Debrecen/Ungarn und ihrer Arbeitsgruppe, besonders Dr. Éva Ana Enyedy, Dr. Katalin Ösz und Dr. Péter Buglyó, für die Hilfe vor, während und nach den Messungen;

Dr. Walter Maringgele und Jörg Teichgräber, sowie der Werkstatt und den Glasbläsern für die Hilfe beim Aufbau der neuen Labore in Göttingen;

Dr. Jens Ackermann und Dr. Sebastian Dechert für die Hilfe und Unterstützung an den Rechnern;

Ilka Varnay, Olaf Jansen, Stamatia Katsiaouni und Michael Hilbert für die engagierte Arbeit während ihrer Forschungspraktika;

Dem DAAD und dem COST-Programm für die finanzielle Förderung der Aufenthalte in Ungarn, sowie der Roche AG und der Fa. Pfizer Inc. für die freundliche Bereitstellung einiger Antibiotika;

meinen Laborkollegen Dr. Jens Ackermann, Dr. Rosana Ferrari und Stamatia Katsiaouni für das heitere Arbeitsklima im Labor;

Dr. Andreas Stasch, Gilles Noël und Dr. Dante Neculai für die kurzweilige Zeit während der Betreuung der AC-F-Praktika;

und Maya Siebenlist für das Korrekturlesen.

Ganz besonders bedanke ich mich bei meiner Frau Julia für die Geduld und das Verständnis während und vor allem in der Endphase dieser Arbeit. 


\section{LEBENSLAUF}

NAME

Geboren

STAATSANGEHÖRIGKEIT

FAMILIENSTAND

KINDER

SCHULAUSBILDUNG

September 1981 -

Juli 1985

September 1985 -

Juni 1994

GRUNDWEHRDIENST

Juli 1994 - Juni 1995

HOCHSCHULSTUDIUM

Oktober 1995 -

September 1997

Oktober 1997 -

Mai 2000

Juni 2000 -

Dezember 2000

PROMOTION

März 2001 -

Oktober 2001

November 2001 -

März 2004

AUSLANDSAUfENTHALTE

April 2002 und

Oktober/November

2003

STIPENDIEN

Oktober/November 2003
Bernhard Bauer-Siebenlist (geb. Bauer)

am 3. Dezember 1974 in München

deutsch

verheiratet, mit Julia Siebenlist

Helena Siebenlist, geb. 22. Januar 1999

Anabel Siebenlist, geb. 13. August 2003

Grundschule Erdweg

Josef-Effner-Gymnasium Dachau
Grundstudium Chemie, Diplom an der TU München

Hauptstudium Chemie, Diplom an der TU München

Diplomarbeit bei Prof. W. A. Herrmann an der TU München „Beiträge zur Immobilisierung von Metallocenen“

Beginn der Doktorarbeit bei Prof. F. Meyer an der Universität Heidelberg

Doktorarbeit an der Universität Göttingen
Forschungsaufenthalte bei Prof. E. Farkas am Institut für Anorganische und Analytische Chemie an der Universität Debrecen/Ungarn

Doktorandenstipendium des DAAD 